\title{
Terminal Trifluoromethylation of Ketones via Selective C-C Cleavage of Cycloalkanols Enabled by Hypervalent Iodine Reagents
}

\author{
Shuang Wu, ${ }^{\dagger,+}$ Junzhao Li $,{ }^{\dagger} \mathrm{Ru} \mathrm{He},{ }^{\dagger *}$ Kunfang Jia, ${ }^{\dagger}$ and Yiyun Chen $* \dagger,+$, \\ ${ }^{\dagger}$ State Key Laboratory of Bioorganic and Natural Products Chemistry, Centre of Excellence in Molecular \\ Synthesis, Shanghai Institute of Organic Chemistry, University of Chinese Academy of Sciences, Chinese \\ Academy of Sciences, Shanghai 200032, China; ${ }^{\$}$ School of Physical Science and Technology, ShanghaiTech \\ University, Shanghai 201210, China; ${ }^{\S}$ School of Chemistry and Material Sciences, Hangzhou Institute for \\ Advanced Study, University of Chinese Academy of Sciences, Hangzhou 310024, China \\ *E-mail: yiyunchen@sioc.ac.cn
}

\section{Supplementary Information}

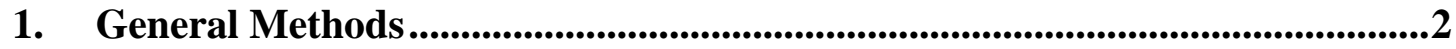

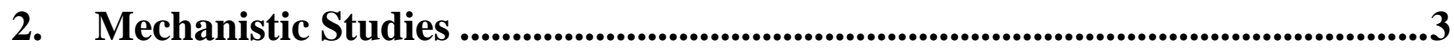

3. Detailed Reaction Optimizations ....................................................................22

4. Substrates Preparation and Characterization ..............................................29

5. Characterization of the Products ...........................................................................44

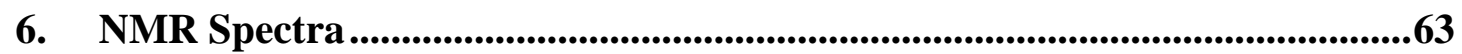

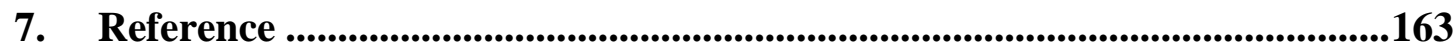




\section{General Methods}

Unless otherwise noted, all reactions of substrates preparation were conducted in flamedried glassware under a nitrogen atmosphere using anhydrous solvent. Commercially available reagents were used without further purification. Thin layer chromatography (TLC) was performed using Jiangyou TLC silica gel plates HSG $F_{254}$ and visualized using UV light, anisaldehyde or potassium permanganate. Flash chromatography was performed on Lisure science EWZ purification system using the Santai technologies silica gel cartridge. Photochemical reactions were carried with 4 W blue LED (468 nm peak wavelength, $25 \mathrm{~nm}$ spectral half-wave width, composed of 55-65 LED units each with $60 \mathrm{~mW}, 3 \mathrm{~V}, 20 \mathrm{~mA}$ ) obtained from Qiding Photo Electric (analyzed by Everfine PMS-50), or blue LED (450 nm peak wavelength, 15 20 nm spectral half-wave width, composed of 1 LED unit with 100W, 220V, 3.5 A) obtained from PerfectLight. ${ }^{1} \mathrm{H}$ and ${ }^{13} \mathrm{C}$ NMR spectra were recorded in $\mathrm{CDCl}_{3}$ unless otherwise noted, on an Agilent 600 $\mathrm{MHz}$ or an Agilent $500 \mathrm{MHz} .{ }^{19} \mathrm{~F}$ NMR spectra were recorded a Bruker $400 \mathrm{MHz}$ spectrometer. Chemical shifts in ${ }^{1} \mathrm{H}$ NMR spectra were reported in parts per million (ppm) on the $\delta$ scale from an internal standard of residual chloroform (7.26 ppm). Data for ${ }^{1} \mathrm{H}$ NMR were reported as follows: chemical shift, multiplicity ( $\mathrm{s}=$ singlet, $\mathrm{d}=$ doublet, $\mathrm{t}=$ triplet, $\mathrm{q}=$ quartet, $\mathrm{m}=$ multiplet, $\mathrm{br}=$ broad), coupling constant in Herts $(\mathrm{Hz})$ and integration. Data for ${ }^{13} \mathrm{C}$ NMR spectra were reported in terms of chemical shift in ppm from the central peak of chloroform (77.16 ppm). IR (neat) spectra were recorded on a Thermo Scientific Nicolet 380 FT-IR (neat) spectrometer. MS experiments were performed on a Bruker maXis $4 \mathrm{G}$ instrument for HRMS-ESI, an Agilent 5973N instrument for EI-MS, and a Waters Micromass GCT Premier instrument for HRMS-EI. Cyclic voltammetry experiments were performed on a $\mathrm{CH}$ Instruments Electrochemical Analyzer. Emission intensities were recorded on the HITACHI F-2700 spectrometer. 


\section{Mechanistic Studies}

\subsection{Radical quenching experiments}

a)

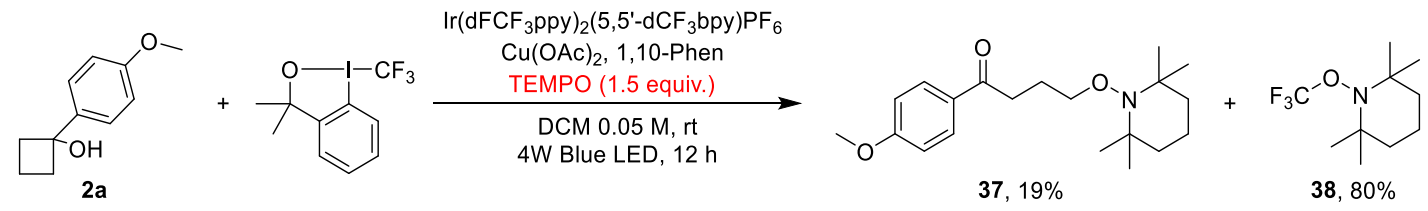

b)

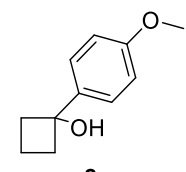

2a

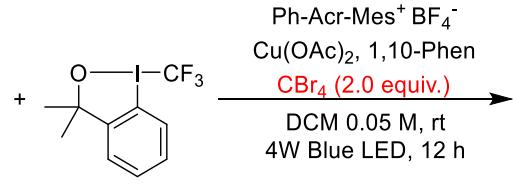

W Blue LED, $12 \mathrm{~h}$

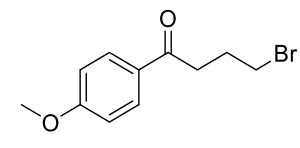

$39,58 \%$

Cyclobutanol 2a (0.15 mmol, 1.5 equiv.), Togni I reagent ( $0.1 \mathrm{mmol}, 1.0$ equiv.), photocatalysts ( $0.002 \mathrm{mmol}, 0.02$ equiv.), $\mathrm{Cu}(\mathrm{OAc})_{2}$ (3.6 mg, $0.02 \mathrm{mmol}, 0.2$ equiv.), 1,10-Phen (5.4 mg, 0.03 mmol, 0.3 equiv.) and the radical quencher (TEMPO, or $\mathrm{CBr}_{4}$ ) were placed in a $4 \mathrm{~mL}$ clear-colored glass vial equipped with a magnetic stir bar. After $2.0 \mathrm{~mL}$ degassed DCM were added, the vial was sealed and exposed to blue LEDs (4W LED light bulb $5 \mathrm{~cm}$ away from the vial) at room temperature for $12 \mathrm{~h}$ with stirring. Conversions and yields were calculated on ${ }^{1} \mathrm{H}$ NMR analysis and ${ }^{19} \mathrm{~F}$ NMR analysis.

When (2,2,6,6-tetramethylpiperidin-1-yl)oxyl (TEMPO) (23.4 mg, $0.15 \mathrm{mmol}, 1.5$ equiv.) was used, the crude ${ }^{19} \mathrm{~F}$ NMR yield of 2,2,6,6-tetramethyl-1(trifluoromethoxy)piperidine $\mathbf{3 8}$ was determined using (trifluoromethyl)benzene as an internal standard ${ }^{1}{ }^{19} \mathrm{~F}$ NMR $\left(376 \mathrm{MHz}, \mathrm{CDCl}_{3}\right) \delta-55.29$; HRMS-EI $(\mathrm{m} / \mathrm{z})[\mathrm{M}]^{+}$cal'd for $\mathrm{C}_{10} \mathrm{H}_{18} \mathrm{~F}_{3} \mathrm{NO}, 225.1335$, found 225.1340. After evaporation of the solvents in vacuo, the crude product was purified by column chromatography (PE:EA = 10:1) to afford 1(4-methoxyphenyl)-4-((2,2,6,6-tetramethylpiperidin-1-yl)oxy)butan-1-one $\mathbf{3 7}$ as a colorless oil (9.4 mg, 19\% yield): IR (neat) 2973, 2932, 1680, 1601, 1510, 1359, 1270, 1170, 1032, $837 \mathrm{~cm}^{-1} ;{ }^{1} \mathrm{H}$ NMR (500 MHz, $\left.\mathrm{CDCl}_{3}\right) \delta 7.96(\mathrm{~d}, J=8.8 \mathrm{~Hz}, 2 \mathrm{H}), 6.93(\mathrm{~d}$, $J=8.9 \mathrm{~Hz}, 2 \mathrm{H}), 3.87(\mathrm{~s}, 3 \mathrm{H}), 3.82(\mathrm{t}, J=6.2 \mathrm{~Hz}, 2 \mathrm{H}), 3.07-3.00(\mathrm{~m}, 2 \mathrm{H}), 1.96(\mathrm{p}, J=$ $6.5 \mathrm{~Hz}, 2 \mathrm{H}), 1.60-1.49(\mathrm{~m}, 1 \mathrm{H}), 1.49-1.39(\mathrm{~m}, 4 \mathrm{H}), 1.31(\mathrm{dt}, J=9.7,5.9 \mathrm{~Hz}, 1 \mathrm{H})$, 1.14 (s, 6H), 1.09 (s, 6H); ${ }^{13} \mathrm{C}$ NMR (126 MHz, $\left.\mathrm{CDCl}_{3}\right) \delta 198.8,163.3,130.3,130.1$, 
113.7, 77.3, 77.0, 76.8, 75.6, 59.7, 55.4, 39.6, 35.1, 33.1, 23.8, 20.1, 17.1; HRMS-ESI $(\mathrm{m} / \mathrm{z})[\mathrm{M}+\mathrm{H}]^{+}$cal'd for $\mathrm{C}_{20} \mathrm{H}_{32} \mathrm{NO}_{3}, 334.2377$, found 334.2379.

When $\mathrm{CBr}_{4}$ (49.7 mg, $0.20 \mathrm{mmol}, 2.0$ equiv.) was used, ${ }^{2}$ the reaction mixture was concentrated and purified by column chromatography (PE:EA = 10:1) to afford 4bromo-1-(4-methoxyphenyl)butan-1-one 39 as a pale yellow oil (14.8 mg, 58\% yield): ${ }^{1} \mathrm{H}$ NMR $\left(500 \mathrm{MHz}, \mathrm{CDCl}_{3}\right) \delta 7.97(\mathrm{~d}, J=8.9 \mathrm{~Hz}, 2 \mathrm{H}), 6.94(\mathrm{~d}, J=8.8 \mathrm{~Hz}, 2 \mathrm{H}), 3.88$ $(\mathrm{s}, 3 \mathrm{H}), 3.55(\mathrm{t}, J=6.3 \mathrm{~Hz}, 2 \mathrm{H}), 3.14(\mathrm{t}, J=7.0 \mathrm{~Hz}, 2 \mathrm{H}), 2.33-2.26(\mathrm{~m}, 2 \mathrm{H}) ;{ }^{13} \mathrm{C} \mathrm{NMR}$ $\left(126 \mathrm{MHz} \mathrm{CDCl}_{3}\right) \delta 197.4,163.5,130.3,129.8,113.8,55.5,36.2,34.0,27.0$. The data is consistent with the literature. ${ }^{2}$

\subsection{Radical clock experiments}

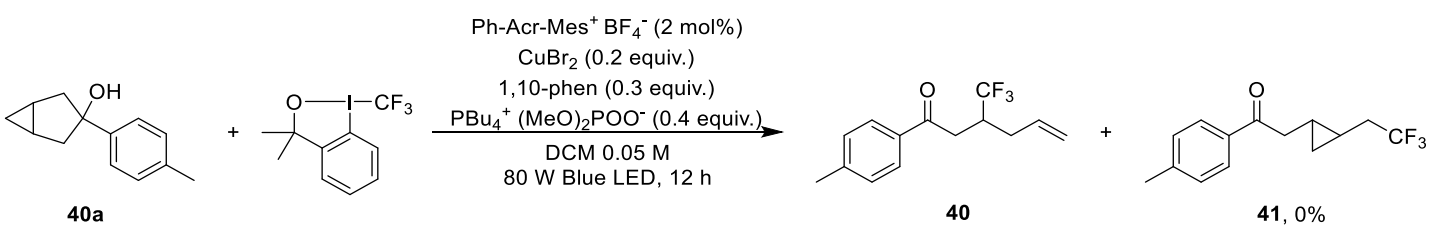

The fused cyclopropyl alkanol 40a $(0.15 \mathrm{mmol}, 1.5$ equiv.), Togni I reagent $(0.1 \mathrm{mmol}$, 1.0 equiv.), $\mathrm{Ph}$-Acr-Mes ${ }^{+} \mathrm{BF}_{4}{ }^{-}$(1.1 mg, $0.002 \mathrm{mmol}, 0.02$ equiv.), $\mathrm{CuBr}_{2}$ (4.5 mg, 0.02 mmol, 0.2 equiv.), 1,10-Phen (5.4 mg, $0.03 \mathrm{mmol}, 0.3$ equiv.) and the ${ }^{\mathrm{n}} \mathrm{Bu}_{4} \mathrm{P}^{+}(\mathrm{OMe})_{2} \mathrm{POO}^{-}$(15.4 mg, $0.04 \mathrm{mmol}, 0.4$ equiv.) were placed in a $4 \mathrm{~mL}$ clearcolored glass vial equipped with a magnetic stir bar. After $2.0 \mathrm{~mL}$ degassed DCM were added, the vial was sealed and exposed to blue LED (80 W LED light bulb $5 \mathrm{~cm}$ away from the vial) at room temperature for $12 \mathrm{~h}$ with stirring. After evaporation of the solvents in vacuo, the crude product was purified by column chromatography (PE:EA $=10: 1)$ to afford 1-(p-tolyl)-3-(trifluoromethyl)hex-5-en-1-one $\mathbf{4 0}$ as a colorless oil $\left({ }^{1} \mathrm{H}\right.$ NMR analysis yield: $28 \%$, isolated $4.0 \mathrm{mg}, 16 \%$ yield): IR (neat) 2924, 2852, 1684, $1608,1380,1272,1254,1130,806,650 \mathrm{~cm}^{-1} ;{ }^{1} \mathrm{H} \mathrm{NMR}\left(600 \mathrm{MHz}, \mathrm{CDCl}_{3}\right) \delta 7.83(\mathrm{~d}, J$ $=8.1 \mathrm{~Hz}, 2 \mathrm{H}), 7.27(\mathrm{~d}, J=6.6 \mathrm{~Hz}, 2 \mathrm{H}), 5.83(\mathrm{dt}, J=17.7,9.3 \mathrm{~Hz}, 1 \mathrm{H}), 5.13-5.03(\mathrm{~m}$, $2 \mathrm{H}), 3.20-3.12(\mathrm{~m}, 1 \mathrm{H}), 3.10(\mathrm{~d}, J=6.8 \mathrm{~Hz}, 2 \mathrm{H}), 2.42(\mathrm{~s}, 3 \mathrm{H}), 2.40-2.21(\mathrm{~m}, 2 \mathrm{H})$; ${ }^{13} \mathrm{C} \mathrm{NMR}\left(151 \mathrm{MHz}, \mathrm{CDCl}_{3}\right) \delta 197.4,144.2,139.2,134.4,129.4,128.1,126.6$ (q, $J=$ $277.6 \mathrm{~Hz}), 115.9,42.6,38.0(\mathrm{q}, J=27.2 \mathrm{~Hz}), 33.7$ (q, $J=2.3 \mathrm{~Hz}), 21.7 ;{ }^{19} \mathrm{~F} \mathrm{NMR}(376$ 
$\left.\mathrm{MHz}, \mathrm{CDCl}_{3}\right) \delta-62.83\left(\mathrm{t}, J=10.5 \mathrm{~Hz}\right.$ ); HRMS-EI $(\mathrm{m} / \mathrm{z})[\mathrm{M}]^{+}$cal'd for $\mathrm{C}_{14} \mathrm{H}_{15} \mathrm{OF}_{3}$, 256.1070 , found 256.1072 .

\subsection{The reaction intermediates experiment}

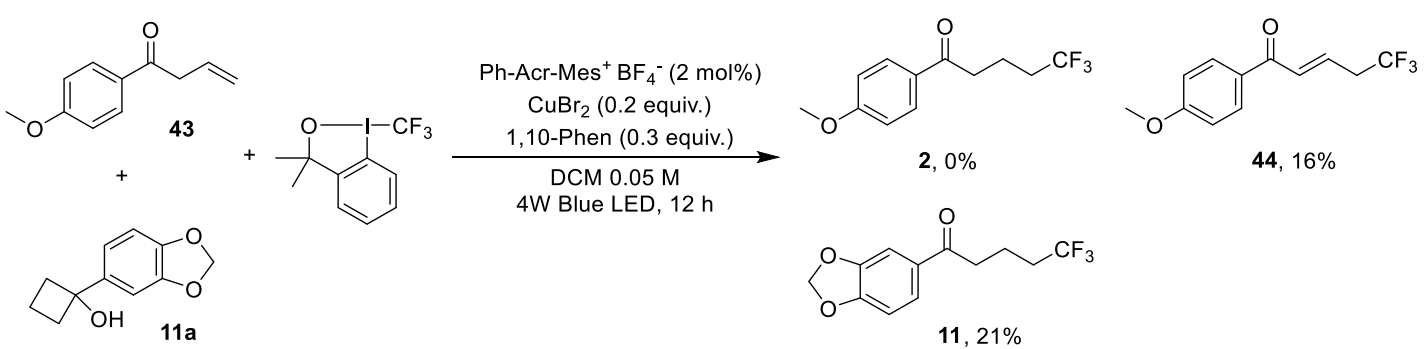

1-(4-methoxyphenyl)but-3-en-1-one 43 (0.15 mmol, 1.5 equiv.), cyclobutanol 11a ( 0.15 mmol, 1.5 equiv.), Togni I reagent ( $0.1 \mathrm{mmol}, 1.0$ equiv.), $\mathrm{Ph}-\mathrm{Acr}-\mathrm{Mes}^{+} \mathrm{BF}_{4}{ }^{-}$(1.1 mg, 0.002 mmol, 0.02 equiv.), $\mathrm{CuBr}_{2}$ (4.5 mg, $0.02 \mathrm{mmol}, 0.2$ equiv.) and 1,10-Phen (5.4 mg, $0.03 \mathrm{mmol}, 0.3$ equiv.) were placed in a $4 \mathrm{~mL}$ clear-colored glass vial equipped with a magnetic stir bar. After $2.0 \mathrm{~mL}$ degassed DCM were added, the vial was sealed and exposed to blue LEDs (4W LED light bulb $5 \mathrm{~cm}$ away from the vial) at room temperature for $12 \mathrm{~h}$ with stirring. After evaporation of the solvents in vacuo, the crude product was purified by column chromatography $(\mathrm{PE}: \mathrm{EA}=20: 1)$ to afford 5,5,5trifluoro-1-(4-methoxyphenyl)pent-2-en-1-one 44 as a pale yellow solid (3.9 mg, 16\% yield): IR (neat) 2939, 2844, 1674, 1628, 1600, 1302, 1261, 1173, 1142, 1017, 976, 812 $\mathrm{cm}^{-1} ;{ }^{1} \mathrm{H}$ NMR $\left(600 \mathrm{MHz}, \mathrm{CDCl}_{3}\right) \delta 7.97-7.93(\mathrm{~m}, 2 \mathrm{H}), 7.09(\mathrm{~d}, J=15.5 \mathrm{~Hz}, 1 \mathrm{H})$, $6.99-6.94(\mathrm{~m}, 2 \mathrm{H}), 6.86(\mathrm{dt}, J=15.4,7.2 \mathrm{~Hz}, 1 \mathrm{H}), 3.88(\mathrm{~s}, 3 \mathrm{H}), 3.14-3.05(\mathrm{~m}, 2 \mathrm{H})$; ${ }^{13} \mathrm{C} \mathrm{NMR}\left(151 \mathrm{MHz}, \mathrm{CDCl}_{3}\right) \delta 187.8,163.8,133.7$ (q, $\left.J=3.5 \mathrm{~Hz}\right), 131.2,131.0,130.0$, $125.3(\mathrm{q}, J=276.9 \mathrm{~Hz}), 114.0,55.5,37.2(\mathrm{q}, J=30.5 \mathrm{~Hz}) ;{ }^{19} \mathrm{~F} \mathrm{NMR}\left(376 \mathrm{MHz}, \mathrm{CDCl}_{3}\right)$ $\delta$-64.75 (t, $J=10.0 \mathrm{~Hz})$; HRMS-EI $(\mathrm{m} / \mathrm{z})[\mathrm{M}]^{+}$cal'd for $\mathrm{C}_{12} \mathrm{H}_{11} \mathrm{O}_{2} \mathrm{~F}_{3}, 244.0706$, found 244.0699 .

\subsection{The cyclic voltammetry experiments}

The MeCN solution of $1 \mathrm{mM}$ solute was prepared with $100 \mathrm{mM}$ of tetrabutylammonium hexafluorophosphate as the supporting electrolyte, using a glassy carbon working electrode, a Pt counter electrode, and a $\mathrm{Hg} / \mathrm{HgCl}_{2}$ reference electrode at room temperature under a nitrogen atmosphere. 


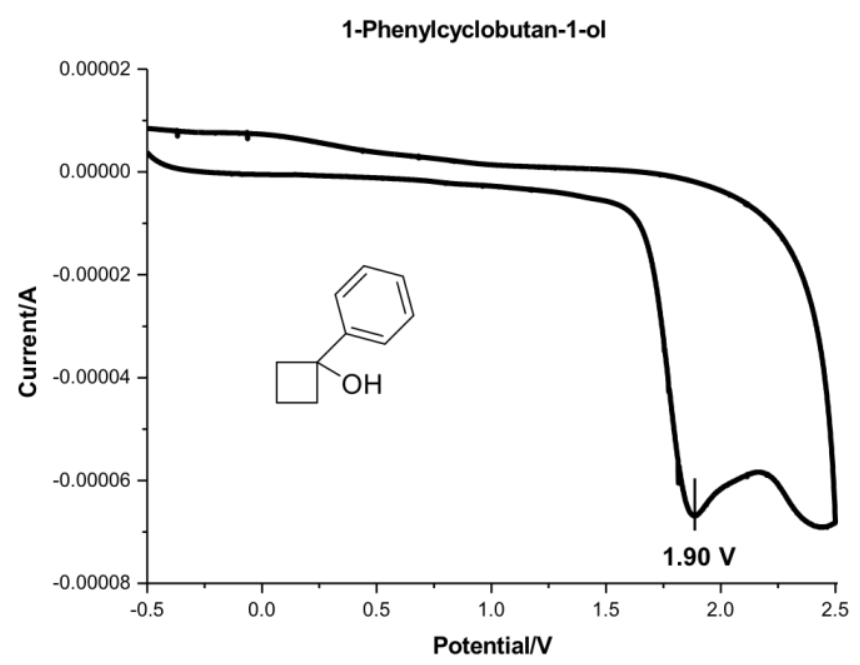

Scheme S1 The Cyclic Voltammetry Experiment of 1-phenylcyclobutan-1-ol 1a.

Scan Rate $(\mathrm{V} / \mathrm{s})=0.05$.

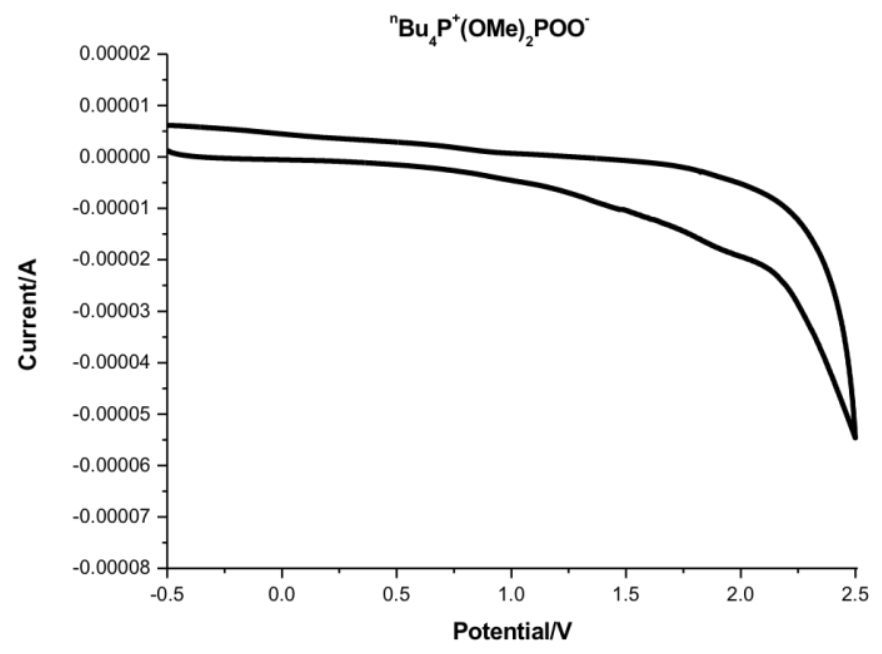

Scheme S2 The Cyclic Voltammetry Experiment of ${ }^{\mathrm{n}} \mathrm{Bu}_{4} \mathrm{P}^{+}(\mathrm{OMe})_{2} \mathrm{POO}^{-}$. Scan Rate $(\mathrm{V} / \mathrm{s})=0.05$. 


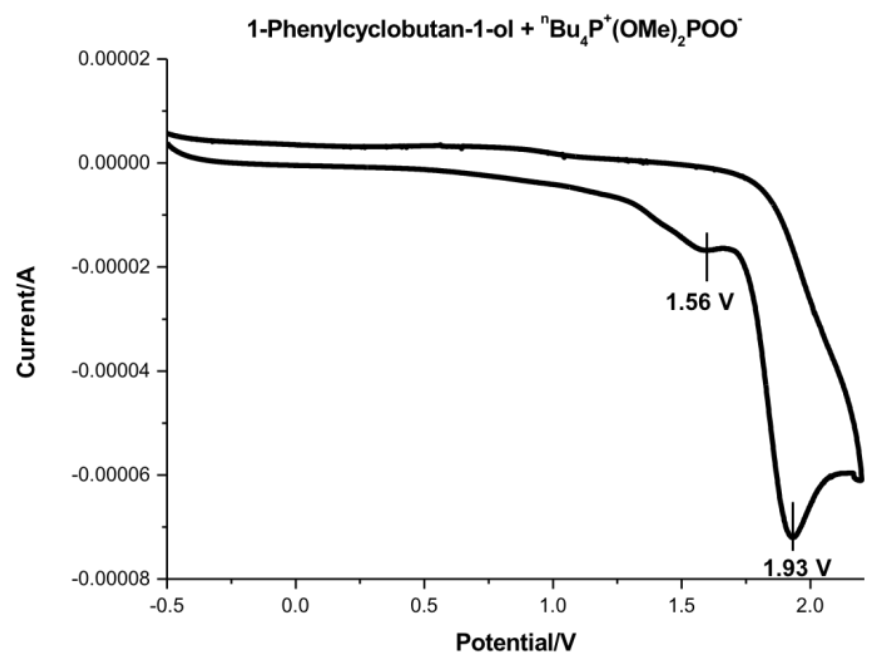

Scheme S3 The Cyclic Voltammetry Experiment of 1-phenylcyclobutan-1-ol 1a with ${ }^{\mathrm{n}} \mathrm{Bu}_{4} \mathrm{P}^{+}(\mathrm{OMe}){ }_{2} \mathrm{POO}^{-}$. Scan Rate $(\mathrm{V} / \mathrm{s})=0.05$.

The unreactive substrates such as decahydronaphthalene-2-ol and 1-(pyridine-3yl)cyclopentan-1-ol had the oxidation potentials up to $2.57 \mathrm{~V}$ vs. SCE in MeCN, or more than $3 \mathrm{~V}$ vs. SCE in $\mathrm{MeCN}$.

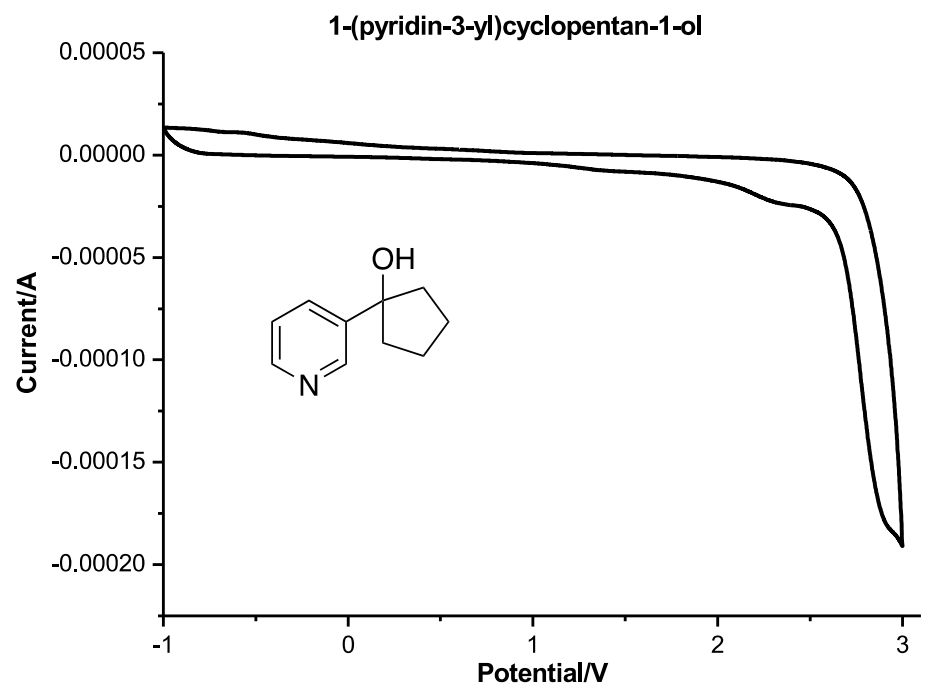

Scheme S4 The Cyclic Voltammetry Experiment of 1-(pyridine-3-yl)cyclopentan-1ol. Scan Rate $(\mathrm{V} / \mathrm{s})=0.05$. 


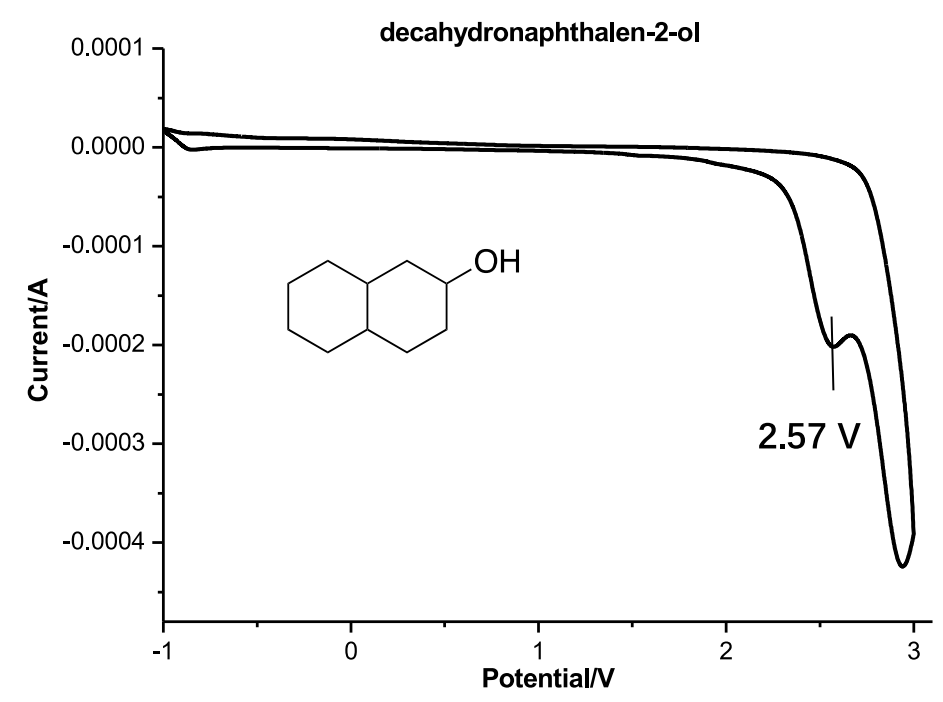

Scheme S5 The Cyclic Voltammetry Experiment of decahydronaphthalene-2-ol. Scan

$$
\text { Rate }(\mathrm{V} / \mathrm{s})=0.05 \text {. }
$$

\subsection{The Stern-Volmer quenching experiments}

As photocatalysts $\mathrm{Ph}-\mathrm{Acr}-\mathrm{Mes}^{+} \mathrm{BF}_{4}{ }^{-}$and $\operatorname{Ir}\left[\mathrm{dF}\left(\mathrm{CF}_{3}\right) \text { ppy }\right]_{2}\left(5,5^{\prime}-\mathrm{dCF}_{3}\right.$ bpy $)\left(\mathrm{PF}_{6}\right)$ afforded product 1 from 1 a in similar yields, $\operatorname{Ir}\left[\mathrm{dF}\left(\mathrm{CF}_{3}\right) \text { ppy }\right]_{2}\left(5,5^{\prime}-\mathrm{dCF}_{3}\right.$ bpy $)\left(\mathrm{PF}_{6}\right)$ was used for the Stern-Volmer quenching experiments in Schemes S6-12. The [Ir] solutions were excited at $400 \mathrm{~nm}$ and the emission intensity at $600 \mathrm{~nm}$ was recorded. In a typical experiment, the DCM solution of $[\mathrm{Ir}](40 \mu \mathrm{M})$ was added the appropriate amount of quencher in a screw-top $1.0 \mathrm{~cm}$ quartz cuvette. After degassing with nitrogen, the emission spectra of the samples were collected. 


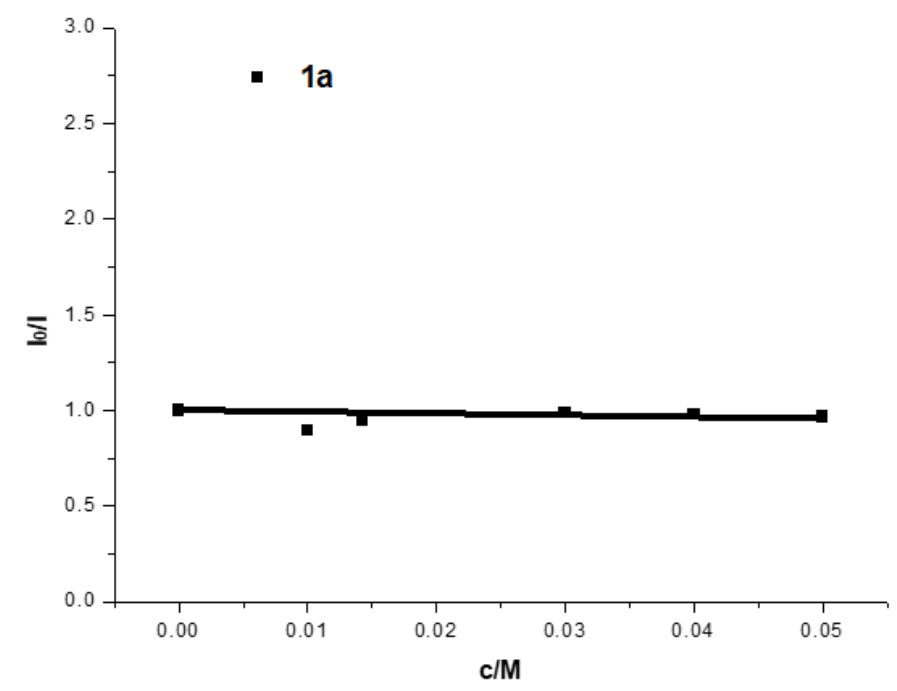

Scheme S6 The Stern-Volmer plot of $\operatorname{Ir}\left[\mathrm{dF}\left(\mathrm{CF}_{3}\right) \mathrm{ppy}\right]_{2}\left(5,5^{\prime}-\mathrm{dCF}_{3}\right.$ bpy $)\left(\mathrm{PF}_{6}\right)$ with $\mathbf{1 a}$.

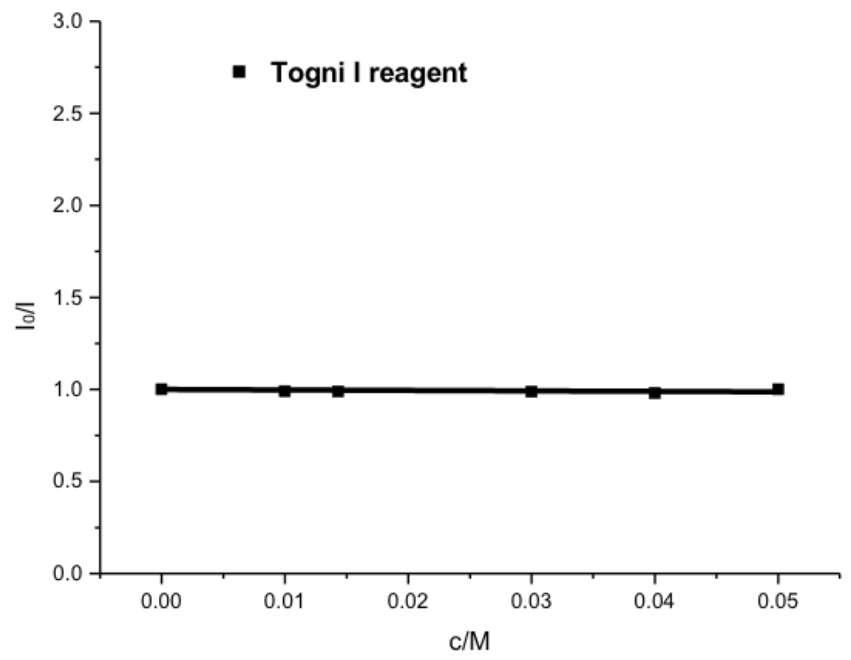

Scheme S7 The Stern-Volmer plot of $\operatorname{Ir}\left[\mathrm{dF}\left(\mathrm{CF}_{3}\right) \text { ppy }\right]_{2}\left(5,5^{\prime}-\mathrm{dCF}_{3}\right.$ bpy $)\left(\mathrm{PF}_{6}\right)$ with Togni I reagent.

The trendlines in Schemes S6-7 were parallel to X-axis, suggesting that the photocatalyst was quenched by neither 1a nor Togni I reagent. 


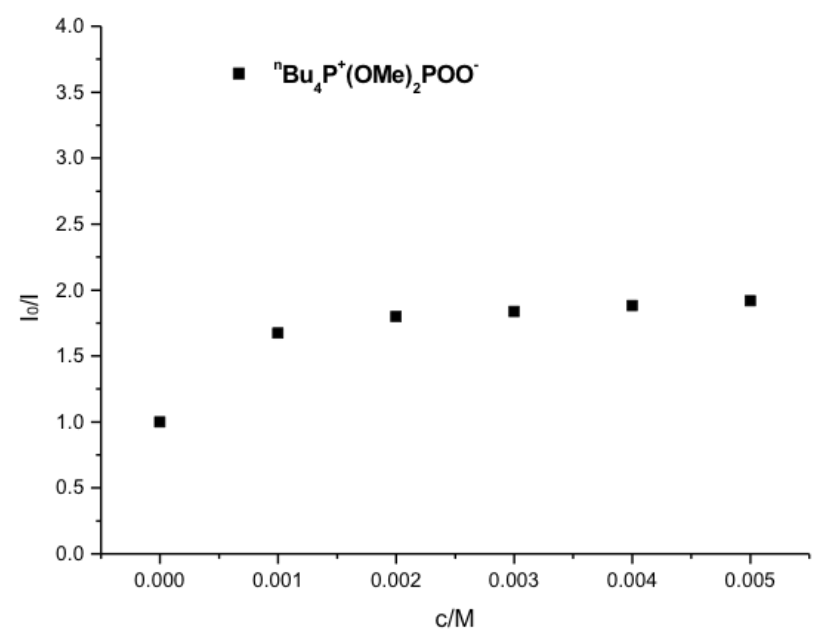

Scheme S8 The Stern-Volmer plot of $\operatorname{Ir}\left[\mathrm{dF}\left(\mathrm{CF}_{3}\right) \text { ppy }\right]_{2}\left(5,5^{\prime}-\mathrm{dCF}_{3}\right.$ bpy $)\left(\mathrm{PF}_{6}\right)$ with ${ }^{\mathrm{n}} \mathrm{Bu}_{4} \mathrm{P}^{+}(\mathrm{OMe}){ }_{2} \mathrm{POO}^{-}$.

As the quenching was non-linear in Scheme S8, the electron transfer very likely did not take place between the base and the photocatalyst. The Stern-Volmer plot like this had little influence on the redox properties of photocatalyst according to literatures. ${ }^{3,4}$

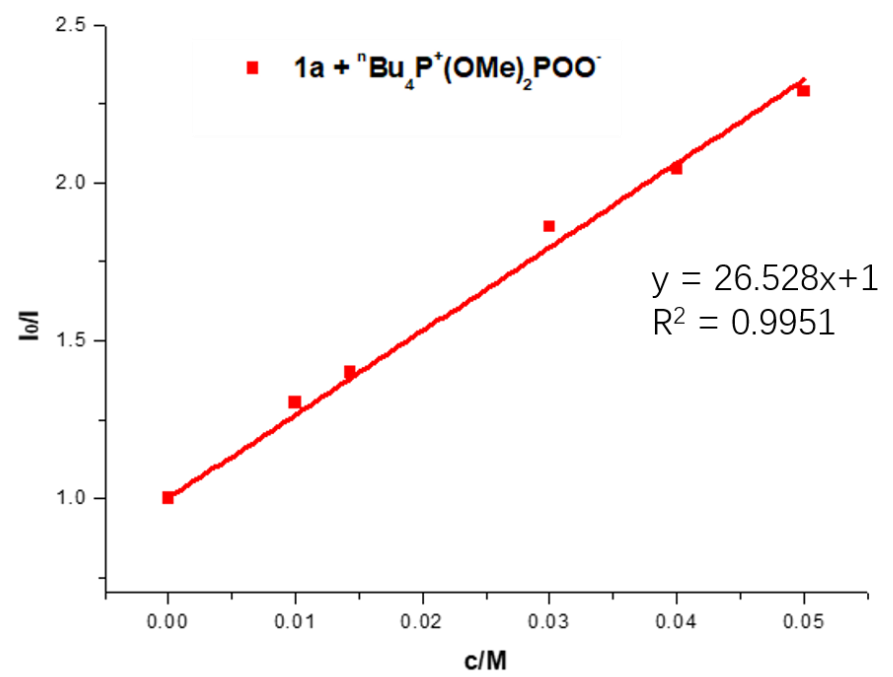

Scheme S9 The Stern-Volmer plot of $\operatorname{Ir}\left[\mathrm{dF}\left(\mathrm{CF}_{3}\right) \text { ppy }\right]_{2}\left(5,5^{\prime}-\mathrm{dCF}_{3}\right.$ bpy $)\left(\mathrm{PF}_{6}\right)$ with $1 \mathbf{a}$ in the presence of a constant concentration of ${ }^{\mathrm{n}} \mathrm{Bu}_{4} \mathrm{P}^{+}(\mathrm{OMe})_{2} \mathrm{POO}^{-}(0.025 \mathrm{M}){ }^{5}$ 


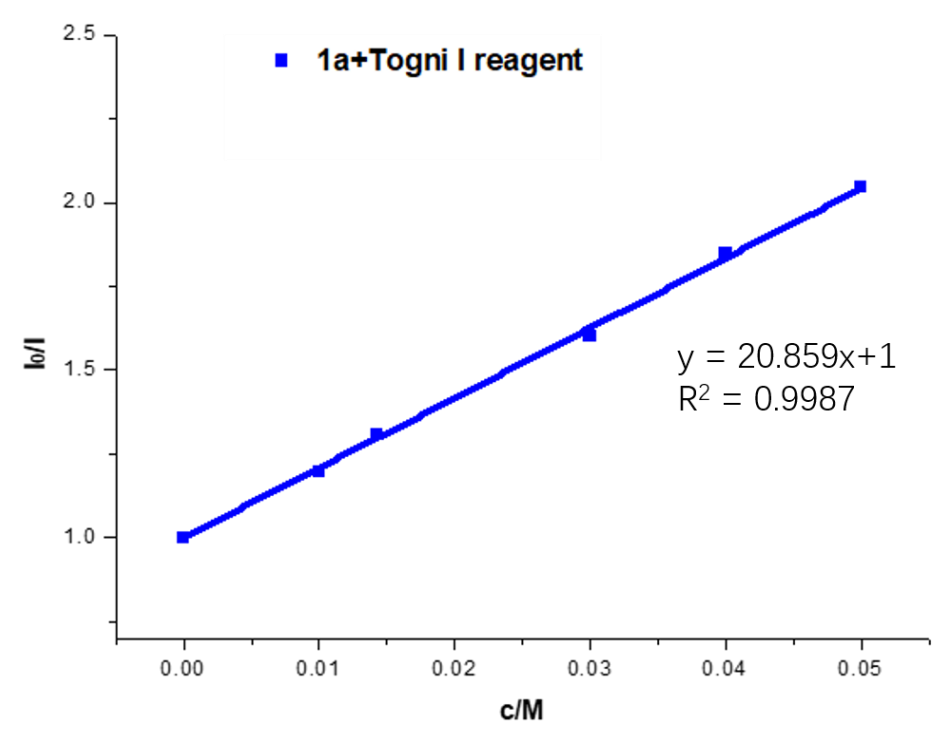

Scheme S10 The Stern-Volmer plot of $\operatorname{Ir}\left[\mathrm{dF}\left(\mathrm{CF}_{3}\right) \mathrm{ppy}\right]_{2}\left(5,5^{\prime}-\mathrm{dCF}_{3}\right.$ bpy $)\left(\mathrm{PF}_{6}\right)$ with $\mathbf{1 a}$ in the presence of a constant concentration of Togni I reagent $(0.025 \mathrm{M})$.

In the Stern-Volmer quenching experiments in Schemes S9-10, the quenching efficiency of $1 \mathbf{a}$ was significantly increased with the addition of ${ }^{\mathrm{n}} \mathrm{Bu}_{4} \mathrm{P}^{+}(\mathrm{OMe})_{2} \mathrm{POO}^{-}$ or Togni I reagent.

MeCN was used for the Stern-Volmer quenching experiment of copper salt and 1,10phen in Scheme S11-12. The MeCN solution of $\operatorname{Ir}\left[\mathrm{dF}\left(\mathrm{CF}_{3}\right) \text { ppy }\right]_{2}\left(5,5^{\prime}-\mathrm{dCF}_{3}\right.$ bpy $)\left(\mathrm{PF}_{6}\right)$ $(40 \mu \mathrm{M})$ was added the appropriate amount of quencher in a screw-top $1.0 \mathrm{~cm}$ quartz cuvette. After degassing with nitrogen, the emission spectra of the samples were collected. 


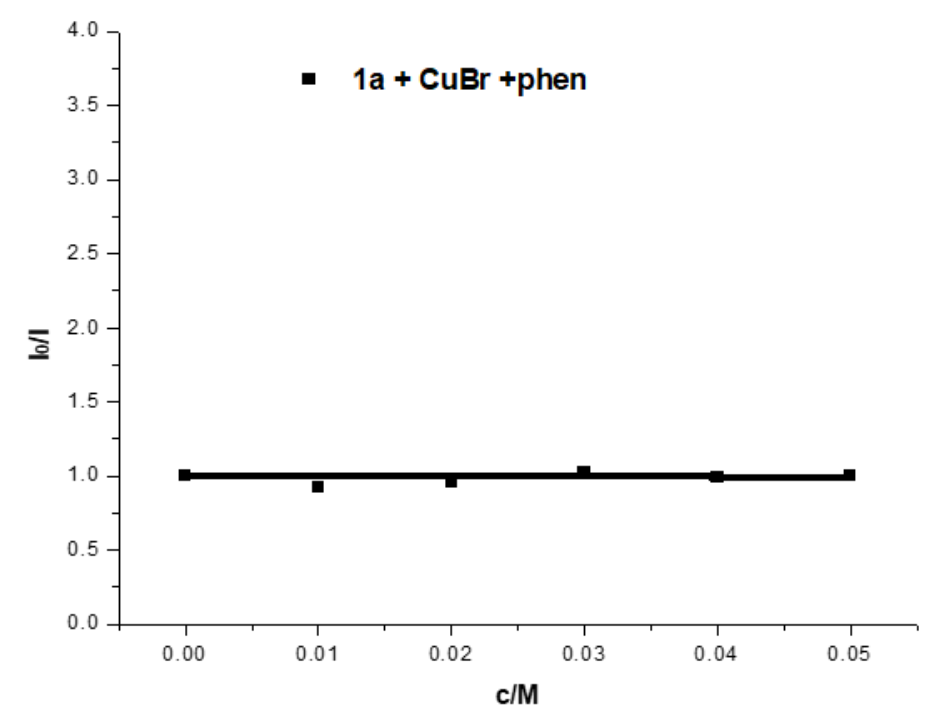

Scheme S11 The Stern-Volmer plot of $\operatorname{Ir}\left[\mathrm{dF}\left(\mathrm{CF}_{3}\right) \mathrm{ppy}\right]_{2}\left(5,5^{\prime}-\mathrm{dCF}_{3}\right.$ bpy $)\left(\mathrm{PF}_{6}\right)$ with $\mathbf{1 a}$ in the presence of a constant concentration of $\mathrm{CuBr}$ and 1,10-phen.

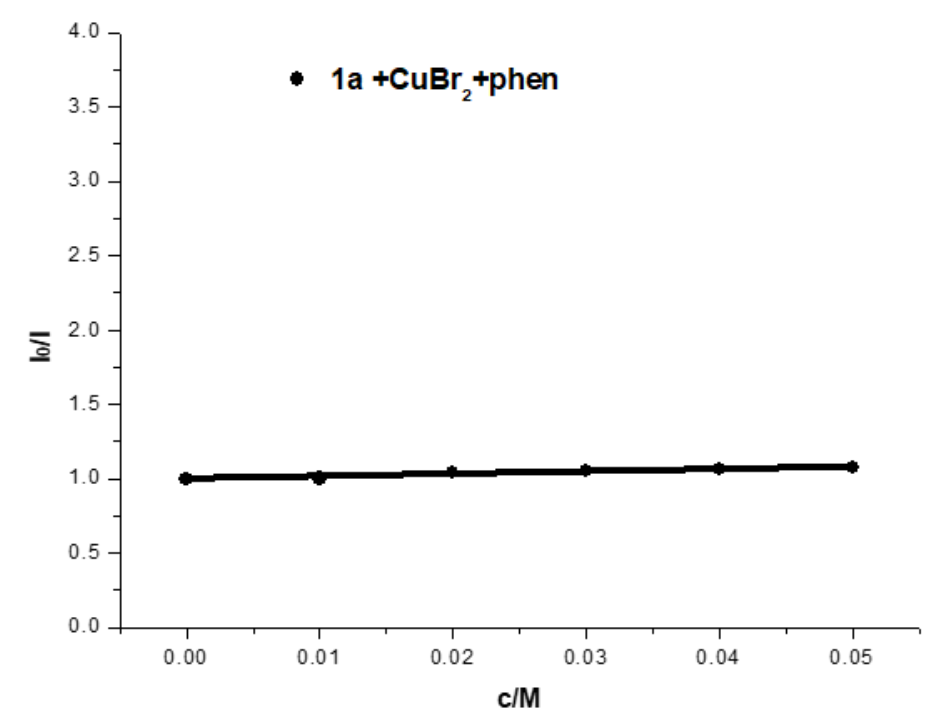

Scheme S12 The Stern-Volmer plot of $\operatorname{Ir}\left[\mathrm{dF}\left(\mathrm{CF}_{3}\right) \mathrm{ppy}\right]_{2}\left(5,5^{\prime}-\mathrm{dCF}_{3} \mathrm{bpy}\right)\left(\mathrm{PF}_{6}\right)$ with $\mathbf{1 a}$ in the presence of a constant concentration of $\mathrm{CuBr}_{2}$ and 1,10-phen.

The trendlines in Schemes S11-12 were parallel to X-axis, suggesting that the quenching efficiency of 1a was not increased with the addition of copper salt $(0.0002$ $\mathrm{M})$ and 1,10-phen (0.0003 M). 


\subsection{The interactions detected by NMR experiments}

\section{${ }^{1}$ H NMR spectra}

Different amounts of Togni I reagent or ${ }^{\mathrm{n}} \mathrm{Bu}_{4} \mathrm{P}^{+}(\mathrm{OMe})_{2} \mathrm{POO}^{-}$were added to the solution of cyclobutanol $1 \mathbf{a}\left(0.05 \mathrm{M}_{\text {in }} \mathrm{CDCl}_{3}\right.$, reference peak $\left.=7.26 \mathrm{ppm}\right)$. The ${ }^{1} \mathrm{H}$ NMR signal of O-H from 1a shifted downfield, while the signal of the aryl C-H and alkyl C-H shifted upfield with the addition of Togni I/II reagent or ${ }^{n} \mathrm{Bu}_{4} \mathrm{P}^{+}(\mathrm{OMe})_{2} \mathrm{POO}^{-}$. At the same time, the signal of Togni I reagent also shifted upfield.

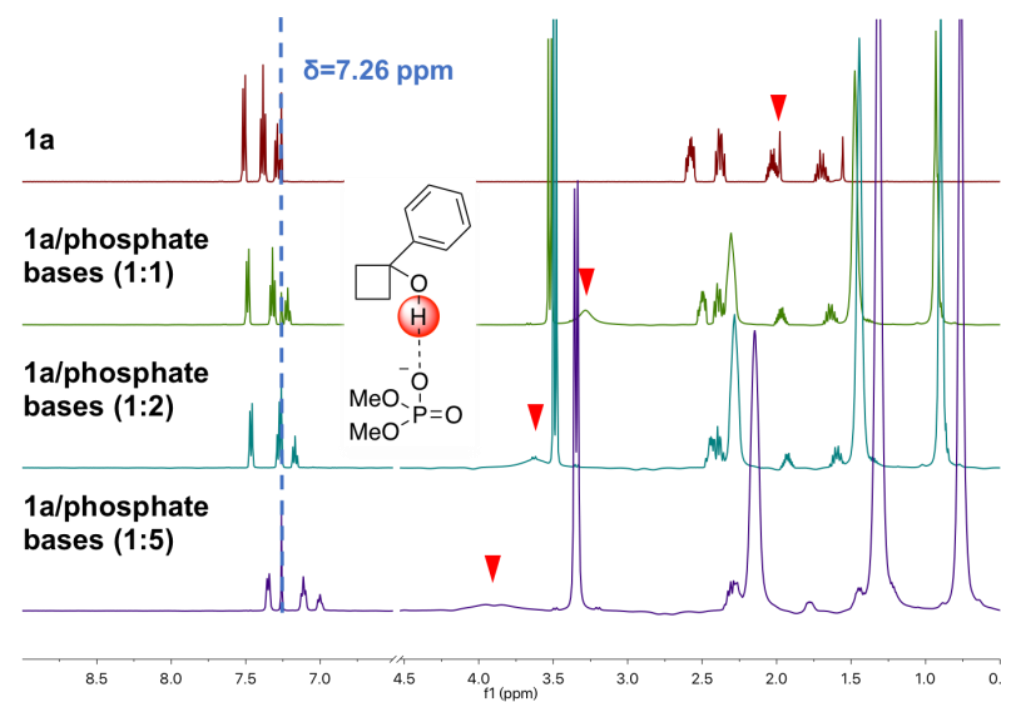

Scheme $\mathbf{S 1 3}{ }^{1} \mathrm{H}$ NMR spectra of $\mathbf{1 a}$ with various amounts of ${ }^{\mathrm{n}} \mathrm{Bu}_{4} \mathrm{P}^{+}(\mathrm{OMe})_{2} \mathrm{POO}^{-}$.

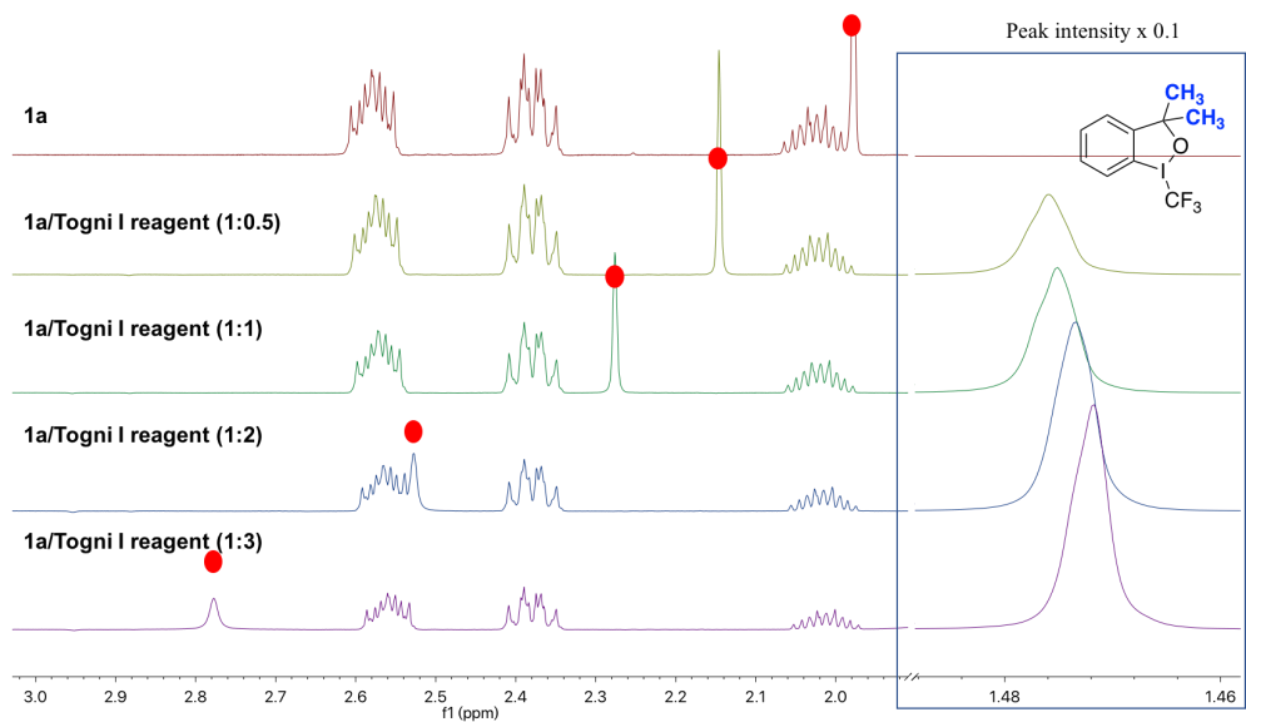

Scheme S14 ${ }^{1}$ H NMR spectra of 1 a with various amounts of Togni I reagent. 


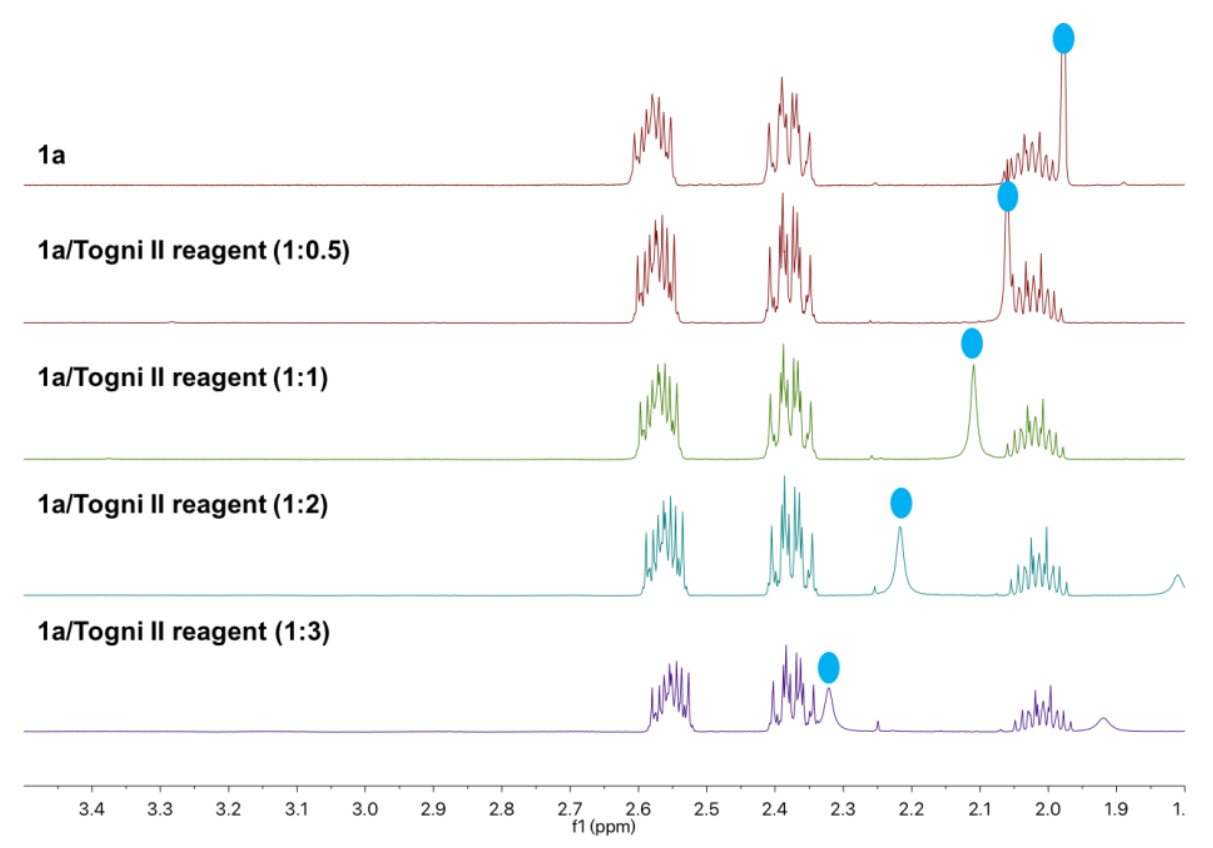

Scheme S15 ${ }^{1} \mathrm{H}$ NMR spectra of $1 \mathrm{a}$ with various amounts of Togni II reagent.

Different amounts of Togni I reagent were added to the solution of (1methoxycyclobutyl) benzene $42\left(0.05 \mathrm{M}\right.$ in $\mathrm{CDCl}_{3}$, reference peak $\left.=7.26 \mathrm{ppm}\right)$. The ${ }^{1} \mathrm{H}$ NMR signal of O-Me from 42 shifted upfield with the addition of Togni I reagent, while the signal of Togni I reagent also shifted upfield.

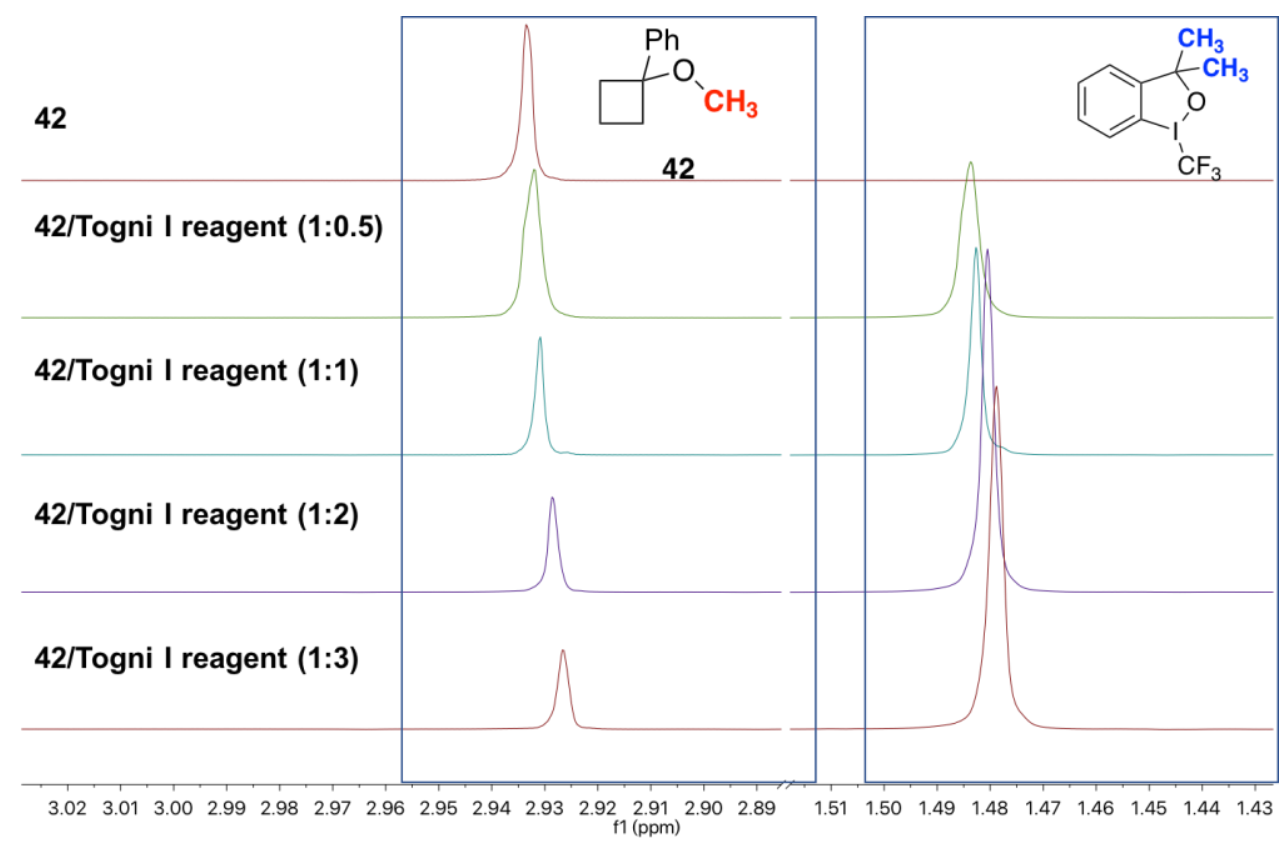

Scheme S16 ${ }^{1} \mathrm{H}$ NMR spectra of $\mathbf{4 2}$ with various amounts of Togni I reagent. 


\section{${ }^{19}$ F NMR spectra}

Different amounts of $\mathbf{1 a}$ were added to the solution of Togni I reagent $\left(0.05 \mathrm{M}^{\text {in }} \mathrm{CDCl}_{3}\right.$, $\mathrm{PhCF}_{3}$ was added as an internal standard). The ${ }^{19} \mathrm{~F}$ NMR signal of $\mathrm{CF}_{3}$ from Togni I reagent shifted downfield with the addition of $\mathbf{1 a}$.

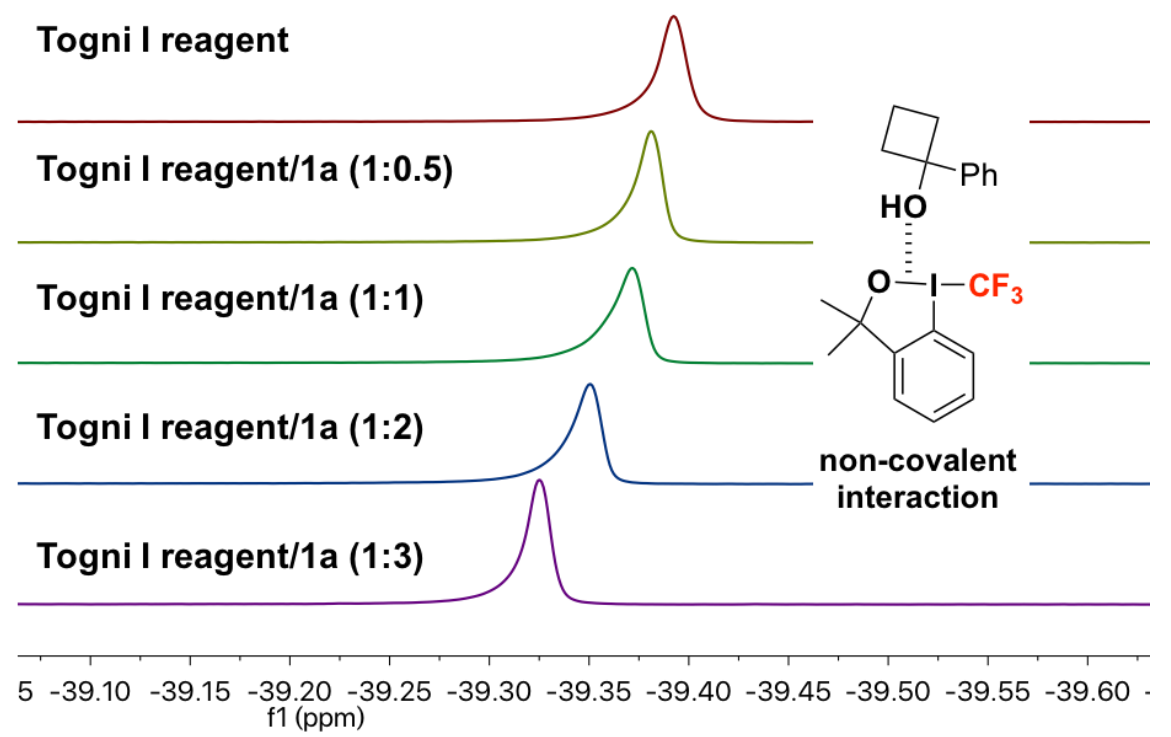

Scheme S17 ${ }^{19}$ F NMR spectra of Togni I reagent with various amounts of $\mathbf{1 a}$.

\subsection{The test of protected $\mathrm{O}-\mathrm{H}$ for the reaction}

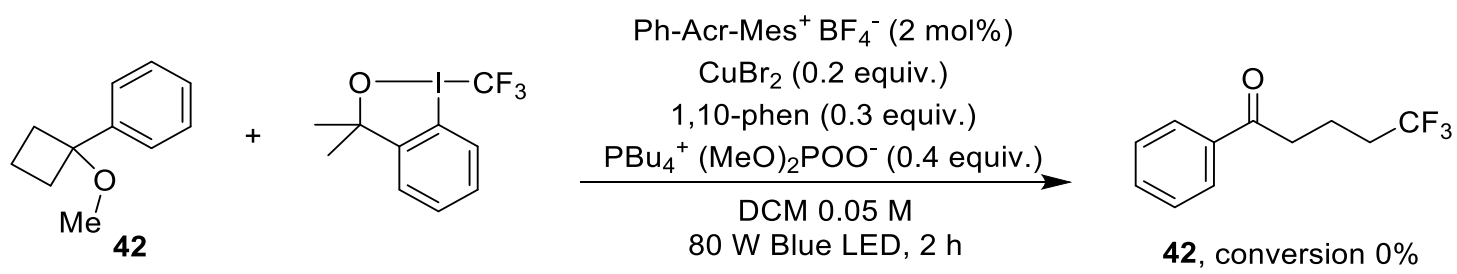

Scheme S18 The test of protected O-H for the reaction.

The methyl ether of cyclobutanol 42 ( $0.15 \mathrm{mmol}, 1.5$ equiv.), Togni I reagent ( $0.1 \mathrm{mmol}$,

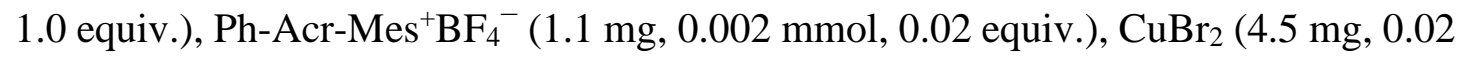
mmol, 0.2 equiv.), 1,10-Phen $(5.4 \mathrm{mg}, 0.03 \mathrm{mmol}, 0.3$ equiv.) and the ${ }^{\mathrm{n}} \mathrm{Bu}_{4} \mathrm{P}^{+}(\mathrm{OMe})_{2} \mathrm{POO}^{-}$(15.4 mg, $0.04 \mathrm{mmol}, 0.4$ equiv.) were placed in a $4 \mathrm{~mL}$ clearcolored glass vial equipped with a magnetic stir bar. After $2.0 \mathrm{~mL}$ degassed DCM were added, the vial was sealed and exposed to blue LED (80 W LED light bulb $5 \mathrm{~cm}$ away from the vial) at room temperature for $2 \mathrm{~h}$ with stirring. ${ }^{1} \mathrm{H}$ NMR analysis found no conversation of $\mathbf{4 2}$. 


\subsection{The optical absorption spectra}

Optical absorption spectra between copper catalyst (0.001 M), 1,10-Phen (0.0015 M) and reactants $(0.001 \mathrm{M})$ in $5.0 \mathrm{~mL} \mathrm{MeCN}$ were recorded in $10 \mathrm{~mm}$ path quartz cuvettes using a Thermo Nanodrop 2000c UV/Vis spectrometer. Upon mixing $\mathrm{CuBr}_{2} / 1,10-P h e n$ with $1 \mathbf{a}$ or Togni I reagent, no new absorption band was observed. Upon mixing $\mathrm{CuBr} / 1,10-\mathrm{Phen}$ with Togni I reagent, a 650-800 nm peak representing $\mathrm{Cu}$ (II) appeared, accompanied with the decrease of the $\mathrm{Cu}(\mathrm{I})$ signal at $350-500 \mathrm{~nm}$.

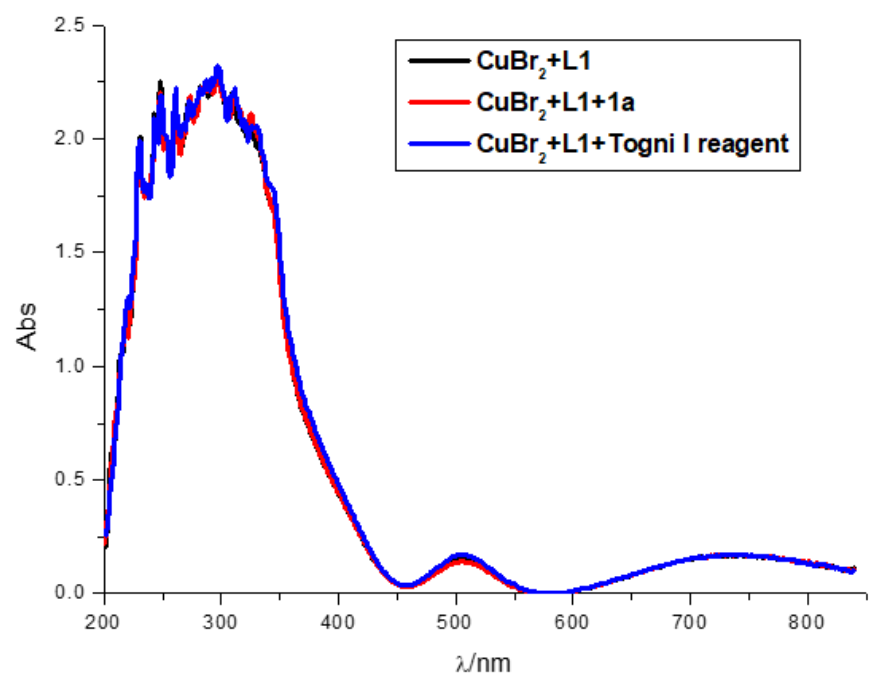

Scheme S19 UV/Vis spectra of $\mathrm{CuBr}_{2} / 1,10-$ Phen and reactants.

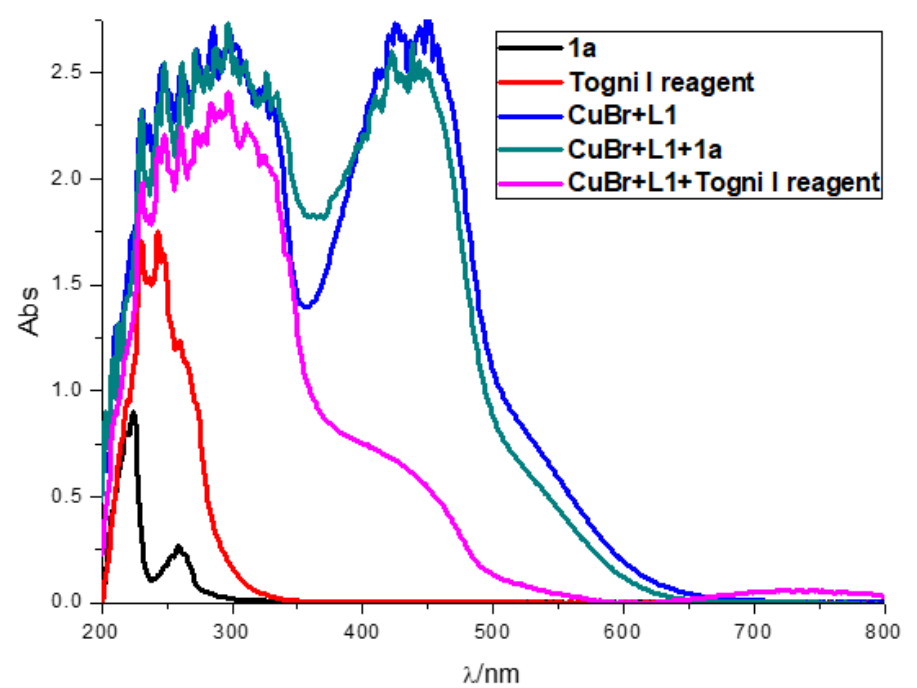

Scheme S20 UV/Vis spectra of $\mathrm{CuBr} / 1,10-\mathrm{Phen}$ and reactants. 
Optical absorption spectra between $\mathrm{CuBr}(0.001 \mathrm{M}), 1,10-\mathrm{Phen}(0.0015 \mathrm{M})$, and Togni I reagent $(0.001 \mathrm{M})$ in $5.0 \mathrm{~mL} \mathrm{MeCN}$ were recorded in $10 \mathrm{~mm}$ path quartz cuvettes using a Thermo Nanodrop 2000c UV/Vis spectrometer. When 1a was added to the mixture, the appearance of the $\mathrm{Cu}(\mathrm{II})$ peak and the decrease of the $\mathrm{Cu}(\mathrm{I})$ peak was greatly accelerated.

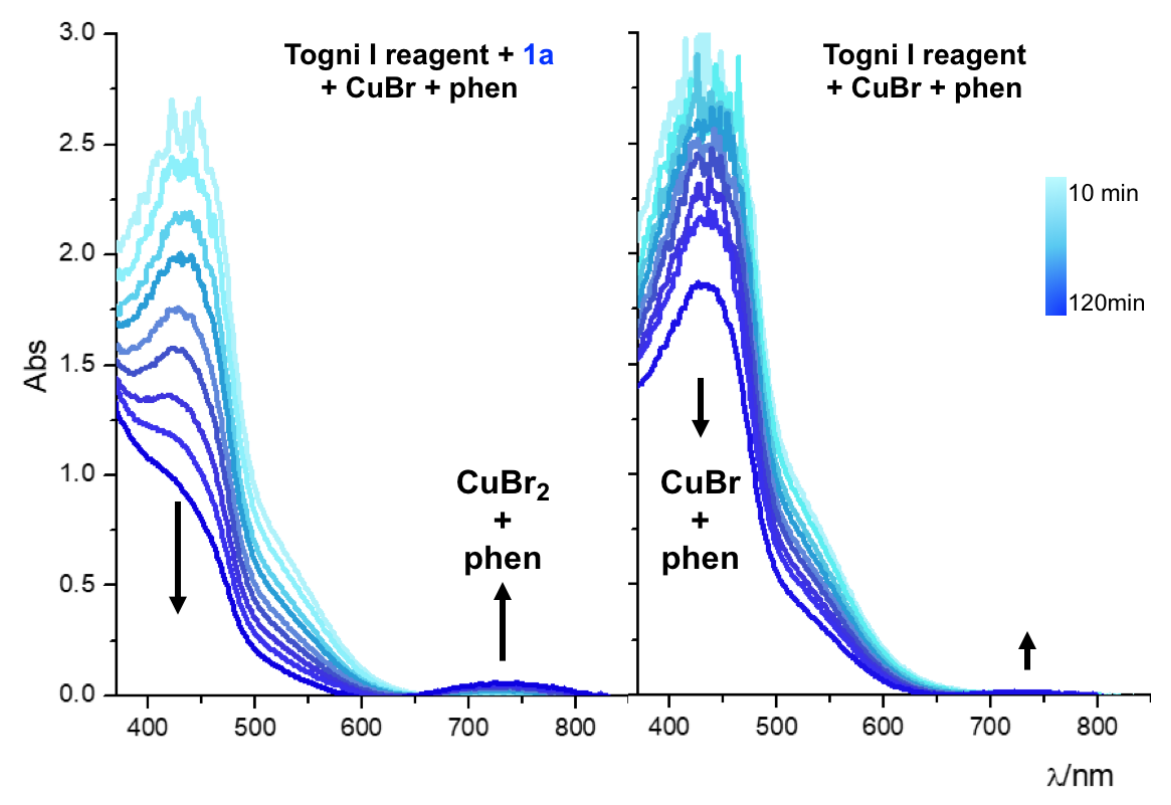

Scheme S21 UV/Vis spectra of cyclobutanol $1 \mathbf{a}$ and the mixture of $\mathrm{CuBr} / 1,10$ Phen/Togni I reagent.

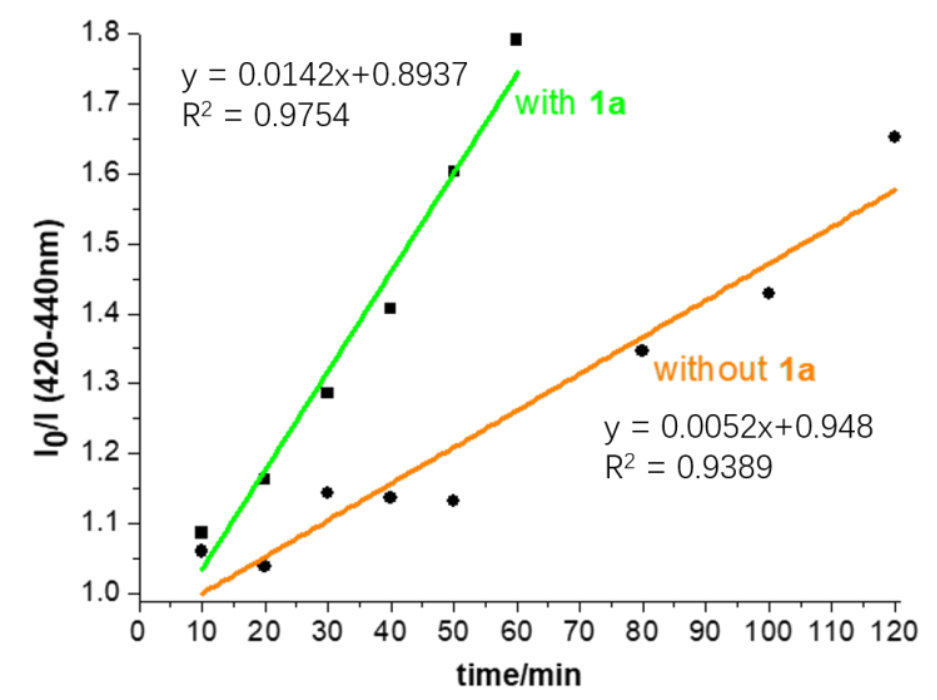

Scheme S22 The decrease rate of signal at 420-440 nm in Scheme 21. 


\subsection{Screen of oxidants for Ph-Acr-Mes ${ }^{+} \mathrm{BF}_{4}{ }^{-}$}

Table S1 Screen of oxidants for Ph-Acr-Mes ${ }^{+} \mathrm{BF}_{4}{ }^{-}$

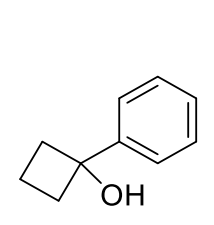

$1 \mathrm{a}$

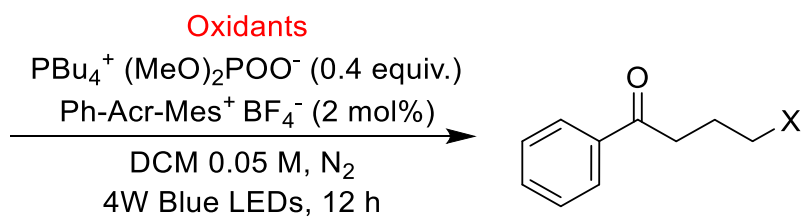

$\mathrm{X}=\mathrm{CF}_{3}, \mathrm{H}, \mathrm{Br}$, I

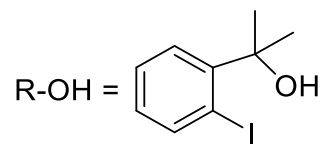

a conv. $(\%)^{b}$

\begin{tabular}{ccc}
\hline Entry & Oxidants & 1a conv. $(\%)^{b}$ \\
\hline 1 & Togni I reagent (1.0 equiv.) & 10 \\
2 & $\mathrm{CuBr}_{2}$ (1.0 equiv.), 1,10-phen (1.2 equiv.) & 9 \\
3 & $\mathrm{CuBr}_{2}$ (1.0 equiv.), 1,10-phen (1.2 equiv.), & 5 \\
& $\mathrm{R}-\mathrm{OH}(1.0$ equiv.) & 6 \\
& $\mathrm{CuBr} 2$ (1.0 equiv.), 1,10-phen (1.2 equiv.), & \\
& $\mathrm{R}-\mathrm{OH}\left(1.0\right.$ equiv.), $\mathrm{Na}_{2} \mathrm{CO}_{3}$ (1.0 equiv.)
\end{tabular}

${ }^{a}$ Reaction conditions: $1 \mathrm{a}(0.15 \mathrm{mmol}, 1.5$ equiv. $)$, Oxidants $(0.10 \mathrm{mmol}, 1.0$ equiv. $)$, Ph-Acr-Mes ${ }^{+} \mathrm{BF}_{4}{ }^{-}$(0.002 mmol, 0.02 equiv. $)$ and ${ }^{\mathrm{n}} \mathrm{Bu}_{4} \mathrm{P}^{+}(\mathrm{OMe}){ }_{2} \mathrm{POO}^{-}(0.04 \mathrm{mmol}, 0.4$ equiv.) in $2.0 \mathrm{~mL}$ DCM under nitrogen with $4 \mathrm{~W}$ blue LED ( $5 \mathrm{~cm}$ away from the vial) irradiation at $25{ }^{\circ} \mathrm{C}$ for $12 \mathrm{~h}$, unless otherwise noted. ${ }^{b}$ Conversions and yields were determined by ${ }^{1} \mathrm{H}$ NMR analysis.

Several possible oxidants were tested in the reaction, but none of them converted 1a successfully, such as Togni I reagent, $\mathrm{Cu}$ (II) salt.

\section{$2.10{ }^{19}$ F NMR analysis}

Cyclobutanol 1a (0.15 mmol, 1.5 equiv.), Togni I reagent (33.0 mg, $0.1 \mathrm{mmol}, 1.0$ equiv.), $\mathrm{Ph}-\mathrm{Acr}-\mathrm{Mes}^{+} \mathrm{BF}_{4}{ }^{-}$(1.1 mg, $0.002 \mathrm{mmol}, 0.02$ equiv.), $\mathrm{CuBr}_{2}$ (3.6 mg, 0.02 mmol, 0.2 equiv.), 1,10-phen (5.4 mg, 0.03 mmol, 0.3 equiv.) and ${ }^{n} \mathrm{Bu}_{4} \mathrm{P}^{+}(\mathrm{OMe})_{2} \mathrm{POO}^{-}$ (15.4 mg, $0.04 \mathrm{mmol}, 0.4$ equiv.) were placed in a $4 \mathrm{~mL}$ clear-colored glass vial equipped with a magnetic stir bar. After $2.0 \mathrm{~mL} \mathrm{CDCl}_{3}$ was added, the vial was sealed in nitrogen atmosphere and exposed to blue LEDs (4 W LED light bulb $5 \mathrm{~cm}$ away from the vial) at room temperature for $0.5 \mathrm{~h}$ with stirring. $\mathrm{PhCF}_{3}$ was added as the internal standard and the solution was subjected to ${ }^{19} \mathrm{~F}$ NMR analysis. The remaining Togni I reagent was observed ( $83 \%$ remaining) at $-40.5 \mathrm{ppm}$, together with the presence 
of a new species at $-27.4 \mathrm{ppm}$, which presumed to be a $\mathrm{Cu}^{\mathrm{III}}-\left(\mathrm{CF}_{3}\right)_{\mathrm{x}}$ complex. ${ }^{6}$ The $17.5 \mathrm{ppm}$ peak (red bar) was unidentified and may belong to the copper trifluoromethyl intermediate.

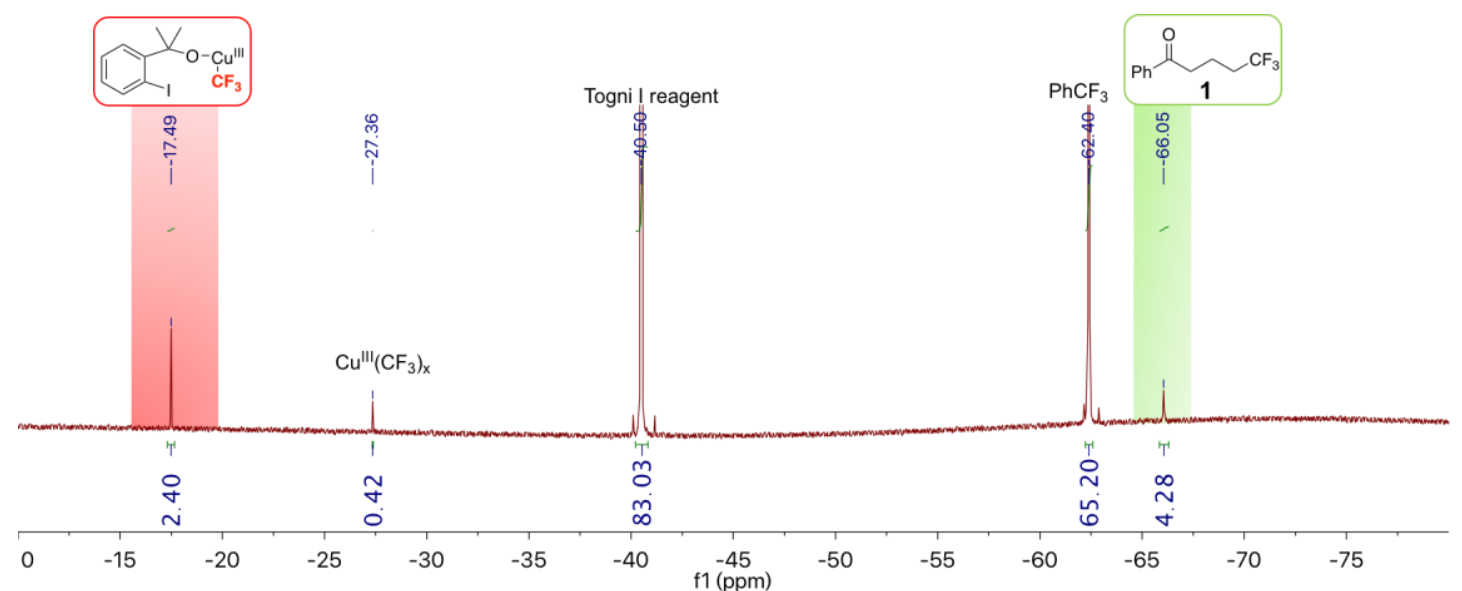

Scheme S23 ${ }^{19}$ F NMR analysis of reaction intermediates.

We then increased the equivalent of $\mathrm{CuBr}_{2}(7.2 \mathrm{mg}, 0.04 \mathrm{mmol}, 0.4$ equiv.) and used bpy (10.8 mg, $0.06 \mathrm{mmol}, 0.6$ equiv.) as the ligand. The vial was sealed in the nitrogen atmosphere and exposed to blue LED (80 W LED light bulb $5 \mathrm{~cm}$ away from the vial) at room temperature for $1 \mathrm{~h}$ with stirring. $\mathrm{PhCF}_{3}$ was added as the internal standard and the solution was subjected to ${ }^{19} \mathrm{~F}$ NMR analysis. New signal peaks were formed (-23.4 ppm and $-36.9 \mathrm{ppm}$ ), which were presumed to the previously reported Grushin reagent, (bpy) $\mathrm{Cu}\left(\mathrm{CF}_{3}\right)_{3}{ }^{7}$

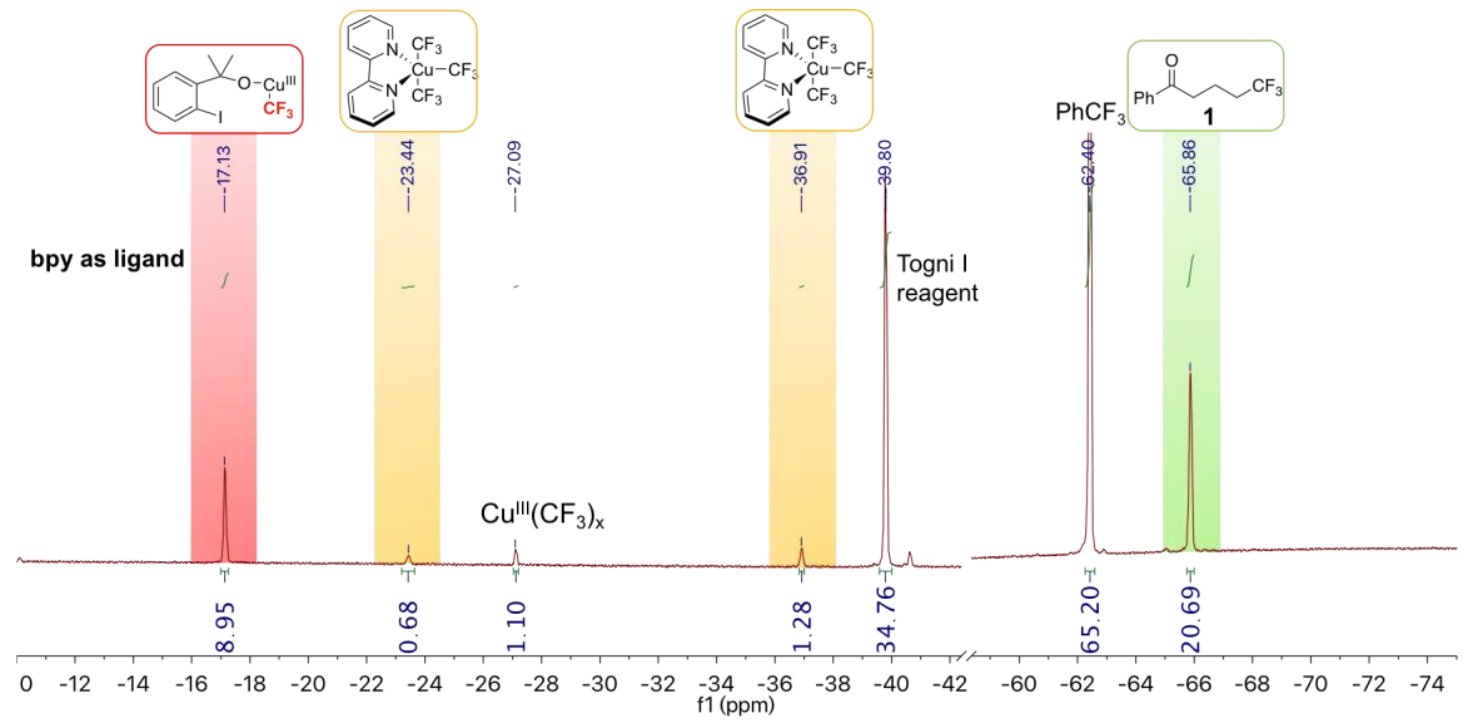

Scheme S24 ${ }^{19}$ F NMR analysis of reaction intermediates with bpy as the ligand. 
We next used $\left(\mathrm{CF}_{3}\right)_{2}-\mathrm{BI}-\mathrm{CF}_{3}(43.8 \mathrm{mg}, 0.1 \mathrm{mmol}, 1.0$ equiv.) as the trifluoromethyl source and increased the equivalent of $\mathrm{CuBr}_{2}(7.2 \mathrm{mg}, 0.04 \mathrm{mmol}, 0.4$ equiv.) and 1,10phen (10.8 mg, $0.06 \mathrm{mmol}, 0.6$ equiv.). The vial was sealed in nitrogen atmosphere and exposed to blue LED (80 W LED light bulb $5 \mathrm{~cm}$ away from the vial) at room temperature for $1 \mathrm{~h}$ with stirring. $\mathrm{PhCF}_{3}$ was added as the internal standard and the solution was subjected to ${ }^{19} \mathrm{~F}$ NMR analysis. New signal peaks were formed (-17.1 ppm, -72.4 ppm, -74.4 ppm), which were presumed to be the new complex. The two $\left(\mathrm{CF}_{3}\right)_{2}-$ benzylic groups at $-72.4 \mathrm{ppm}$ (red bar) and -74.4 ppm (blue bar) were supposedly the $\left(\mathrm{CF}_{3}\right)_{2}$-benzylic groups complexed to the copper as the ligand. By integral analysis, the consistent 2:1 ratio between the $-72.4 \mathrm{ppm}$ peak and the $-17.1 \mathrm{ppm}$ peak (red bars) was observed, suggesting the 1:1:1 ratio of the $\left(\mathrm{CF}_{3}\right)_{2}$-benzylic-O-Cu(III)-CF 3 complex formation. The other $-74.4 \mathrm{ppm}$ peak (blue bar) should attribute to the $\mathrm{Cu}(\mathrm{II})$ complexed $\left(\mathrm{CF}_{3}\right)_{2}$-benzylic group after the trifluoromethyl radical elimination.

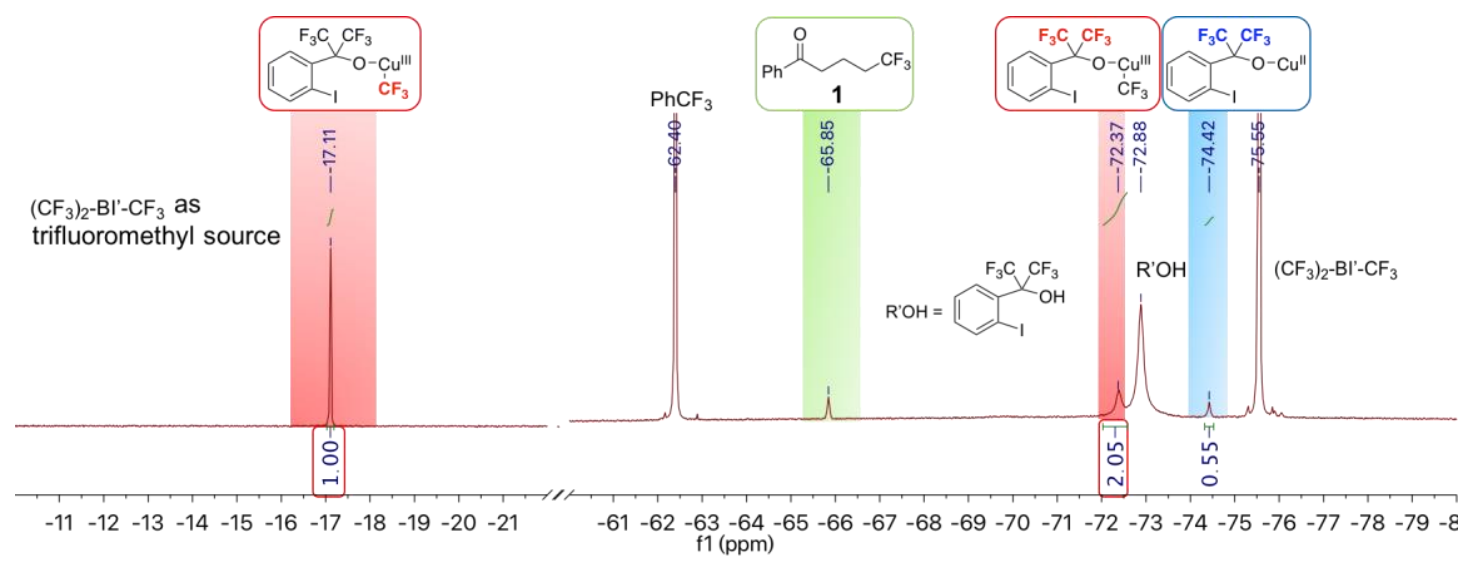

Scheme $\mathbf{S 2 5}{ }^{19} \mathrm{~F}$ NMR analysis of reaction intermediates with $\left(\mathrm{CF}_{3}\right)_{2}-\mathrm{BI}{ }^{\prime}-\mathrm{CF}_{3}$ as the trifluoromethyl source.

$\left(\mathrm{CF}_{3}\right)_{2}-\mathrm{BI}$ '- $\mathrm{CF}_{3}$ (43.8 mg, $0.1 \mathrm{mmol}, 1.0$ equiv.) was then mixed with $\mathrm{CuBr}$ (18.0 mg, $0.1 \mathrm{mmol}, 1.0$ equiv.) and 1,10-phen (27.0 mg, $0.15 \mathrm{mmol}, 1.5$ equiv.) in a $4 \mathrm{~mL}$ clearcolored glass vial equipped with a magnetic stir bar. After $2.0 \mathrm{~mL} \mathrm{CDCl}_{3}$ was added, the vial was sealed in the nitrogen atmosphere at room temperature for $1 \mathrm{~h}$ with stirring. $\mathrm{PhCF}_{3}$ was added as the internal standard and the solution was subjected to ${ }^{19} \mathrm{~F}$ NMR analysis. New signal peaks were formed, which were presumed to be the new complex however shifted upfield comparing to Scheme S25. Due to the condition changes, the number of anions coordinated to copper may be different and resulted in the signal peaks shift. 


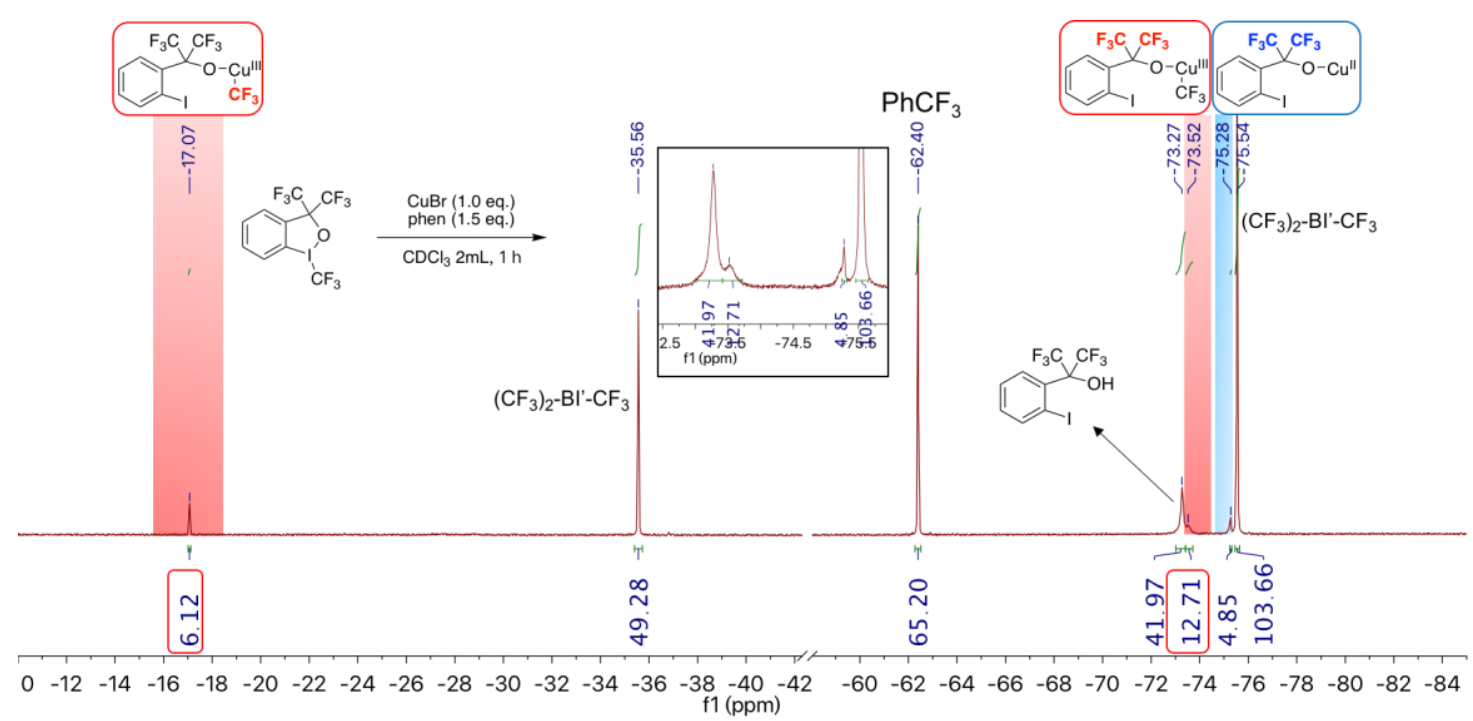

Scheme S26 ${ }^{19} \mathrm{~F}$ NMR analysis of $\left(\mathrm{CF}_{3}\right)_{2}-\mathrm{BI}$ '- $\mathrm{CF}_{3}$ reacting with $\mathrm{CuBr}$ and 1,10-phen.

We further added $1 \mathrm{a}(0.15 \mathrm{mmol}, 1.5$ equiv. $)$ and ${ }^{\mathrm{n}} \mathrm{Bu}_{4} \mathrm{P}^{+}(\mathrm{OMe})_{2} \mathrm{POO}^{-}(15.4 \mathrm{mg}, 0.04$ mmol, 0.4 equiv.) to the mixture of $\left(\mathrm{CF}_{3}\right)_{2}-\mathrm{BI}^{\prime}-\mathrm{CF}_{3}(43.8 \mathrm{mg}, 0.1 \mathrm{mmol}, 1.0$ equiv.), $\mathrm{CuBr}$ (18.0 mg, $0.1 \mathrm{mmol}, 1.0$ equiv.) and phen (27.0 mg, $0.15 \mathrm{mmol}, 1.5$ equiv.) in a $4 \mathrm{~mL}$ clear-colored glass vial equipped with a magnetic stir bar. After $2.0 \mathrm{~mL} \mathrm{CDCl} 3$ was added, the vial was sealed in the nitrogen atmosphere at room temperature for $1 \mathrm{~h}$ with stirring. $\mathrm{PhCF}_{3}$ was added as the internal standard and the solution was subjected to ${ }^{19} \mathrm{~F}$ NMR analysis. New signal peaks were formed and shifted downfield comparing to Scheme S26.

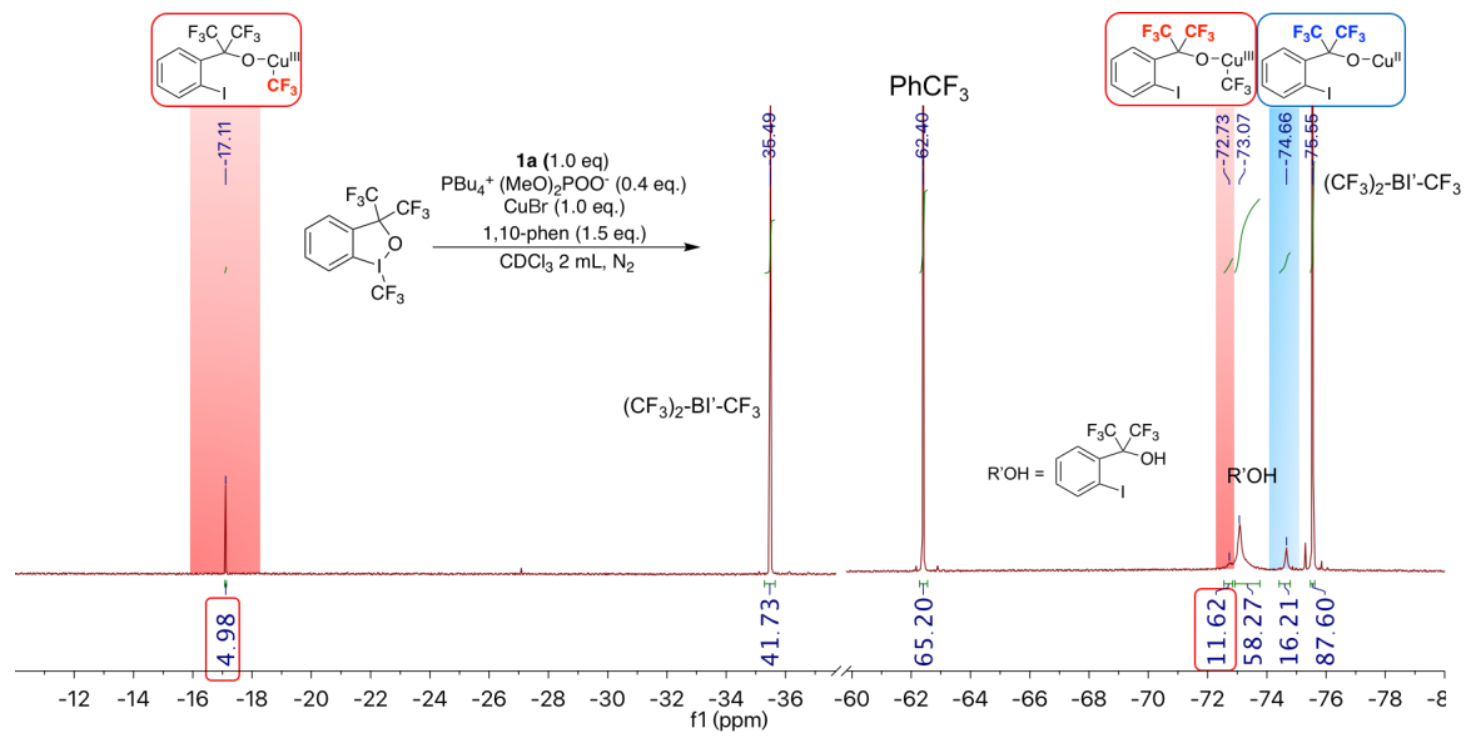

Scheme S27 ${ }^{19} \mathrm{~F}$ NMR analysis of $\left(\mathrm{CF}_{3}\right)_{2}-\mathrm{BI}^{\prime}-\mathrm{CF}_{3}$ react with $\mathrm{CuBr}$ and 1,10-phen in different ionic enviroments. 


\section{Detailed Reaction Optimizations}

\subsection{Screen of photocatalysts}

Table S2 Screen of photocatalysts

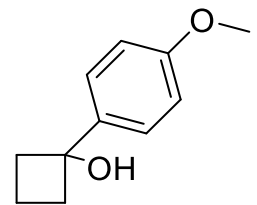

2a<smiles>CC1(C)OI(C(F)(F)F)c2ccccc21</smiles>

Entry Conditions ${ }^{a}$ photocatalysts

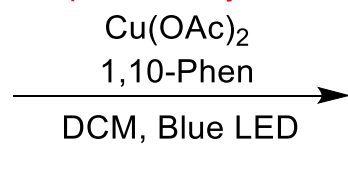<smiles>COc1ccc(C(=O)CCCC(F)(F)F)cc1</smiles>

2

\begin{tabular}{ccccc} 
& & vs SCE & $(\%)$ & \\
\hline 1 & $\mathrm{Ph}-\mathrm{Acr}_{-} \mathrm{Mes}^{+} \mathrm{BF}_{4}^{-}$ & 2.08 & 107 & $51(48)$ \\
2 & $\mathrm{Ir}\left(\mathrm{dF}\left(\mathrm{CF}_{3}\right) \mathrm{ppy}\right)_{2}\left(5,5^{\prime}-\mathrm{dCF}_{3} \mathrm{bpy}^{-} \mathrm{PF}_{6}\right.$ & 1.68 & 81 & $55(52)$ \\
3 & $\mathrm{Ru}(\mathrm{bpy})_{3}\left(\mathrm{PF}_{6}\right)_{2}$ & 0.77 & $<5$ & 0 \\
4 & $\operatorname{Ir}(\mathrm{ppy})_{3}$ & 0.31 & 18 & 0 \\
5 & $4 \mathrm{CzIPN}_{6}$ & 1.49 & 6 & 6 \\
& $\mathrm{Ir}(\mathrm{dtbbpy})(\mathrm{ppy})_{2} \mathrm{PF}_{6}$ & 0.66 & 15 & $<5$
\end{tabular}

${ }^{a}$ Reaction conditions: $2 \mathbf{a}(0.15 \mathrm{mmol}, 1.5$ equiv. $)$, Togni I reagent $(0.10 \mathrm{mmol}, 1.0$ equiv.), photocatalyst (0.002 mmol, 0.02 equiv.), $\mathrm{Cu}(\mathrm{OAc})_{2}(0.02 \mathrm{mmol}, 0.2$ equiv.) and 1,10-phen ( $0.03 \mathrm{mmol}, 0.3$ equiv.) in $2.0 \mathrm{~mL}$ DCM under nitrogen with $4 \mathrm{~W}$ blue LED ( $5 \mathrm{~cm}$ away from the vial) irradiation at $25^{\circ} \mathrm{C}$ for $12 \mathrm{~h}$, unless otherwise noted. ${ }^{b}$ Conversions and yields were determined by ${ }^{1} \mathrm{H}$ NMR analysis.<smiles>Cc1cc(C)c(-c2c3ccc(C(C)(C)C)cc3[n+](-c3ccccc3)c3cc(C(C)(C)C)ccc23)c(C)c1</smiles>

Ph-Acr-MesBF 4<smiles></smiles>

$\operatorname{Ir}\left(\mathrm{dF}\left(\mathrm{CF}_{3}\right) \mathrm{ppy}\right)_{2}\left(5,5^{\prime}-\mathrm{dCF}_{3}\right.$ bpy $) \mathrm{PF}_{6}$<smiles></smiles>

$\operatorname{Ir}(\mathrm{dtbbpy})(\mathrm{ppy}){ }_{2} \mathrm{PF}_{6}$ 


\subsection{Screen of solvents}

Table S3 Screen of solvents

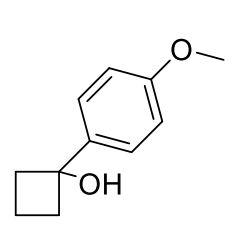

$2 a$
$\operatorname{Ir}\left(\mathrm{dF}\left(\mathrm{CF}_{3}\right) \mathrm{ppy}\right)_{2}\left(5,5^{\prime}-\mathrm{dCF} \mathrm{F}_{3} \mathrm{bpy}\right) \mathrm{PF}_{6}$

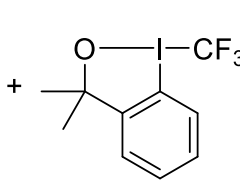

$\mathrm{Cu}(\mathrm{OAc})_{2}$

1,10-Phen

Solvent, Blue LED

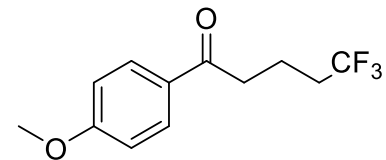

\begin{tabular}{|c|c|c|c|c|}
\hline Entry & Solvent $^{a}$ & $\mathbf{2 a} \operatorname{conv}(\%)$ & Togni I reagent conv (\%) & Yield $2(\%)^{b}$ \\
\hline 1 & DCM & 81 & $>95$ & $55(52)$ \\
\hline 2 & DCE & 71 & 76 & 38 \\
\hline 3 & $\mathrm{PhCl}$ & 9 & 75 & $<5$ \\
\hline 4 & $\mathrm{PhCH}_{3}$ & 30 & 77 & $<5$ \\
\hline 5 & EtOAc & 36 & 28 & $<5$ \\
\hline 6 & $\mathrm{MeCN}$ & 53 & $>95$ & 25 \\
\hline 7 & $\mathrm{CHCl}_{3}$ & 99 & $>95$ & 20 \\
\hline 8 & THF & 8 & 26 & $<5$ \\
\hline 9 & Dioxane & 12 & 47 & $<5$ \\
\hline 10 & $\mathrm{MeOH}$ & 80 & $>95$ & 11 \\
\hline 11 & HFIP & $>140$ & $>95$ & $<5$ \\
\hline 12 & HFIP/DCM (v:v = 1:5) & 104 & $>95$ & $<12$ \\
\hline 13 & $\mathrm{DCM} / \mathrm{H}_{2} \mathrm{O}(\mathrm{v}: \mathrm{v}=1: 1)$ & 107 & $>95$ & 55 \\
\hline 14 & $\mathrm{MeOH} / \mathrm{H}_{2} \mathrm{O}(\mathrm{v}: \mathrm{v}=1: 1)$ & 52 & 77 & 0 \\
\hline 15 & $\mathrm{DCM} / \mathrm{MeOH}(\mathrm{v}: \mathrm{v}=1: 1)$ & 99 & $>95$ & 30 \\
\hline
\end{tabular}

${ }^{a}$ Reaction conditions: $2 \mathrm{a}(0.15 \mathrm{mmol}, 1.5$ equiv. $)$, Togni I reagent $(0.10 \mathrm{mmol}, 1.0$ equiv.), $\operatorname{Ir}\left(\mathrm{dF}\left(\mathrm{CF}_{3}\right) \text { ppy }\right)_{2}\left(5,5^{\prime}-\mathrm{dCF}_{3}\right.$ bpy $) \mathrm{PF}_{6}(0.002 \mathrm{mmol}, 0.02$ equiv. $), \mathrm{Cu}(\mathrm{OAc})_{2}(0.02$ mmol, 0.2 equiv.) and 1,10-phen (0.03 mmol, 0.3 equiv.) in $2.0 \mathrm{~mL}$ solvent under nitrogen with $4 \mathrm{~W}$ blue LED ( $5 \mathrm{~cm}$ away from the vial) irradiation at $25^{\circ} \mathrm{C}$ for $12 \mathrm{~h}$, unless otherwise noted. ${ }^{b}$ Conversions and yields were determined by ${ }^{1} \mathrm{H}$ NMR analysis. 


\subsection{Screen of copper catalysts}

Table S4 Screen of copper catalysts

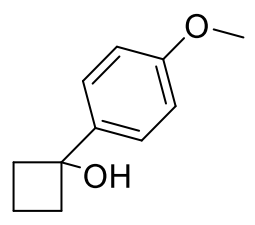

$2 a$

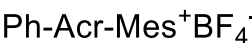

Catalysts

1,10-Phen

DCM, Blue LED<smiles>COc1ccc(C(=O)CCCC(F)(F)F)cc1</smiles>

2

\begin{tabular}{ccccc}
\hline Entry & Conditions $^{a}$ & 2a conv. (\%) & Togni I reagent conv. (\%) & ${\text { Yield 2(\% })^{b}}^{b}$ \\
\hline 1 & $\mathrm{Cu}(\mathrm{OAc})_{2}$ & 107 & $>95$ & $51(48)$ \\
2 & $\mathrm{CuCl}$ & 105 & $>95$ & 65 \\
3 & $\mathrm{CuBr}$ & 98 & $>95$ & 66 \\
4 & $\mathrm{CuI}$ & 116 & $>95$ & 62 \\
5 & $\mathrm{Cu}\left(\mathrm{MeCN}_{4}\right)_{\mathrm{BF}_{4}}$ & 126 & $>95$ & $<10$ \\
6 & $\mathrm{Cu}\left(\mathrm{MeCN}_{4} \mathrm{PF}_{6}\right.$ & 118 & $>95$ & $<10$ \\
7 & $\mathrm{Cu}\left(\mathrm{BF}_{4}\right)_{2} 6 \mathrm{H}_{2} \mathrm{O}$ & 107 & $>95$ & $<10$ \\
8 & $\mathrm{CuBr}_{2}$ & 140 & $>95$ & $76(71)$ \\
9 & $\mathrm{CuO}_{2}$ & 107 & $>95$ & 73 \\
\hline
\end{tabular}

${ }^{a}$ Reaction conditions: $2 \mathrm{a}(0.15 \mathrm{mmol}, 1.5$ equiv. $)$, Togni I reagent $(0.10 \mathrm{mmol}, 1.0$ equiv.), $\mathrm{Ph}$-Acr-Mes ${ }^{+} \mathrm{BF}_{4}{ }^{-}$(0.002 mmol, 0.02 equiv.), catalysts ( $0.02 \mathrm{mmol}, 0.2$ equiv.) and 1,10-phen ( $0.03 \mathrm{mmol}, 0.3$ equiv.) in $2.0 \mathrm{~mL}$ DCM under nitrogen with $4 \mathrm{~W}$ blue LED ( $5 \mathrm{~cm}$ away from the vial) irradiation at $25{ }^{\circ} \mathrm{C}$ for $12 \mathrm{~h}$, unless otherwise noted. ${ }^{b}$ Conversions and yields were determined by ${ }^{1} \mathrm{H}$ NMR analysis. 


\subsection{Screen of trifluoromethyl sources}

Table S5 Screen of trifluoromethyl sources

\begin{tabular}{|c|c|c|c|c|}
\hline & $\mathrm{O}-\mathrm{CF}_{3}$ & $\begin{array}{c}\text { Ph-Acr-Mes }{ }^{+} \mathrm{BF}_{4}^{-} \\
\mathrm{CuBr}_{2}, 1,10-\text { phen } \\
\mathrm{PBu}_{4}^{+}(\mathrm{MeO})_{2} \mathrm{POO}^{-}\end{array}$ & \multirow{2}{*}{\multicolumn{2}{|c|}{1}} \\
\hline $1 a$ & & & & \\
\hline Entry & \multicolumn{2}{|c|}{ Conditions $^{a}$} & 1a conv. $(\%)$ & Yield $\mathbf{1}(\%)^{b}$ \\
\hline 1 & \multicolumn{2}{|c|}{ I } & 89 & $66(65)$ \\
\hline 2 & \multicolumn{2}{|c|}{ Togni II reagent instead of Togni I reagent } & 75 & 35 \\
\hline 3 & \multicolumn{2}{|c|}{ Umemoto's reagent instead of Togni I reagent } & 116 & $<5$ \\
\hline 4 & \multicolumn{2}{|c|}{$\left(\mathrm{CF}_{3}\right)_{2}$-BI'-CF $\mathbf{C F}_{3}$ instead of Togni I reagent } & 59 & 26 \\
\hline
\end{tabular}

${ }^{a}$ Reaction conditions: $1 \mathbf{a}(0.15 \mathrm{mmol}, 1.5$ equiv. $)$, trifluoromethylation reagent $(0.10$ mmol, 1.0 equiv.), $\mathrm{Ph}-\mathrm{Acr}-\mathrm{Mes}^{+} \mathrm{BF}_{4}^{-}$(0.002 mmol, 0.02 equiv.), $\mathrm{Cu}(\mathrm{OAc})_{2}(0.02 \mathrm{mmol}$, 0.2 equiv.) and 1,10-phen ( $0.03 \mathrm{mmol}, 0.3$ equiv.) in $2.0 \mathrm{~mL}$ DCM under nitrogen with $4 \mathrm{~W}$ blue LED ( $5 \mathrm{~cm}$ away from the vial) irradiation at $25^{\circ} \mathrm{C}$ for $12 \mathrm{~h}$, unless otherwise noted. ${ }^{b}$ Conversions and yields were determined by ${ }^{1} \mathrm{H}$ NMR analysis.<smiles>CC1(C)OI(C(F)(F)F)c2ccccc21</smiles>

Togni I reagent Togni II reagent<smiles>O=C1OI(C(F)(F)F)c2ccccc21</smiles><smiles></smiles>

$\left(\mathrm{CF}_{3}\right)_{2}-\mathrm{Bl}^{\prime}-\mathrm{CF}_{3}$<smiles>FC(F)(F)c1ccccc1-c1ccccc1[Si](F)(F)F</smiles>

Umemoto's reagent 


\subsection{Screen of additives}

Table S6 Screen of additives

\begin{tabular}{|c|c|c|c|}
\hline 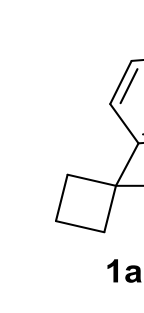 & \multicolumn{2}{|c|}{$\begin{array}{c}\text { Ph-Acr-Mes }{ }^{+} \mathrm{BF}_{4}^{-} \\
\mathrm{Cu}(\mathrm{OAc})_{2} \\
1,10-\mathrm{Phen} \\
\text { Additive } \\
\text { DCM, Blue LED }\end{array}$} & 1 \\
\hline Entry & Additive & 1a conv. (\%) & Yield $\mathbf{1}(\%)$ \\
\hline 1 & I & 32 & 24 \\
\hline 2 & $\mathrm{KF}(30 \%)$ & 35 & 25 \\
\hline 3 & $\mathrm{Et}_{3} \mathrm{~N}(30 \%)$ & 31 & 18 \\
\hline 4 & TMEDA $(30 \%)$ & 14 & 14 \\
\hline 5 & BTMG (30\%) & 13 & 13 \\
\hline 6 & ${ }^{\mathrm{n}} \mathrm{Bu}_{4} \mathrm{NPF}_{6}(30 \%)$ & 15 & 20 \\
\hline 7 & ${ }^{\mathrm{n}} \mathrm{Bu} 4 \mathrm{NCl}(30 \%)$ & 16 & 15 \\
\hline 8 & $\mathrm{~K}_{3} \mathrm{PO}_{4}$ (1.0 equiv.) & 29 & 17 \\
\hline 9 & DBU (1.0 equiv.) & 16 & 5 \\
\hline $10^{\mathrm{c}}$ & l & 35 & 25 \\
\hline $12^{\mathrm{c}}$ & BI-OAc (1.0 equiv.) & 60 & 8 \\
\hline $11^{\mathrm{c}}$ & ${ }^{\mathrm{n}} \mathrm{Bu}_{4} \mathrm{P}^{+}(\mathrm{OMe})_{2} \mathrm{POO}^{-}$(0.4 equiv. & 69 & 60 \\
\hline $12^{\mathrm{c}}$ & collidines ( 0.4 equiv.) & 38 & 32 \\
\hline
\end{tabular}

${ }^{a}$ Reaction conditions: 1a $(0.15 \mathrm{mmol}, 1.5$ equiv. $)$, Togni I reagent $(0.10 \mathrm{mmol}, 1.0$ equiv.), Ph-Acr-Mes ${ }^{+} \mathrm{BF}_{4}{ }^{-}$(0.002 mmol, 0.02 equiv.), $\mathrm{Cu}(\mathrm{OAc})_{2}(0.02 \mathrm{mmol}, 0.2$ equiv.), 1,10-phen (0.03 mmol, 0.3 equiv.) and additives in $2.0 \mathrm{~mL}$ DCM under nitrogen with $4 \mathrm{~W}$ blue LED ( $5 \mathrm{~cm}$ away from the vial) irradiation at $25{ }^{\circ} \mathrm{C}$ for $12 \mathrm{~h}$, unless otherwise noted. ${ }^{b}$ Conversions and yields were determined by ${ }^{1} \mathrm{H}$ NMR analysis. ${ }^{\mathrm{c}} 80 \mathrm{~W}$ blue LED, $2 \mathrm{~h}$. 


\subsection{Screen of ligands}

Table S7 Screen of ligands

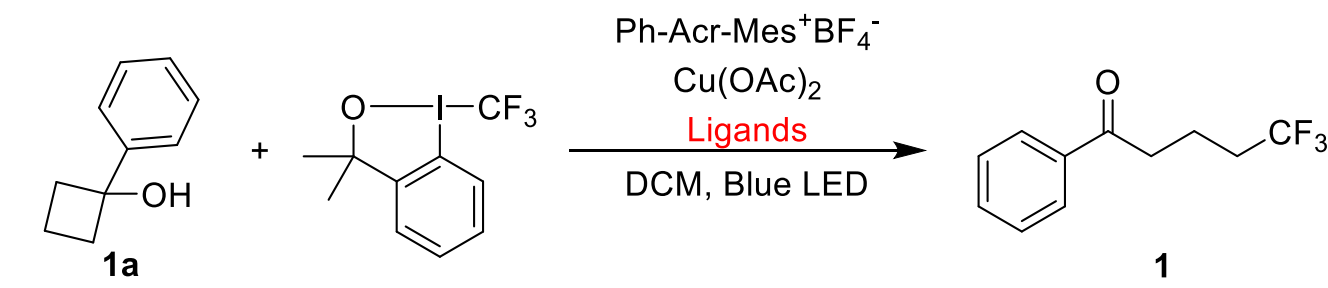

\begin{tabular}{cccc}
\hline Entry & Ligand $^{a}$ & 1a conv. & Yield $\mathbf{1}(\%)^{b}$ \\
\hline 1 & $\mathbf{L 1}$ & 32 & 24 \\
2 & $\mathbf{L 2}$ & 18 & 16 \\
3 & $\mathbf{L 3}$ & 27 & 15 \\
4 & $\mathbf{L 4}$ & $<5$ & 0
\end{tabular}

${ }^{a}$ Reaction conditions: $1 \mathrm{a}(0.15 \mathrm{mmol}, 1.5$ equiv. $)$, Togni I reagent $(0.10 \mathrm{mmol}, 1.0$ equiv.), $\mathrm{Ph}$-Acr-Mes ${ }^{+} \mathrm{BF}_{4}{ }^{-}$(0.002 mmol, 0.02 equiv.), $\mathrm{Cu}(\mathrm{OAc})_{2}(0.02 \mathrm{mmol}, 0.2$ equiv.) and Ligands ( $0.03 \mathrm{mmol}, 0.3$ equiv.) in $2.0 \mathrm{~mL}$ DCM under nitrogen with $4 \mathrm{~W}$ blue LED ( $5 \mathrm{~cm}$ away from the vial) irradiation at $25{ }^{\circ} \mathrm{C}$ for $12 \mathrm{~h}$, unless otherwise noted. ${ }^{b}$ Conversions and yields were determined by ${ }^{1} \mathrm{H}$ NMR analysis. 


\subsection{Control experiments}

Table S8 Control experiments

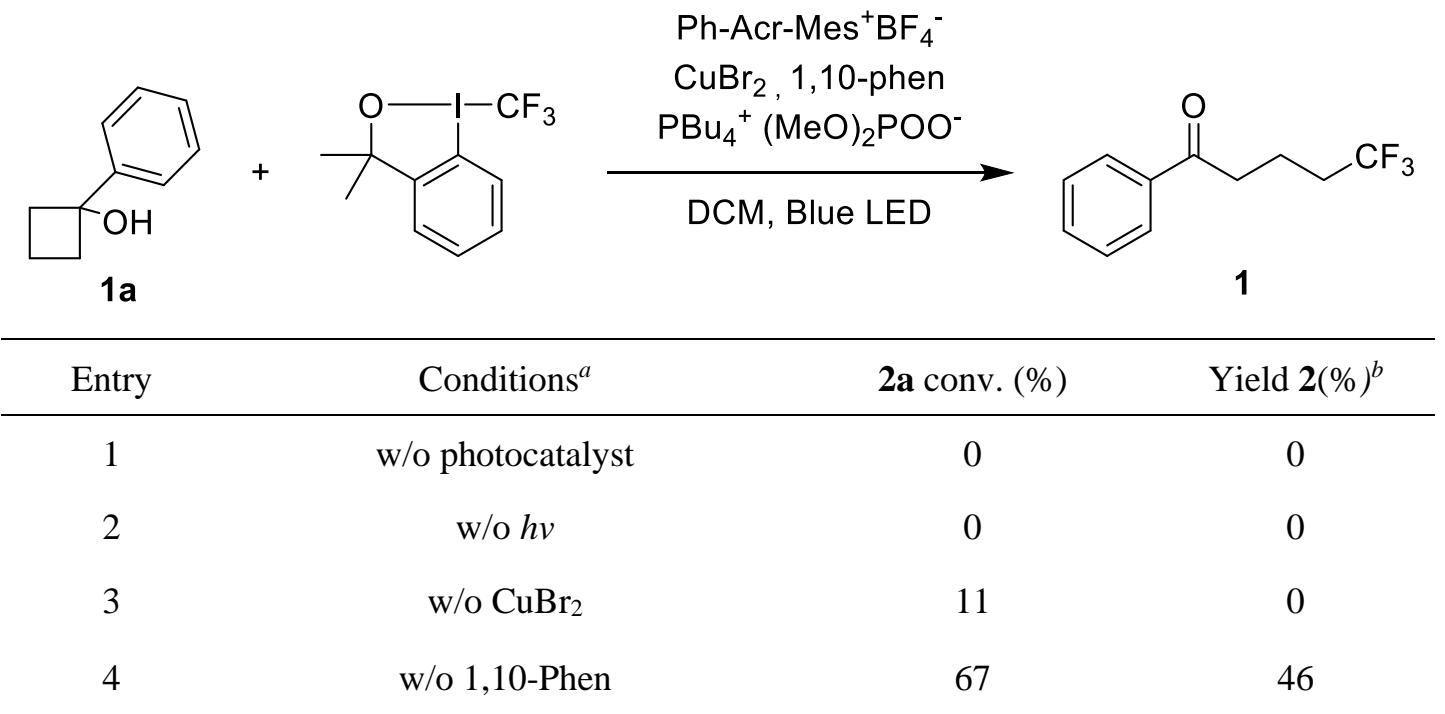

${ }^{a}$ Reaction conditions: 1a $(0.15 \mathrm{mmol}, 1.5$ equiv. $)$, Togni I reagent $(0.10 \mathrm{mmol}, 1.0$ equiv.), $\mathrm{Ph}-\mathrm{Acr}-\mathrm{Mes}^{+} \mathrm{BF}_{4}{ }^{-}$(0.002 mmol, 0.02 equiv.), $\mathrm{CuBr}_{2}$ (0.02 mmol, 0.2 equiv.) and 1,10-phen ( $0.03 \mathrm{mmol}, 0.3$ equiv.) in $2.0 \mathrm{~mL}$ DCM under nitrogen with $4 \mathrm{~W}$ blue LED ( $5 \mathrm{~cm}$ away from the vial) irradiation at $25^{\circ} \mathrm{C}$ for $12 \mathrm{~h}$, unless otherwise noted. ${ }^{b}$ Conversions and yields were determined by ${ }^{1} \mathrm{H}$ NMR analysis. 


\section{Substrates Preparation and Characterization}

\subsection{Synthesis of cycloalkanols}

\section{General procedure A with Grignard reactions:}

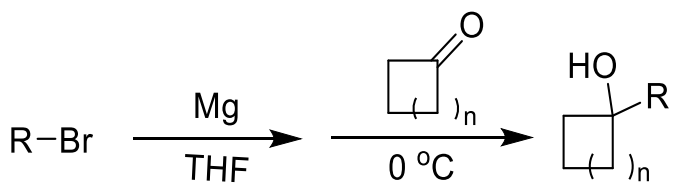

Following the literature procedure with slight modifications. ${ }^{8}$ In a flame-dried threeneck bottle, a mixture of $\mathrm{Mg}$ (15.5 mmol, 1.55 equiv) and a catalytic amount of iodine in anhydrous THF $(20 \mathrm{~mL})$ under a $\mathrm{N}_{2}$ atmosphere was added and a small amount of aryl/alkyl bromide was added to initiate reaction. After successfully initiating, the remained aryl/alkyl bromide $(15.5 \mathrm{mmol}, 1.55$ equiv, commercially available reagents were used without further purification) was added dropwise via syringe with stirring, after which the temperature was increased to $40{ }^{\circ} \mathrm{C}$ when the reaction was no longer so intense. The mixture was next heated to $70{ }^{\circ} \mathrm{C}$ by an oil bath and stirred over $2 \mathrm{~h}$ until the complete formation of the aryl Grignard reagent. The corresponding ketone (10 mmol, 1.00 equiv) in $10 \mathrm{~mL}$ THF was added to the reaction mixture at $0{ }^{\circ} \mathrm{C}$, after which the cooling was removed and kept at room temperature overnight. The ammonium chloride solution was then added to quench the reaction and extracted with ethyl acetate ( 3 x $30 \mathrm{~mL}$ ), washed with sodium chloride solution, and dried over $\mathrm{Na}_{2} \mathrm{SO}_{4}$. After filtration and concentration, the residue was purified by column chromatography (PE/EA) to afford the products.

\section{General procedure B with lithiation reaction:}

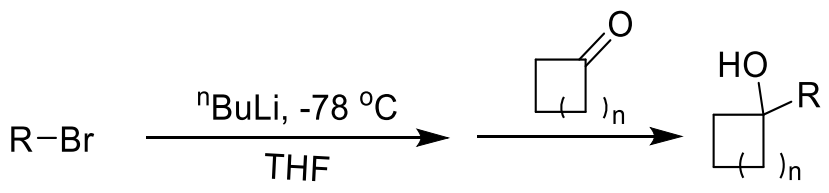

Following the literature procedure ${ }^{9}$, a flame-dried round bottom flask charged with 12 mmol of aryl/alkyl bromide (commercially available reagents were used without further purification) in $25 \mathrm{~mL}$ THF was cooled to $-78{ }^{\circ} \mathrm{C}$ and $4.5 \mathrm{~mL}$ of $\mathrm{n}-\mathrm{BuLi}(2.5 \mathrm{M}$ in hexane, 12 mmol, 1.2 eq.) was added dropwise via syringe. The mixture was stirred for $0.5 \mathrm{~h}$ at $-78^{\circ} \mathrm{C}$ before the addition of the corresponding ketone (10 mmol, 1.0 eq.), then 
the mixture was warmed to room temperature and stirred over $3 \mathrm{~h}$. The ammonium chloride solution was then added to quench the reaction and extracted with ethyl acetate (3 x $30 \mathrm{~mL}$ ), washed with sodium chloride solution, and dried over $\mathrm{Na}_{2} \mathrm{SO}_{4}$. After filtration and concentration, the residue was purified by column chromatography (PE/EA) to afford the products.<smiles>OC1(c2ccccc2)CCC1</smiles>

1-phenyl-1-cyclobutanol 1a Following the general procedure A, the reaction of bromobenzene and cyclobutanone afforded 1a as a colorless solid (1.36 g, 91\% yield) after flash chromatography (PE:EA = 10:1): ${ }^{1} \mathrm{H}$ NMR $\left(500 \mathrm{MHz}, \mathrm{CDCl}_{3}\right) \delta 7.51(\mathrm{dt}, J$ $=8.2,1.7 \mathrm{~Hz}, 2 \mathrm{H}), 7.41-7.36(\mathrm{~m}, 2 \mathrm{H}), 7.31-7.26(\mathrm{~m}, 1 \mathrm{H}), 2.62-2.54(\mathrm{~m}, 2 \mathrm{H}), 2.38$ (tdd, $J=9.5,7.5,2.9 \mathrm{~Hz}, 2 \mathrm{H}), 2.08-1.98(\mathrm{~m}, 1 \mathrm{H}), 1.75-1.64(\mathrm{~m}, 1 \mathrm{H}) .{ }^{13} \mathrm{C}$ NMR $(126$ $\left.\mathrm{MHz}_{\mathrm{CDCl}}\right) \delta 146.2,128.4,127.2,124.9,77.0,36.8,13.0$. The data is consistent with the literature. ${ }^{10}$<smiles>COc1ccc(C2(O)CCC2)cc1</smiles>

1-(4-methoxyphenyl)cyclobutan-1-ol 2a Following the general procedure A, the reaction of 1-bromo-4-methoxybenzene and cyclobutanone afforded $\mathbf{2 a}$ as a colorless oil (1.52 g, 84\% yield) after flash chromatography (PE:EA = 10:1): ${ }^{1} \mathrm{H}$ NMR (500 MHz, $\left.\mathrm{CDCl}_{3}\right) \delta 7.44-7.40(\mathrm{~m}, 2 \mathrm{H}), 6.92-6.88(\mathrm{~m}, 2 \mathrm{H}), 3.81(\mathrm{~s}, 3 \mathrm{H}), 2.58-2.50(\mathrm{~m}, 2 \mathrm{H})$, $2.40-2.32(\mathrm{~m}, 2 \mathrm{H}), 2.02-1.93(\mathrm{~m}, 1 \mathrm{H}), 1.69-1.58(\mathrm{~m}, 1 \mathrm{H}) ;{ }^{13} \mathrm{C} \mathrm{NMR}(126 \mathrm{MHz}$, $\left.\mathrm{CDCl}_{3}\right) \delta 158.7,138.5,126.3,113.7,76.7,55.3,36.8,12.9$. The data is consistent with the literature. ${ }^{10}$<smiles>COc1cccc(C2(O)CCC2)c1</smiles>

1-(3-methoxyphenyl)cyclobutan-1-ol 3a Following the general procedure A, the reaction of 1-bromo-3-methoxybenzene and cyclobutanone afforded $\mathbf{3 a}$ as a white solid (1.56 g, 86\% yield) after flash chromatography (PE:EA = 10:1): ${ }^{1} \mathrm{H}$ NMR (500 MHz, 
$\left.\mathrm{CDCl}_{3}\right) \delta 7.30(\mathrm{t}, J=7.9 \mathrm{~Hz}, 1 \mathrm{H}), 7.09(\mathrm{~d}, J=7.7 \mathrm{~Hz}, 1 \mathrm{H}), 7.07-7.04(\mathrm{~m}, 1 \mathrm{H}), 6.83$ $(\mathrm{dd}, J=8.2,2.5 \mathrm{~Hz}, 1 \mathrm{H}), 3.83(\mathrm{~s}, 3 \mathrm{H}), 2.55(\mathrm{~m}, J=11.3,7.9,5.6,2.6 \mathrm{~Hz}, 2 \mathrm{H}), 2.37$ (m, $J=12.6,6.3,2.4 \mathrm{~Hz}, 2 \mathrm{H}), 2.07-1.97(\mathrm{~m}, 2 \mathrm{H}), 1.70(\mathrm{~m}, J=16.3,11.2,8.7,7.6 \mathrm{~Hz}$, 1H). ${ }^{13} \mathrm{C} \mathrm{NMR}\left(126 \mathrm{MHz}, \mathrm{CDCl}_{3}\right) \delta 159.7,148.0,129.5,117.2,112.5,110.9,77.2$, $55.3,36.8,13.0$. The data is consistent with the literature. ${ }^{10}$<smiles>COc1ccccc1C1(O)CCC1</smiles>

1-(2-methoxyphenyl)cyclobutan-1-ol 4a Following the general procedure A, the reaction of 1-bromo-2-methoxybenzene and cyclobutanone afforded $\mathbf{4 a}$ as a colorless oil (0.84 g, $48 \%$ yield) after flash chromatography (PE:EA = 10:1): ${ }^{1} \mathrm{H}$ NMR $(500 \mathrm{MHz}$, $\left.\mathrm{CDCl}_{3}\right) \delta 7.33(\mathrm{~d}, J=7.5 \mathrm{~Hz}, 1 \mathrm{H}), 7.30-7.25(\mathrm{~m}, 1 \mathrm{H}), 6.97(\mathrm{t}, J=7.5 \mathrm{~Hz}, 1 \mathrm{H}), 6.92$ $(\mathrm{d}, J=8.2 \mathrm{~Hz}, 1 \mathrm{H}), 3.88(\mathrm{~s}, 3 \mathrm{H}), 3.68(\mathrm{~s}, 1 \mathrm{H}), 2.57-2.49(\mathrm{~m}, 2 \mathrm{H}), 2.41-2.33(\mathrm{~m}, 2 \mathrm{H})$, $2.10-2.00(\mathrm{~m}, 1 \mathrm{H}), 1.71-1.61(\mathrm{~m}, 1 \mathrm{H}) ;{ }^{13} \mathrm{C} \mathrm{NMR}\left(126 \mathrm{MHz}, \mathrm{CDCl}_{3}\right) \delta 157.3,133.2$, $128.5,125.5,120.6,110.9,76.7,55.2,35.1,14.1$. The data is consistent with the literature. $^{10}$<smiles>COc1ccc(C2(O)CCC2)cc1OC</smiles>

1-(3,4-dimethoxyphenyl)cyclobutan-1-ol 5a Following the general procedure B, the reaction of 4-bromo-1,2-dimethoxybenzene and cyclobutanone afforded $\mathbf{5} \mathbf{a}$ as a white solid (0.67 g, 32\% yield) after flash chromatography (PE:EA = 10:1): ${ }^{1} \mathrm{H}$ NMR (500 $\left.\mathrm{MHz} \mathrm{CDCl}_{3}\right) \delta 7.03(\mathrm{~d}, J=7.4 \mathrm{~Hz}, 2 \mathrm{H}), 6.85(\mathrm{~d}, J=8.1 \mathrm{~Hz}, 1 \mathrm{H}), 3.91(\mathrm{~s}, 3 \mathrm{H}), 3.88(\mathrm{~s}$, $3 \mathrm{H}), 2.59-2.51(\mathrm{~m}, 2 \mathrm{H}), 2.40-2.31(\mathrm{~m}, 2 \mathrm{H}), 2.03-1.94(\mathrm{~m}, 1 \mathrm{H}), 1.71-1.61(\mathrm{~m}$, $1 \mathrm{H}) ;{ }^{13} \mathrm{C} \mathrm{NMR}\left(126 \mathrm{MHz}, \mathrm{CDCl}_{3}\right) \delta 149.0,148.2,138.9,117.0,110.6,108.7,76.9$, $55.9,55.9,36.8,12.9$. The data is consistent with the literature. ${ }^{11}$<smiles>Cc1ccc(C2(O)CCC2)cc1</smiles>

1-(p-tolyl)cyclobutan-1-ol 6a Following the general procedure A, the reaction of 1bromo-4-methylbenzene and cyclobutanone afforded $\mathbf{6 a}$ as a (1.56 g, 96\% yield) after 
flash chromatography (PE:EA = 10:1): ${ }^{1} \mathrm{H}$ NMR $\left(500 \mathrm{MHz}, \mathrm{CDCl}_{3}\right) \delta 7.40(\mathrm{~d}, J=8.1$ $\mathrm{Hz}, 2 \mathrm{H}), 7.19$ (d, $J=7.9 \mathrm{~Hz}, 2 \mathrm{H}), 2.55(\mathrm{~m}, J=11.4,7.7,5.4,2.7 \mathrm{~Hz}, 2 \mathrm{H}), 2.40-2.33$ $(\mathrm{m}, 5 \mathrm{H}), 2.05-1.95(\mathrm{~m}, 1 \mathrm{H}), 1.68(\mathrm{~m}, J=11.2,8.6,5.6 \mathrm{~Hz}, 1 \mathrm{H}) .{ }^{13} \mathrm{C} \mathrm{NMR}(126 \mathrm{MHz}$, $\left.\mathrm{CDCl}_{3}\right) \delta 143.4,136.9,129.1,125.0,76.9,36.8,21.0,13.0$. The data is consistent with the literature. ${ }^{10}$<smiles>OC1(c2ccc(-c3ccccc3)cc2)CCC1</smiles>

1-([1,1'-biphenyl]-4-yl)cyclobutan-1-ol 7a Following the general procedure A, the reaction of 4-bromo-1,1'-biphenyl and cyclobutanone afforded $\mathbf{7 a}$ as a white solid (1.99 $\mathrm{g}, 89 \%$ yield) after flash chromatography (PE:EA = 20:1): ${ }^{1} \mathrm{H} \mathrm{NMR}\left(500 \mathrm{MHz}, \mathrm{CDCl}_{3}\right)$ $\delta 7.71-7.56(\mathrm{~m}, 6 \mathrm{H}), 7.46(\mathrm{t}, J=7.5 \mathrm{~Hz}, 2 \mathrm{H}), 7.37(\mathrm{t}, J=7.2 \mathrm{~Hz}, 1 \mathrm{H}), 2.69-2.57(\mathrm{~m}$, 2H), $2.49-2.36(\mathrm{~m}, 2 \mathrm{H}), 2.24(\mathrm{~s}, 1 \mathrm{H}), 2.06(\mathrm{~m}, J=14.6,9.8,5.0 \mathrm{~Hz}, 1 \mathrm{H}), 1.75(\mathrm{~m}, J$ $=16.4,8.2 \mathrm{~Hz}, 1 \mathrm{H}) ;{ }^{13} \mathrm{C} \mathrm{NMR}\left(126 \mathrm{MHz}, \mathrm{CDCl}_{3}\right) \delta 145.3,140.8,140.2,128.8,127.3$, $127.2,127.1,125.4,76.9,37.0,13.0$. The data is consistent with the literature. ${ }^{10}$<smiles>OC1(c2ccc(Cl)cc2)CCC1</smiles>

1-(4-chlorophenyl)cyclobutan-1-ol 8a Following the general procedure A, the reaction of 1-bromo-4-chlorobenzene and cyclobutanone afforded $\mathbf{8 a}$ as a pale yellow oil (1.51 g, 46\% yield) after flash chromatography (PE:EA = 10:1): ${ }^{1} \mathrm{H}$ NMR (500 MHz, $\left.\mathrm{CDCl}_{3}\right) \delta 7.45-7.41(\mathrm{~m}, 2 \mathrm{H}), 7.36-7.31(\mathrm{~m}, 2 \mathrm{H}), 2.56-2.48(\mathrm{~m}, 2 \mathrm{H}), 2.40-2.32$ (m, 2H), $2.02(\mathrm{~m}, J=16.5,10.3,7.3,4.9 \mathrm{~Hz}, 2 \mathrm{H}), 1.69(\mathrm{~m}, J=11.3,8.8,7.5 \mathrm{~Hz}, 1 \mathrm{H})$. ${ }^{13} \mathrm{C} \mathrm{NMR}\left(126 \mathrm{MHz}, \mathrm{CDCl}_{3}\right) \delta 144.8,133.0,128.5,126.5,76.6,37.0,12.9$. The data is consistent with the literature. ${ }^{10}$<smiles>OC1(c2ccc(Br)cc2)CCC1</smiles>

1-(4-bromophenyl)cyclobutan-1-ol 9a Following the general procedure $B$, the reaction of 1, 4-dibromobenzene and cyclobutanone afforded 9a as a white solid (1.41 
g, 83\% yield) after flash chromatography (PE:EA = 10:1): ${ }^{1} \mathrm{H}$ NMR (500 MHz, $\mathrm{CDCl}_{3}$ ) $\delta 7.51-7.47(\mathrm{~m}, 2 \mathrm{H}), 7.40-7.36(\mathrm{~m}, 2 \mathrm{H}), 2.56-2.49(\mathrm{~m}, 2 \mathrm{H}), 2.40-2.32(\mathrm{~m}, 2 \mathrm{H})$, $2.08-1.97(\mathrm{~m}, 2 \mathrm{H}), 1.70(\mathrm{dtt}, J=11.4,8.8,7.5 \mathrm{~Hz}, 1 \mathrm{H}) .{ }^{13} \mathrm{C} \mathrm{NMR}\left(126 \mathrm{MHz}, \mathrm{CDCl}_{3}\right)$ $\delta 145.3,131.5,126.8,121.1,76.6,37.0,12.9$. The data is consistent with the literature. ${ }^{10}$<smiles>OC1(c2ccc3c(c2)OCCO3)CCC1</smiles>

1-(2,3-dihydrobenzo[b] $[1,4]$ dioxin-6-yl)cyclobutan-1-ol 10a Following the general procedure $\mathrm{A}$, the reaction of 6-bromo-2,3-dihydrobenzo[b][1,4]dioxine and cyclobutanone afforded 10a as a colorless oil (0.63 g, 95\% yield) after flash chromatography (PE:EA = 10:1): ${ }^{1} \mathrm{H}$ NMR $\left(500 \mathrm{MHz}, \mathrm{CDCl}_{3}\right) \delta 7.01(\mathrm{~d}, J=2.2 \mathrm{~Hz}$, 1H), 6.97 (dd, $J=8.4,2.2 \mathrm{~Hz}, 1 \mathrm{H}), 6.86(\mathrm{~d}, J=8.4 \mathrm{~Hz}, 1 \mathrm{H}), 4.26(\mathrm{~s}, 4 \mathrm{H}), 2.51$ (m, $J=$ 11.2, 9.1, 5.3, 2.3 Hz, 2H), 2.33 (m, $J=9.5,7.7,2.9 \mathrm{~Hz}, 2 \mathrm{H}), 1.97$ (m, $J=9.5,6.2,4.8$ $\mathrm{Hz}, 2 \mathrm{H}), 1.69-1.58$ (m, 1H). ${ }^{13} \mathrm{C} \mathrm{NMR}\left(126 \mathrm{MHz}, \mathrm{CDCl}_{3}\right) \delta 143.3,142.7,139.8,118.2$, $117.1,114.2,76.6,64.4,64.4,36.8,12.9$. The data is consistent with the literature. ${ }^{11}$<smiles>OC1(c2ccc3c(c2)OCO3)CCC1</smiles>

1-(benzo[d][1,3]dioxol-5-yl)cyclobutan-1-ol 11a Following the general procedure B, the reaction of 5-bromobenzo[d][1,3]dioxole and cyclobutanone afforded 11a as a pale yellow oil (1.62 g, 84\% yield) after flash chromatography (PE:EA = 10:1): ${ }^{1} \mathrm{H}$ NMR $\left(500 \mathrm{MHz}, \mathrm{CDCl}_{3}\right) \delta 6.98(\mathrm{~d}, J=1.7 \mathrm{~Hz}, 1 \mathrm{H}), 6.95(\mathrm{dd}, J=8.0,1.8 \mathrm{~Hz}, 1 \mathrm{H}), 6.78(\mathrm{~d}, J$ $=8.0 \mathrm{~Hz}, 1 \mathrm{H}), 5.95(\mathrm{~s}, 2 \mathrm{H}), 2.53-2.46(\mathrm{~m}, 2 \mathrm{H}), 2.36-2.29(\mathrm{~m}, 2 \mathrm{H}), 2.10(\mathrm{~s}, 1 \mathrm{H}), 2.03$ $-1.93(\mathrm{~m}, 1 \mathrm{H}), 1.69-1.59(\mathrm{~m}, 1 \mathrm{H}) ;{ }^{13} \mathrm{C} \mathrm{NMR}\left(126 \mathrm{MHz}, \mathrm{CDCl}_{3}\right) \delta 147.8,146.6,140.4$, $118.1,107.9,106.2,101.0,77.0,36.9,13.0$. The data is consistent with the literature. ${ }^{11}$<smiles>OC1(c2cc3ccccc3o2)CCC1</smiles>

1-(benzofuran-2-yl)cyclobutan-1-ol 12a Following the general procedure $B$, the reaction of benzofuran and cyclobutanone afforded 12a as a white solid (0.67 g, 71\% yield) after flash chromatography (PE:EA = 10:1): ${ }^{1} \mathrm{H} \mathrm{NMR}\left(500 \mathrm{MHz}, \mathrm{CDCl}_{3}\right) \delta 7.56$ 
(d, $J=7.6 \mathrm{~Hz}, 1 \mathrm{H}), 7.49-7.46(\mathrm{~m}, 1 \mathrm{H}), 7.30-7.25(\mathrm{~m}, 1 \mathrm{H}), 7.25-7.20(\mathrm{~m}, 1 \mathrm{H}), 6.70$ - $6.67(\mathrm{~m}, 1 \mathrm{H}), 2.66-2.59(\mathrm{~m}, 2 \mathrm{H}), 2.46-2.38(\mathrm{~m}, 3 \mathrm{H}), 2.02-1.91(\mathrm{~m}, 1 \mathrm{H}), 1.85-$ $1.74(\mathrm{~m}, 1 \mathrm{H}) ;{ }^{13} \mathrm{C} \mathrm{NMR}\left(126 \mathrm{MHz}, \mathrm{CDCl}_{3}\right) \delta 160.7,155.0,128.2,124.2,122.8,121.1$, $111.3,101.5,72.7,35.6,12.8$. The data is consistent with the literature. ${ }^{12}$<smiles>OC1(c2csc3ccccc23)CCC1</smiles>

1-(benzo[b]thiophen-3-yl)cyclobutan-1-ol 13a Following the general procedure A, the reaction of 3-bromothianaphthene and cyclobutanone afforded 13a as a white solid (1.35 g, 43\% yield) after flash chromatography (PE:EA = 10:1): ${ }^{1} \mathrm{H}$ NMR (500 MHz, $\left.\mathrm{CDCl}_{3}\right) \delta 8.01(\mathrm{dd}, J=6.7,2.0 \mathrm{~Hz}, 1 \mathrm{H}), 7.87(\mathrm{dd}, J=6.6,2.0 \mathrm{~Hz}, 1 \mathrm{H}), 7.41-7.33(\mathrm{~m}$, $3 \mathrm{H}), 2.72-2.64(\mathrm{~m}, 2 \mathrm{H}), 2.56-2.47(\mathrm{~m}, 2 \mathrm{H}), 2.20(\mathrm{~s}, 1 \mathrm{H}), 2.02-1.94(\mathrm{~m}, 1 \mathrm{H}), 1.73$ $-1.62(\mathrm{~m}, 1 \mathrm{H}) ;{ }^{13} \mathrm{C} \mathrm{NMR}\left(126 \mathrm{MHz}, \mathrm{CDCl}_{3}\right) \delta 141.3,140.0,137.2,124.4,124.0,123.9$, $122.9,122.0,74.7,36.3,13.5$. The data is consistent with the literature. ${ }^{12}$<smiles>OC1(c2cccs2)CCC1</smiles>

1-(thiophen-2-yl)cyclobutan-1-ol 14a Following the general procedure A, the reaction of 2-bromothiophene and cyclobutanone afforded 14a as a pale yellow solid (3.01 g, $81 \%$ yield) after flash chromatography (PE:EA = 20:1): ${ }^{1} \mathrm{H} \mathrm{NMR}\left(500 \mathrm{MHz}, \mathrm{CDCl}_{3}\right) \delta$ $7.25(\mathrm{dd}, J=5.1,1.2 \mathrm{~Hz}, 1 \mathrm{H}), 7.07(\mathrm{dd}, J=3.5,1.2 \mathrm{~Hz}, 1 \mathrm{H}), 6.98(\mathrm{dd}, J=5.1,3.5 \mathrm{~Hz}$, 1H), $2.54(\mathrm{~m}, J=10.6,8.7,4.5,2.2 \mathrm{~Hz}, 2 \mathrm{H}), 2.48-2.40(\mathrm{~m}, 2 \mathrm{H}), 2.32(\mathrm{~s}, 1 \mathrm{H}), 1.94(\mathrm{~m}$, $J=11.2,9.5,4.8 \mathrm{~Hz}, 1 \mathrm{H}), 1.75(\mathrm{dp}, J=11.3,8.5 \mathrm{~Hz}, 1 \mathrm{H}) .{ }^{13} \mathrm{C} \mathrm{NMR}\left(126 \mathrm{MHz}, \mathrm{CDCl}_{3}\right)$ $\delta 151.7,126.7,124.6,122.8,74.8,38.6,12.7$. The data is consistent with the literature. ${ }^{10}$<smiles>Cc1ccc2ccccc2c1C1(O)CCC1</smiles>

1-(2-methylnaphthalen-1-yl)cyclobutan-1-ol 15a Following the general procedure A, the reaction of 1-bromo-2-methylnaphthalene and cyclobutanone afforded $\mathbf{1 5} \mathbf{a}$ as a pale yellow solid (2.70 g, 64\% yield) after flash chromatography (PE:EA = 20:1): IR (neat) 
3314, 2989, 2947, 1508, 1132, 1098, 1008, 902, 809, $741 \mathrm{~cm}^{-1} ;{ }^{1} \mathrm{H}$ NMR (500 MHz, $\left.\mathrm{CDCl}_{3}\right) \delta 8.13(\mathrm{~d}, J=8.5 \mathrm{~Hz}, 1 \mathrm{H}), 7.81-7.77(\mathrm{~m}, 1 \mathrm{H}), 7.66(\mathrm{~d}, J=8.3 \mathrm{~Hz}, 1 \mathrm{H}), 7.46$ $-7.37(\mathrm{~m}, 2 \mathrm{H}), 7.23(\mathrm{~d}, J=8.3 \mathrm{~Hz}, 1 \mathrm{H}), 2.93-2.84(\mathrm{~m}, 2 \mathrm{H}), 2.84-2.76(\mathrm{~m}, 2 \mathrm{H}), 2.52$ (s, 3H), $2.47-2.36(\mathrm{~m}, 1 \mathrm{H}), 1.89-1.81(\mathrm{~m}, 1 \mathrm{H}) ;{ }^{13} \mathrm{C} \mathrm{NMR}\left(126 \mathrm{MHz}, \mathrm{CDCl}_{3}\right) \delta 138.8$, 134.0, 132.9, 131.4, 130.4, 128.5, 127.9, 125.4, 125.0, 124.5, 81.1, 40.1, 22.0, 16.8; HRMS-ESI (m/z) [M+Na] $]^{+}$cal'd for $\mathrm{C}_{15} \mathrm{H}_{16} \mathrm{ONa}, 235.1093$, found 235.1094.<smiles>OC1(c2cccc3ccccc23)CCC1</smiles>

1-(naphthalen-1-yl)cyclobutan-1-ol 16a Following the general procedure $B$, the reaction of 1-bromo-naphthalene and cyclobutanone afforded 16a as a white solid ( 0.47 g, 47\% yield) after flash chromatography (PE:EA = 20:1): ${ }^{1} \mathrm{H} \mathrm{NMR}\left(500 \mathrm{MHz}, \mathrm{CDCl}_{3}\right)$ $\delta 8.31-8.27(\mathrm{~m}, 1 \mathrm{H}), 7.91-7.86(\mathrm{~m}, 1 \mathrm{H}), 7.80(\mathrm{~d}, J=8.2 \mathrm{~Hz}, 1 \mathrm{H}), 7.55-7.47(\mathrm{~m}$, $3 \mathrm{H}), 7.44(\mathrm{dd}, J=8.1,7.2 \mathrm{~Hz}, 1 \mathrm{H}), 2.91-2.82(\mathrm{~m}, 2 \mathrm{H}), 2.67-2.58(\mathrm{~m}, 2 \mathrm{H}), 2.24(\mathrm{~s}$, $1 \mathrm{H}), 2.20-2.11(\mathrm{~m}, 1 \mathrm{H}), 1.76-1.65(\mathrm{~m}, 1 \mathrm{H}) ;{ }^{13} \mathrm{C} \mathrm{NMR}\left(126 \mathrm{MHz}, \mathrm{CDCl}_{3}\right) \delta 140.3$, $134.7,130.8,128.9,128.7,126.2,125.7,125.6,124.8,122.9,78.5,36.7,14.5$. The data is consistent with the literature. ${ }^{13}$<smiles>COc1ccc(C2(O)CCCC2)cc1</smiles>

1-(4-methoxyphenyl)cyclopentan-1-ol 17a Following the general procedure A, the reaction of 1-bromo-4-methoxybenzene and cyclopentanone afforded $\mathbf{1 7} \mathbf{a}$ as a white solid ( $2.3 \mathrm{~g}, 59 \%$ yield) after flash chromatography (PE:EA = 10:1): ${ }^{1} \mathrm{H}$ NMR (500 $\left.\mathrm{MHz}, \mathrm{CDCl}_{3}\right) \delta 7.42(\mathrm{dt}, J=9.2,2.7 \mathrm{~Hz}, 2 \mathrm{H}), 6.88(\mathrm{dd}, J=8.9,2.2 \mathrm{~Hz}, 2 \mathrm{H}), 3.81(\mathrm{~s}$, $3 \mathrm{H}), 1.98(\mathrm{~s}, 6 \mathrm{H}), 1.87-1.77(\mathrm{~m}, 2 \mathrm{H}) ;{ }^{13} \mathrm{C} \mathrm{NMR}\left(126 \mathrm{MHz}, \mathrm{CDCl}_{3}\right) \delta 158.4,139.2$, $126.3,113.5,83.2,55.3,41.5,23.7$. The data is consistent with the literature. ${ }^{5}$<smiles>Cc1ccc(C2(O)CCCC2)cc1</smiles> 
1-(p-tolyl)cyclopentan-1-ol 18a Following the general procedure A, the reaction of 1bromo-4-methylbenzene and cyclopentanone afforded 18a as a yellow oil ( $0.51 \mathrm{~g}, 58 \%$ yield) after flash chromatography (PE:EA $=10: 1):{ }^{1} \mathrm{H} \mathrm{NMR}\left(500 \mathrm{MHz}, \mathrm{CDCl}_{3}\right) \delta 7.39$ $(\mathrm{d}, J=8.1 \mathrm{~Hz}, 2 \mathrm{H}), 7.16(\mathrm{~d}, J=8.1 \mathrm{~Hz}, 2 \mathrm{H}), 2.35(\mathrm{~s}, 3 \mathrm{H}), 2.05-1.94(\mathrm{~m}, 6 \mathrm{H}), 1.83(\mathrm{q}$, $J=9.0,6.6 \mathrm{~Hz}, 2 \mathrm{H}) .{ }^{13} \mathrm{C} \mathrm{NMR}\left(126 \mathrm{MHz}, \mathrm{CDCl}_{3}\right) \delta 144.1,136.4,128.9,125.0,83.4$, $41.7,23.8,21.0$. The data is consistent with the literature. ${ }^{14}$<smiles>OC1(c2ccc(-c3ccccc3)cc2)CCCC1</smiles>

1-([1,1'-biphenyl]-4-yl)cyclopentan-1-ol 19a Following the general procedure $\mathrm{A}$, the reaction of 4-bromo-1,1'-biphenyl and cyclopentanone afforded 19a as a pale yellow solid (0.47 g, 39\% yield) after flash chromatography (PE:EA = 10:1): ${ }^{1} \mathrm{H}$ NMR (500 $\left.\mathrm{MHz}, \mathrm{CDCl}_{3}\right) \delta 7.63-7.56(\mathrm{~m}, 6 \mathrm{H}), 7.44(\mathrm{t}, J=7.6 \mathrm{~Hz}, 2 \mathrm{H}), 7.35(\mathrm{t}, J=7.6 \mathrm{~Hz}, 1 \mathrm{H})$, $2.11-1.98(\mathrm{~m}, 6 \mathrm{H}), 1.87(\mathrm{~m}, J=4.6 \mathrm{~Hz}, 2 \mathrm{H}), 1.61(\mathrm{~s}, 1 \mathrm{H}) .{ }^{13} \mathrm{C} \mathrm{NMR}\left(126 \mathrm{MHz}, \mathrm{CDCl}_{3}\right)$ $\delta 146.1,140.9,139.8,128.7,127.2,127.1,127.0,125.6,83.4,41.9,23.9$. The data is consistent with the literature. ${ }^{15}$<smiles>OC1(c2ccc(Cl)cc2)CCCC1</smiles>

1-(4-chlorophenyl)cyclopentan-1-ol 20a Following the general procedure B, the reaction of 1-bromo-4-chlorobenzene and cyclopentanone afforded 20a as a yellow oil (1.76 g, 50\% yield) after flash chromatography (PE:EA = 10:1): ${ }^{1} \mathrm{H}$ NMR (500 MHz, $\left.\mathrm{CDCl}_{3}\right) \delta 7.42(\mathrm{~d}, J=8.2 \mathrm{~Hz}, 2 \mathrm{H}), 7.32-7.27(\mathrm{~m}, 2 \mathrm{H}), 2.02-1.92(\mathrm{~m}, 6 \mathrm{H}), 1.88-$ $1.80(\mathrm{~m}, 2 \mathrm{H}), 1.63(\mathrm{~s}, 1 \mathrm{H}) ;{ }^{13} \mathrm{C} \mathrm{NMR}\left(126 \mathrm{MHz}, \mathrm{CDCl}_{3}\right) \delta 145.6,132.5,128.2,126.6$, 83.1, 42.0, 23.8. The data is consistent with the literature. ${ }^{2}$

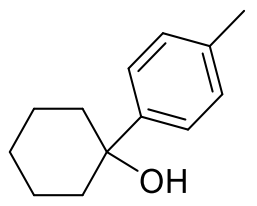


1-(p-tolyl)cyclohexan-1-ol 21a Following the general procedure A, the reaction of 1bromo-4-methylbenzene and cyclohexanone afforded 21a as a pale yellow solid (1.17 $\mathrm{g}, 62 \%$ yield) after flash chromatography (PE:EA = 10:1): ${ }^{1} \mathrm{H} \mathrm{NMR}\left(500 \mathrm{MHz}, \mathrm{CDCl}_{3}\right)$ $\delta 7.40(\mathrm{~d}, J=8.2 \mathrm{~Hz}, 2 \mathrm{H}), 7.16(\mathrm{~d}, J=8.4 \mathrm{~Hz}, 2 \mathrm{H}), 2.34(\mathrm{~s}, 3 \mathrm{H}), 1.89-1.70(\mathrm{~m}, 7 \mathrm{H})$, $1.67-1.60(\mathrm{~m}, 3 \mathrm{H}), 1.35-1.24(\mathrm{~m}, 1 \mathrm{H}) .{ }^{13} \mathrm{C} \mathrm{NMR}\left(126 \mathrm{MHz}, \mathrm{CDCl}_{3}\right) \delta 146.5,136.3$, $128.9,124.5,73.0,38.9,25.5,22.2,20.9$. The data is consistent with the literature. ${ }^{14}$

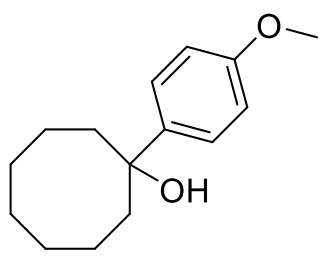

1-(4-methoxyphenyl)cyclooctan-1-ol 22a Following the general procedure A, the reaction of 1-1-bromo-4-methylbenzene and cyclooctanone afforded 22a as a white solid (1.75 g, 75\% yield) after flash chromatography (PE:EA = 10:1): ${ }^{1} \mathrm{H}$ NMR (500 $\left.\mathrm{MHz}, \mathrm{CDCl}_{3}\right) \delta 7.45-7.41(\mathrm{~m}, 2 \mathrm{H}), 6.89-6.85(\mathrm{~m}, 2 \mathrm{H}), 3.80(\mathrm{~s}, 3 \mathrm{H}), 2.08-1.92(\mathrm{~m}$, 4H), $1.78-1.65(\mathrm{~m}, 5 \mathrm{H}), 1.57-1.48(\mathrm{~m}, 6 \mathrm{H}) ;{ }^{13} \mathrm{C} \mathrm{NMR}\left(126 \mathrm{MHz}, \mathrm{CDCl}_{3}\right) \delta 158.3$, $141.2,126.3,113.3,76.4,55.2,37.7,28.4,24.5,22.1$. The data is consistent with the literature. $^{5}$

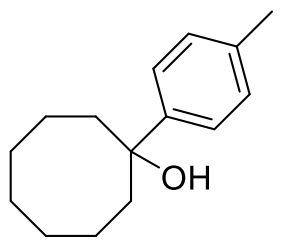

1-(p-tolyl)cyclooctan-1-ol 23a Following the general procedure A, the reaction of 11-bromo-4-methylbenzene and cyclooctanone afforded 23a as a white solid (1.55 g, 71\% yield) after flash chromatography (PE:EA = 10:1): IR (neat) 3423, 2921, 2854, 1512, 1472, 1447, 1058, 990, 820, $803 \mathrm{~cm}^{-1} ;{ }^{1} \mathrm{H}$ NMR (500 MHz, $\left.\mathrm{CDCl}_{3}\right) \delta 7.42-7.39(\mathrm{~m}$, 2H), $7.16(\mathrm{~d}, J=8.0 \mathrm{~Hz}, 2 \mathrm{H}), 2.43-2.39(\mathrm{~m}, 1 \mathrm{H}), 2.34(\mathrm{~s}, 3 \mathrm{H}), 2.07-2.00(\mathrm{~m}, 2 \mathrm{H})$, $1.99-1.92(\mathrm{~m}, 2 \mathrm{H}), 1.91-1.85(\mathrm{~m}, 1 \mathrm{H}), 1.77-1.67(\mathrm{~m}, 4 \mathrm{H}), 1.58-1.50(\mathrm{~m}, 4 \mathrm{H}) ;{ }^{13} \mathrm{C}$ NMR $\left(126 \mathrm{MHz}, \mathrm{CDCl}_{3}\right) \delta 146.1,136.2,128.8,125.0,76.5,42.0,37.7,28.4,27.2,25.7$, 24.4, 22.0, 21.0; HRMS-ESI (m/z) $[\mathrm{M}+\mathrm{Na}]^{+}$cal'd for $\mathrm{C}_{15} \mathrm{H}_{22} \mathrm{ONa}, 241.1563$, found 241.1564 . 
<smiles>COc1ccc(C2(O)CCCCCCCCCC2)cc1</smiles>

1-(4-methoxyphenyl)cyclododecan-1-ol 24a Following the general procedure B, the reaction of 1-bromo-4-methoxybenzene and cyclododecanone afforded 24a as a white solid (1.00 g, 69\% yield) after flash chromatography (PE:EA = 10:1): ${ }^{1} \mathrm{H}$ NMR (500 $\left.\mathrm{MHz}, \mathrm{CDCl}_{3}\right) \delta 7.42-7.37(\mathrm{~m}, 2 \mathrm{H}), 6.88-6.83(\mathrm{~m}, 2 \mathrm{H}), 3.80(\mathrm{~s}, 3 \mathrm{H}), 1.90-1.82(\mathrm{~m}$, $4 \mathrm{H}), 1.57(\mathrm{~s}, 1 \mathrm{H}), 1.37(\mathrm{~d}, J=12.5 \mathrm{~Hz}, 18 \mathrm{H}) ;{ }^{13} \mathrm{C} \mathrm{NMR}\left(126 \mathrm{MHz}, \mathrm{CDCl}_{3}\right) \delta 158.3$, $140.6,126.4,113.2,76.1,55.2,35.5,26.3,26.1,22.5,22.2,19.9$. The data is consistent with the literature. ${ }^{5}$

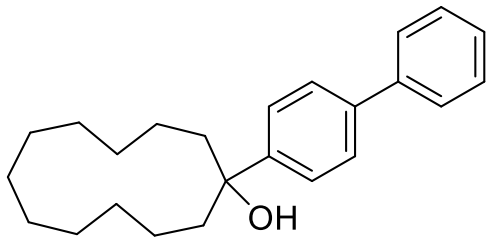

1-([1,1'-biphenyl]-4-yl)cyclododecan-1-ol 25a Following the general procedure B, the reaction of 4-bromo-1,1'-biphenyl and cyclododecanone afforded 25a as a white solid (0.55 g, 66\% yield) after flash chromatography (PE:EA = 10:1): IR (neat) 3345, 2944, 2861, 2848, 1487, 1471, 1445, 1006, 845, 768, 751, 735, $697 \mathrm{~cm}^{-1} ;{ }^{1} \mathrm{H}$ NMR (500 $\left.\mathrm{MHz}_{\mathrm{CDCl}}\right) \delta 7.60(\mathrm{~d}, J=7.4 \mathrm{~Hz}, 2 \mathrm{H}), 7.56(\mathrm{~s}, 4 \mathrm{H}), 7.44(\mathrm{t}, J=7.7 \mathrm{~Hz}, 2 \mathrm{H}), 7.36-$ $7.31(\mathrm{~m}, 1 \mathrm{H}), 1.94-1.87(\mathrm{~m}, 4 \mathrm{H}), 1.64(\mathrm{~s}, 1 \mathrm{H}), 1.44-1.32(\mathrm{~m}, 18 \mathrm{H}) ;{ }^{13} \mathrm{C}$ NMR $(126$ $\left.\mathrm{MHz}_{\mathrm{CDCl}}\right) \delta 147.4,140.9,139.5,128.7,127.2,127.0,126.6,125.7,76.4,35.5,26.3$, 26.1, 22.5, 22.2, 20.0; HRMS-EI (m/z) [M] ${ }^{+}$cal'd for $\mathrm{C}_{27} \mathrm{H}_{32} \mathrm{O}$ 336.2448, found 336.2452 .

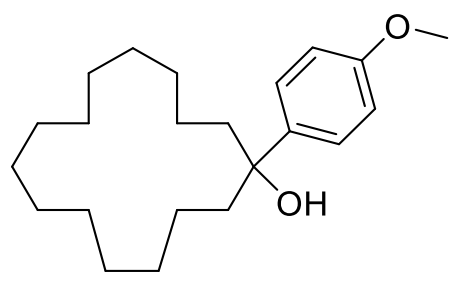

1-(4-methoxyphenyl)cyclopentadecan-1-ol 26a Following the general procedure B, the reaction of 1-bromo-4-methoxybenzene and cyclopentadecanone afforded 26a as a white solid (0.55 g, 66\% yield) after flash chromatography (PE:EA = 10:1): ${ }^{1} \mathrm{H}$ NMR $\left(500 \mathrm{MHz}, \mathrm{CDCl}_{3}\right) \delta 7.42-7.38(\mathrm{~m}, 2 \mathrm{H}), 6.89-6.85(\mathrm{~m}, 2 \mathrm{H}), 3.80$ (s, 3H), $1.88(\mathrm{~m}, J$ $=15.5,10.9,4.9 \mathrm{~Hz}, 2 \mathrm{H}), 1.80-1.72(\mathrm{~m}, 2 \mathrm{H}), 1.58(\mathrm{~s}, 1 \mathrm{H}), 1.42-1.30(\mathrm{~m}, 22 \mathrm{H}), 1.27$ 
$-1.16(\mathrm{~m}, 2 \mathrm{H}) ;{ }^{13} \mathrm{C} \mathrm{NMR}\left(126 \mathrm{MHz}, \mathrm{CDCl}_{3}\right) \delta 158.3,140.5,126.5,113.3,76.1,55.2$, $39.3,27.8,27.0,26.8,26.7,26.4,22.0$. The data is consistent with the literature. ${ }^{16}$<smiles>Cc1ccc(C2(O)CCCC2C)cc1</smiles>

2-methyl-1-(p-tolyl)cyclopentan-1-ol 28a Following the general procedure A, the reaction of 1-bromo-4-methylbenzene and 2-methylcyclopentan-1-one afforded 28a as a pale yellow oil (4.13 g, 43\% yield) after flash chromatography (PE:EA = 10:1): ${ }^{1} \mathrm{H}$ NMR (500 MHz, $\left.\mathrm{CDCl}_{3}\right) \delta 7.35(\mathrm{~d}, J=8.2 \mathrm{~Hz}, 2 \mathrm{H}), 7.16(\mathrm{~d}, J=8.0 \mathrm{~Hz}, 2 \mathrm{H}), 2.34(\mathrm{~s}$, $3 \mathrm{H}), 2.21(\mathrm{~m}, J=11.2,6.9 \mathrm{~Hz}, 1 \mathrm{H}), 2.13-2.03(\mathrm{~m}, 1 \mathrm{H}), 2.01-1.86(\mathrm{~m}, 3 \mathrm{H}), 1.78(\mathrm{~m}$, $J=18.2,12.9,9.2,4.6 \mathrm{~Hz}, 1 \mathrm{H}), 1.67-1.57(\mathrm{~m}, 2 \mathrm{H}), 0.85(\mathrm{~d}, J=6.8 \mathrm{~Hz}, 3 \mathrm{H}) ;{ }^{13} \mathrm{C} \mathrm{NMR}$ $\left(126 \mathrm{MHz}, \mathrm{CDCl}_{3}\right) \delta 143.0,136.0,128.8,124.9,83.8,45.1,43.2,31.8,21.6,21.0,12.1$. The data is consistent with the literature. ${ }^{17}$

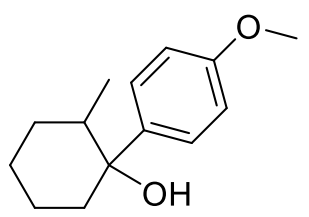

1-(4-methoxyphenyl)-2-methylcyclohexan-1-ol 29a Following the general procedure A, the reaction of 1-bromo-4-methoxybenzene and 2-methylcyclohexan-1-one afforded 29a as a colorless oil (3.29 g, 62\% yield) after flash chromatography (PE:EA = 10:1): ${ }^{1} \mathrm{H}$ NMR (500 MHz, $\left.\mathrm{CDCl}_{3}\right) \delta 7.39-7.34(\mathrm{~m}, 2 \mathrm{H}), 6.89-6.85(\mathrm{~m}, 2 \mathrm{H}), 3.80(\mathrm{~s}, 3 \mathrm{H})$, $1.94-1.85(\mathrm{~m}, 1 \mathrm{H}), 1.82-1.75(\mathrm{~m}, 1 \mathrm{H}), 1.75-1.69(\mathrm{~m}, 2 \mathrm{H}), 1.69-1.57(\mathrm{~m}, 4 \mathrm{H})$, $1.52-1.34(\mathrm{~m}, 2 \mathrm{H}), 0.63(\mathrm{~d}, J=6.8 \mathrm{~Hz}, 3 \mathrm{H}) ;{ }^{13} \mathrm{C} \mathrm{NMR}\left(126 \mathrm{MHz}, \mathrm{CDCl}_{3}\right) \delta 157.9$, $140.7,125.8,113.3,75.5,55.2,41.4,40.0,30.5,26.2,22.1,15.6$. The data is consistent with the literature. ${ }^{16}$

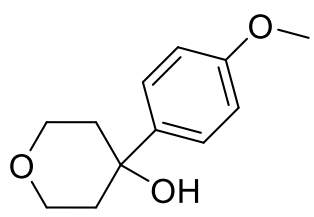

4-(4-methoxyphenyl)tetrahydro-2H-pyran-4-ol 30a Following the general procedure $\mathrm{B}$, the reaction of 1-bromo-4-methoxybenzene and tetrahydro-4H-pyran-4one afforded 30a as a white solid (0.88 g, 84\% yield) after flash chromatography $(\mathrm{PE}: \mathrm{EA}=10: 1):{ }^{1} \mathrm{H}$ NMR $\left(500 \mathrm{MHz}, \mathrm{CDCl}_{3}\right) \delta 7.43-7.39(\mathrm{~m}, 2 \mathrm{H}), 6.92-6.88(\mathrm{~m}$, 
2H), $3.92(\mathrm{td}, J=11.7,2.2 \mathrm{~Hz}, 2 \mathrm{H}), 3.87-3.82(\mathrm{~m}, 2 \mathrm{H}), 3.81(\mathrm{~s}, 3 \mathrm{H}), 2.13(\mathrm{td}, J=$ 13.0, $5.1 \mathrm{~Hz}, 2 \mathrm{H}), 1.75(\mathrm{~s}, 1 \mathrm{H}), 1.69(\mathrm{dd}, J=13.9,2.0 \mathrm{~Hz}, 2 \mathrm{H}) ;{ }^{13} \mathrm{C} \mathrm{NMR}(126 \mathrm{MHz}$, $\left.\mathrm{CDCl}_{3}\right) \delta 158.7,140.3,125.7,113.7,70.2,64.0,55.3,38.9$. The data is consistent with the literature. ${ }^{18}$

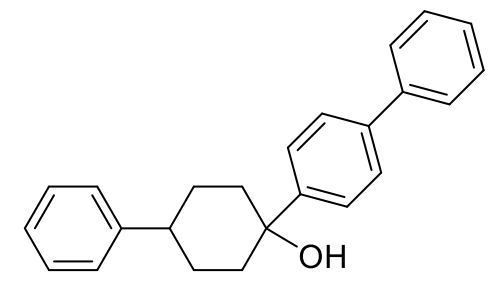

1-([1,1'-biphenyl]-4-yl)-4-phenylcyclohexan-1-ol 31a Following the general procedure $\mathrm{A}$, the reaction of 4-bromo-1,1'-biphenyl and 4-phenylcyclohexan-1-one afforded 31a as a white solid $(0.47 \mathrm{~g}, 29 \%$ yield $)$ after flash chromatography (PE:EA = 10:1): ${ }^{1} \mathrm{H}$ NMR (500 MHz, $\left.\mathrm{CDCl}_{3}\right) \delta 7.64(\mathrm{~m}, J=10.2,6.8,3.7 \mathrm{~Hz}, 6 \mathrm{H}), 7.49-7.44$ $(\mathrm{m}, 2 \mathrm{H}), 7.39-7.34(\mathrm{~m}, 1 \mathrm{H}), 7.29-7.23(\mathrm{~m}, 2 \mathrm{H}), 7.19-7.14(\mathrm{~m}, 3 \mathrm{H}), 2.77(\mathrm{t}, J=$ $10.8 \mathrm{~Hz}, 1 \mathrm{H}), 2.61(\mathrm{~d}, J=12.6 \mathrm{~Hz}, 2 \mathrm{H}), 2.05-1.91(\mathrm{~m}, 4 \mathrm{H}), 1.84(\mathrm{~s}, 1 \mathrm{H}), 1.64$ (q, $J=$ 12.1, $11.7 \mathrm{~Hz}, 2 \mathrm{H}) ;{ }^{13} \mathrm{C} \mathrm{NMR}\left(126 \mathrm{MHz}, \mathrm{CDCl}_{3}\right) \delta 146.0,143.6,140.6,140.3,128.8$, $128.3,127.4,127.4,127.1,126.8,126.7,126.0,73.1,42.9,38.1,31.0$. The data is consistent with the literature. ${ }^{16}$

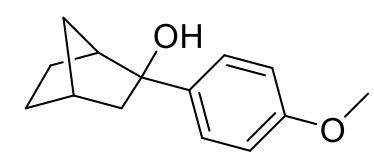

(1S,2S,4R)-2-(4-methoxyphenyl)bicyclo[2.2.1]heptan-2-ol 32a Following the general procedure $\mathrm{B}$, the reaction of 1-1-bromo-4-methylbenzene and $(1 S, 4 R)$ bicyclo[2.2.1] heptan-2-one afforded 32a as a white solid (1.71 g, 78\% yield) after flash chromatography (PE:EA = 10:1): ${ }^{1} \mathrm{H}$ NMR $\left(500 \mathrm{MHz}, \mathrm{CDCl}_{3}\right) \delta 7.44(\mathrm{~d}, J=8.7 \mathrm{~Hz}$, $2 \mathrm{H}), 6.87(\mathrm{~d}, J=8.7 \mathrm{~Hz}, 2 \mathrm{H}), 3.80(\mathrm{~s}, 3 \mathrm{H}), 2.57(\mathrm{~d}, J=3.0 \mathrm{~Hz}, 1 \mathrm{H}), 2.29$ (dd, $J=10.4$, $2.9 \mathrm{~Hz}, 2 \mathrm{H}), 2.20-2.12(\mathrm{~m}, 1 \mathrm{H}), 1.67-1.59(\mathrm{~m}, 2 \mathrm{H}), 1.54-1.42(\mathrm{~m}, 4 \mathrm{H}), 1.34-1.29$ $(\mathrm{m}, 1 \mathrm{H}) ;{ }^{13} \mathrm{C}$ NMR $\left(126 \mathrm{MHz}, \mathrm{CDCl}_{3}\right) \delta 158.3,141.3,127.1,113.5,80.5,55.3,47.3$, $46.5,38.7,37.6,29.2,22.3$. The data is consistent with the literature. ${ }^{16}$<smiles>OC1(CCc2ccccc2)CCC1</smiles> 
1-phenethylcyclobutan-1-ol 33a Following the general procedure A, the reaction of benzofuran and cyclobutanone afforded 33a as a white solid (2.4 g, 67\% yield) after flash chromatography $(\mathrm{PE}: \mathrm{EA}=20: 1):{ }^{1} \mathrm{H} \mathrm{NMR}\left(500 \mathrm{MHz}, \mathrm{CDCl}_{3}\right) \delta 7.34-7.29(\mathrm{~m}$, $2 \mathrm{H}), 7.25(\mathrm{~d}, J=7.2 \mathrm{~Hz}, 2 \mathrm{H}), 7.23-7.19(\mathrm{~m}, 1 \mathrm{H}), 2.77-2.71(\mathrm{~m}, 2 \mathrm{H}), 2.17-2.10(\mathrm{~m}$, 2H), $2.10-2.02(\mathrm{~m}, 2 \mathrm{H}), 1.98-1.93(\mathrm{~m}, 2 \mathrm{H}), 1.91(\mathrm{~s}, 1 \mathrm{H}), 1.85-1.76(\mathrm{~m}, 1 \mathrm{H}), 1.63$ $-1.52(\mathrm{~m}, 1 \mathrm{H}) ;{ }^{13} \mathrm{C} \mathrm{NMR}\left(126 \mathrm{MHz}, \mathrm{CDCl}_{3}\right) \delta 142.6,128.5,128.4,125.8,75.3,41.4$, $36.1,30.0,12.2$. The data is consistent with the literature. ${ }^{19}$<smiles>COc1ccc(CCCC2(O)CCC2)cc1</smiles>

1-(3-(4-methoxyphenyl)propyl)cyclobutan-1-ol 34a Following the general procedure A, the reaction of benzofuran and cyclobutanone afforded $\mathbf{3 4 a}$ as a white solid $(1.1 \mathrm{~g}$, 97\% yield) after flash chromatography (PE:EA = 20:1): IR (neat) 3387, 2933, 2836, 1612, 1513, 1463, 1300, 1246, 1177, 1037, $830 \mathrm{~cm}-1 ;{ }^{1} \mathrm{H}$ NMR $\left(500 \mathrm{MHz}, \mathrm{CDCl}_{3}\right) \delta$ $7.11(\mathrm{~d}, J=8.7 \mathrm{~Hz}, 2 \mathrm{H}), 6.85-6.80(\mathrm{~m}, 2 \mathrm{H}), 3.79(\mathrm{~s}, 3 \mathrm{H}), 2.60(\mathrm{t}, J=7.4 \mathrm{~Hz}, 2 \mathrm{H})$, $2.07-1.93(\mathrm{~m}, 4 \mathrm{H}), 1.77-1.59(\mathrm{~m}, 6 \mathrm{H}), 1.54-1.44(\mathrm{~m}, 1 \mathrm{H}) ;{ }^{13} \mathrm{C}$ NMR $(126 \mathrm{MHz}$, $\left.\mathrm{CDCl}_{3}\right) \delta 157.7,134.5,129.3,113.7,75.3,55.3,39.0,36.0,35.2,25.5,12.1$; HRMS-FI $(\mathrm{m} / \mathrm{z})[\mathrm{M}]+$ cal'd for $\mathrm{C}_{14} \mathrm{H}_{20} \mathrm{O}_{2} 220.1458$, found 220.1456 .

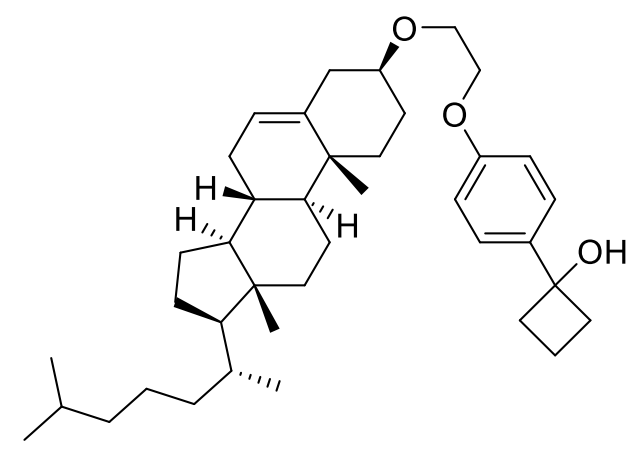

1-(4-(2-(((3S,8S,9S,10R,13R,14S,17R)-10,13-dimethyl-17-((R)-6-methylheptan-2yl)-2,3,4,7,8,9,10,11,12,13,14,15,16,17-tetradecahydro- $1 H$ cyclopenta $[a]$ phenanthren-3-yl)oxy)ethoxy)phenyl)cyclobutan-1-ol 35a The synthesis was previously reported. ${ }^{11}$

\subsection{Synthesis of linear alcohols}

\section{General procedure:}




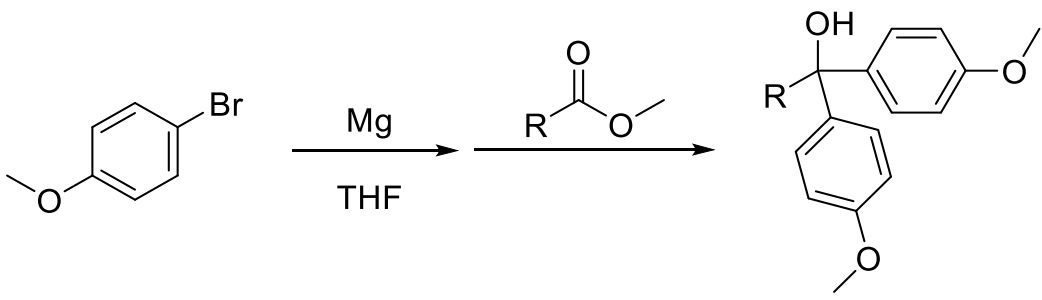

Following the literature procedure with slight modifications. ${ }^{20}$ In a flame-dried threeneck bottle, a mixture of $\mathrm{Mg}$ (31.0 mmol, 3.10 equiv) and a catalytic amount of iodine in anhydrous THF (40 mL) under a $\mathrm{N}_{2}$ atmosphere was added and a small amount of aryl bromide was added to initiate reaction. After successfully initiating, the remained aryl bromide ( $15.5 \mathrm{mmol}, 1.55$ equiv) was added dropwise via syringe with stirring, after which the temperature was increased to $40^{\circ} \mathrm{C}$ when the reaction was no longer so intense. The mixture was next heated to $70{ }^{\circ} \mathrm{C}$ by an oil bath and stirred over $2 \mathrm{~h}$ until the complete formation of the aryl Grignard reagent. The corresponding ketone (10 mmol, 1.00 equiv) in $10 \mathrm{~mL}$ THF was added into the reaction mixture at $0{ }^{\circ} \mathrm{C}$, after which the cooling was removed and kept at room temperature overnight. The ammonium chloride solution was then added to quench the reaction and extracted with ethyl acetate $(3 \times 30 \mathrm{~mL})$, washed with sodium chloride solution, and dried over $\mathrm{Na}_{2} \mathrm{SO}_{4}$. After filtration and concentration, the residue was purified by column chromatography (PE/EA) to afford the products.<smiles>COc1ccc(C(O)(c2ccc(OC)cc2)C2CCN(S(=O)(=O)c3ccc(C)cc3)CC2)cc1</smiles>

bis(4-methoxyphenyl)(1-tosylpiperidin-4-yl)methanol 27a Following the general procedure, the reaction of methyl 1-tosylpiperidine-4-carboxylate and 1-bromo-4methoxybenzene afforded 27a as a white solid (5.46 g, 89\% yield) after flash chromatography (PE:EA = 5:1): IR (neat) 3522, 2955, 2934, 2837, 1608, 1509, 1465, $1337,1249,1179,1163,1093,1034,934,826,729,647,580,549 \mathrm{~cm}^{-1} ;{ }^{1} \mathrm{H}$ NMR (500 $\left.\mathrm{MHz} \mathrm{CDCl}_{3}\right) \delta 7.62(\mathrm{~d}, J=8.2 \mathrm{~Hz}, 2 \mathrm{H}), 7.32(\mathrm{~d}, J=8.1 \mathrm{~Hz}, 2 \mathrm{H}), 7.28-7.24(\mathrm{~m}, 4 \mathrm{H})$, $6.81-6.77(\mathrm{~m}, 4 \mathrm{H}), 3.81(\mathrm{~d}, J=11.4 \mathrm{~Hz}, 2 \mathrm{H}), 3.75(\mathrm{~s}, 6 \mathrm{H}), 2.44(\mathrm{~s}, 3 \mathrm{H}), 2.25-2.15$ $(\mathrm{m}, 3 \mathrm{H}), 2.01(\mathrm{~s}, 1 \mathrm{H}), 1.59(\mathrm{~d}, J=13.0 \mathrm{~Hz}, 2 \mathrm{H}), 1.48(\mathrm{qd}, J=12.7,3.9 \mathrm{~Hz}, 2 \mathrm{H}) ;{ }^{13} \mathrm{C}$ NMR $\left(126 \mathrm{MHz} \mathrm{CDCl}_{3}\right) \delta 158.2,143.5,137.6,132.8,129.6,127.8,127.0,113.5,78.9$, 
55.2, 46.7, 43.8, 26.0, 21.6; HRMS-ESI $(\mathrm{m} / \mathrm{z})[\mathrm{M}+\mathrm{Na}]^{+}$cal'd for $\mathrm{C}_{27} \mathrm{H}_{31} \mathrm{O}_{5} \mathrm{NNaS}$, 504.1815, found 504.1815.

\subsection{Synthesis of 3-(p-tolyl)bicyclo[3.1.0]hexan-3-ol}

\section{General procedure:}
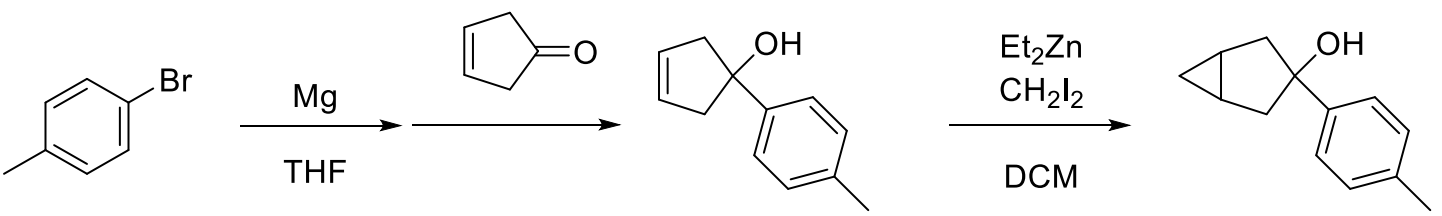

Following the literature procedure with slight modifications. ${ }^{8,21}$ In a flame-dried threeneck bottle, a mixture of $\mathrm{Mg}$ ( $86 \mathrm{mmol}, 2.0$ equiv) and a catalytic amount of iodine in anhydrous THF $(150 \mathrm{~mL})$ under a $\mathrm{N}_{2}$ atmosphere was added and a small amount of aryl bromide was added to initiate reaction. After successfully initiating, the remained aryl bromide ( $86 \mathrm{mmol}, 2.0$ equiv, commercially available reagents were used without further purification) was added dropwise via syringe with stirring, after which the temperature was increased to $40{ }^{\circ} \mathrm{C}$ when the reaction was no longer so intense. The mixture was next heated to $70{ }^{\circ} \mathrm{C}$ by an oil bath and stirred over $2 \mathrm{~h}$ until the complete formation of the aryl Grignard reagent. The cyclopent-3-en-1-one (43 mmol, 1.0 equiv) in $20 \mathrm{~mL}$ THF was added into the reaction mixture at $0{ }^{\circ} \mathrm{C}$, after which the cooling was removed and kept at room temperature overnight. The ammonium chloride solution was then added to quench the reaction and extracted with ethyl acetate $(3 \times 50 \mathrm{~mL})$, washed with sodium chloride solution, and dried over $\mathrm{Na}_{2} \mathrm{SO}_{4}$. After filtration and concentration, the residue was purified by column chromatography $(\mathrm{PE}: \mathrm{EA}=20: 1)$ to afford 1-(p-tolyl)cyclopent-3-en-1-ol as a yellow oil (2.46 g, 33\% yield). To a stirred solution of diiodomethane $(2.04 \mathrm{~g}, 11.7 \mathrm{mmol})$ in DCM $(16 \mathrm{~mL})$ under $\mathrm{N}_{2}$ atmosphere at $0{ }^{\circ} \mathrm{C}$ was added dropwise a solution of diethyl zinc in toluene $(2.0 \mathrm{M}, 11.7 \mathrm{~mL}, 23.4$ mmol) and stirred over $30 \mathrm{~min}$. 1-(p-tolyl)cyclopent-3-en-1-ol was added to the solution at $0{ }^{\circ} \mathrm{C}$. The reaction mixture was allowed to warm to room temperature and stirred for $12 \mathrm{~h}$. The ammonium chloride solution was then added to quench the reaction and the precipitated solid was removed by filtration. The filtrate was extracted with DCM $(3 \mathrm{x}$ $50 \mathrm{~mL}$ ), washed with sodium chloride solution, and dried over $\mathrm{Na}_{2} \mathrm{SO}_{4}$. After filtration and concentration, the residue was purified by column chromatography (PE:EA = 20:1) to afford 3-( $p$-tolyl)bicyclo[3.1.0]hexan-3-ol 40a as a pale yellow solid $(0.35 \mathrm{~g}, 16 \%$ 
yield): IR (neat) 3552, 3421, 3025, 2923, 2854, 1513, 1438, 1247, 1103, 1042, 945, 817, 763, $542 \mathrm{~cm}^{-1} ;{ }^{1} \mathrm{H}$ NMR $\left(500 \mathrm{MHz}, \mathrm{CDCl}_{3}\right) \delta 7.33(\mathrm{~d}, J=8.2 \mathrm{~Hz}, 2 \mathrm{H}), 7.14(\mathrm{~d}, J$ $=7.9 \mathrm{~Hz}, 2 \mathrm{H}), 2.42(\mathrm{dd}, J=14.1,3.4 \mathrm{~Hz}, 2 \mathrm{H}), 2.33(\mathrm{~s}, 3 \mathrm{H}), 2.07(\mathrm{~d}, J=14.1 \mathrm{~Hz}, 2 \mathrm{H})$, $1.45(\mathrm{dd}, J=7.8,4.2 \mathrm{~Hz}, 3 \mathrm{H}), 1.04(\mathrm{q}, J=4.0 \mathrm{~Hz}, 1 \mathrm{H}), 0.53(\mathrm{td}, J=8.3,4.9 \mathrm{~Hz}, 1 \mathrm{H})$; ${ }^{13} \mathrm{C} \mathrm{NMR}\left(126 \mathrm{MHz}, \mathrm{CDCl}_{3}\right) \delta 145.6,136.3,128.9,124.7,83.1,44.6,20.9,17.2,11.0$; HRMS-FI (m/z) [M] $]^{+}$cal'd for $\mathrm{C}_{13} \mathrm{H}_{16} \mathrm{O}, 188.1196$, found 188.1198 .

\section{Characterization of the Products}

\section{General procedure A:}

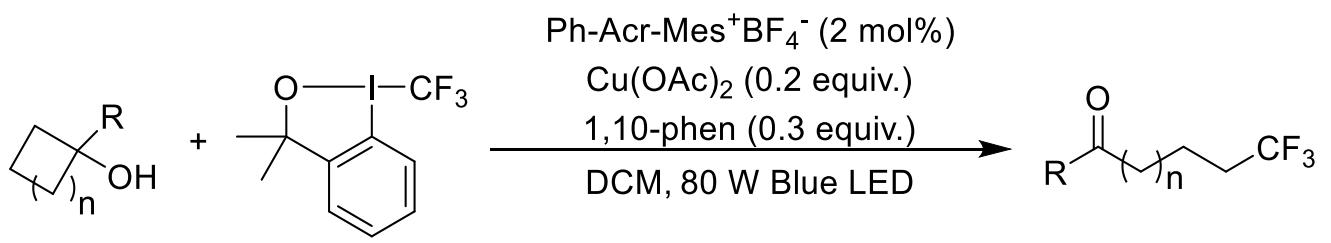

Cyclobutanol ( $0.15 \mathrm{mmol}, 1.5$ equiv.), Togni I reagent (33.0 mg, $0.1 \mathrm{mmol}, 1.0$ equiv.), $\mathrm{Ph}-\mathrm{Acr}-\mathrm{Mes}^{+} \mathrm{BF}_{4}{ }^{-}$(1.1 mg, $0.002 \mathrm{mmol}, 0.02$ equiv.), $\mathrm{CuBr}_{2}$ (3.6 mg, $0.02 \mathrm{mmol}, 0.2$ equiv.), 1,10-Phen (5.4 mg, $0.03 \mathrm{mmol}, 0.3$ equiv.) and ${ }^{n} \mathrm{Bu}_{4} \mathrm{P}^{+}(\mathrm{OMe})_{2} \mathrm{POO}^{-}(15.4 \mathrm{mg}$, $0.04 \mathrm{mmol}, 0.4$ equiv.) were placed in a $4 \mathrm{~mL}$ clear-colored glass vial equipped with a magnetic stir bar. After $2.0 \mathrm{~mL}$ degassed $\mathrm{CH}_{2} \mathrm{Cl}_{2}$ was added, the vial was sealed in the nitrogen atmosphere and exposed to blue LED (80 W LED light bulb $5 \mathrm{~cm}$ away from the vial) at room temperature for $2 \mathrm{~h}$ with stirring. After TLC indicated the complete consumption of starting materials, the reaction mixture was concentrated and purified directly by column chromatogram (PE/EA) to afford the products.

\section{General procedure B:}

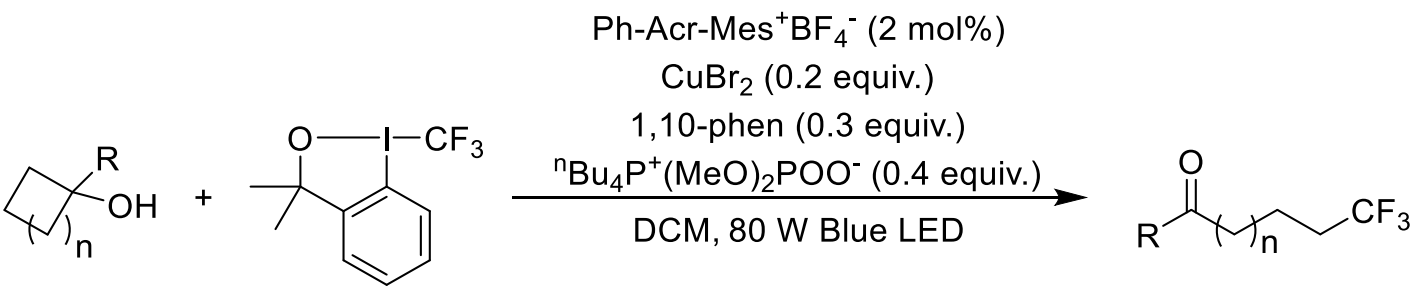

Cyclobutanol (0.15 mmol, 1.5 equiv.), Togni I reagent (33.0 mg, $0.1 \mathrm{mmol}, 1.0$ equiv.), $\mathrm{Ph}-\mathrm{Acr}-\mathrm{Mes}^{+} \mathrm{BF}_{4}{ }^{-}$(1.1 mg, $0.002 \mathrm{mmol}, 0.02$ equiv.), $\mathrm{Cu}(\mathrm{OAc})_{2}$ (3.6 mg, $0.02 \mathrm{mmol}$, 0.2 equiv.), and 1,10-Phen ( $5.4 \mathrm{mg}, 0.03 \mathrm{mmol}, 0.3$ equiv.) were placed in a $4 \mathrm{~mL}$ clearcolored glass vial equipped with a magnetic stir bar. After $2.0 \mathrm{~mL}$ degassed $\mathrm{CH}_{2} \mathrm{Cl}_{2}$ 
was added, the vial was sealed in the nitrogen atmosphere and exposed to blue LED (80 W LED light bulb $5 \mathrm{~cm}$ away from the vial) at room temperature for $2 \mathrm{~h}$ with stirring. After TLC indicated the complete consumption of starting materials, the reaction mixture was concentrated and purified directly by column chromatogram (PE/EA) to afford the products.<smiles>O=C(CCCC(F)(F)F)c1ccccc1</smiles>

5,5,5-trifluoro-1-phenylpentan-1-one 1 Following the general procedure A, the reaction of $1 \mathbf{a}(22.2 \mathrm{mg}, 0.15 \mathrm{mmol})$ afforded 1 as a white solid $(14.0 \mathrm{mg}, 65 \%$ yield $)$ after flash chromatography $(\mathrm{PE}: \mathrm{EA}=20: 1)$ : IR (neat) 1683, 1448, 1393, 1316, 1293, 1208, 1128, 1100, 1058, 1012, 736, $690 \mathrm{~cm}^{-1} ;{ }^{1} \mathrm{H} \mathrm{NMR}\left(600 \mathrm{MHz}, \mathrm{CDCl}_{3}\right) \delta 7.96(\mathrm{dt}$, $J=8.5,1.5 \mathrm{~Hz}, 2 \mathrm{H}), 7.58(\mathrm{tt}, J=7.0,1.3 \mathrm{~Hz}, 1 \mathrm{H}), 7.50-7.46(\mathrm{~m}, 2 \mathrm{H}), 3.09(\mathrm{t}, J=7.0$ $\mathrm{Hz}, 2 \mathrm{H}), 2.27-2.17(\mathrm{~m}, 2 \mathrm{H}), 2.07-2.00(\mathrm{~m}, 2 \mathrm{H}) ;{ }^{13} \mathrm{C} \mathrm{NMR}\left(151 \mathrm{MHz}, \mathrm{CDCl}_{3}\right) \delta$ 198.6, 136.7, 133.3, 128.7, 128.0, 127.1 (q, $J=276.4 \mathrm{~Hz}), 36.8,33.0$ (q, $J=28.7 \mathrm{~Hz}$ ), $16.5(\mathrm{q}, J=3.0 \mathrm{~Hz}) ;{ }^{19} \mathrm{~F}$ NMR $\left(376 \mathrm{MHz}, \mathrm{CDCl}_{3}\right) \delta-66.18(\mathrm{t}, J=10.7 \mathrm{~Hz})$; HRMS-EI $(\mathrm{m} / \mathrm{z})[\mathrm{M}]^{+}$cal'd for $\mathrm{C}_{11} \mathrm{H}_{11} \mathrm{OF}_{3}, 216.0757$, found 216.0760 .<smiles>COc1ccc(C(=O)CCCC(F)(F)F)cc1</smiles>

5,5,5-trifluoro-1-(4-methoxyphenyl)pentan-1-one 2 Following the general procedure $\mathrm{B}, \mathrm{CuBr}_{2}$ (3.6 mg, $0.02 \mathrm{mmol}, 0.2$ equiv.) as the catalyst, the reaction of $\mathbf{2 a}(26.7 \mathrm{mg}$, $0.15 \mathrm{mmol}$ ) afforded 2 as a white solid ( $17.5 \mathrm{mg}, 71 \%$ yield) after flash chromatography (PE:EA = 20:1): IR (neat) 3056, 2967, 2844, 1671, 1601, 1512, 1258, 1211, 1133, 1015, 834, $741 \mathrm{~cm}^{-1} ;{ }^{1} \mathrm{H}$ NMR (600 MHz, $\left.\mathrm{CDCl}_{3}\right) \delta 7.95-7.92(\mathrm{~m}, 2 \mathrm{H}), 6.96-6.93(\mathrm{~m}, 2 \mathrm{H})$, $3.87(\mathrm{~s}, 3 \mathrm{H}), 3.03(\mathrm{t}, J=7.0 \mathrm{~Hz}, 2 \mathrm{H}), 2.26-2.16(\mathrm{~m}, 2 \mathrm{H}), 2.05-1.98(\mathrm{~m}, 2 \mathrm{H}) ;{ }^{13} \mathrm{C}$ NMR $\left(151 \mathrm{MHz}, \mathrm{CDCl}_{3}\right) \delta 197.2,163.6,130.2,129.7,127.1(\mathrm{q}, J=276.5 \mathrm{~Hz}), 113.8$, $55.5,36.4,33.0(\mathrm{q}, J=28.6 \mathrm{~Hz}), 16.6(\mathrm{q}, J=2.9 \mathrm{~Hz}) ;{ }^{19} \mathrm{~F} \mathrm{NMR}\left(376 \mathrm{MHz}, \mathrm{CDCl}_{3}\right) \delta$ $66.20(\mathrm{t}, J=10.8 \mathrm{~Hz})$; HRMS-EI $(\mathrm{m} / \mathrm{z})[\mathrm{M}]^{+}$cal'd for $\mathrm{C}_{12} \mathrm{H}_{13} \mathrm{O}_{2} \mathrm{~F}_{3}, 246.0862$, found 246.0867 . 
<smiles>COc1cccc(C(=O)CCCC(F)(F)F)c1</smiles>

5,5,5-trifluoro-1-(3-methoxyphenyl)pentan-1-one 3 Following the general procedure A, the reaction of $\mathbf{3 a}(26.7 \mathrm{mg}, 0.15 \mathrm{mmol})$ afforded $\mathbf{3}$ as a white solid (15.9 $\mathrm{mg}, 65 \%$ yield) after flash chromatography (PE:EA = 20:1): IR (neat) 2942, 2835, 1687, 1598, 1584, 1454, 1431, 1291, 1259, 1133, 1031, 1011, 786, $686 \mathrm{~cm}^{-1} ;{ }^{1} \mathrm{H}$ NMR (600 MHz, $\left.\mathrm{CDCl}_{3}\right) \delta 7.52(\mathrm{~d}, J=7.6 \mathrm{~Hz}, 1 \mathrm{H}), 7.49-7.47(\mathrm{~m}, 1 \mathrm{H}), 7.38(\mathrm{t}, J=7.9 \mathrm{~Hz}, 1 \mathrm{H}), 7.12$ $(\mathrm{dd}, J=8.2,2.6 \mathrm{~Hz}, 1 \mathrm{H}), 3.86(\mathrm{~s}, 3 \mathrm{H}), 3.07(\mathrm{t}, J=7.0 \mathrm{~Hz}, 2 \mathrm{H}), 2.21(\mathrm{~m}, J=9.5,8.2$, $4.5 \mathrm{~Hz}, 2 \mathrm{H}), 2.06$ - $1.99(\mathrm{~m}, 2 \mathrm{H}) ;{ }^{13} \mathrm{C} \mathrm{NMR}\left(151 \mathrm{MHz}, \mathrm{CDCl}_{3}\right) \delta$ 197.8, 159.3, 137.3, 129.0, $126.4(\mathrm{q}, J=276.4 \mathrm{~Hz}), 119.9,119.0,111.6,54.8,36.3,32.3(\mathrm{q}, J=28.7 \mathrm{~Hz})$, $15.9(\mathrm{q}, J=3.0 \mathrm{~Hz}) ;{ }^{19} \mathrm{~F}$ NMR $\left(376 \mathrm{MHz}, \mathrm{CDCl}_{3}\right) \delta-66.20(\mathrm{t}, J=10.8 \mathrm{~Hz})$; HRMS-EI $(\mathrm{m} / \mathrm{z})[\mathrm{M}]^{+}$cal'd for $\mathrm{C}_{12} \mathrm{H}_{13} \mathrm{O}_{2} \mathrm{~F}_{3}, 246.0862$, found 246.0865 .<smiles>COc1ccccc1C(=O)CCCC(F)(F)F</smiles>

5,5,5-trifluoro-1-(2-methoxyphenyl)pentan-1-one 4 Following the general procedure A, the reaction of $\mathbf{4 a}(54.6 \mathrm{mg}, 0.30 \mathrm{mmol})$ in $12 \mathrm{~h}$ afforded 4 as a white solid (15.8 $\mathrm{mg}$, $64 \%$ yield) after flash chromatography (PE:EA = 20:1): IR (neat) 2947, 2841, 1674, 1598, 1486, 1467, 1439, 1298, 1286, 1249, 1133, 1102, 1027, $758 \mathrm{~cm}^{-1} ;{ }^{1} \mathrm{H}$ NMR (600 $\left.\mathrm{MHz} \mathrm{CDCl}_{3}\right) \delta 7.70(\mathrm{~d}, J=7.6 \mathrm{~Hz}, 1 \mathrm{H}), 7.49-7.44(\mathrm{~m}, 1 \mathrm{H}), 7.01(\mathrm{t}, J=7.5 \mathrm{~Hz}, 1 \mathrm{H})$, $6.97(\mathrm{~d}, J=8.4 \mathrm{~Hz}, 1 \mathrm{H}), 3.91(\mathrm{~s}, 3 \mathrm{H}), 3.07(\mathrm{t}, J=7.1 \mathrm{~Hz}, 2 \mathrm{H}), 2.23-2.13(\mathrm{~m}, 2 \mathrm{H})$, $1.97(\mathrm{~m}, J=7.2 \mathrm{~Hz}, 2 \mathrm{H}) ;{ }^{13} \mathrm{C} \mathrm{NMR}\left(151 \mathrm{MHz}, \mathrm{CDCl}_{3}\right) \delta 200.7,158.3,133.4,130.0$, $127.5,126.9$ (q, $J=276.5 \mathrm{~Hz}), 120.4,111.2,55.1,41.9,32.8(\mathrm{q}, J=28.5 \mathrm{~Hz}), 16.4(\mathrm{q}$, $J=3.1 \mathrm{~Hz}) ;{ }^{19} \mathrm{~F}$ NMR $\left(376 \mathrm{MHz}, \mathrm{CDCl}_{3}\right) \delta-66.30(\mathrm{t}, J=10.8 \mathrm{~Hz}) ; \mathrm{HRMS}-\mathrm{EI}(\mathrm{m} / \mathrm{z})$ $[\mathrm{M}]^{+}$cal'd for $\mathrm{C}_{12} \mathrm{H}_{13} \mathrm{O}_{2} \mathrm{~F}_{3}, 246.0862$, found 246.0864.<smiles>COc1ccc(C(=O)CCCC(F)(F)F)cc1OC</smiles>

1-(3,4-dimethoxyphenyl)-5,5,5-trifluoropentan-1-one 5 Following the general procedure A, the reaction of $\mathbf{5 a}(31.2 \mathrm{mg}, 0.15 \mathrm{mmol})$ afforded $\mathbf{5}$ as a yellow oil (16.9 mg, 61\% yield) after flash chromatography (PE:EA = 5:1): IR (neat) 2942, 2841, 1677, 
1596, 1587, 1516, 1465, 1419, 1261, 1166, 1131, 1024, $766 \mathrm{~cm}^{-1}$; ${ }^{1} \mathrm{H}$ NMR $(600 \mathrm{MHz}$, $\left.\mathrm{CDCl}_{3}\right) \delta 7.57(\mathrm{dd}, J=8.4,2.0 \mathrm{~Hz}, 1 \mathrm{H}), 7.52(\mathrm{~d}, J=2.0 \mathrm{~Hz}, 1 \mathrm{H}), 6.89(\mathrm{~d}, J=8.4 \mathrm{~Hz}$, $1 \mathrm{H}), 3.95(\mathrm{~s}, 3 \mathrm{H}), 3.93(\mathrm{~s}, 3 \mathrm{H}), 3.04(\mathrm{t}, J=7.0 \mathrm{~Hz}, 2 \mathrm{H}), 2.25-2.16(\mathrm{~m}, 2 \mathrm{H}), 2.05-$ $1.98(\mathrm{~m}, 2 \mathrm{H}) ;{ }^{13} \mathrm{C} \mathrm{NMR}\left(151 \mathrm{MHz}, \mathrm{CDCl}_{3}\right) \delta 197.3,153.4,149.1,129.9,127.1$ (q, $J=$ $276.4 \mathrm{~Hz}), 122.6,110.0,110.0,56.1,56.0,36.3,33.0$ (q, $J=28.6 \mathrm{~Hz}), 16.7$ (q, $J=3.0$ $\mathrm{Hz}) ;{ }^{19} \mathrm{~F} \mathrm{NMR}\left(376 \mathrm{MHz}, \mathrm{CDCl}_{3}\right) \delta-66.18(\mathrm{t}, J=10.9 \mathrm{~Hz}) ; \mathrm{HRMS}-\mathrm{EI}(\mathrm{m} / \mathrm{z})[\mathrm{M}]^{+} \mathrm{cal}^{\prime} \mathrm{d}$ for $\mathrm{C}_{13} \mathrm{H}_{15} \mathrm{O}_{3} \mathrm{~F}_{3}, 276.0968$, found 276.0969 .<smiles>Cc1ccc(C(=O)CCCC(F)(F)F)cc1</smiles>

5,5,5-trifluoro-1-( $p$-tolyl)pentan-1-one 6 Following the general procedure A, the reaction of $\mathbf{6 a}(24.3 \mathrm{mg}, 0.15 \mathrm{mmol})$ afforded $\mathbf{6}$ as a light yellow solid (16.9 mg, 73\% yield) after flash chromatography (PE:EA = 20:1): IR (neat) 1676, 1258, 1210, 1132, 1103, 1016, 816, $750 \mathrm{~cm}^{-1} ;{ }^{1} \mathrm{H}$ NMR $\left(600 \mathrm{MHz}, \mathrm{CDCl}_{3}\right) \delta 7.85(\mathrm{~d}, J=8.2 \mathrm{~Hz}, 2 \mathrm{H})$, $7.27(\mathrm{~d}, J=8.0 \mathrm{~Hz}, 2 \mathrm{H}), 3.05(\mathrm{t}, J=7.0 \mathrm{~Hz}, 2 \mathrm{H}), 2.42(\mathrm{~s}, 3 \mathrm{H}), 2.26-2.16(\mathrm{~m}, 2 \mathrm{H})$, $2.02(\mathrm{~m}, J=7.2 \mathrm{~Hz}, 2 \mathrm{H}) ;{ }^{13} \mathrm{C} \mathrm{NMR}\left(151 \mathrm{MHz}, \mathrm{CDCl}_{3}\right) \delta 198.3,144.1,134.2,129.4$, 128.1, 127.1 (q, $J=276.6 \mathrm{~Hz}), 36.7,33.0$ (q, $J=28.6 \mathrm{~Hz}), 21.7,16.5$ (q, $J=3.0 \mathrm{~Hz})$; ${ }^{19} \mathrm{~F}$ NMR $\left(376 \mathrm{MHz}, \mathrm{CDCl}_{3}\right) \delta-66.19(\mathrm{t}, J=10.8 \mathrm{~Hz})$; HRMS-EI $(\mathrm{m} / \mathrm{z})[\mathrm{M}]^{+}$cal'd for $\mathrm{C}_{12} \mathrm{H}_{13} \mathrm{OF}_{3}, 230.0913$, found 230.0916 .<smiles>O=C(CCCC(F)(F)F)c1ccc(-c2ccccc2)cc1</smiles>

1-([1,1'-biphenyl]-4-yl)-5,5,5-trifluoropentan-1-one 7 Following the general procedure A, the reaction of $7 \mathbf{a}(33.6 \mathrm{mg}, 0.15 \mathrm{mmol})$ afforded 7 as a white solid (20.7 mg, 71\% yield) after flash chromatography (PE:EA = 20:1): IR (neat) 1681, 1397, 1261, 1206, 1128, 1099, 1061, 1011, 827, 766, 747, $\left.688 \mathrm{~cm}^{-1} ;{ }^{1} \mathrm{H} \mathrm{NMR} \mathrm{(600} \mathrm{MHz,} \mathrm{CDCl}_{3}\right) \delta$ $8.03(\mathrm{~d}, J=8.3 \mathrm{~Hz}, 2 \mathrm{H}), 7.70(\mathrm{~d}, J=8.3 \mathrm{~Hz}, 2 \mathrm{H}), 7.63(\mathrm{~d}, J=7.3 \mathrm{~Hz}, 2 \mathrm{H}), 7.48(\mathrm{t}, J=$ $7.6 \mathrm{~Hz}, 2 \mathrm{H}), 7.41(\mathrm{t}, J=7.4 \mathrm{~Hz}, 1 \mathrm{H}), 3.12(\mathrm{t}, J=7.0 \mathrm{~Hz}, 2 \mathrm{H}), 2.29-2.20(\mathrm{~m}, 2 \mathrm{H}), 2.09$ $-2.03(\mathrm{~m}, 2 \mathrm{H}) ;{ }^{13} \mathrm{C} \mathrm{NMR}\left(151 \mathrm{MHz}, \mathrm{CDCl}_{3}\right) \delta 198.3,146.0,139.8,135.3,129.0,128.6$, $128.3,127.3,127.3,127.1(\mathrm{q}, J=276.5 \mathrm{~Hz}), 36.9,33.0(\mathrm{q}, J=28.6 \mathrm{~Hz}), 16.5$ (q, $J=$ 
$3.1 \mathrm{~Hz}) ;{ }^{19} \mathrm{~F}$ NMR $\left(376 \mathrm{MHz}, \mathrm{CDCl}_{3}\right) \delta-66.12(\mathrm{t}, J=10.8 \mathrm{~Hz}) ; \mathrm{HRMS}-\mathrm{EI}(\mathrm{m} / \mathrm{z})[\mathrm{M}]^{+}$ cal'd for $\mathrm{C}_{17} \mathrm{H}_{15} \mathrm{OF}_{3}, 292.1070$, found 292.1073.<smiles>O=C(CCCC(F)(F)F)c1ccc(Cl)cc1</smiles>

1-(4-chlorophenyl)-5,5,5-trifluoropentan-1-one 8 Following the general procedure A, the reaction of $8 \mathbf{a}(54.6 \mathrm{mg}, 0.30 \mathrm{mmol})$ in $12 \mathrm{~h}$ afforded $\mathbf{8}$ as a yellow oil (16.2 $\mathrm{mg}$, $65 \%$ yield) after flash chromatography (PE:EA = 20:1): IR (neat) 2949, 1688, 1590, 1400, 1255, 1210, 1133, 1093, 1013, $815 \mathrm{~cm}^{-1}$; ${ }^{1} \mathrm{H}$ NMR $\left(600 \mathrm{MHz}, \mathrm{CDCl}_{3}\right) \delta 7.90-$ $7.88(\mathrm{~m}, 2 \mathrm{H}), 7.46-7.43(\mathrm{~m}, 2 \mathrm{H}), 3.05$ (t, $J=7.0 \mathrm{~Hz}, 2 \mathrm{H}), 2.26-2.17(\mathrm{~m}, 2 \mathrm{H}), 2.05-$ $2.00(\mathrm{~m}, 2 \mathrm{H}) ;{ }^{13} \mathrm{C} \mathrm{NMR}\left(151 \mathrm{MHz}, \mathrm{CDCl}_{3}\right) \delta 197.4,139.8,134.9,129.4,129.0,127.1$ $(\mathrm{q}, J=276.6 \mathrm{~Hz}), 36.8,32.9(\mathrm{q}, J=28.7 \mathrm{~Hz}), 16.4(\mathrm{q}, J=3.0 \mathrm{~Hz}) ;{ }^{19} \mathrm{~F}$ NMR $(376 \mathrm{MHz}$, $\left.\mathrm{CDCl}_{3}\right) \delta-66.14(\mathrm{t}, J=10.7 \mathrm{~Hz}) ; \operatorname{HRMS}-\mathrm{EI}(\mathrm{m} / \mathrm{z})[\mathrm{M}]^{+}$cal'd for $\mathrm{C}_{11} \mathrm{H}_{10} \mathrm{OClF}_{3}$, 250.0367 , found 250.0368 .<smiles>O=C(CCCC(F)(F)F)c1ccc(Br)cc1</smiles>

1-(4-bromophenyl)-5,5,5-trifluoropentan-1-one 9 Following the general procedure A, the reaction of $9 \mathbf{a}(68.2 \mathrm{mg}, 0.30 \mathrm{mmol})$ in $12 \mathrm{~h}$ afforded 9 as a yellow oil (20.1 mg, $68 \%$ yield) after flash chromatography (PE:EA = 20:1): IR (neat) 2958, ,2914, 2847, 1686, 1586, 1394, 1255, 1209, 1132, 1071, 1011, 811, $751 \mathrm{~cm}^{-1}$; ${ }^{1} \mathrm{H}$ NMR (600 MHz, $\left.\mathrm{CDCl}_{3}\right) \delta 7.83-7.80(\mathrm{~m}, 2 \mathrm{H}), 7.63-7.60(\mathrm{~m}, 2 \mathrm{H}), 3.05(\mathrm{t}, J=7.0 \mathrm{~Hz}, 2 \mathrm{H}), 2.26-2.17$ (m, 2H), $2.06-1.99(\mathrm{~m}, 2 \mathrm{H}) ;{ }^{13} \mathrm{C} \mathrm{NMR}\left(151 \mathrm{MHz}, \mathrm{CDCl}_{3}\right) \delta$ 196.6, 134.3, 131.0, 128.4, 127.5, 126.0 (q, $J=276.5 \mathrm{~Hz}), 35.7,31.9(\mathrm{q}, J=28.7 \mathrm{~Hz}), 15.3(\mathrm{q}, J=3.0 \mathrm{~Hz}) ;{ }^{19} \mathrm{~F}$ NMR $\left(376 \mathrm{MHz}, \mathrm{CDCl}_{3}\right) \delta-66.14(\mathrm{t}, J=10.6 \mathrm{~Hz})$; HRMS-EI $(\mathrm{m} / \mathrm{z})[\mathrm{M}]^{+}$cal'd for $\mathrm{C}_{16} \mathrm{H}_{21} \mathrm{OF}_{3}, 293.9862$, found 293.9856.<smiles>O=C(CCCC(F)(F)F)c1ccc2c(c1)OCCO2</smiles>

1-(2,3-dihydrobenzo[b][1,4]dioxin-6-yl)-5,5,5-trifluoropentan-1-one 10 Following the general procedure $\mathrm{A}$, the reaction of $\mathbf{1 0 a}(30.9 \mathrm{mg}, 0.15 \mathrm{mmol})$ afforded $\mathbf{1 0}$ as a 
white solid (18.6 mg, 68\% yield) after flash chromatography (PE:EA = 20:1): IR (neat) 2946, 2892, 1670, 1608, 1582, 1511, 1296, 1254, 1204, 1120, 1066, 1011, $818 \mathrm{~cm}^{-1}$; ${ }^{1} \mathrm{H}$ NMR (600 MHz, $\left.\mathrm{CDCl}_{3}\right) \delta 7.50-7.47(\mathrm{~m}, 2 \mathrm{H}), 6.92-6.89(\mathrm{~m}, 1 \mathrm{H}), 4.33-4.30$ (m, 2H), $4.29-4.27(\mathrm{~m}, 2 \mathrm{H}), 2.99(\mathrm{t}, J=7.0 \mathrm{~Hz}, 2 \mathrm{H}), 2.24-2.15(\mathrm{~m}, 2 \mathrm{H}), 2.03-1.96$ (m, 2H); ${ }^{13} \mathrm{C} \mathrm{NMR}\left(151 \mathrm{MHz}, \mathrm{CDCl}_{3}\right) \delta 197.1,148.2,143.4,130.6,127.1$ (q, $J=276.4$ $\mathrm{Hz}), 122.1,117.5,117.3,64.7,64.1,36.5,33.0$ (q, $J=28.6 \mathrm{~Hz}), 16.6$ (q, $J=3.0 \mathrm{~Hz}$ ); ${ }^{19} \mathrm{~F}$ NMR $\left(376 \mathrm{MHz}, \mathrm{CDCl}_{3}\right) \delta-66.31(\mathrm{t}, J=10.8 \mathrm{~Hz})$; HRMS-EI $(\mathrm{m} / \mathrm{z})[\mathrm{M}]^{+}$cal'd for $\mathrm{C}_{13} \mathrm{H}_{13} \mathrm{O}_{3} \mathrm{~F}_{3}, 274.0811$, found 274.0809.<smiles>O=C(CCCC(F)(F)F)c1ccc2c(c1)OCO2</smiles>

1-(benzo[d][1,3]dioxol-5-yl)-5,5,5-trifluoropentan-1-one 11 Following the general procedure $\mathrm{B}$, the reaction of $\mathbf{1 1 a}(28.8 \mathrm{mg}, 0.15 \mathrm{mmol})$ afforded $\mathbf{1 1}$ as a white solid (16.3 mg, 63\% yield) after flash chromatography (PE:EA = 20:1): IR (neat) 2955, 1667, 1602, 1442, 1299, 1249, 1204, 1146, 1097, 1007, 931, 824, $745 \mathrm{~cm}^{-1} ;{ }^{1} \mathrm{H}$ NMR (600 $\left.\mathrm{MHz} \mathrm{CDCl}_{3}\right) \delta 7.55(\mathrm{dd}, J=8.2,1.7 \mathrm{~Hz}, 1 \mathrm{H}), 7.43(\mathrm{~d}, J=1.7 \mathrm{~Hz}, 1 \mathrm{H}), 6.85(\mathrm{~d}, J=8.2$ $\mathrm{Hz}, 1 \mathrm{H}), 6.05$ (s, 2H), 3.00 (t, $J=7.0 \mathrm{~Hz}, 2 \mathrm{H}), 2.20$ (m, $J=10.9,9.5,6.7 \mathrm{~Hz}, 2 \mathrm{H}), 2.00$ $(\mathrm{dt}, J=14.5,7.2 \mathrm{~Hz}, 2 \mathrm{H}) ;{ }^{13} \mathrm{C} \mathrm{NMR}\left(151 \mathrm{MHz}, \mathrm{CDCl}_{3}\right) \delta 196.7,151.9,148.3,131.5$, 127.1 (q, $J=276.5 \mathrm{~Hz}), 124.2,108.0,107.8,101.9,36.6,33.0$ (q, $J=28.6 \mathrm{~Hz}), 16.7$ (q, $J=2.9 \mathrm{~Hz}) ;{ }^{19} \mathrm{~F}$ NMR $\left(376 \mathrm{MHz}, \mathrm{CDCl}_{3}\right) \delta-62.45(\mathrm{t}, J=10.7 \mathrm{~Hz}) ;$ HRMS-EI $(\mathrm{m} / \mathrm{z})$ $[\mathrm{M}]^{+}$cal'd for $\mathrm{C}_{12} \mathrm{H}_{11} \mathrm{O}_{3} \mathrm{~F}_{3}, 260.0655$, found 260.0654 .

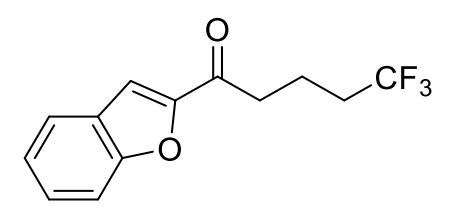

1-(benzofuran-2-yl)-5,5,5-trifluoropentan-1-one 12 Following the general procedure A, the reaction of $\mathbf{1 2 a}(28.3 \mathrm{mg}, 0.15 \mathrm{mmol})$ afforded $\mathbf{1 2}$ as a white solid (15.4 $\mathrm{mg}, 60 \%$ yield) after flash chromatography (PE:EA = 20:1): IR (neat) 2984, 1958, 2927, 1683, 1391, 1262, 1208, 1148, 1129, 1102, 1008, 835, 755, $435 \mathrm{~cm}^{-1} ;{ }^{1} \mathrm{H}$ NMR (600 MHz, $\left.\mathrm{CDCl}_{3}\right) \delta 7.72(\mathrm{~d}, J=7.9 \mathrm{~Hz}, 1 \mathrm{H}), 7.58(\mathrm{~d}, J=8.4 \mathrm{~Hz}, 1 \mathrm{H}), 7.52(\mathrm{~s}, 1 \mathrm{H}), 7.51-7.47$ (m, 1H), $7.34-7.30(\mathrm{~m}, 1 \mathrm{H}), 3.09(\mathrm{t}, J=7.2 \mathrm{~Hz}, 2 \mathrm{H}), 2.29-2.19(\mathrm{~m}, 2 \mathrm{H}), 2.10-2.03$ $(\mathrm{m}, 2 \mathrm{H}) ;{ }^{13} \mathrm{C} \mathrm{NMR}\left(151 \mathrm{MHz}, \mathrm{CDCl}_{3}\right) \delta 189.7,155.4,152.1,128.2,126.8(\mathrm{q}, J=276.5$ $\mathrm{Hz}), 126.8,123.8,123.1,112.5,112.3,36.9,32.7$ (q, $J=28.9 \mathrm{~Hz}), 16.1$ (q, $J=3.2 \mathrm{~Hz})$; 
${ }^{19} \mathrm{~F}$ NMR $\left(376 \mathrm{MHz}, \mathrm{CDCl}_{3}\right) \delta-70.92(\mathrm{t}, J=10.4 \mathrm{~Hz})$; HRMS-EI $(\mathrm{m} / \mathrm{z})[\mathrm{M}]^{+}$cal'd for $\mathrm{C}_{13} \mathrm{H}_{11} \mathrm{O}_{2} \mathrm{~F}_{3}, 256.0706$, found 256.0711.<smiles>O=C(CCCC(F)(F)F)c1csc2ccccc12</smiles>

1-(benzo[b]thiophen-3-yl)-5,5,5-trifluoropentan-1-one 13 Following the general procedure A, the reaction of $\mathbf{1 3 a}(30.7 \mathrm{mg}, 0.15 \mathrm{mmol})$ afforded $\mathbf{1 3}$ as a yellow oil (13.8 mg, 51\% yield) after flash chromatography (PE:EA = 20:1): IR (neat) 3101, 2949, 1671, 1494, 1459, 1425, 1258, 1134, 1102, 1012, 762, $735 \mathrm{~cm}^{-1} ;{ }^{1} \mathrm{H}$ NMR (600 MHz, $\mathrm{CDCl}_{3}$ ) $\delta 8.76(\mathrm{dt}, J=8.2,0.9 \mathrm{~Hz}, 1 \mathrm{H}), 8.29(\mathrm{~s}, 1 \mathrm{H}), 7.87(\mathrm{dt}, J=8.0,0.8 \mathrm{~Hz}, 1 \mathrm{H}), 7.50(\mathrm{~m}, J$ = 8.2, 7.1, 1.1 Hz, 1H), $7.43(\mathrm{td}, J=7.7,7.3,1.1 \mathrm{~Hz}, 1 \mathrm{H}), 3.12(\mathrm{t}, J=7.1 \mathrm{~Hz}, 2 \mathrm{H}), 2.30$ $-2.21(\mathrm{~m}, 2 \mathrm{H}), 2.11-2.04(\mathrm{~m}, 2 \mathrm{H}) ;{ }^{13} \mathrm{C} \mathrm{NMR}\left(151 \mathrm{MHz}, \mathrm{CDCl}_{3}\right) \delta 193.9,139.8,136.7$, $136.5,134.9,127.1$ (q, $J=276.5 \mathrm{~Hz}), 126.0,125.6,125.6,122.3,38.2,33.0$ (q, $J=28.6$ $\mathrm{Hz}), 16.6(\mathrm{q}, J=3.1 \mathrm{~Hz}) ;{ }^{19} \mathrm{~F} \mathrm{NMR}\left(376 \mathrm{MHz}, \mathrm{CDCl}_{3}\right) \delta-66.11$ (t, $\left.J=10.2 \mathrm{~Hz}\right)$; HRMS$\mathrm{EI}(\mathrm{m} / \mathrm{z})[\mathrm{M}]^{+}$cal'd for $\mathrm{C}_{13} \mathrm{H}_{11} \mathrm{OF}_{3} \mathrm{~S}, 272.0477$, found 272.0480 .<smiles>O=C(CCCC(F)(F)F)c1cccs1</smiles>

5,5,5-trifluoro-1-(thiophen-2-yl)pentan-1-one 14 Following the general procedure A, the reaction of $\mathbf{1 4 a}(23.1 \mathrm{mg}, 0.15 \mathrm{mmol})$ afforded $\mathbf{1 4}$ as a yellow oil $(11.6 \mathrm{mg}, 53 \%$ yield) after flash chromatography (PE:EA = 20:1): IR (neat) 3094, 2946, 1666, 1519, 1417, 1256, 1222, 1209, 1134, 1015, 853, $725 \mathrm{~cm}^{-1} ;{ }^{1} \mathrm{H}$ NMR (600 MHz, $\left.\mathrm{CDCl}_{3}\right) \delta 7.72$ $(\mathrm{dd}, J=3.8,1.1 \mathrm{~Hz}, 1 \mathrm{H}), 7.65(\mathrm{dd}, J=4.9,1.1 \mathrm{~Hz}, 1 \mathrm{H}), 7.14(\mathrm{dd}, J=4.9,3.8 \mathrm{~Hz}, 1 \mathrm{H})$, $3.02(\mathrm{t}, J=7.1 \mathrm{~Hz}, 2 \mathrm{H}), 2.26-2.17(\mathrm{~m}, 2 \mathrm{H}), 2.06-2.00(\mathrm{~m}, 2 \mathrm{H}) ;{ }^{13} \mathrm{C} \mathrm{NMR}(151 \mathrm{MHz}$, $\left.\mathrm{CDCl}_{3}\right) \delta 191.6,143.9,133.8,131.9,128.2,127.0(\mathrm{q}, J=276.5 \mathrm{~Hz}), 37.5,32.9(\mathrm{q}, J=$ $28.6 \mathrm{~Hz}), 16.7(\mathrm{q}, J=3.2 \mathrm{~Hz}) ;{ }^{19} \mathrm{~F}$ NMR $\left(376 \mathrm{MHz}, \mathrm{CDCl}_{3}\right) \delta-66.18(\mathrm{t}, J=10.7 \mathrm{~Hz})$; HRMS-EI (m/z) [M] $]^{+}$cal'd for $\mathrm{C}_{9} \mathrm{H}_{9} \mathrm{OSF}_{3}, 222.0321$, found 222.0324 .<smiles>Cc1ccc2ccccc2c1C(=O)CCCC(F)(F)F</smiles> 
5,5,5-trifluoro-1-(2-methylnaphthalen-1-yl)pentan-1-one 15 Following the general procedure A, the reaction of $\mathbf{1 5 a}(31.8 \mathrm{mg}, 0.15 \mathrm{mmol})$ afforded 15 as a colorless oil (16.1 mg, 58\% yield) after flash chromatography (PE:EA = 20:1): IR (neat) 3053, 2952, 1702, 1509, 1391, 1253, 1203, 1189, 1133, 1092, 1019, 814, 744, $444 \mathrm{~cm}^{-1} ;{ }^{1} \mathrm{H}$ NMR $\left(600 \mathrm{MHz}, \mathrm{CDCl}_{3}\right) \delta 7.85-7.82(\mathrm{~m}, 1 \mathrm{H}), 7.78(\mathrm{~d}, J=8.4 \mathrm{~Hz}, 1 \mathrm{H}), 7.50-7.44(\mathrm{~m}, 3 \mathrm{H})$, $7.30(\mathrm{~d}, J=8.4 \mathrm{~Hz}, 1 \mathrm{H}), 2.95(\mathrm{t}, J=7.1 \mathrm{~Hz}, 2 \mathrm{H}), 2.40(\mathrm{~s}, 3 \mathrm{H}), 2.33-2.23(\mathrm{~m}, 2 \mathrm{H})$, $2.14-2.07(\mathrm{~m}, 2 \mathrm{H}) ;{ }^{13} \mathrm{C} \mathrm{NMR}\left(151 \mathrm{MHz}, \mathrm{CDCl}_{3}\right) \delta 209.0,138.0,131.7,130.2,129.1$, $129.0,128.5,128.4,127.0,127.0$ (q, $J=276.4 \mathrm{~Hz}), 125.6,123.6,43.7,33.0$ (q, $J=28.8$ $\mathrm{Hz}), 19.3,16.1$ (q, $J=3.0 \mathrm{~Hz}) ;{ }^{19} \mathrm{~F}$ NMR $\left(376 \mathrm{MHz}, \mathrm{CDCl}_{3}\right) \delta-66.18(\mathrm{t}, J=10.7 \mathrm{~Hz})$; HRMS-EI (m/z) [M] $]^{+}$cal'd for $\mathrm{C}_{16} \mathrm{H}_{15} \mathrm{OF}_{3}, 280.1070$, found 280.1071 .<smiles>O=C(CCCC(F)(F)F)c1cccc2ccccc12</smiles>

5,5,5-trifluoro-1-(naphthalen-1-yl)pentan-1-one 16 Following the general procedure A, the reaction of $\mathbf{1 6 a}(28.5 \mathrm{mg}, 0.15 \mathrm{mmol})$ afforded $\mathbf{1 6}$ as a colorless oil (12.9 $\mathrm{mg}$, $48 \%$ yield) after flash chromatography (PE:EA = 20:1): IR (neat) 3050, 2946, 1682, 1509, 1391, 1289, 1254, 1133, 1091, 1013, 932, 802, $776 \mathrm{~cm}^{-1} ;{ }^{1} \mathrm{H}$ NMR (600 MHz, $\left.\mathrm{CDCl}_{3}\right) \delta 8.60(\mathrm{~d}, J=8.6 \mathrm{~Hz}, 1 \mathrm{H}), 8.01(\mathrm{~d}, J=8.2 \mathrm{~Hz}, 1 \mathrm{H}), 7.90-7.85(\mathrm{~m}, 2 \mathrm{H}), 7.60$ (ddd, $J=8.5,6.8,1.4 \mathrm{~Hz}, 1 \mathrm{H}), 7.57-7.49(\mathrm{~m}, 2 \mathrm{H}), 3.17$ (t, $J=7.1 \mathrm{~Hz}, 2 \mathrm{H}), 2.31-$ $2.21(\mathrm{~m}, 2 \mathrm{H}), 2.13-2.06(\mathrm{~m}, 2 \mathrm{H}) ;{ }^{13} \mathrm{C} \mathrm{NMR}\left(151 \mathrm{MHz}, \mathrm{CDCl}_{3}\right) \delta 202.8,135.5,134.0$, $132.9,130.1,128.5,128.1,127.1$ (q, $J=276.3 \mathrm{~Hz}), 127.5,126.6,125.6,124.4,40.2$, $33.0(\mathrm{q}, J=28.8 \mathrm{~Hz}), 16.9(\mathrm{q}, J=3.3 \mathrm{~Hz}) ;{ }^{19} \mathrm{~F} \mathrm{NMR}\left(376 \mathrm{MHz}, \mathrm{CDCl}_{3}\right) \delta-66.13(\mathrm{t}, J$ $=10.7 \mathrm{~Hz})$; HRMS-DART $(\mathrm{m} / \mathrm{z})[\mathrm{M}+\mathrm{H}]^{+}$cal'd for $\mathrm{C}_{15} \mathrm{H}_{14} \mathrm{OF}_{3}, 267.0991$, found 267.0992 .<smiles>O=C1CCCc2ccc3ccccc3c21</smiles>

2,3-dihydrophenanthren-4(1H)-one 16-1 Following the general procedure $A$, the reaction of 16a $(28.5 \mathrm{mg}, 0.15 \mathrm{mmol})$ afforded $\mathbf{1 6 - 1}$ as a pale yellow oil $(6.7 \mathrm{mg}, 34 \%$ yield) after flash chromatography (PE:EA $=20: 1):{ }^{1} \mathrm{H} \mathrm{NMR}\left(500 \mathrm{MHz}, \mathrm{CDCl}_{3}\right) \delta 9.41$ $(\mathrm{d}, J=8.8 \mathrm{~Hz}, 1 \mathrm{H}), 7.92(\mathrm{~d}, J=8.4 \mathrm{~Hz}, 1 \mathrm{H}), 7.81(\mathrm{~d}, J=8.1 \mathrm{~Hz}, 1 \mathrm{H}), 7.63(\mathrm{~s}, 1 \mathrm{H}), 7.52$ $-7.46(\mathrm{~m}, 1 \mathrm{H}), 7.32(\mathrm{~d}, J=8.4 \mathrm{~Hz}, 1 \mathrm{H}), 3.13(\mathrm{t}, J=6.1 \mathrm{~Hz}, 2 \mathrm{H}), 2.84-2.77(\mathrm{~m}, 2 \mathrm{H})$, 
2.24 - $2.16(\mathrm{~m}, 2 \mathrm{H}) ;{ }^{13} \mathrm{C} \mathrm{NMR}\left(126 \mathrm{MHz}, \mathrm{CDCl}_{3}\right) \delta 200.5,146.8,134.2,132.8,131.4$, $128.8,128.3,127.3,127.0,126.7,125.8,41.1,31.6,23.0$. The data is consistent with the literature. ${ }^{13}$<smiles>COc1ccc(C(=O)CCCCC(F)(F)F)cc1</smiles>

6,6,6-trifluoro-1-(4-methoxyphenyl)hexan-1-one 17 Following the general procedure $\mathrm{A}$, the reaction of $\mathbf{1 7 a}(28.8 \mathrm{mg}, 0.15 \mathrm{mmol})$ afforded 17 as a white solid (19.0 mg, 73\% yield) after flash chromatography (PE:EA = 20:1): IR (neat) 3055, 2944, 2870, 2843, 1673, 1601, 1511, 1464, 1265, 1171, 1029, 836, 739, 704; ${ }^{1} \mathrm{H}$ NMR (600 $\left.\mathrm{MHz} \mathrm{CDCl}_{3}\right) \delta 7.96-7.91(\mathrm{~m}, 2 \mathrm{H}), 6.95-6.92(\mathrm{~m}, 2 \mathrm{H}), 3.87(\mathrm{~s}, 3 \mathrm{H}), 2.95(\mathrm{t}, J=7.2$ $\mathrm{Hz}, 2 \mathrm{H}), 2.13(\mathrm{~m}, J=18.7,8.0,5.3 \mathrm{~Hz}, 2 \mathrm{H}), 1.81$ (p, $J=7.3 \mathrm{~Hz}, 2 \mathrm{H}), 1.65$ (m, $J=12.2$, 4.6, $2.5 \mathrm{~Hz}, 3 \mathrm{H}) ;{ }^{13} \mathrm{C} \mathrm{NMR}\left(151 \mathrm{MHz}, \mathrm{CDCl}_{3}\right) \delta 197.9,163.3,130.1,129.8,126.9$ (q, $J=276.4 \mathrm{~Hz}), 113.6,55.3,37.4,33.6(\mathrm{q}, J=28.5 \mathrm{~Hz}), 23.2,21.6(\mathrm{q}, J=2.8 \mathrm{~Hz}) ;{ }^{19} \mathrm{~F}$ NMR $\left(376 \mathrm{MHz}, \mathrm{CDCl}_{3}\right) \delta-66.40(\mathrm{t}, J=10.9 \mathrm{~Hz})$; HRMS-EI $(\mathrm{m} / \mathrm{z})[\mathrm{M}]^{+}$cal'd for $\mathrm{C}_{13} \mathrm{H}_{15} \mathrm{O}_{2} \mathrm{~F}_{3}, 260.1019$, found 260.1023 .<smiles>Cc1ccc(C(=O)CCCCC(F)(F)F)cc1</smiles>

6,6,6-trifluoro-1-(p-tolyl)hexan-1-one 18 Following the general procedure $A$, the reaction of $\mathbf{1 8 a}$ ( $26.4 \mathrm{mg}, 0.15 \mathrm{mmol}$ ) afforded 18 as a white solid (17.5 $\mathrm{mg}, 72 \%$ yield) after flash chromatography (PE:EA = 20:1): IR (neat) 2943, 2917, 2870, 2847, 1673, 1606, 1465, 1270, 1248, 1202, 1153, 1031, $786 \mathrm{~cm}^{-1} ;{ }^{1} \mathrm{H} \mathrm{NMR}\left(600 \mathrm{MHz}, \mathrm{CDCl}_{3}\right) \delta$ $7.85(\mathrm{~d}, J=8.2 \mathrm{~Hz}, 2 \mathrm{H}), 7.26(\mathrm{~d}, J=8.0 \mathrm{~Hz}, 2 \mathrm{H}), 2.98(\mathrm{t}, J=7.2 \mathrm{~Hz}, 2 \mathrm{H}), 2.41(\mathrm{~s}, 3 \mathrm{H})$, $2.19-2.07(\mathrm{~m}, 2 \mathrm{H}), 1.82(\mathrm{p}, J=7.3 \mathrm{~Hz}, 2 \mathrm{H}), 1.69-1.62(\mathrm{~m}, 2 \mathrm{H}) ;{ }^{13} \mathrm{C} \mathrm{NMR}(151 \mathrm{MHz}$, $\left.\mathrm{CDCl}_{3}\right) \delta 197.6,142.4,132.8,127.8,126.6,125.5(\mathrm{q}, J=276.3 \mathrm{~Hz}), 36.3,32.2(\mathrm{q}, J=$ $28.5 \mathrm{~Hz}), 28.2,21.7,20.2(\mathrm{q}, J=2.9 \mathrm{~Hz}) ;{ }^{19} \mathrm{~F} \mathrm{NMR}\left(376 \mathrm{MHz}, \mathrm{CDCl}_{3}\right) \delta-66.92(\mathrm{t}, J=$ 10.4 Hz); HRMS-EI (m/z) [M] ${ }^{+}$cal'd for $\mathrm{C}_{13} \mathrm{H}_{15} \mathrm{OF}_{3}, 244.1070$, found 244.1069. 
<smiles>O=C(CCCCC(F)(F)F)c1ccc(-c2ccccc2)cc1</smiles>

1-([1,1'-biphenyl]-4-yl)-6,6,6-trifluorohexan-1-one 19 Following the general procedure A, the reaction of 19a $(35.8 \mathrm{mg}, 0.15 \mathrm{mmol})$ afforded 19 as a white solid (20.1 mg, 66\% yield) after flash chromatography (PE:EA = 20:1): IR (neat) 2955, 1676, 1399, 1272, 1248, 1193, 1133, 1100, 1020, 838, 760, $689 \mathrm{~cm}^{-1} ;{ }^{1} \mathrm{H}$ NMR (600 MHz, $\left.\mathrm{CDCl}_{3}\right) \delta 8.05-8.01(\mathrm{~m}, 2 \mathrm{H}), 7.71-7.67(\mathrm{~m}, 2 \mathrm{H}), 7.65-7.61(\mathrm{~m}, 2 \mathrm{H}), 7.48(\mathrm{t}, J=7.6$ $\mathrm{Hz}, 2 \mathrm{H}), 7.43-7.39$ (m, 1H), 3.04 (t, $J=7.2 \mathrm{~Hz}, 2 \mathrm{H}), 2.21-2.11(\mathrm{~m}, 2 \mathrm{H}), 1.86$ (p, $J$ $=7.3 \mathrm{~Hz}, 2 \mathrm{H}), 1.68(\mathrm{~m}, J=12.2,4.7,2.7 \mathrm{~Hz}, 2 \mathrm{H}) ;{ }^{13} \mathrm{C} \mathrm{NMR}\left(151 \mathrm{MHz}, \mathrm{CDCl}_{3}\right) \delta 199.0$, 145.8, 139.8, 135.5, 129.0, 128.6, 128.3, 127.3, 127.3, 127.1 (q, $J=276.3 \mathrm{~Hz}$ ), 38.0, $33.8(\mathrm{q}, J=28.5 \mathrm{~Hz}), 23.2,21.7(\mathrm{q}, J=2.9 \mathrm{~Hz}) ;{ }^{19} \mathrm{~F} \mathrm{NMR}\left(376 \mathrm{MHz}, \mathrm{CDCl}_{3}\right) \delta-66.36$ $(\mathrm{t}, J=10.8 \mathrm{~Hz})$; HRMS-EI (m/z) [M] $]^{+}$cal'd for $\mathrm{C}_{18} \mathrm{H}_{17} \mathrm{OF}_{3}, 306.1226$, found 306.1221.<smiles>O=C(CCCCC(F)(F)F)c1ccc(Cl)cc1</smiles>

1-(4-chlorophenyl)-6,6,6-trifluorohexan-1-one 20 Following the general procedure A, the reaction of $\mathbf{2 0 a}(59.0 \mathrm{mg}, 0.30 \mathrm{mmol})$ in $12 \mathrm{~h}$ afforded 20 as a yellow oil (16.7 $\mathrm{mg}, 63 \%$ yield) after flash chromatography (PE:EA = 20:1): IR (neat) 2950, 2876, 1689, 1590, 1571, 1488, 1400, 1254, 1137, 1093, 1029, 1014, $835 \mathrm{~cm}^{-1} ;{ }^{1} \mathrm{H}$ NMR (600 MHz, $\left.\mathrm{CDCl}_{3}\right) \delta 7.91-7.88(\mathrm{~m}, 2 \mathrm{H}), 7.46-7.43(\mathrm{~m}, 2 \mathrm{H}), 2.98(\mathrm{t}, J=7.2 \mathrm{~Hz}, 2 \mathrm{H}), 2.18-2.09$ $(\mathrm{m}, 2 \mathrm{H}), 1.82(\mathrm{dt}, J=15.3,7.3 \mathrm{~Hz}, 2 \mathrm{H}), 1.68-1.62(\mathrm{~m}, 2 \mathrm{H}) ;{ }^{13} \mathrm{C}$ NMR $(151 \mathrm{MHz}$, $\left.\mathrm{CDCl}_{3}\right) \delta 198.2,139.6,135.1,129.4,129.0,127.0(\mathrm{q}, J=276.3 \mathrm{~Hz}), 37.9,33.7(\mathrm{q}, J=$ 28.6 Hz), 23.0, 21.7 (q, $J=2.9 \mathrm{~Hz}) ;{ }^{19} \mathrm{~F} \mathrm{NMR}\left(376 \mathrm{MHz}, \mathrm{CDCl}_{3}\right) \delta-66.39(\mathrm{t}, J=10.8$ $\mathrm{Hz})$; HRMS-FI (m/z) [M] $]^{+}$cal'd for $\mathrm{C}_{12} \mathrm{H}_{12} \mathrm{ClOF}_{3}, 264.0523$, found 264.0527.<smiles>Cc1ccc(C(=O)CCCCCC(F)(F)F)cc1</smiles>

7,7,7-trifluoro-1-(p-tolyl)heptan-1-one 21 Following the general procedure A, the reaction of $21 \mathrm{a}(28.5 \mathrm{mg}, 0.15 \mathrm{mmol})$ afforded 21 in $12 \mathrm{~h}$ as a white solid (14.8 $\mathrm{mg}$, $58 \%$ yield) after flash chromatography (PE:EA = 20:1): IR (neat) 2940, 2867, 1673, 
1608, 1466, 1260, 1151, 1034, 810, $749 \mathrm{~cm}^{-1} ;{ }^{1} \mathrm{H}$ NMR (600 MHz, $\left.\mathrm{CDCl}_{3}\right) \delta 7.85(\mathrm{~d}, J$ $=8.2 \mathrm{~Hz}, 2 \mathrm{H}), 7.26(\mathrm{~d}, J=7.9 \mathrm{~Hz}, 2 \mathrm{H}), 2.96(\mathrm{t}, J=7.3 \mathrm{~Hz}, 2 \mathrm{H}), 2.41(\mathrm{~s}, 3 \mathrm{H}), 2.09(\mathrm{~m}$, $J=10.9,8.0,5.6 \mathrm{~Hz}, 2 \mathrm{H}), 1.76(\mathrm{dt}, J=15.1,7.3 \mathrm{~Hz}, 2 \mathrm{H}), 1.65-1.58(\mathrm{~m}, 2 \mathrm{H}), 1.46(\mathrm{~m}$, $J=7.7 \mathrm{~Hz}, 2 \mathrm{H}) ;{ }^{13} \mathrm{C} \mathrm{NMR}\left(151 \mathrm{MHz}, \mathrm{CDCl}_{3}\right) \delta 198.6,142.8,133.5,128.3,127.1$, $126.2(\mathrm{q}, J=276.5 \mathrm{~Hz}), 37.0,32.6(\mathrm{q}, J=28.4 \mathrm{~Hz}), 27.4,22.8,20.8(\mathrm{q}, J=2.7 \mathrm{~Hz})$, 20.6; ${ }^{19} \mathrm{~F}$ NMR (376 MHz, $\left.\mathrm{CDCl}_{3}\right) \delta$-68.16; HRMS-EI (m/z) $[\mathrm{M}]^{+}$cal'd for $\mathrm{C}_{14} \mathrm{H}_{17} \mathrm{OF}_{3}$, 258.1226, found 258.1223.<smiles>COc1ccc(C(=O)CCCCCCCC(F)(F)F)cc1</smiles>

9,9,9-trifluoro-1-(4-methoxyphenyl)nonan-1-one 22 Following the general procedure A, the reaction of $\mathbf{2 2 a}(35.1 \mathrm{mg}, 0.15 \mathrm{mmol})$ in $12 \mathrm{~h}$ afforded $\mathbf{2 2}$ as a white solid (22.0 mg, 73\% yield) after flash chromatography (PE:EA = 20:1): IR (neat) 2935, $2854,1667,1603,1471,1461,1255,1178,1141,1045,834,814,732 ;{ }^{1} \mathrm{H}$ NMR (600 $\left.\mathrm{MHz}, \mathrm{CDCl}_{3}\right) \delta 7.94(\mathrm{~d}, J=8.8 \mathrm{~Hz}, 2 \mathrm{H}), 6.93(\mathrm{~d}, J=8.8 \mathrm{~Hz}, 2 \mathrm{H}), 3.87(\mathrm{~s}, 3 \mathrm{H}), 2.91(\mathrm{t}$, $J=7.4 \mathrm{~Hz}, 2 \mathrm{H}), 2.10-2.00(\mathrm{~m}, 2 \mathrm{H}), 1.75-1.69(\mathrm{~m}, 2 \mathrm{H}), 1.58-1.51(\mathrm{~m}, 2 \mathrm{H}), 1.42-$ $1.33(\mathrm{~m}, 6 \mathrm{H}) ;{ }^{13} \mathrm{C}$ NMR $\left(151 \mathrm{MHz}, \mathrm{CDCl}_{3}\right) \delta 199.0,163.4,130.3,130.2,127.3$ (q, $J=$ $276.3 \mathrm{~Hz}), 113.7,55.5,38.2,33.7$ (q, $J=28.3 \mathrm{~Hz}), 29.1,29.1,28.6,24.4,21.8$ (q, $J=$ $2.7 \mathrm{~Hz}) ;{ }^{19} \mathrm{~F} \mathrm{NMR}\left(376 \mathrm{MHz}, \mathrm{CDCl}_{3}\right) \delta-66.26(\mathrm{t}, J=10.2 \mathrm{~Hz}) ; \mathrm{HRMS}-\mathrm{EI}(\mathrm{m} / \mathrm{z})[\mathrm{M}]^{+}$ cal'd for $\mathrm{C}_{16} \mathrm{H}_{21} \mathrm{O}_{2} \mathrm{~F}_{3}, 302.1488$, found 302.1485 .<smiles>Cc1ccc(C(=O)CCCCCCCC(F)(F)F)cc1</smiles>

9,9,9-trifluoro-1-(p-tolyl)nonan-1-one 23 Following the general procedure A, the reaction of 23a ( $32.8 \mathrm{mg}, 0.15 \mathrm{mmol}$ ) in $12 \mathrm{~h}$ afforded 23 as a white solid (16.0 $\mathrm{mg}$, $56 \%$ yield) after flash chromatography (PE:EA = 20:1): IR (neat) 2939, 2914, 2852, 1672, 1610, 1468, 1248, 1181, 1133, 1044, $807 \mathrm{~cm}^{-1} ;{ }^{1} \mathrm{H}$ NMR (600 MHz, $\left.\mathrm{CDCl}_{3}\right) \delta$ $7.86(\mathrm{~d}, J=8.1 \mathrm{~Hz}, 2 \mathrm{H}), 7.25(\mathrm{~d}, J=7.5 \mathrm{~Hz}, 2 \mathrm{H}), 2.94(\mathrm{t}, J=7.4 \mathrm{~Hz}, 2 \mathrm{H}), 2.41(\mathrm{~s}, 3 \mathrm{H})$, $2.10-2.01(\mathrm{~m}, 2 \mathrm{H}), 1.76-1.70(\mathrm{~m}, 2 \mathrm{H}), 1.58-1.52(\mathrm{~m}, 2 \mathrm{H}), 1.42-1.35(\mathrm{~m}, 6 \mathrm{H}) ;{ }^{13} \mathrm{C}$ NMR (151 MHz, $\left.\mathrm{CDCl}_{3}\right) \delta 200.1,143.7,134.6,129.3,130.3$ - 124.1 (m), 128.2, 38.4, $33.7(\mathrm{q}, J=28.3 \mathrm{~Hz}), 29.1,29.1,28.6,24.3,21.8(\mathrm{q}, J=2.8 \mathrm{~Hz}), 21.6 ;{ }^{19} \mathrm{~F}$ NMR $(376$ 
$\left.\mathrm{MHz}, \mathrm{CDCl}_{3}\right) \delta-66.45(\mathrm{t}, J=11.0 \mathrm{~Hz})$; HRMS-FI $(\mathrm{m} / \mathrm{z})[\mathrm{M}]^{+}$cal'd for $\mathrm{C}_{16} \mathrm{H}_{21} \mathrm{OF}_{3}$, 286.1539 , found 286.1537.<smiles>COc1ccc(C(=O)CCCCCCCCCCCC(F)(F)F)cc1</smiles>

13,13,13-trifluoro-1-(4-methoxyphenyl)tridecan-1-one 24 Following the general procedure A, the reaction of $\mathbf{2 4 a}(87.1 \mathrm{mg}, 0.3 \mathrm{mmol})$ in $12 \mathrm{~h}$ afforded $\mathbf{2 4}$ as a white solid (27.6 mg, 77\% yield) after flash chromatography (PE:EA = 20:1): IR (neat) 2928, 2855, 1679, 1602, 1510, 1466, 1258, 1170, 1032,. $831 \mathrm{~cm}^{-1} ;{ }^{1} \mathrm{H} \mathrm{NMR}\left(600 \mathrm{MHz}, \mathrm{CDCl}_{3}\right)$ $\delta 7.96-7.92(\mathrm{~m}, 2 \mathrm{H}), 6.95-6.91(\mathrm{~m}, 2 \mathrm{H}), 3.86(\mathrm{~s}, 3 \mathrm{H}), 2.90(\mathrm{t}, 2 \mathrm{H}), 2.10-2.00(\mathrm{~m}$, $2 \mathrm{H}), 1.75-1.68(\mathrm{~m}, 2 \mathrm{H}), 1.57-1.51(\mathrm{~m}, 2 \mathrm{H}), 1.40-1.24(\mathrm{~m}, 14 \mathrm{H}) ;{ }^{13} \mathrm{C}$ NMR $(151$ $\left.\mathrm{MHz}, \mathrm{CDCl}_{3}\right) \delta 199.2,163.3,130.3,130.2,127.3(\mathrm{q}, J=276.4 \mathrm{~Hz}), 113.7,55.5,38.3$, 33.7 (q, $J=28.2 \mathrm{~Hz}), 29.5,29.5,29.5,29.4,29.4,29.2,28.7,24.6,21.9$ (q, $J=2.8 \mathrm{~Hz})$; ${ }^{19} \mathrm{~F}$ NMR $\left(376 \mathrm{MHz}, \mathrm{CDCl}_{3}\right) \delta-66.44(\mathrm{t}, J=11.0 \mathrm{~Hz})$; HRMS-EI $(\mathrm{m} / \mathrm{z})[\mathrm{M}]^{+}$cal'd for $\mathrm{C}_{20} \mathrm{H}_{29} \mathrm{O}_{2} \mathrm{~F}_{3}, 358.2114$, found 358.2109.<smiles>O=C(CCCCCCCCCCCC(F)(F)F)c1ccc(-c2ccccc2)cc1</smiles>

1-([1,1'-biphenyl]-4-yl)-13,13,13-trifluorotridecan-1-one 25 Following the general procedure A, the reaction of $\mathbf{2 5 a}(101.0 \mathrm{mg}, 0.3 \mathrm{mmol})$ in $12 \mathrm{~h}$ afforded 25 as a white solid (27.5 mg, 68\% yield) after flash chromatography (PE:EA = 20:1): IR (neat) 2929, 2853, 1675, 1604, 1467, 1404, 1255, 1136, 909, $734 \mathrm{~cm}^{-1} ;{ }^{1} \mathrm{H}$ NMR (600 MHz, $\mathrm{CDCl}_{3}$ ) $\delta 8.05-8.01(\mathrm{~m}, 2 \mathrm{H}), 7.70-7.67(\mathrm{~m}, 2 \mathrm{H}), 7.65-7.62(\mathrm{~m}, 2 \mathrm{H}), 7.47$ (t, $J=7.6 \mathrm{~Hz}$, 2H), $7.42-7.38(\mathrm{~m}, 1 \mathrm{H}), 2.99(\mathrm{t}, J=7.4 \mathrm{~Hz}, 2 \mathrm{H}), 2.10-2.00(\mathrm{~m}, 2 \mathrm{H}), 1.76(\mathrm{p}, J=7.4$ $\mathrm{Hz}, 2 \mathrm{H}), 1.59-1.50(\mathrm{~m}, 2 \mathrm{H}), 1.43-1.23(\mathrm{~m}, 14 \mathrm{H}) ;{ }^{13} \mathrm{C} \mathrm{NMR}\left(151 \mathrm{MHz}, \mathrm{CDCl}_{3}\right) \delta$ 198.3, 143.6, 138.0, 133.8, 127.0, 126.7, 126.2, 125.4 (q, $J=276.2 \mathrm{~Hz}), 125.3,125.3$, 36.7, $31.8(\mathrm{q}, J=28.2 \mathrm{~Hz}), 27.5,27.5,27.5,27.4,27.4,27.2,26.7,22.5,19.9$ (q, $J=$ $2.8 \mathrm{~Hz}) ;{ }^{19} \mathrm{~F} \mathrm{NMR}\left(376 \mathrm{MHz}, \mathrm{CDCl}_{3}\right) \delta-66.39(\mathrm{t}, J=11.0 \mathrm{~Hz}) ; \mathrm{HRMS}-\mathrm{EI}(\mathrm{m} / \mathrm{z})[\mathrm{M}]^{+}$ cal'd for $\mathrm{C}_{25} \mathrm{H}_{31} \mathrm{OF}_{3}, 404.2322$, found 404.2328.

\section{Gram Scale experiments}




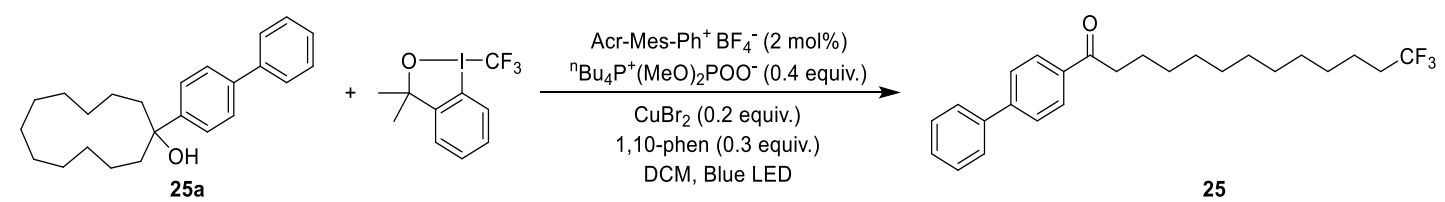

Scheme S28 Gram Scale experiments

Cycloalkanol 25a (2.02 g, 6.0 mmol, 1.50 equiv.), Togni I reagent (1.32 g, 4.0 mmol, 1.00 equiv.), $\mathrm{Ph}-\mathrm{Acr}-\mathrm{Mes}^{+} \mathrm{BF}_{4}{ }^{-}$(48 mg, $0.08 \mathrm{mmol}, 0.02$ equiv.), $\mathrm{CuBr}_{2}$ (180 mg, 0.8 mmol, 0.2 equiv.), 1,10-Phen (216 mg, $1.2 \mathrm{mmol}, 0.3$ equiv.) and ${ }^{\mathrm{n}} \mathrm{Bu}_{4} \mathrm{P}^{+}(\mathrm{OMe})_{2} \mathrm{POO}^{-}$ (616 mg, $1.6 \mathrm{mmol}, 0.4$ equiv.) were placed in a $100 \mathrm{~mL}$ Schlenk tube equipped with a magnetic stir bar. After $40 \mathrm{~mL} \mathrm{CH}_{2} \mathrm{Cl}_{2}$ was added, the vial was sealed in the nitrogen atmosphere and exposed to blue LED (80 W LED light bulb $5 \mathrm{~cm}$ away from the vial) at room temperature for $18 \mathrm{~h}$ with stirring. The reaction mixture was concentrated and purified directly by column chromatogram $(\mathrm{PE} / \mathrm{EA}=20: 1)$ to afford 25 as a white solid $(1.12 \mathrm{~g}, 70 \%$ yield $)$.<smiles>COc1ccc(C(=O)CCCCCCCCCCCCCCC(F)(F)F)cc1</smiles>

16,16,16-trifluoro-1-(4-methoxyphenyl)hexadecan-1-one 26 Following the general procedure A, the reaction of $\mathbf{2 6 a}(99.8 \mathrm{mg}, 0.3 \mathrm{mmol})$ in $12 \mathrm{~h}$ afforded $\mathbf{2 6}$ as a white solid (27.9 mg, 70\% yield) after flash chromatography (PE:EA = 20:1): IR (neat) 2915, 2847, 1667, 1601, 1468, 1258, 1246, 1143, 1051, 1027, 843, 812, 722, $656 \mathrm{~cm}^{-1} ;{ }^{1} \mathrm{H}$ NMR (600 MHz, $\left.\mathrm{CDCl}_{3}\right) \delta 7.96-7.92(\mathrm{~m}, 2 \mathrm{H}), 6.95-6.91(\mathrm{~m}, 2 \mathrm{H}), 3.86(\mathrm{~s}, 3 \mathrm{H}), 2.92$ $-2.88(\mathrm{~m}, 2 \mathrm{H}), 2.10-2.00(\mathrm{~m}, 2 \mathrm{H}), 1.71(\mathrm{p}, J=7.5 \mathrm{~Hz}, 2 \mathrm{H}), 1.54(\mathrm{p}, J=7.6 \mathrm{~Hz}, 2 \mathrm{H})$, $1.40-1.25(\mathrm{~m}, 20 \mathrm{H}) ;{ }^{13} \mathrm{C}$ NMR $\left(151 \mathrm{MHz}, \mathrm{CDCl}_{3}\right) \delta 199.0,163.1,130.1,130.0,127.1$ (q, $J=276.3 \mathrm{~Hz}), 113.4,55.2,38.1,33.5$ (q, $J=28.2 \mathrm{~Hz}), 29.4,29.4,29.4,29.3,29.3$, 29.2, 29.1, 28.9, 28.5, 24.4, 21.6 (q, $J=2.7 \mathrm{~Hz}) ;{ }^{19} \mathrm{~F} \mathrm{NMR}\left(376 \mathrm{MHz}, \mathrm{CDCl}_{3}\right) \delta-66.44$ (t, $J=11.0 \mathrm{~Hz})$; HRMS-DART $(\mathrm{m} / \mathrm{z})[\mathrm{M}+\mathrm{H}]^{+}$cal'd for $\mathrm{C}_{23} \mathrm{H}_{36} \mathrm{O}_{2} \mathrm{~F}_{3}, 401.2662$, found 401.2660 .<smiles>Cc1ccc(S(=O)(=O)N2CCC(C(F)(F)F)CC2)cc1</smiles> 
4-((difluoro- $\lambda^{3}$-methyl)- $\lambda^{2}$-fluoraneyl)-1-tosylpiperidine 27 Following the general procedure B, the reaction of $\mathbf{2 7} \mathbf{a}(144.5 \mathrm{mg}, 0.30 \mathrm{mmol})$ in $12 \mathrm{~h}$ afforded 27 as a white solid (17.8 mg, 58\% yield) after flash chromatography (PE:EA = 20:1): IR (neat) 3047, 2968, 1404, 1336, 1305, 1257, 1160, 1126, 1082, 929, 815, 741, 650, 584, 552, 422 $\mathrm{cm}^{-1} ;{ }^{1} \mathrm{H}$ NMR $\left(600 \mathrm{MHz}, \mathrm{CDCl}_{3}\right) \delta 7.64(\mathrm{~d}, J=8.2 \mathrm{~Hz}, 2 \mathrm{H}), 7.33(\mathrm{~d}, J=8.1 \mathrm{~Hz}, 2 \mathrm{H})$, $3.89(\mathrm{~d}, J=12.0 \mathrm{~Hz}, 2 \mathrm{H}), 2.44(\mathrm{~s}, 3 \mathrm{H}), 2.25(\mathrm{td}, J=12.2,2.0 \mathrm{~Hz}, 2 \mathrm{H}), 2.01-1.88(\mathrm{~m}$, $3 \mathrm{H}), 1.73-1.64(\mathrm{~m}, 2 \mathrm{H}) ;{ }^{13} \mathrm{C}$ NMR $\left(151 \mathrm{MHz}, \mathrm{CDCl}_{3}\right) \delta 142.5,131.6,128.4,126.4$, $125.5(\mathrm{q}, J=278.3 \mathrm{~Hz}), 43.7,38.4(\mathrm{q}, J=27.8 \mathrm{~Hz}), 22.7(\mathrm{~d}, J=2.4 \mathrm{~Hz}), 20.2 ;{ }^{19} \mathrm{~F} \mathrm{NMR}$ $\left(376 \mathrm{MHz}, \mathrm{CDCl}_{3}\right) \delta-73.77(\mathrm{~d}, J=7.3 \mathrm{~Hz})$; HRMS-ESI $(\mathrm{m} / \mathrm{z})[\mathrm{M}+\mathrm{H}]^{+}$cal'd for $\mathrm{C}_{13} \mathrm{H}_{17} \mathrm{O}_{2} \mathrm{NF}_{3} \mathrm{~S}, 308.0927$, found 308.0924.<smiles>Cc1ccc(C(=O)CCCC(C)(F)F)cc1</smiles>

1-([1,1'-biphenyl]-4-yl)-6,6,6-trifluorohexan-1-one 28 Following the general procedure A, the reaction of $\mathbf{2 8 a}(28.5 \mathrm{mg}, 0.15 \mathrm{mmol})$ in $12 \mathrm{~h}$ afforded $\mathbf{2 8}$ as a white solid (19.8 mg, 77\% yield) after flash chromatography (PE:EA = 20:1): IR (neat) 2950, 2870, 1682, 1468, 1266, 1171, 1134, 1015, 813, $744 \mathrm{~cm}^{-1} ;{ }^{1} \mathrm{H}$ NMR (600 MHz, $\left.\mathrm{CDCl}_{3}\right)$ $\delta 7.86(\mathrm{dd}, J=8.3,1.8 \mathrm{~Hz}, 2 \mathrm{H}), 7.26(\mathrm{~d}, J=7.9 \mathrm{~Hz}, 2 \mathrm{H}), 2.99-2.95(\mathrm{~m}, 2 \mathrm{H}), 2.41(\mathrm{~s}$, $3 \mathrm{H}), 2.23-2.14(\mathrm{~m}, 1 \mathrm{H}), 1.95-1.85(\mathrm{~m}, 1 \mathrm{H}), 1.79-1.69(\mathrm{~m}, 2 \mathrm{H}), 1.44-1.36(\mathrm{~m}$, $1 \mathrm{H}), 1.14(\mathrm{~d}, J=7.0 \mathrm{~Hz}, 3 \mathrm{H}) ;{ }^{13} \mathrm{C} \mathrm{NMR}\left(151 \mathrm{MHz}, \mathrm{CDCl}_{3}\right) \delta 199.2,143.9,134.4,128.4$ $(\mathrm{q}, J=279.3 \mathrm{~Hz}), 129.3,128.1,38.1,38.0(\mathrm{q}, J=26.2 \mathrm{~Hz}), 29.1(\mathrm{q}, J=2.1 \mathrm{~Hz}), 21.6$, 21.3, $12.6(\mathrm{q}, J=3.1 \mathrm{~Hz}) ;{ }^{19} \mathrm{~F} \mathrm{NMR}\left(376 \mathrm{MHz}, \mathrm{CDCl}_{3}\right) \delta-73.33(\mathrm{~d}, J=9.0 \mathrm{~Hz})$; HRMS$\mathrm{EI}(\mathrm{m} / \mathrm{z})[\mathrm{M}]^{+}$cal'd for $\mathrm{C}_{14} \mathrm{H}_{17} \mathrm{OF}_{3}, 258.1226$, found 258.1222 .<smiles>COc1ccc(C(=O)CCCCC(C)(F)F)cc1</smiles>

7,7,7-trifluoro-1-(4-methoxyphenyl)-6-methylheptan-1-one 29 Following the general procedure A, the reaction of $\mathbf{2 9 a}(33.0 \mathrm{mg}, 0.15 \mathrm{mmol})$ in $12 \mathrm{~h}$ afforded 29 as a yellow oil (17.4 mg, 61\% yield) after flash chromatography (PE:EA = 20:1): IR (neat) 2946, 2866, 1678, 1601, 1576, 1510, 1468, 1260, 1171, 1128, 1031, $837 \mathrm{~cm}^{-1} ;{ }^{1} \mathrm{H}$ NMR $\left(600 \mathrm{MHz} \mathrm{CDCl}_{3}\right) \delta 7.94(\mathrm{~d}, J=8.8 \mathrm{~Hz}, 2 \mathrm{H}), 6.94(\mathrm{~d}, 2 \mathrm{H}), 3.87(\mathrm{~s}, 3 \mathrm{H}), 2.93(\mathrm{td}, J=$ 7.4, $1.5 \mathrm{~Hz}, 2 \mathrm{H}), 2.20-2.10(\mathrm{~m}, 1 \mathrm{H}), 1.80-1.67(\mathrm{~m}, 3 \mathrm{H}), 1.51(\mathrm{~m}, J=16.2,6.4,3.8$ 
$\mathrm{Hz}, 1 \mathrm{H}), 1.43-1.31(\mathrm{~m}, 2 \mathrm{H}), 1.09(\mathrm{~d}, J=7.0 \mathrm{~Hz}, 3 \mathrm{H}) ;{ }^{13} \mathrm{C} \mathrm{NMR}\left(151 \mathrm{MHz}, \mathrm{CDCl}_{3}\right) \delta$ 198.5, 163.4, 130.2, 130.0, 128.4 (q, $J=279.3 \mathrm{~Hz}), 113.7,55.4,37.8,37.7$ (q, $J=27.2$ $\mathrm{Hz}), 29.2(\mathrm{q}, J=2.0 \mathrm{~Hz}), 26.3,24.2,12.5(\mathrm{q}, J=2.9 \mathrm{~Hz}) ;{ }^{19} \mathrm{~F}$ NMR $(376 \mathrm{MHz}$, Methanol- $\left.d_{4}\right) \delta-75.89(\mathrm{~d}, J=8.9 \mathrm{~Hz})$; HRMS-FI $(\mathrm{m} / \mathrm{z})[\mathrm{M}]^{+}$cal'd for $\mathrm{C}_{15} \mathrm{H}_{19} \mathrm{O}_{2} \mathrm{~F}_{3}$, 288.1332 , found 288.1334 .<smiles>COc1ccc(C(=O)CCOCCC(F)(F)F)cc1</smiles>

1-(4-methoxyphenyl)-3-(3,3,3-trifluoropropoxy)propan-1-one 30 Following the general procedure A, the reaction of $\mathbf{3 0 a}(31.2 \mathrm{mg}, 0.15 \mathrm{mmol})$ afforded $\mathbf{3 0}$ as a yellow oil (17.0 mg, 62\% yield) after flash chromatography (PE:EA = 20:1): IR (neat) 2904, 2843, 1677, 1602, 1577, 1511, 1421, 1384, 1258, 1145, 1112, 1030, 1006, 987, 842 $\mathrm{cm}^{-1} ;{ }^{1} \mathrm{H} \mathrm{NMR}\left(600 \mathrm{MHz}, \mathrm{CDCl}_{3}\right) \delta 7.96-7.93(\mathrm{~m}, 2 \mathrm{H}), 6.95-6.92(\mathrm{~m}, 2 \mathrm{H}), 3.89-$ $3.86(\mathrm{~m}, 5 \mathrm{H}), 3.69(\mathrm{t}, J=6.7 \mathrm{~Hz}, 2 \mathrm{H}), 3.19(\mathrm{t}, J=6.5 \mathrm{~Hz}, 2 \mathrm{H}), 2.43-2.33(\mathrm{~m}, 2 \mathrm{H}) ;{ }^{13} \mathrm{C}$ NMR (151 MHz, $\left.\mathrm{CDCl}_{3}\right) \delta 196.0,163.0,129.8,129.4,125.5$ (q, $\left.J=276.5 \mathrm{~Hz}\right), 113.1$, $63.3(\mathrm{q}, J=3.5 \mathrm{~Hz}), 54.8,37.6,33.6(\mathrm{q}, J=28.3 \mathrm{~Hz}) ;{ }^{19} \mathrm{~F} \mathrm{NMR}\left(376 \mathrm{MHz}, \mathrm{CDCl}_{3}\right) \delta$ $64.84(\mathrm{t}, J=10.8 \mathrm{~Hz})$; HRMS-FI $(\mathrm{m} / \mathrm{z})[\mathrm{M}]^{+}$cal'd for $\mathrm{C}_{13} \mathrm{H}_{15} \mathrm{O}_{3} \mathrm{~F}_{3}, 276.0968$, found 276.0966.<smiles>O=C(CCC(CCC(F)(F)F)c1ccccc1)c1ccc(-c2ccccc2)cc1</smiles>

1-([1,1'-biphenyl]-4-yl)-7,7,7-trifluoro-4-phenylheptan-1-one 31 Following the general procedure A, the reaction of $\mathbf{3 1 a}(49.3 \mathrm{mg}, 0.15 \mathrm{mmol})$ in $12 \mathrm{~h}$ afforded $\mathbf{3 1}$ as a white solid (24.0 mg, 61\% yield) after flash chromatography (PE:EA = 20:1): IR (neat) 3034, 2941, 1675, 1604, 1408, 1387, 1273, 1254, 1129, 1009, 764, 754, 702, $555 \mathrm{~cm}^{-1}$; ${ }^{1} \mathrm{H}$ NMR (600 MHz, $\left.\mathrm{CDCl}_{3}\right) \delta 7.89(\mathrm{~d}, J=8.4 \mathrm{~Hz}, 2 \mathrm{H}), 7.64-7.57(\mathrm{~m}, 4 \mathrm{H}), 7.46(\mathrm{t}, J$ $=7.6 \mathrm{~Hz}, 2 \mathrm{H}), 7.39(\mathrm{t}, J=7.4 \mathrm{~Hz}, 1 \mathrm{H}), 7.33(\mathrm{t}, J=7.6 \mathrm{~Hz}, 2 \mathrm{H}), 7.27-7.23(\mathrm{~m}, 1 \mathrm{H})$, $7.19-7.15(\mathrm{~m}, 2 \mathrm{H}), 2.89-2.75(\mathrm{~m}, 2 \mathrm{H}), 2.71-2.64(\mathrm{~m}, 1 \mathrm{H}), 2.25-2.17(\mathrm{~m}, 1 \mathrm{H})$, $2.04-1.95$ (m, 3H), $1.92-1.83(\mathrm{~m}, 2 \mathrm{H}) ;{ }^{13} \mathrm{C} \mathrm{NMR}\left(151 \mathrm{MHz}, \mathrm{CDCl}_{3}\right) \delta$ 199.5, 145.7, 142.9, 139.9, 135.5, 129.0, 128.9, 128.6, 128.2, 127.6, 127.2, 127.2 (q, $J=276.3 \mathrm{~Hz})$, 127.2, 127.0, 44.5, 36.3, $32.0(\mathrm{q}, J=28.5 \mathrm{~Hz}), 31.0,29.0(\mathrm{q}, J=2.4 \mathrm{~Hz}) ;{ }^{19} \mathrm{~F}$ NMR $(376$ 
$\left.\mathrm{MHz}, \mathrm{CDCl}_{3}\right) \delta-66.30(\mathrm{t}, J=9.8 \mathrm{~Hz}) ; \operatorname{HRMS}-\mathrm{FI}(\mathrm{m} / \mathrm{z})[\mathrm{M}]^{+}$cal'd for $\mathrm{C}_{25} \mathrm{H}_{23} \mathrm{OF}_{3}$, 396.1696, found 396.1702.<smiles>COc1ccc(C(=O)CC2CCC(C(F)(F)F)C2)cc1</smiles>

\section{1-(4-methoxyphenyl)-2-(3-(trifluoromethyl)cyclopentyl)ethan-1-one}

Following the general procedure A, the reaction of 32a $(65.4 \mathrm{mg}, 0.30 \mathrm{mmol})$ in $12 \mathrm{~h}$ afforded 32-1 as a yellow oil (10.9mg, 38\% yield) after flash chromatography (PE:EA = 20:1): IR (neat) 2961, 2882, 2841, 1678, 1601, 1510, 1389, 1262, 1171, 1097, 986, $831 \mathrm{~cm}^{-1}$; HRMS-EI (m/z) [M] $]^{+}$cal'd for $\mathrm{C}_{15} \mathrm{H}_{17} \mathrm{O}_{2} \mathrm{~F}_{3}, 286.1175$, found 286.1182.

Major product: ${ }^{1} \mathrm{H} \mathrm{NMR}\left(600 \mathrm{MHz}, \mathrm{CDCl}_{3}\right) \delta 7.93(\mathrm{dd}, J=8.8,3.4 \mathrm{~Hz}, 2 \mathrm{H}), 6.93(\mathrm{~d}, J$ $=8.8 \mathrm{~Hz}, 2 \mathrm{H}), 3.87(\mathrm{~s}, 3 \mathrm{H}), 3.00(\mathrm{dd}, J=6.9,3.8 \mathrm{~Hz}, 2 \mathrm{H}), 2.73-2.62(\mathrm{~m}, 2 \mathrm{H}), 2.51-$ $2.43(\mathrm{~m}, 1 \mathrm{H}), 2.06-2.00(\mathrm{~m}, 1 \mathrm{H}), 1.99-1.93(\mathrm{~m}, 1 \mathrm{H}), 1.84(\mathrm{td}, J=8.8,4.4 \mathrm{~Hz}, 2 \mathrm{H})$, $1.33-1.22(\mathrm{~m}, 1 \mathrm{H}) ;{ }^{13} \mathrm{C}$ NMR $\left(151 \mathrm{MHz}, \mathrm{CDCl}_{3}\right) \delta 197.8,163.4,130.2,129.9,128.3$ $(\mathrm{q}, J=277.0 \mathrm{~Hz}), 113.6,55.3,43.2,42.3(\mathrm{q}, J=27.4 \mathrm{~Hz}), 35.9,32.8(\mathrm{~d}, J=2.1 \mathrm{~Hz})$, 31.8, $25.0(\mathrm{~d}, J=2.2 \mathrm{~Hz}) ;{ }^{19} \mathrm{~F} \mathrm{NMR}\left(376 \mathrm{MHz}, \mathrm{CDCl}_{3}\right) \delta-76.29(\mathrm{~d}, J=9.9 \mathrm{~Hz})$.

Minor product: ${ }^{1} \mathrm{H} \mathrm{NMR}\left(600 \mathrm{MHz}, \mathrm{CDCl}_{3}\right) \delta 7.93(\mathrm{dd}, J=8.8,3.4 \mathrm{~Hz}, 2 \mathrm{H}), 6.93(\mathrm{~d}$, $J=8.8 \mathrm{~Hz}, 2 \mathrm{H}), 3.87(\mathrm{~s}, 3 \mathrm{H}), 2.95(\mathrm{dd}, J=7.0,3.1 \mathrm{~Hz}, 2 \mathrm{H}), 2.55(\mathrm{tt}, J=12.8,6.3 \mathrm{~Hz}$, $1 \mathrm{H}), 2.20(\mathrm{dt}, J=14.2,7.4 \mathrm{~Hz}, 2 \mathrm{H}), 2.08(\mathrm{ddd}, J=13.7,7.9,5.8 \mathrm{~Hz}, 1 \mathrm{H}), 2.00-1.94$ (m, 1H), 1.70 (ddt, $J=13.3,10.5,7.9 \mathrm{~Hz}, 1 \mathrm{H}), 1.55-1.48$ (m, 1H), 1.32 (ddt, $J=14.5$, 9.7, $5.6 \mathrm{~Hz}, 1 \mathrm{H}) ;{ }^{13} \mathrm{C} \mathrm{NMR}\left(151 \mathrm{MHz}, \mathrm{CDCl}_{3}\right) \delta 197.7,163.3,130.2,129.9,128.4$ (q, $J=277.2 \mathrm{~Hz}), 113.6,55.3,43.4,41.8(\mathrm{q}, J=27.4 \mathrm{~Hz}), 35.4,32.7,32.1(\mathrm{~d}, J=2.0 \mathrm{~Hz})$, $25.8(\mathrm{~d}, J=2.3 \mathrm{~Hz}) ;{ }^{19} \mathrm{~F}$ NMR $\left(376 \mathrm{MHz}, \mathrm{CDCl}_{3}\right) \delta-76.29(\mathrm{~d}, J=9.9 \mathrm{~Hz})$.

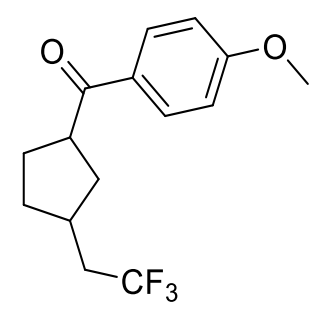

(4-methoxyphenyl)(3-(2,2,2-trifluoroethyl)cyclopentyl)methanone 32-2 Following the general procedure A, the reaction of $\mathbf{3 2 a}(65.4 \mathrm{mg}, 0.30 \mathrm{mmol})$ in $12 \mathrm{~h}$ afforded $\mathbf{3 2}$ - 
2 as a yellow oil $(6.3 \mathrm{mg}, 22 \%$ yield) after flash chromatography (PE:EA = 20:1): IR (neat) 2960, 2866, 1675, 1062, 1510, 1258, 1171, 1140, 1094, 1032, $842 \mathrm{~cm}^{-1} ;{ }^{1} \mathrm{H}$ NMR (600 MHz, $\left.\mathrm{CDCl}_{3}\right) \delta 7.94(\mathrm{~d}, J=8.8 \mathrm{~Hz}, 2 \mathrm{H}), 6.94(\mathrm{~d}, J=8.8 \mathrm{~Hz}, 2 \mathrm{H}), 3.87$ (s, $3 \mathrm{H}), 3.80-3.73(\mathrm{~m}, 1 \mathrm{H}), 2.29-2.15(\mathrm{~m}, 4 \mathrm{H}), 2.05-1.90(\mathrm{~m}, 3 \mathrm{H}), 1.72-1.65(\mathrm{~m}$, 1H), $1.43-1.34(\mathrm{~m}, 1 \mathrm{H}) ;{ }^{13} \mathrm{C} \mathrm{NMR}\left(151 \mathrm{MHz}, \mathrm{CDCl}_{3}\right) \delta 200.5,163.4,130.7,129.5$, $127.0(\mathrm{q}, J=277.0 \mathrm{~Hz}), 113.8,55.5,45.3,38.7(\mathrm{q}, J=27.7 \mathrm{~Hz}), 36.1,34.7$ (q, $J=2.1$ $\mathrm{Hz}), 32.4,29.3 ;{ }^{19} \mathrm{~F}$ NMR $\left(376 \mathrm{MHz}, \mathrm{CDCl}_{3}\right) \delta-68.88(\mathrm{t}, J=10.7 \mathrm{~Hz})$; HRMS-EI $(\mathrm{m} / \mathrm{z})$ $[\mathrm{M}]^{+}$cal'd for $\mathrm{C}_{15} \mathrm{H}_{17} \mathrm{O}_{2} \mathrm{~F}_{3}, 286.1175$, found 286.1177 .<smiles>O=C(CCCC(F)(F)F)CCc1ccccc1</smiles>

7,7,7-trifluoro-1-phenylheptan-3-one 33 Following the general procedure $\mathrm{A}$, the reaction of 33a $(52.8 \mathrm{mg}, 0.30 \mathrm{mmol})$ in $12 \mathrm{~h}$ afforded $\mathbf{3 3}$ as a colorless oil (16.6 mg, $68 \%$ yield) after flash chromatography (PE:EA = 20:1): IR (neat) 3028, 2952, 1716, 1497, 1455, 1392, 1255, 1134, 750, $700 \mathrm{~cm}^{-1} ;{ }^{1} \mathrm{H}$ NMR $\left(600 \mathrm{MHz}, \mathrm{CDCl}_{3}\right) \delta 7.30-$ $7.26(\mathrm{~m}, 2 \mathrm{H}), 7.22-7.16(\mathrm{~m}, 3 \mathrm{H}), 2.90(\mathrm{t}, J=7.6 \mathrm{~Hz}, 2 \mathrm{H}), 2.73(\mathrm{t}, J=7.6 \mathrm{~Hz}, 2 \mathrm{H})$, $2.47(\mathrm{t}, J=7.1 \mathrm{~Hz}, 2 \mathrm{H}), 2.11-2.02(\mathrm{~m}, 2 \mathrm{H}), 1.82(\mathrm{dt}, J=14.6,7.2 \mathrm{~Hz}, 2 \mathrm{H}) ;{ }^{13} \mathrm{C} \mathrm{NMR}$ $\left(151 \mathrm{MHz}, \mathrm{CDCl}_{3}\right) \delta 208.5,140.8,128.6,128.3,127.0(\mathrm{q}, J=276.5 \mathrm{~Hz}), 126.2,44.3$, $41.1,32.8(\mathrm{q}, J=28.6 \mathrm{~Hz}), 29.8,16.0(\mathrm{q}, J=3.2 \mathrm{~Hz}) ;{ }^{19} \mathrm{~F} \mathrm{NMR}\left(376 \mathrm{MHz}, \mathrm{CDCl}_{3}\right) \delta-$ $66.26(\mathrm{t}, J=10.7 \mathrm{~Hz})$; HRMS-EI $(\mathrm{m} / \mathrm{z})[\mathrm{M}]^{+}$cal'd for $\mathrm{C}_{13} \mathrm{H}_{15} \mathrm{OF}_{3}, 244.1070$, found 244.1069.<smiles>COc1ccc(CCCC(=O)CCCC(F)(F)F)cc1</smiles>

8,8,8-trifluoro-1-(4-methoxyphenyl)octan-4-one 34 Following the general procedure A, the reaction of $\mathbf{3 4 a}(66.2 \mathrm{mg}, 0.30 \mathrm{mmol})$ in $12 \mathrm{~h}$ afforded 34 as a colorless oil (14.9 mg, 52\% yield) after flash chromatography (PE:EA = 10:1): IR (neat) 2941, 2859, 2838, 1715, 1612, 1513, 1456, 1391, 1250, 1134, 1036, $831 \mathrm{~cm}^{-1} ;{ }^{1} \mathrm{H}$ NMR (600 MHz, $\left.\mathrm{CDCl}_{3}\right)$ $\delta 7.08(\mathrm{~d}, J=8.6 \mathrm{~Hz}, 2 \mathrm{H}), 6.85-6.81(\mathrm{~m}, 2 \mathrm{H}), 3.79(\mathrm{~s}, 3 \mathrm{H}), 2.56(\mathrm{t}, J=7.5 \mathrm{~Hz}, 2 \mathrm{H})$, $2.46(\mathrm{t}, J=7.1 \mathrm{~Hz}, 2 \mathrm{H}), 2.39(\mathrm{t}, J=7.4 \mathrm{~Hz}, 2 \mathrm{H}), 2.13-2.04(\mathrm{~m}, 2 \mathrm{H}), 1.88$ (p, $J=7.4$ $\mathrm{Hz}, 2 \mathrm{H}), 1.82(\mathrm{p}, J=7.2 \mathrm{~Hz}, 2 \mathrm{H}) ;{ }^{13} \mathrm{C} \mathrm{NMR}\left(151 \mathrm{MHz}, \mathrm{CDCl}_{3}\right) \delta 209.3,157.9,133.5$, 129.4, $127.0(\mathrm{q}, J=276.5 \mathrm{~Hz}), 113.8,55.3,41.9,40.9,34.1,32.8(\mathrm{q}, J=28.7 \mathrm{~Hz}), 25.4$, 
$16.0(\mathrm{q}, J=3.2 \mathrm{~Hz}) ;{ }^{19} \mathrm{~F}$ NMR $\left(376 \mathrm{MHz}, \mathrm{CDCl}_{3}\right) \delta-66.24(\mathrm{t}, J=10.9 \mathrm{~Hz})$; HRMS-EI $(\mathrm{m} / \mathrm{z})[\mathrm{M}]^{+}$cal'd for $\mathrm{C}_{15} \mathrm{H}_{19} \mathrm{O}_{2} \mathrm{~F}_{3}, 288.1332$, found 288.1334.

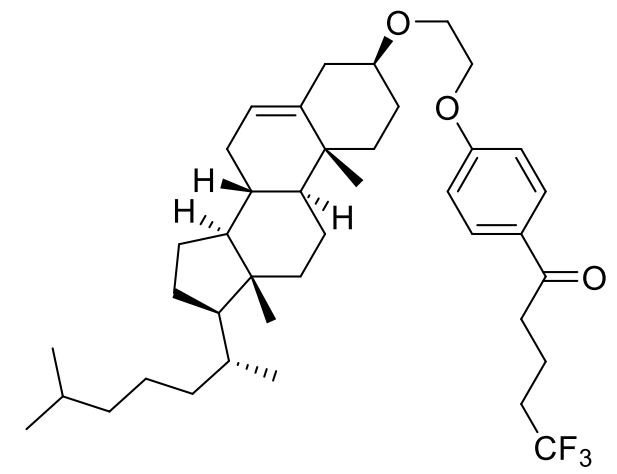

1-(4-(2-(((3S,8S,9S,10R,13R,14S,17R)-10,13-dimethyl-17-((R)-6-methylheptan-2yl)-2,3,4,7,8,9,10,11,12,13,14,15,16,17-tetradecahydro-1H-cyclopenta[a]

phenanthren-3-yl)oxy)ethoxy)phenyl)-5,5,5-trifluoropentan-1-one 35 Following the general procedure $\mathrm{A}$, the reaction of $\mathbf{3 5 a}(88.6 \mathrm{mg}, 0.15 \mathrm{mmol})$ in $12 \mathrm{~h}$ afforded 35 as a white solid (35.4 mg, 55\% yield) after flash chromatography (PE:EA = 20:1): IR (neat) 2968, 2901, 2869, 1674, 1602, 1577, 1509, 1468, 1289, 1257, 1211, 1132, 1111, 1065, 1043, 1013, 834, 805, $739 \mathrm{~cm}^{-1} ;{ }^{1} \mathrm{H}$ NMR (600 MHz, $\left.\mathrm{CDCl}_{3}\right) \delta 7.94-7.90(\mathrm{~m}$, $2 \mathrm{H}), 6.98-6.94(\mathrm{~m}, 2 \mathrm{H}), 5.38-5.34(\mathrm{~m}, 1 \mathrm{H}), 4.17(\mathrm{t}, J=5.0 \mathrm{~Hz}, 2 \mathrm{H}), 3.86(\mathrm{td}, J=$ 4.7, $1.7 \mathrm{~Hz}, 2 \mathrm{H}), 3.30-3.22(\mathrm{~m}, 1 \mathrm{H}), 3.02(\mathrm{t}, J=7.0 \mathrm{~Hz}, 2 \mathrm{H}), 2.42-2.37(\mathrm{~m}, 1 \mathrm{H})$, $2.28-2.16(\mathrm{~m}, 3 \mathrm{H}), 2.05-1.91(\mathrm{~m}, 5 \mathrm{H}), 1.90-1.79(\mathrm{~m}, 2 \mathrm{H}), 1.61-1.42(\mathrm{~m}, 8 \mathrm{H})$, $1.40-1.31(\mathrm{~m}, 3 \mathrm{H}), 1.26(\mathrm{td}, J=14.3,13.7,6.6 \mathrm{~Hz}, 1 \mathrm{H}), 1.19-1.04(\mathrm{~m}, 7 \mathrm{H}), 1.04-$ $0.96(\mathrm{~m}, 5 \mathrm{H}), 0.91(\mathrm{~d}, J=6.5 \mathrm{~Hz}, 3 \mathrm{H}), 0.86(\mathrm{dd}, J=6.6,2.7 \mathrm{~Hz}, 6 \mathrm{H}), 0.68(\mathrm{~s}, 3 \mathrm{H}) ;{ }^{13} \mathrm{C}$ NMR (151 MHz, $\left.\mathrm{CDCl}_{3}\right) \delta 197.2,163.0,140.8,130.2,129.8,127.1$ (q, $J=276.5 \mathrm{~Hz}$ ), $121.8,114.5,79.9,68.0,66.3,56.8,56.2,50.2$, 42.3, 39.8, 39.5, 39.1, 37.2 , 36.9, 36.4, 36.2, 35.8, 33.1 (q, $J=28.6 \mathrm{~Hz}$ ), 32.0, 31.9, 28.4, 28.2, 28.0, 24.3, 23.8, 22.8, 22.6, $21.1,19.4,18.7,16.6$ (q, $J=2.9 \mathrm{~Hz}), 11.9 ;{ }^{19} \mathrm{~F} \mathrm{NMR}\left(376 \mathrm{MHz}, \mathrm{CDCl}_{3}\right) \delta-66.18(\mathrm{t}, J$ $=10.8 \mathrm{~Hz})$; HRMS-DART $(\mathrm{m} / \mathrm{z})[\mathrm{M}+\mathrm{H}]^{+}$cal'd for $\mathrm{C}_{40} \mathrm{H}_{60} \mathrm{O}_{3} \mathrm{~F}_{3}, 645.4489$, found 645.4480 .<smiles>O=C(CCCC(F)(F)F)c1ccc(-c2ccccc2)cc1</smiles> 
1-([1,1'-biphenyl]-4-yl)-5,5,6,6,6-pentafluorohexan-1-one 36 Following the general procedure A, the reaction of $\mathbf{7 a}(88.6 \mathrm{mg}, 0.15 \mathrm{mmol})$ afforded $\mathbf{3 6}$ as a white solid (22.9 mg, 67\% yield) after flash chromatography (PE:EA = 20:1): IR (neat) 1681, 1459, 1403, 1205, 1191, 1097, 1057, 1006, 827, 764, 718, $692 \mathrm{~cm}^{-1} ;{ }^{1} \mathrm{H}$ NMR (600 MHz, $\left.\mathrm{CDCl}_{3}\right) \delta$ $8.05-8.01(\mathrm{~m}, 2 \mathrm{H}), 7.72-7.68(\mathrm{~m}, 2 \mathrm{H}), 7.65-7.62(\mathrm{~m}, 2 \mathrm{H}), 7.50-7.46(\mathrm{~m}, 2 \mathrm{H})$, $7.43-7.39(\mathrm{~m}, 1 \mathrm{H}), 3.14(\mathrm{t}, J=6.8 \mathrm{~Hz}, 2 \mathrm{H}), 2.24-2.14(\mathrm{~m}, 2 \mathrm{H}), 2.14-2.06(\mathrm{~m}, 2 \mathrm{H})$; ${ }^{13} \mathrm{C} \mathrm{NMR}\left(151 \mathrm{MHz}, \mathrm{CDCl}_{3}\right) \delta 197.6,145.4,139.2,134.7,128.4,128.0,127.7,126.8$, 126.7, $118.6(\mathrm{qt}, J=285.5,36.3 \mathrm{~Hz}), 115.2(\mathrm{tq}, J=252.2,37.7 \mathrm{~Hz}), 36.6,29.3(\mathrm{t}, J=$ $22.0 \mathrm{~Hz}), 14.5(\mathrm{t}, J=3.8 \mathrm{~Hz}),{ }^{19} \mathrm{~F}$ NMR $\left(376 \mathrm{MHz} \mathrm{CDCl}_{3}\right) \delta-85.47,-118.23(\mathrm{t}, J=$ $18.0 \mathrm{~Hz})$; HRMS-EI (m/z) [M] ${ }^{+}$cal'd for $\mathrm{C}_{18} \mathrm{H}_{15} \mathrm{OF}_{5}, 342.1038$, found 342.1041.<smiles>O=C(CCCC(F)(F)F)c1ccco1</smiles>

5,5,5-trifluoro-1-(furan-2-yl)pentan-1-one 45 Following the general procedure $\mathrm{A}$, the reaction of 1-(furan-2-yl)cyclobutan-1-ol (20.7 mg, $0.15 \mathrm{mmol})$ afforded 45 as a white solid (7.4 mg, 37\% yield) after flash chromatography (PE:EA = 20:1): IR (neat) 3132, 3100, 2960, 2924, 1674, 1474, 1442, 1409, 1313, 1263, 1239, 1208, 1146, 1035, 1009 , 983, 919, 843, 774, $596 \mathrm{~cm}^{-1}$; ${ }^{1} \mathrm{H}$ NMR (600 MHz, Chloroform-d) $\delta 7.62-7.57$ (m, $1 \mathrm{H}), 7.20(\mathrm{~d}, J=3.6 \mathrm{~Hz}, 1 \mathrm{H}), 6.55(\mathrm{dd}, J=3.5,1.7 \mathrm{~Hz}, 1 \mathrm{H}), 2.94(\mathrm{t}, J=7.2 \mathrm{~Hz}, 2 \mathrm{H})$, $2.25-2.15$ (m, 2H), $2.04-1.97$ (m, 2H) ${ }^{13} \mathrm{C}$ NMR (151 MHz, Chloroform- $d$ ) $\delta 187.9$, $152.5,146.4,127.0(\mathrm{q}, J=276.4 \mathrm{~Hz}), 117.0,112.3,36.7,33.0(\mathrm{q}, J=28.7 \mathrm{~Hz}), 16.3(\mathrm{q}$, $J=3.2 \mathrm{~Hz}) ;{ }^{19} \mathrm{~F}$ NMR $(376 \mathrm{MHz}$, Chloroform- $d$ ) $\delta-66.24(\mathrm{t}, J=10.7 \mathrm{~Hz})$; HRMS-EI $(\mathrm{m} / \mathrm{z})[\mathrm{M}]^{+}$cal'd for $\mathrm{C}_{9} \mathrm{H}_{9} \mathrm{O}_{2} \mathrm{~F}_{3}, 206.0549$, found 206.0554 . 


\section{NMR Spectra}

${ }^{1} \mathrm{H}$ NMR (500 MHz, $\mathrm{CDCl}_{3}$ )

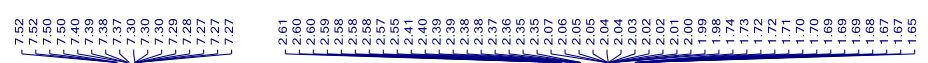
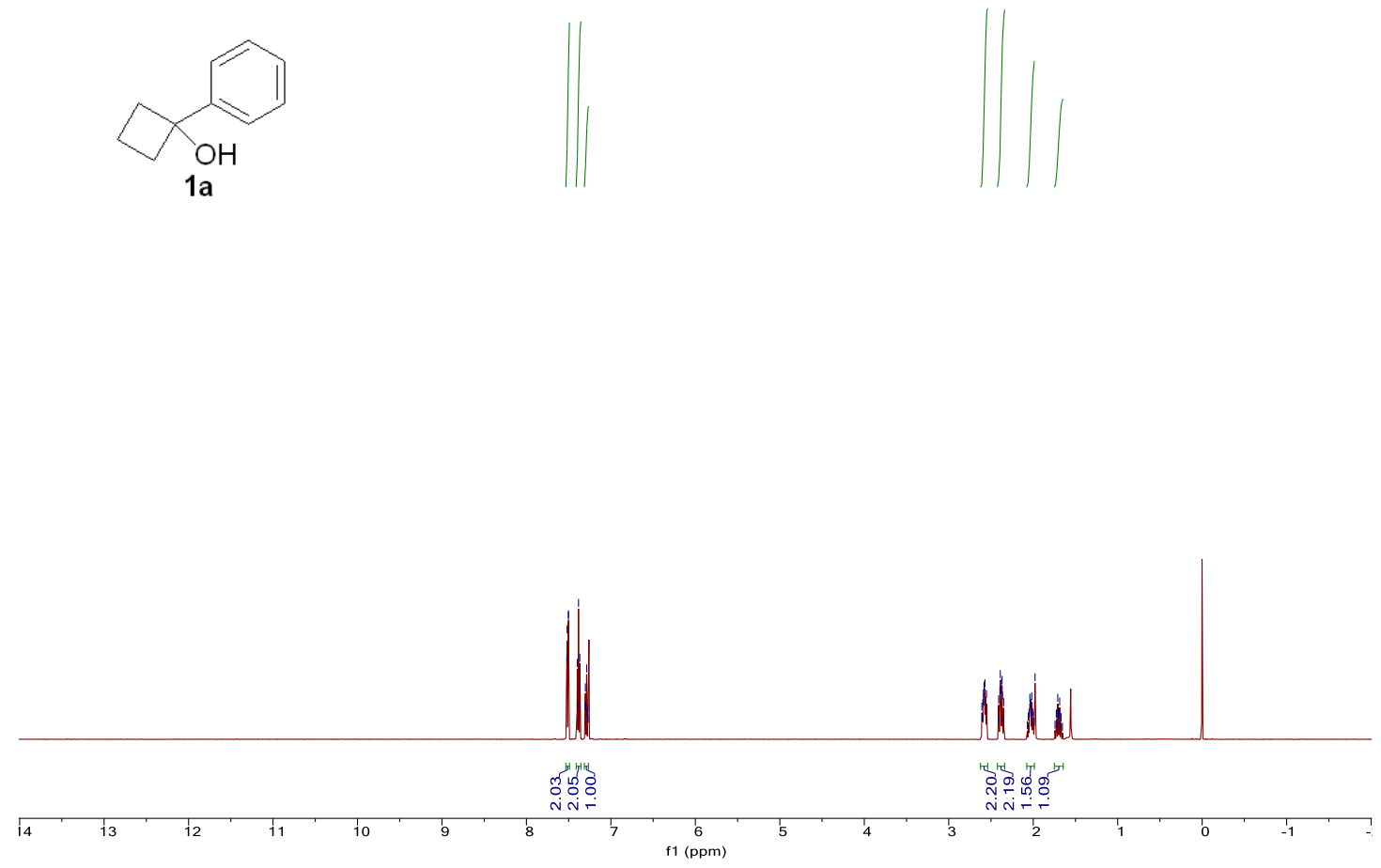

${ }^{13} \mathrm{C} \mathrm{NMR}\left(126 \mathrm{MHz}, \mathrm{CDCl}_{3}\right)$

$\sqrt{1 / 1}$

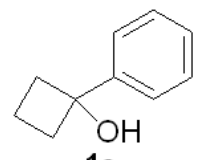

1a

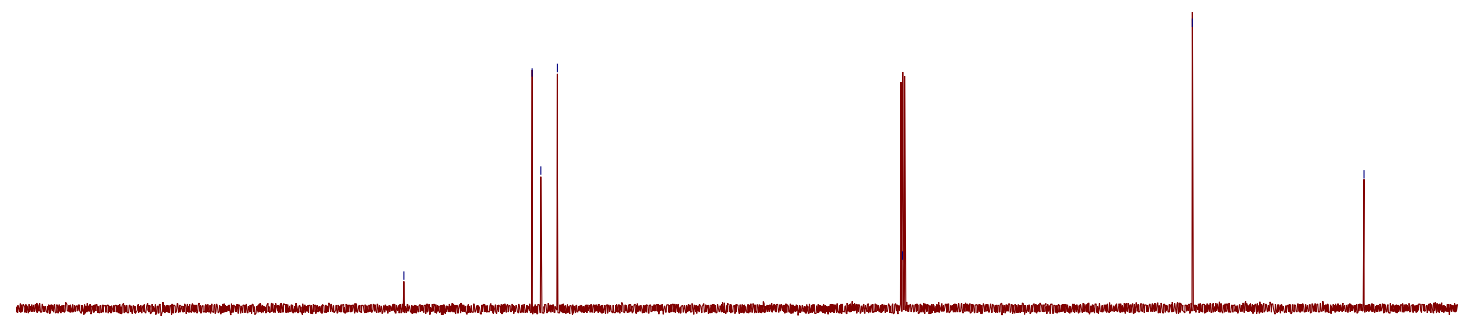

\begin{tabular}{lllllllllllllllllllll}
\hline 00 & 190 & 180 & 170 & 160 & 150 & 140 & 130 & 120 & 110 & $\begin{array}{c}100 \\
\mathrm{f} 1(\mathrm{ppm})\end{array}$ & 90 & 80 & 70 & 60 & 50 & 40 & 30 & 10 & 10 & 1 \\
\hline
\end{tabular} 
${ }^{1} \mathrm{H}$ NMR (500 MHz, $\mathrm{CDCl}_{3}$ )

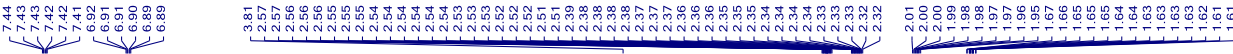

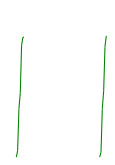<smiles>COc1ccc(C2(O)CCC2)cc1</smiles>

$2 \mathrm{a}$
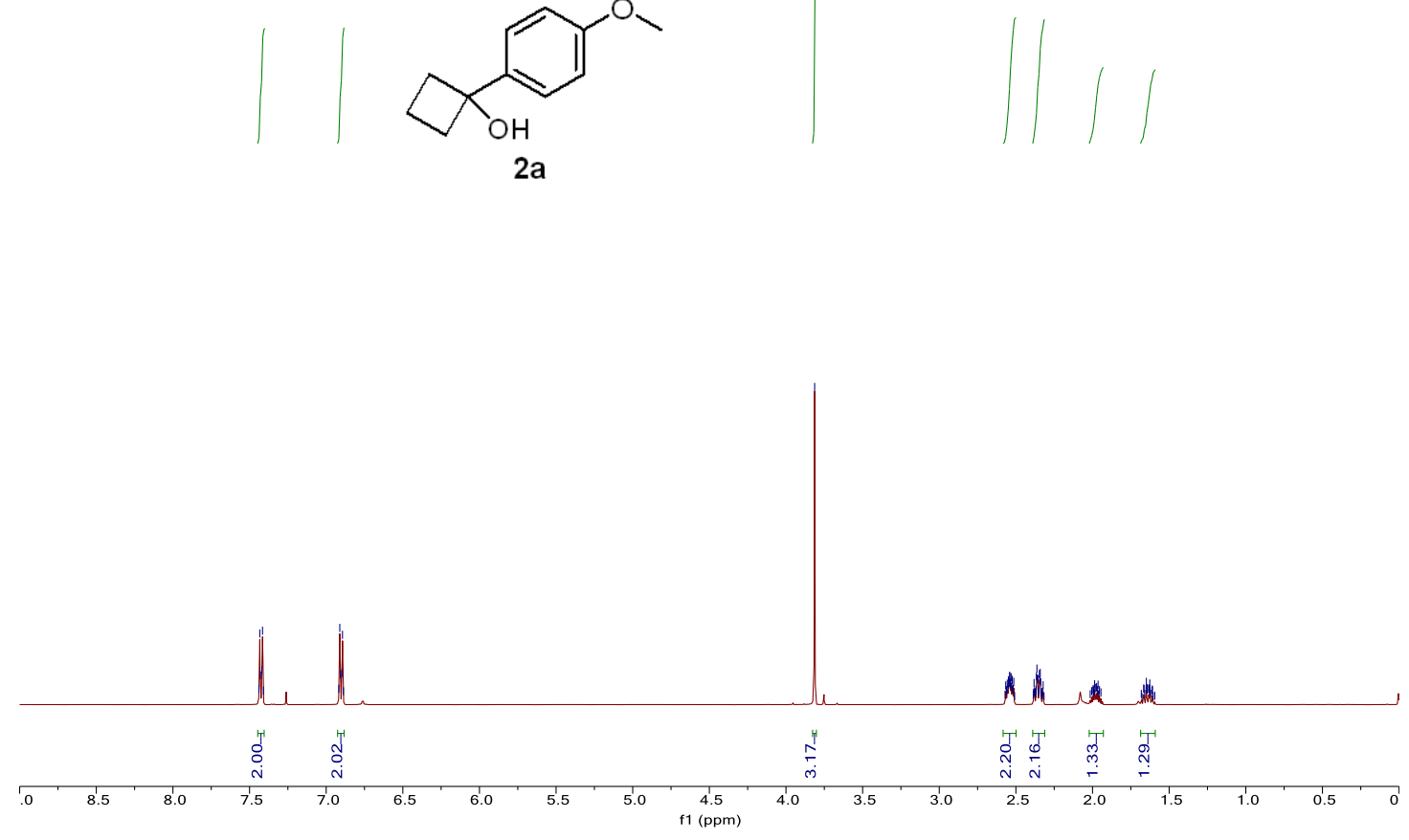

${ }^{13} \mathrm{C} \mathrm{NMR}\left(126 \mathrm{MHz}, \mathrm{CDCl}_{3}\right)$

(<smiles>COc1ccc(C2(O)CCC2)cc1</smiles>

$2 \mathrm{a}$

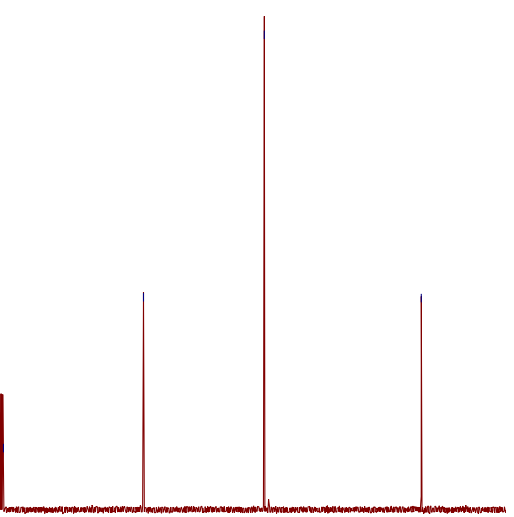

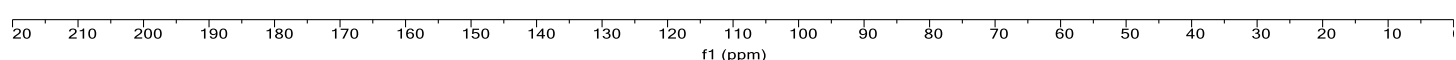


${ }^{1} \mathrm{H}$ NMR (500 MHz, $\mathrm{CDCl}_{3}$ )

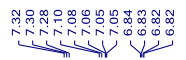

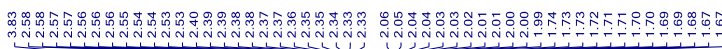

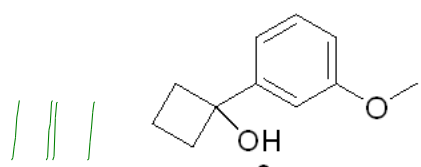

3a
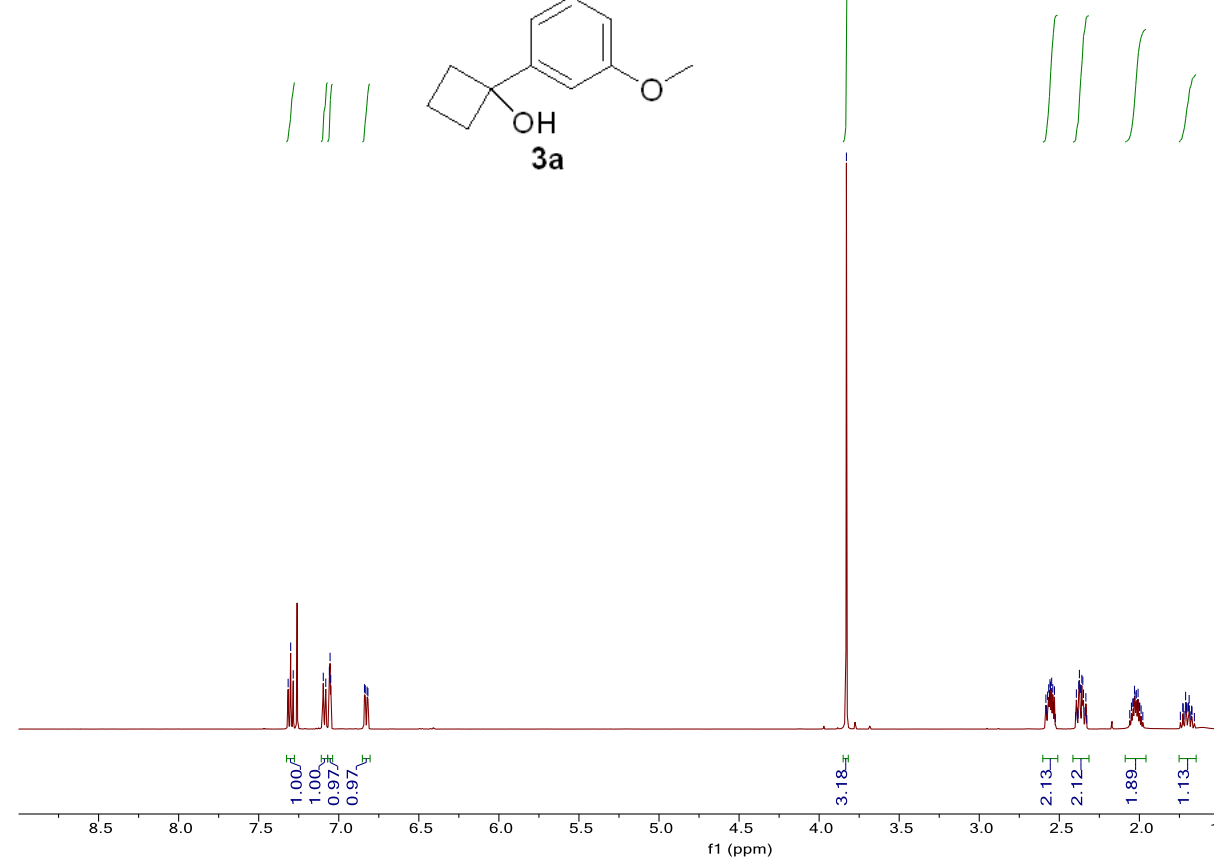

Mh

${ }^{13} \mathrm{C}$ NMR $\left(126 \mathrm{MHz}, \mathrm{CDCl}_{3}\right)$

i

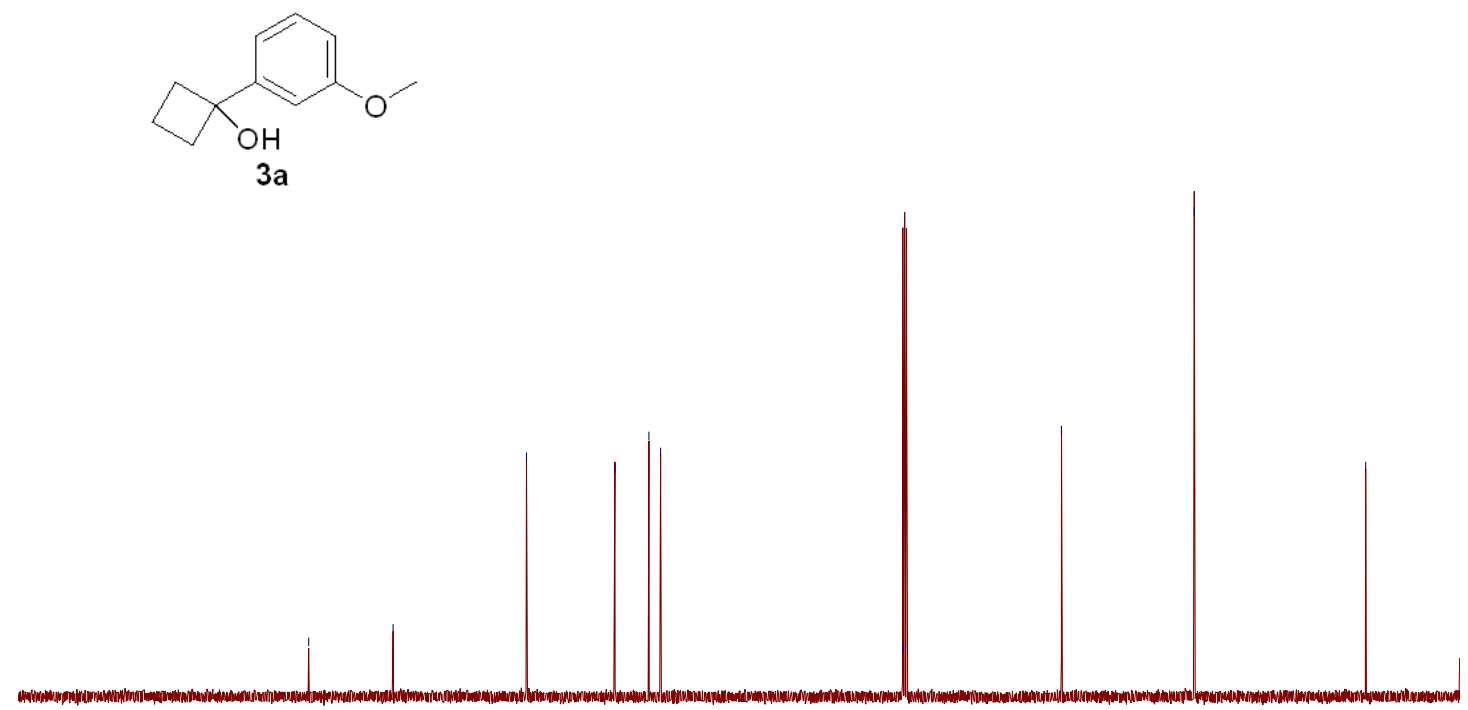

3a

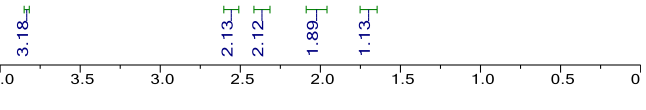

oo

190

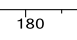

160

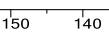

100
$\mathrm{f} 1(\mathrm{ppm})$ 
${ }^{1} \mathrm{H}$ NMR (500 MHz, $\mathrm{CDCl}_{3}$ )

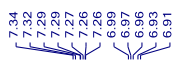

mong
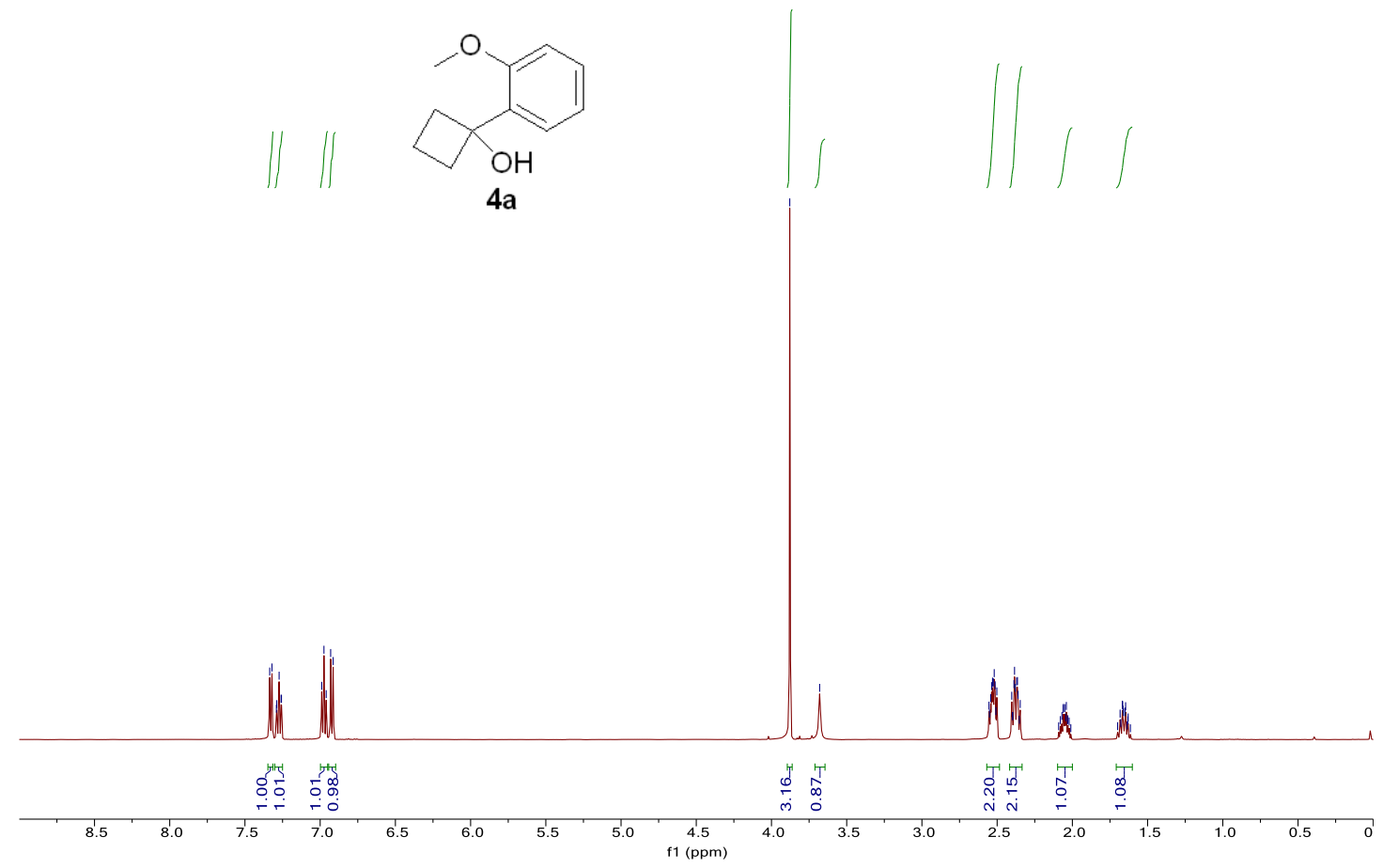

$4 a$

${ }^{13} \mathrm{C}$ NMR $\left(126 \mathrm{MHz}, \mathrm{CDCl}_{3}\right)$<smiles>C1=CCCC=C1</smiles>

$4 a$
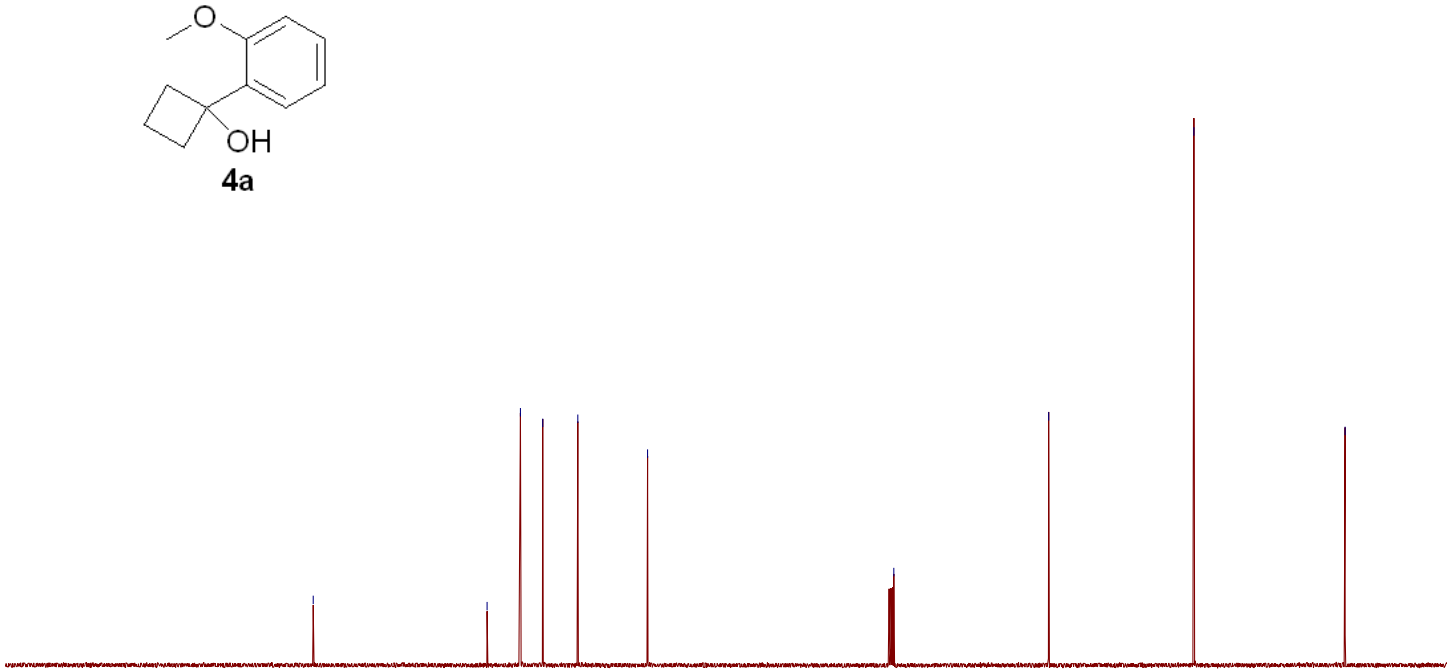

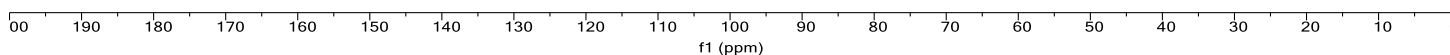


${ }^{1} \mathrm{H}$ NMR (500 MHz, $\left.\mathrm{CDCl}_{3}\right)$

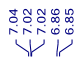

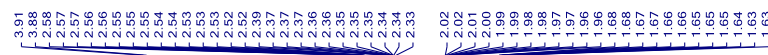<smiles>COc1ccc(C2(O)CCC2)cc1OC</smiles>

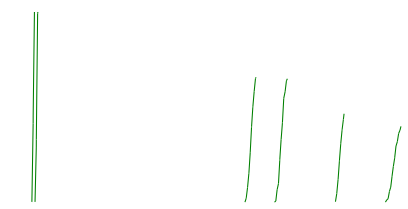

$5 a$

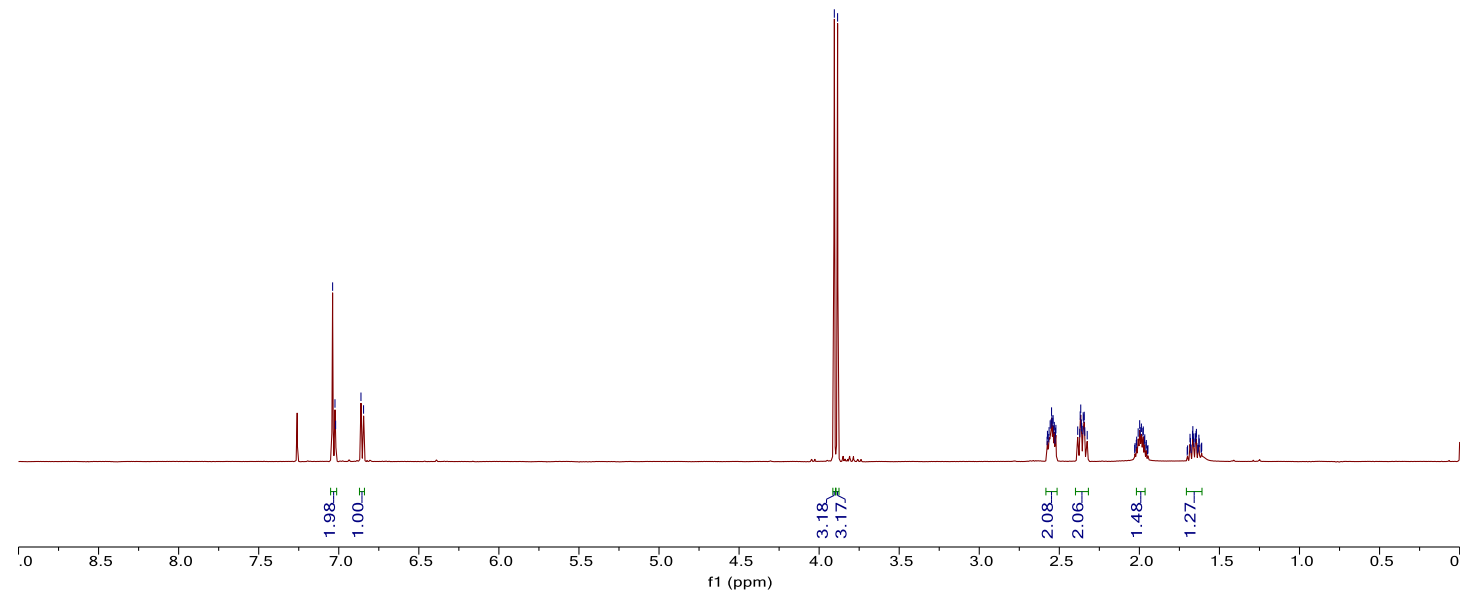

${ }^{13} \mathrm{C}$ NMR (126 MHz, $\left.\mathrm{CDCl}_{3}\right)$

突品

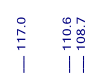

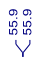<smiles>COc1ccc(C2(O)CCC2)cc1OC</smiles>

$5 a$

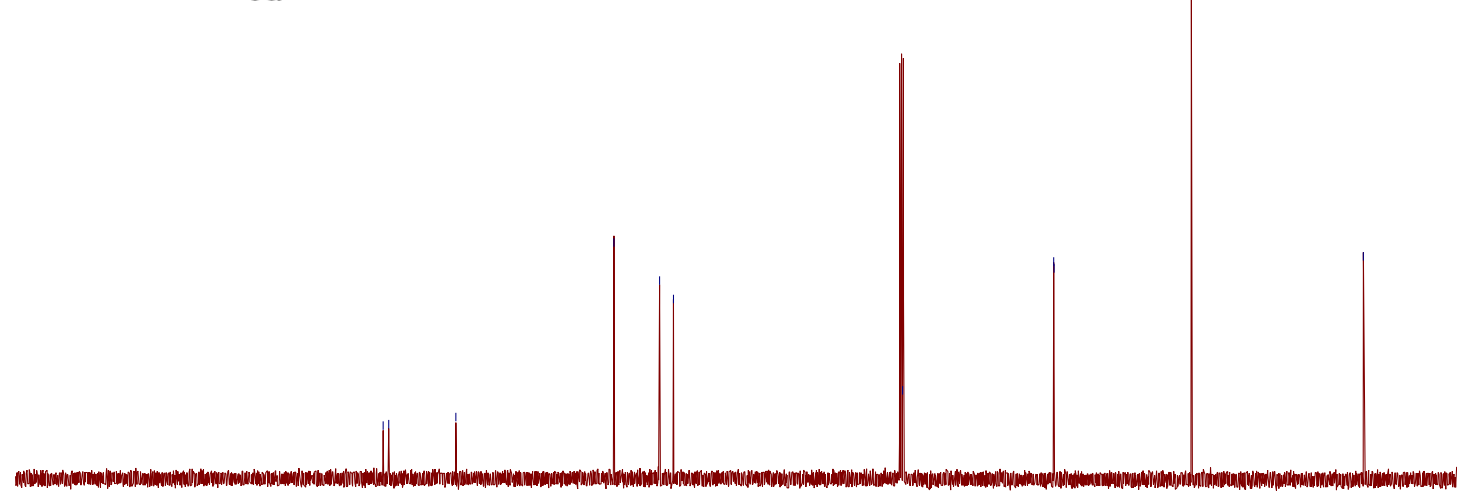

\begin{tabular}{llllll}
\hline oo & 190 & 180 & 170 & 160 & 150
\end{tabular} 100
$f 1(\mathrm{ppm})$ 
${ }^{1} \mathrm{H}$ NMR (500 MHz, $\mathrm{CDCl}_{3}$ )

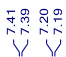

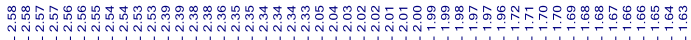
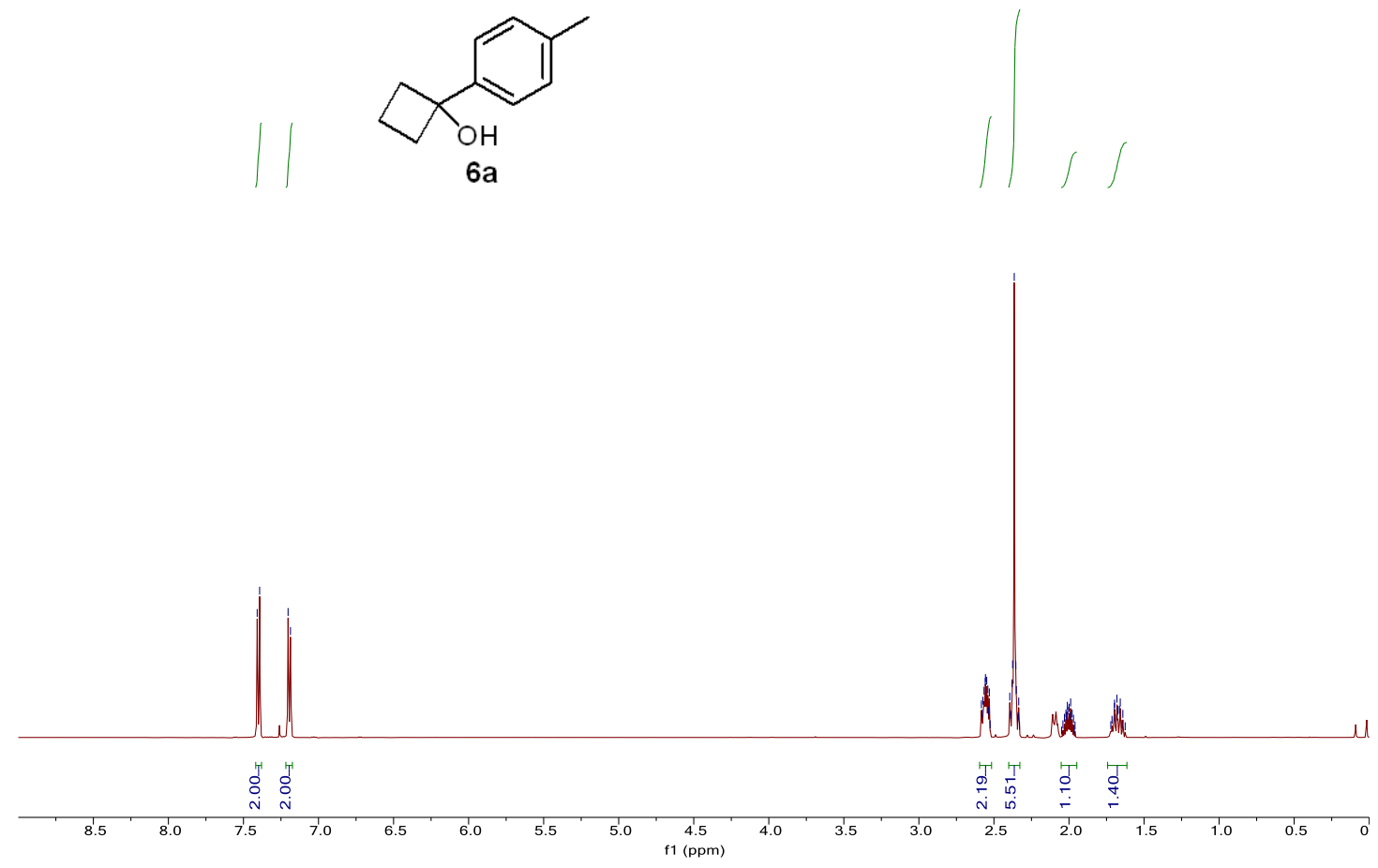

${ }^{13} \mathrm{C}$ NMR $\left(126 \mathrm{MHz}, \mathrm{CDCl}_{3}\right)$

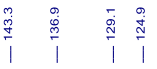

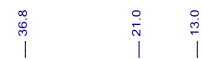

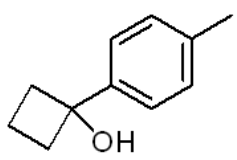

$6 a$

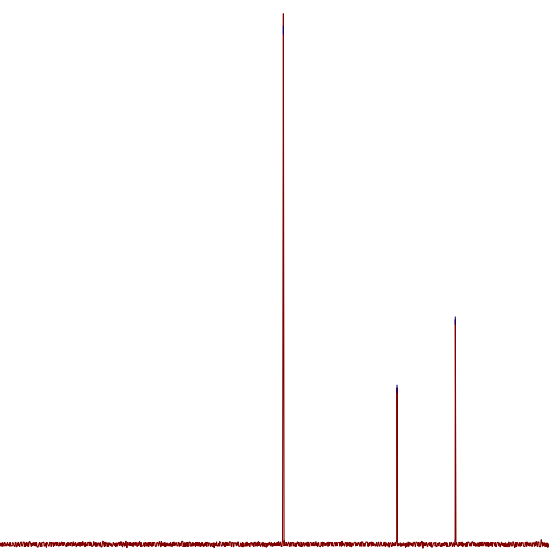

oo 100
$\mathrm{f} 1(\mathrm{ppm})$ 
${ }^{1} \mathrm{H}$ NMR (500 MHz, $\mathrm{CDCl}_{3}$ )

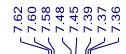

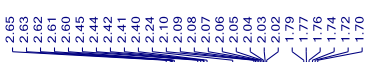
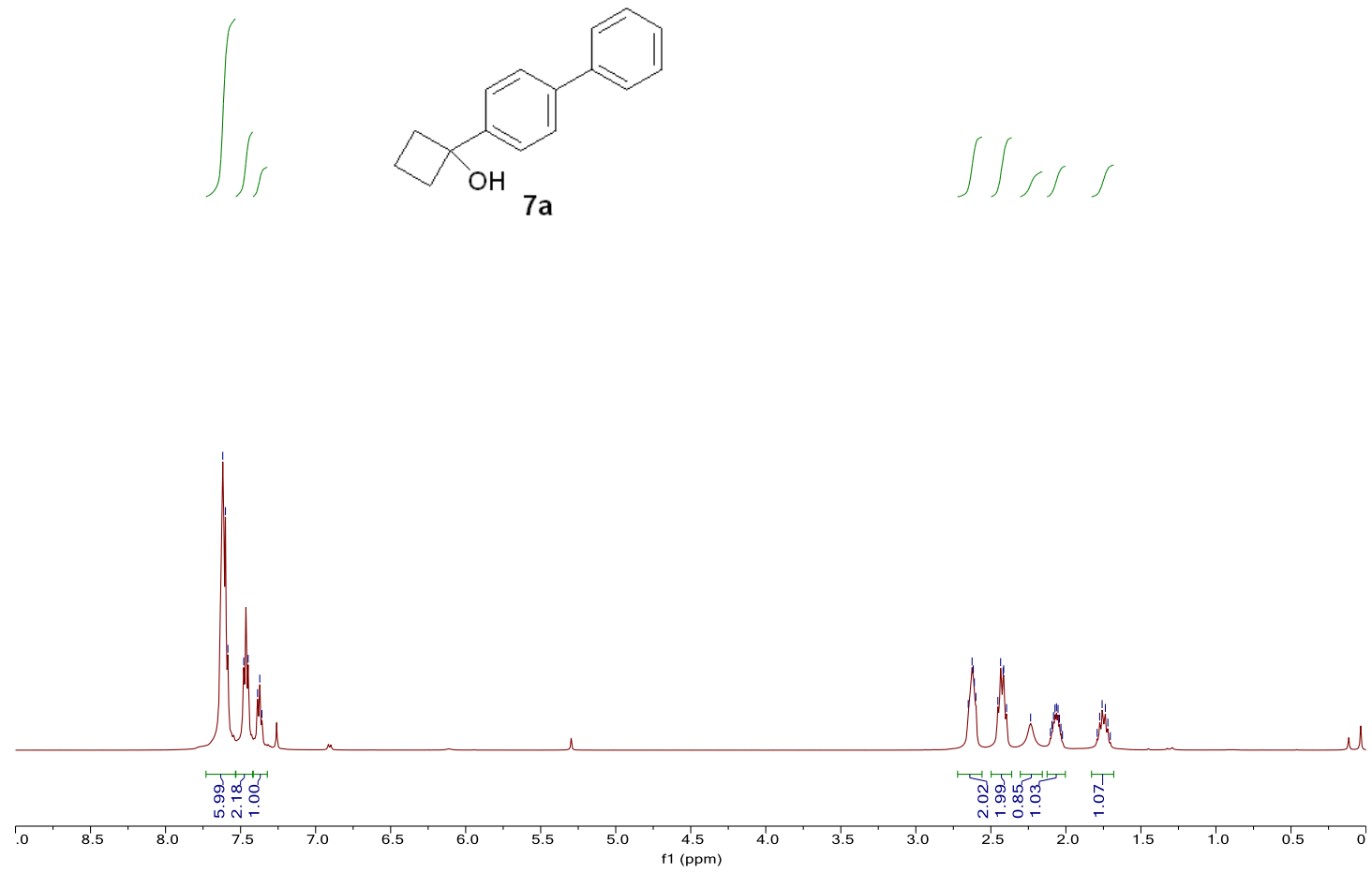

${ }^{13} \mathrm{C}$ NMR $\left(126 \mathrm{MHz}, \mathrm{CDCl}_{3}\right)$

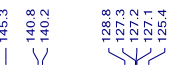
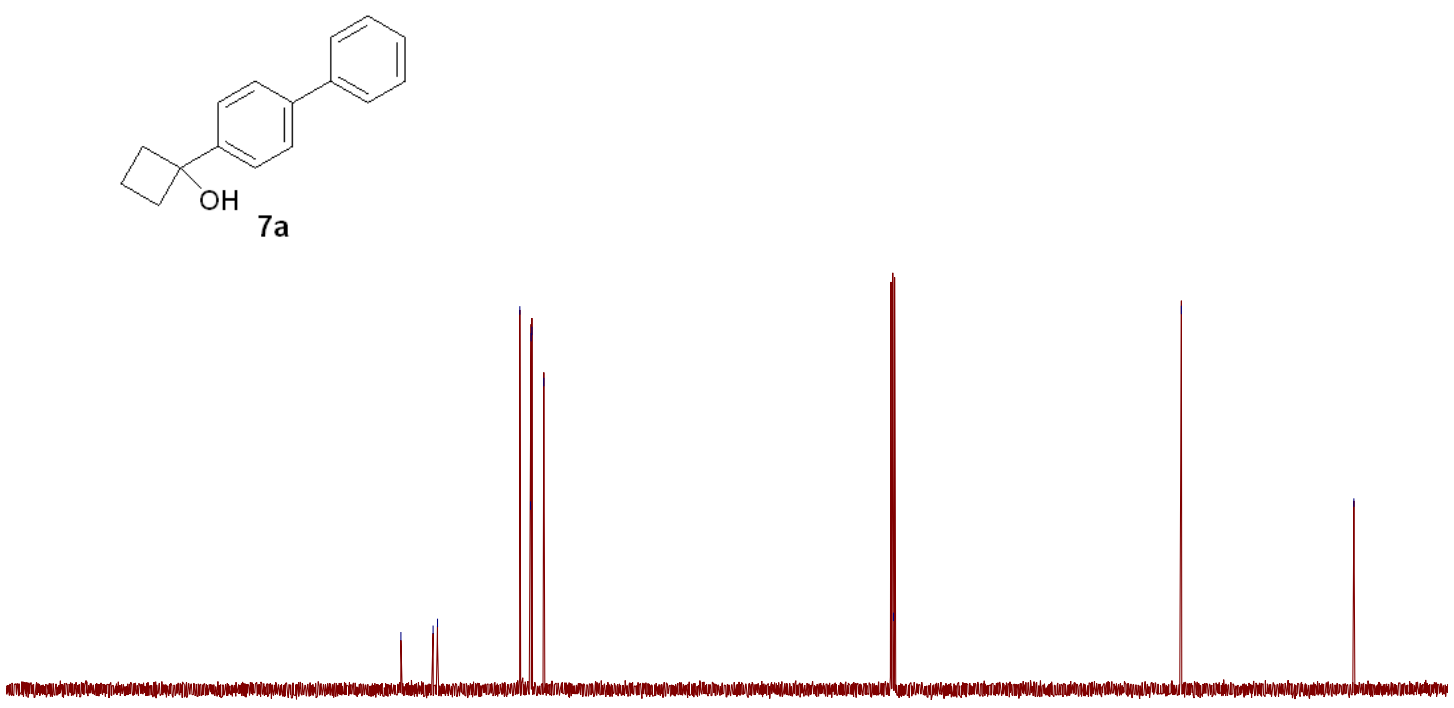

\begin{tabular}{lllll}
\hline oo & 190 & 180 & 170 & 160
\end{tabular} 
${ }^{1} \mathrm{H}$ NMR (500 MHz, $\mathrm{CDCl}_{3}$ )

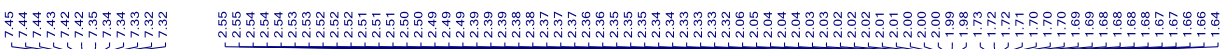

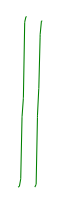<smiles>OC1(c2ccc(Cl)cc2)CCC1</smiles>

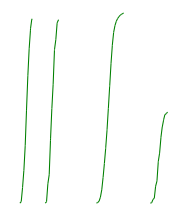

$8 a$

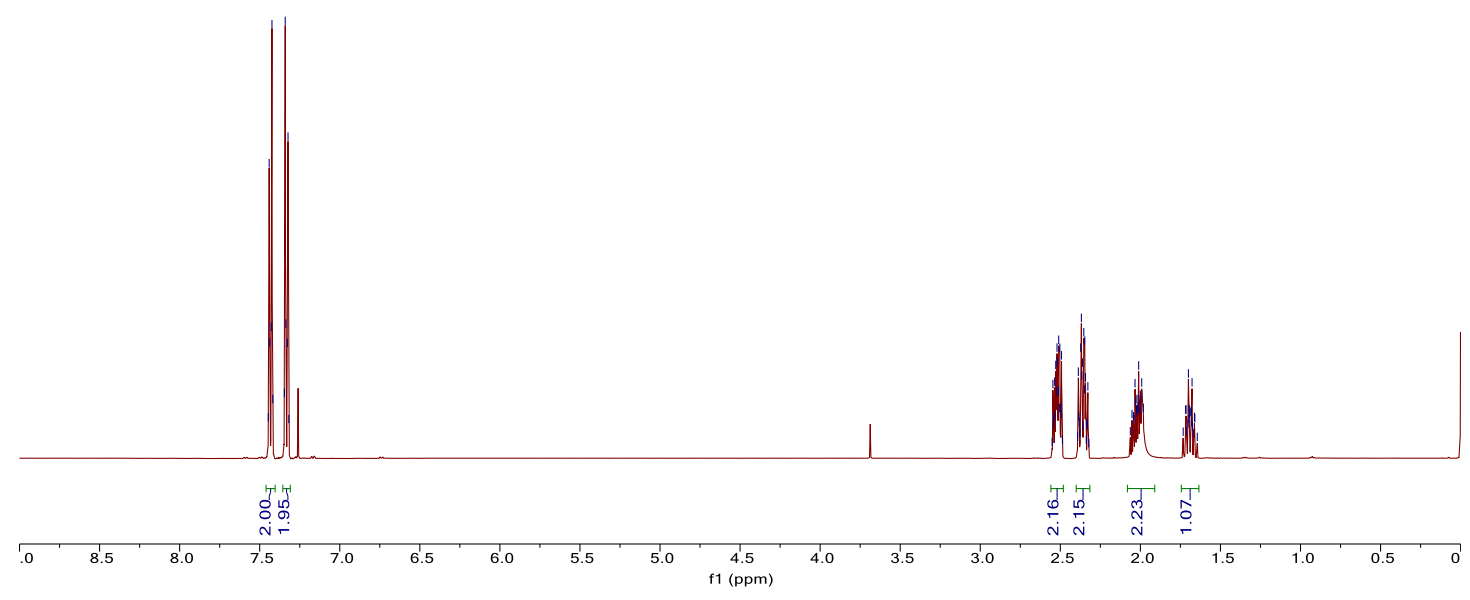

${ }^{13} \mathrm{C}$ NMR $\left(126 \mathrm{MHz}, \mathrm{CDCl}_{3}\right)$<smiles>OC1(c2ccc(Cl)cc2)CCC1</smiles>

$8 a$

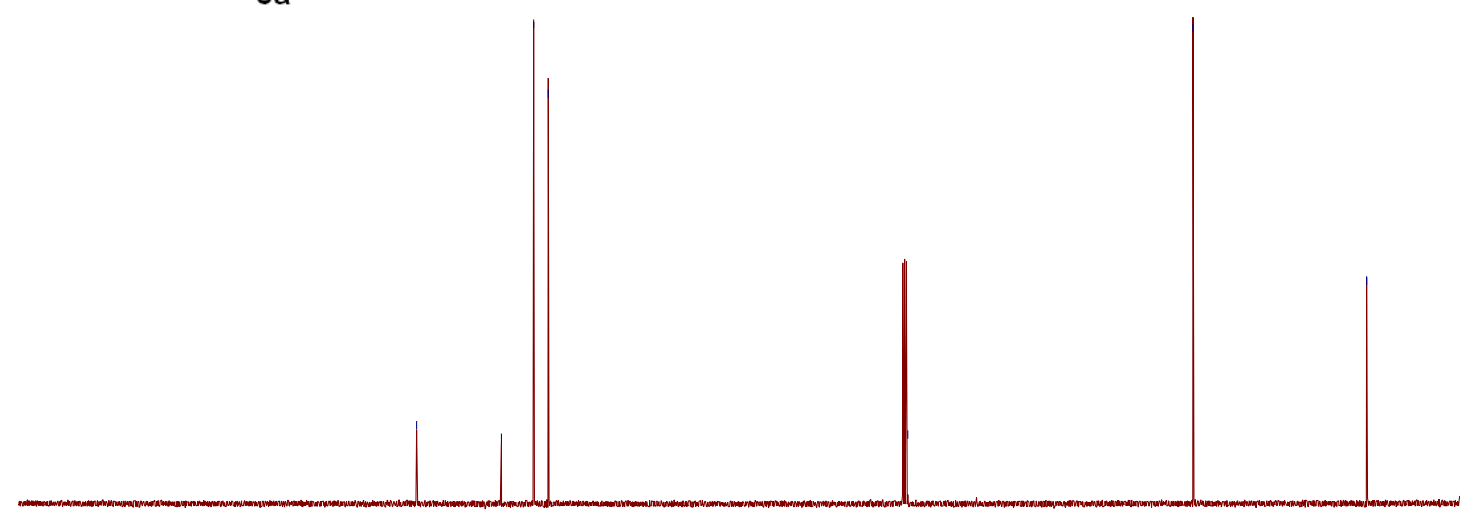

oo
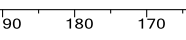
${ }^{1} \mathrm{H}$ NMR (500 MHz, $\left.\mathrm{CDCl}_{3}\right)$

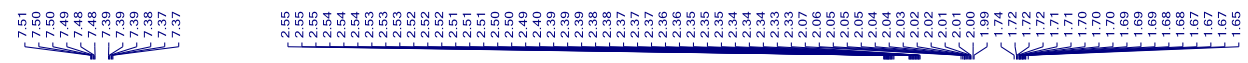

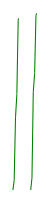<smiles>OC1(c2ccc(Br)cc2)CCC1</smiles>

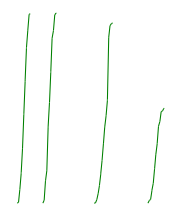

$9 a$

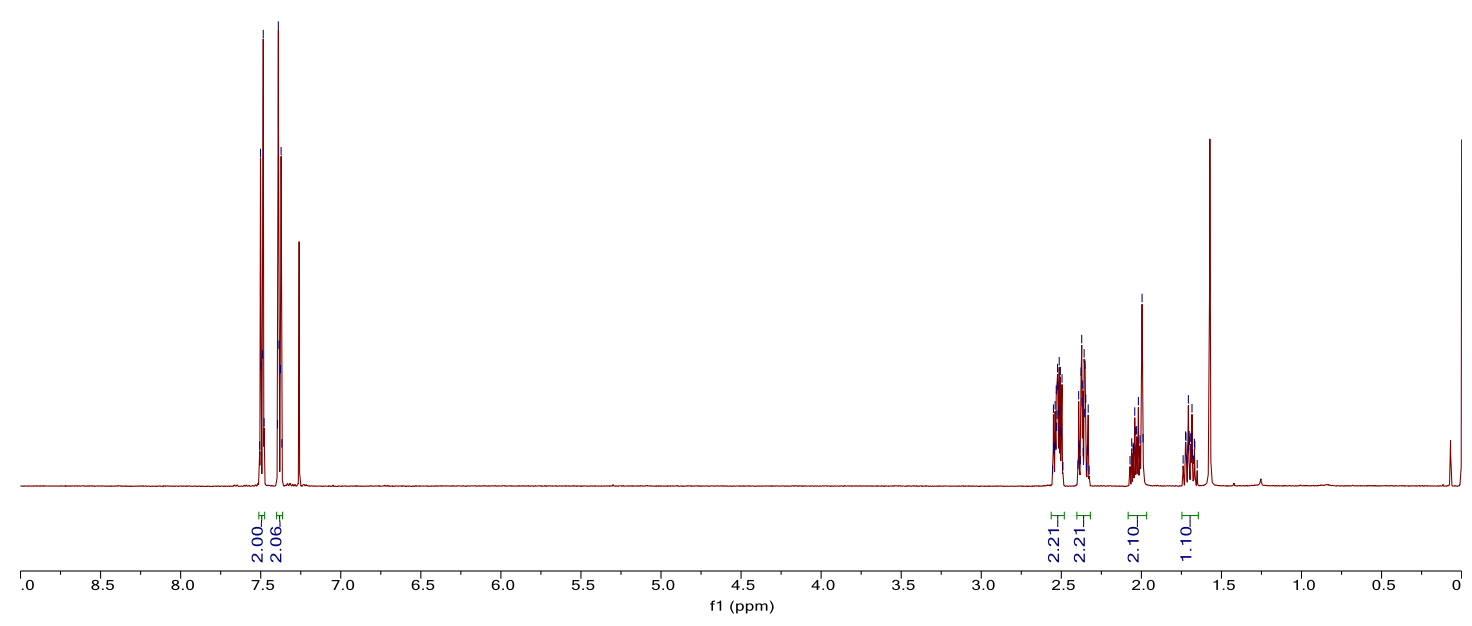

${ }^{13} \mathrm{C}$ NMR $\left(126 \mathrm{MHz}, \mathrm{CDCl}_{3}\right)$<smiles>OC1(c2ccc(Br)cc2)CCC1</smiles>

$9 a$

oo

oo 190

$180 \quad 170$ 100
$f 1(\mathrm{ppm})$ 
${ }^{1} \mathrm{H}$ NMR (500 MHz, $\left.\mathrm{CDCl}_{3}\right)$

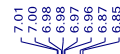

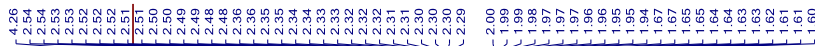
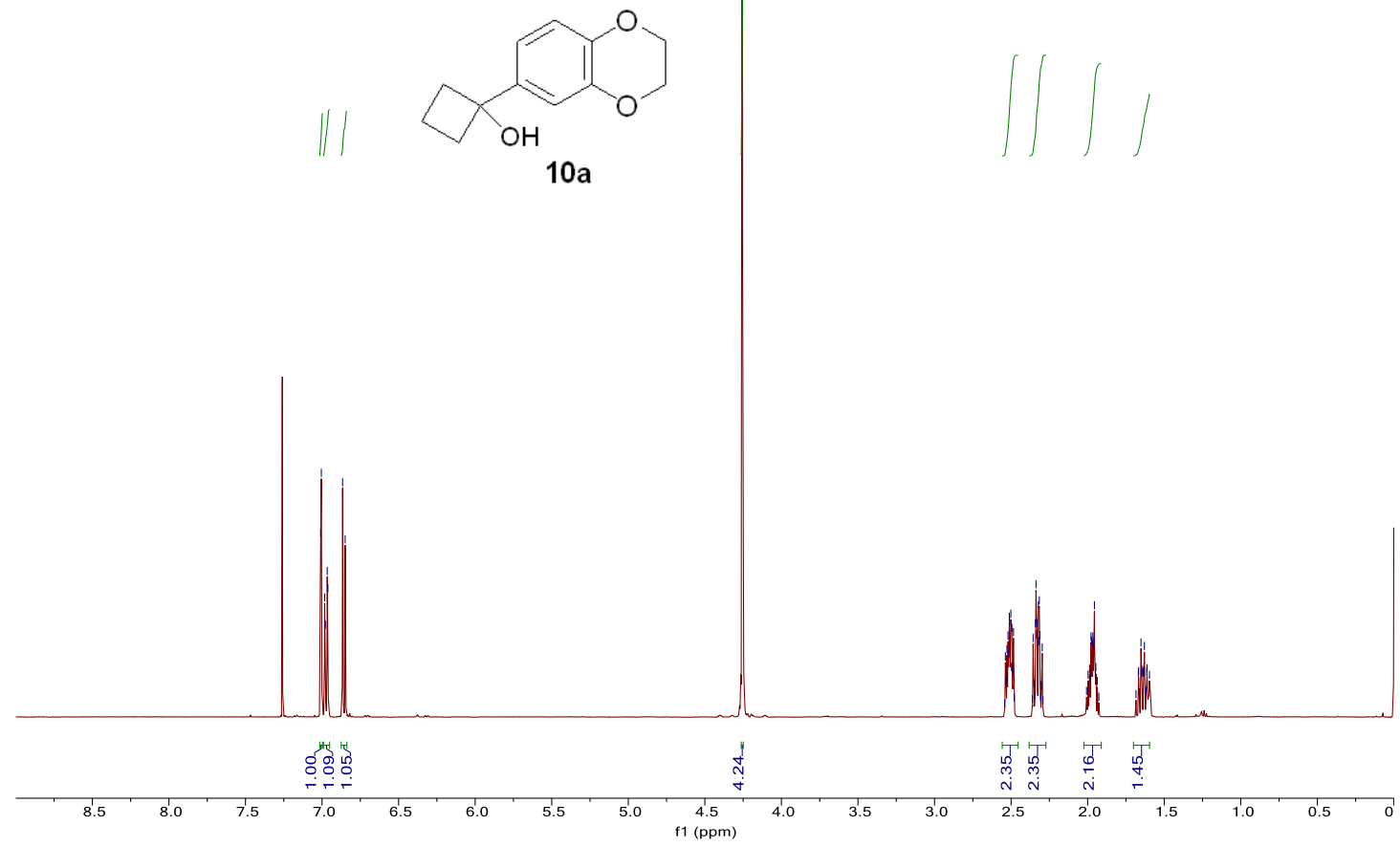

${ }^{13} \mathrm{C}$ NMR $\left(126 \mathrm{MHz}, \mathrm{CDCl}_{3}\right)$

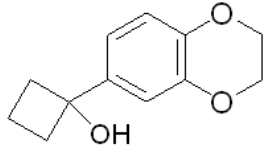

$10 \mathrm{a}$

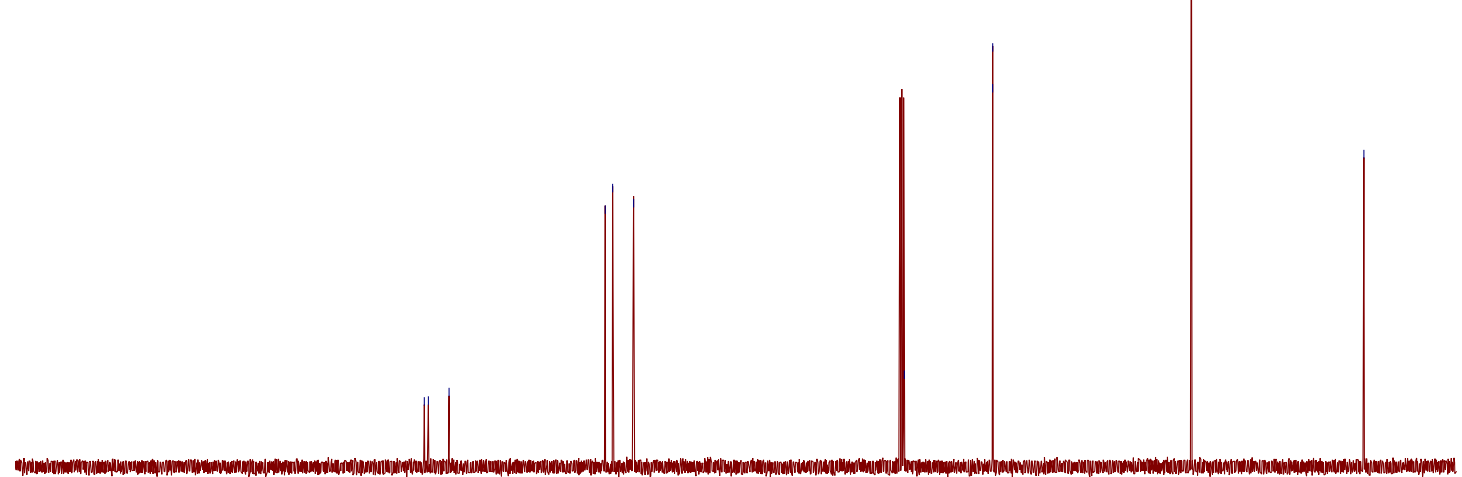
100
$\mathrm{f} 1(\mathrm{ppm})$ 
${ }^{1} \mathrm{H}$ NMR (500 MHz, $\left.\mathrm{CDCl}_{3}\right)$

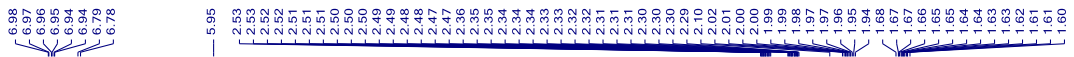
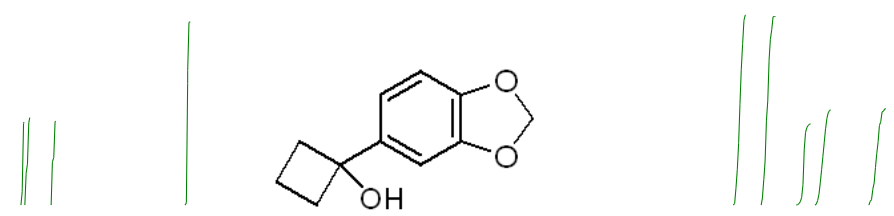

11a

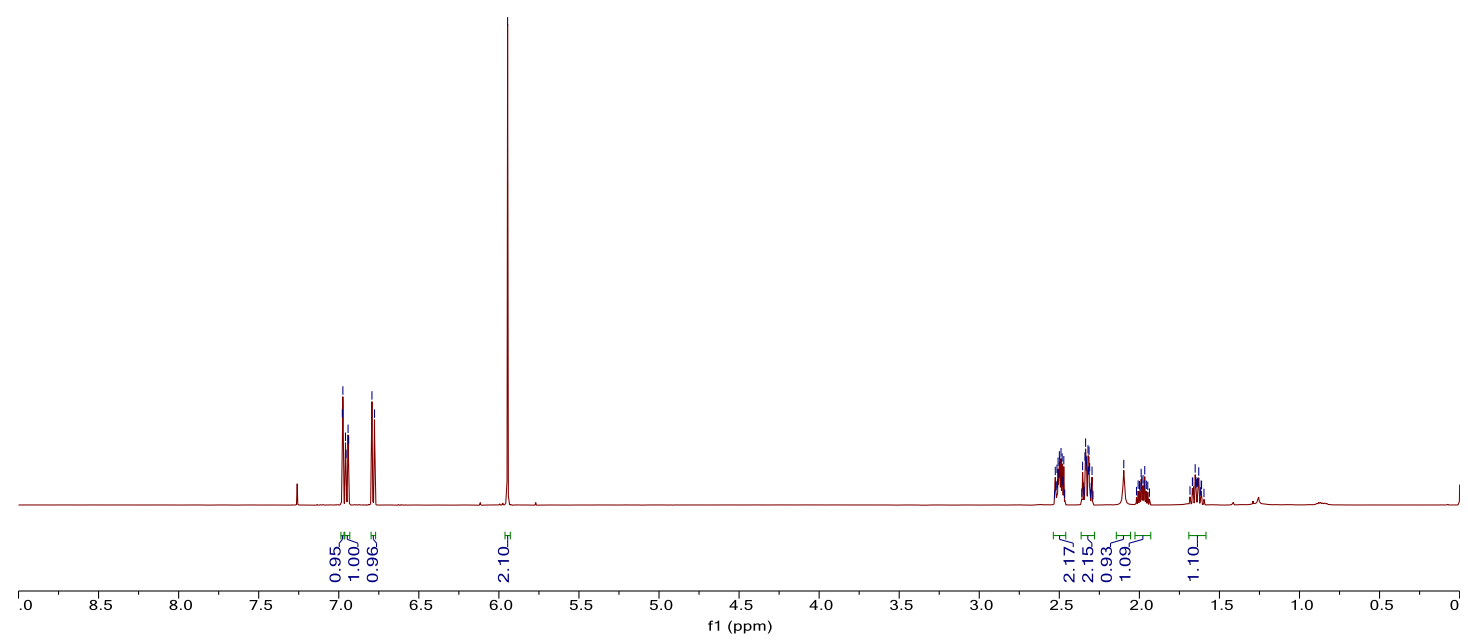

${ }^{13} \mathrm{C} \mathrm{NMR}\left(126 \mathrm{MHz}, \mathrm{CDCl}_{3}\right)$

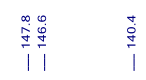<smiles>OC1(c2ccc3c(c2)OCO3)CCC1</smiles>

$11 \mathrm{a}$

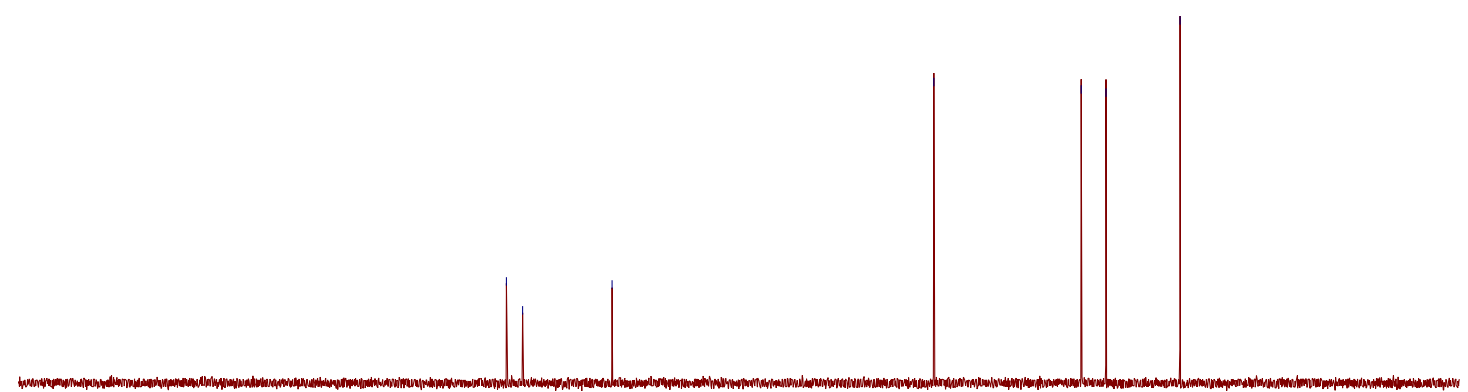

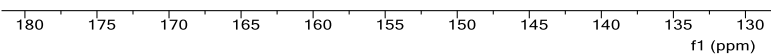


${ }^{1} \mathrm{H}$ NMR (500 MHz, $\left.\mathrm{CDCl}_{3}\right)$

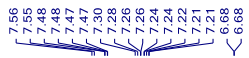

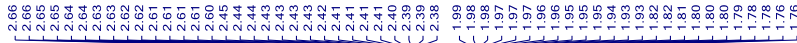

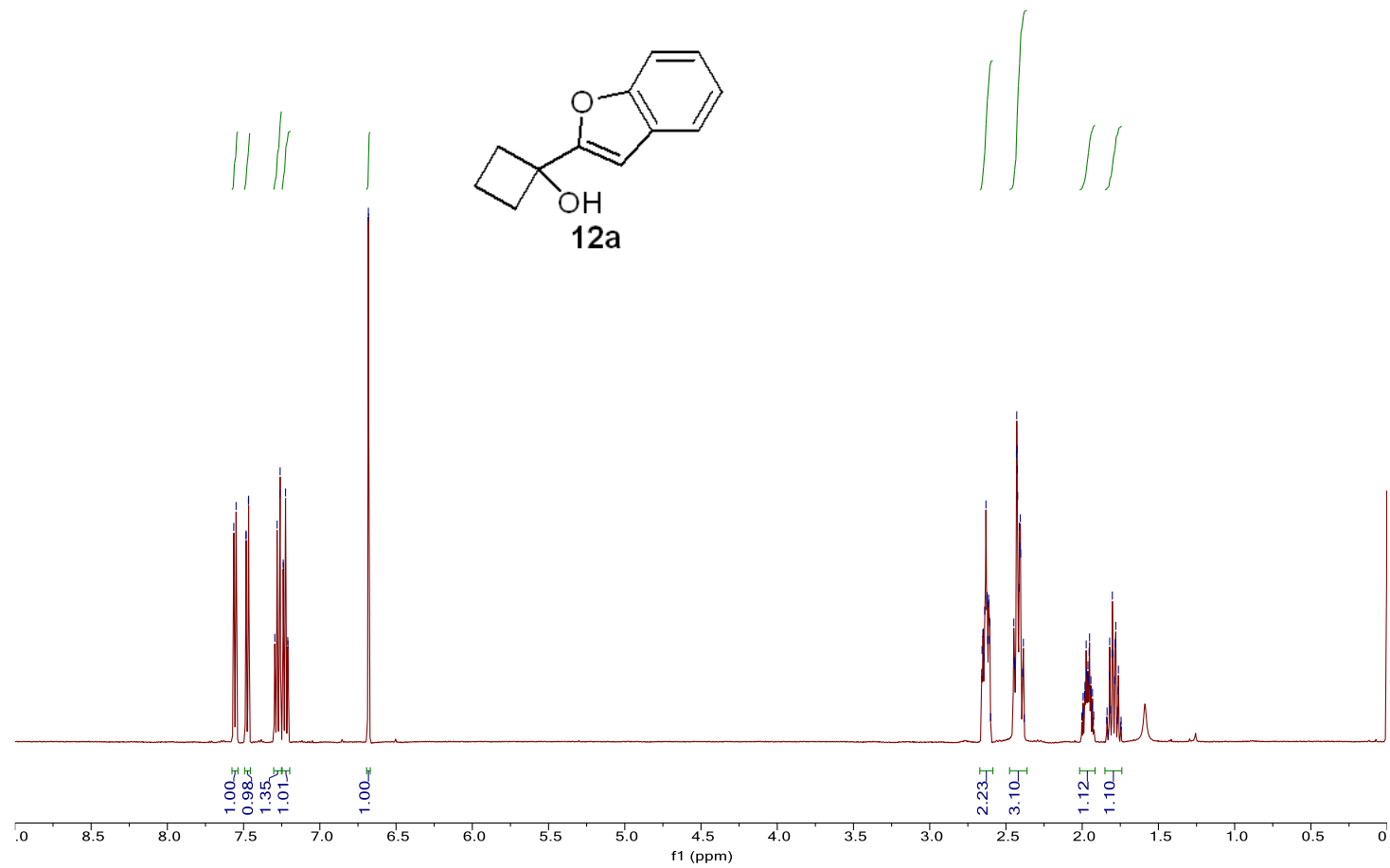

${ }^{13} \mathrm{C}$ NMR (126 MHz, $\left.\mathrm{CDCl}_{3}\right)$

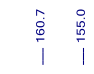

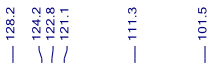<smiles>OC1(c2cc3ccccc3o2)CCC1</smiles>

$12 \mathrm{a}$

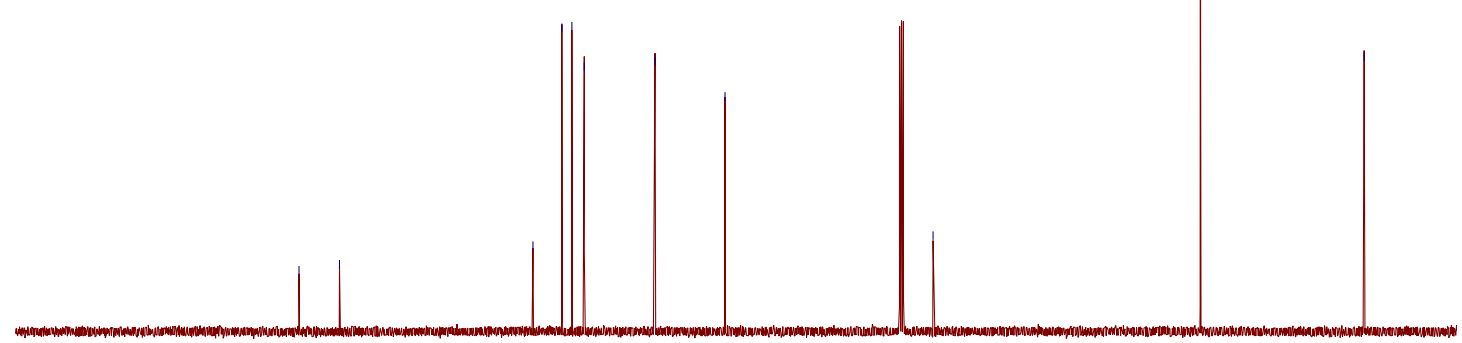

00 f1 $(\mathrm{ppm})$ 
${ }^{1} \mathrm{H}$ NMR (500 MHz, $\left.\mathrm{CDCl}_{3}\right)$

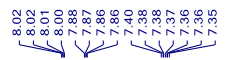

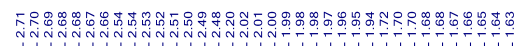
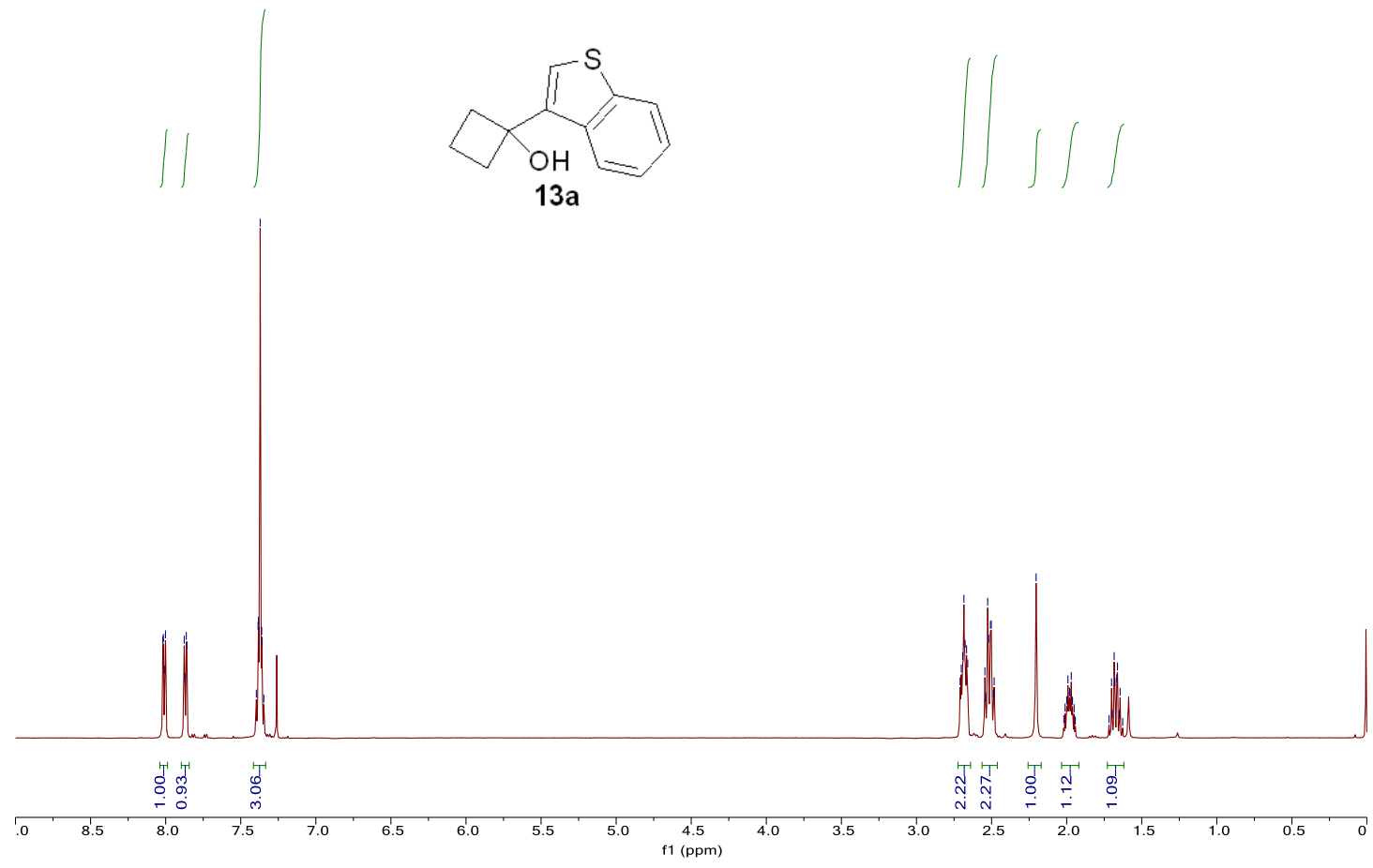

${ }^{13} \mathrm{C} \mathrm{NMR}\left(126 \mathrm{MHz}, \mathrm{CDCl}_{3}\right)$

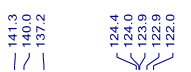

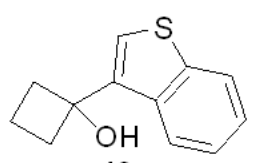

$13 a$

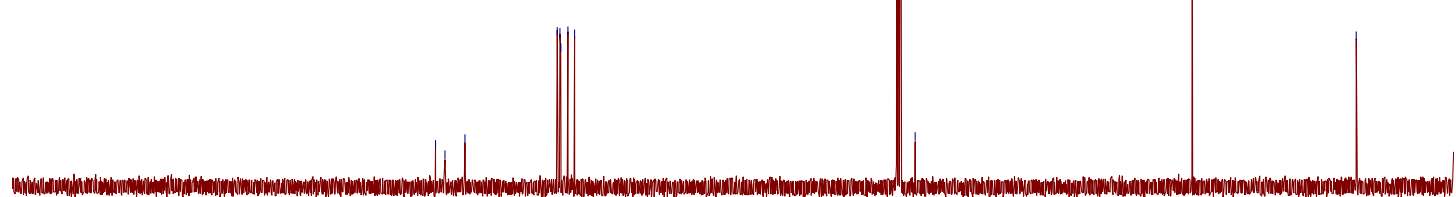

oo

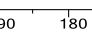

170

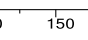

$10 \begin{gathered}100 \\ f 1(\mathrm{ppm})\end{gathered}$ 
${ }^{1} \mathrm{H}$ NMR (500 MHz, $\mathrm{CDCl}_{3}$ )

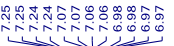
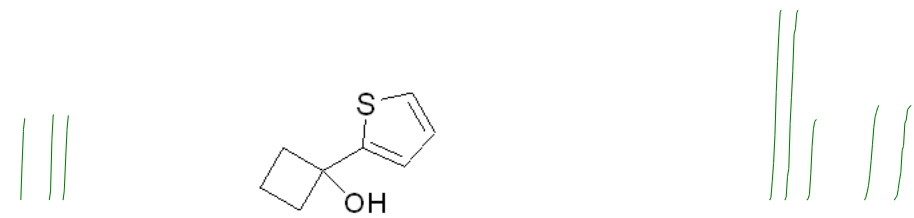

$14 \mathrm{a}$

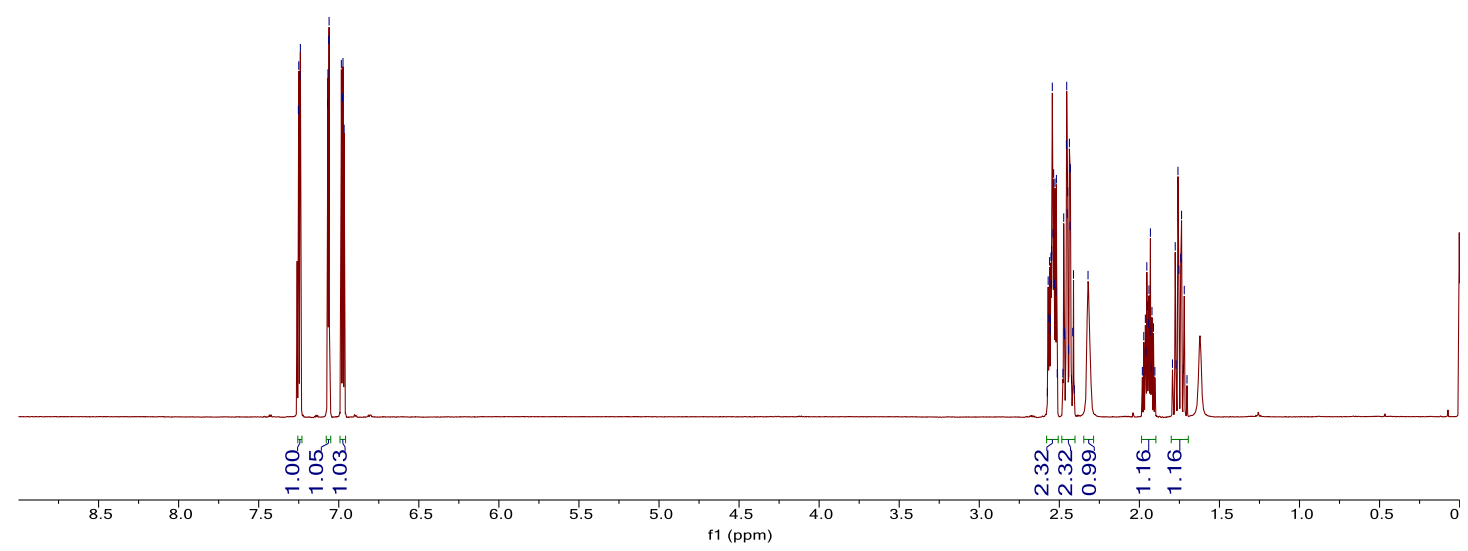

${ }^{13} \mathrm{C}$ NMR $\left(126 \mathrm{MHz}, \mathrm{CDCl}_{3}\right)$

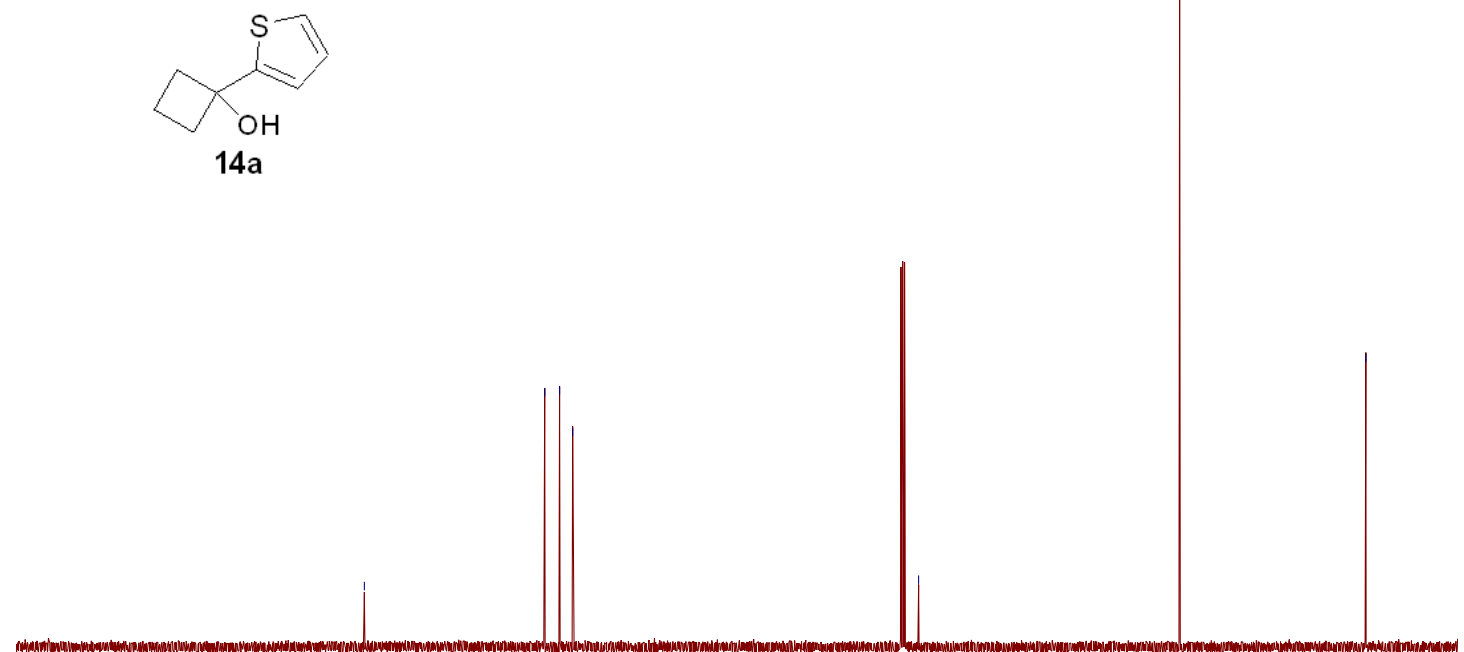

$14 a$

\begin{tabular}{llllll}
\hline 00 & 190 & 180 & 170 & 160 & 150
\end{tabular} 100
$\mathrm{f} 1(\mathrm{ppm})$ 
${ }^{1} \mathrm{H}$ NMR (500 MHz, $\mathrm{CDCl}_{3}$ )

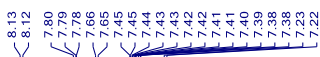

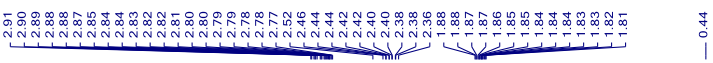
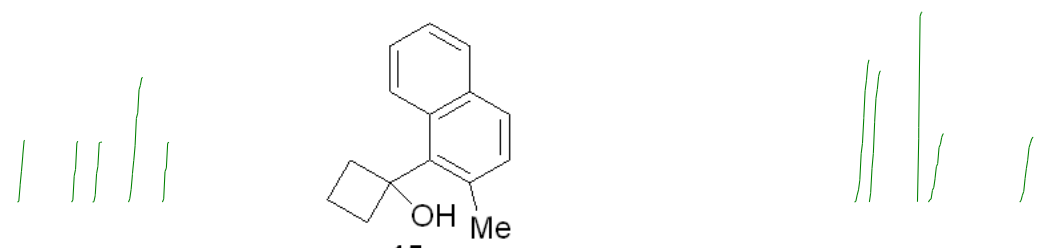

$15 a$

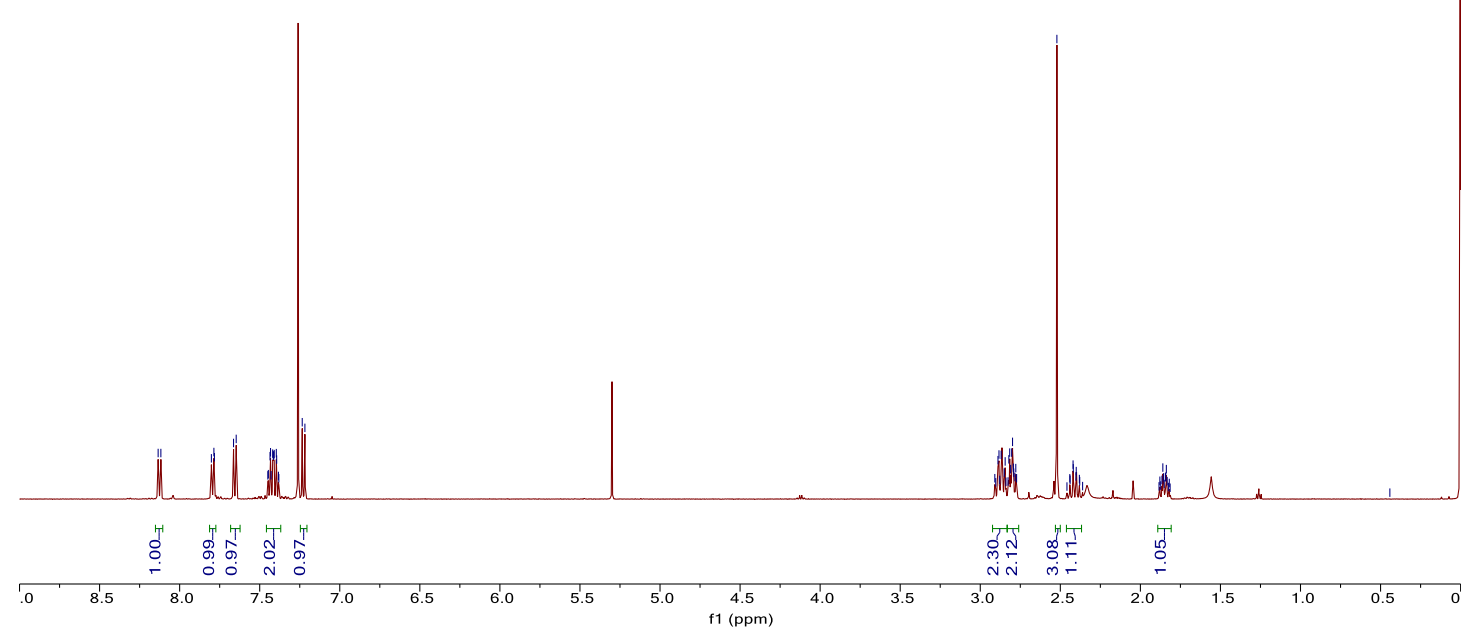

${ }^{13} \mathrm{C}$ NMR (126 MHz, $\left.\mathrm{CDCl}_{3}\right)$

ำ

MI1एक

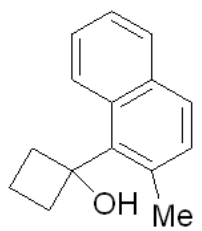

$15 a$

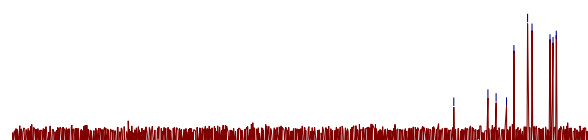

\begin{tabular}{llllll}
\hline 00 & 190 & 180 & 170 & 160 & 150
\end{tabular} 100
$\mathrm{f} 1(\mathrm{ppm})$ 
${ }^{1} \mathrm{H}$ NMR (500 MHz, $\left.\mathrm{CDCl}_{3}\right)$

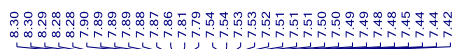

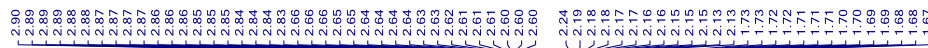
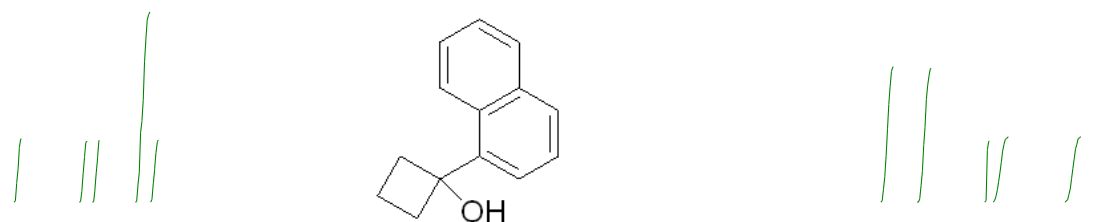

$16 \mathrm{a}$

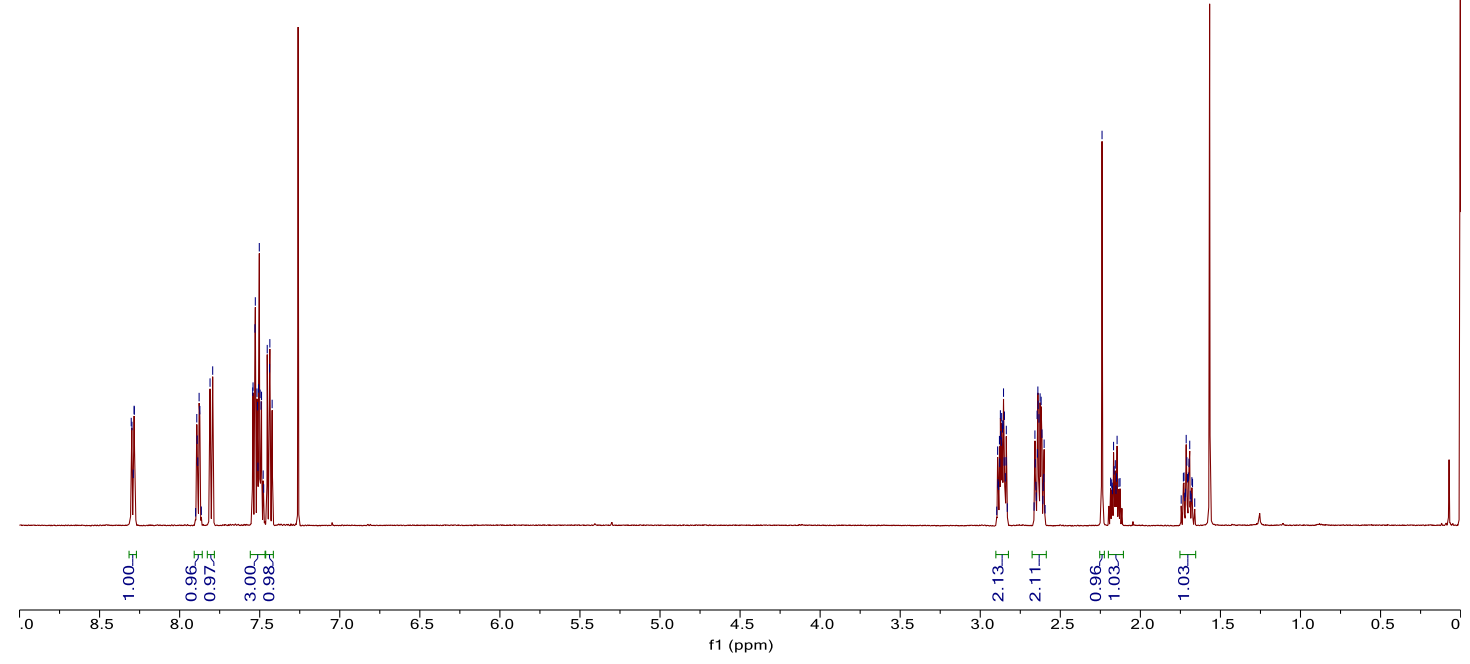

${ }^{13} \mathrm{C} \mathrm{NMR}\left(126 \mathrm{MHz}, \mathrm{CDCl}_{3}\right)$

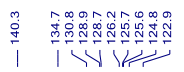

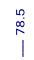<smiles>OC1(c2cccc3ccccc23)CCC1</smiles>

$16 \mathrm{a}$

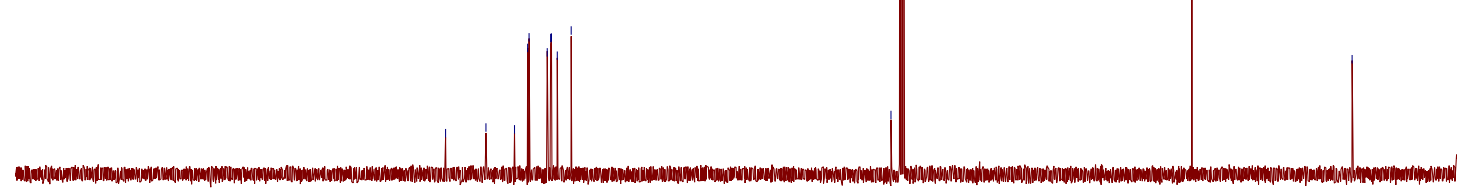

oo $190 \quad 180$

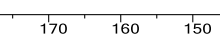

$150 \quad 140$

$10 \begin{gathered}100 \\ f 1(\mathrm{ppm})\end{gathered}$ 
${ }^{1} \mathrm{H} \mathrm{NMR}\left(500 \mathrm{MHz}, \mathrm{CDCl}_{3}\right)$

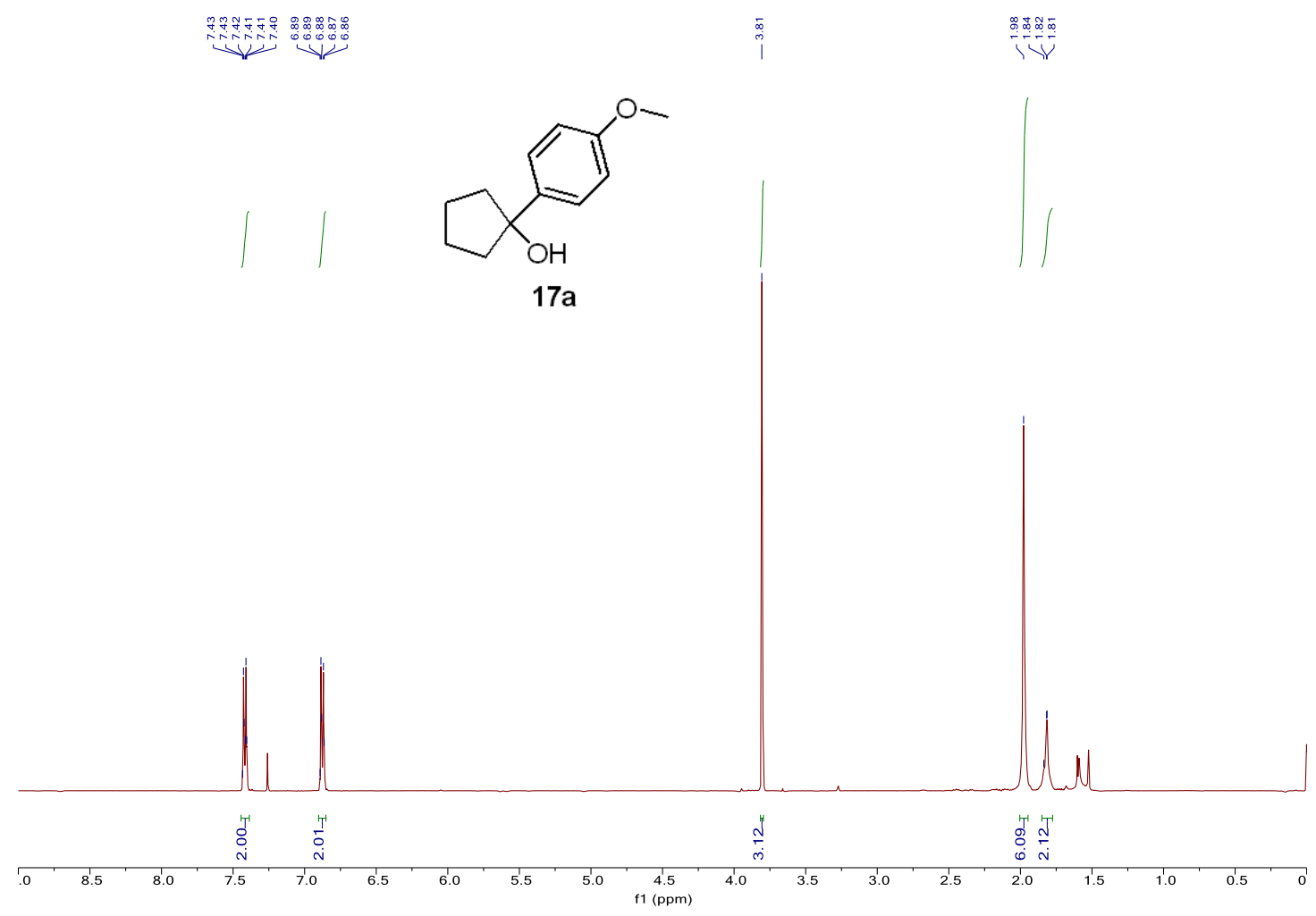

${ }^{13} \mathrm{C} \mathrm{NMR}\left(126 \mathrm{MHz}, \mathrm{CDCl}_{3}\right)$

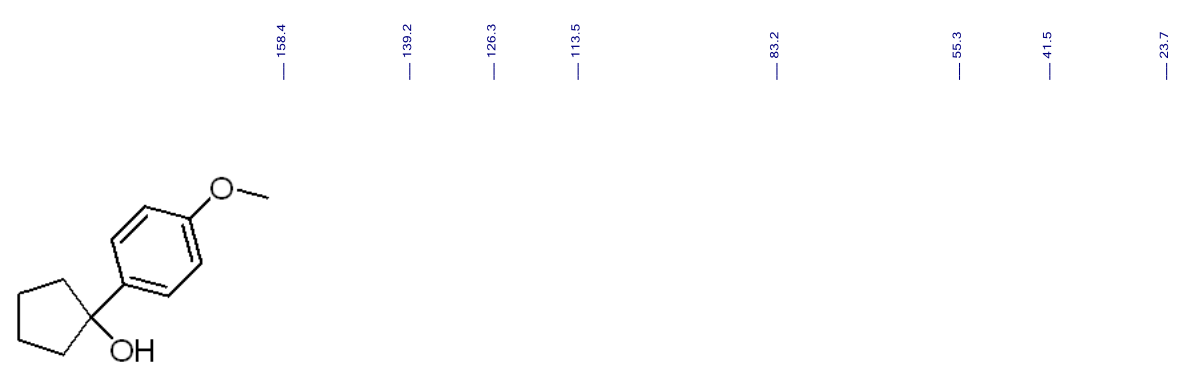

17a

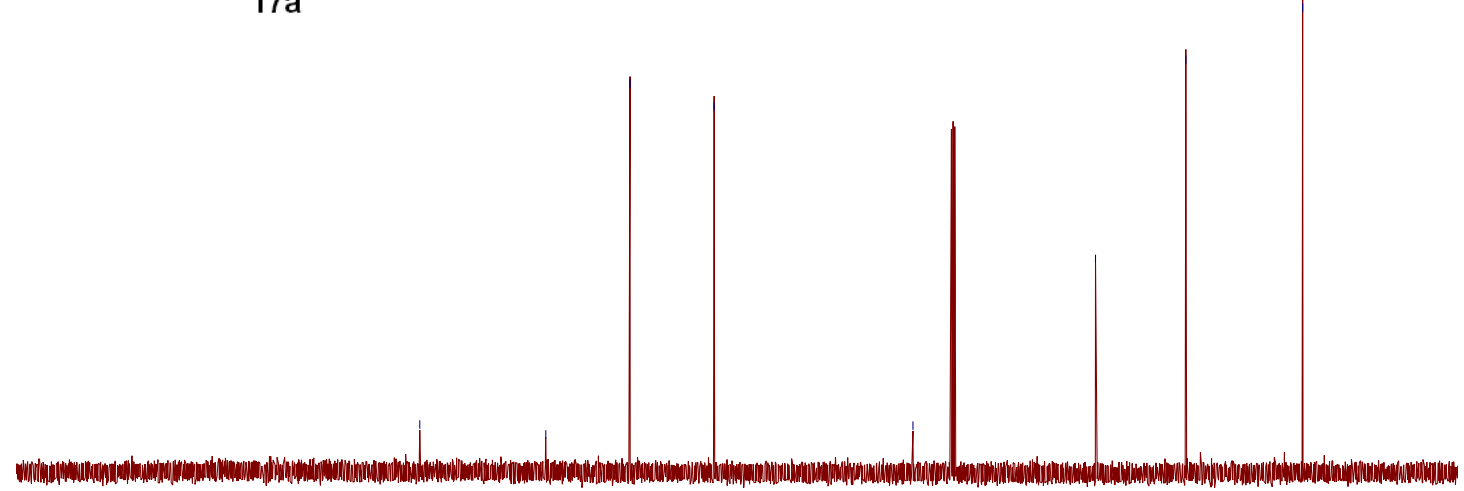

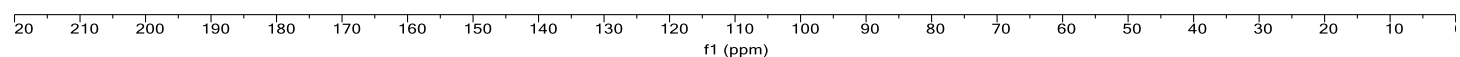


${ }^{1} \mathrm{H} \mathrm{NMR}\left(500 \mathrm{MHz}, \mathrm{CDCl}_{3}\right)$

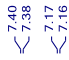

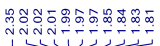
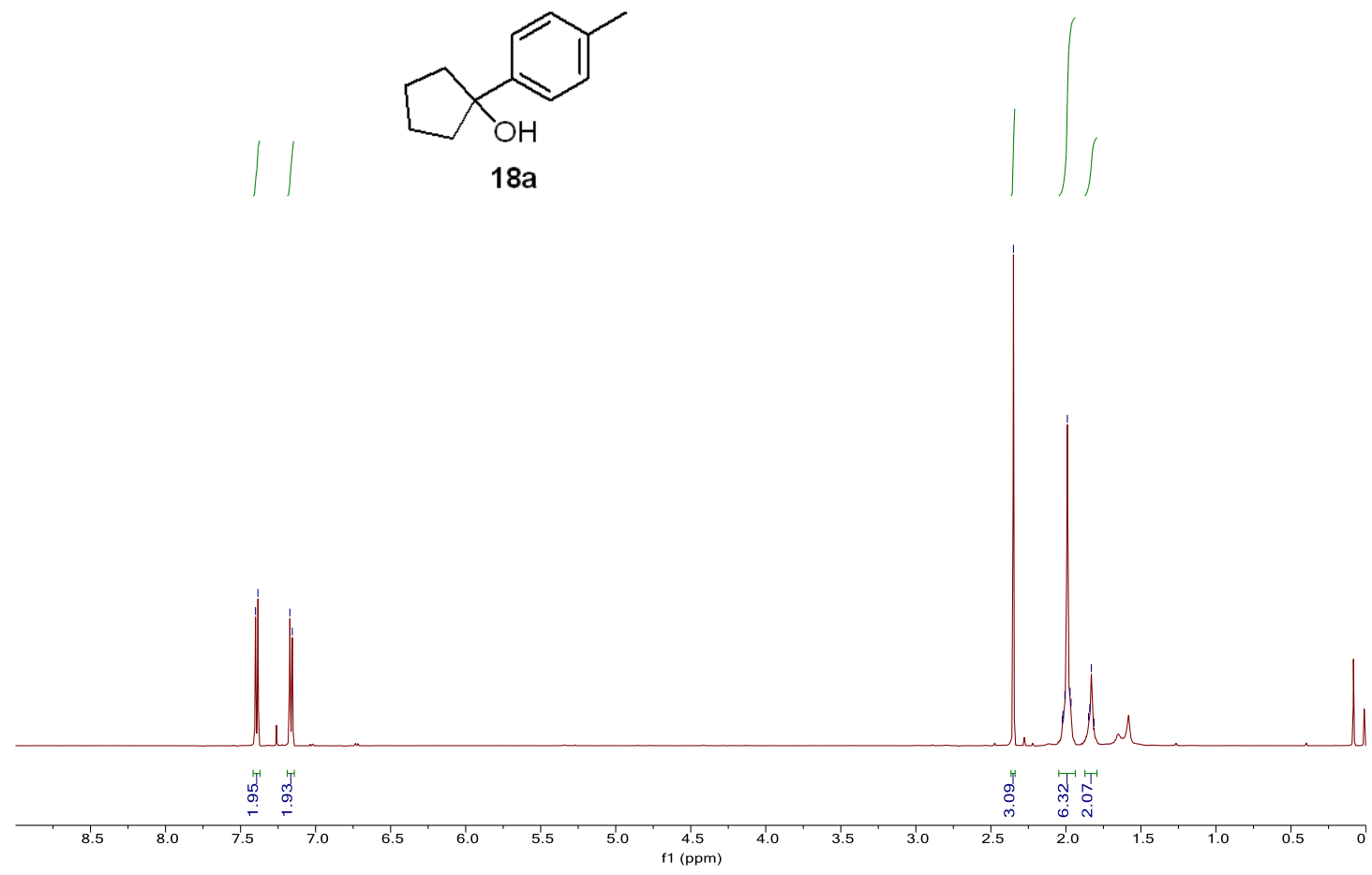

${ }^{13} \mathrm{C}$ NMR $\left(126 \mathrm{MHz}, \mathrm{CDCl}_{3}\right)$

$\underset{⿱}{1}$
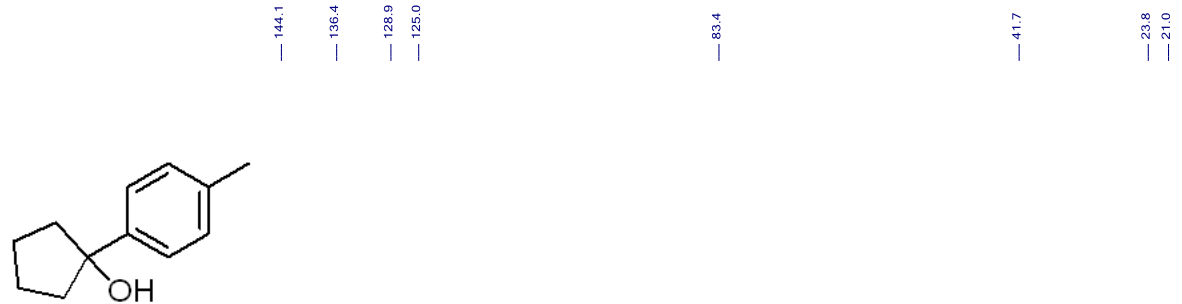

$18 a$

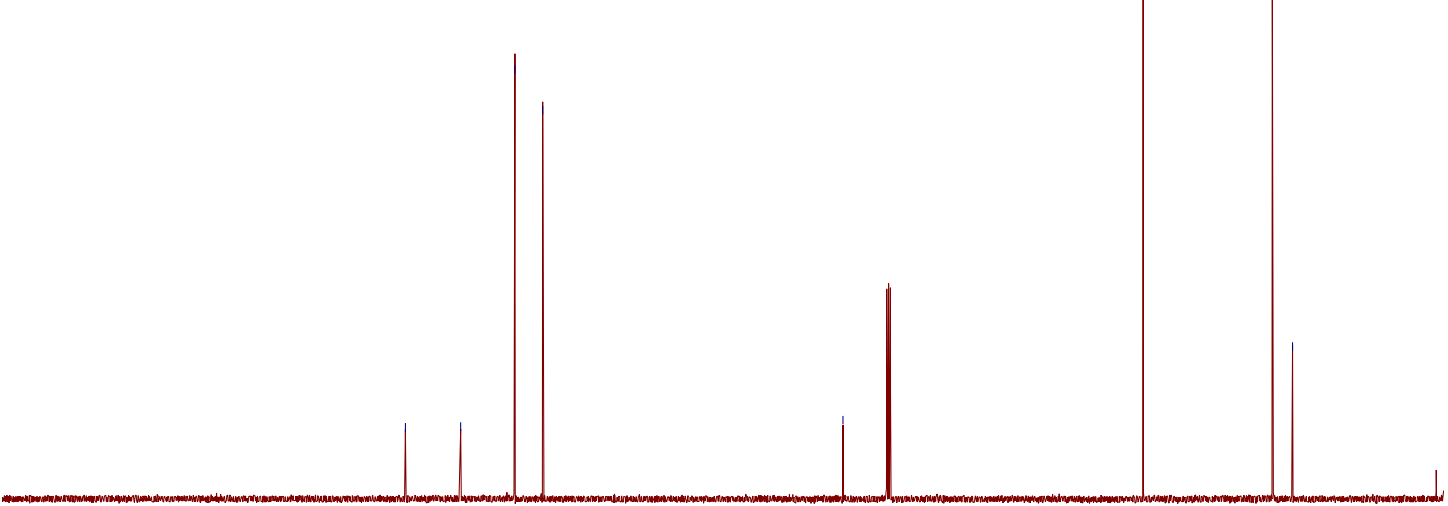

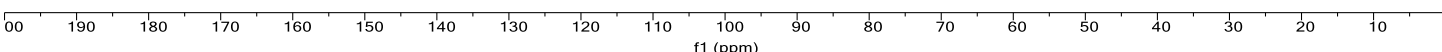


${ }^{1} \mathrm{H} \mathrm{NMR}\left(500 \mathrm{MHz}, \mathrm{CDCl}_{3}\right)$

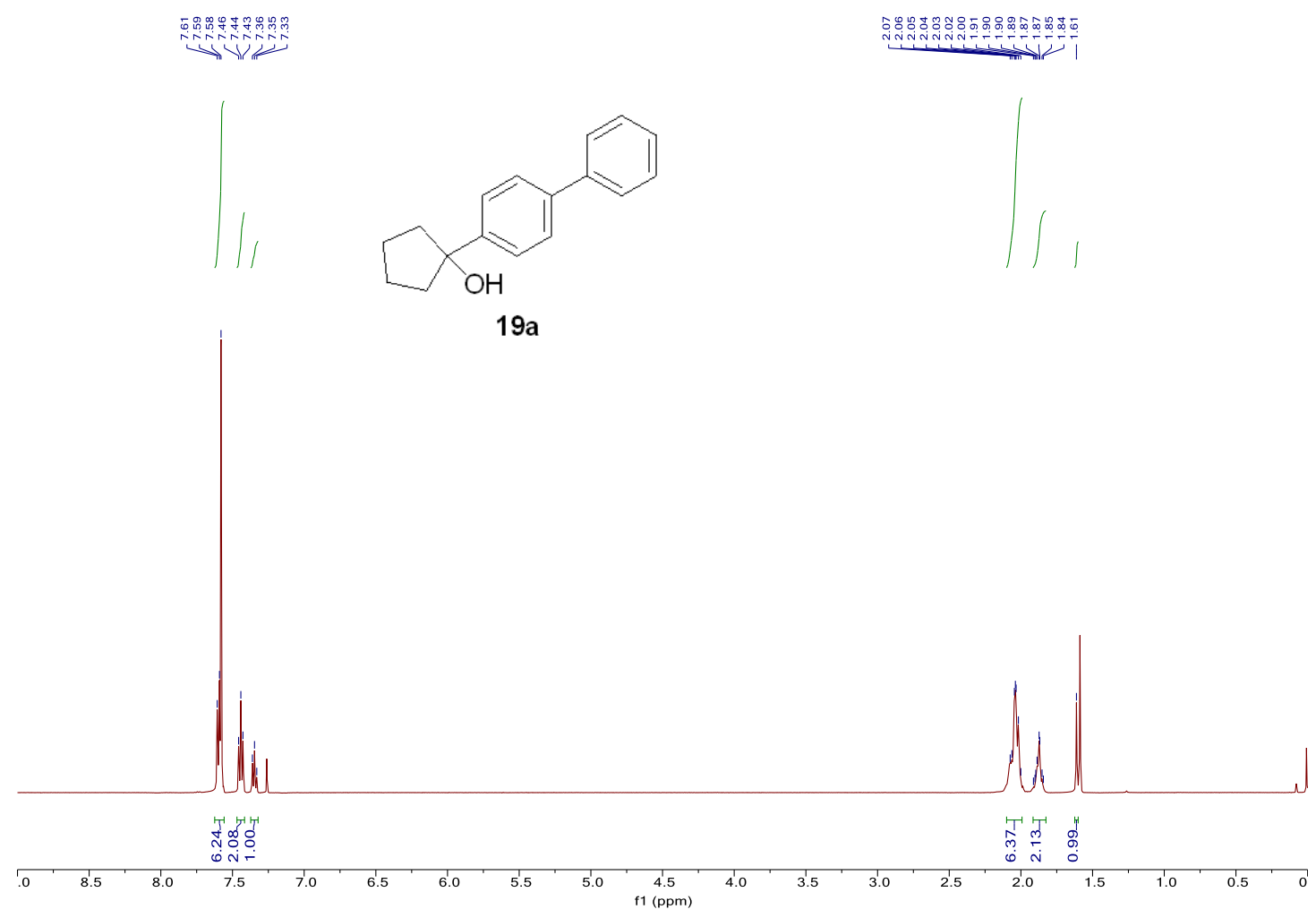

${ }^{13} \mathrm{C} \mathrm{NMR}\left(126 \mathrm{MHz}, \mathrm{CDCl}_{3}\right)$

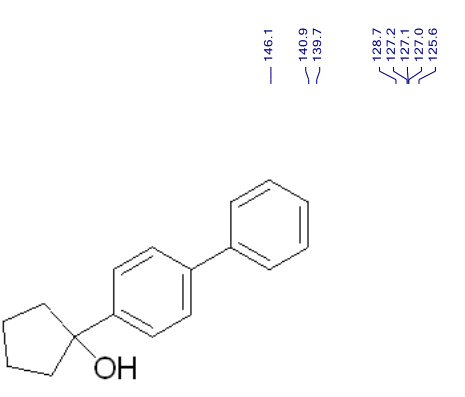

$19 a$

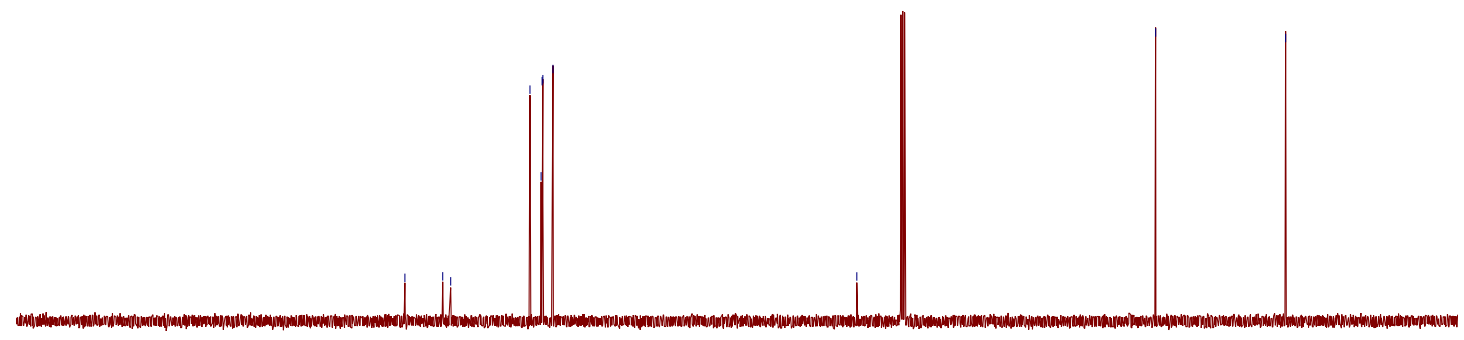

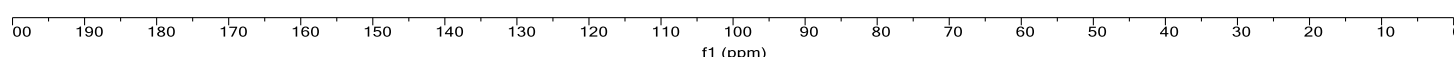


${ }^{1} \mathrm{H} \mathrm{NMR}\left(500 \mathrm{MHz}, \mathrm{CDCl}_{3}\right)$

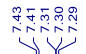

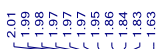

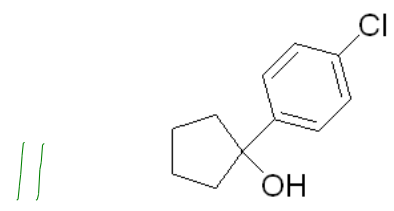

$20 \mathrm{a}$

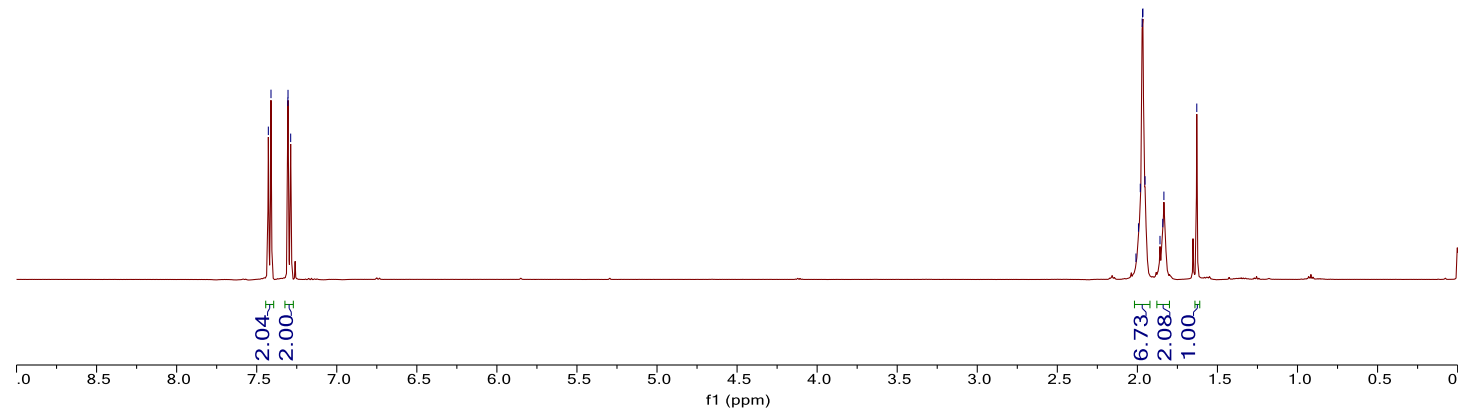

${ }^{13} \mathrm{C}$ NMR $\left(126 \mathrm{MHz}, \mathrm{CDCl}_{3}\right)$

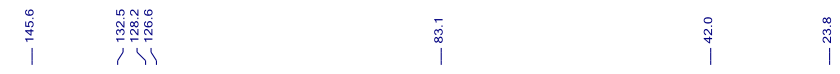

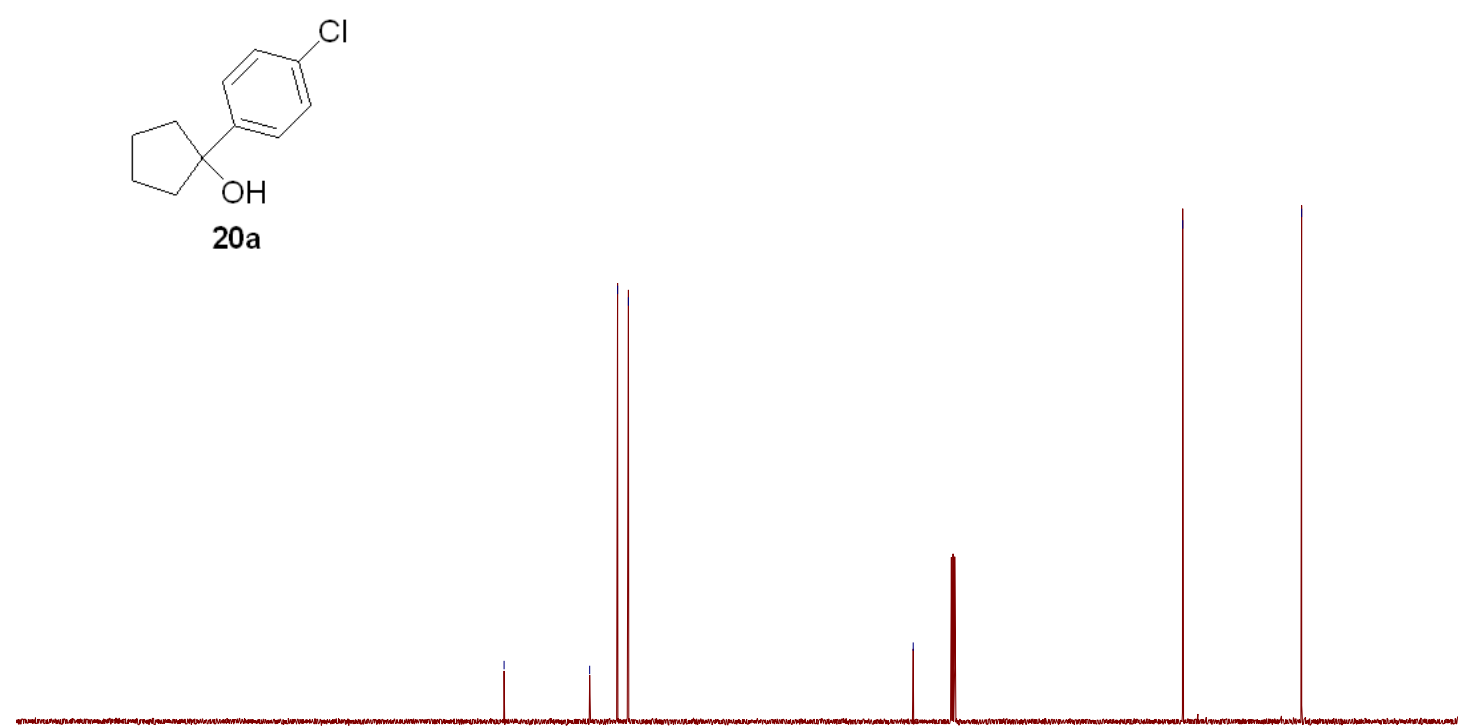

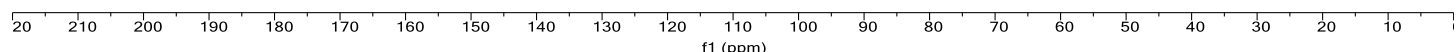


${ }^{1} \mathrm{H}$ NMR (500 MHz, $\mathrm{CDCl}_{3}$ )

琴铝

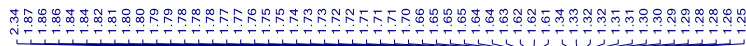
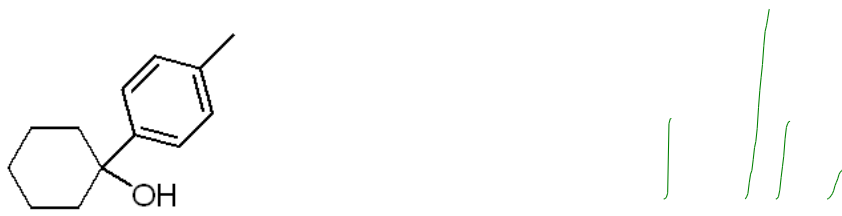

21a

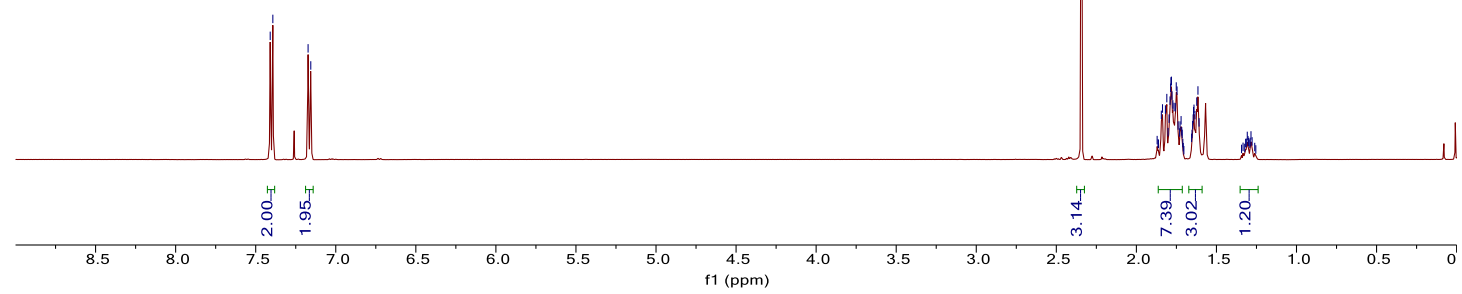

${ }^{13} \mathrm{C}$ NMR $\left(126 \mathrm{MHz}, \mathrm{CDCl}_{3}\right)$

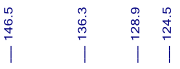

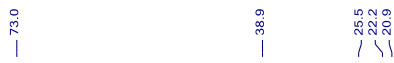

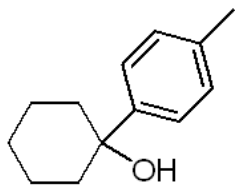

21a

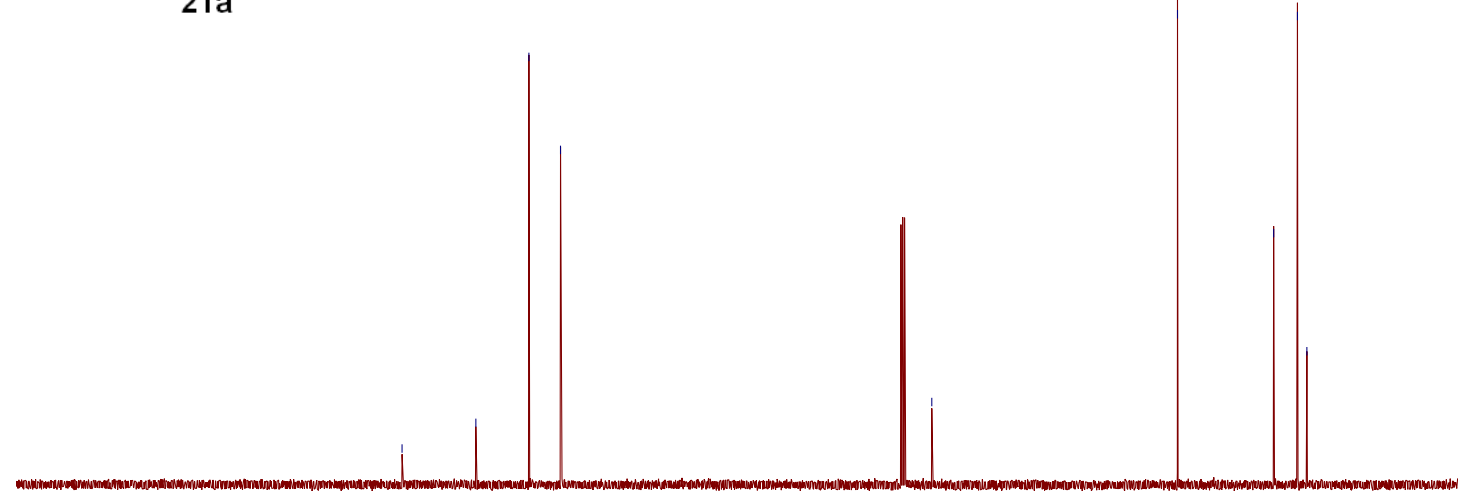

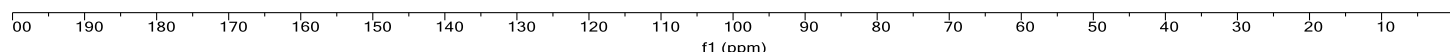


${ }^{1} \mathrm{H}$ NMR (500 MHz, $\mathrm{CDCl}_{3}$ )
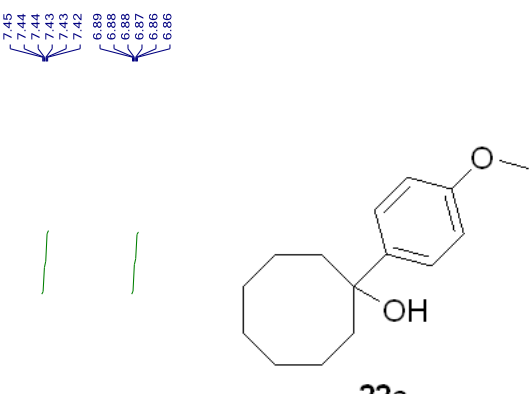

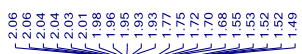
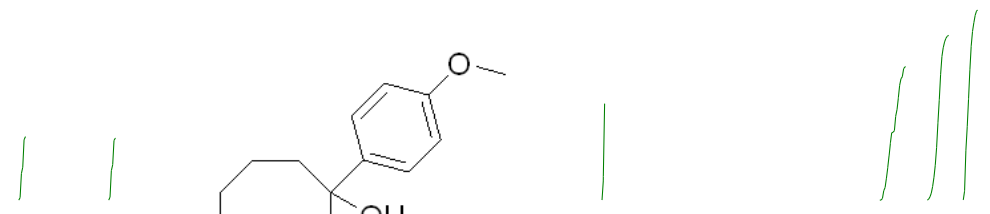

22a
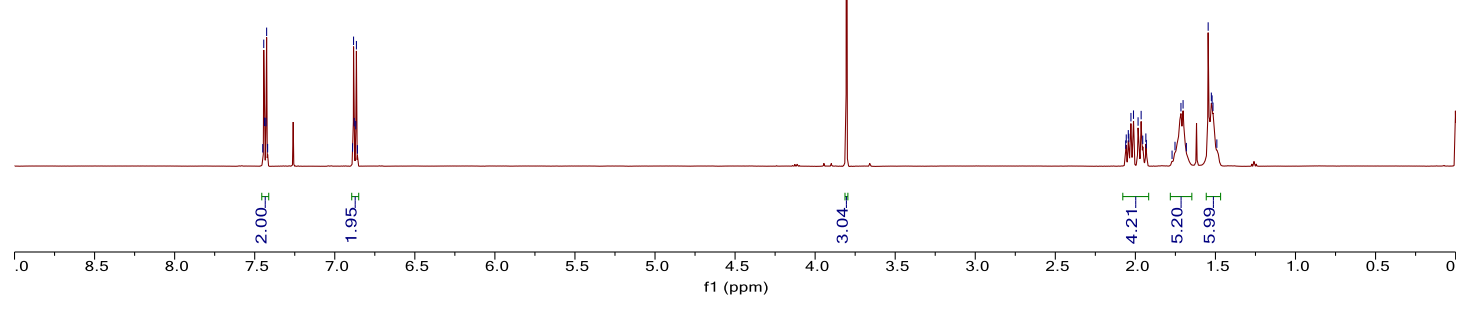

${ }^{13} \mathrm{C} \mathrm{NMR}\left(126 \mathrm{MHz}, \mathrm{CDCl}_{3}\right)$

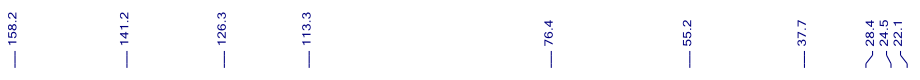

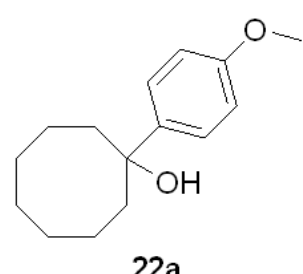

$22 a$

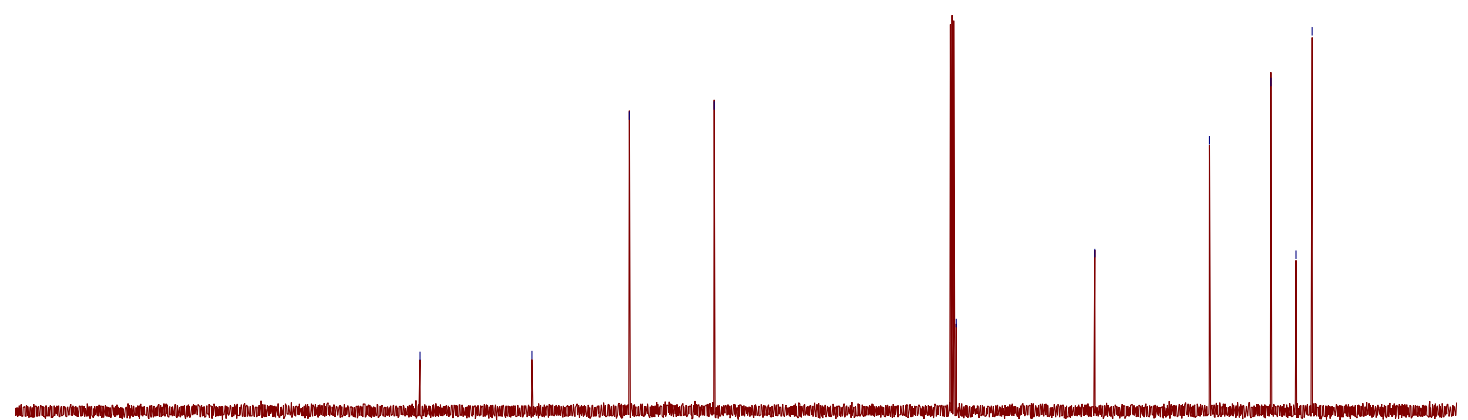

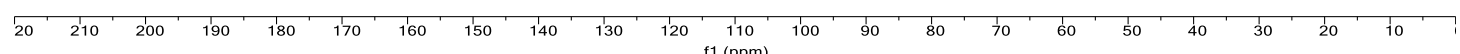


${ }^{1} \mathrm{H}$ NMR (500 MHz, $\left.\mathrm{CDCl}_{3}\right)$

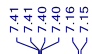

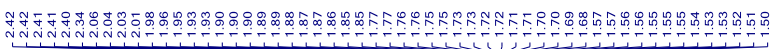
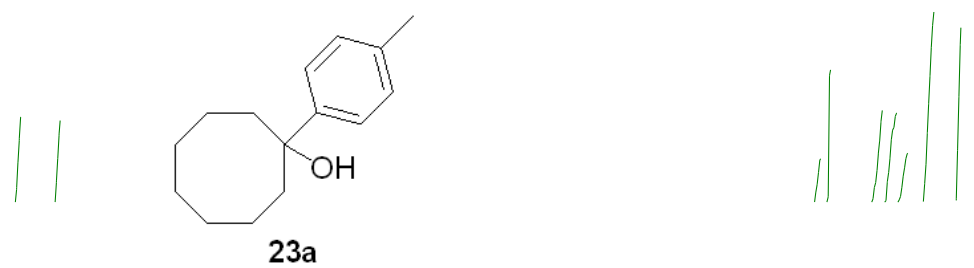

$23 a$

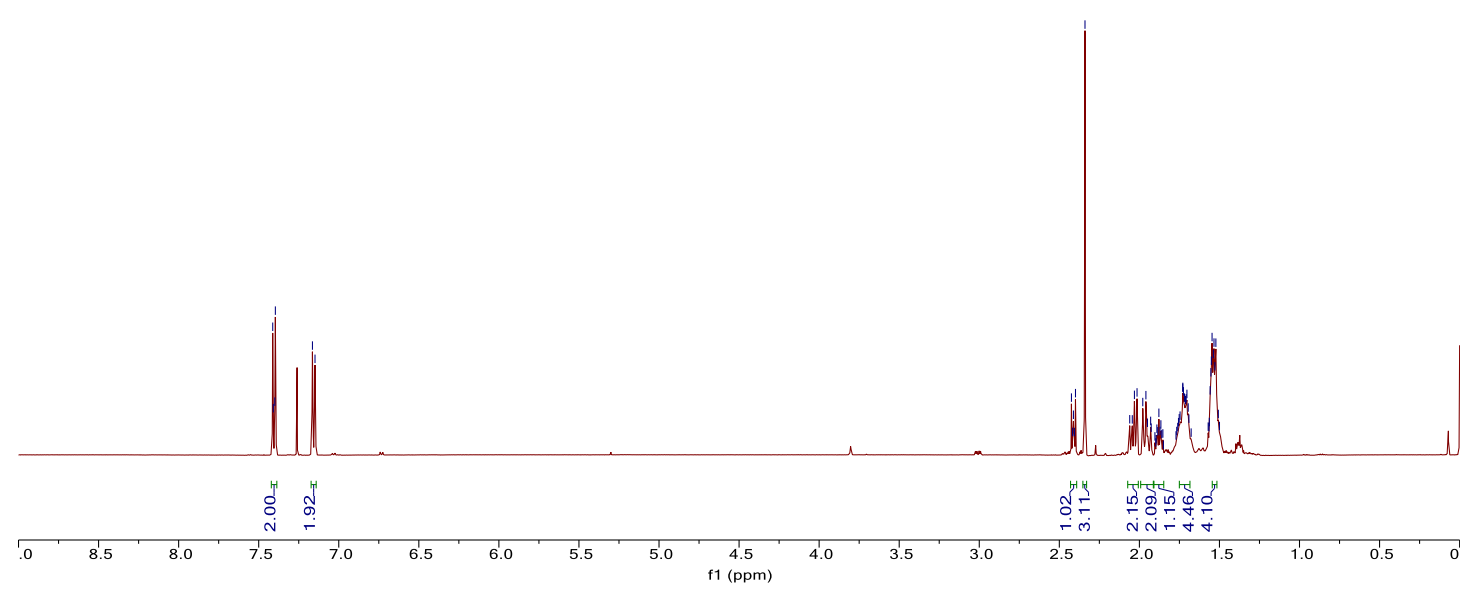

${ }^{13} \mathrm{C}$ NMR $\left(126 \mathrm{MHz}, \mathrm{CDCl}_{3}\right)$

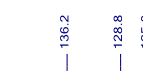

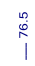

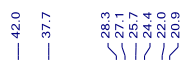
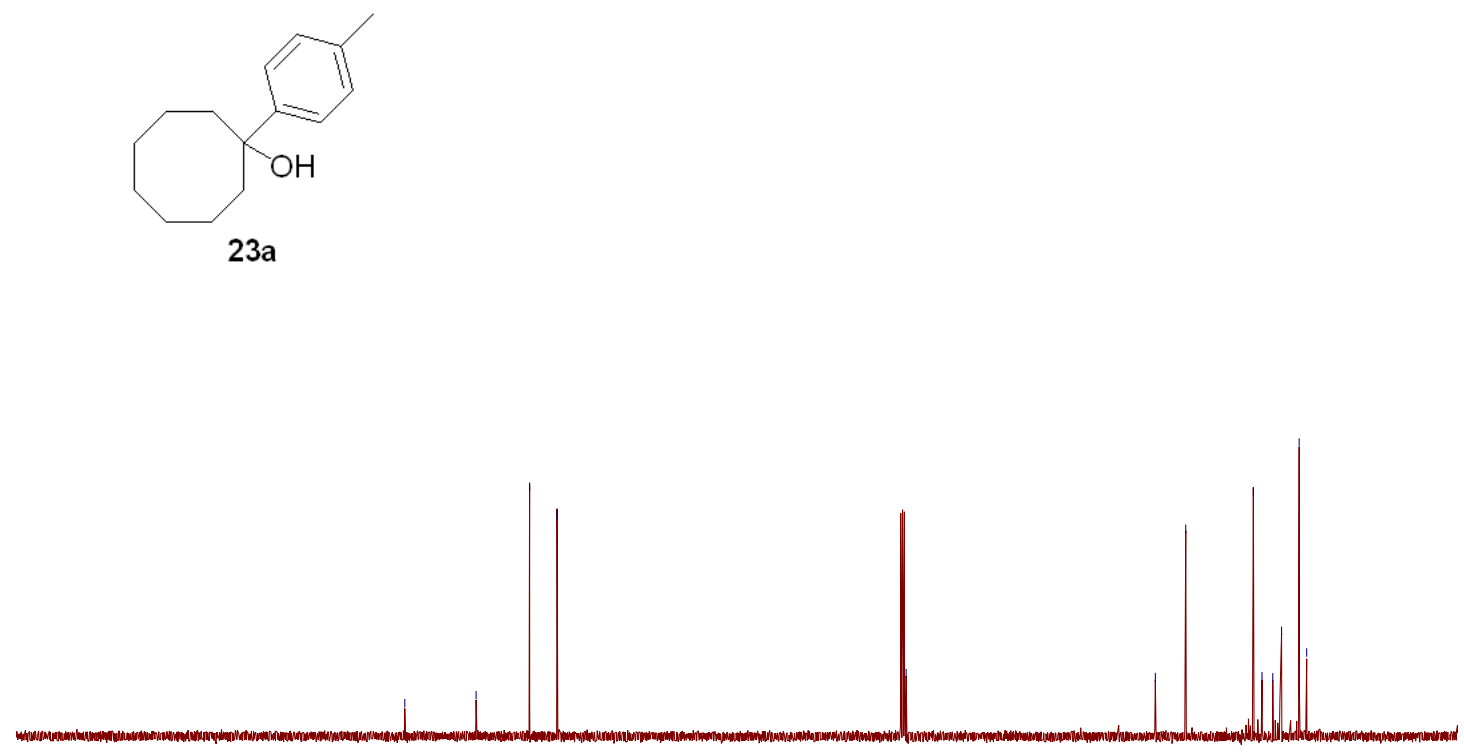

oo

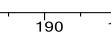

$180 \quad 170$

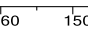

140

$110 \quad 100$

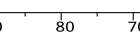

60

$\begin{array}{llll}10 & 10 & 10 & 10\end{array}$ 
${ }^{1} \mathrm{H} \mathrm{NMR}\left(500 \mathrm{MHz}, \mathrm{CDCl}_{3}\right)$

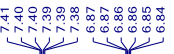

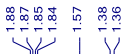

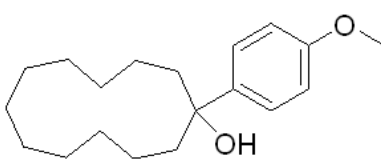

$24 a$

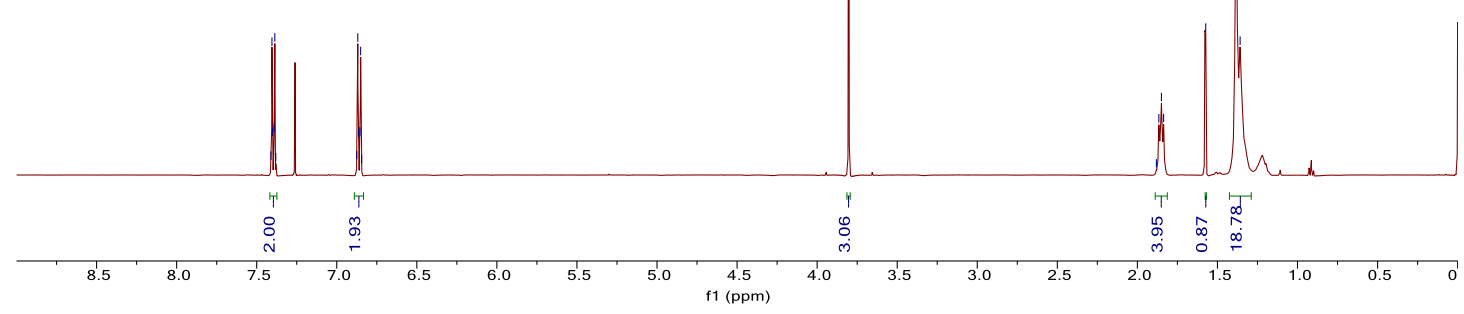

${ }^{13} \mathrm{C}$ NMR (126 MHz, $\left.\mathrm{CDCl}_{3}\right)$

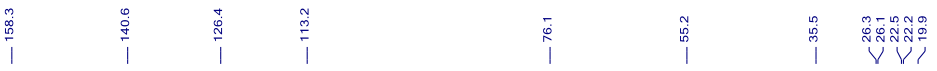

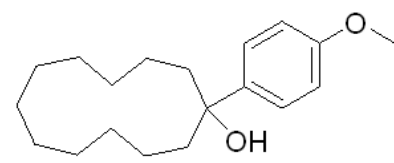

$24 a$

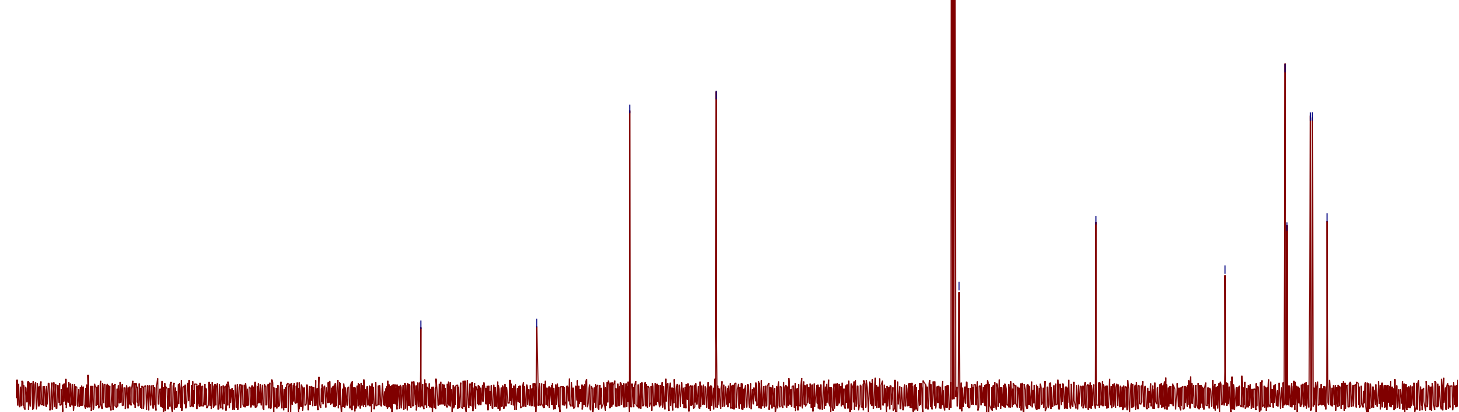

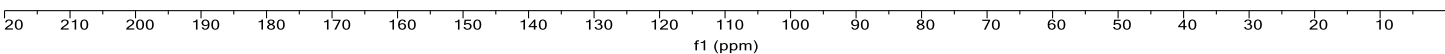


${ }^{1} \mathrm{H}$ NMR (500 MHz, $\left.\mathrm{CDCl}_{3}\right)$

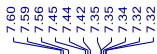

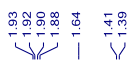
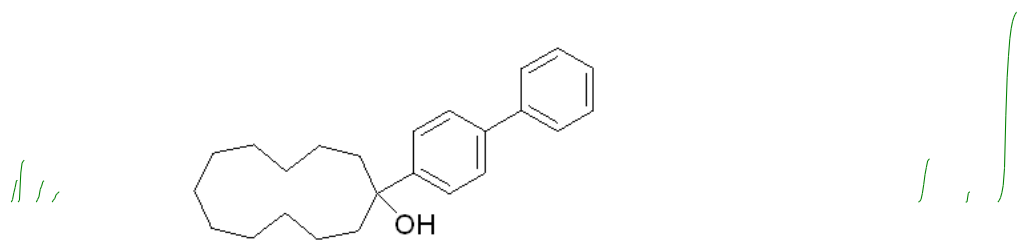

$25 a$

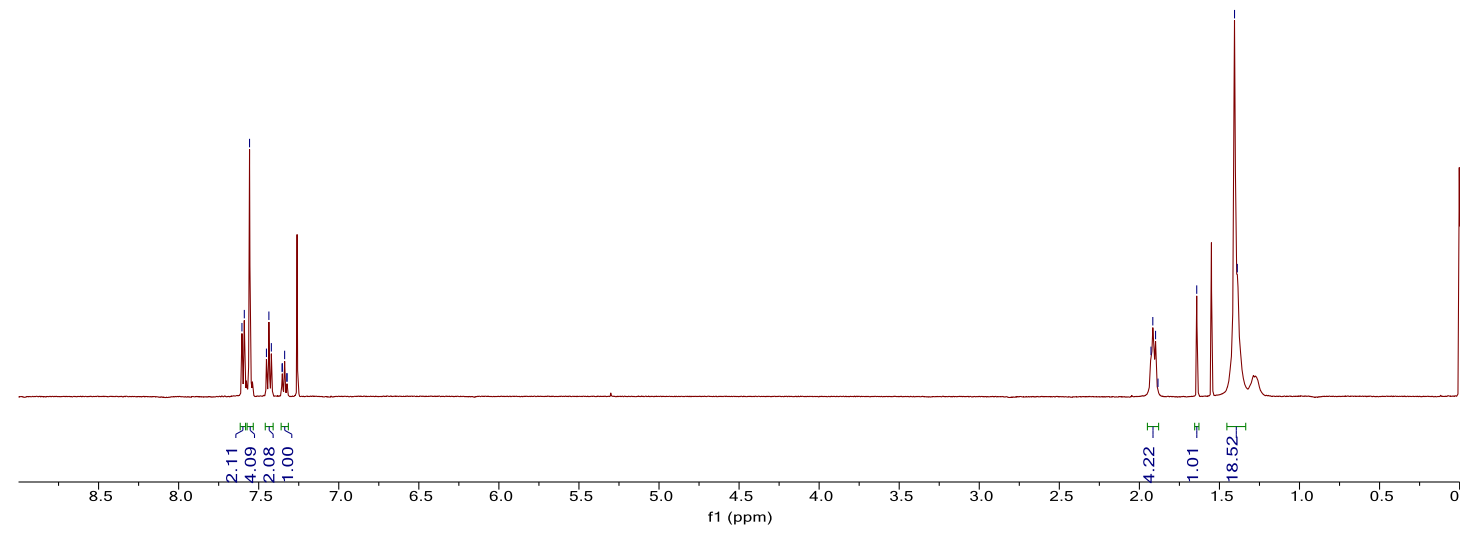

${ }^{13} \mathrm{C} \mathrm{NMR}\left(126 \mathrm{MHz}, \mathrm{CDCl}_{3}\right)$

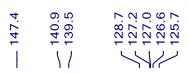

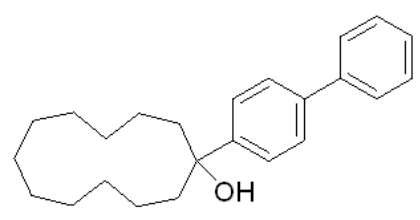

$25 a$

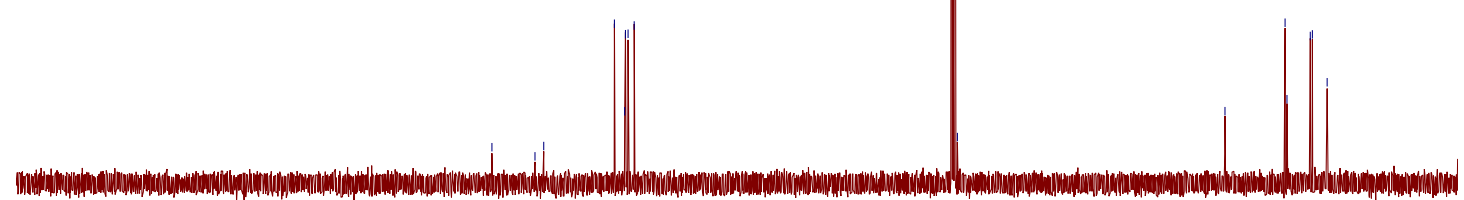

\begin{tabular}{lllllll}
\hline 20 & 1 & 1 & 1 & 1 & & \\
200 & 190 & 180 & 170 & 160
\end{tabular} $120 \begin{array}{cc}110 \\ f 1(\mathrm{ppm})\end{array}$ 
${ }^{1} \mathrm{H} \mathrm{NMR}\left(500 \mathrm{MHz}, \mathrm{CDCl}_{3}\right)$

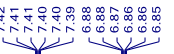

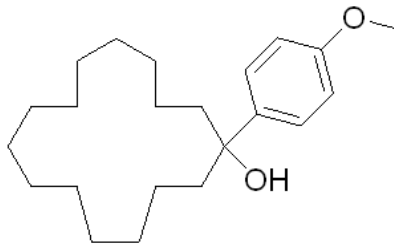

$26 a$

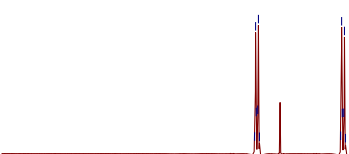

N.

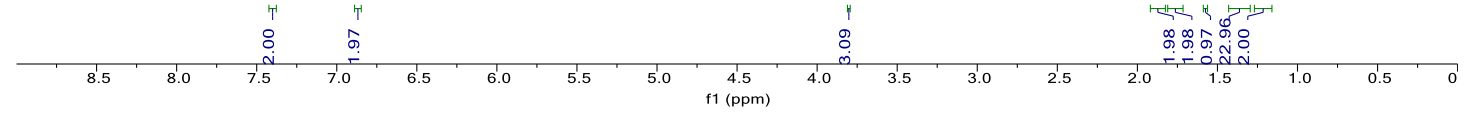

${ }^{13} \mathrm{C}$ NMR (126 MHz, $\left.\mathrm{CDCl}_{3}\right)$

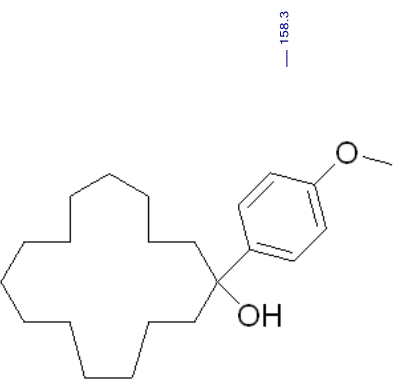

$26 a$

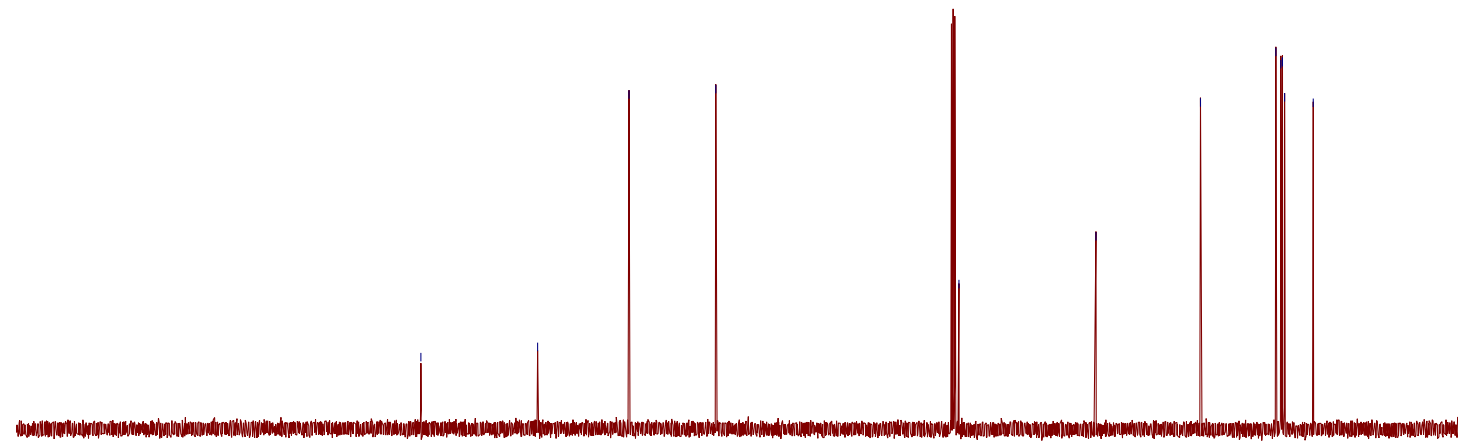

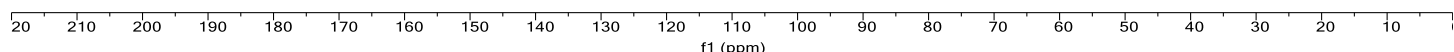


${ }^{1} \mathrm{H}$ NMR (500 MHz, $\mathrm{CDCl}_{3}$ )

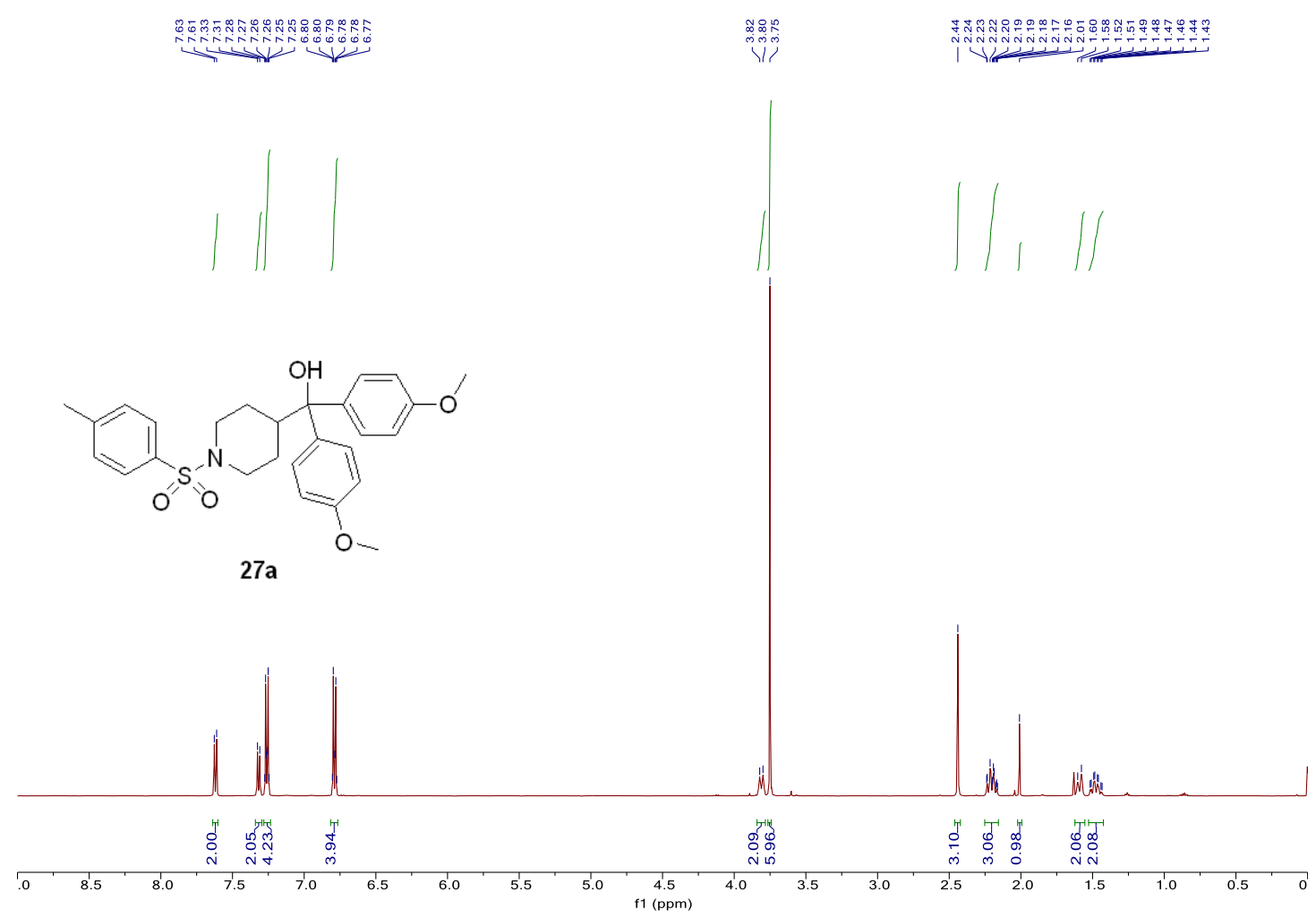

${ }^{13} \mathrm{C}$ NMR $\left(126 \mathrm{MHz}, \mathrm{CDCl}_{3}\right)$

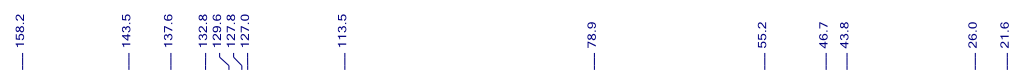
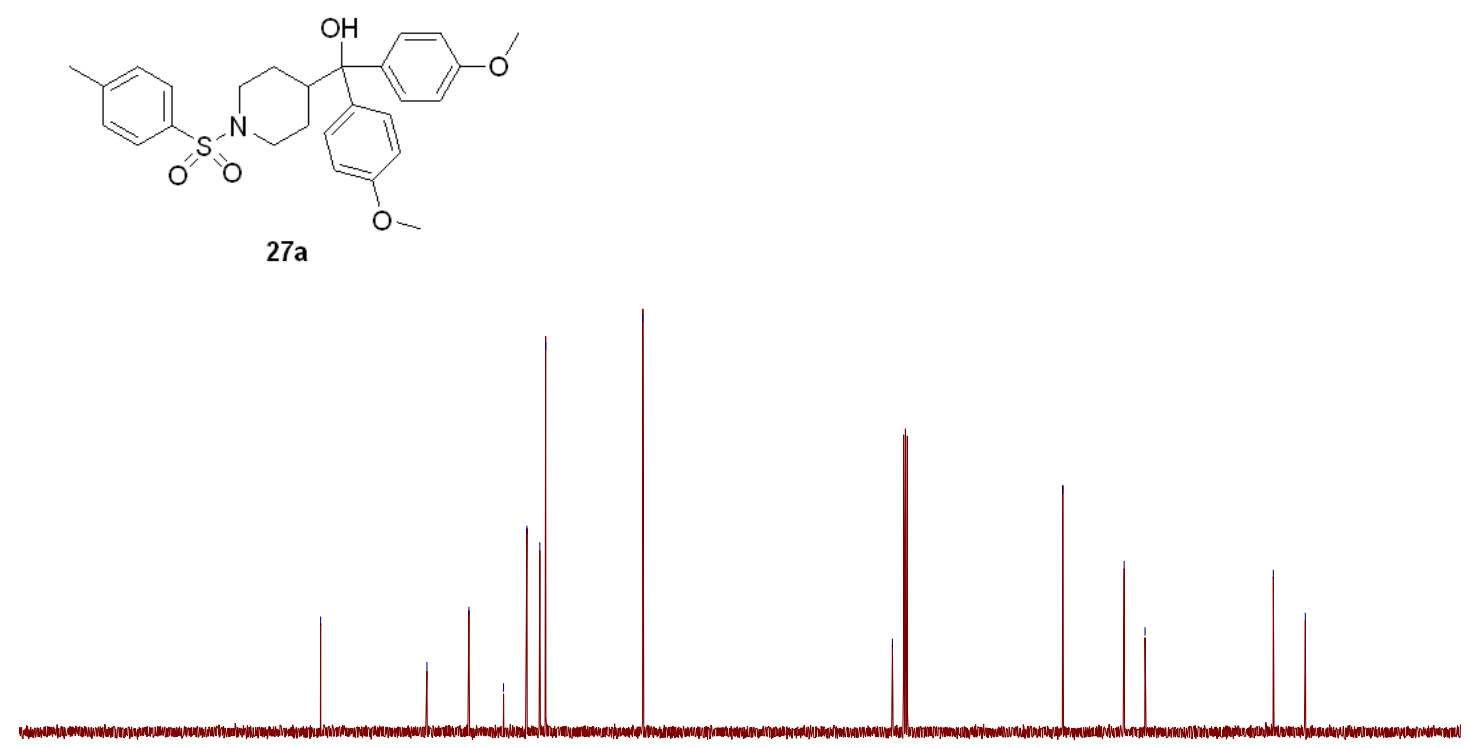

\begin{tabular}{lllllllllllllllllllll}
\hline oo & 190 & 180 & 170 & 160 & 150 & 140 & 130 & 120 & 110 & 100 & 90 & 80 & 70 & 60 & 50 & 40 & 30 & 10 & 10 & 10
\end{tabular} 
${ }^{1} \mathrm{H}$ NMR (500 MHz, $\mathrm{CDCl}_{3}$ )
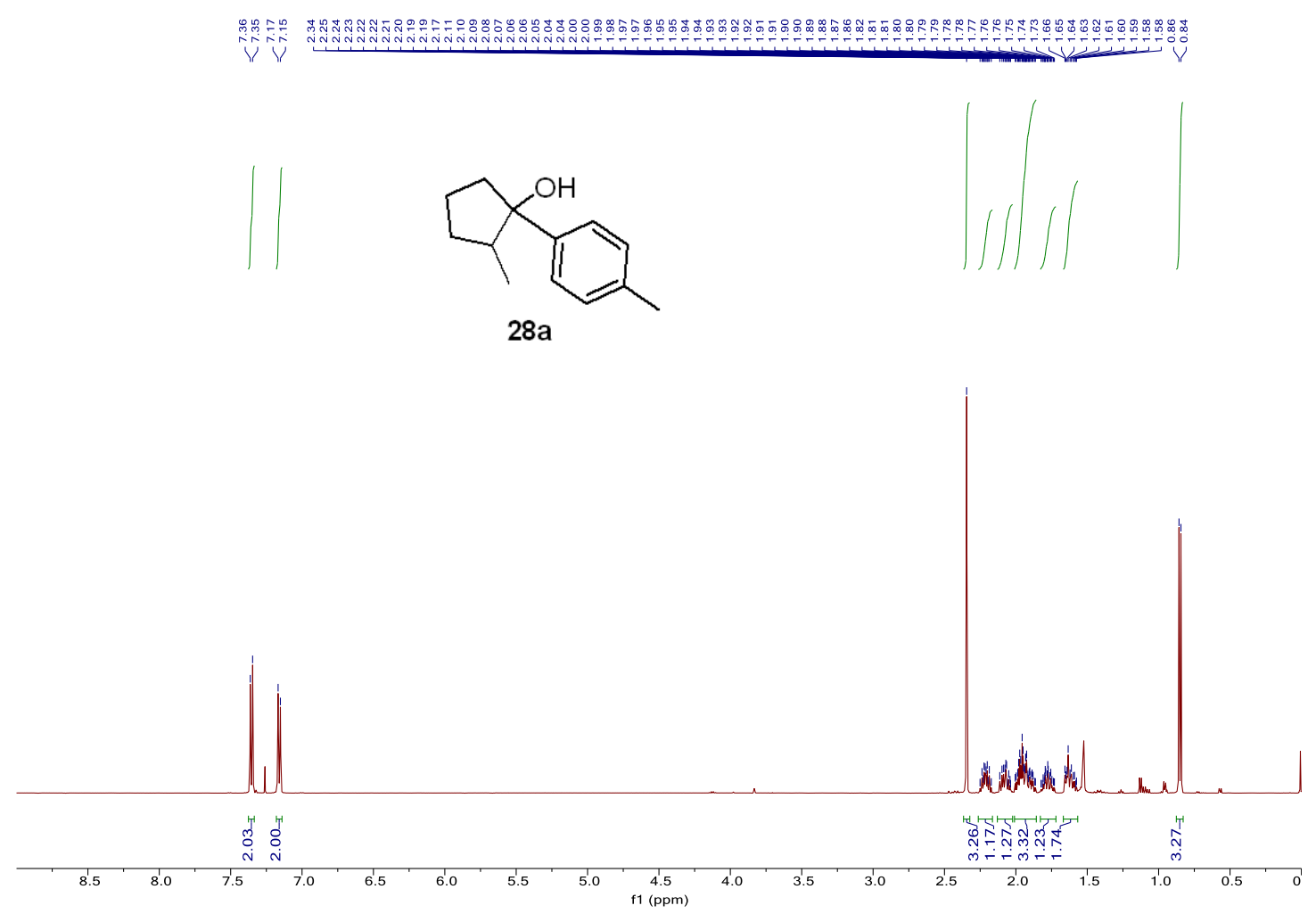

${ }^{13} \mathrm{C}$ NMR (126 MHz, $\left.\mathrm{CDCl}_{3}\right)$
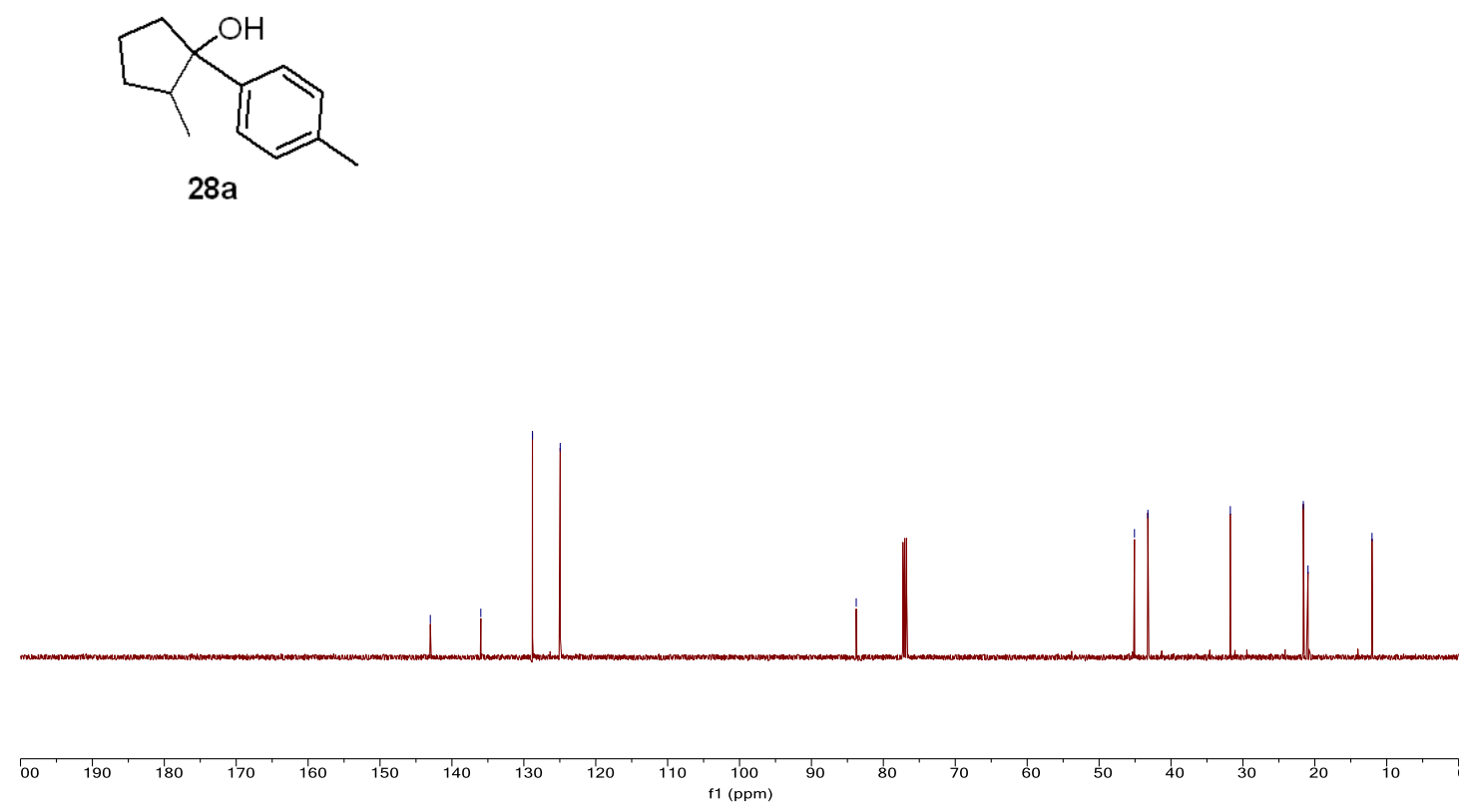

S90 
${ }^{1} \mathrm{H}$ NMR $\left(500 \mathrm{MHz}, \mathrm{CDCl}_{3}\right)$

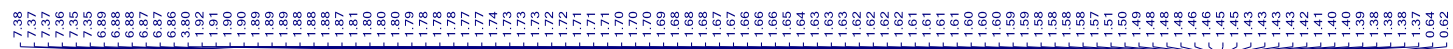

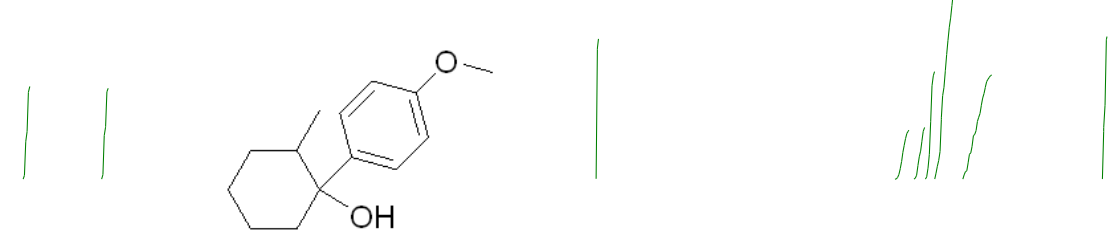

$29 a$

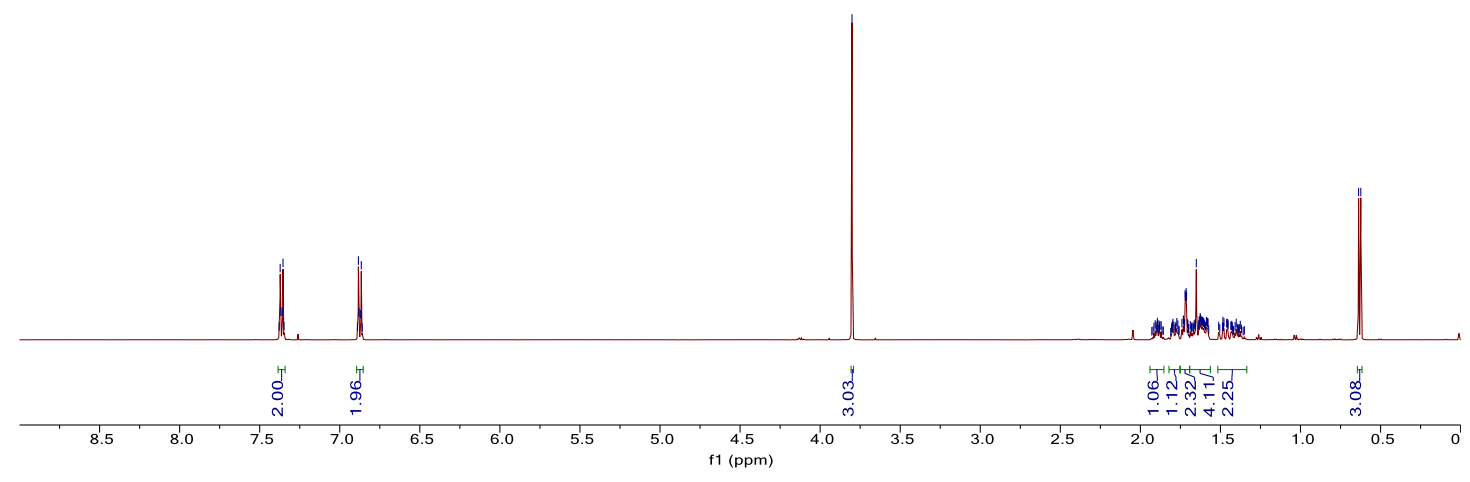

${ }^{13} \mathrm{C} \mathrm{NMR}\left(126 \mathrm{MHz}, \mathrm{CDCl}_{3}\right)$

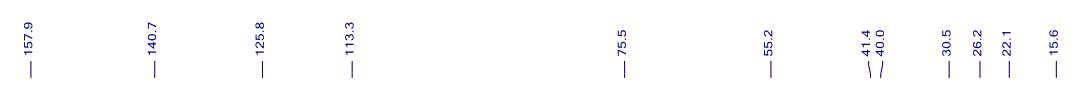

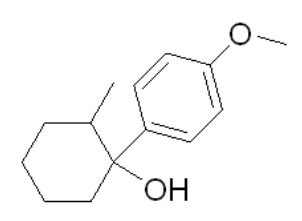

29a

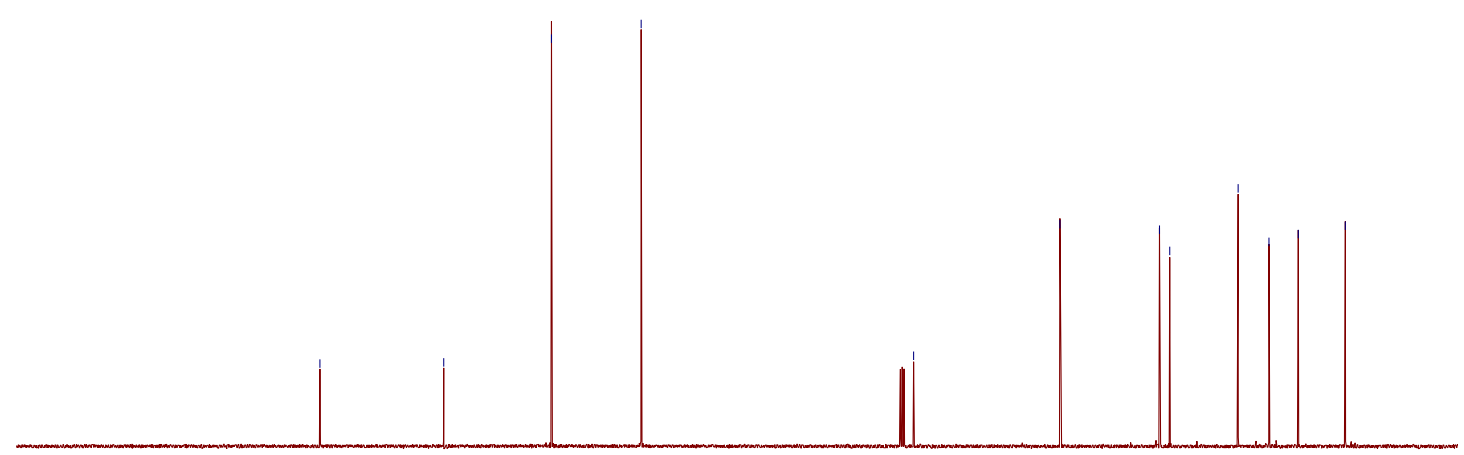

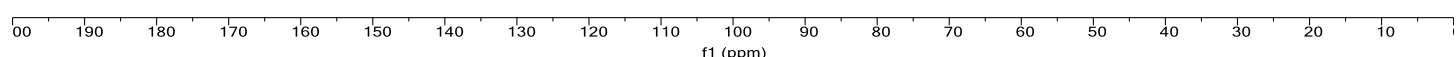


${ }^{1} \mathrm{H} \mathrm{NMR}\left(500 \mathrm{MHz}, \mathrm{CDCl}_{3}\right)$

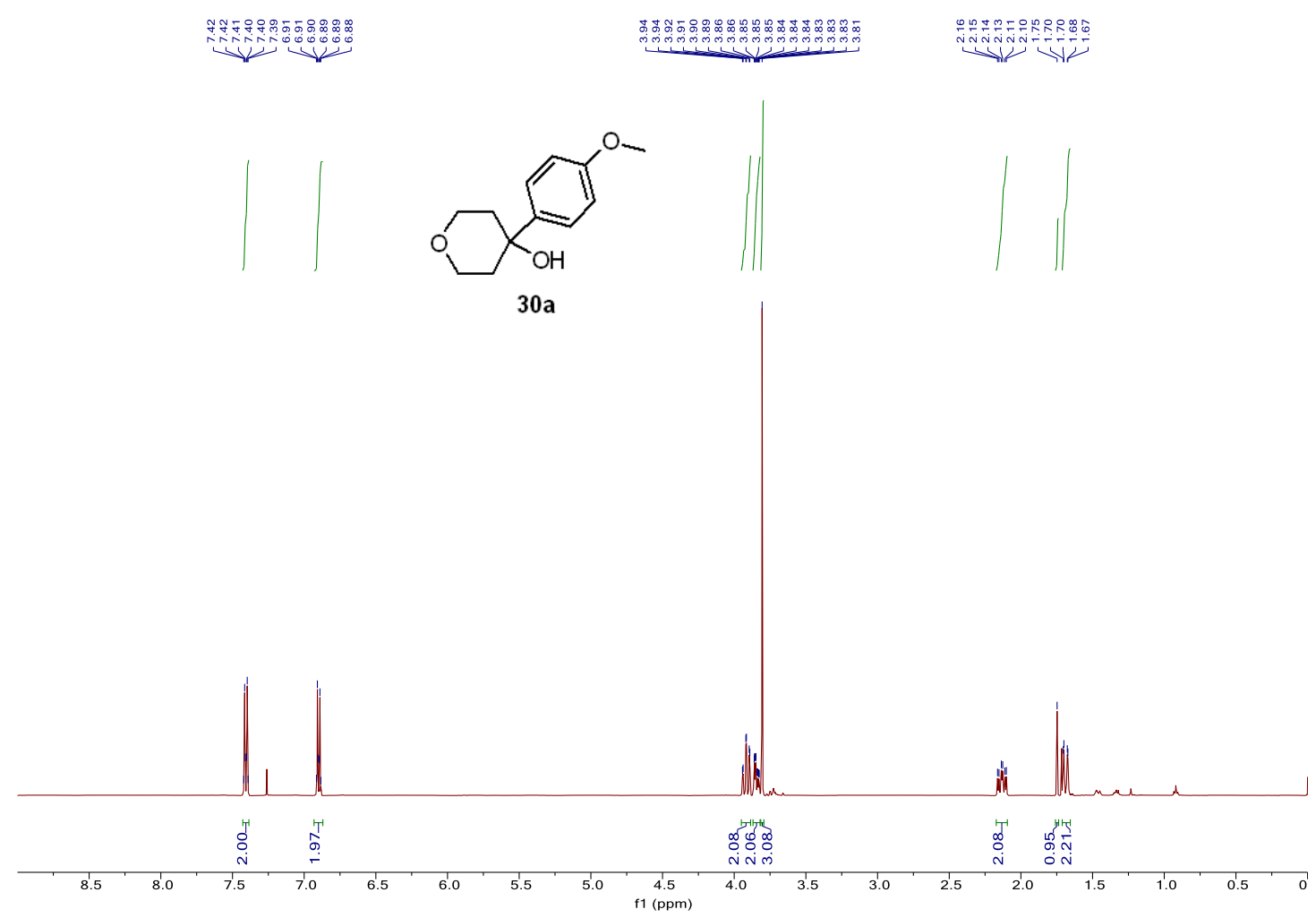

${ }^{13} \mathrm{C}$ NMR $\left(126 \mathrm{MHz}, \mathrm{CDCl}_{3}\right)$

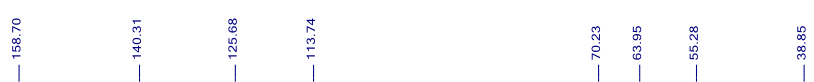

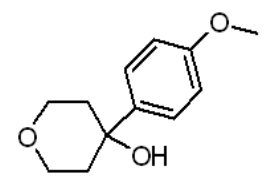

$30 \mathrm{a}$

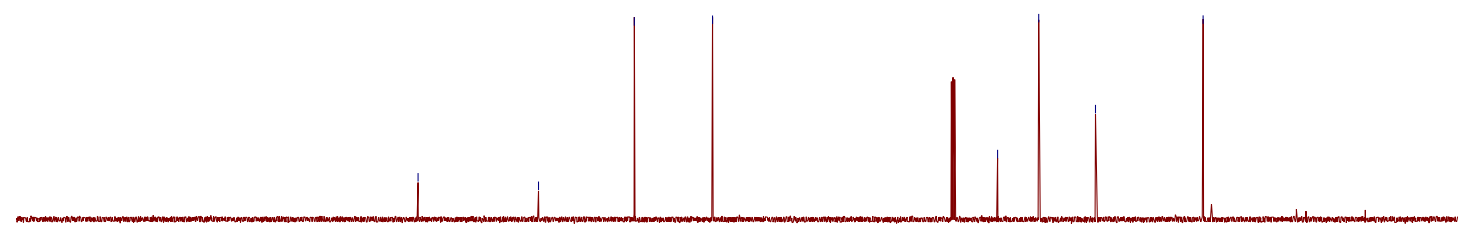

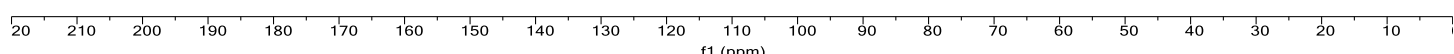


${ }^{1} \mathrm{H}$ NMR (500 MHz, $\mathrm{CDCl}_{3}$ )

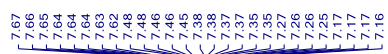

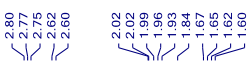
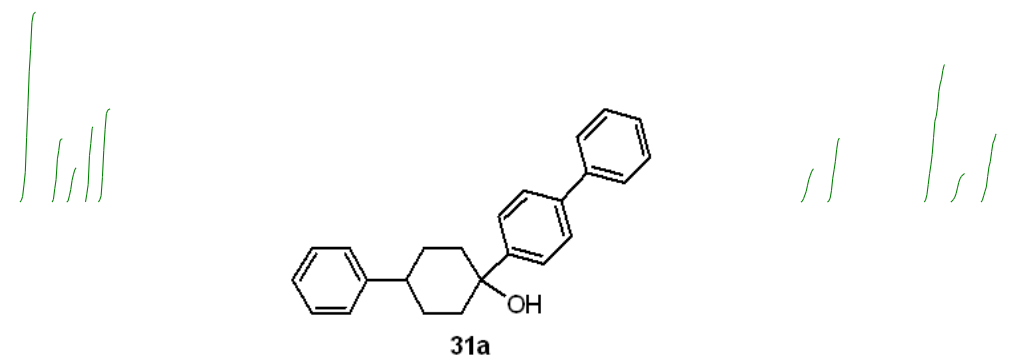

31a

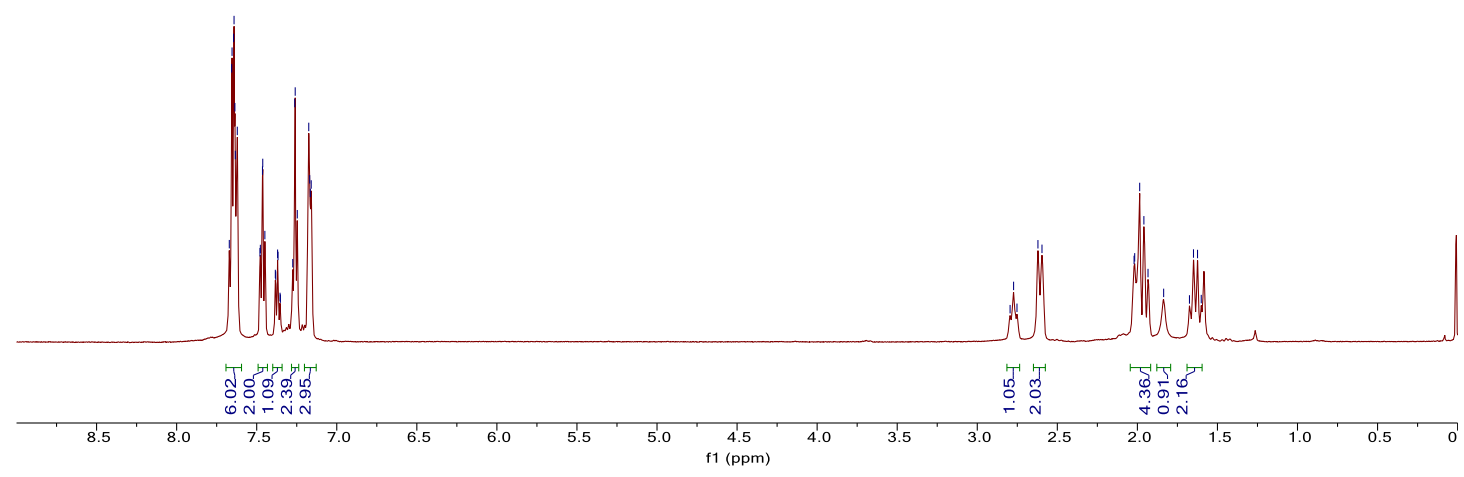

${ }^{13} \mathrm{C}$ NMR $\left(126 \mathrm{MHz}, \mathrm{CDCl}_{3}\right)$

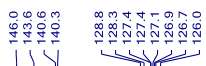

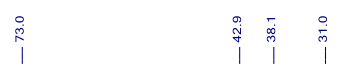

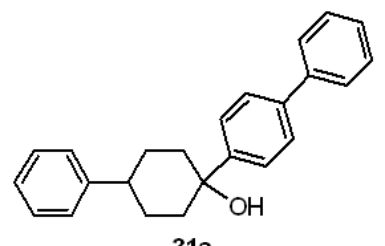

31a

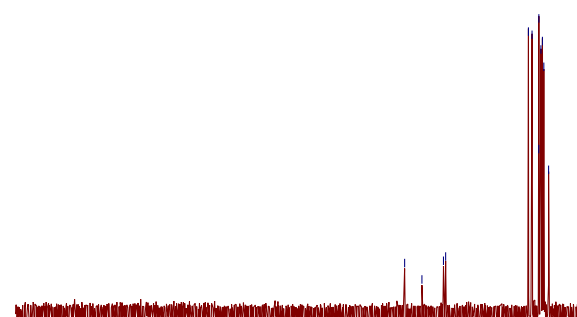

oo

$190 \quad 180$

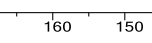

100
$\mathrm{f} 1(\mathrm{ppm})$ 
${ }^{1} \mathrm{H} \mathrm{NMR}\left(500 \mathrm{MHz}, \mathrm{CDCl}_{3}\right)$

跂

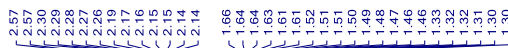

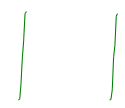

$\mathrm{Ax}^{\circ}$

$32 \mathrm{a}$

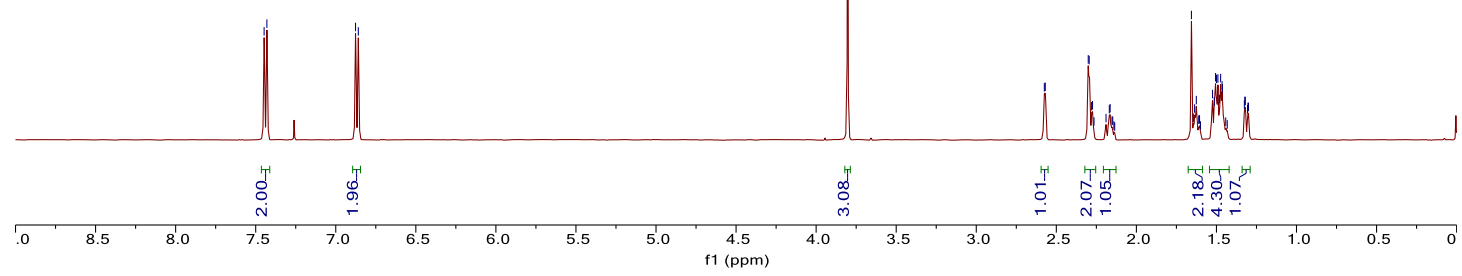

${ }^{13} \mathrm{C} \mathrm{NMR}\left(126 \mathrm{MHz}, \mathrm{CDCl}_{3}\right)$

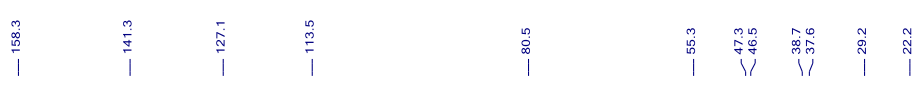<smiles>COc1ccc(C2(O)CC3CCC3C2)cc1</smiles>

32a

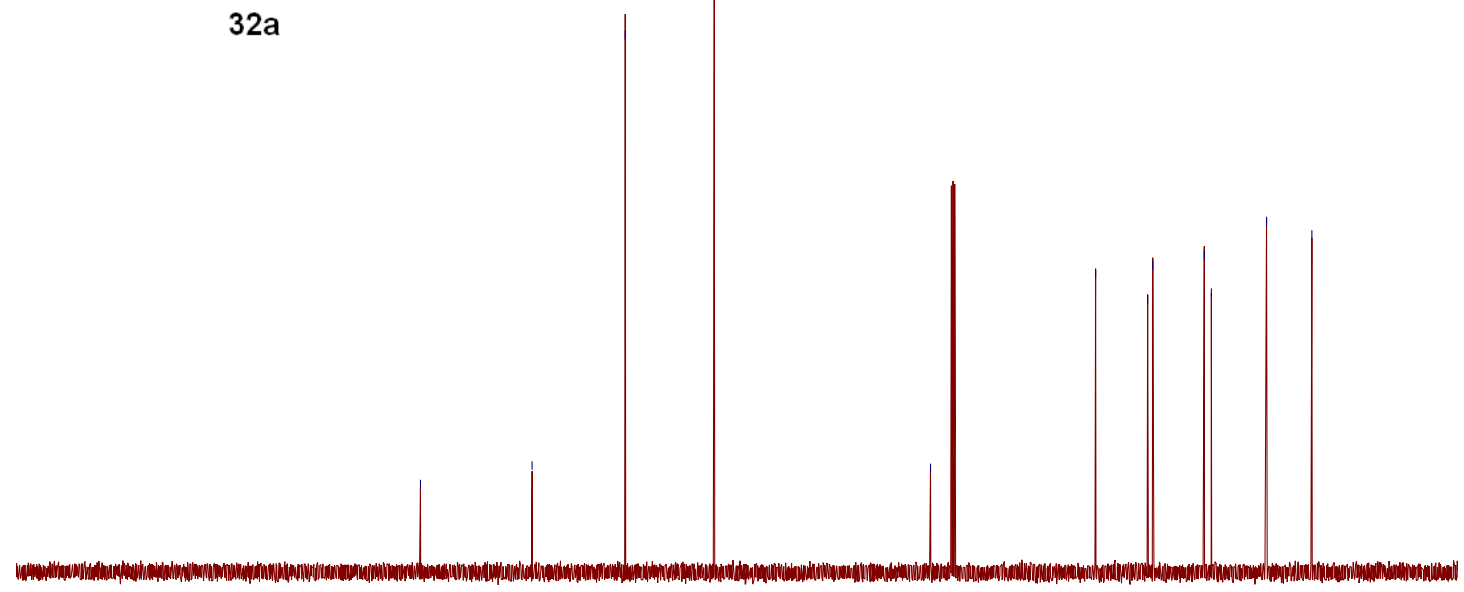

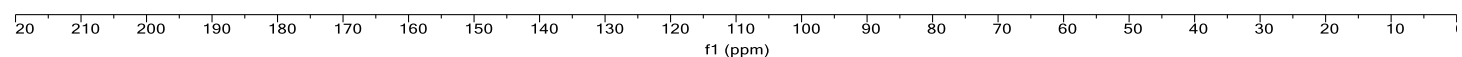


${ }^{1} \mathrm{H} \mathrm{NMR}\left(500 \mathrm{MHz}, \mathrm{CDCl}_{3}\right)$

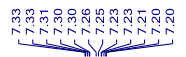

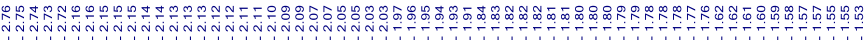

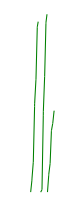<smiles>OC1(CCc2ccccc2)CCC1</smiles>

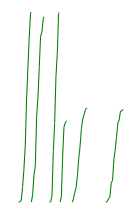

$33 a$

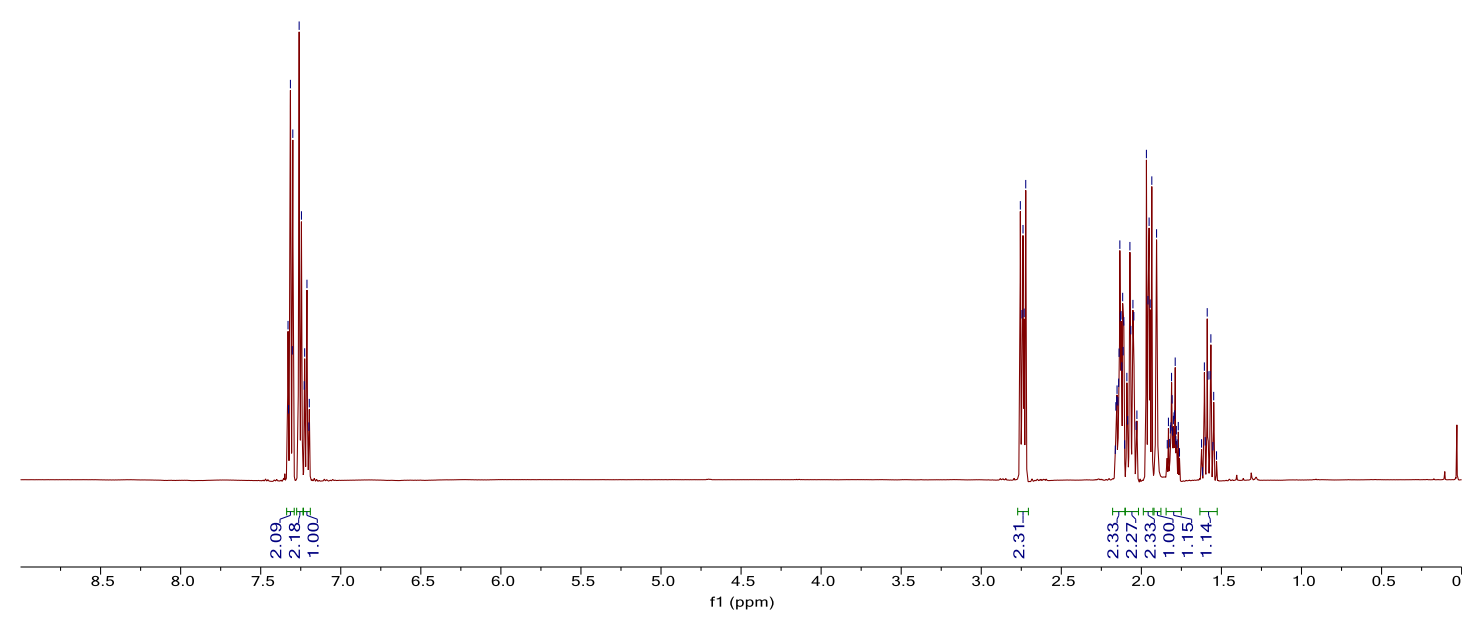

${ }^{13} \mathrm{C} \mathrm{NMR}\left(126 \mathrm{MHz}, \mathrm{CDCl}_{3}\right)$

站<smiles>OC1(CCc2ccccc2)CCC1</smiles>

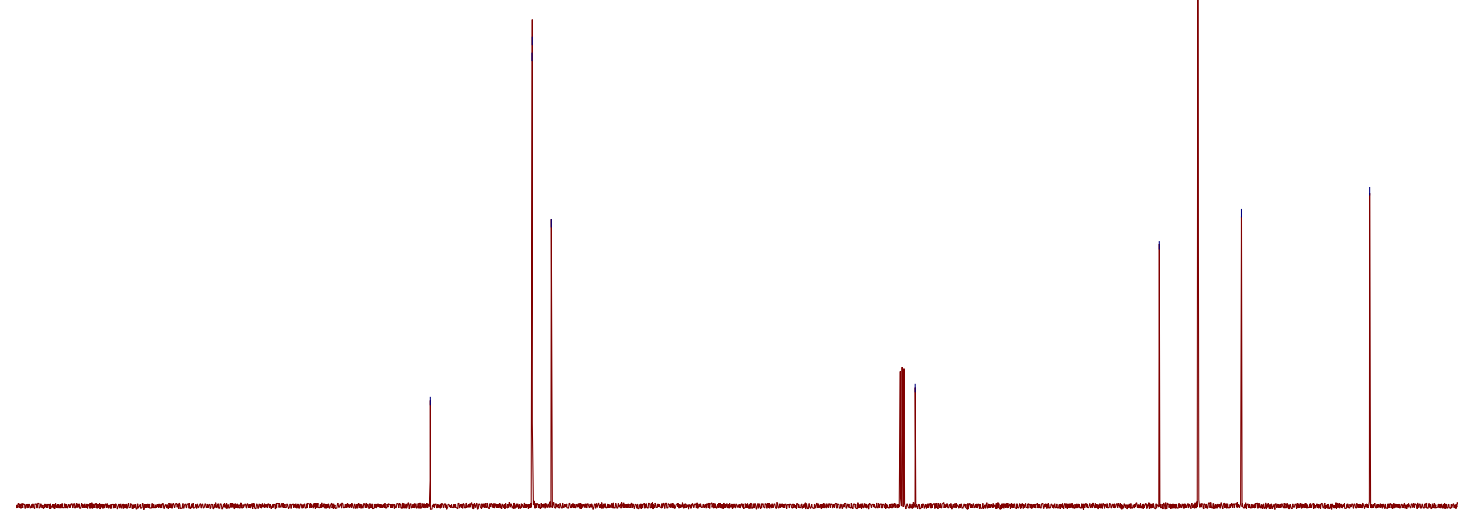

oo
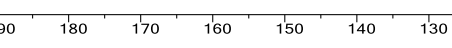
${ }^{1} \mathrm{H}$ NMR (500 MHz, $\mathrm{CDCl}_{3}$ )

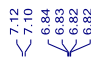

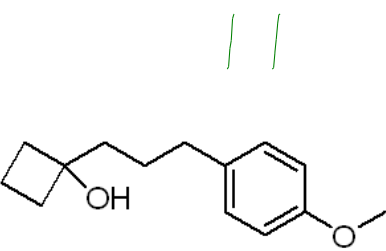

$34 a$

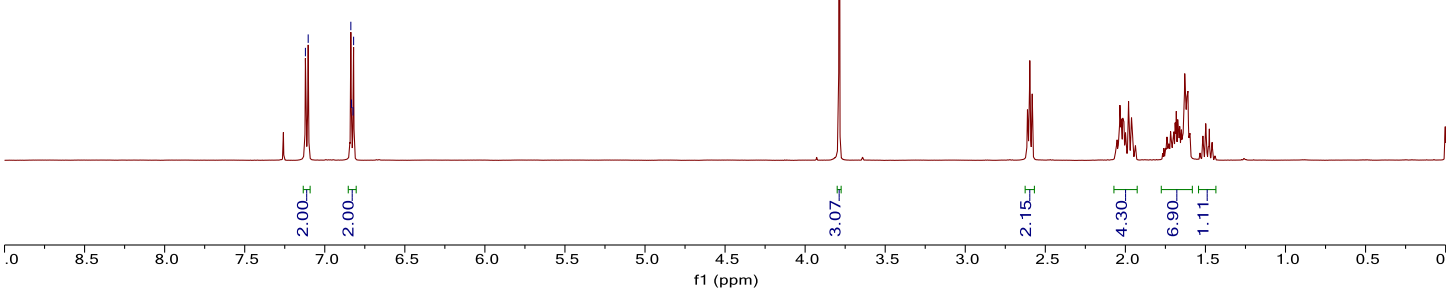

${ }^{13} \mathrm{C}$ NMR $\left(126 \mathrm{MHz}, \mathrm{CDCl}_{3}\right)$

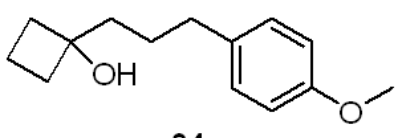
$34 a$
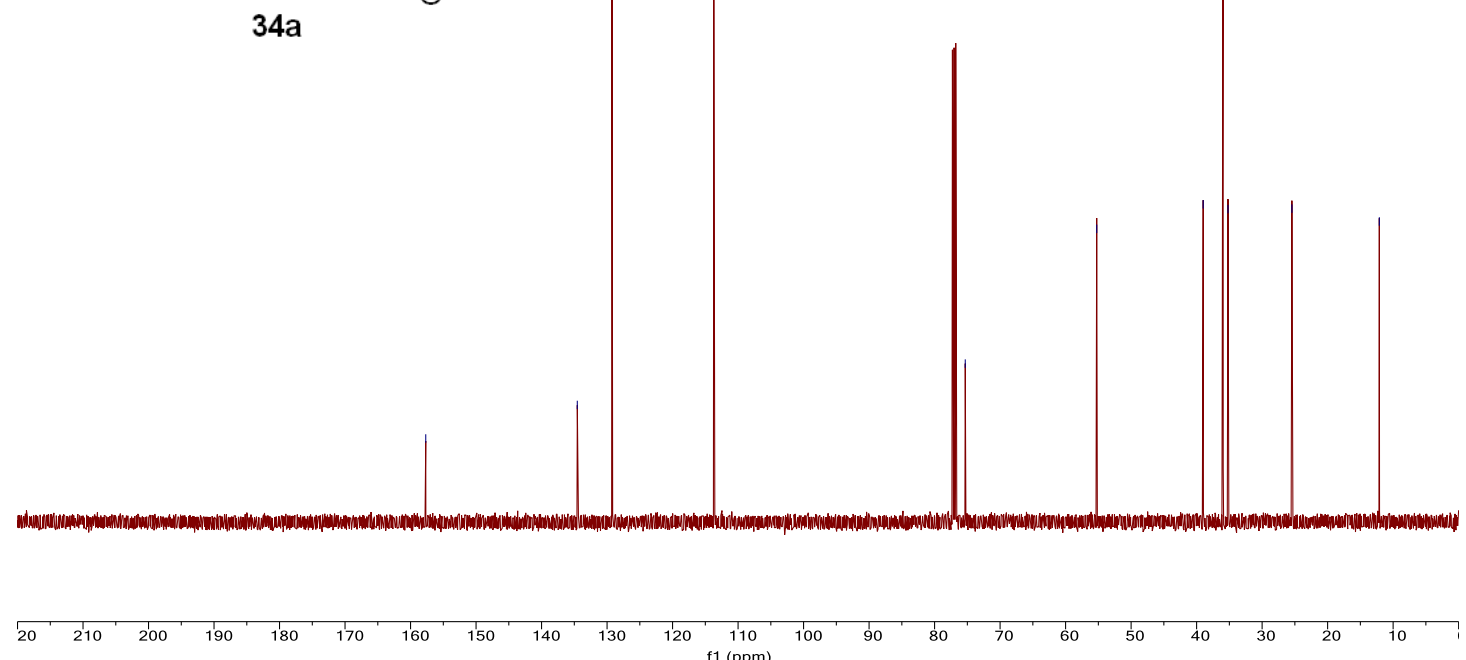
${ }^{1} \mathrm{H}$ NMR (500 MHz, $\mathrm{CDCl}_{3}$ )

约

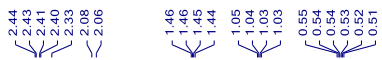
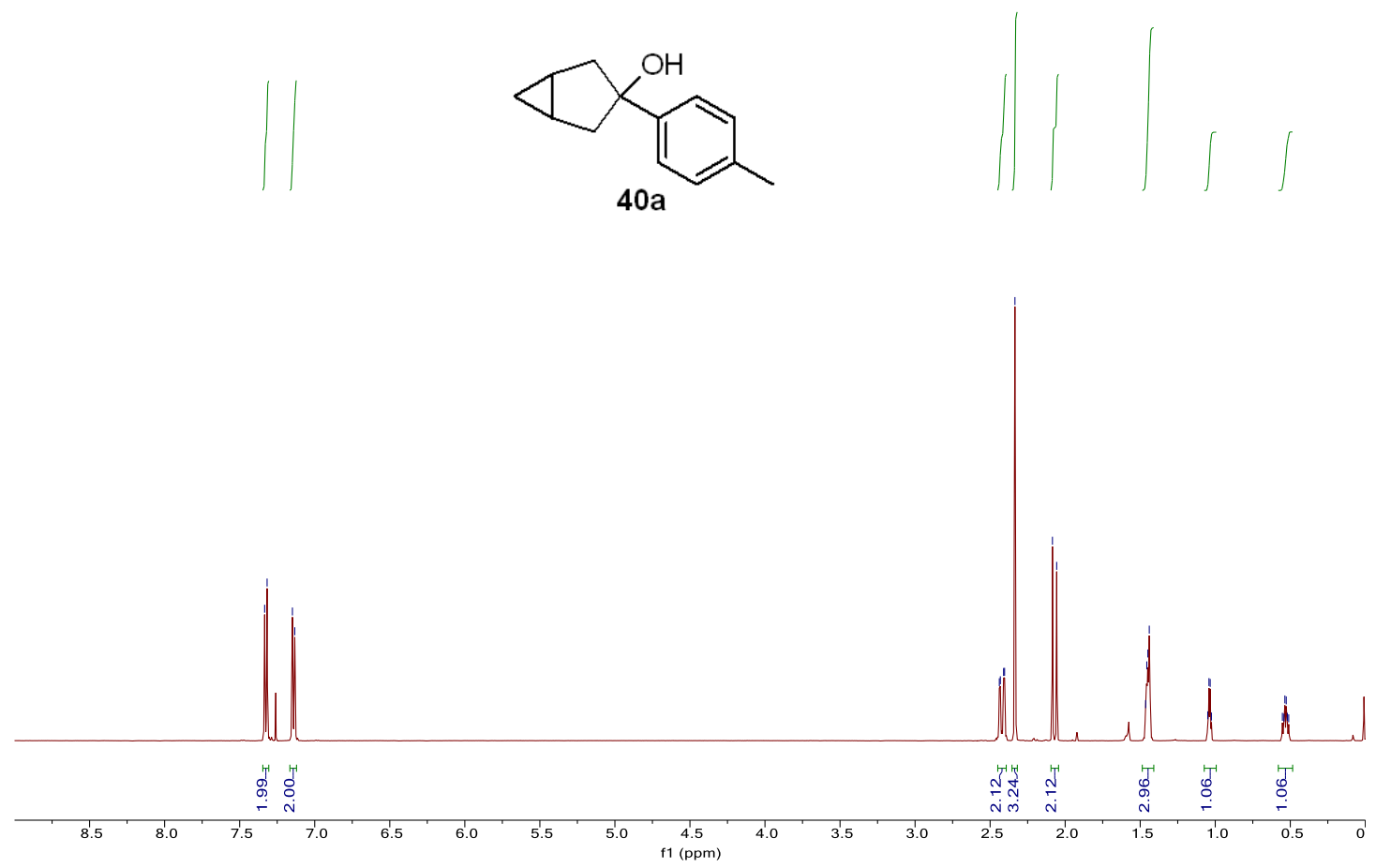

${ }^{13} \mathrm{C}$ NMR $\left(126 \mathrm{MHz}, \mathrm{CDCl}_{3}\right)$
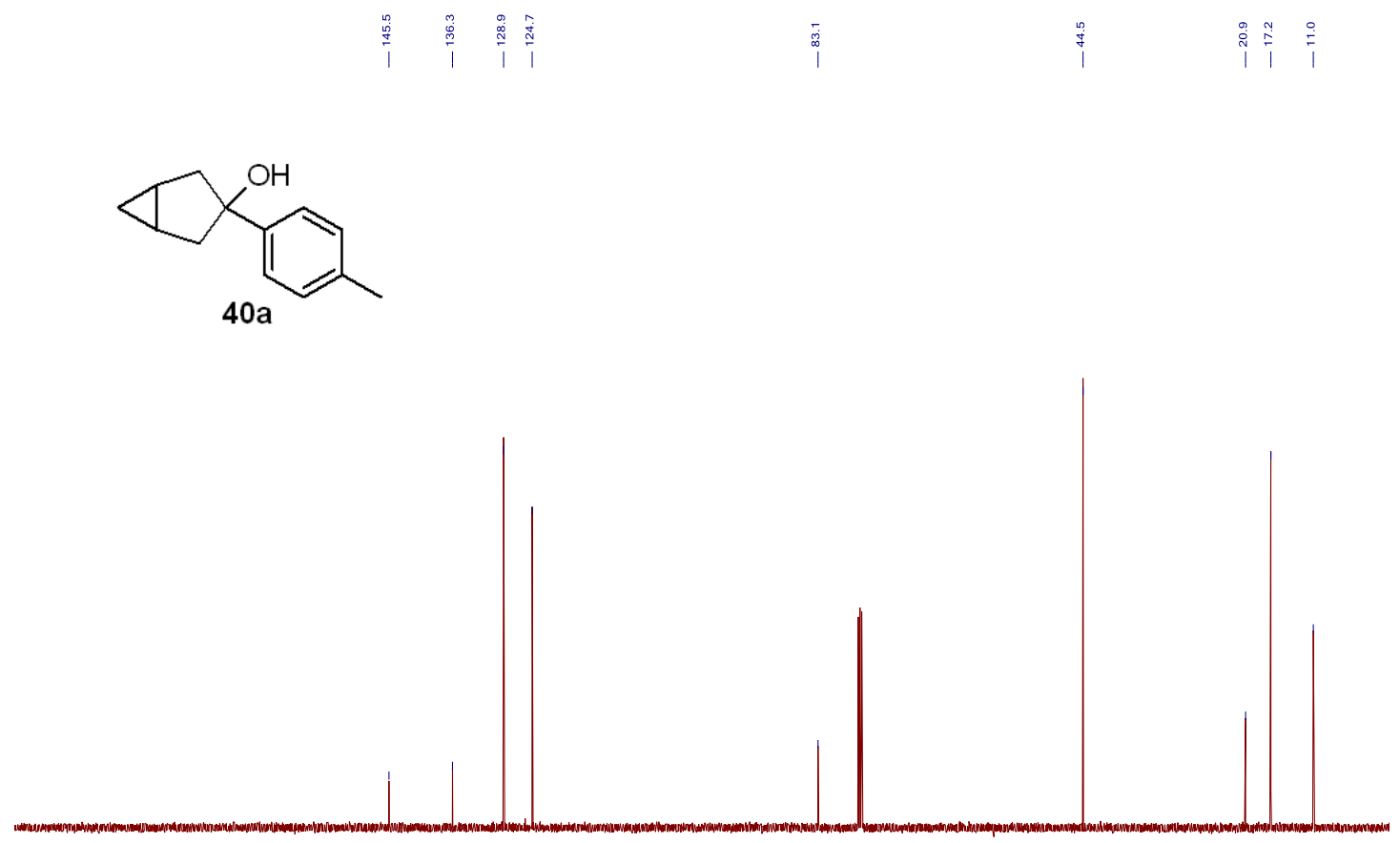

oo
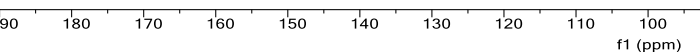
${ }^{1} \mathrm{H}$ NMR (600 MHz, $\left.\mathrm{CDCl}_{3}\right)$

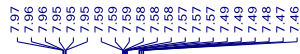

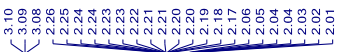

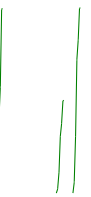<smiles>O=C(CCCC(F)(F)F)c1ccccc1</smiles>
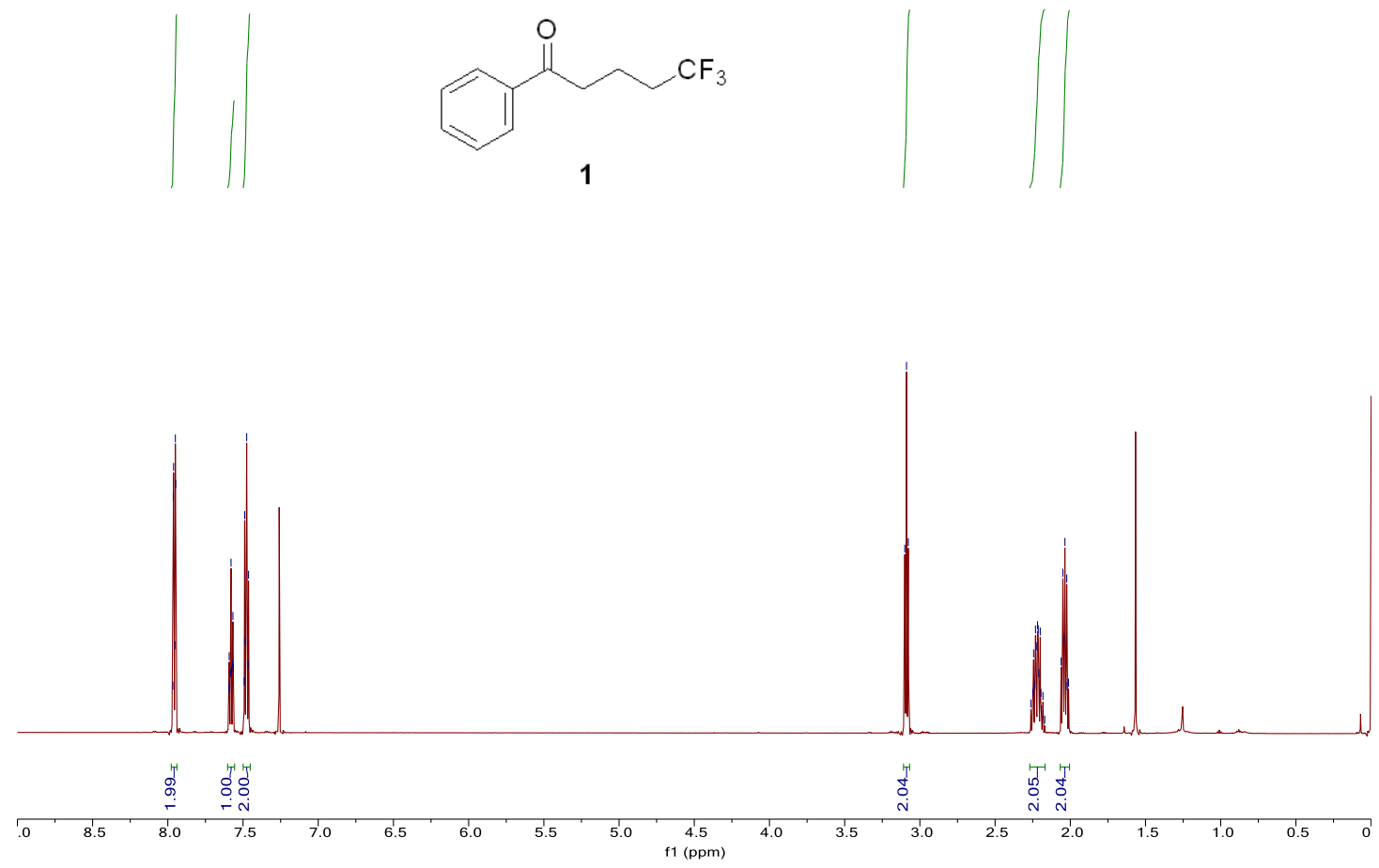

${ }^{13} \mathrm{C}$ NMR $\left(151 \mathrm{MHz}, \mathrm{CDCl}_{3}\right)$
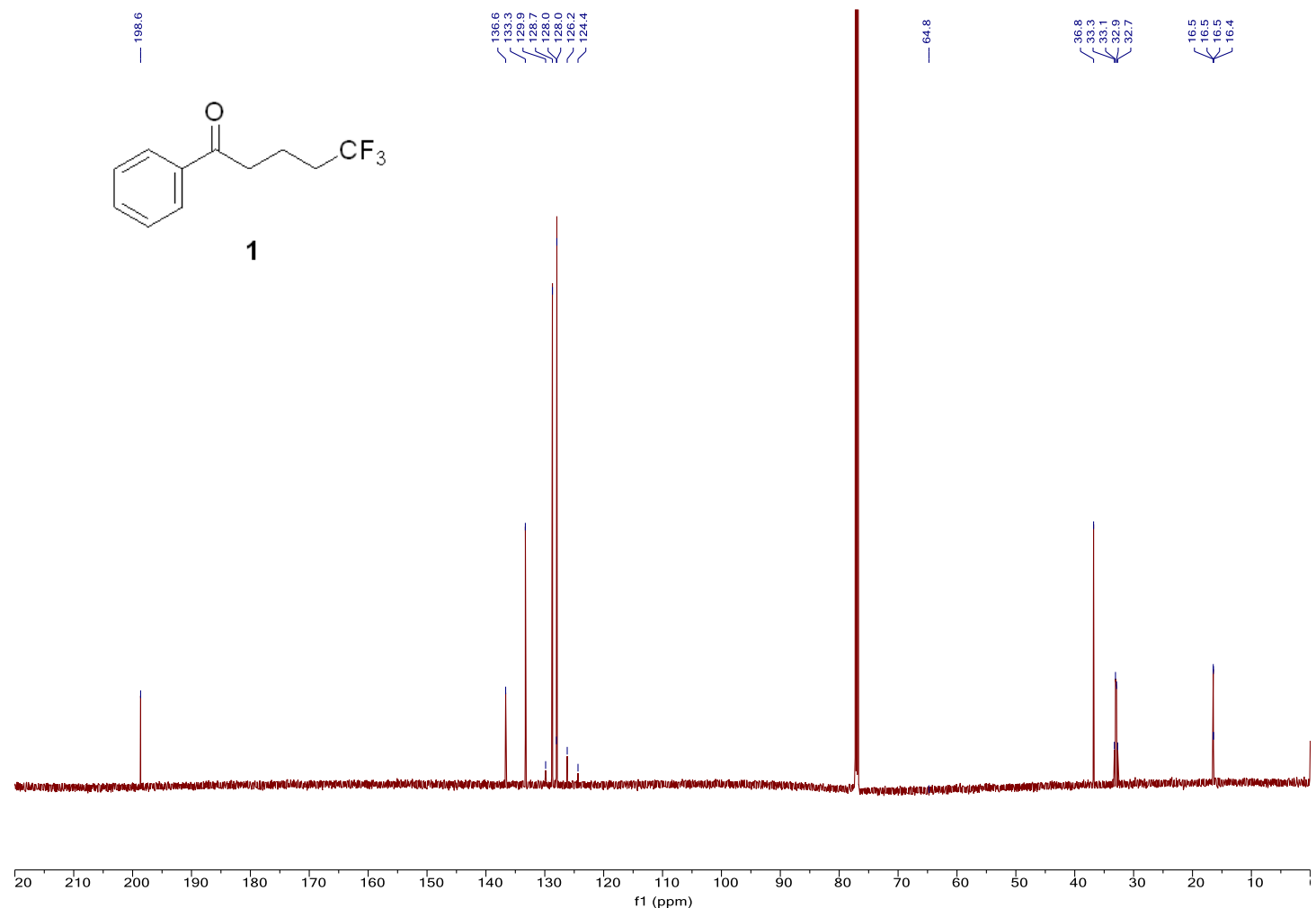
${ }^{19} \mathrm{~F}$ NMR $\left(376 \mathrm{MHz}, \mathrm{CDCl}_{3}\right)$

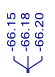<smiles>O=C(CCCC(F)(F)F)c1ccccc1</smiles>
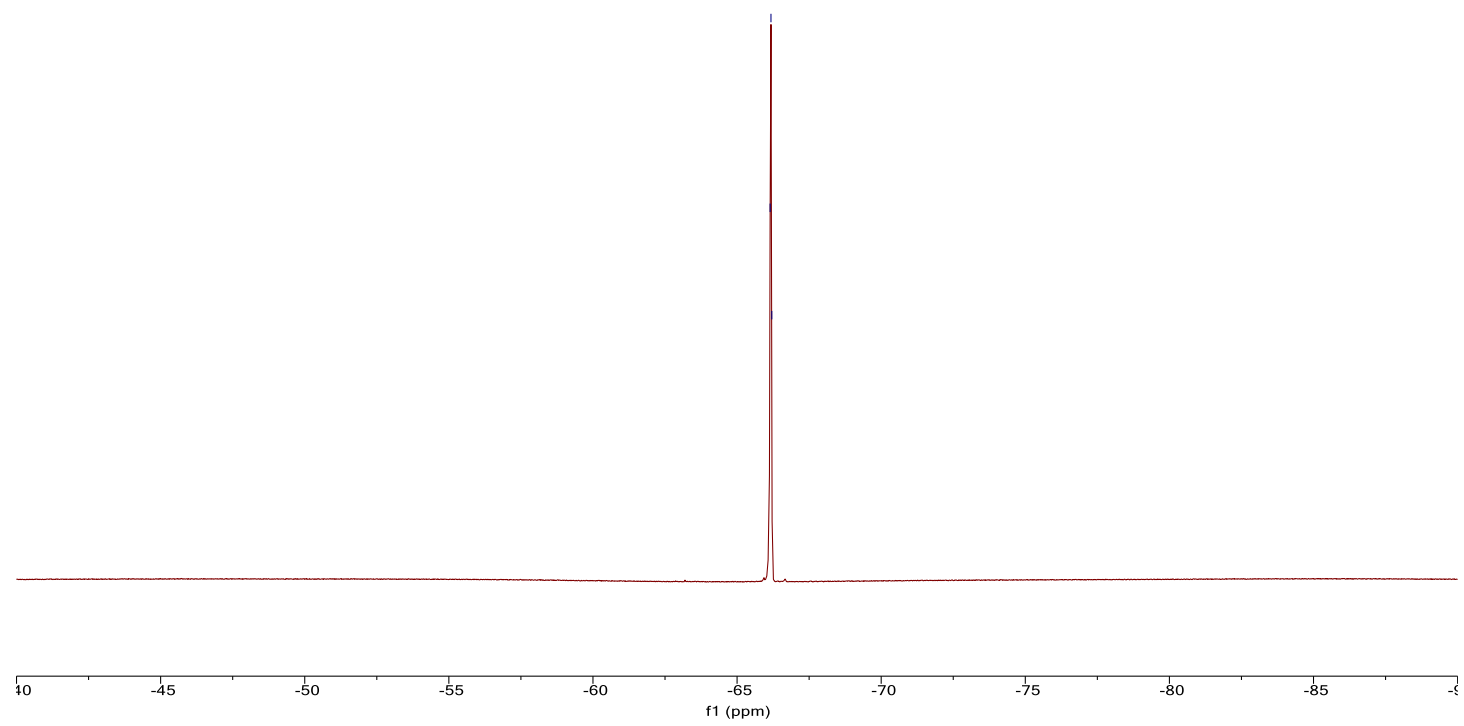

${ }^{1} \mathrm{H}$ NMR (600 MHz, $\left.\mathrm{CDCl}_{3}\right)$

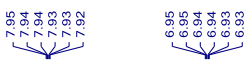

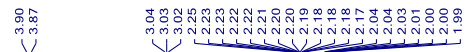

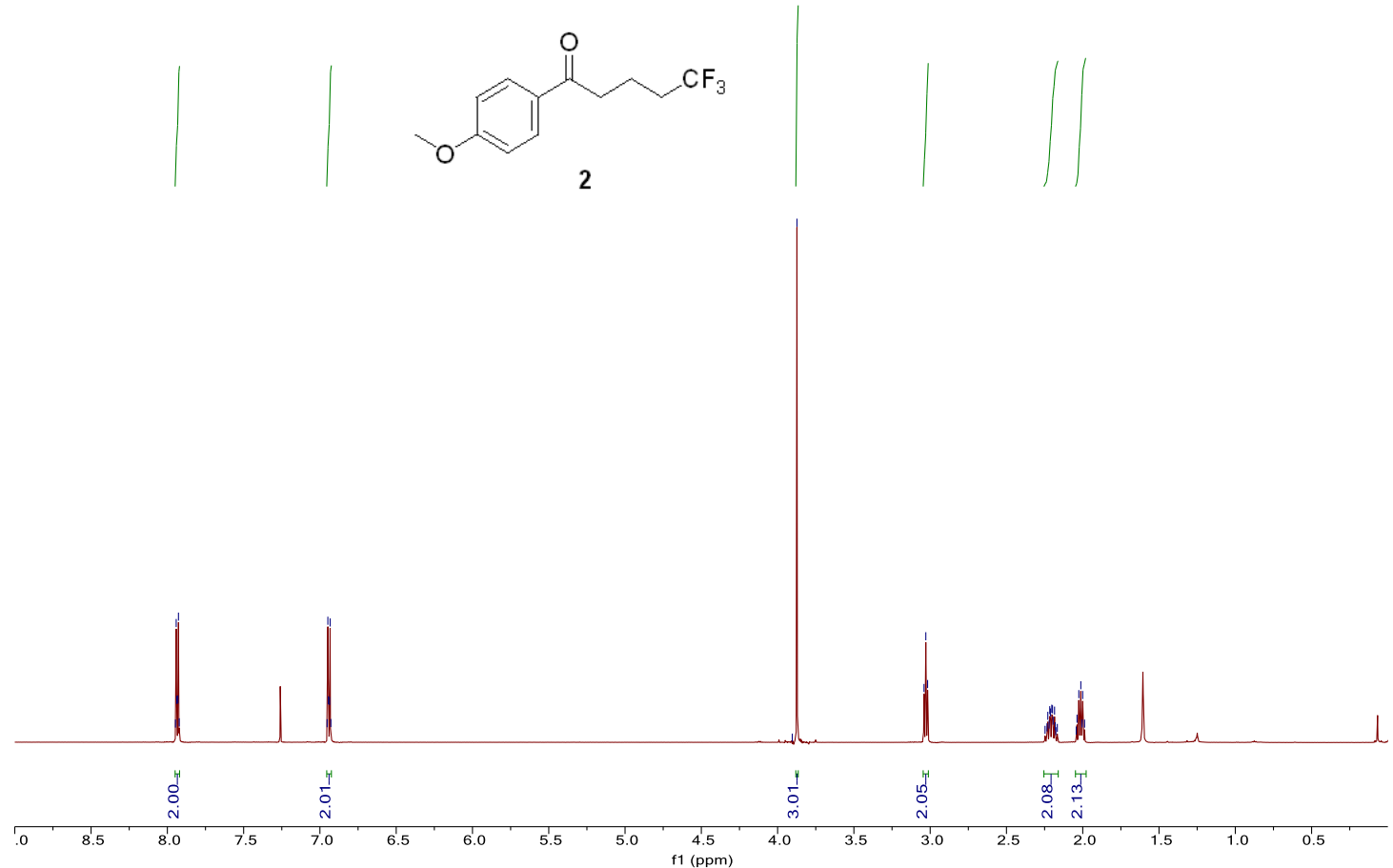


${ }^{13} \mathrm{C} \mathrm{NMR}\left(151 \mathrm{MHz}, \mathrm{CDCl}_{3}\right)$

$$
\text { is }
$$

$$
\stackrel{\circ}{\stackrel{\circ}{\circ}}
$$

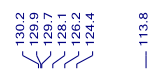

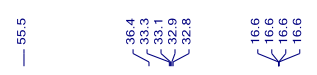

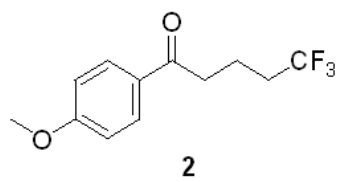

2

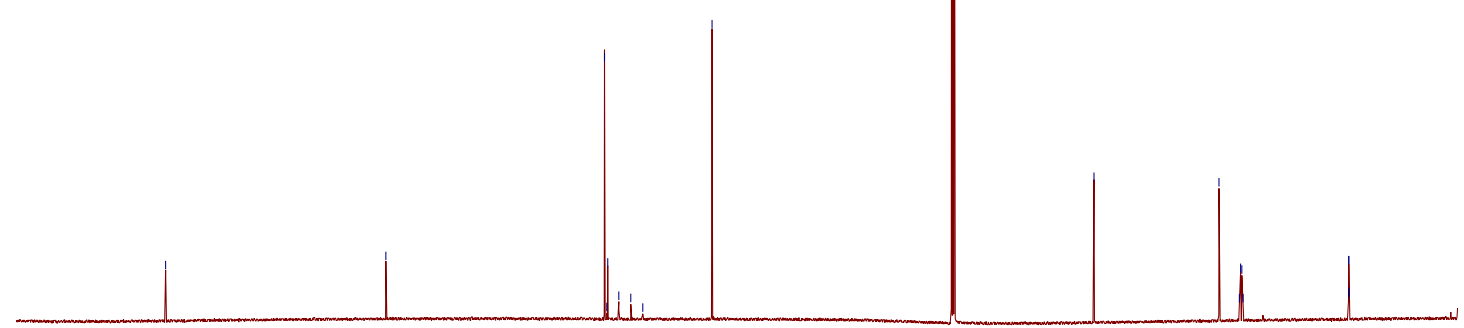

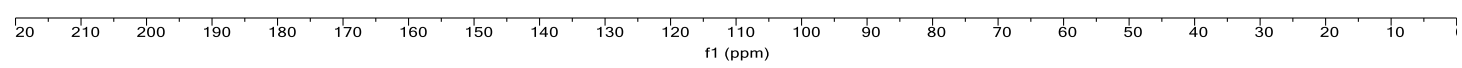

${ }^{19} \mathrm{~F}$ NMR (376 MHz, $\left.\mathrm{CDCl}_{3}\right)$

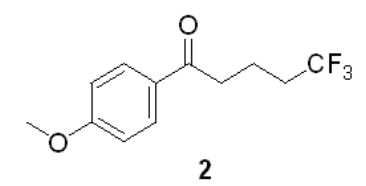

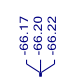

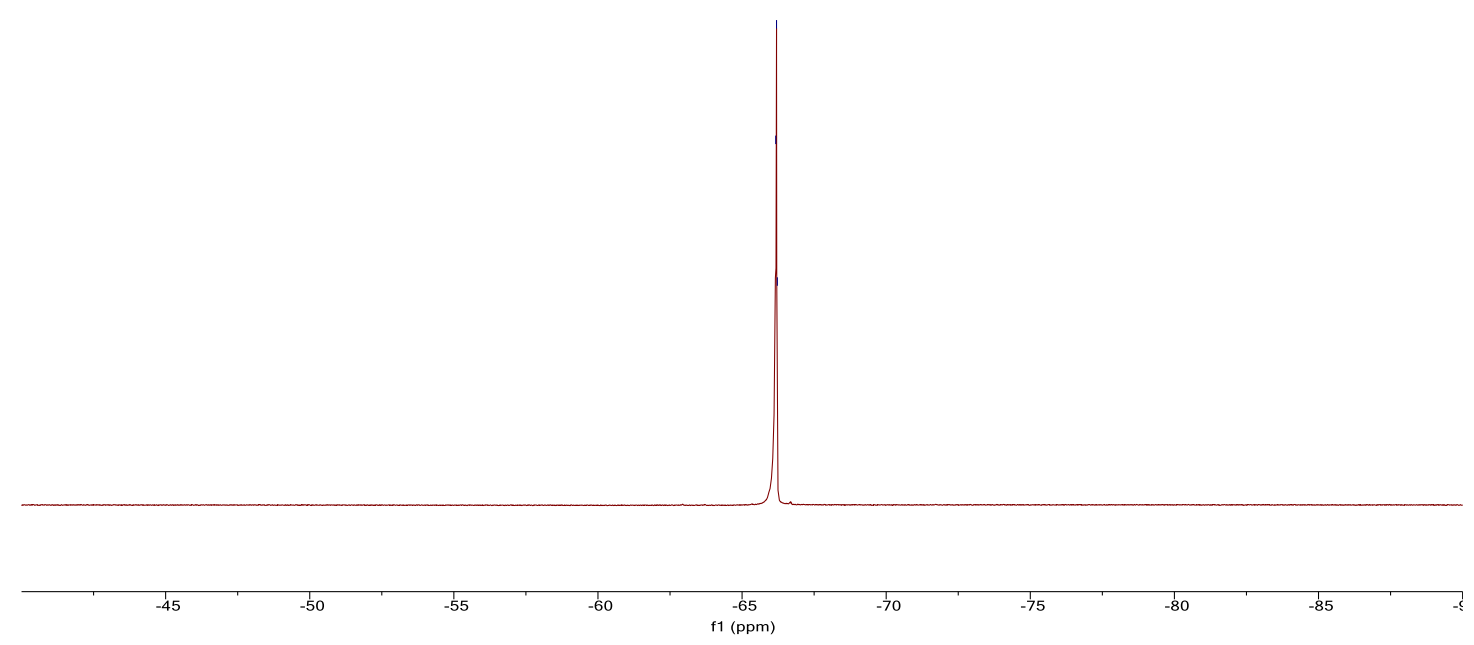


${ }^{1} \mathrm{H}$ NMR (600 MHz, $\left.\mathrm{CDCl}_{3}\right)$

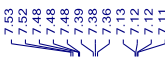

幽
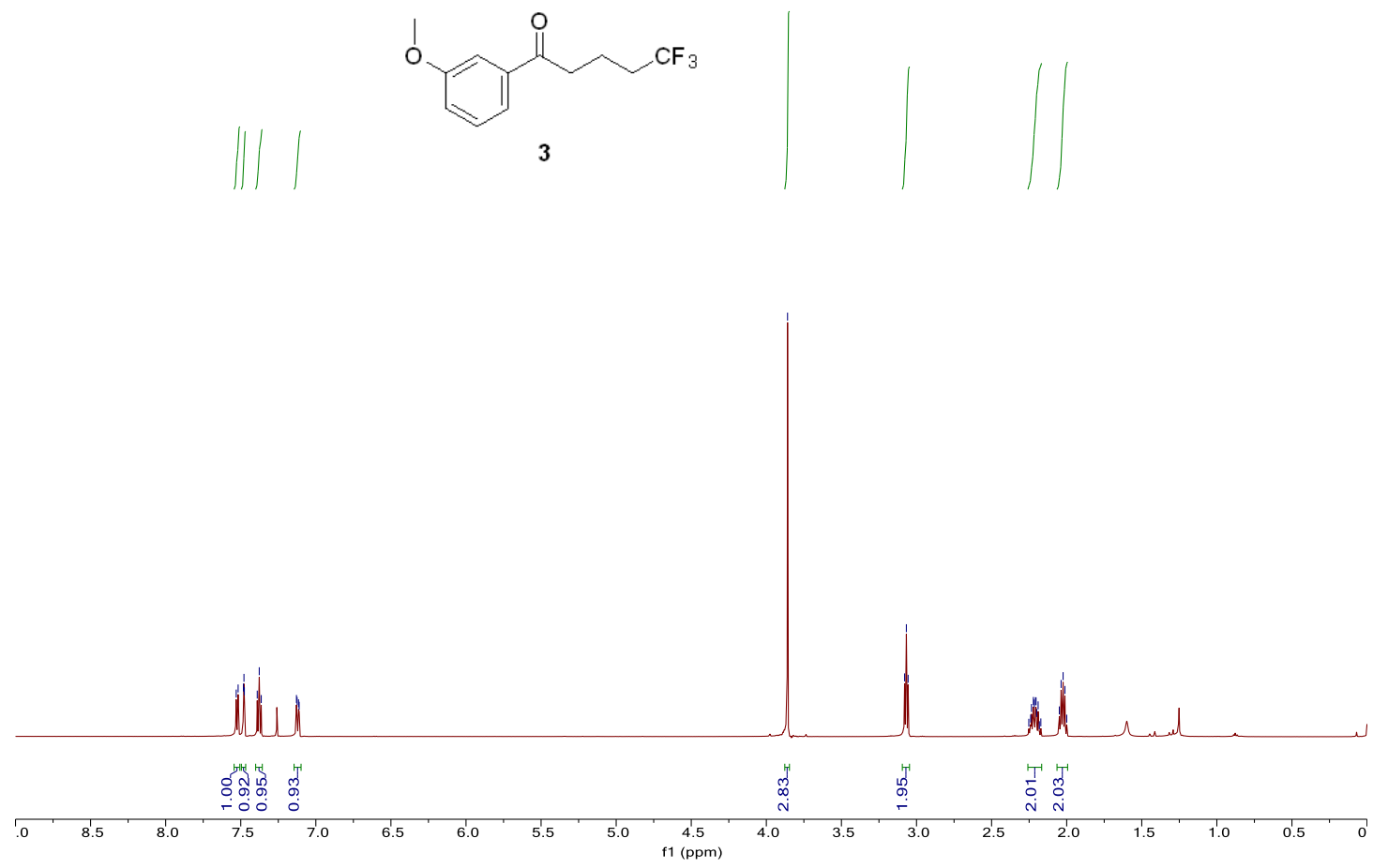

${ }^{13} \mathrm{C} \mathrm{NMR}\left(151 \mathrm{MHz}, \mathrm{CDCl}_{3}\right)$

$\stackrel{\substack{\infty \\ \stackrel{\infty}{i}}}{1}$
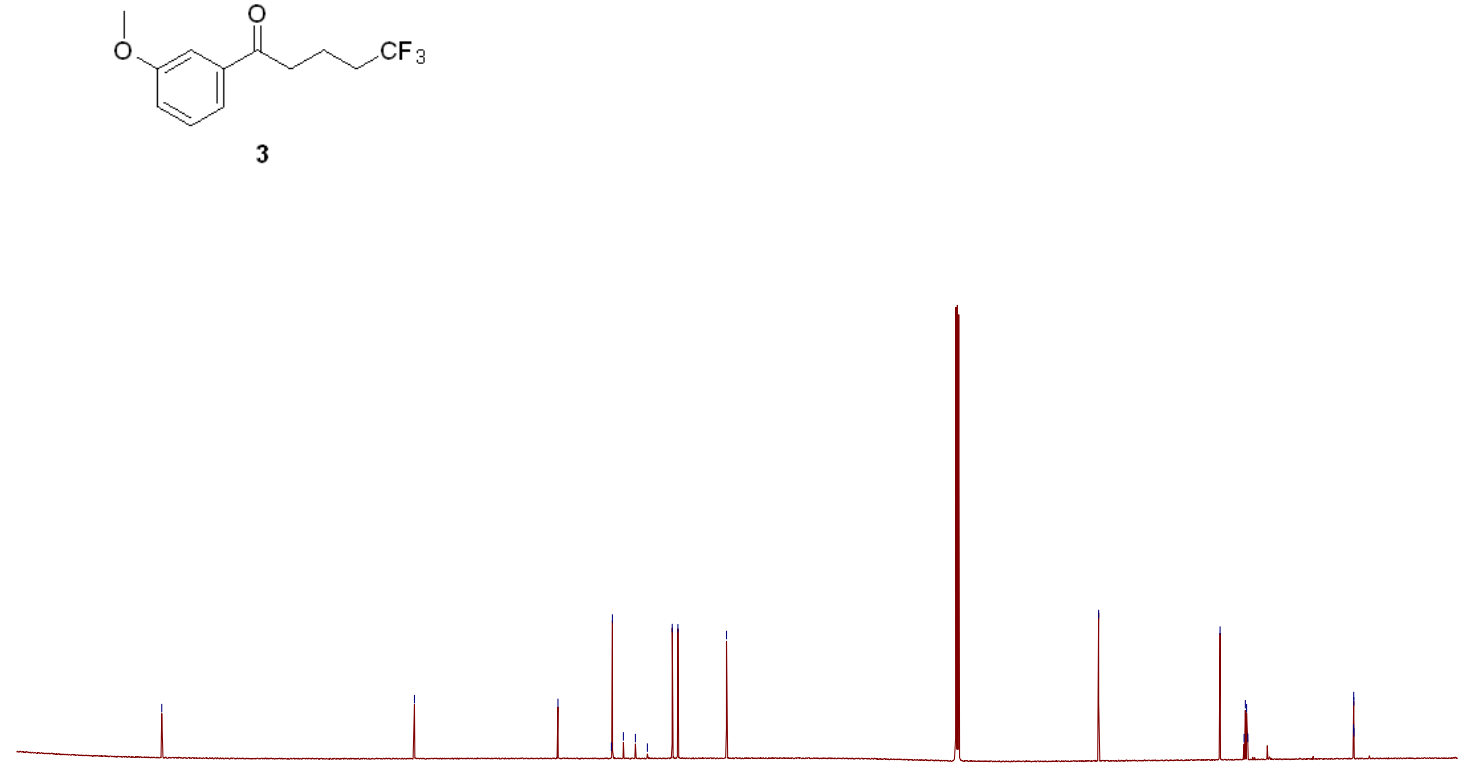

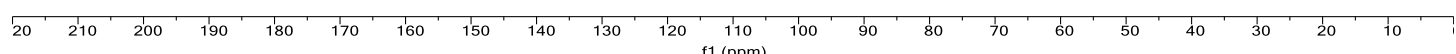


${ }^{19} \mathrm{~F}$ NMR $\left(376 \mathrm{MHz}, \mathrm{CDCl}_{3}\right)$

บับ

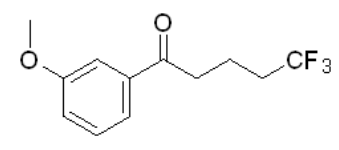

3

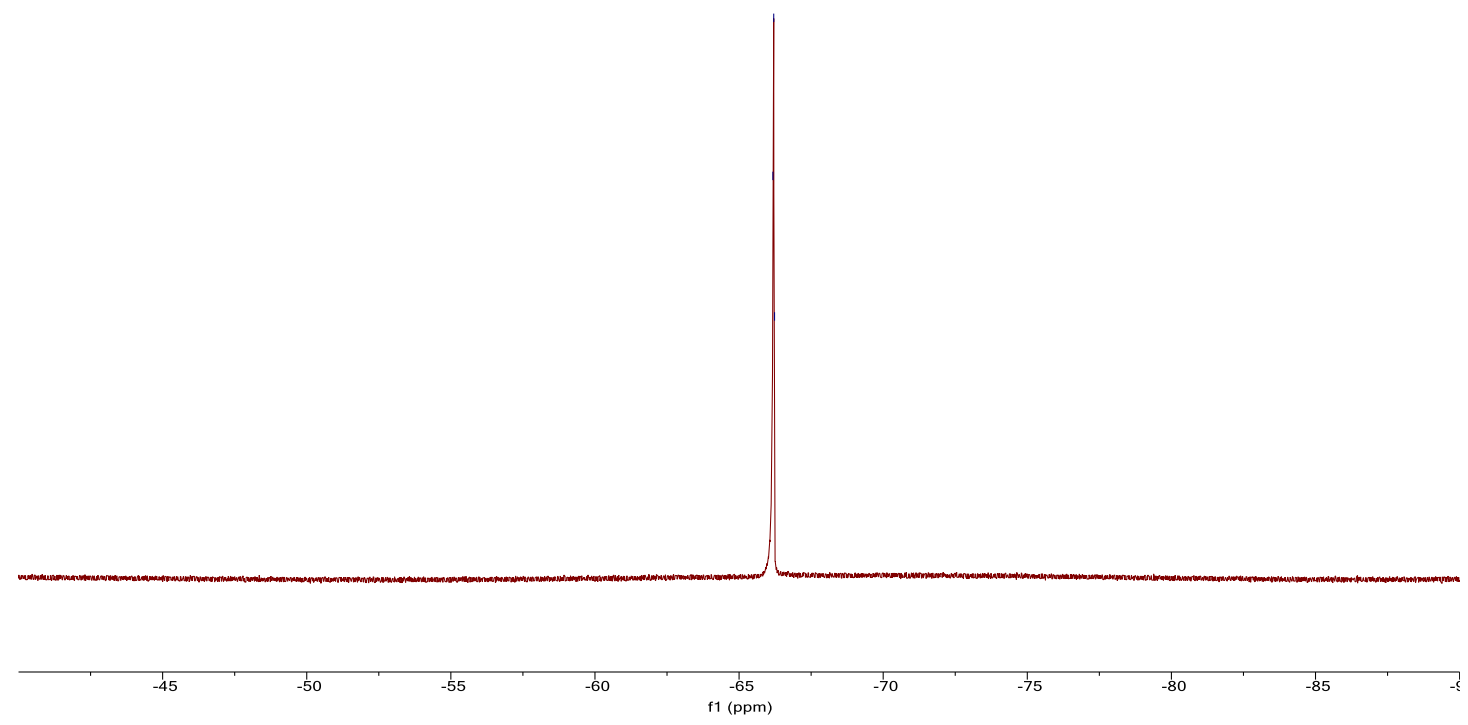

${ }^{1} \mathrm{H}$ NMR (600 MHz, $\left.\mathrm{CDCl}_{3}\right)$

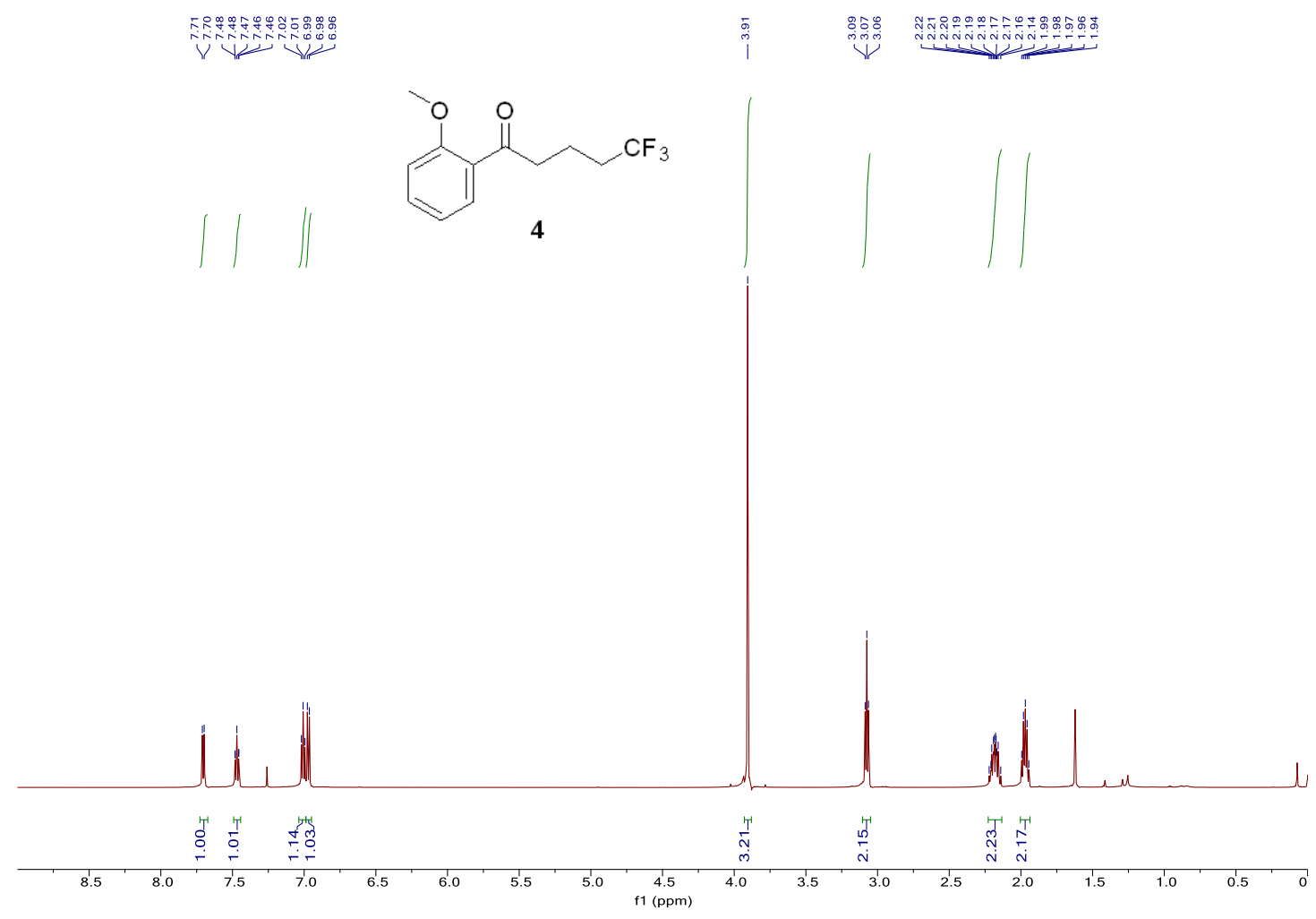


${ }^{13} \mathrm{C} \mathrm{NMR}\left(151 \mathrm{MHz}, \mathrm{CDCl}_{3}\right)$

ڤั̀

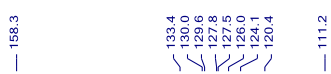

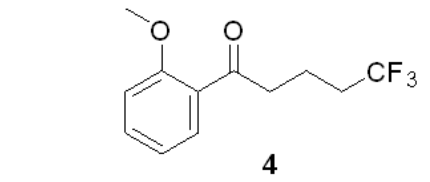

4
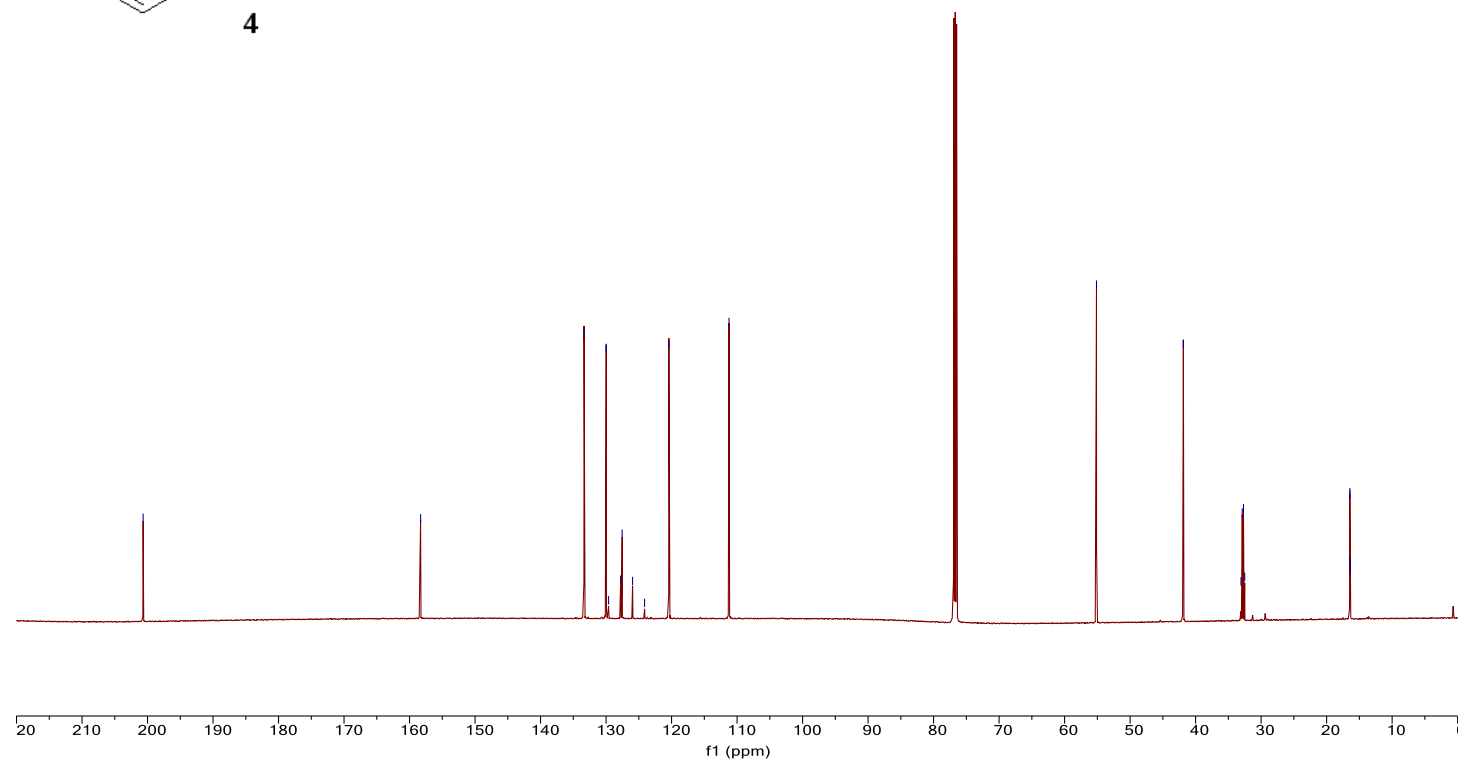

${ }^{19} \mathrm{~F} \mathrm{NMR}\left(376 \mathrm{MHz}, \mathrm{CDCl}_{3}\right)$<smiles>COc1ccccc1C(=O)CCCC(F)(F)F</smiles>

4

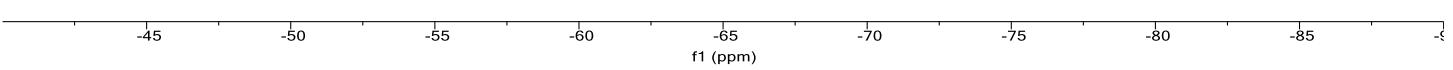


${ }^{1} \mathrm{H}$ NMR (600 MHz, $\left.\mathrm{CDCl}_{3}\right)$

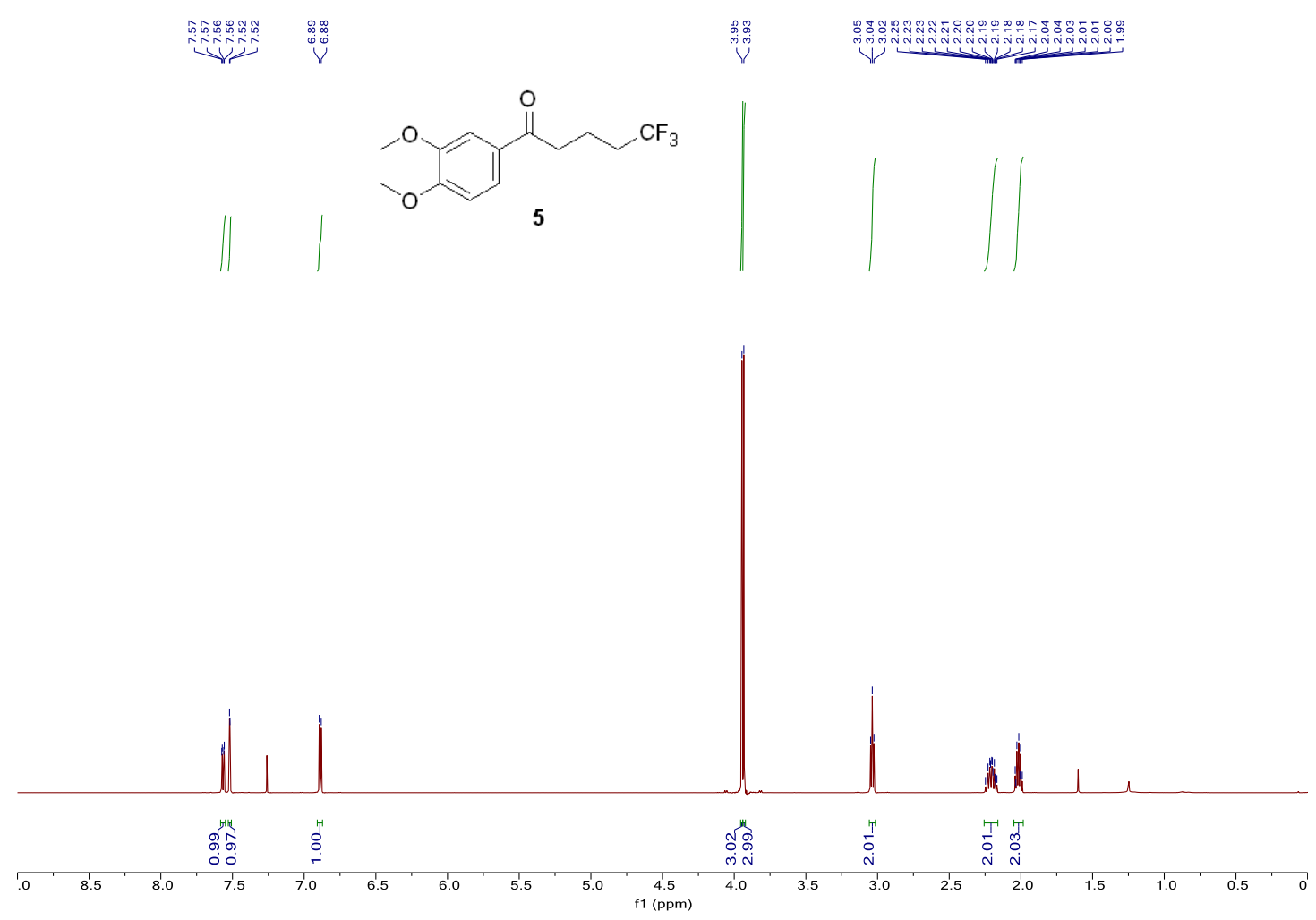

${ }^{13} \mathrm{C}$ NMR $\left(151 \mathrm{MHz}, \mathrm{CDCl}_{3}\right)$

i<smiles>COc1ccc(C(=O)CCCC(F)(F)F)cc1OC</smiles>

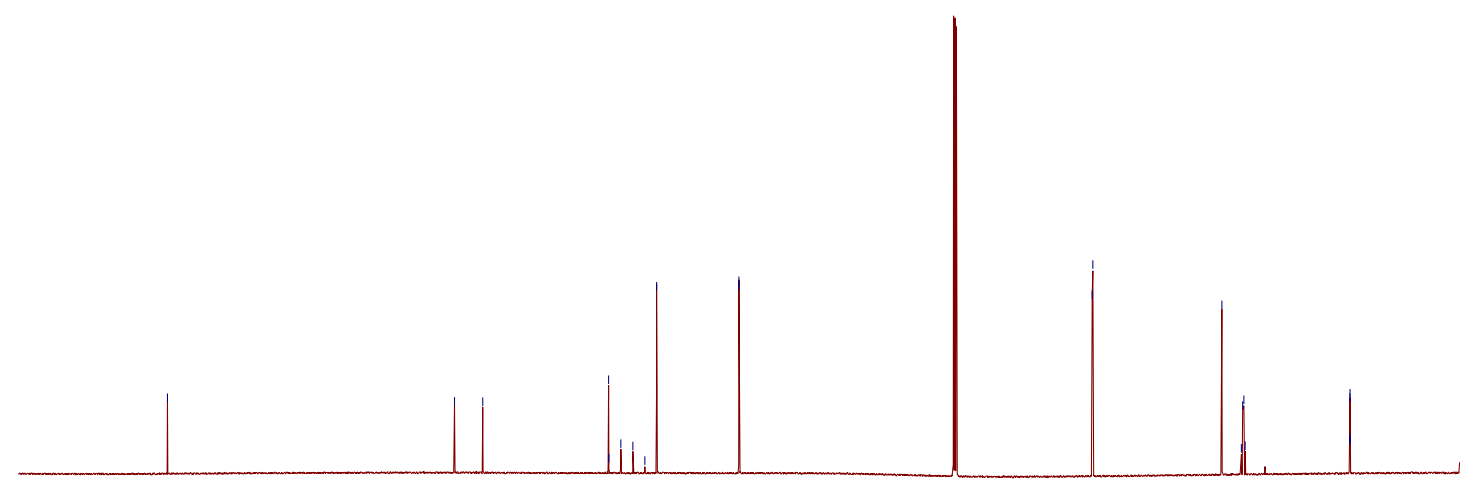

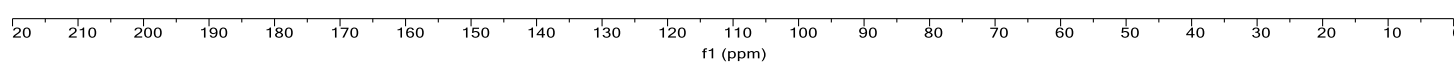


${ }^{19} \mathrm{~F}$ NMR $\left(376 \mathrm{MHz}, \mathrm{CDCl}_{3}\right)$

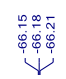<smiles>COc1ccc(C(=O)CCCC(F)(F)F)cc1OC</smiles>

5

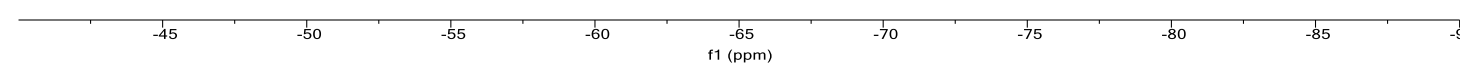

${ }^{1} \mathrm{H}$ NMR $\left(600 \mathrm{MHz}, \mathrm{CDCl}_{3}\right)$

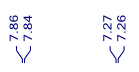

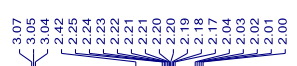

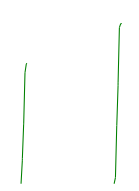<smiles>Cc1ccc(C(=O)CCCC(F)(F)F)cc1</smiles>
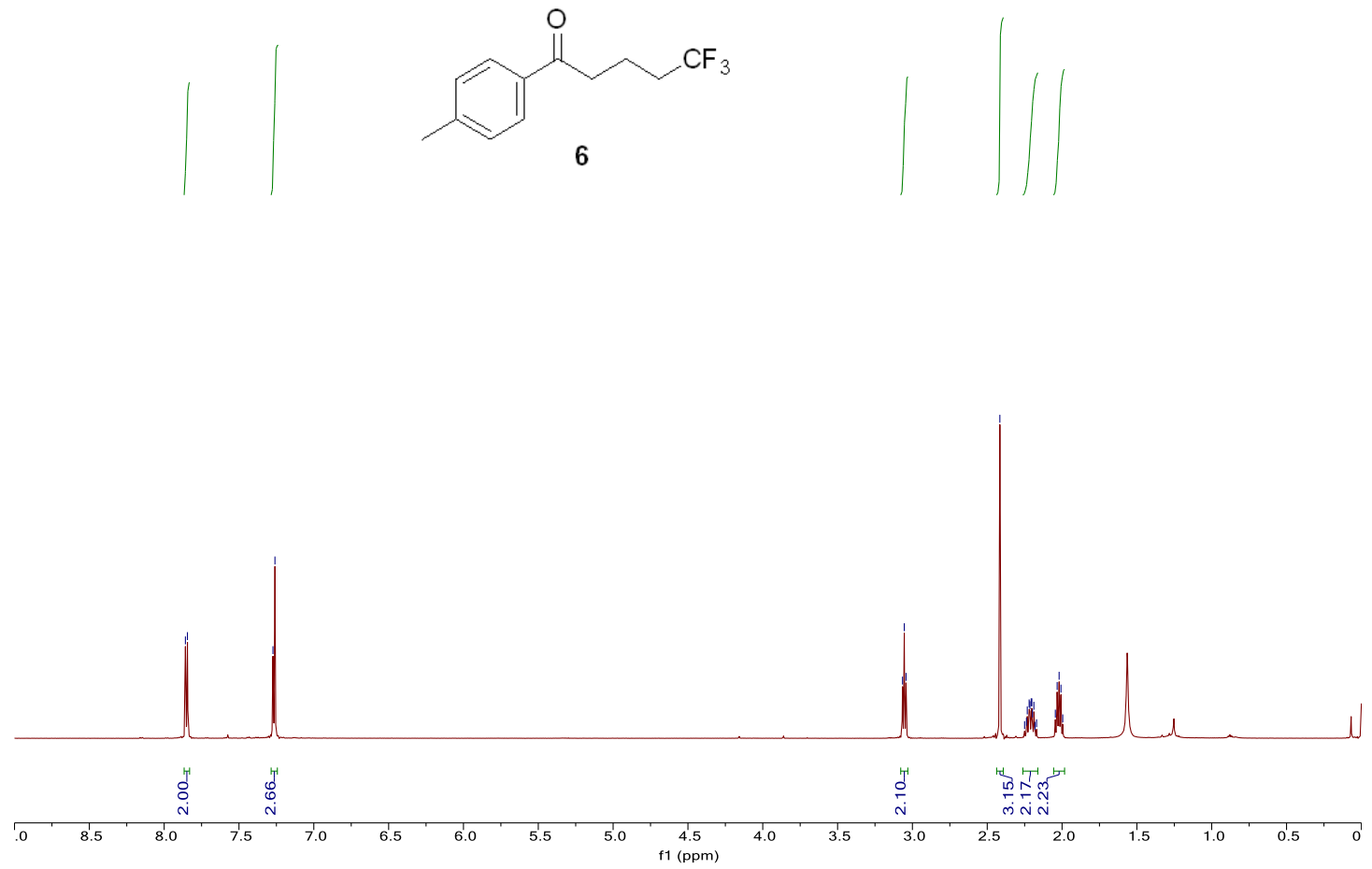
${ }^{13} \mathrm{C} \mathrm{NMR}\left(151 \mathrm{MHz}, \mathrm{CDCl}_{3}\right)$

$\stackrel{\substack{\infty \\ \stackrel{\infty}{\prime}}}{i}$

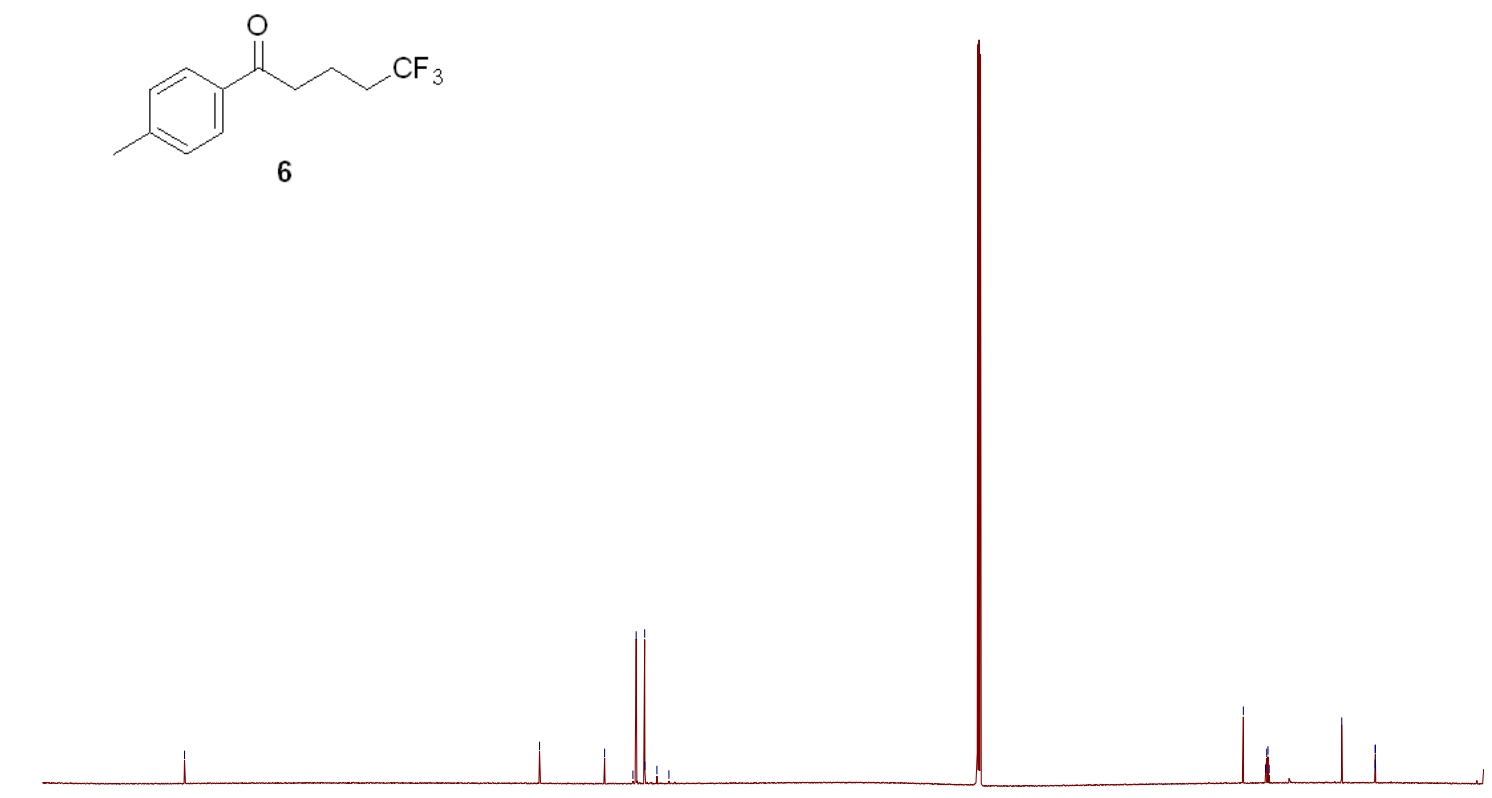

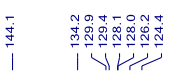

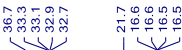$$
\text { ( }
$$

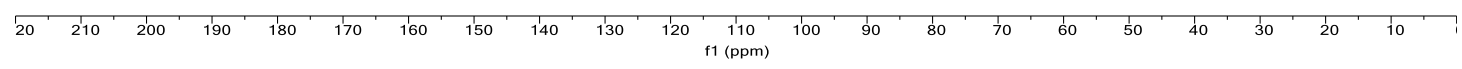

${ }^{19} \mathrm{~F}$ NMR (376 MHz, $\left.\mathrm{CDCl}_{3}\right)$

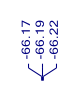<smiles>Cc1ccc(C(=O)CCCC(F)(F)F)cc1</smiles>

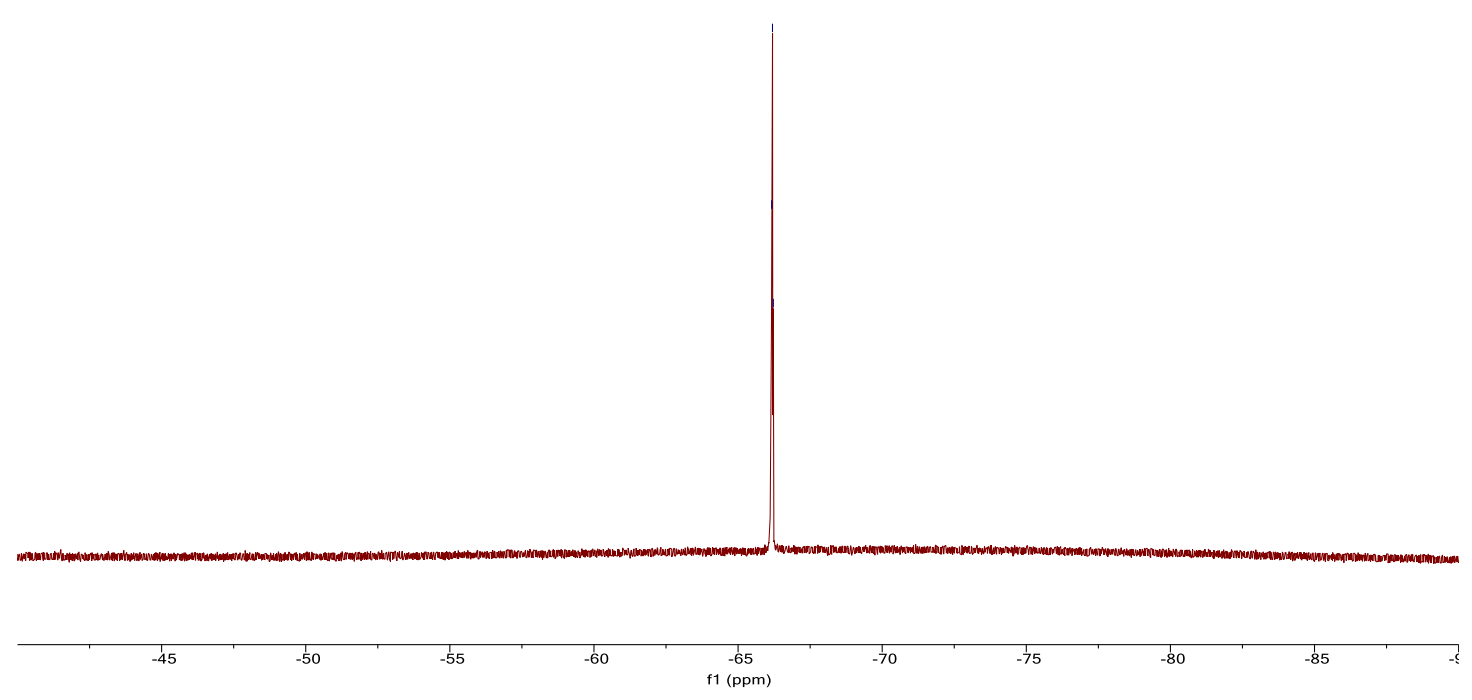


${ }^{1} \mathrm{H}$ NMR (600 MHz, $\left.\mathrm{CDCl}_{3}\right)$

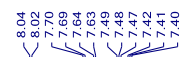

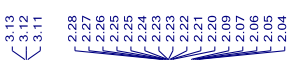
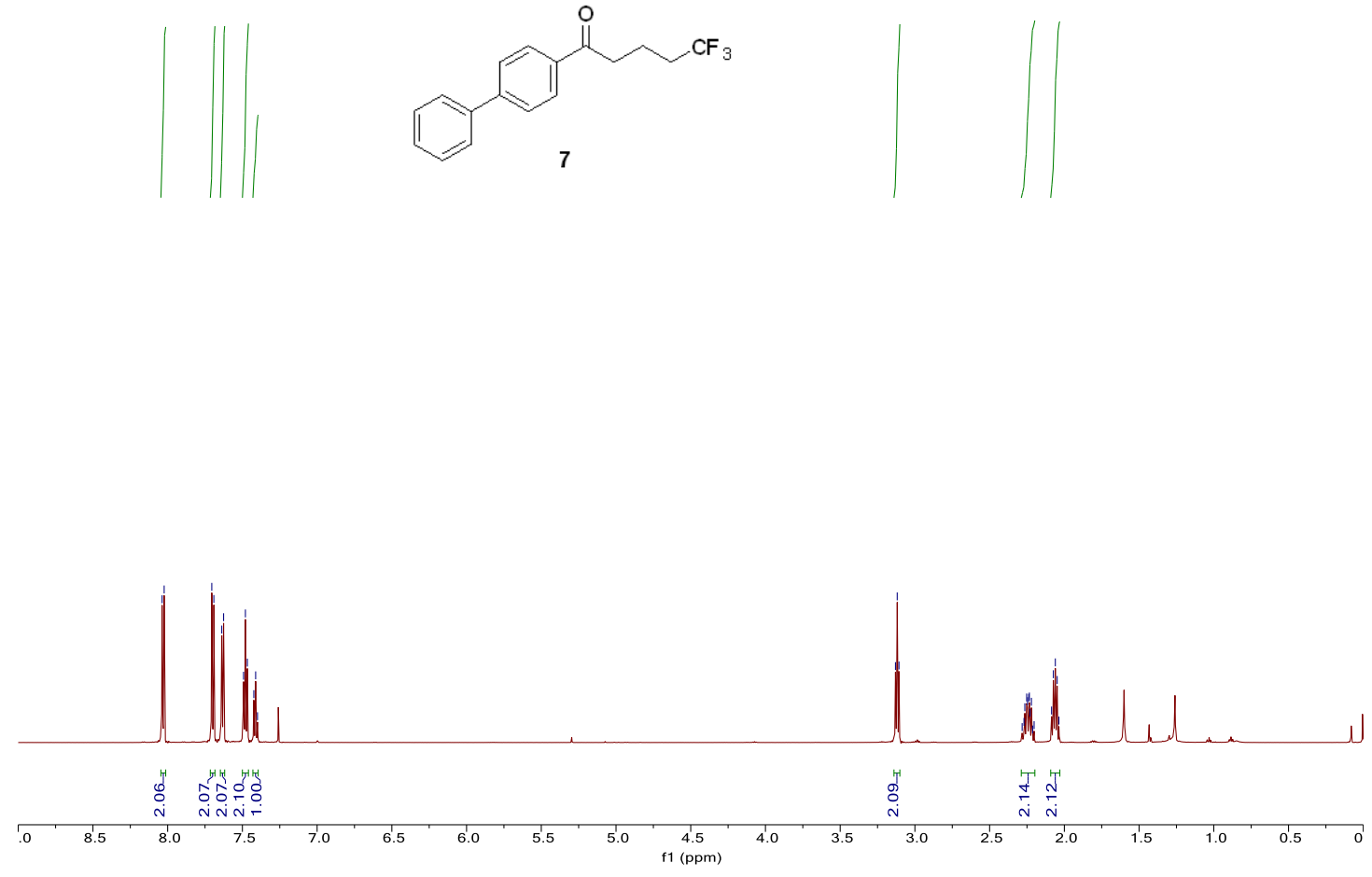

${ }^{13} \mathrm{C} \mathrm{NMR}\left(151 \mathrm{MHz}, \mathrm{CDCl}_{3}\right)$
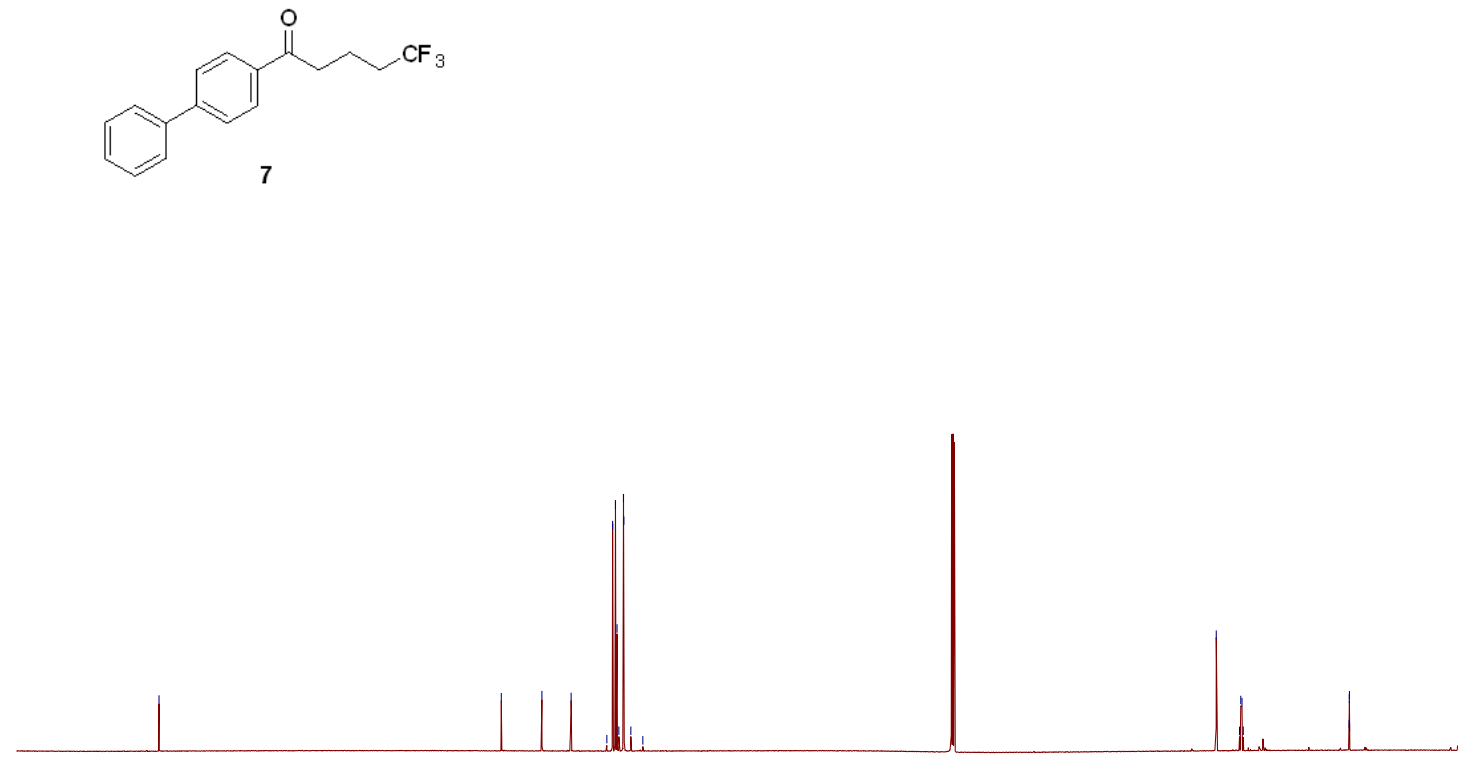

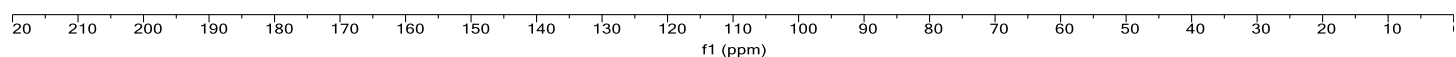


${ }^{19} \mathrm{~F}$ NMR $\left(376 \mathrm{MHz}, \mathrm{CDCl}_{3}\right)$

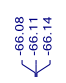
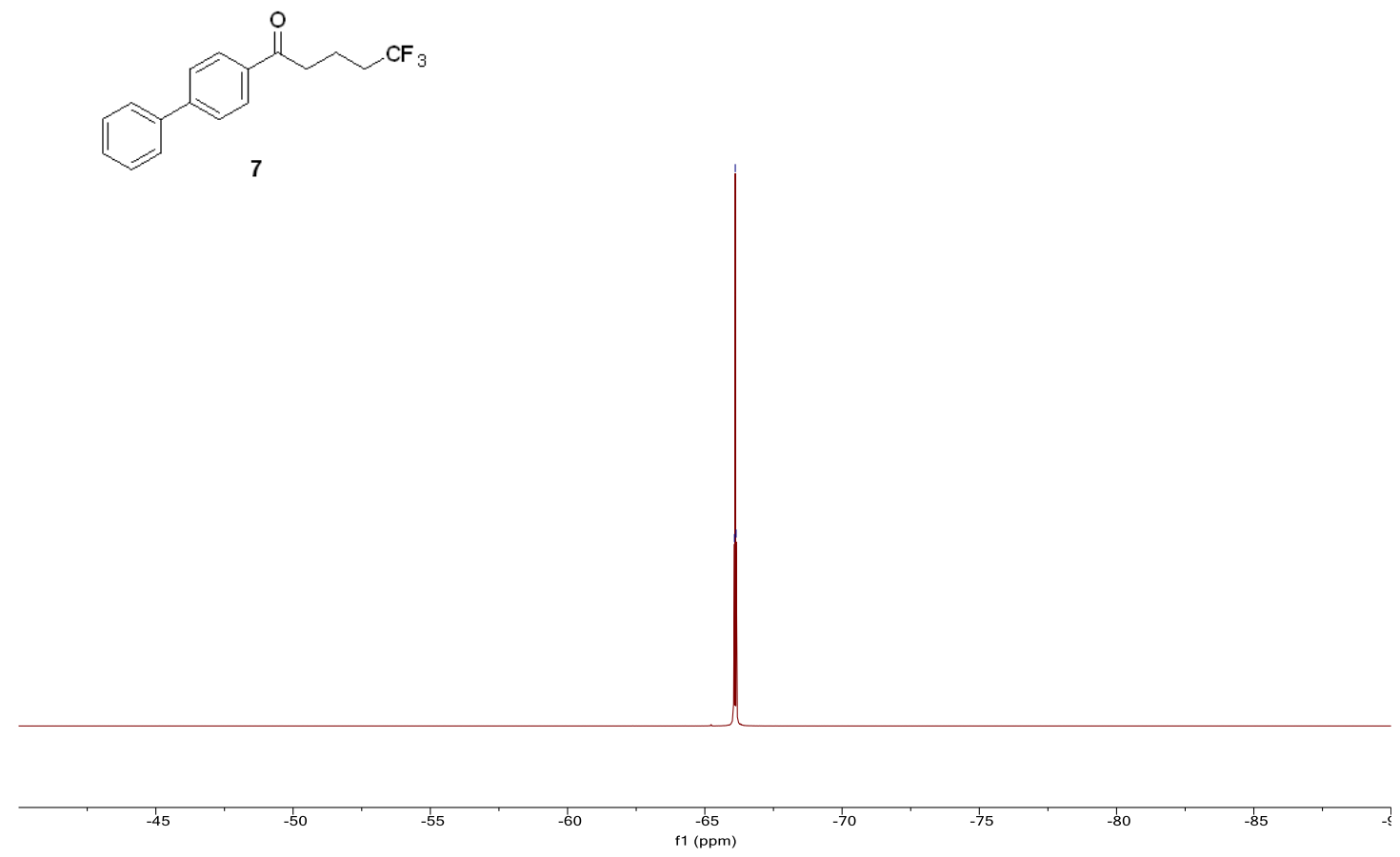

${ }^{1} \mathrm{H}$ NMR (600 MHz, $\left.\mathrm{CDCl}_{3}\right)$

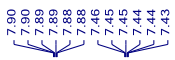

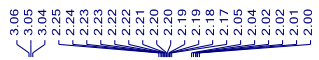
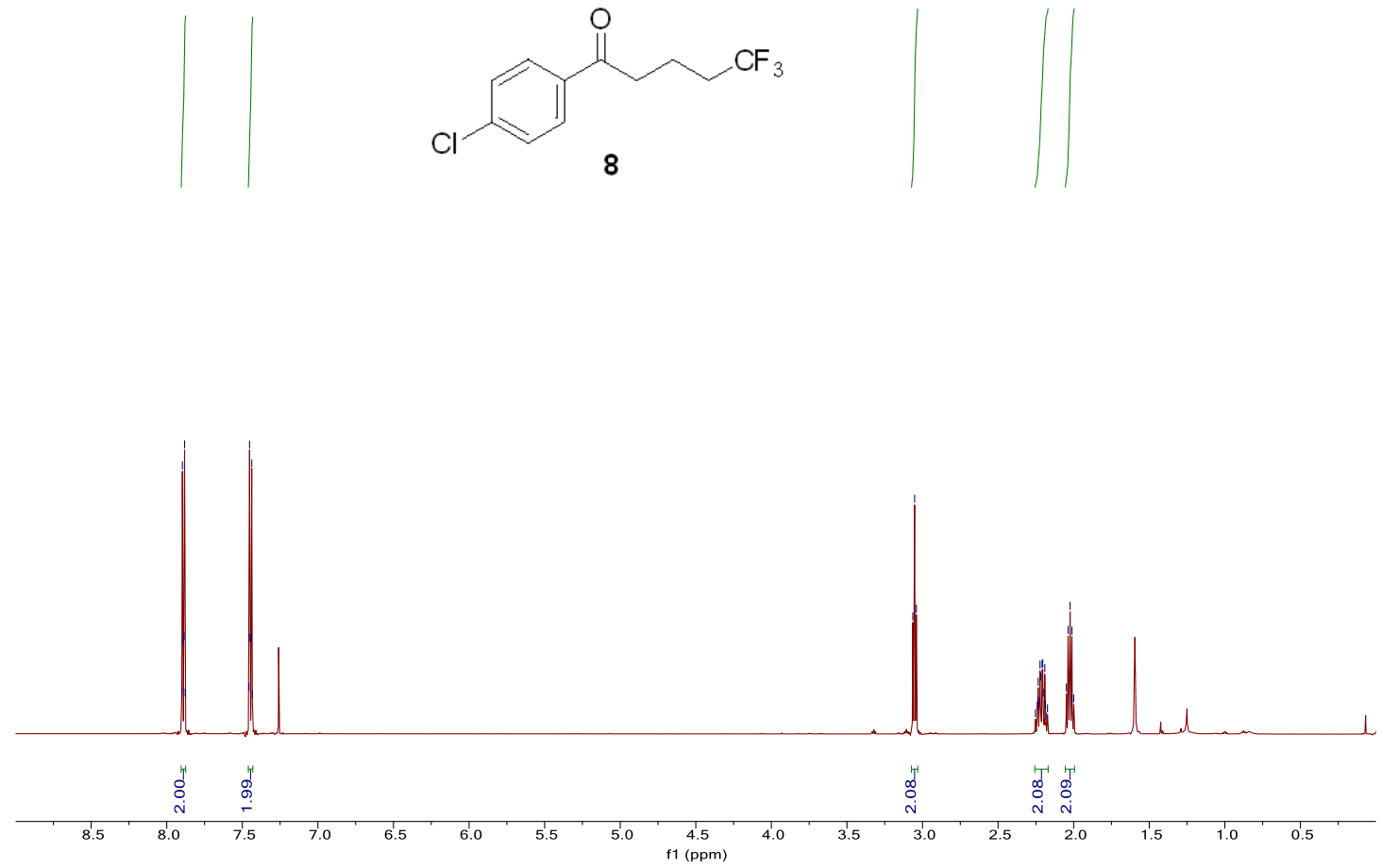
${ }^{13} \mathrm{C} \mathrm{NMR}\left(151 \mathrm{MHz}, \mathrm{CDCl}_{3}\right)$<smiles>O=C(CCCC(F)(F)F)c1ccc(Cl)cc1</smiles>
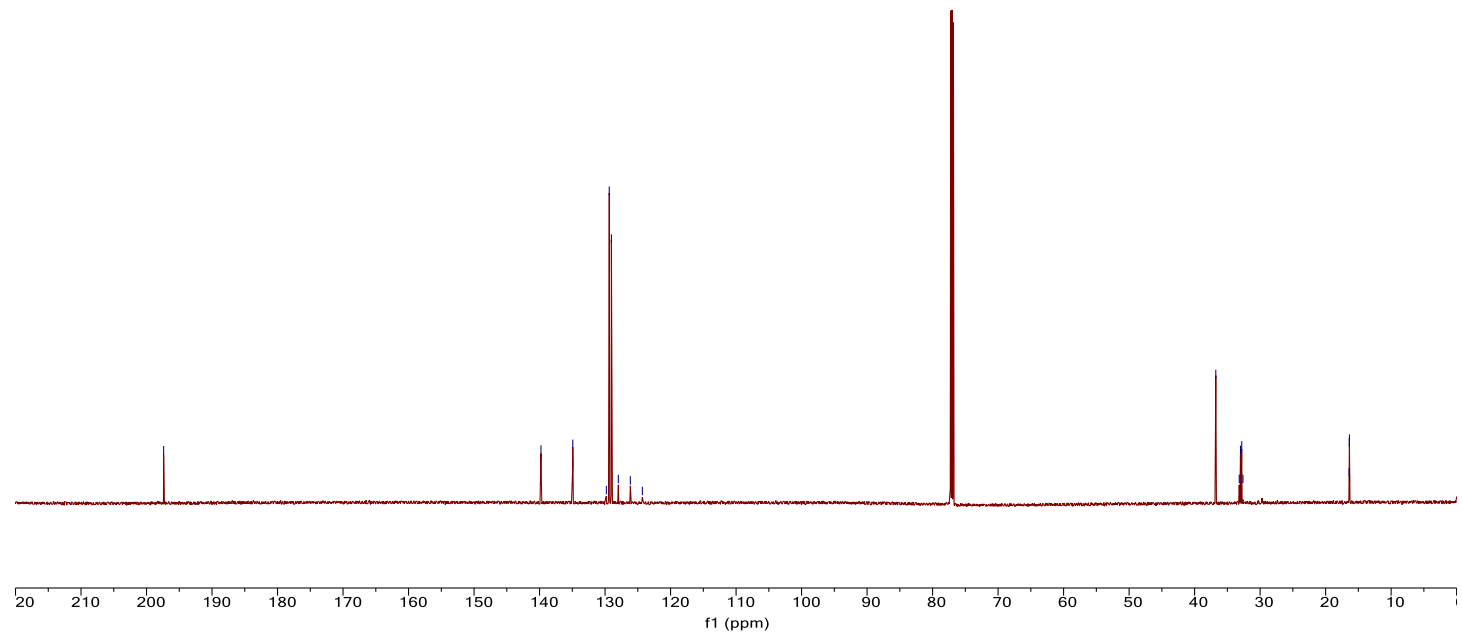

${ }^{19} \mathrm{~F} \mathrm{NMR}\left(376 \mathrm{MHz}, \mathrm{CDCl}_{3}\right)$

ข้<smiles>O=C(CCCC(F)(F)F)c1ccc(Cl)cc1</smiles>

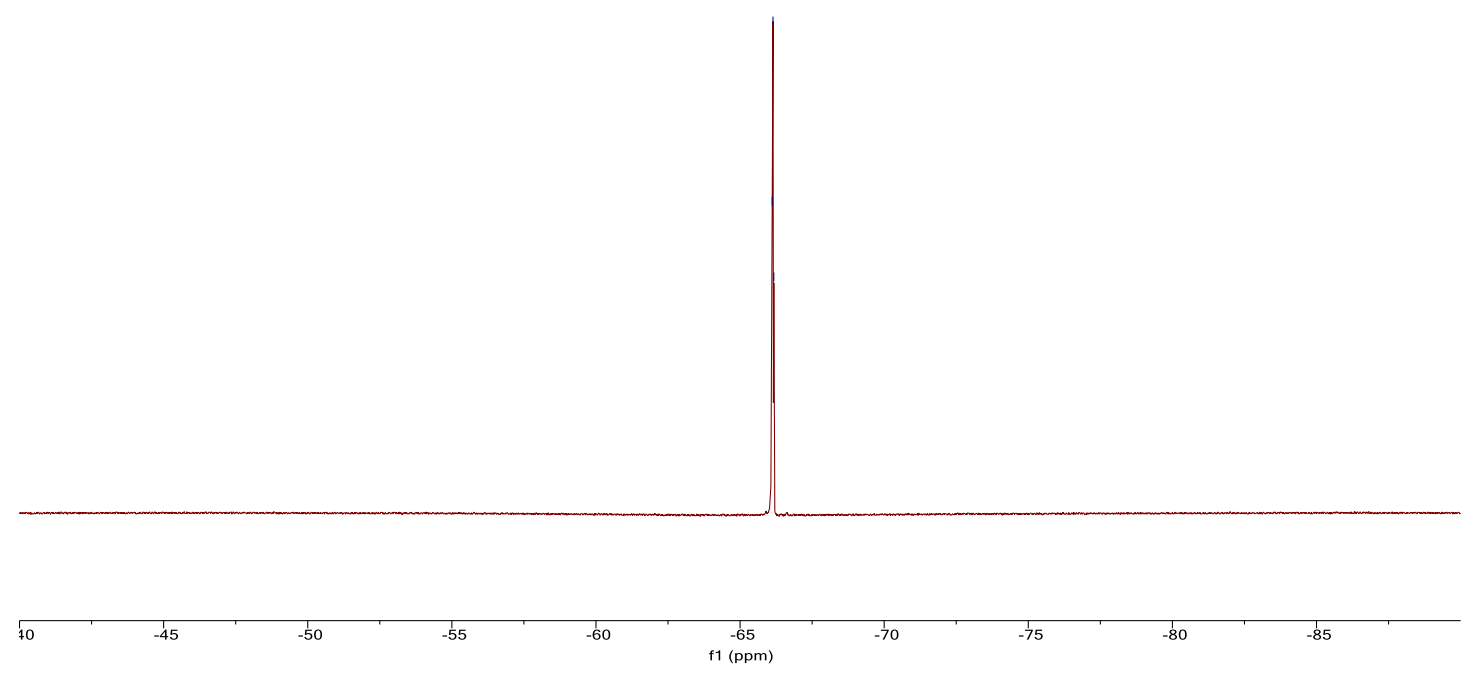


${ }^{1} \mathrm{H} \mathrm{NMR}\left(600 \mathrm{MHz}, \mathrm{CDCl}_{3}\right)$

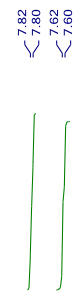<smiles>O=C(CCCC(F)(F)F)c1ccc(Br)cc1</smiles>

\section{告:}
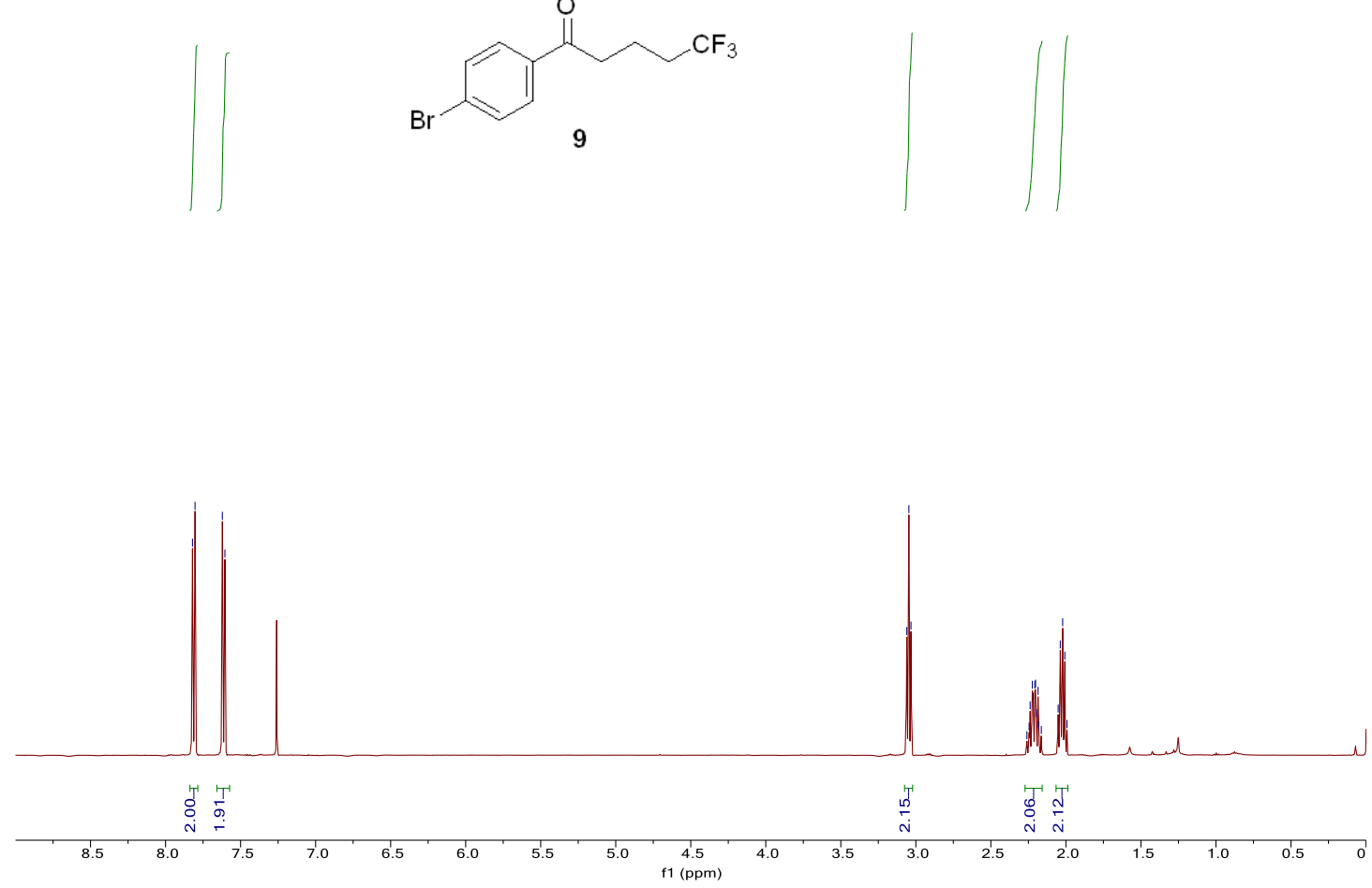

${ }^{13} \mathrm{C} \mathrm{NMR}\left(151 \mathrm{MHz}, \mathrm{CDCl}_{3}\right)$

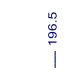

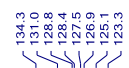

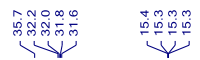
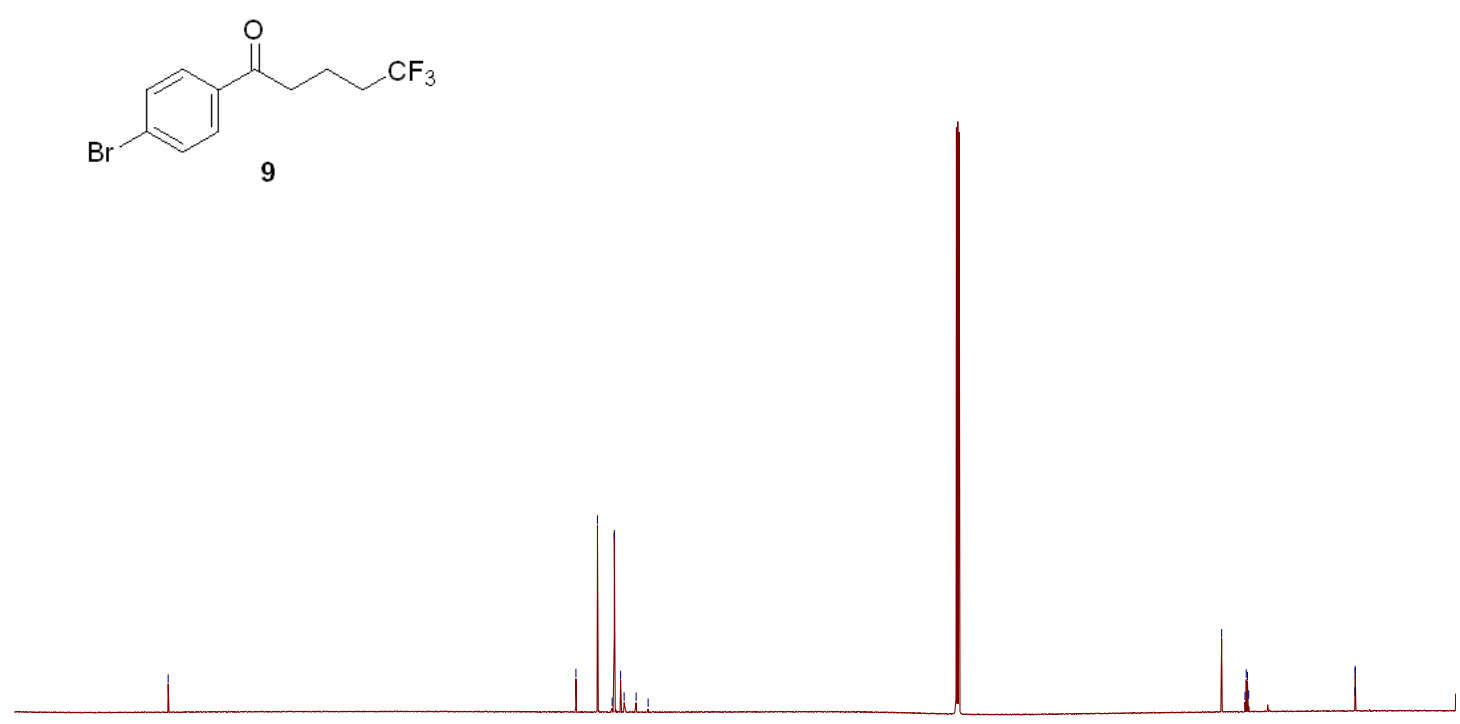

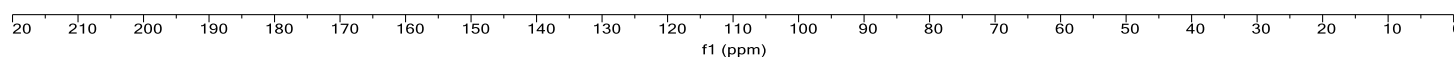


${ }^{19} \mathrm{~F}$ NMR $\left(376 \mathrm{MHz}, \mathrm{CDCl}_{3}\right)$<smiles>O=C(CCCC(F)(F)F)c1ccc(Br)cc1</smiles>
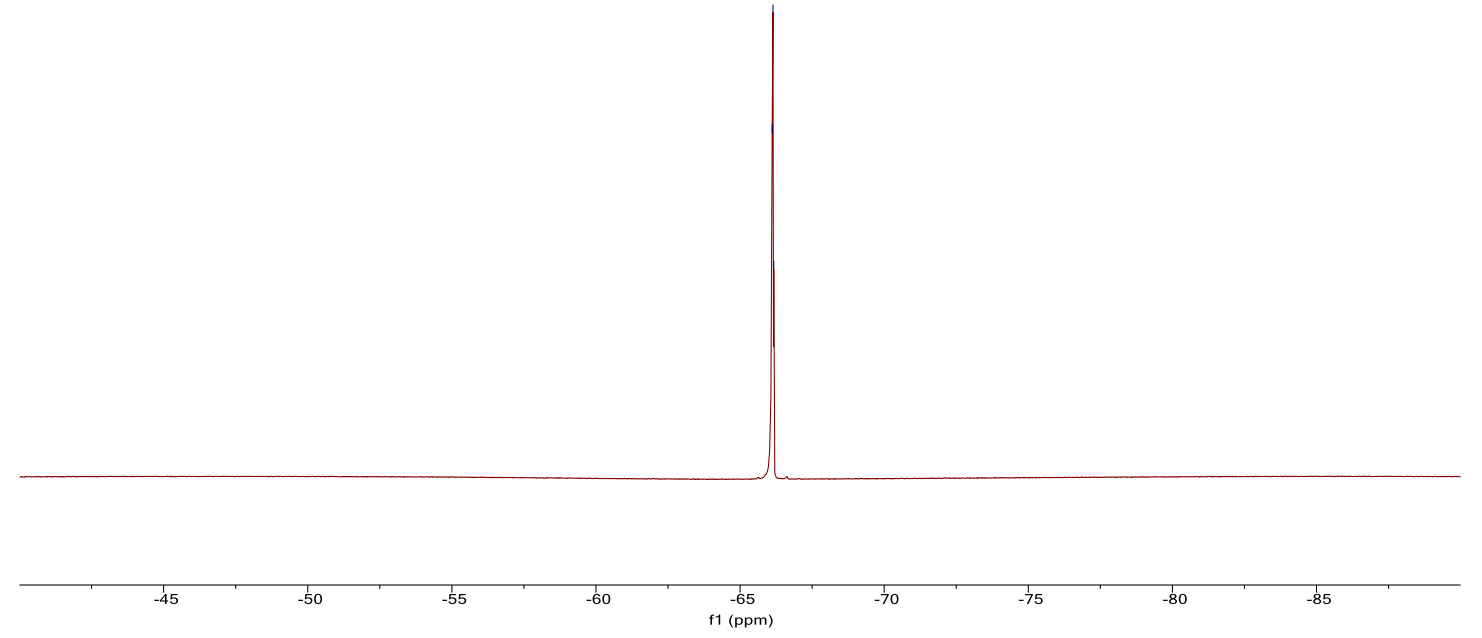

${ }^{1} \mathrm{H}$ NMR (600 MHz, $\left.\mathrm{CDCl}_{3}\right)$

幽

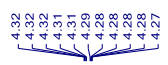

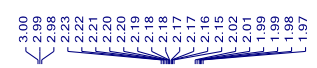
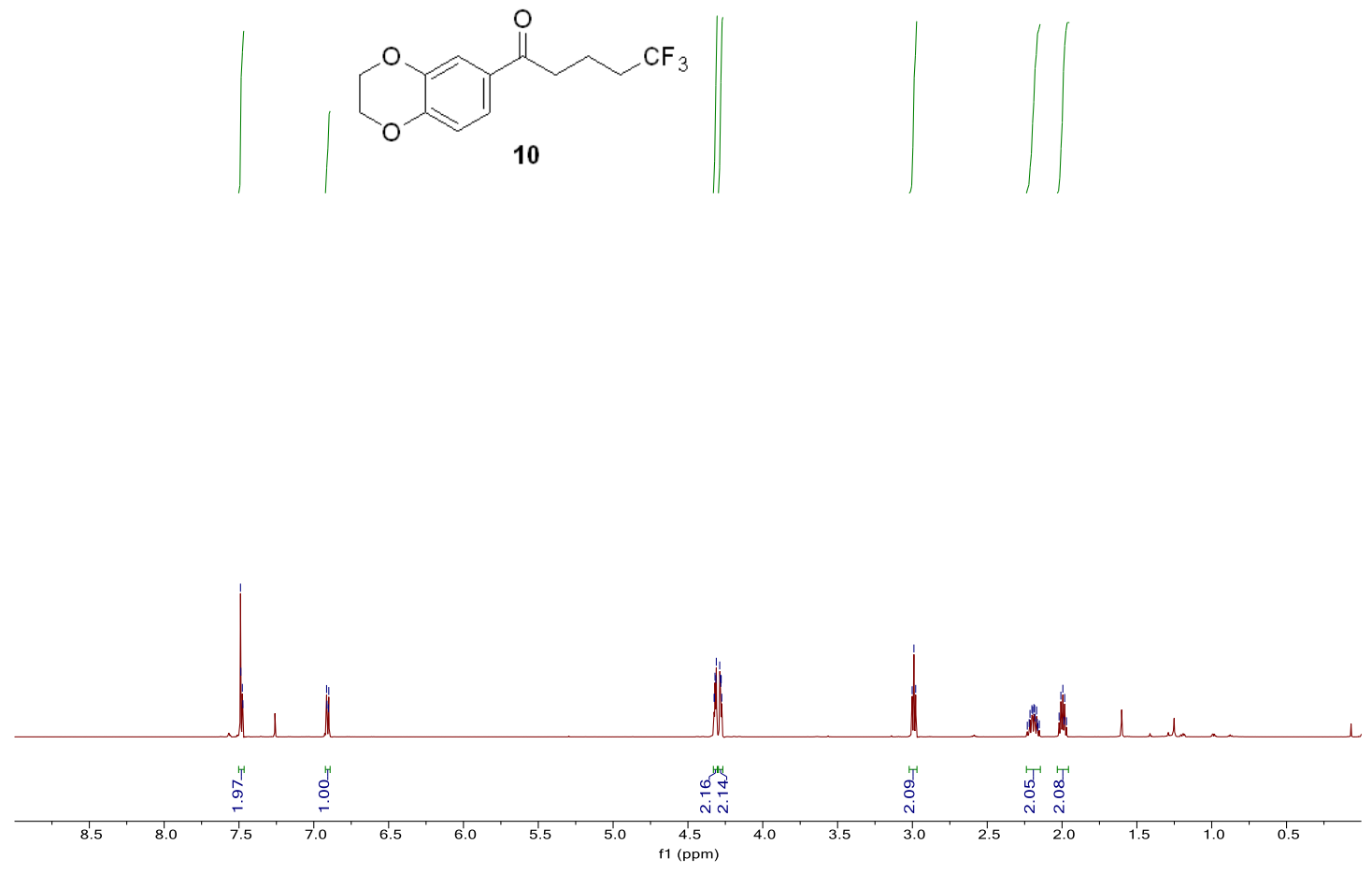
${ }^{13} \mathrm{C} \mathrm{NMR}\left(151 \mathrm{MHz}, \mathrm{CDCl}_{3}\right)$
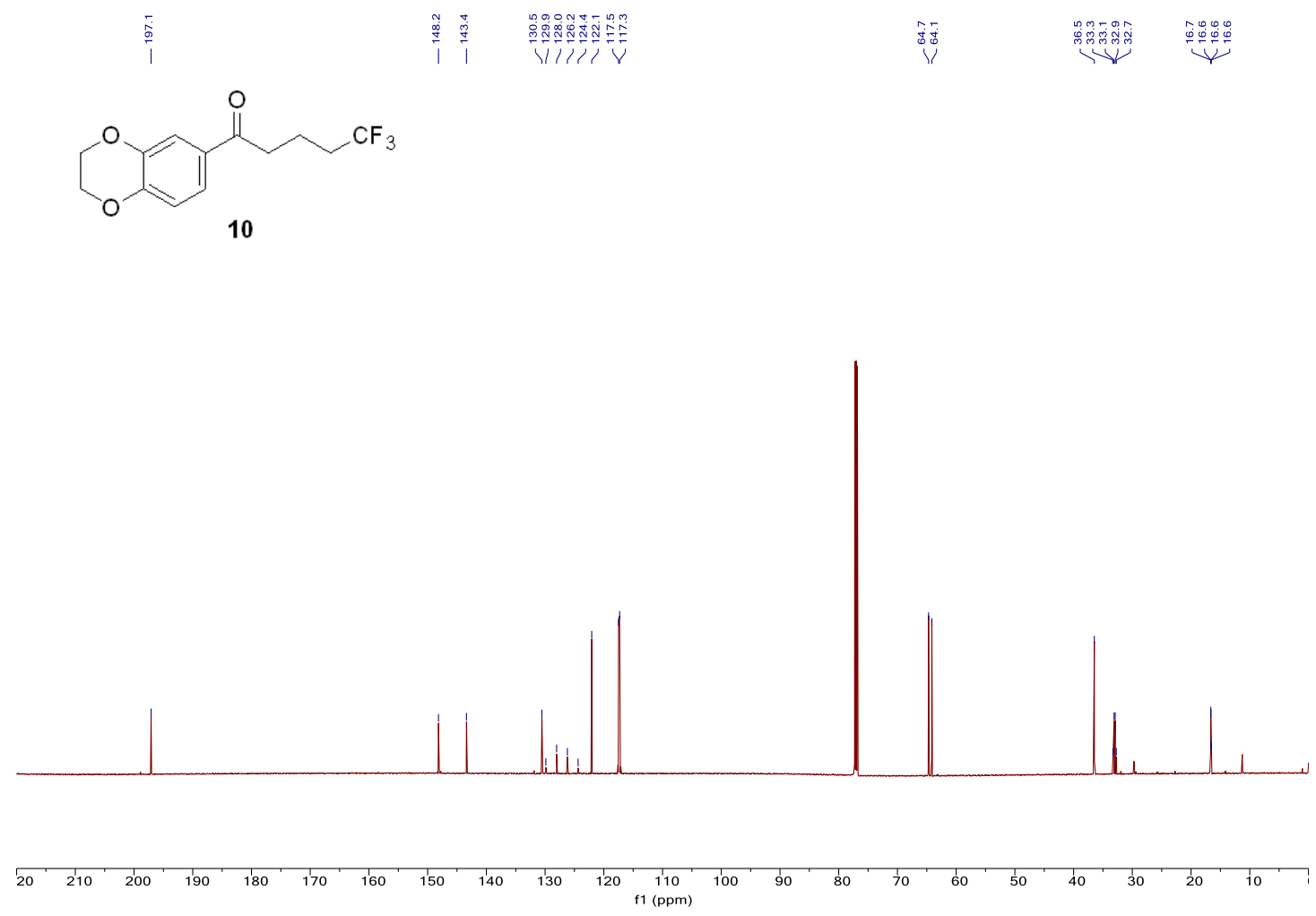

${ }^{19} \mathrm{~F}$ NMR (376 MHz, $\mathrm{CDCl}_{3}$ )

韄<smiles>O=C(CCCC(F)(F)F)c1ccc2c(c1)OCCO2</smiles>

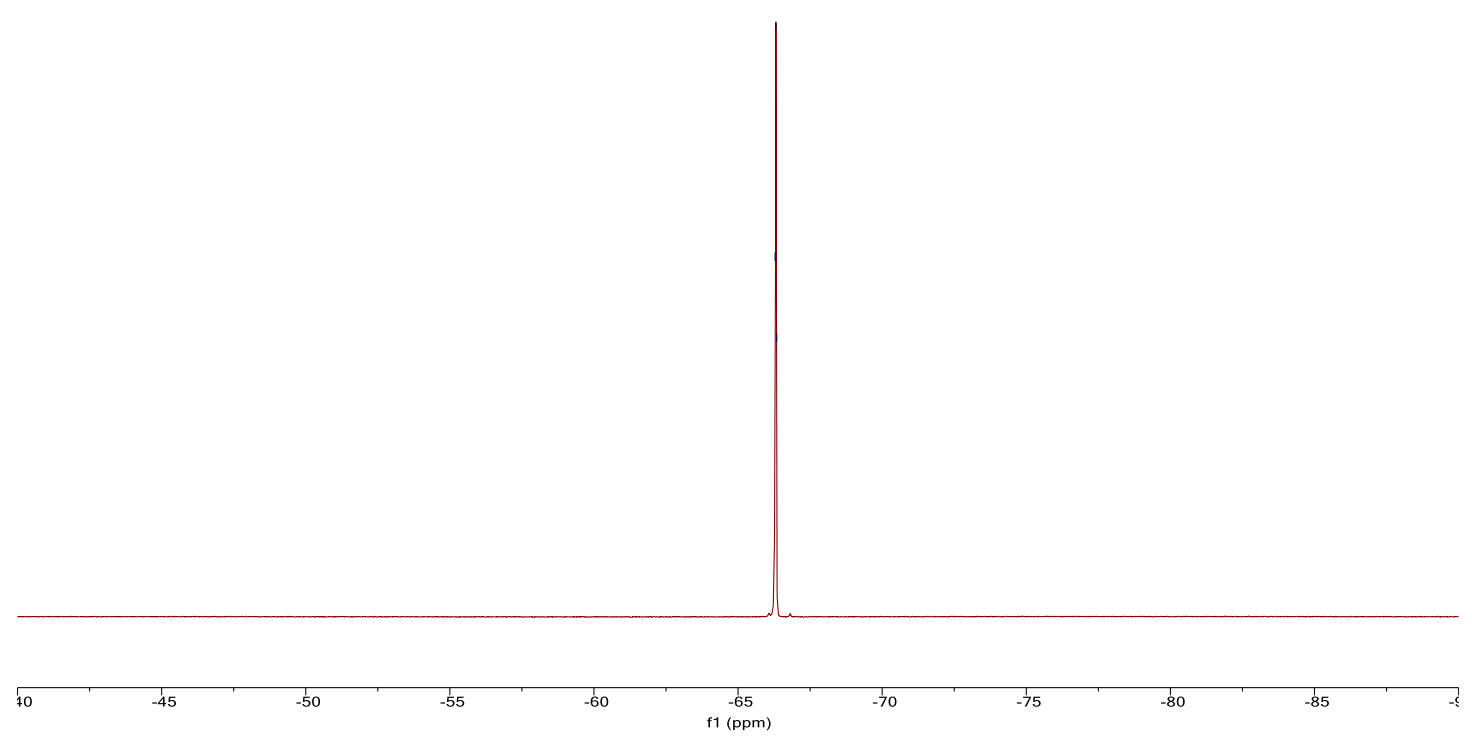


${ }^{1} \mathrm{H}$ NMR (600 MHz, $\left.\mathrm{CDCl}_{3}\right)$

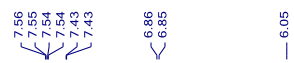

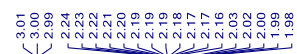
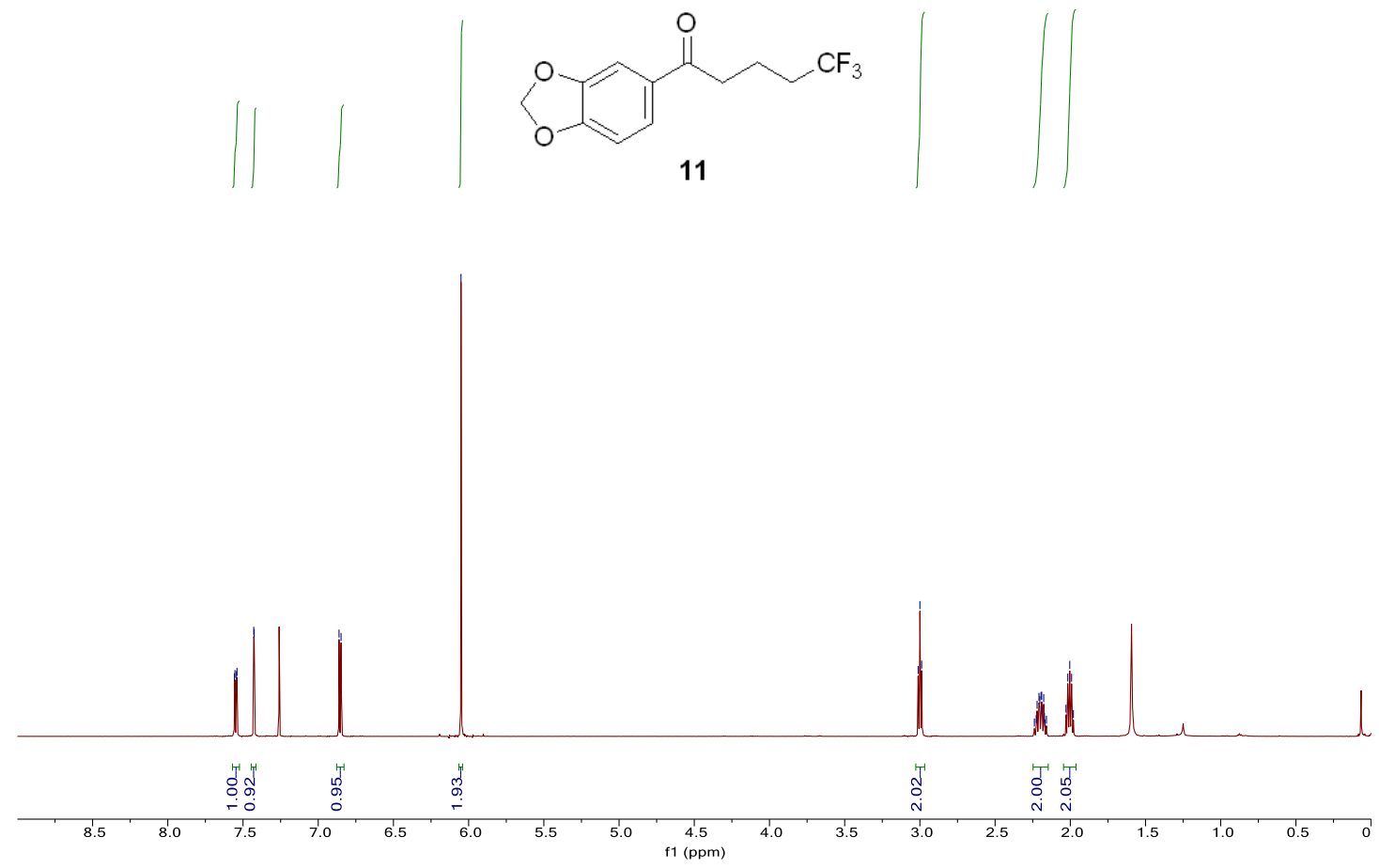

${ }^{13} \mathrm{C} \mathrm{NMR}\left(151 \mathrm{MHz}, \mathrm{CDCl}_{3}\right)$

$\stackrel{\stackrel{0}{\circ}}{i}$

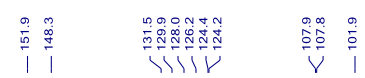

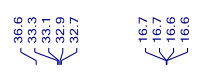<smiles>O=C(CCCC(F)(F)F)c1ccc2c(c1)OCO2</smiles>

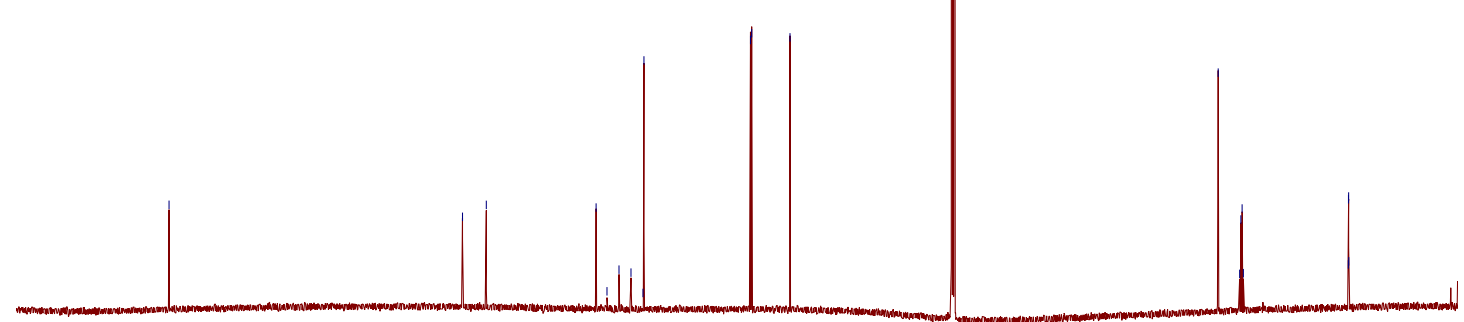

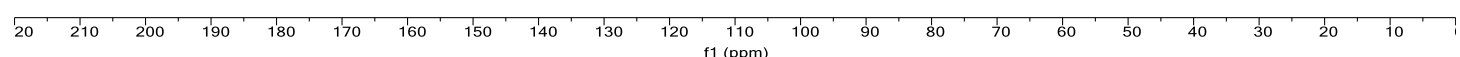


${ }^{19} \mathrm{~F}$ NMR $\left(376 \mathrm{MHz}, \mathrm{CDCl}_{3}\right)$

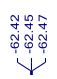<smiles>O=C(CCCC(F)(F)F)c1ccc2c(c1)OCO2</smiles>

11

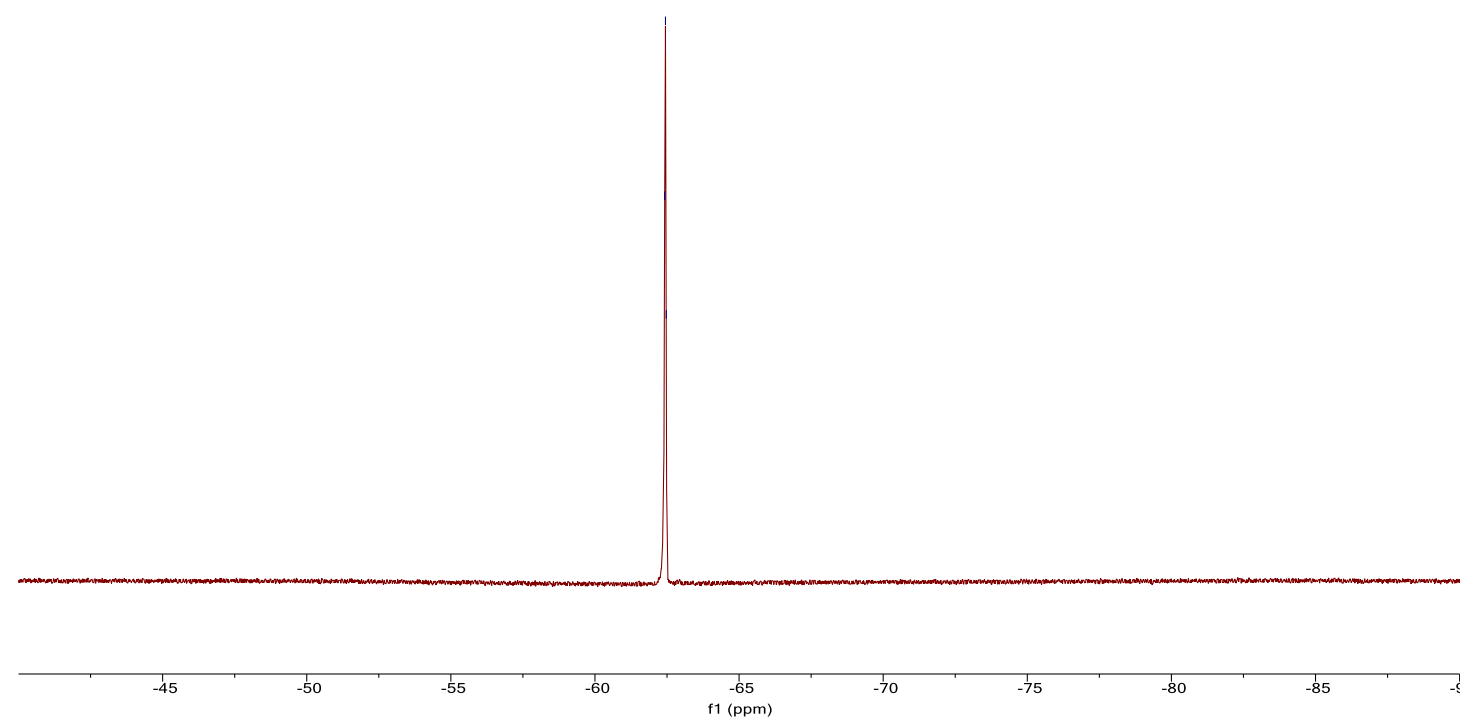

${ }^{1} \mathrm{H}$ NMR (600 MHz, $\left.\mathrm{CDCl}_{3}\right)$

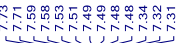

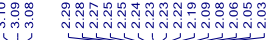
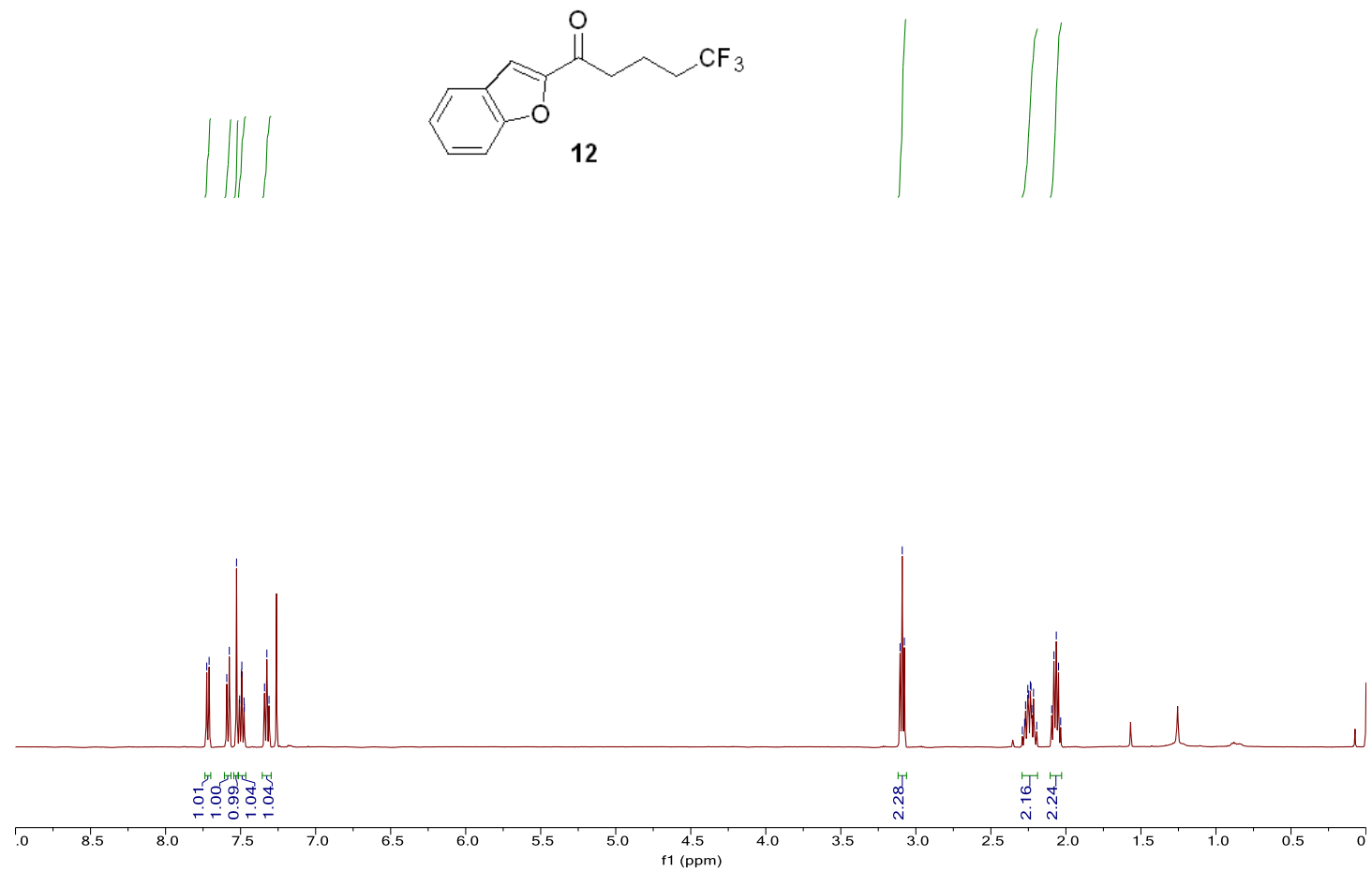
${ }^{13} \mathrm{C} \mathrm{NMR}\left(151 \mathrm{MHz}, \mathrm{CDCl}_{3}\right)$

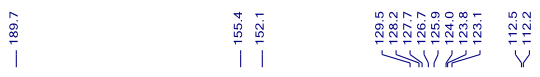

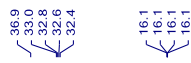
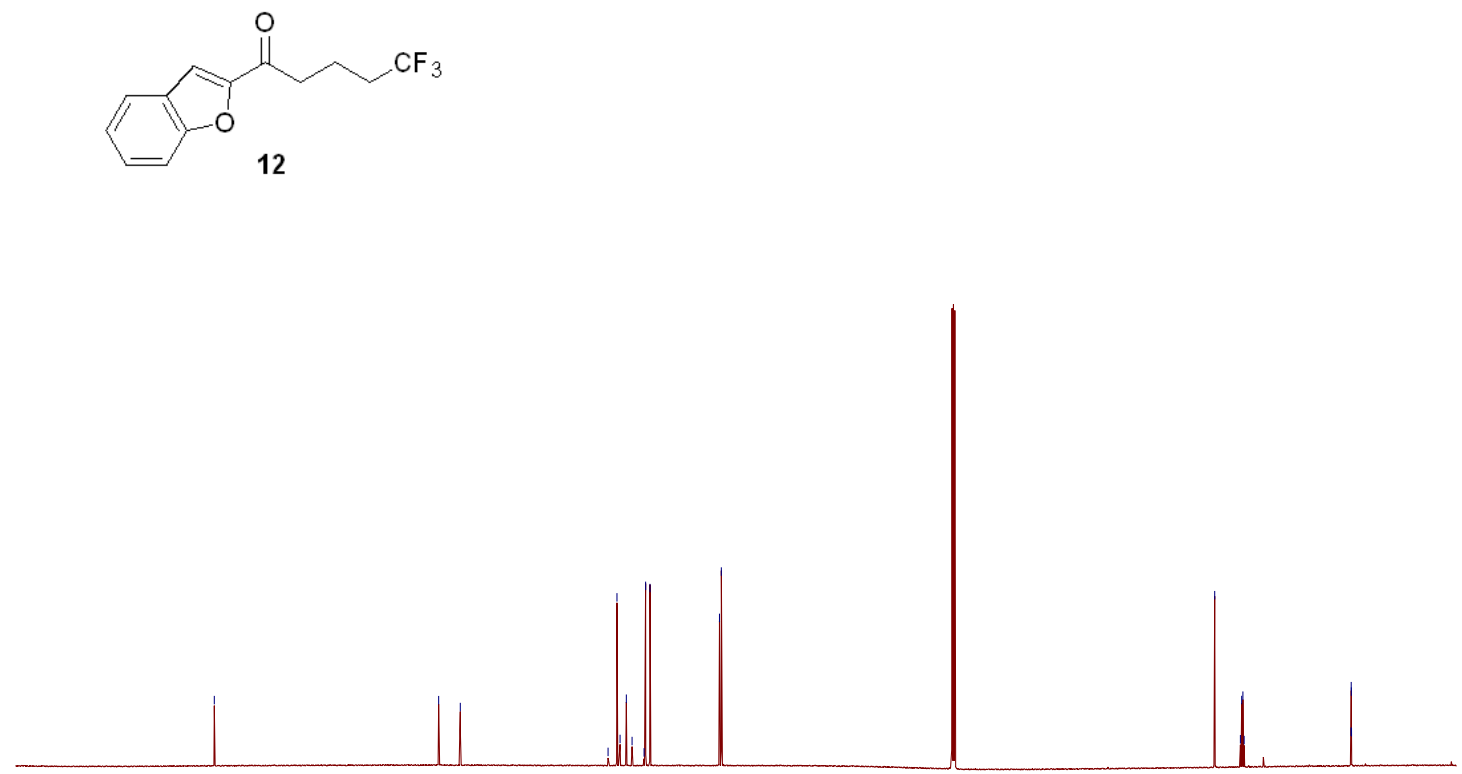

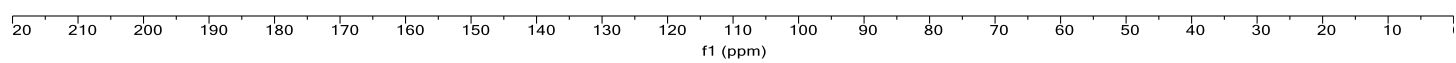

${ }^{19} \mathrm{~F}$ NMR $\left(376 \mathrm{MHz}, \mathrm{CDCl}_{3}\right)$

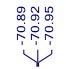<smiles>O=C(CCCC(F)(F)F)c1cc2ccccc2o1</smiles> 
${ }^{1} \mathrm{H} \mathrm{NMR}\left(600 \mathrm{MHz}, \mathrm{CDCl}_{3}\right)$

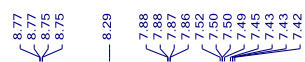

产ำ
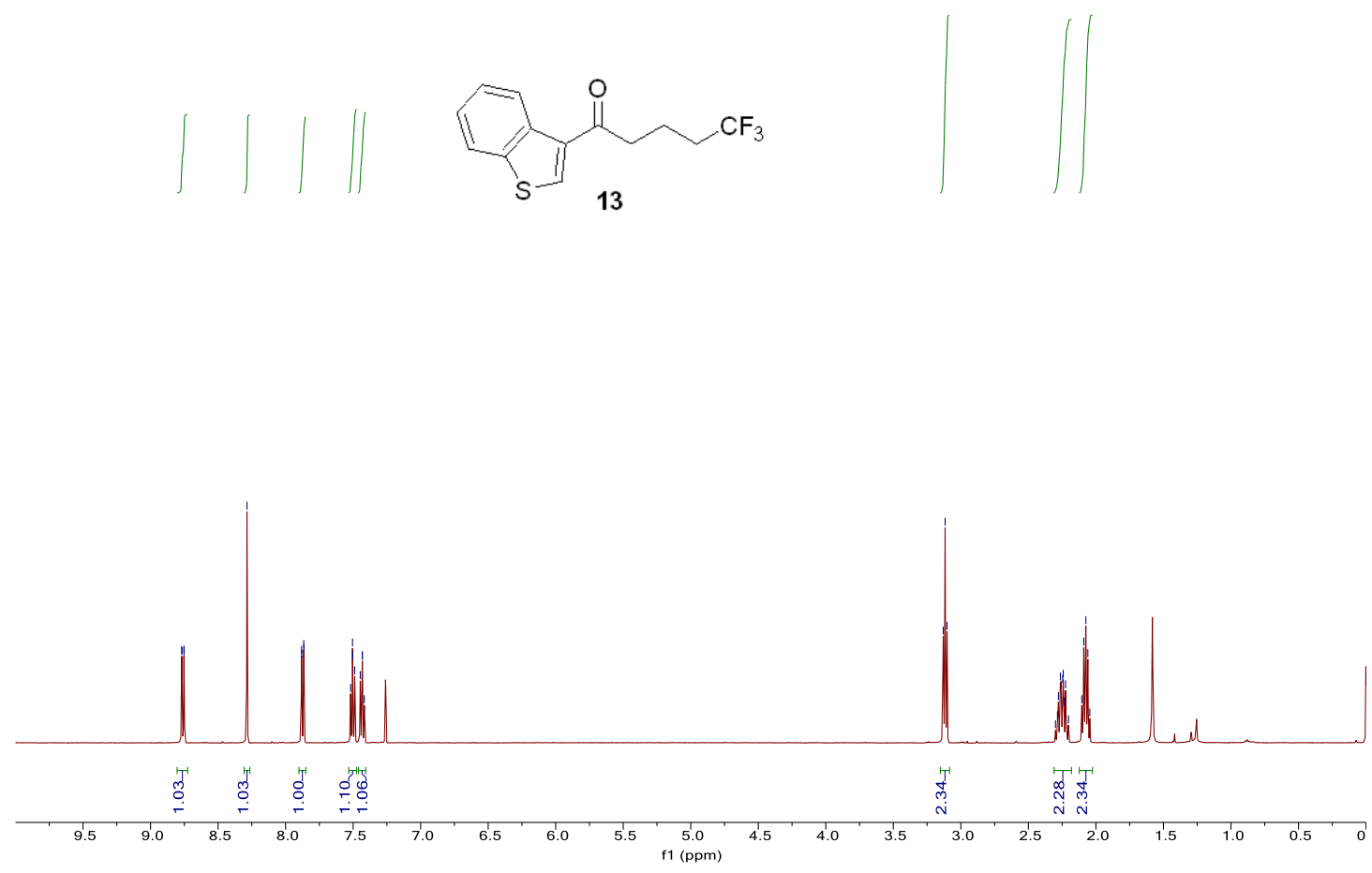

${ }^{13} \mathrm{C}$ NMR $\left(151 \mathrm{MHz}, \mathrm{CDCl}_{3}\right)$

$\stackrel{\substack{\infty \\ \infty}}{\substack{\infty \\ \Gamma}}$

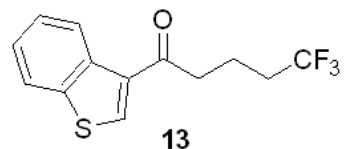

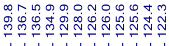

ivi परिए

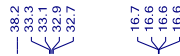

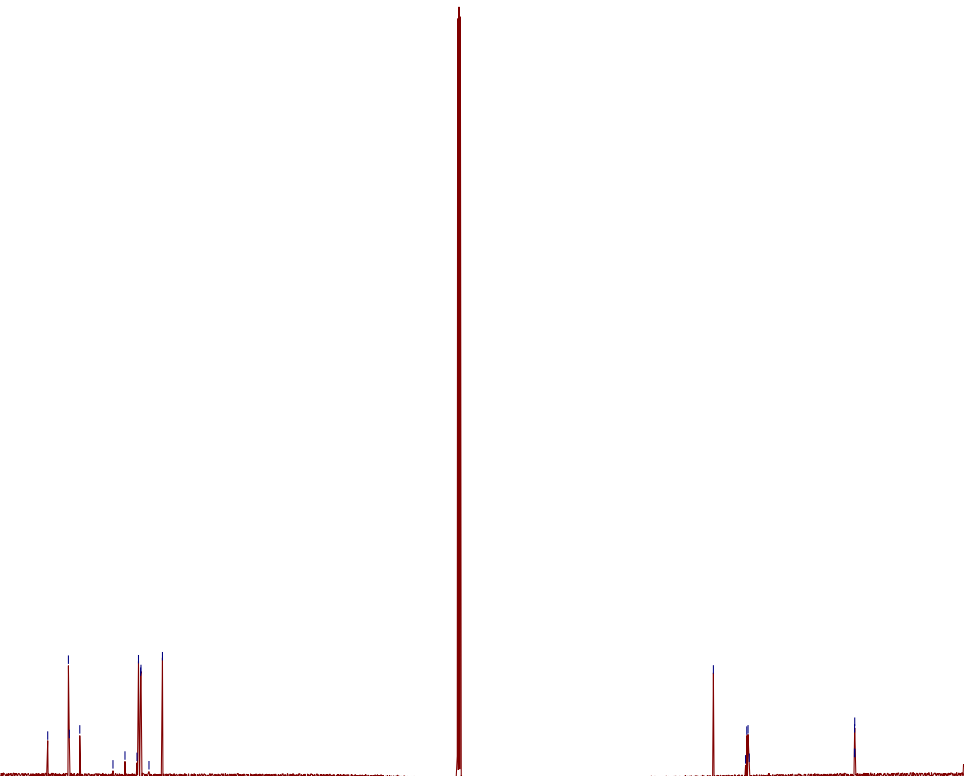

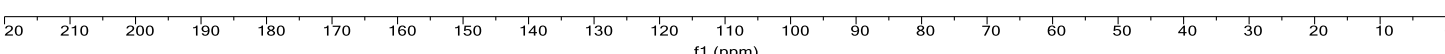


${ }^{19} \mathrm{~F}$ NMR $\left(376 \mathrm{MHz}, \mathrm{CDCl}_{3}\right)$

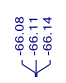

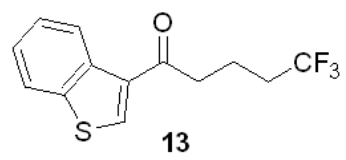

13

$-45$

$-50$

$-55$

$-60$

-65
$f 1(\mathrm{ppm})$

$-70$

$-80$

${ }^{1} \mathrm{H}$ NMR (600 MHz, $\left.\mathrm{CDCl}_{3}\right)$

|

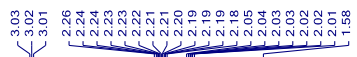
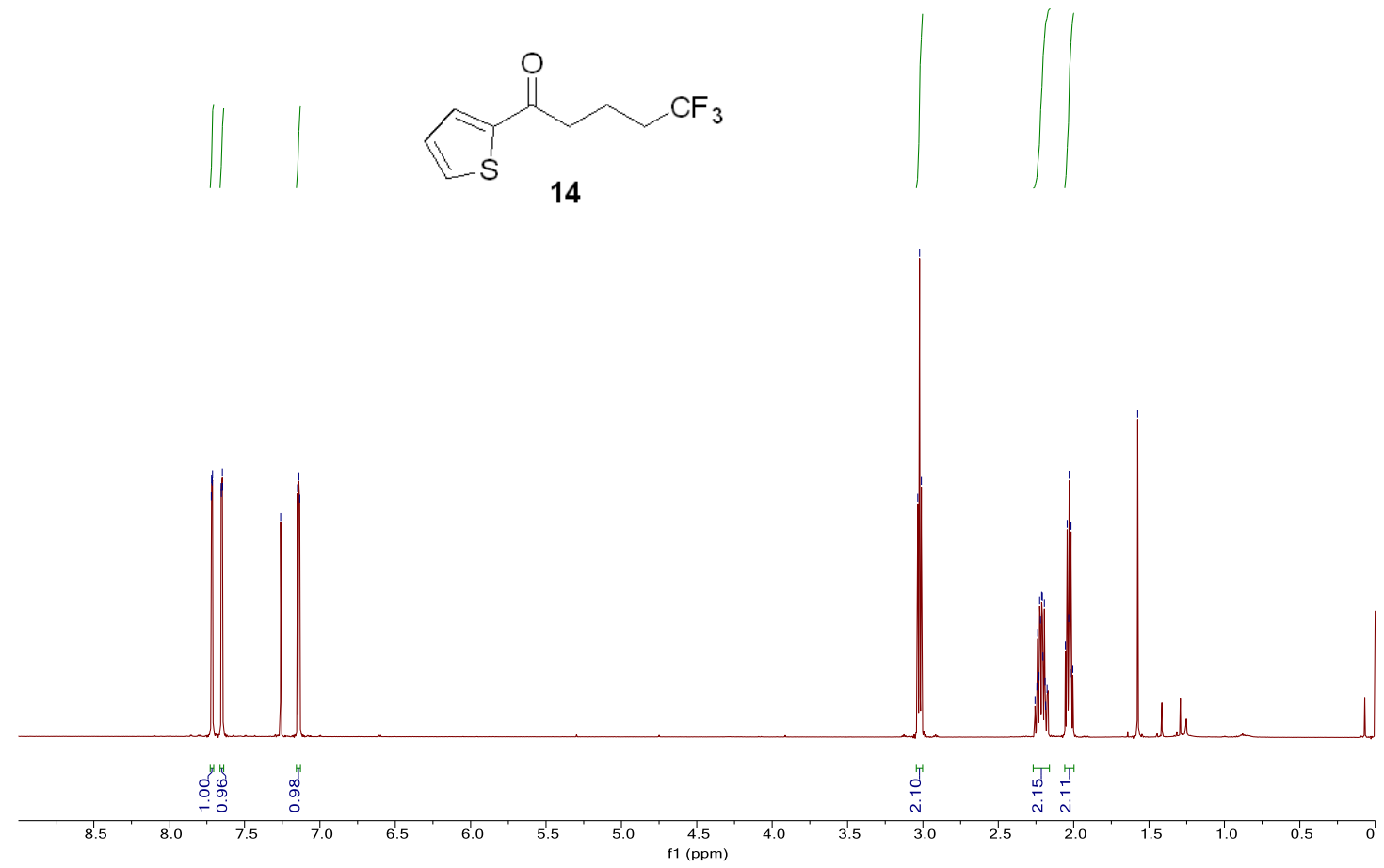
${ }^{13} \mathrm{C} \mathrm{NMR}\left(151 \mathrm{MHz}, \mathrm{CDCl}_{3}\right)$

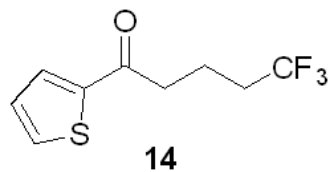

14
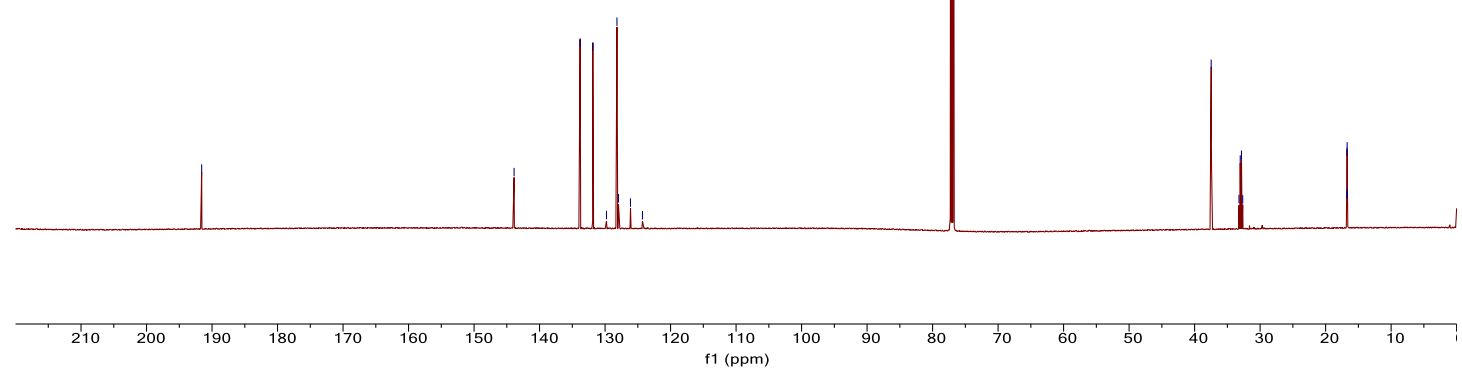

${ }^{19} \mathrm{~F}$ NMR (376 MHz, $\mathrm{CDCl}_{3}$ )

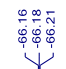<smiles>O=C(CCCC(F)(F)F)c1cccs1</smiles>

14 
${ }^{1} \mathrm{H}$ NMR (600 MHz, $\left.\mathrm{CDCl}_{3}\right)$

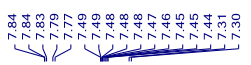

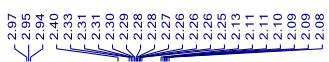

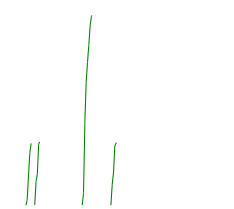<smiles>Cc1ccc2ccccc2c1C(=O)CCCC(F)(F)F</smiles>
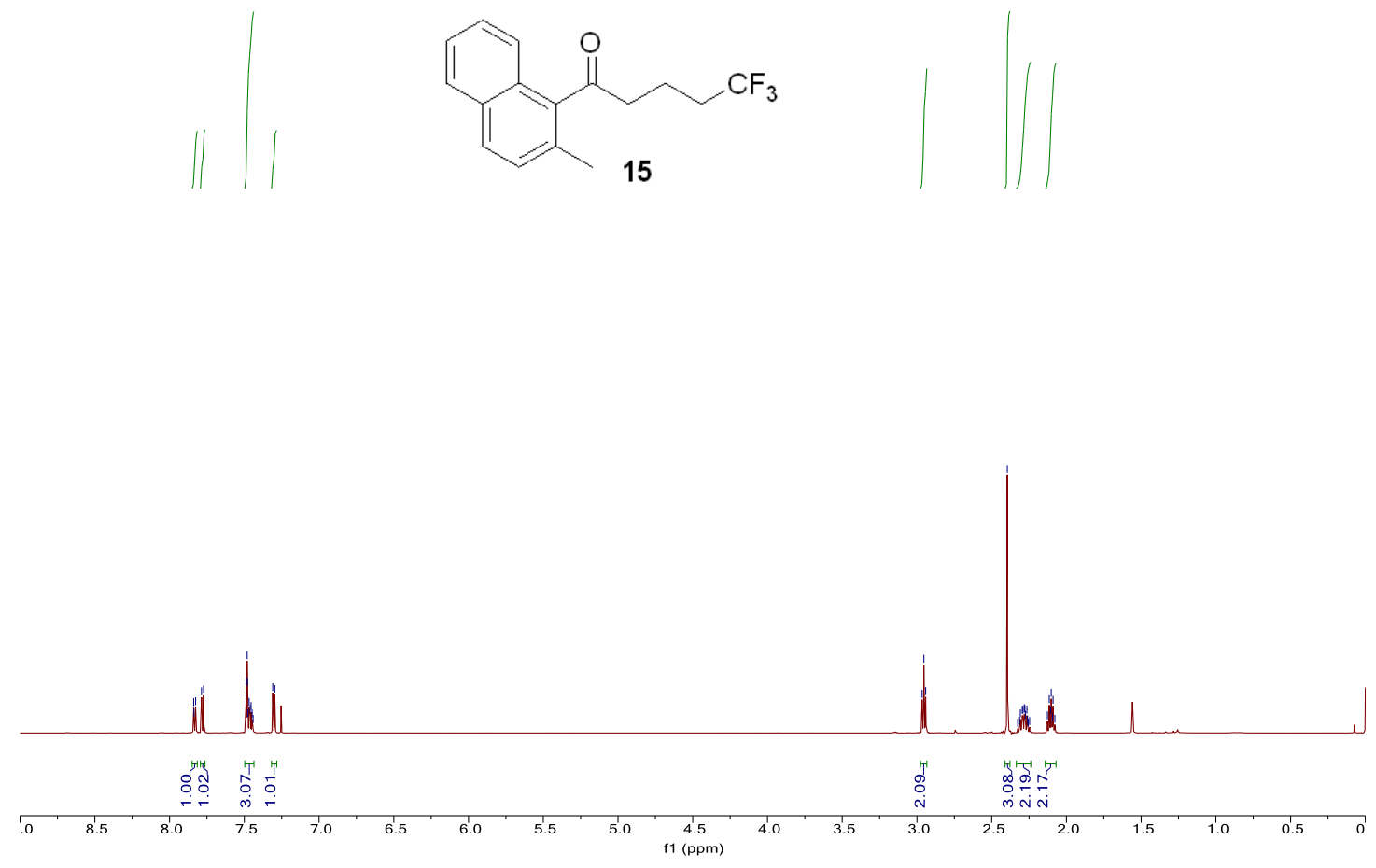

${ }^{13} \mathrm{C} \mathrm{NMR}\left(151 \mathrm{MHz}, \mathrm{CDCl}_{3}\right)$

禹

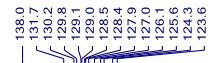<smiles>Cc1ccc2ccccc2c1C(=O)CCCC(F)(F)F</smiles>

15

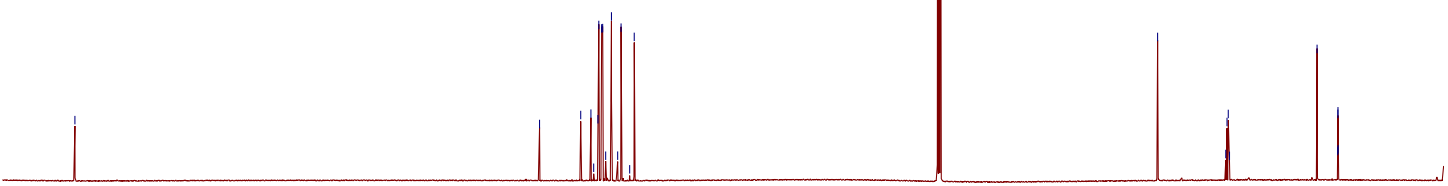

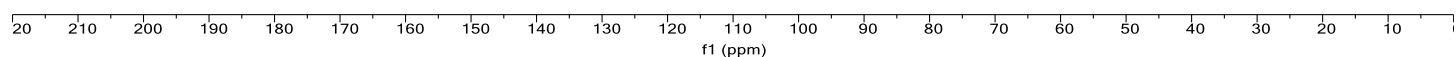


${ }^{19} \mathrm{~F}$ NMR $\left(376 \mathrm{MHz}, \mathrm{CDCl}_{3}\right)$

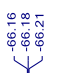<smiles>Cc1ccc2ccccc2c1C(=O)CCCC(F)(F)F</smiles>

15

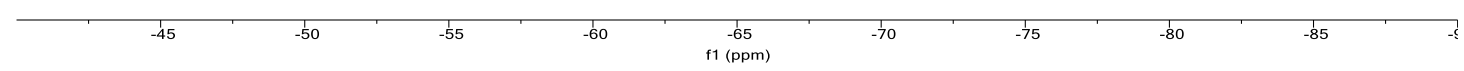

${ }^{1} \mathrm{H}$ NMR (500 MHz, $\left.\mathrm{CDCl}_{3}\right)$

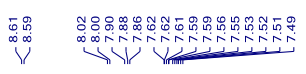

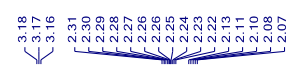
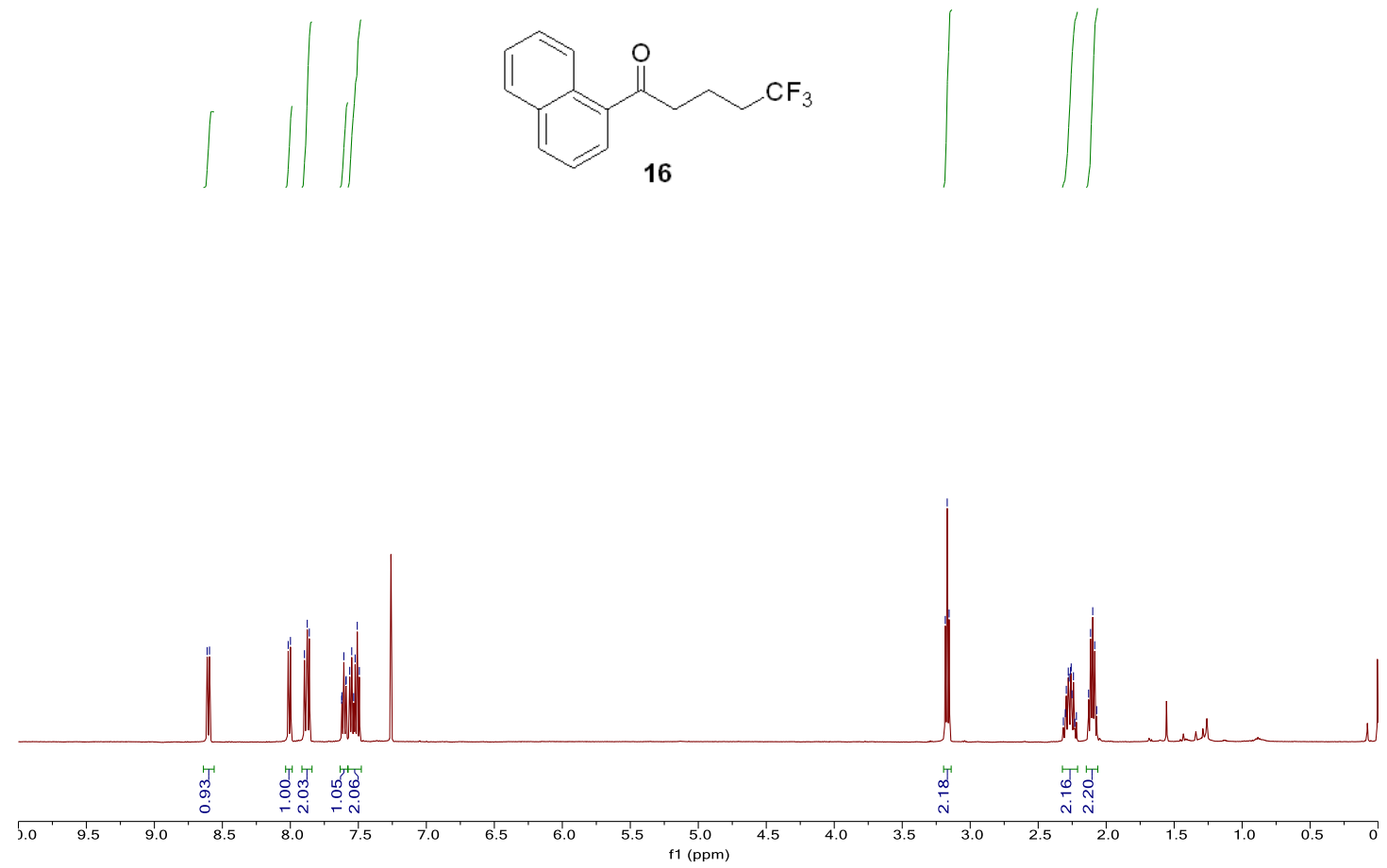
${ }^{13} \mathrm{C} \mathrm{NMR}\left(151 \mathrm{MHz}, \mathrm{CDCl}_{3}\right)$

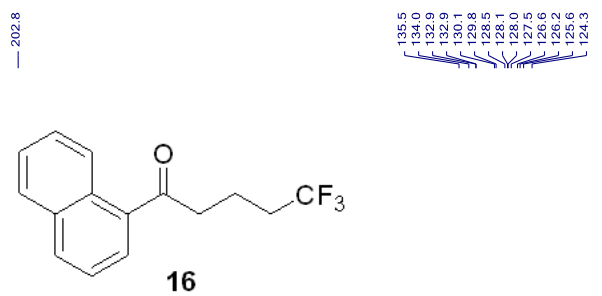

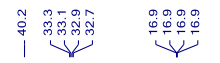

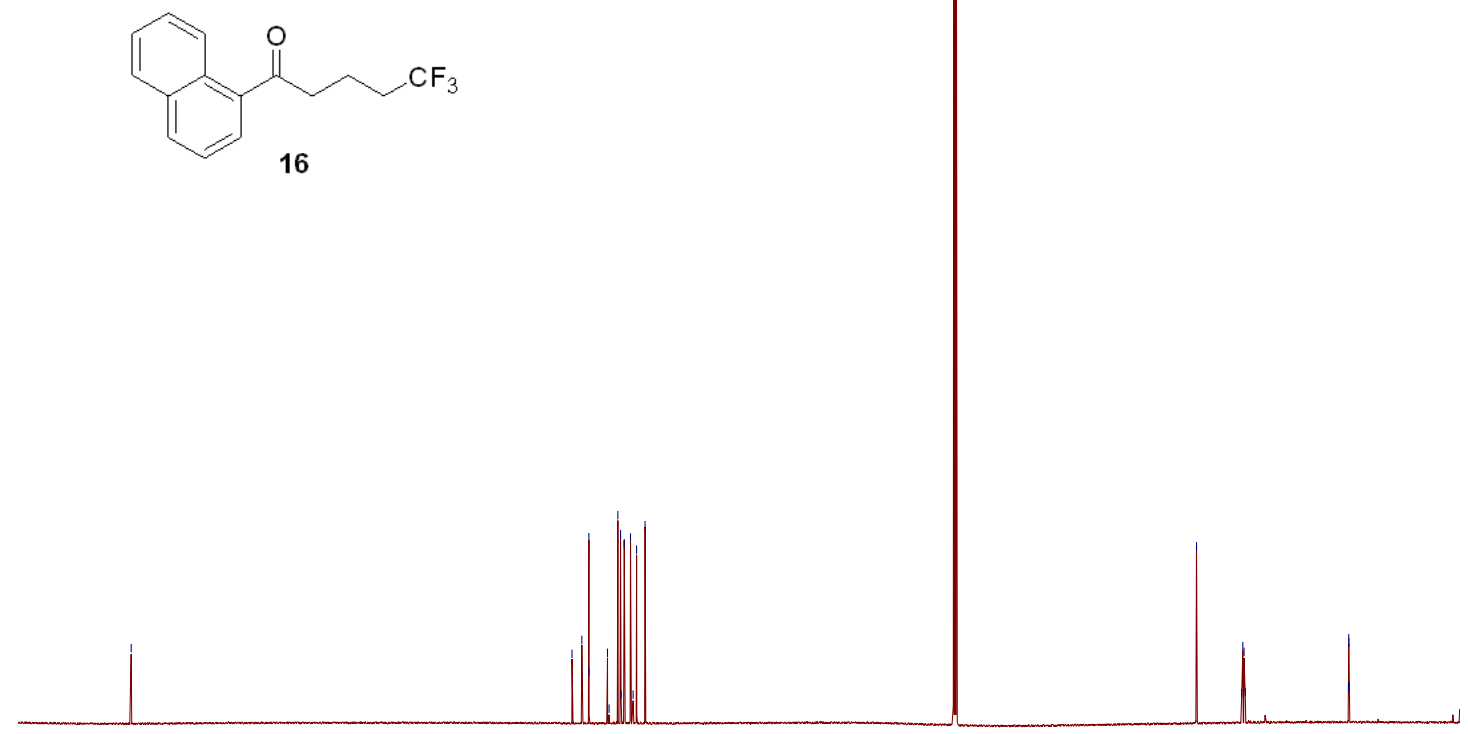

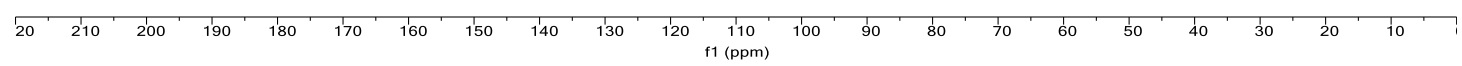

${ }^{19} \mathrm{~F}$ NMR (376 MHz, $\left.\mathrm{CDCl}_{3}\right)$

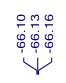<smiles>O=C(CCCC(F)(F)F)c1cccc2ccccc12</smiles>

16 
${ }^{1} \mathrm{H}$ NMR (500 MHz, $\left.\mathrm{CDCl}_{3}\right)$

無孚

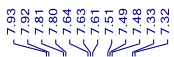

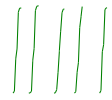

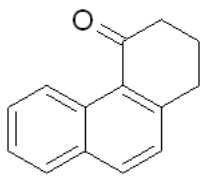

16-1
ข้ำ

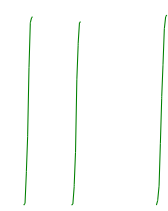

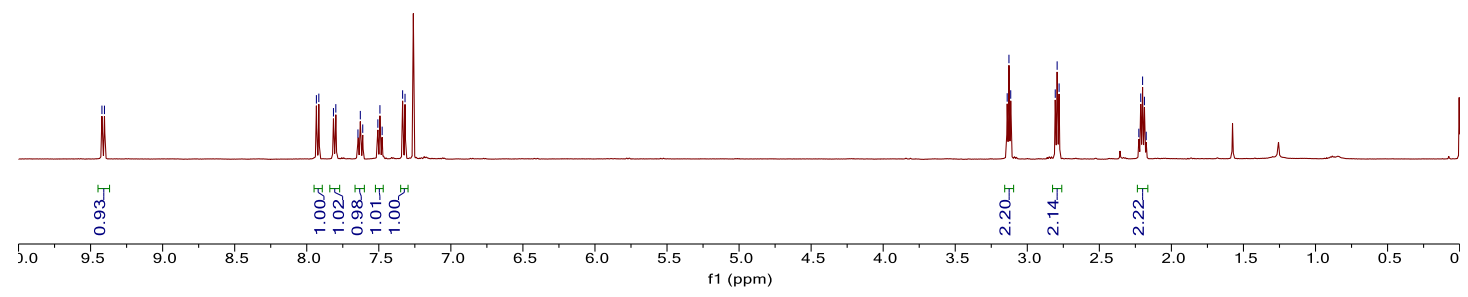

${ }^{13} \mathrm{C} \mathrm{NMR}\left(151 \mathrm{MHz}, \mathrm{CDCl}_{3}\right)$<smiles>O=C1CCCc2ccc3ccccc3c21</smiles>

16-1 
${ }^{1} \mathrm{H}$ NMR (600 MHz, $\left.\mathrm{CDCl}_{3}\right)$

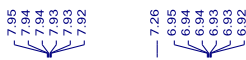

D.<smiles>COc1ccc(C(=O)CCCCC(F)(F)F)cc1</smiles>

17
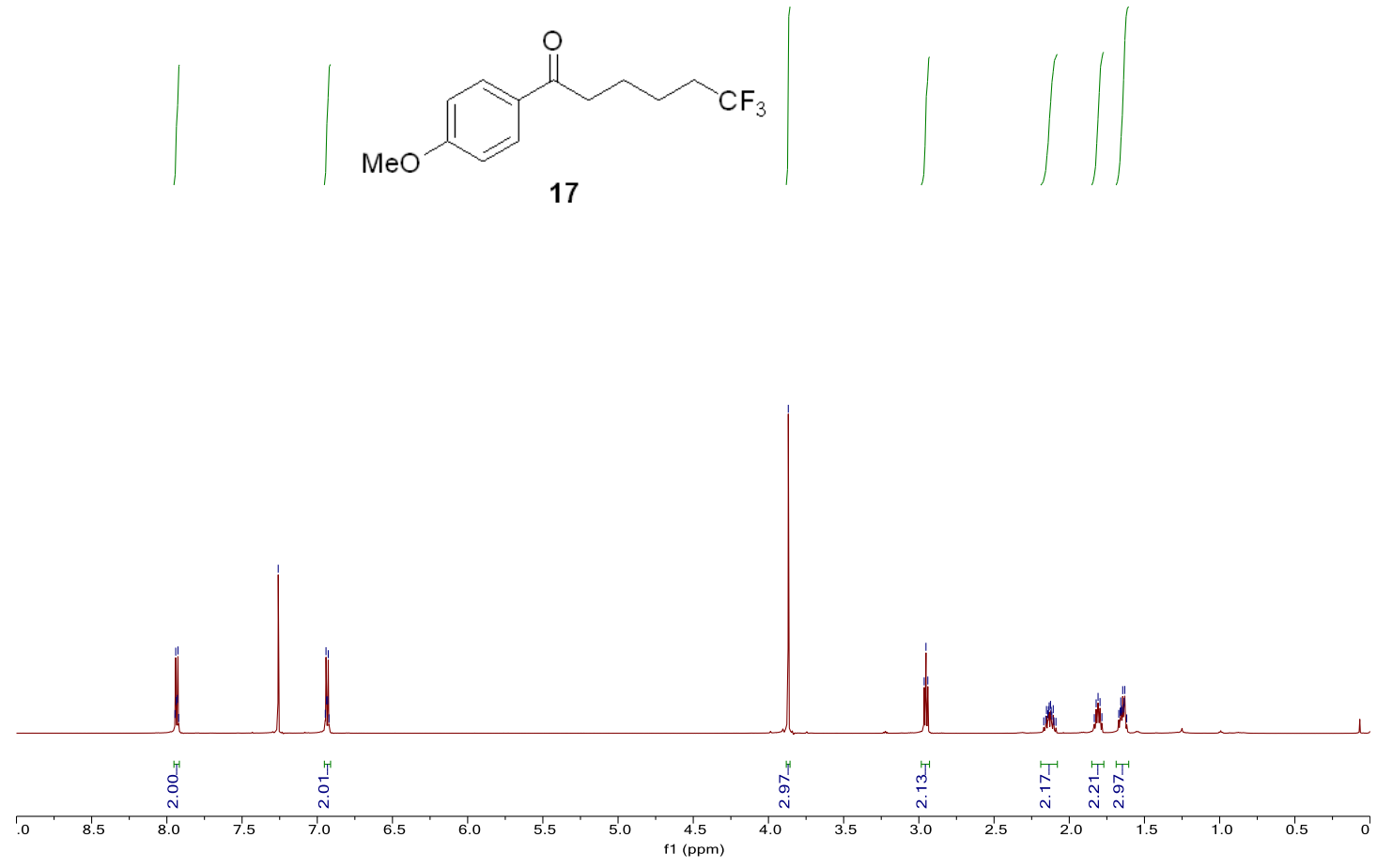

${ }^{13} \mathrm{C}$ NMR $\left(151 \mathrm{MHz}, \mathrm{CDCl}_{3}\right)$<smiles>COc1ccc(C(=O)CCCCC(F)(F)F)cc1</smiles>

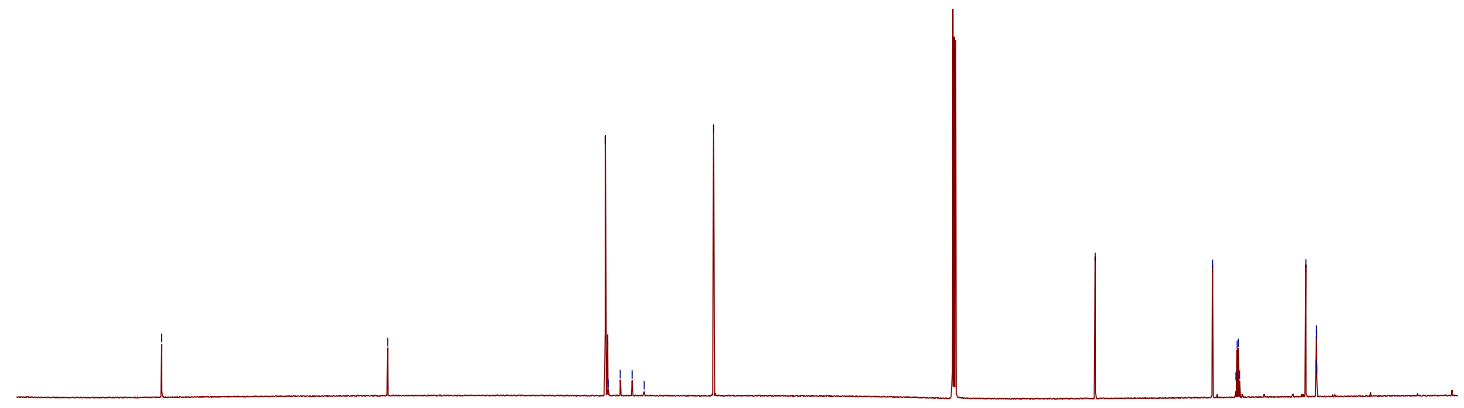

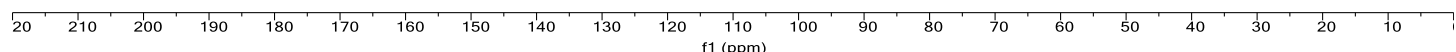


${ }^{19} \mathrm{~F}$ NMR $\left(376 \mathrm{MHz}, \mathrm{CDCl}_{3}\right)$

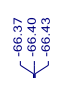<smiles>COc1ccc(C(=O)CCCCC(F)(F)F)cc1</smiles>

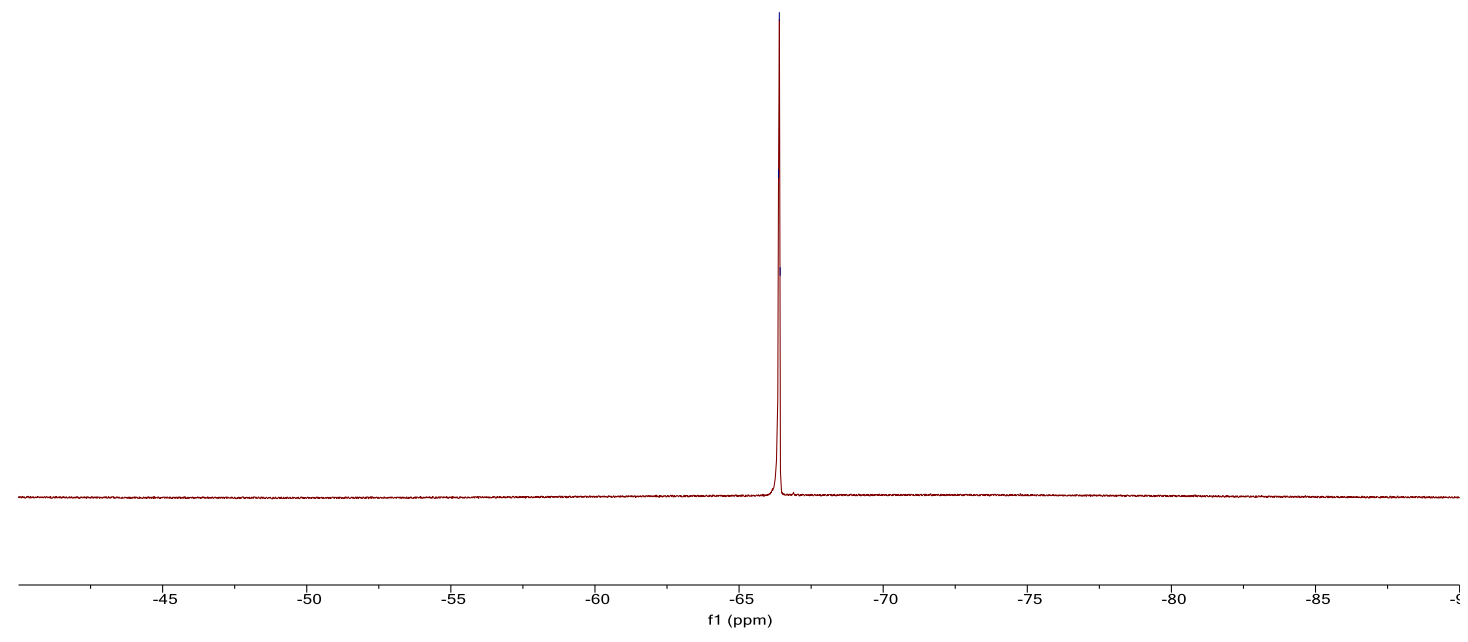

${ }^{1} \mathrm{H}$ NMR $\left(500 \mathrm{MHz}, \mathrm{CDCl}_{3}\right)$

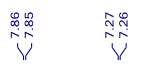

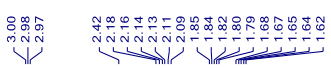

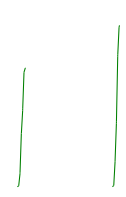<smiles>Cc1ccc(C(=O)CCCCC(F)(F)F)cc1</smiles>

18
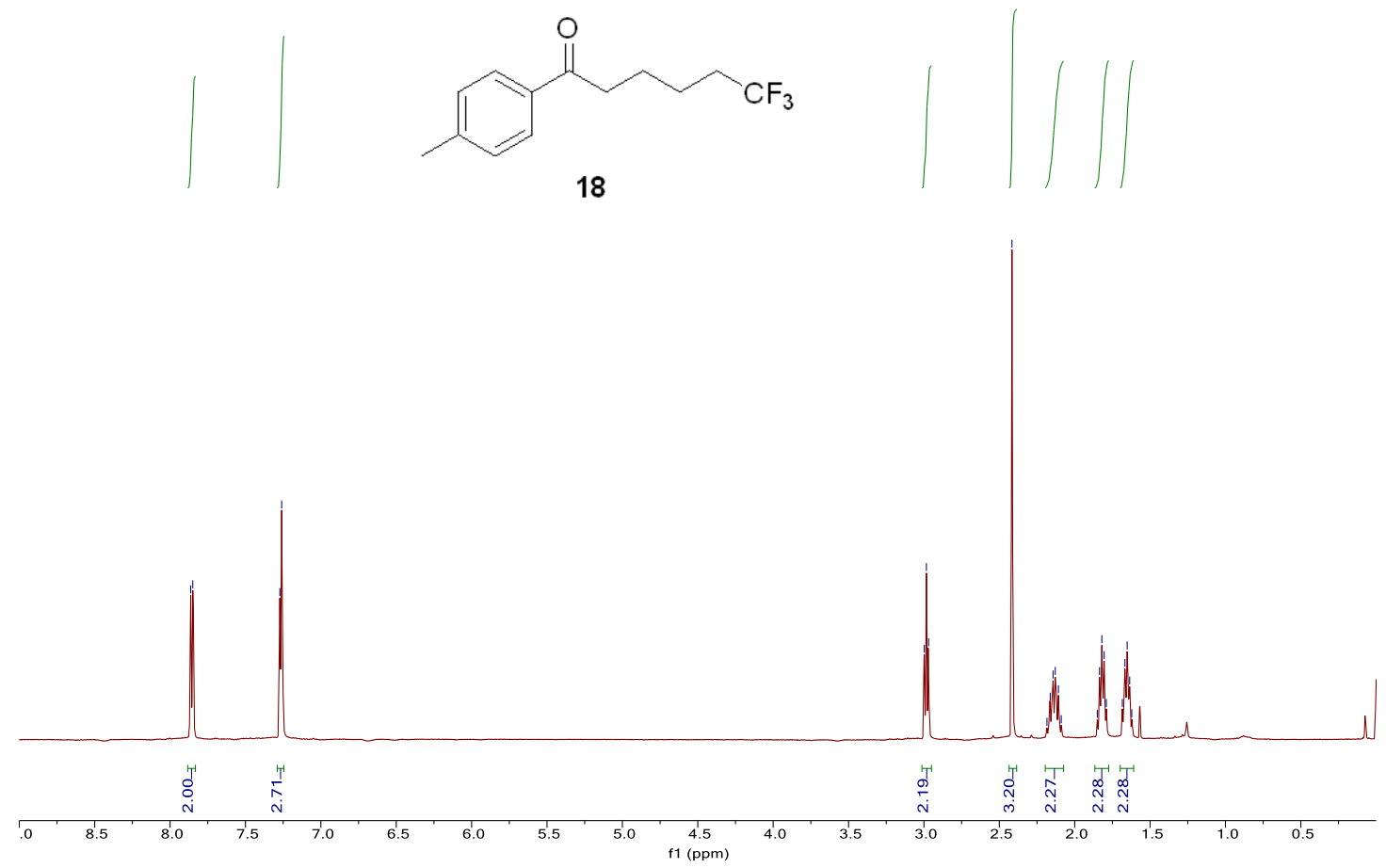
${ }^{13} \mathrm{C} \mathrm{NMR}\left(151 \mathrm{MHz}, \mathrm{CDCl}_{3}\right)$<smiles>Cc1ccc(C(=O)CCCCC(F)(F)F)cc1</smiles>

18

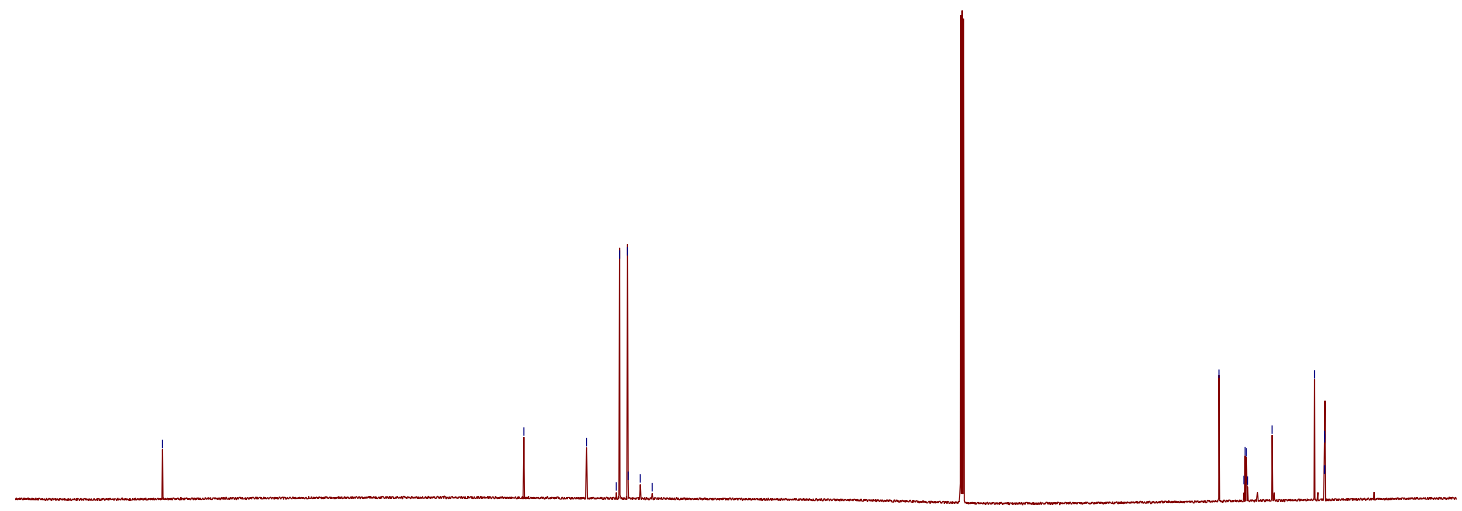

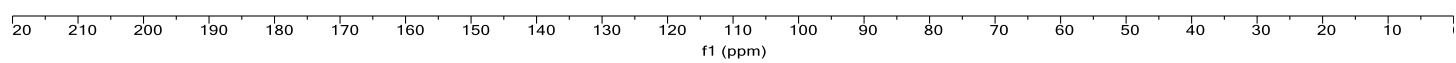
${ }^{19} \mathrm{~F}$ NMR (376 MHz, $\left.\mathrm{CDCl}_{3}\right)$<smiles>Cc1ccc(C(=O)CCCCC(F)(F)F)cc1</smiles>

18 
${ }^{1} \mathrm{H}$ NMR (600 MHz, $\left.\mathrm{CDCl}_{3}\right)$

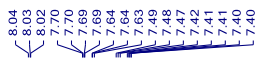

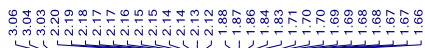
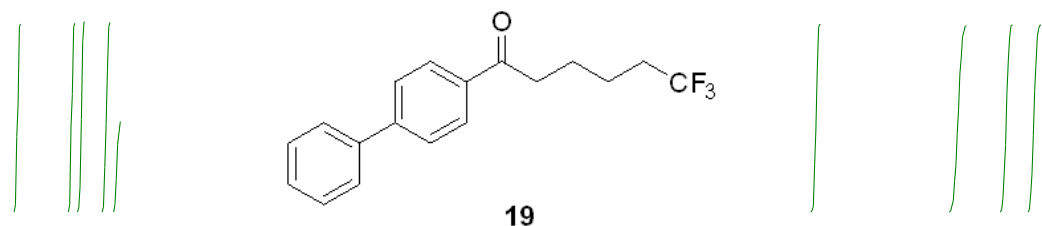

19

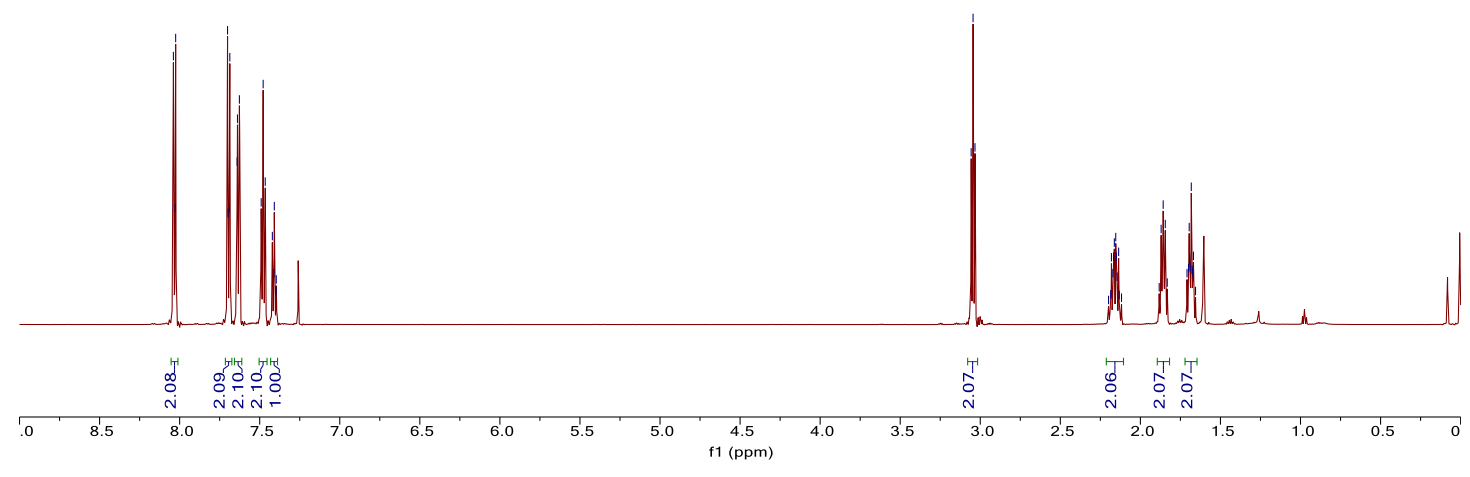

${ }^{13} \mathrm{C} \mathrm{NMR}\left(151 \mathrm{MHz}, \mathrm{CDCl}_{3}\right)$

$\stackrel{\circ}{\stackrel{\circ}{9}}$

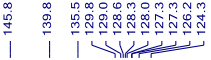

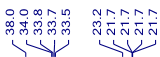<smiles>O=C(CCCCC(F)(F)F)c1ccc(-c2ccccc2)cc1</smiles>

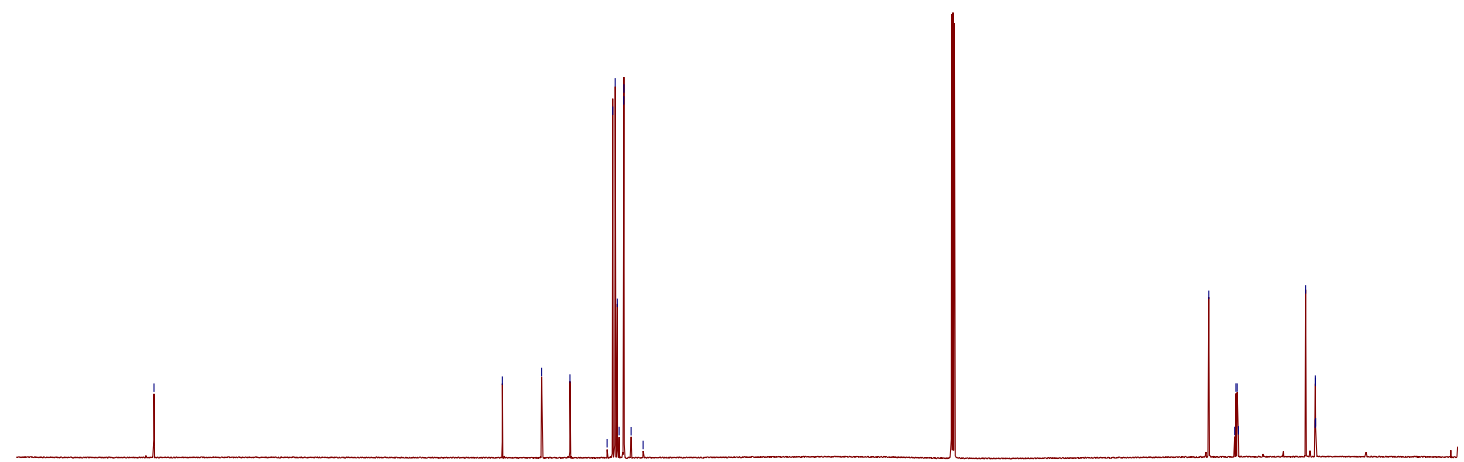

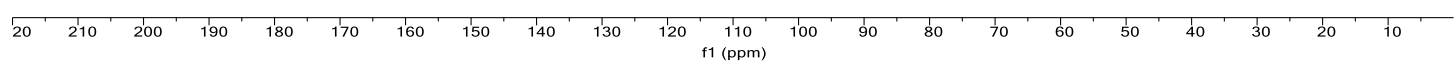


${ }^{19} \mathrm{~F} \mathrm{NMR} \mathrm{(376} \mathrm{MHz,} \mathrm{CDCl}_{3}$ )

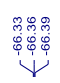<smiles>O=C(CCCCC(F)(F)F)c1ccc(-c2ccccc2)cc1</smiles>

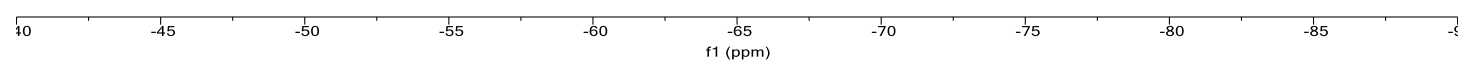

${ }^{1} \mathrm{H}$ NMR $\left(500 \mathrm{MHz}, \mathrm{CDCl}_{3}\right)$

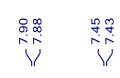

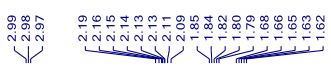

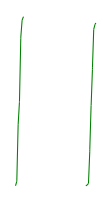<smiles>O=C(CCCCC(F)(F)F)c1ccc(Cl)cc1</smiles>

20
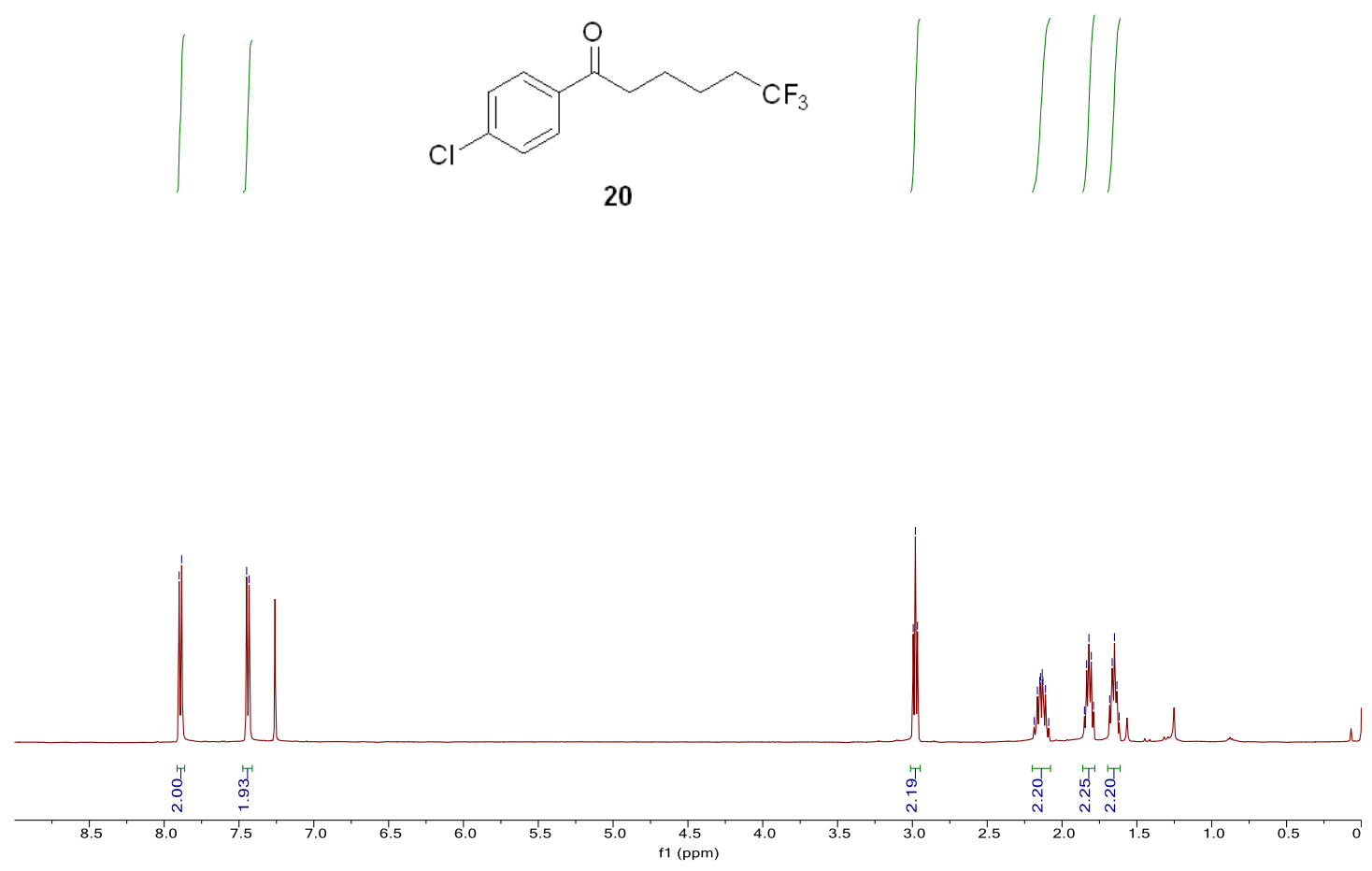
${ }^{13} \mathrm{C} \mathrm{NMR}\left(151 \mathrm{MHz}, \mathrm{CDCl}_{3}\right)$<smiles>O=C(CCCCC(F)(F)F)c1ccc(Cl)cc1</smiles>

20
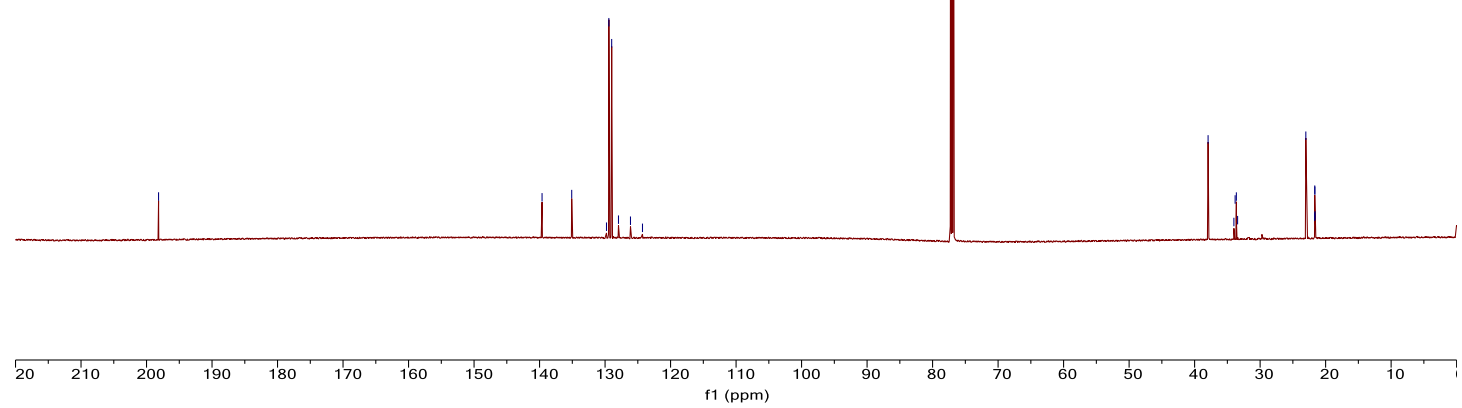

${ }^{19} \mathrm{~F}$ NMR (376 MHz, $\mathrm{CDCl}_{3}$ )<smiles>O=C(CCCCC(F)(F)F)c1ccc(Cl)cc1</smiles>

20 
${ }^{1} \mathrm{H}$ NMR (600 MHz, $\left.\mathrm{CDCl}_{3}\right)$

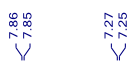

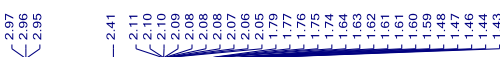

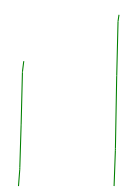<smiles>Cc1ccc(C(=O)CCCCCC(F)(F)F)cc1</smiles>

21
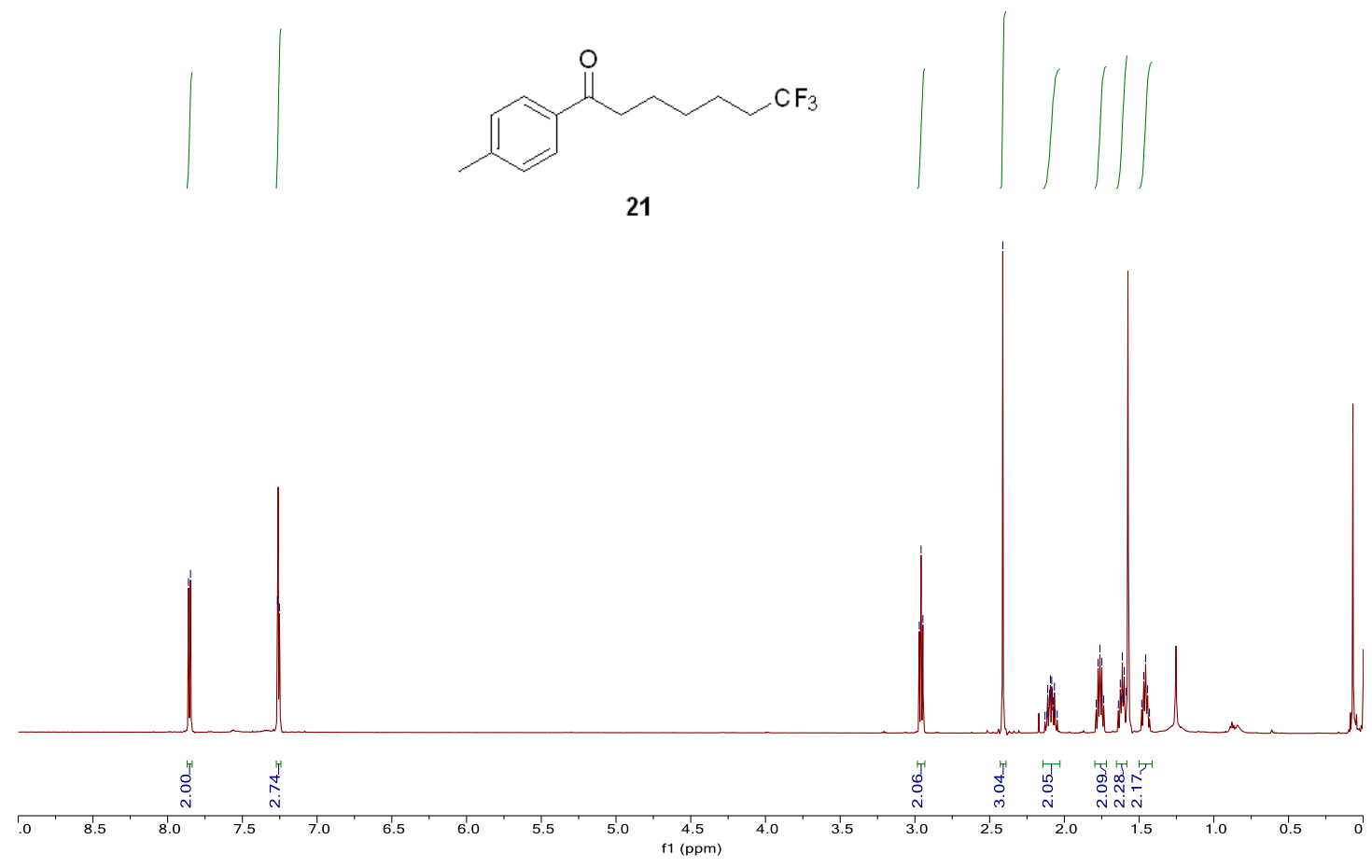

${ }^{13} \mathrm{C}$ NMR (151 MHz, $\mathrm{CDCl}_{3}$ )

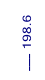

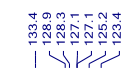

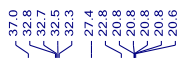

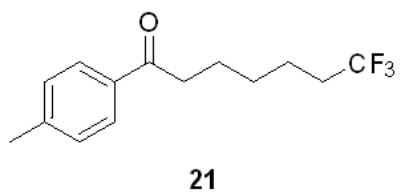

21

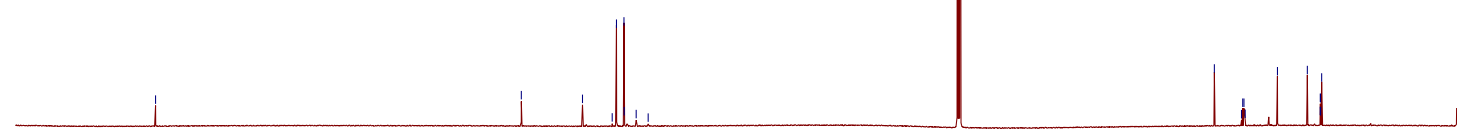

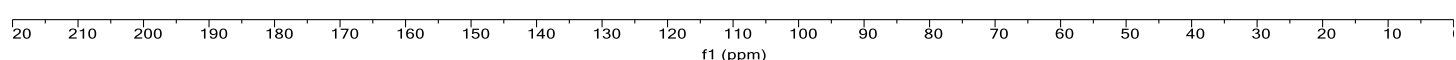


${ }^{19} \mathrm{~F}$ NMR $\left(376 \mathrm{MHz}, \mathrm{CDCl}_{3}\right)$
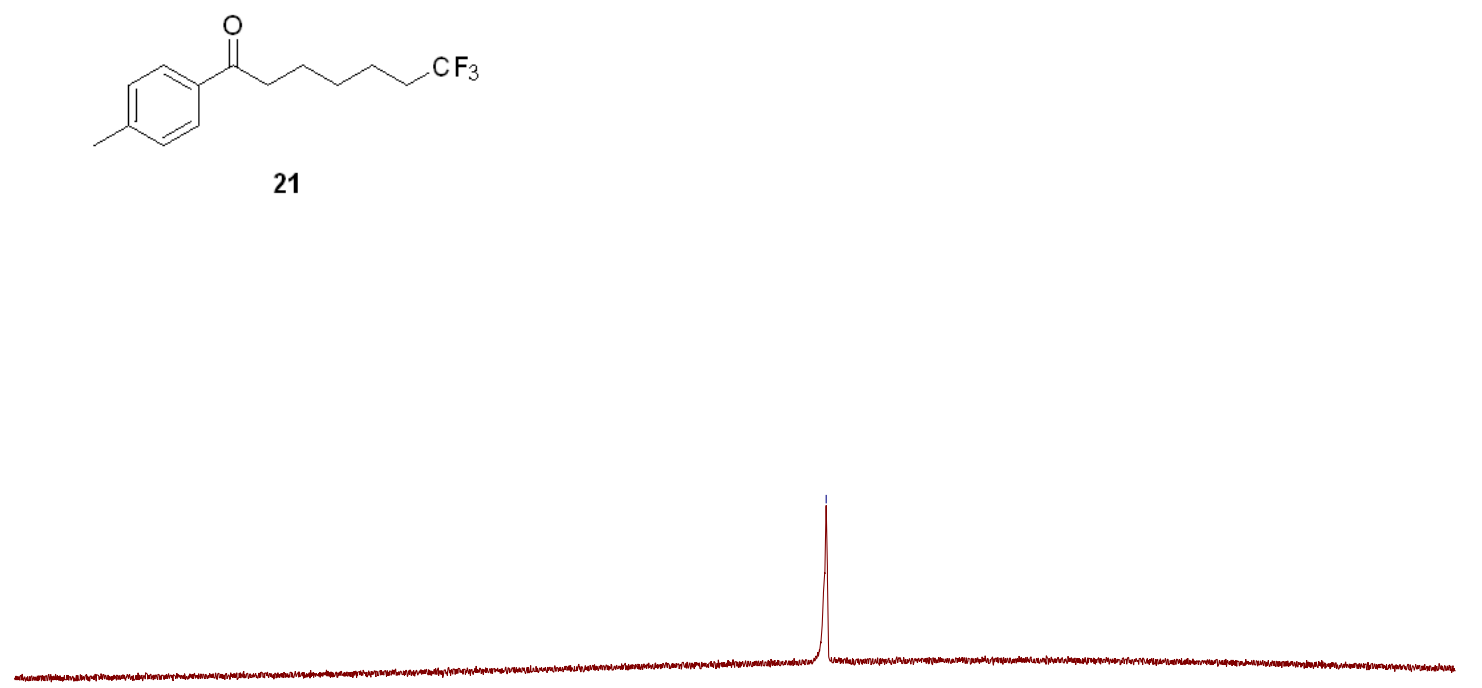

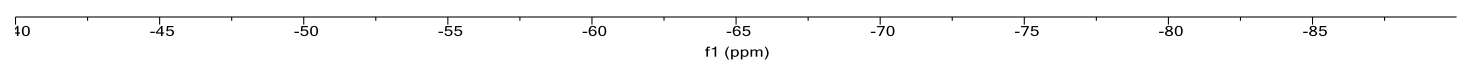

${ }^{1} \mathrm{H} \mathrm{NMR}\left(600 \mathrm{MHz}, \mathrm{CDCl}_{3}\right)$

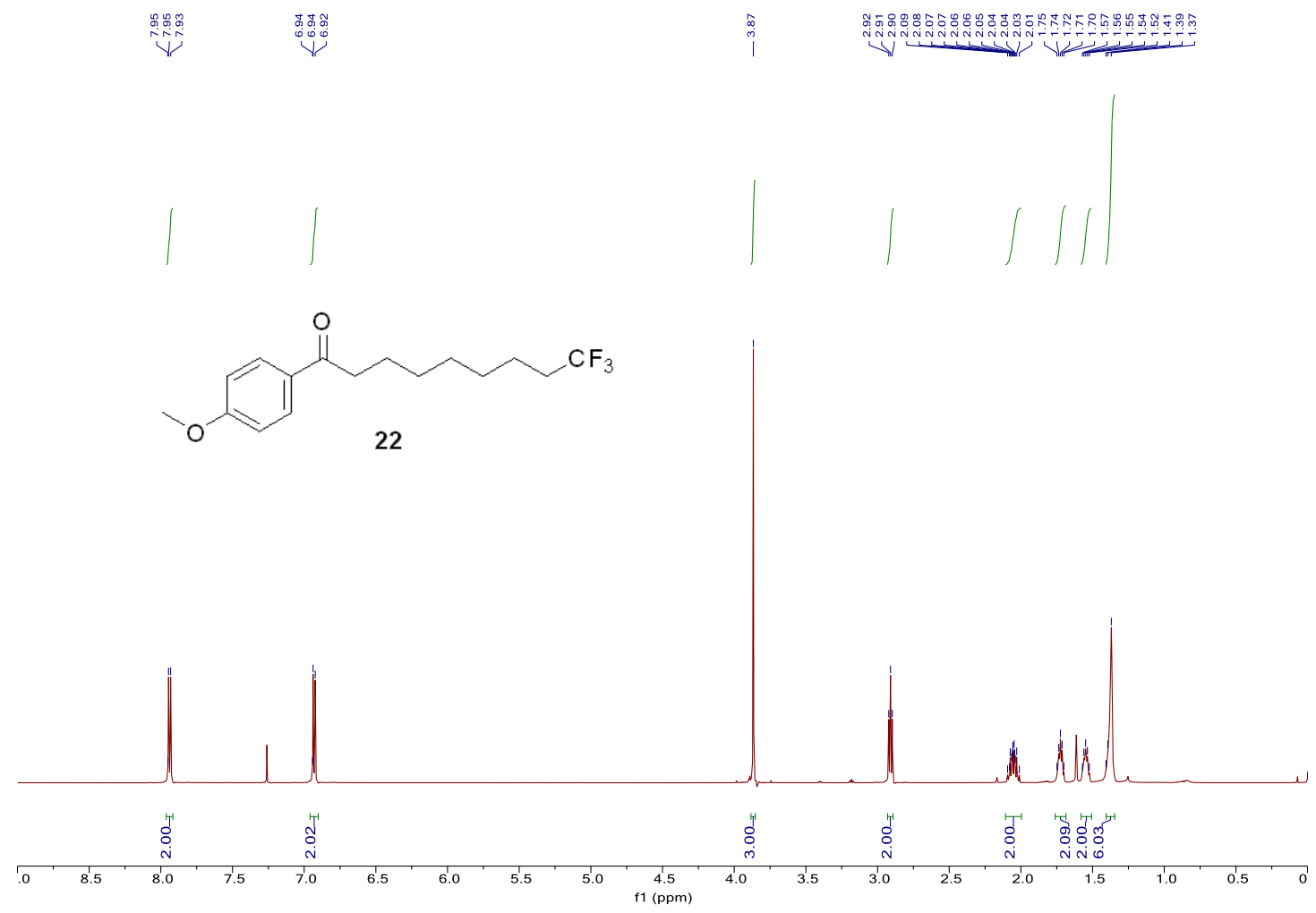


${ }^{13} \mathrm{C} \mathrm{NMR}\left(151 \mathrm{MHz}, \mathrm{CDCl}_{3}\right)$

i<smiles>COc1ccc(C(=O)CCCCCCCC(F)(F)F)cc1</smiles>
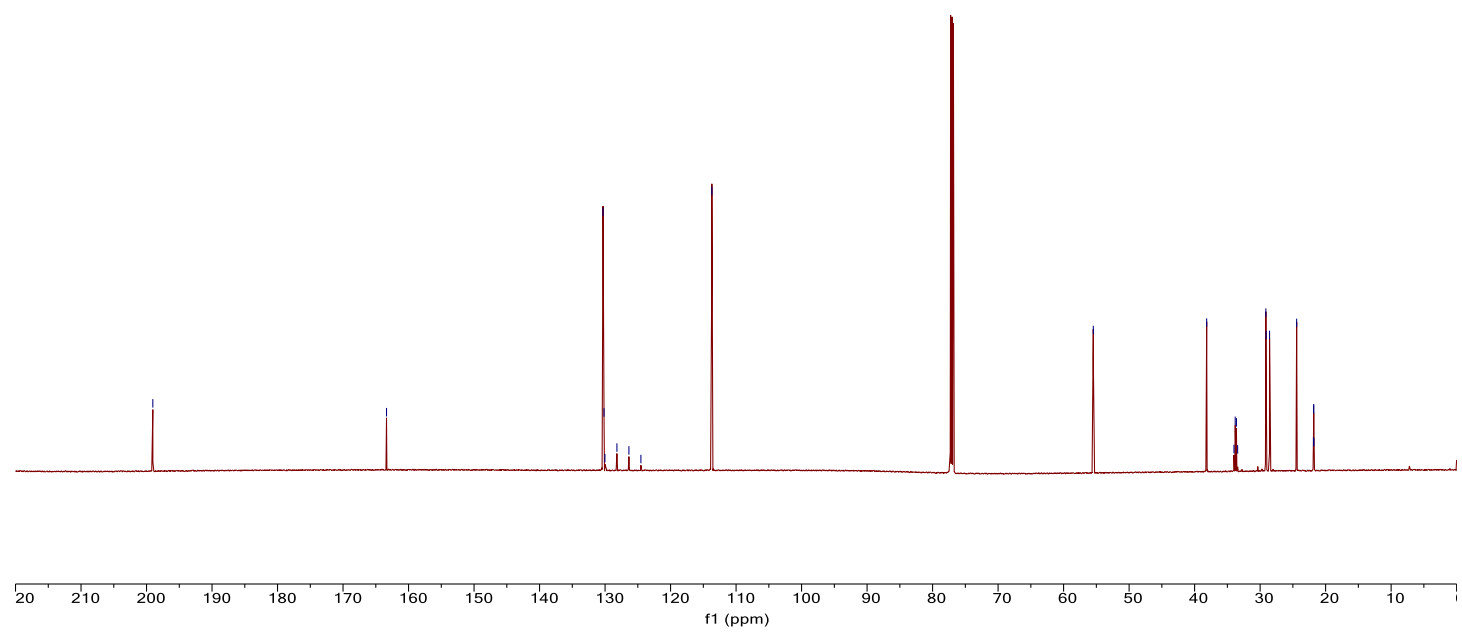

${ }^{19} \mathrm{~F}$ NMR (376 MHz, $\left.\mathrm{CDCl}_{3}\right)$

บัฐ<smiles>COc1ccc(C(=O)CCCCCCCC(F)(F)F)cc1</smiles>

22 
${ }^{1} \mathrm{H}$ NMR (600 MHz, $\left.\mathrm{CDCl}_{3}\right)$

ทั

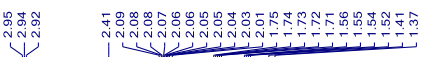

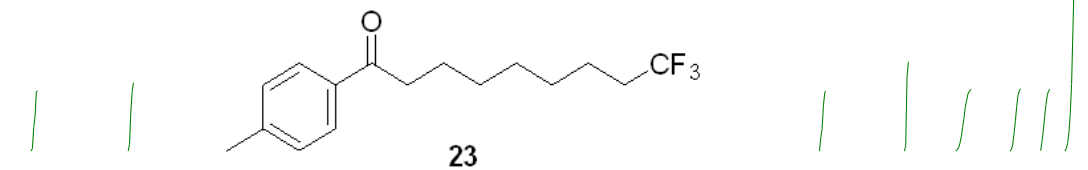

23

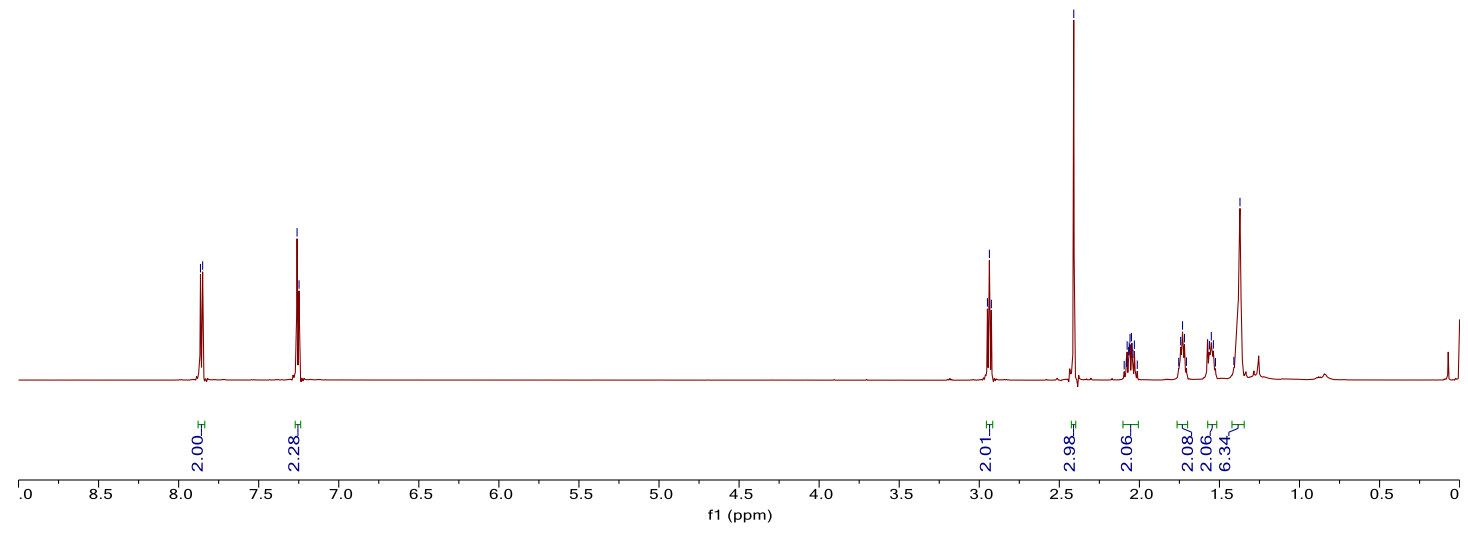

${ }^{13} \mathrm{C}$ NMR $\left(151 \mathrm{MHz}, \mathrm{CDCl}_{3}\right)$

ìำ

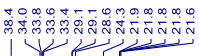<smiles>Cc1ccc(C(=O)CCCCCCCC(F)(F)F)cc1</smiles>

23

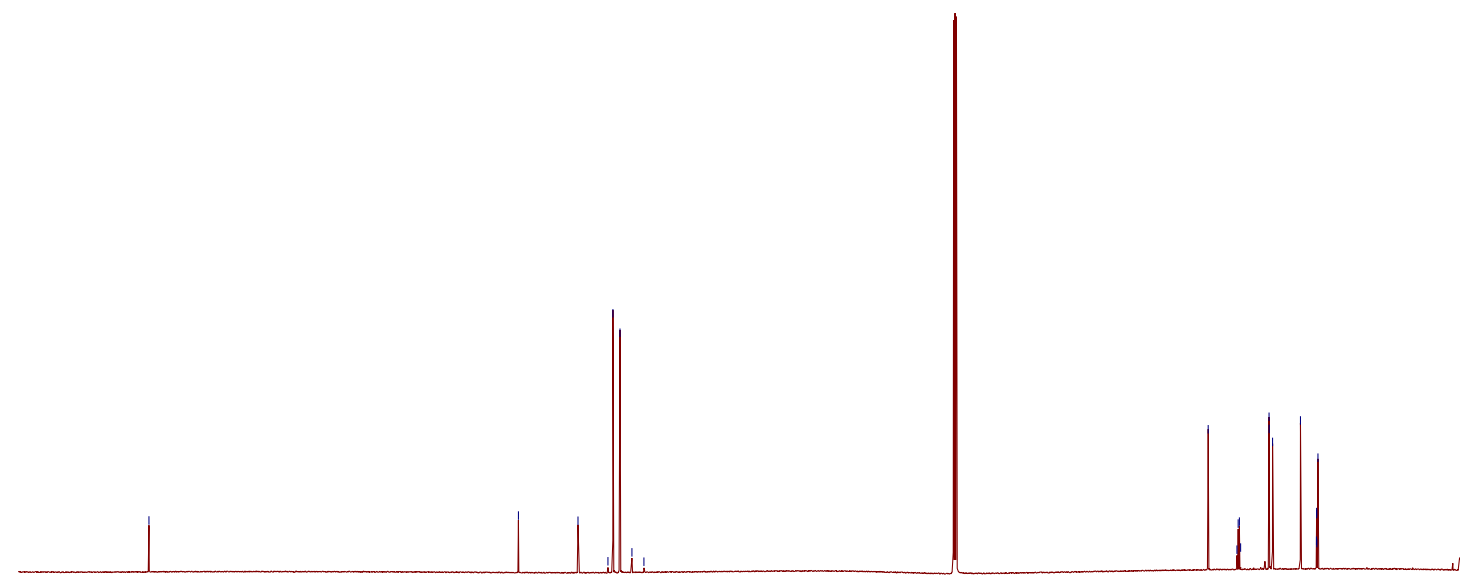

\begin{tabular}{llllllllllllllllllllll}
\hline 20 & 210 & 200 & 190 & 180 & 170 & 160 & 150 & 140 & 130 & 120 & 110 & 100 & 10 & 80 & $\frac{1}{70}$ & 60 & $\frac{1}{50}$ & 40 & $\frac{1}{30}$ & $\frac{1}{20}$ & 10 \\
\hline
\end{tabular} 
${ }^{19} \mathrm{~F} \mathrm{NMR} \mathrm{(376} \mathrm{MHz,} \mathrm{CDCl}_{3}$ )<smiles>Cc1ccc(C(=O)CCCCCCCC(F)(F)F)cc1</smiles>

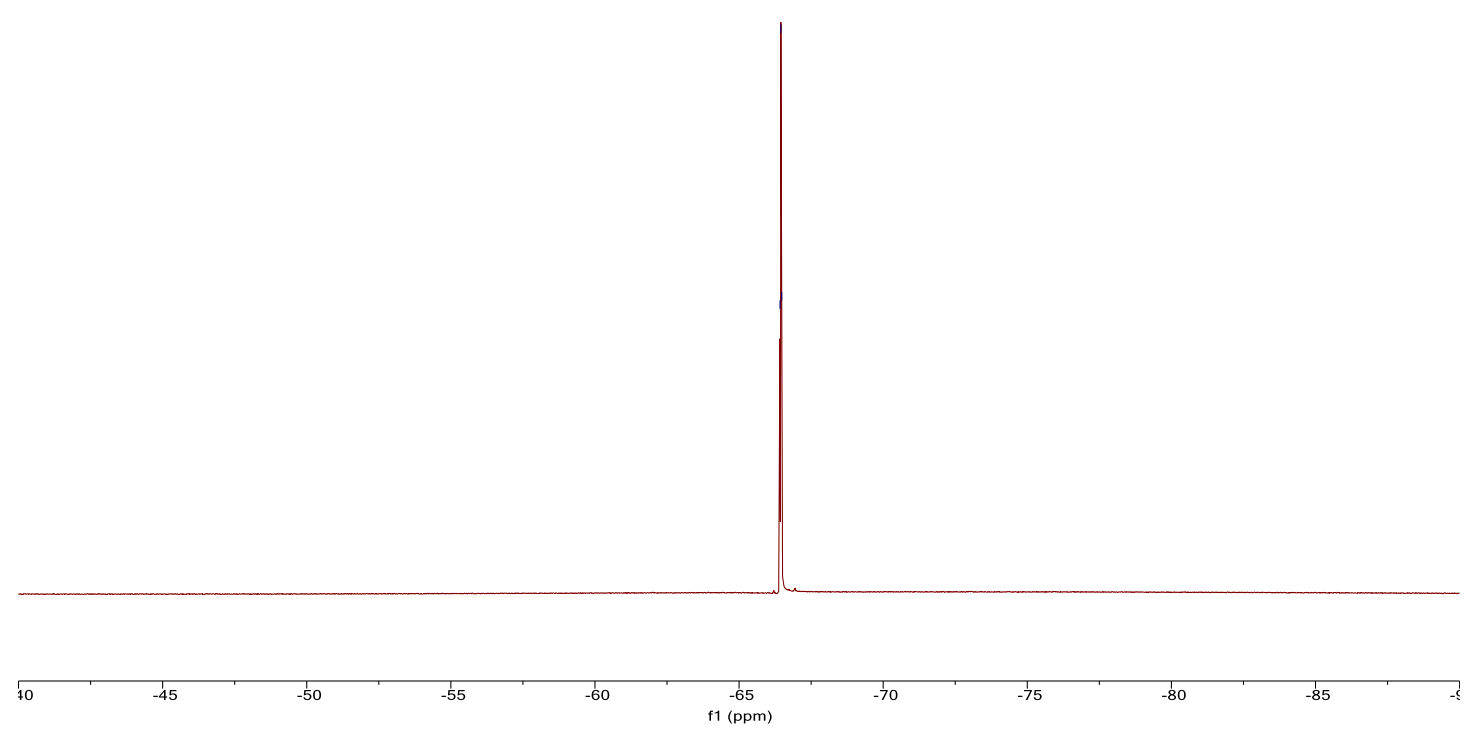

${ }^{1} \mathrm{H}$ NMR (600 MHz, $\left.\mathrm{CDCl}_{3}\right)$

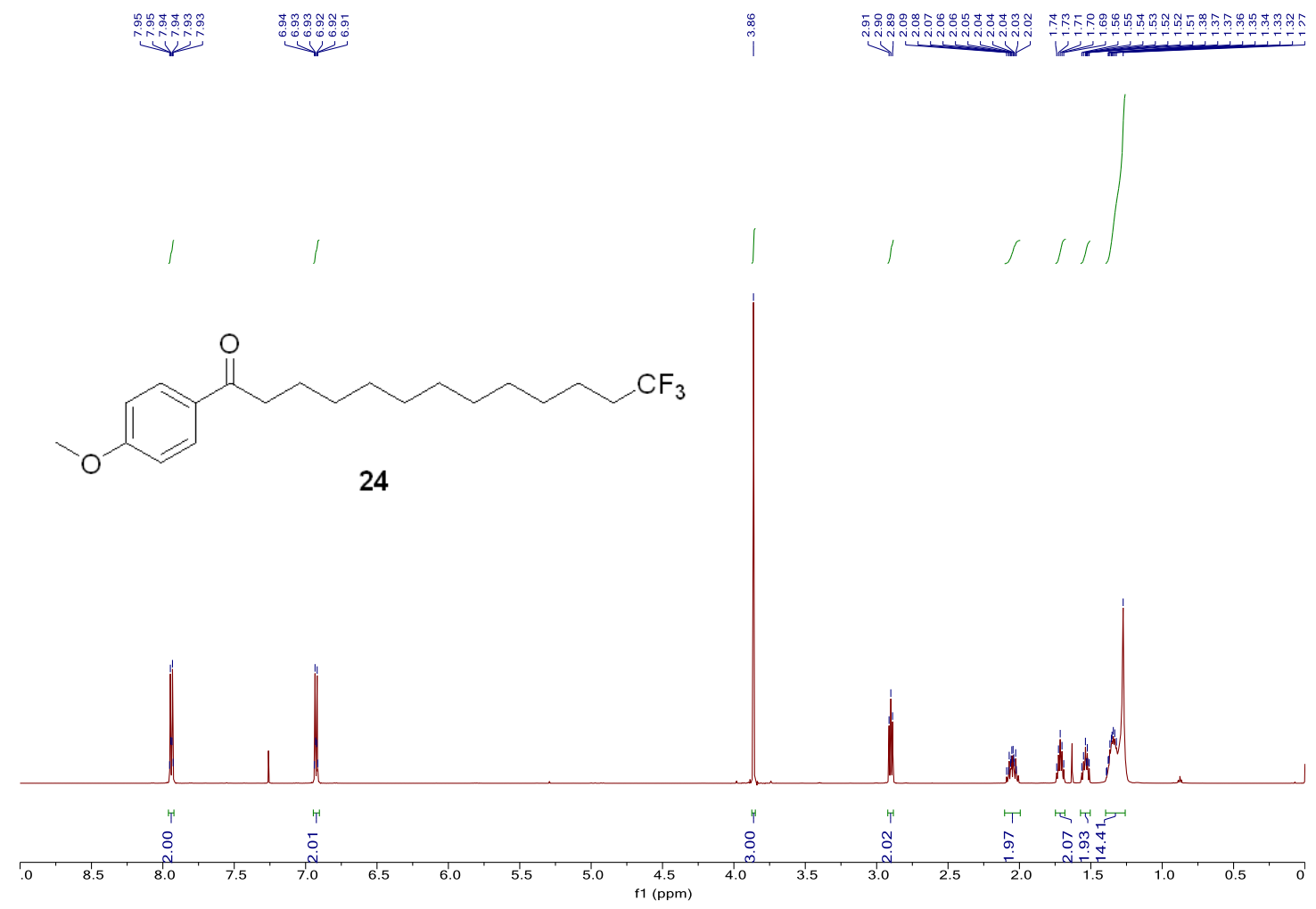


${ }^{13} \mathrm{C} \mathrm{NMR}\left(151 \mathrm{MHz}, \mathrm{CDCl}_{3}\right)$

(n)<smiles>C1CCC1</smiles><smiles>COc1ccc(C(=O)CCCCCCCCCCCC(F)(F)F)cc1</smiles>

24
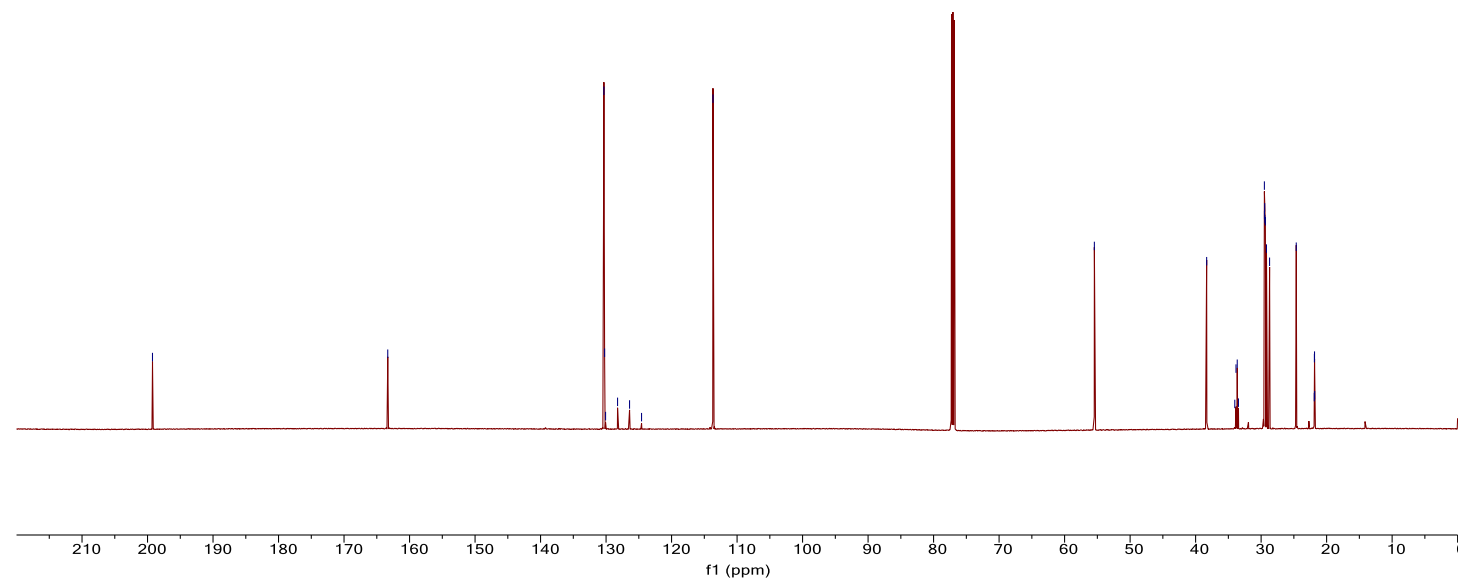

${ }^{19} \mathrm{~F}$ NMR (376 MHz, $\mathrm{CDCl}_{3}$ )

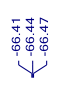<smiles>COc1ccc(C(=O)CCCCCCCCCCCC(F)(F)F)cc1</smiles>

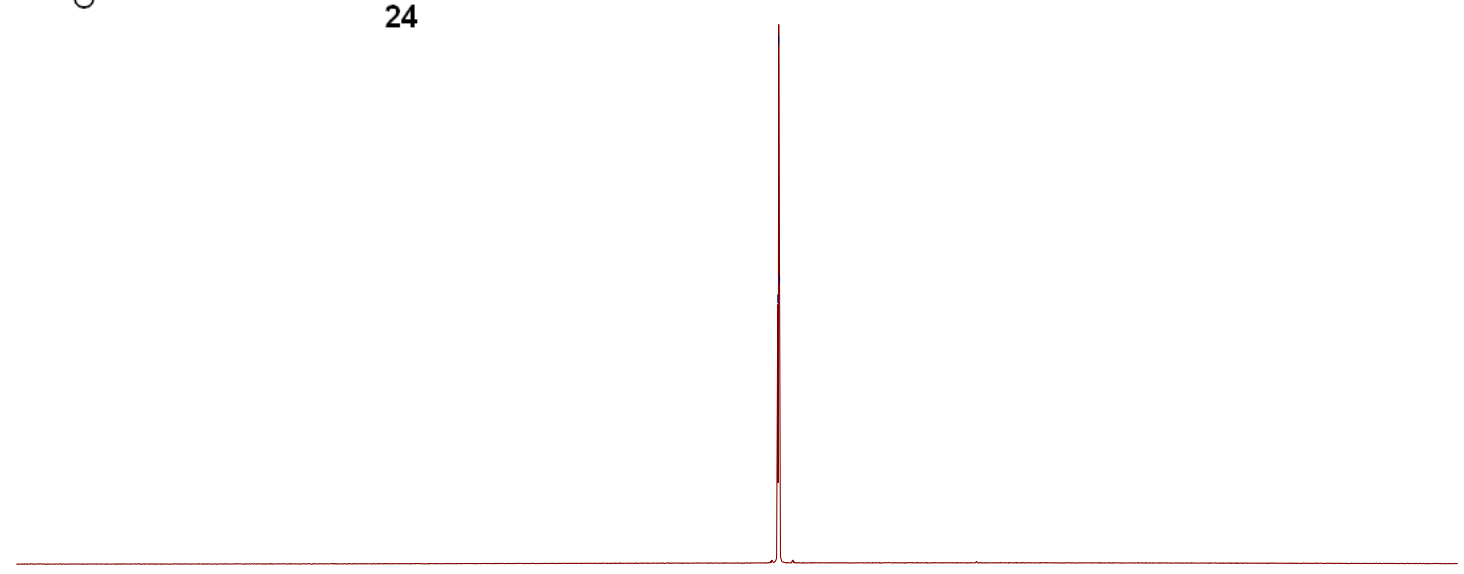

10 $-50$ -65
$-61(\mathrm{ppm})$ $-85$ 
${ }^{1} \mathrm{H} \mathrm{NMR}\left(600 \mathrm{MHz}, \mathrm{CDCl}_{3}\right)$

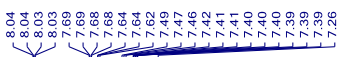

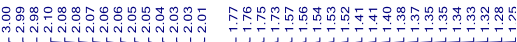

$1 / 1,1 / 1 / 4$

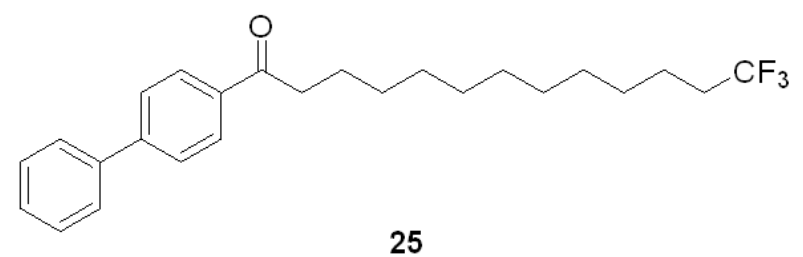

25

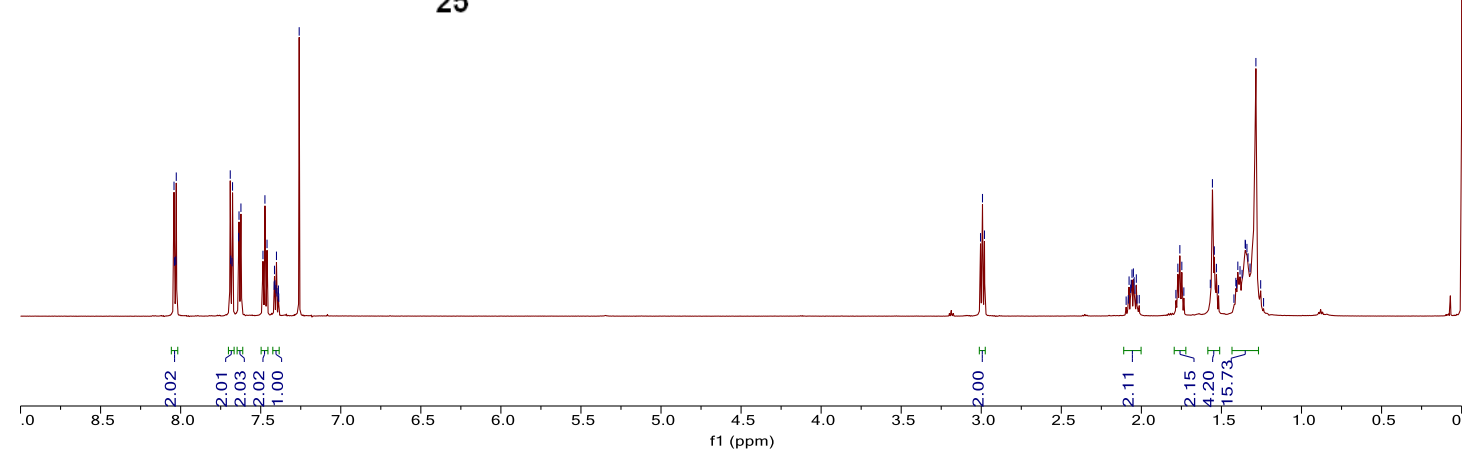

${ }^{13} \mathrm{C}$ NMR $\left(151 \mathrm{MHz}, \mathrm{CDCl}_{3}\right)$

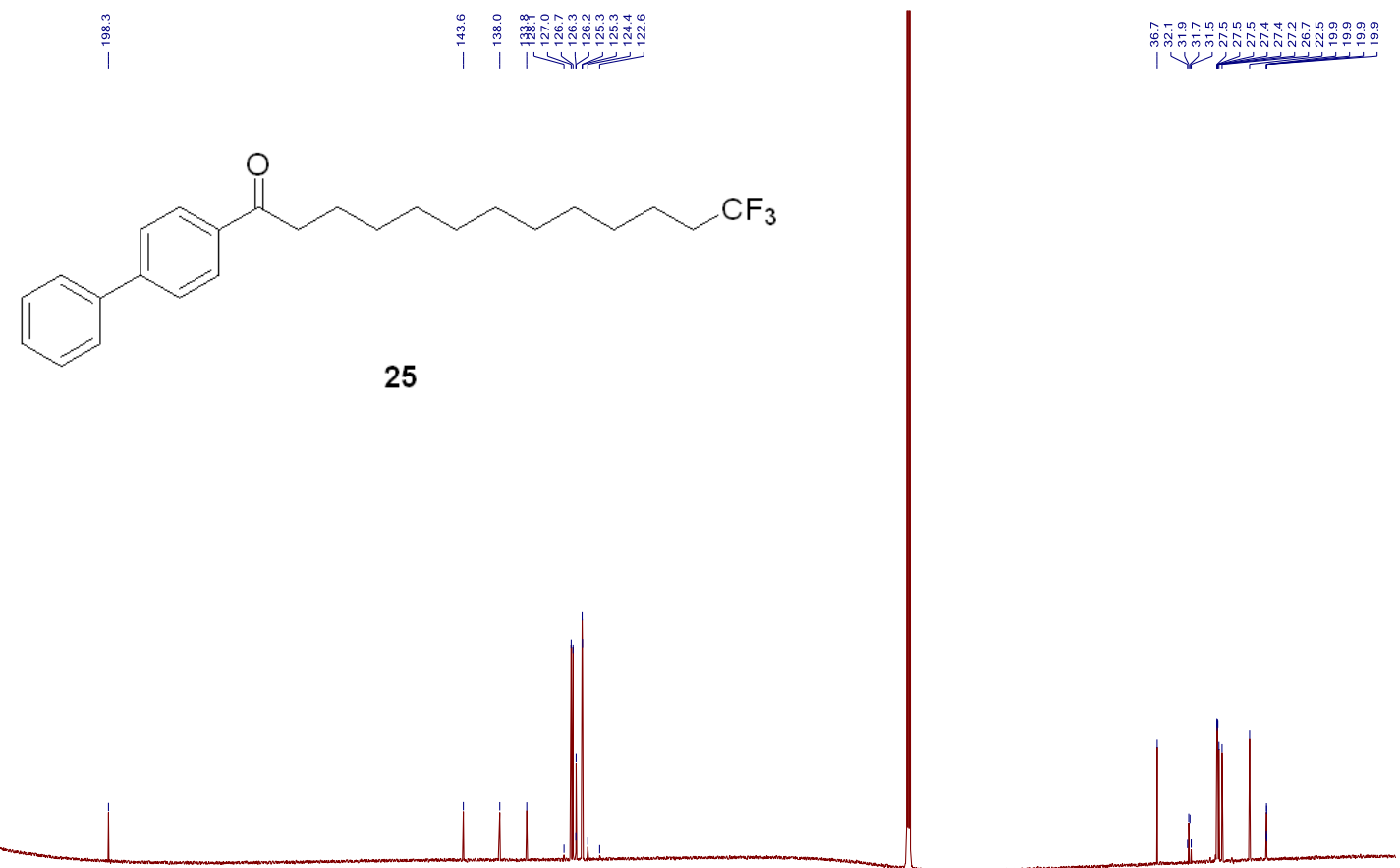

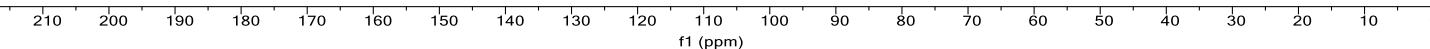


${ }^{19} \mathrm{~F}$ NMR $\left(376 \mathrm{MHz}, \mathrm{CDCl}_{3}\right)$
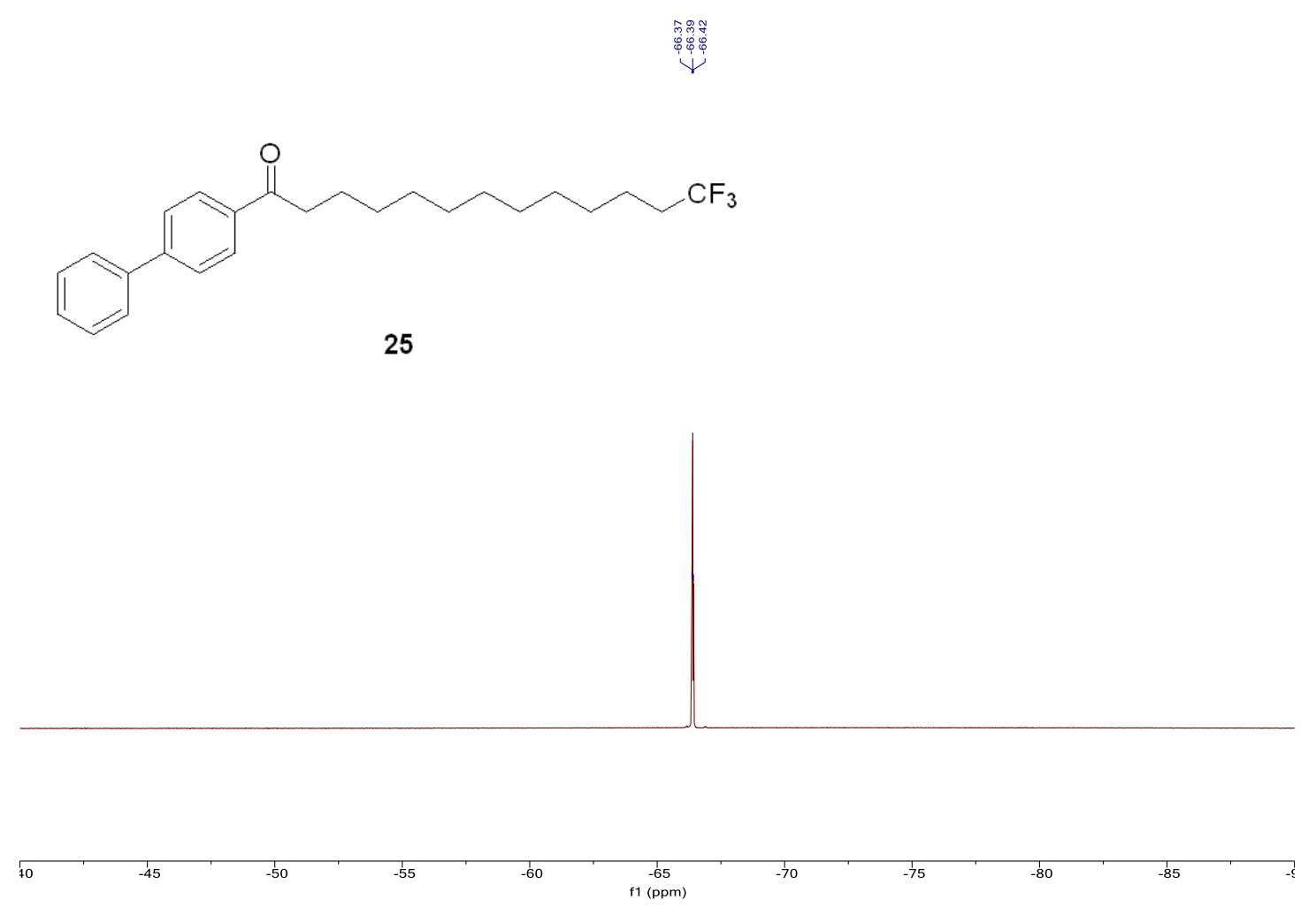

${ }^{1} \mathrm{H}$ NMR (600 MHz, $\left.\mathrm{CDCl}_{3}\right)$

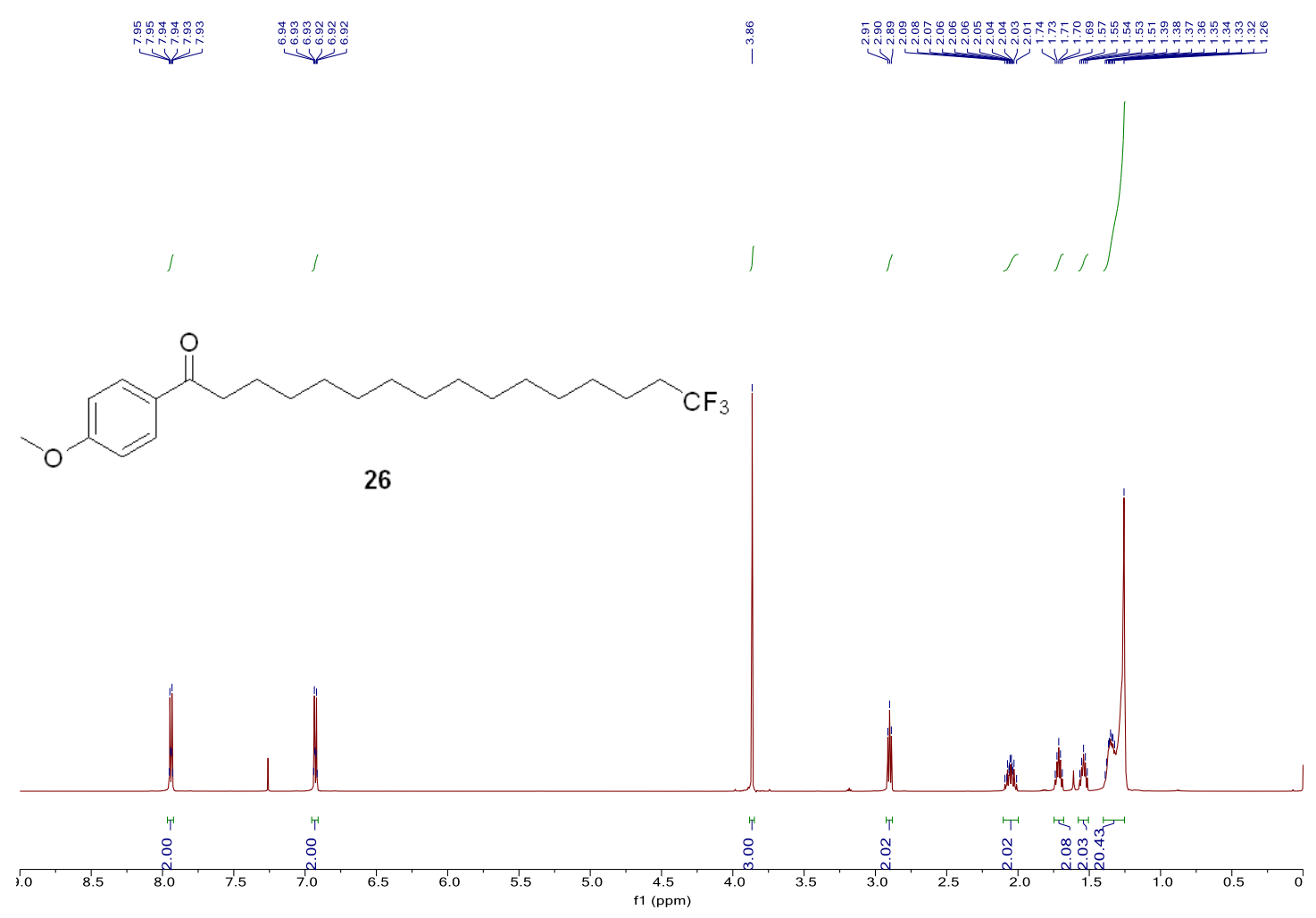


${ }^{13} \mathrm{C} \mathrm{NMR}\left(151 \mathrm{MHz}, \mathrm{CDCl}_{3}\right)$
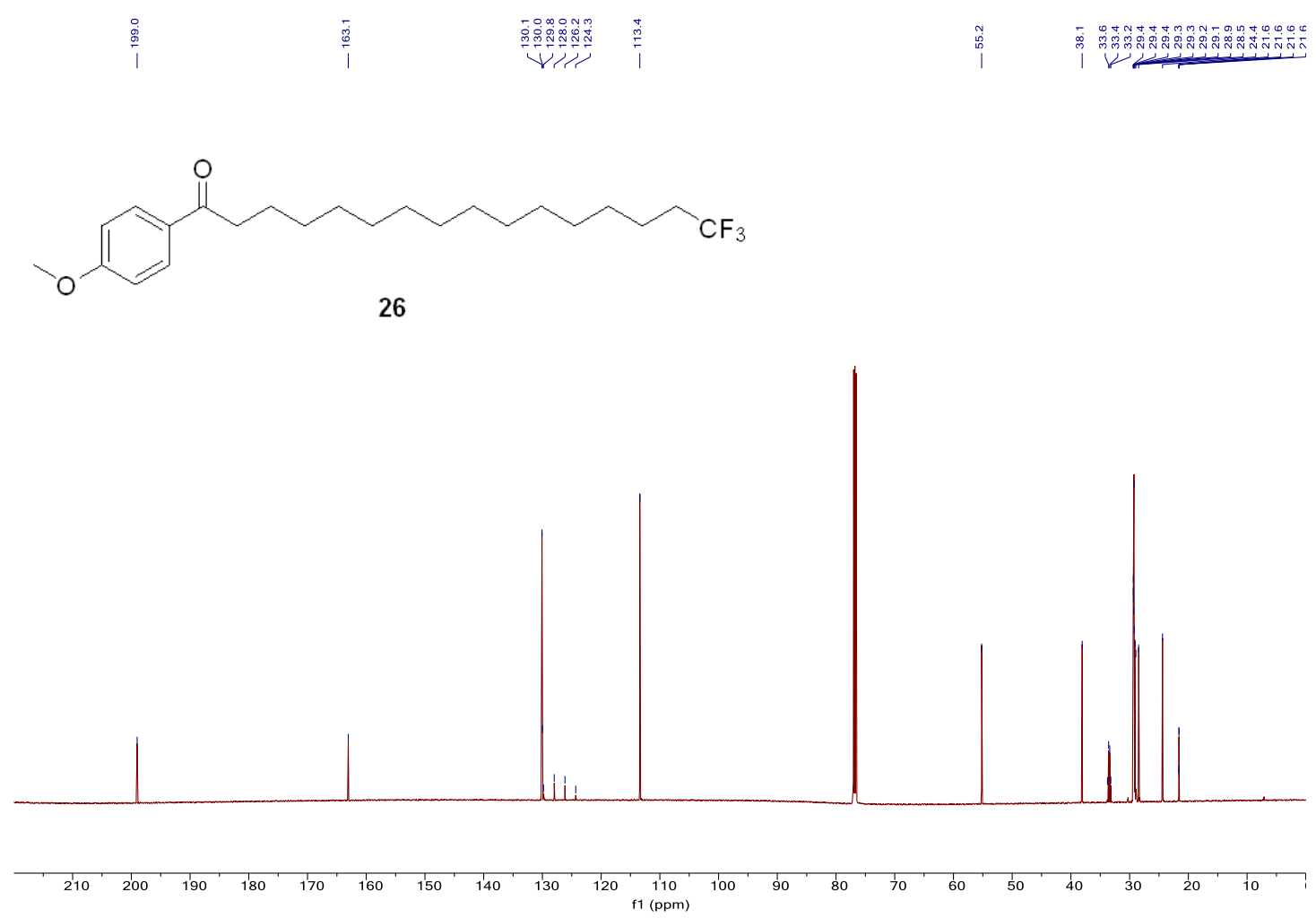

${ }^{19} \mathrm{~F} \mathrm{NMR}\left(376 \mathrm{MHz}, \mathrm{CDCl}_{3}\right)$

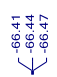

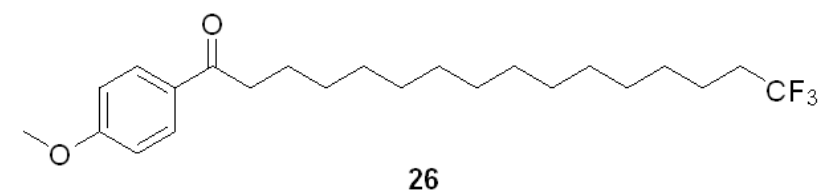

26

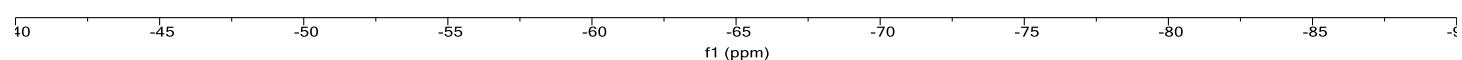


${ }^{1} \mathrm{H} \mathrm{NMR}\left(600 \mathrm{MHz}, \mathrm{CDCl}_{3}\right)$

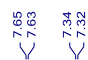

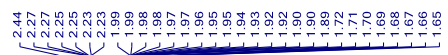<smiles>Cc1ccc(S(=O)(=O)N2CCC(C(F)(F)F)CC2)cc1</smiles>
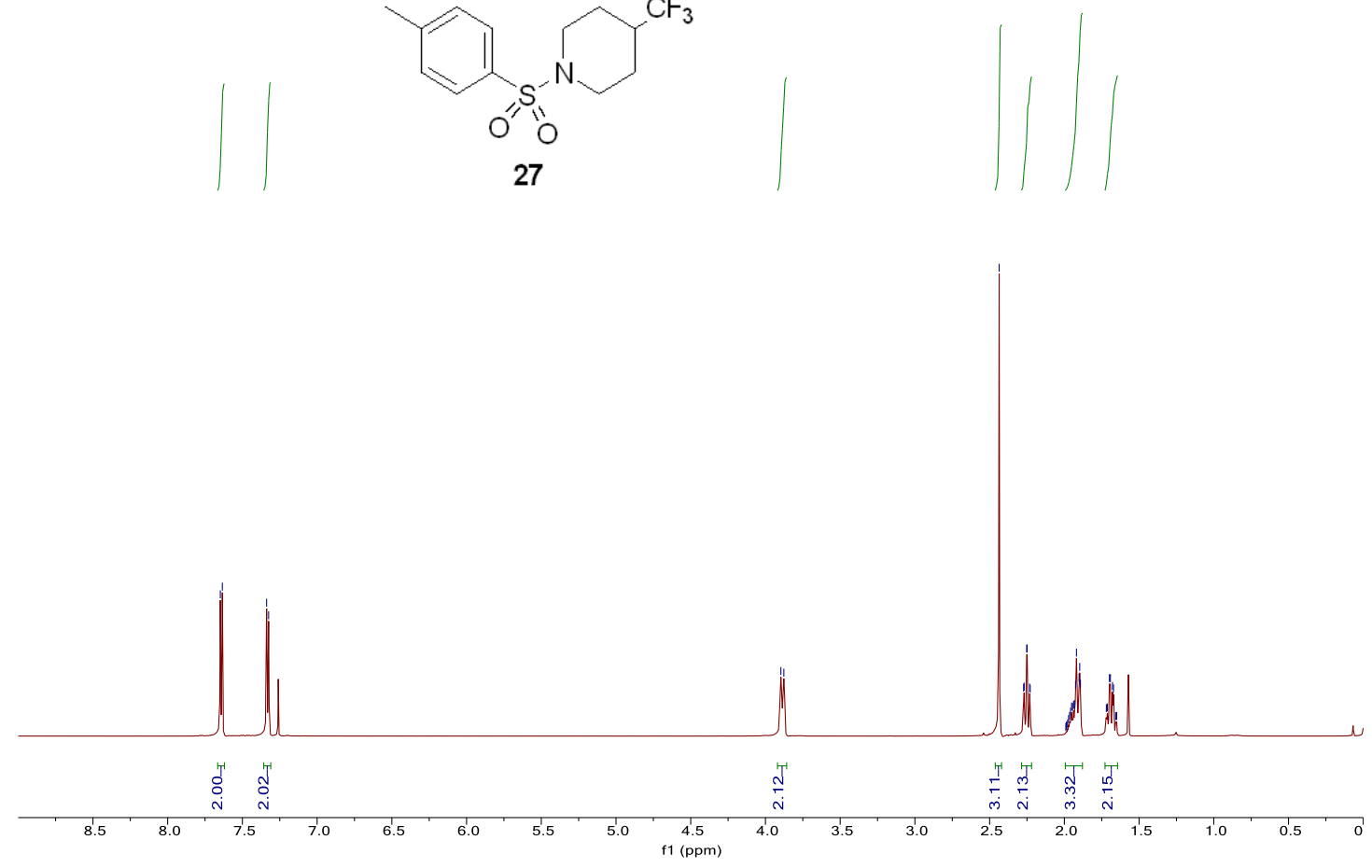

${ }^{13} \mathrm{C} \mathrm{NMR}\left(151 \mathrm{MHz}, \mathrm{CDCl}_{3}\right)$

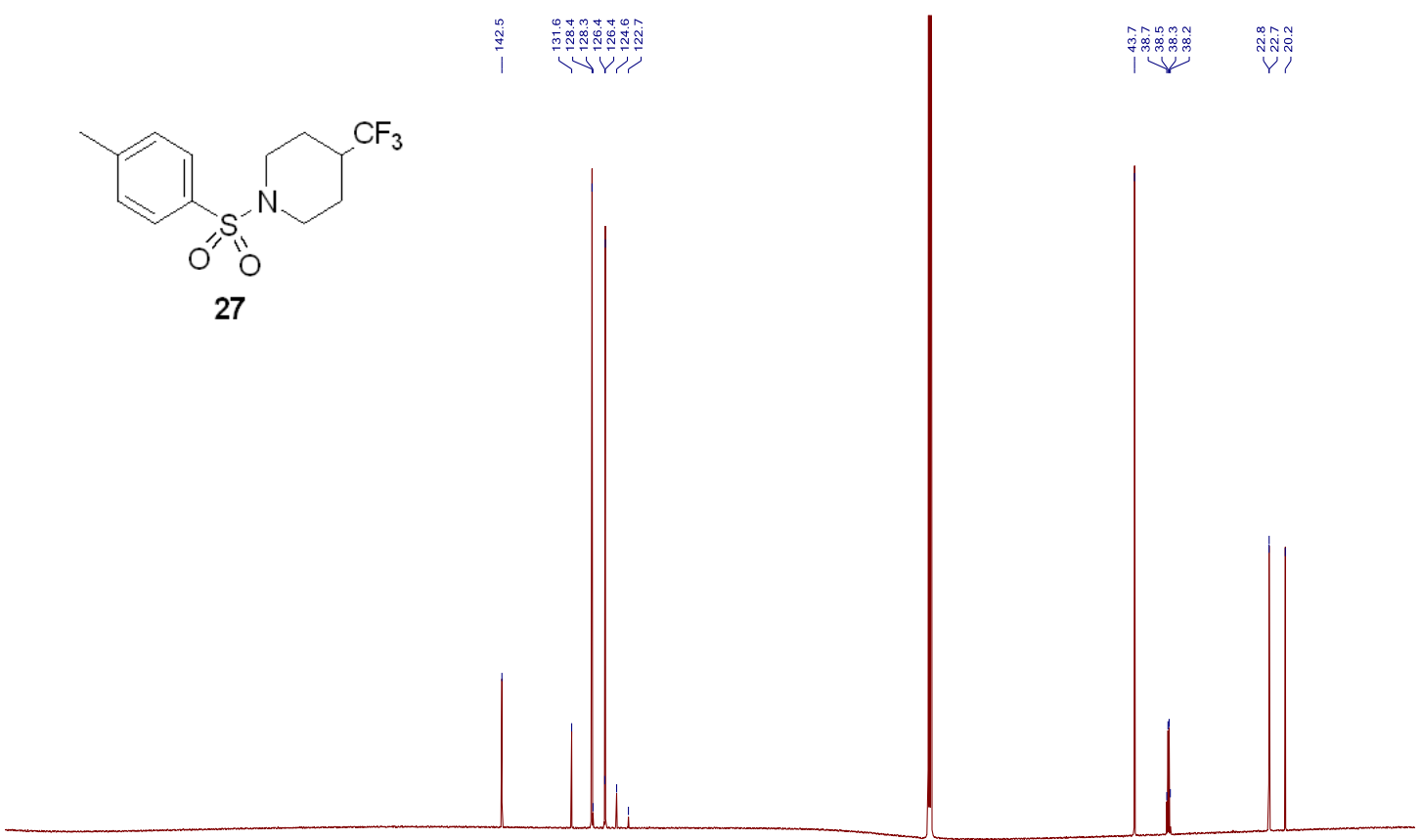

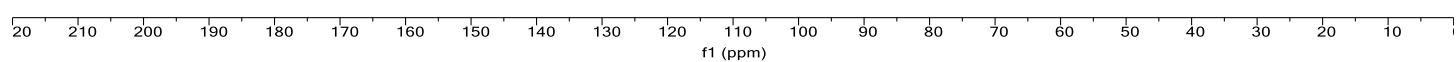


${ }^{19} \mathrm{~F}$ NMR $\left(376 \mathrm{MHz}, \mathrm{CDCl}_{3}\right)$<smiles>CCCOS(=O)(=O)N1CCC(C(F)(F)F)CC1</smiles>

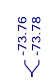

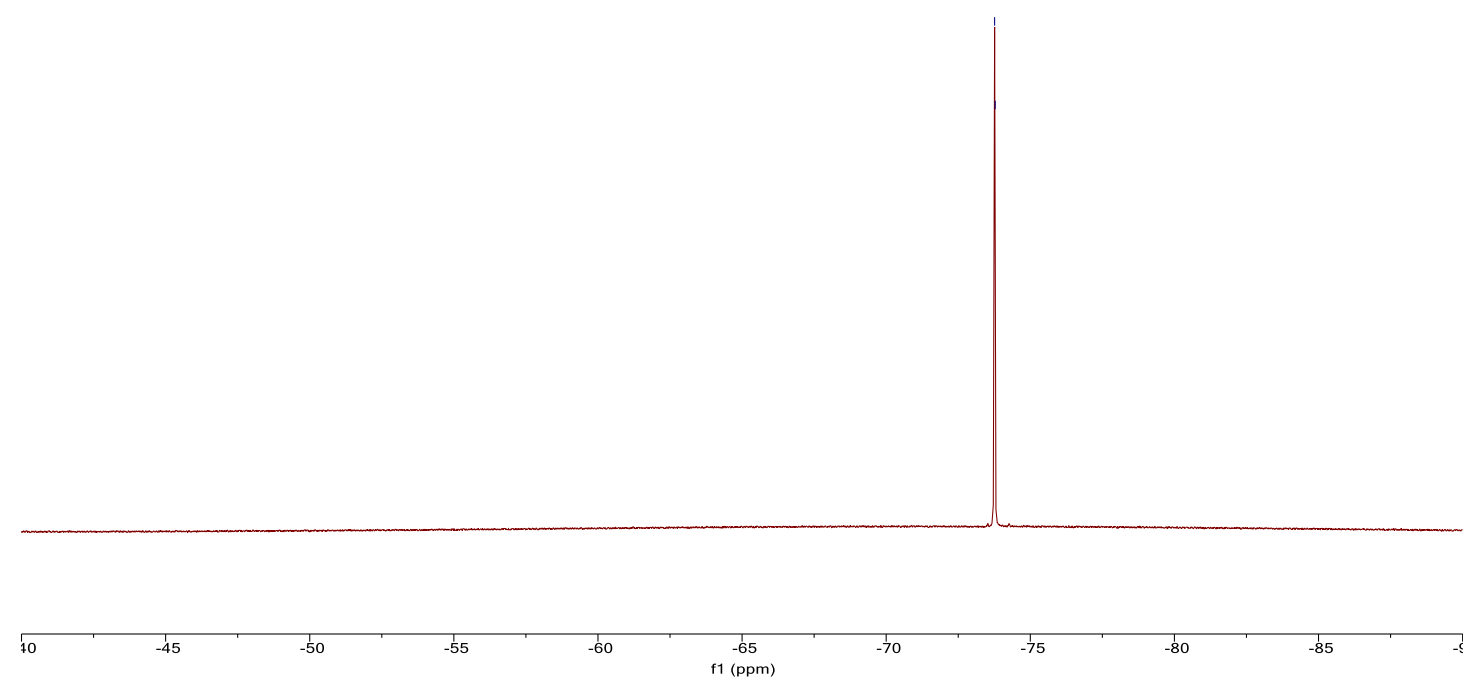

${ }^{1} \mathrm{H}$ NMR $\left(600 \mathrm{MHz}, \mathrm{CDCl}_{3}\right)$

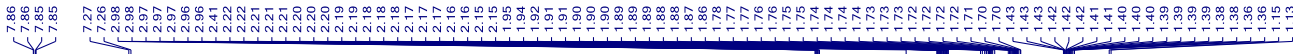

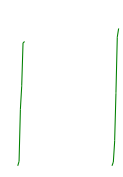<smiles>Cc1ccc(C(=O)CCCC(C)C(F)(F)F)cc1</smiles>
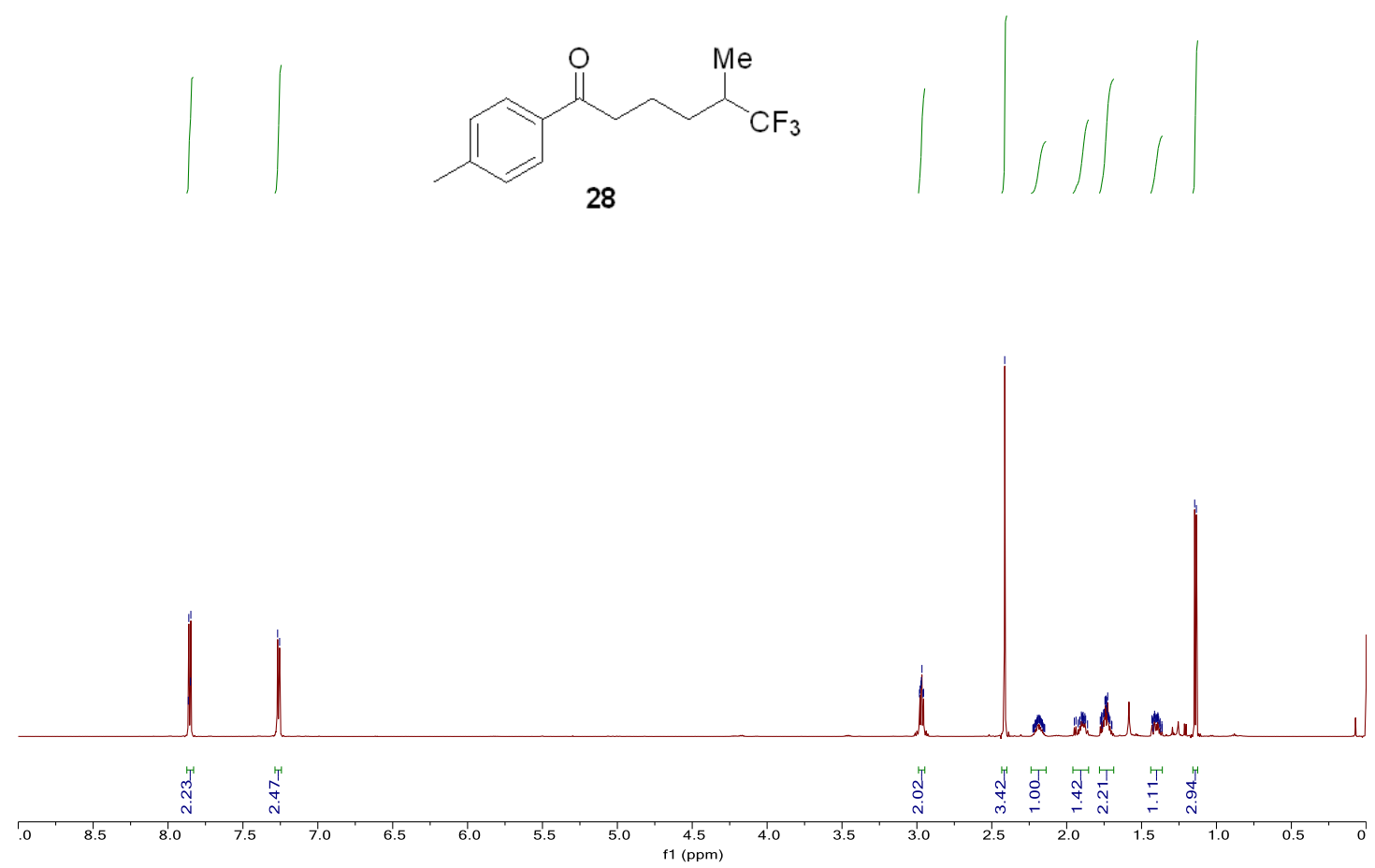
${ }^{13} \mathrm{C} \mathrm{NMR}\left(151 \mathrm{MHz}, \mathrm{CDCl}_{3}\right)$

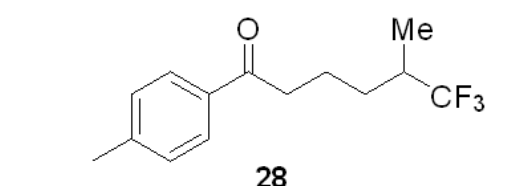

28

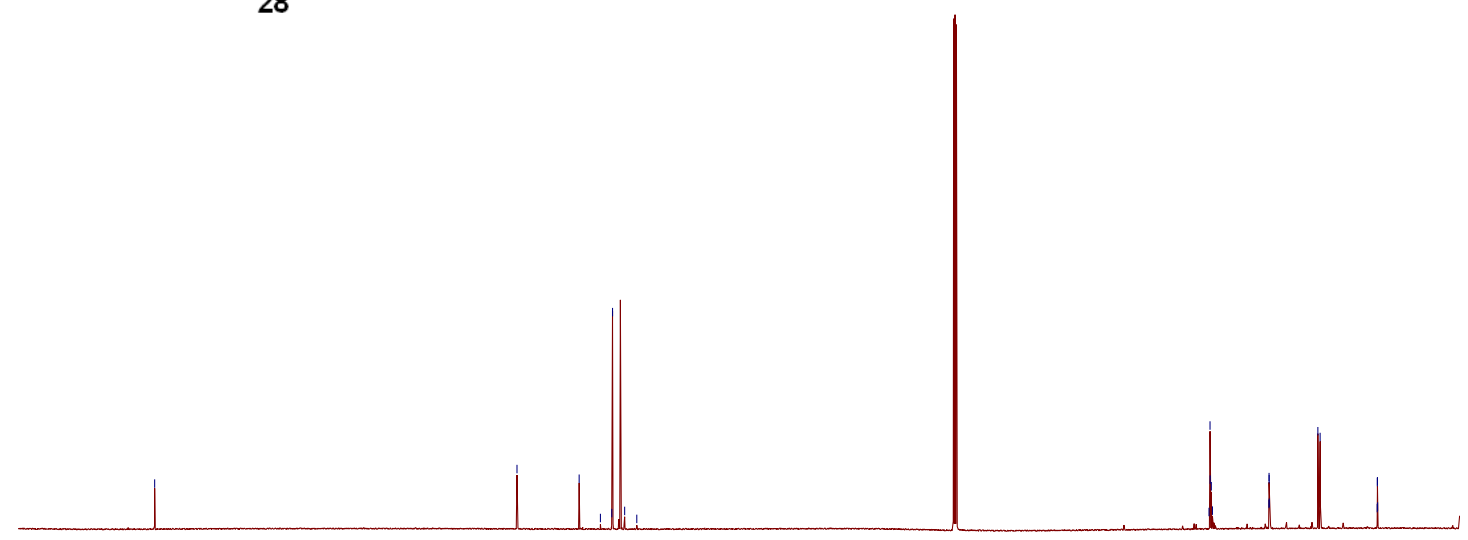

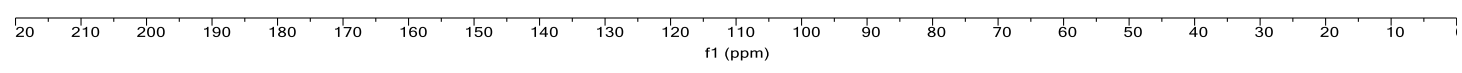

${ }^{19} \mathrm{~F}$ NMR (376 MHz, $\mathrm{CDCl}_{3}$ )<smiles>Cc1ccc(C(=O)CCCC(C)C(F)(F)F)cc1</smiles>

28

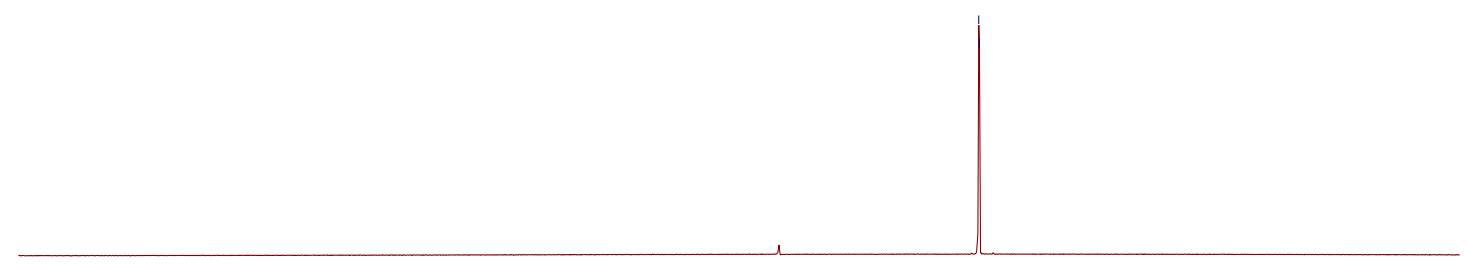

10 -65
$\mathrm{f} 1(\mathrm{ppm})$ $-85$ 
${ }^{1} \mathrm{H}$ NMR $\left(600 \mathrm{MHz}, \mathrm{CDCl}_{3}\right)$

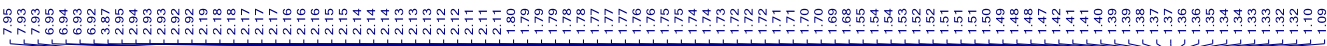

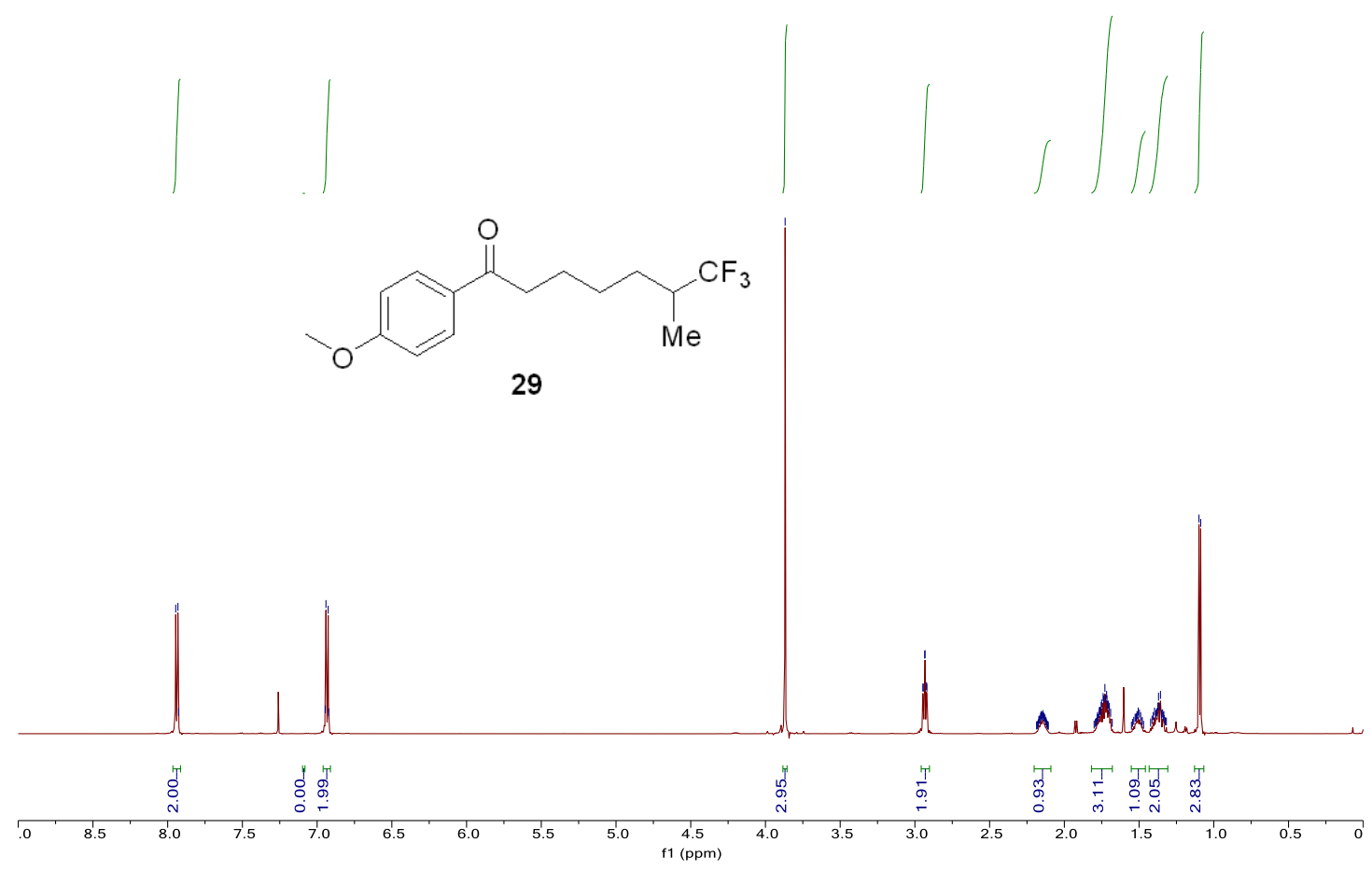

${ }^{13} \mathrm{C}$ NMR (151 MHz, $\left.\mathrm{CDCl}_{3}\right)$

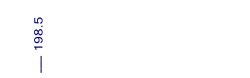<smiles>COc1ccc(C(=O)CCCCC(C)C(F)(F)F)cc1</smiles>

29

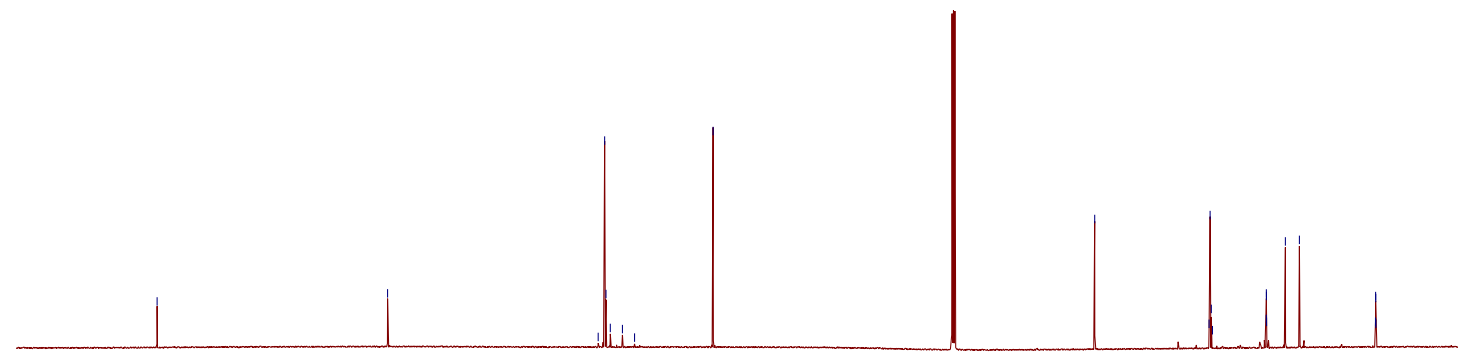

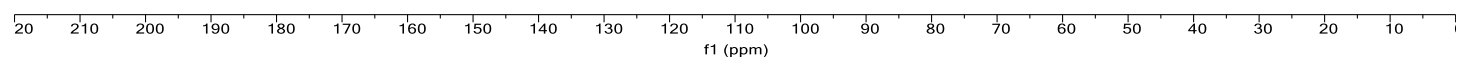


${ }^{19} \mathrm{~F}$ NMR (376 MHz, $\left.\mathrm{CDCl}_{3}\right)$<smiles>COc1ccc(C(=O)CCCCC(C)C(F)(F)F)cc1</smiles>

29

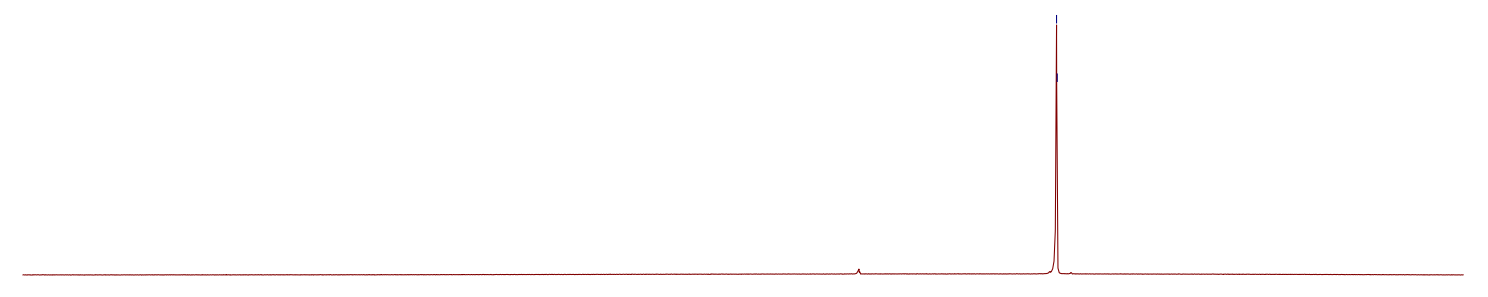

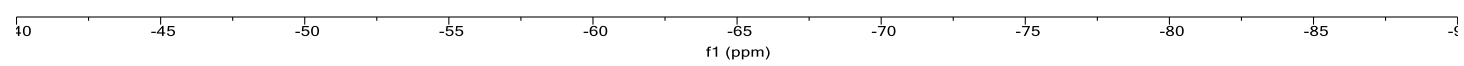

${ }^{1} \mathrm{H}$ NMR $\left(600 \mathrm{MHz}, \mathrm{CDCl}_{3}\right)$

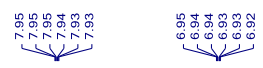

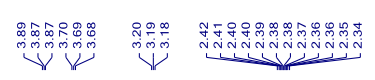
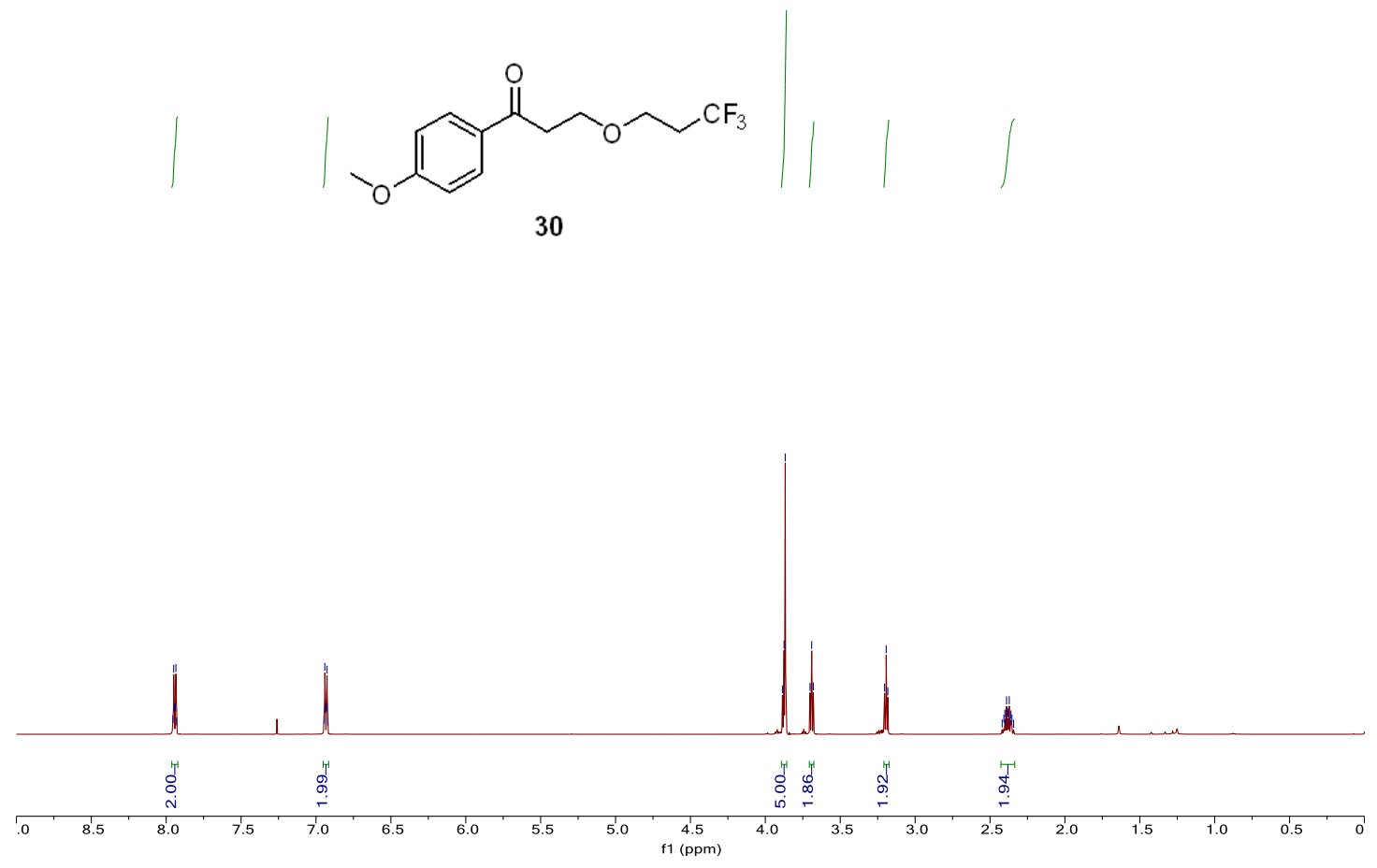
${ }^{13} \mathrm{C} \mathrm{NMR}\left(151 \mathrm{MHz}, \mathrm{CDCl}_{3}\right)$<smiles>COc1ccc(C(=O)CCOCCC(F)(F)F)cc1</smiles>

30
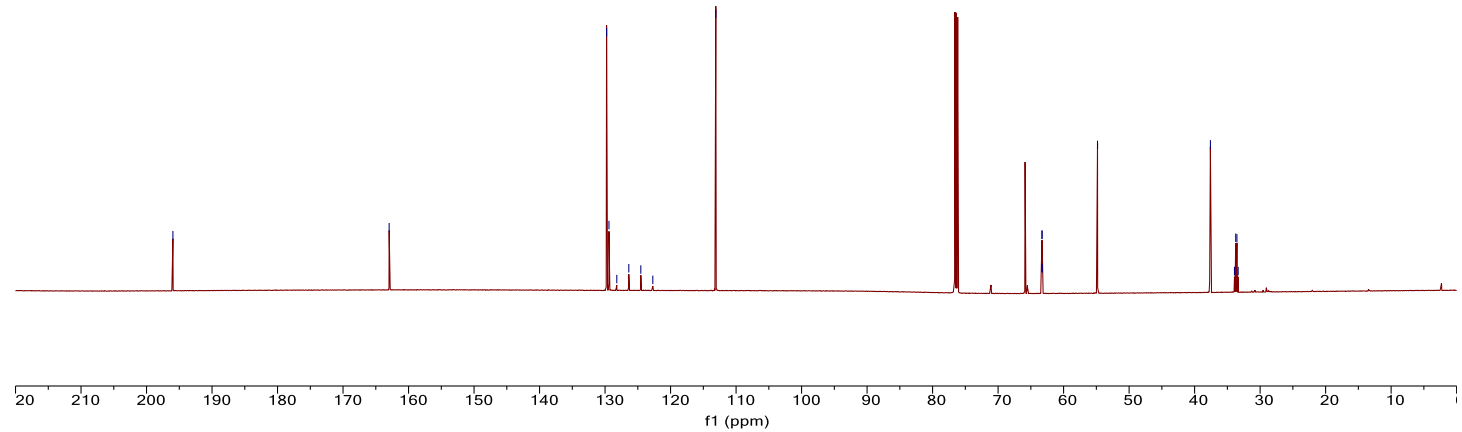

${ }^{19} \mathrm{~F}$ NMR $\left(376 \mathrm{MHz}, \mathrm{CDCl}_{3}\right)$

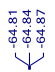<smiles>COc1ccc(C(=O)CCOCCC(F)(F)F)cc1</smiles>

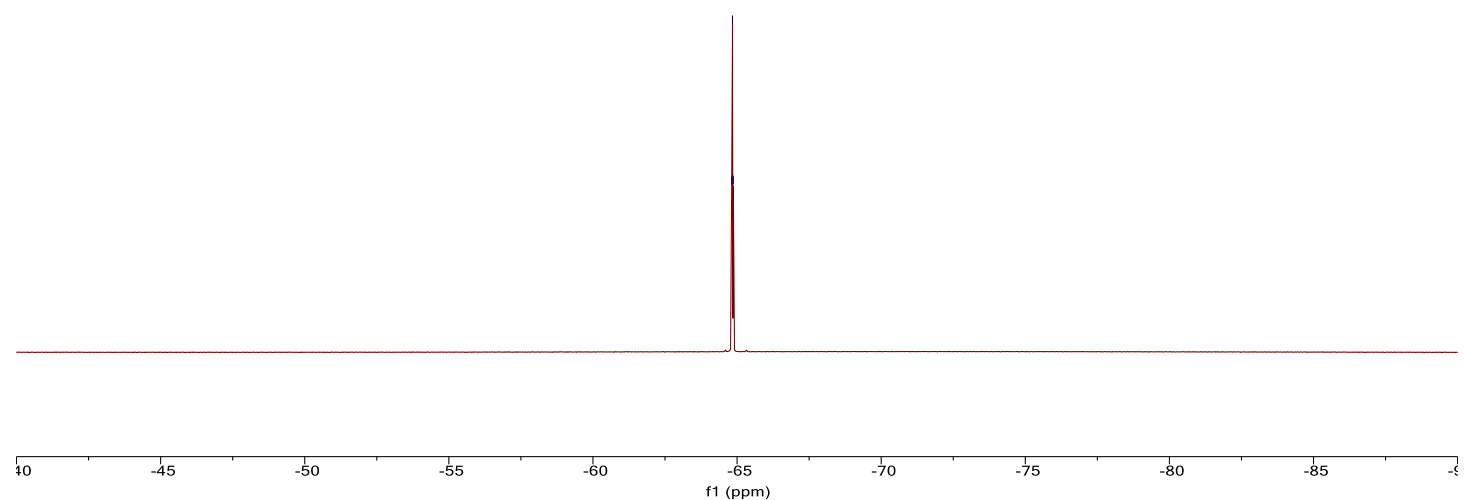


${ }^{1} \mathrm{H}$ NMR $\left(600 \mathrm{MHz}, \mathrm{CDCl}_{3}\right)$

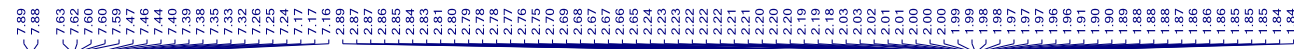

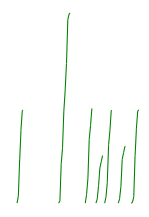<smiles>CCCC(CCC(=O)c1ccc(-c2ccccc2)cc1)c1ccccc1</smiles>

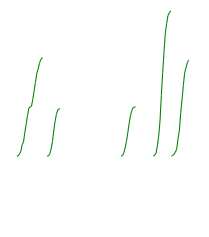

31

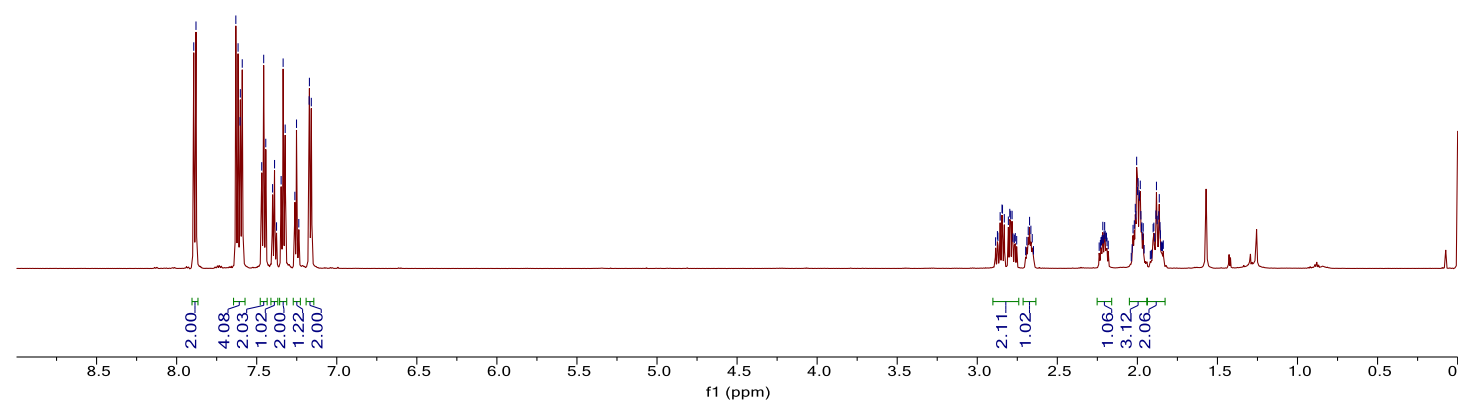

${ }^{13} \mathrm{C}$ NMR $\left(151 \mathrm{MHz}, \mathrm{CDCl}_{3}\right)$<smiles>CCCC(CCCC(=O)c1ccc(-c2ccccc2)cc1)c1ccccc1</smiles>

31
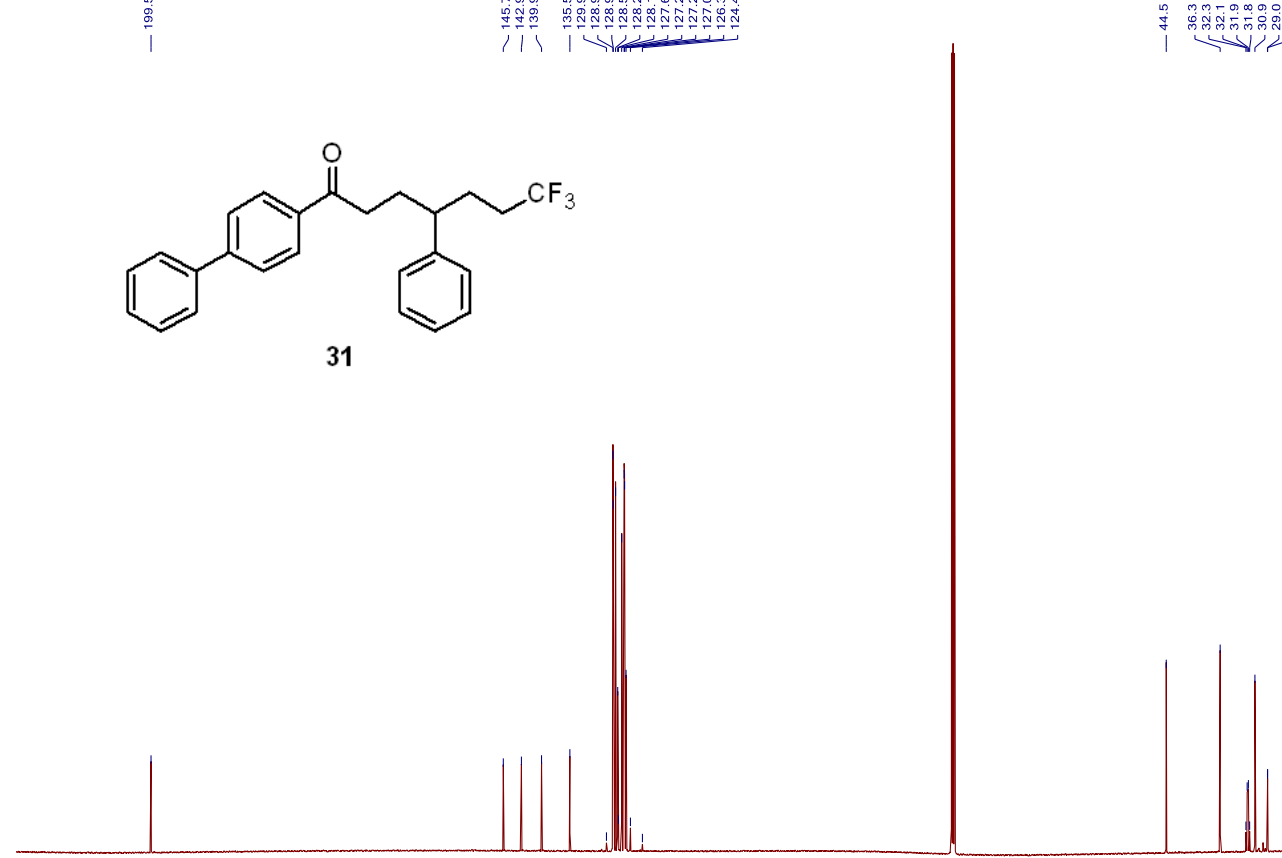

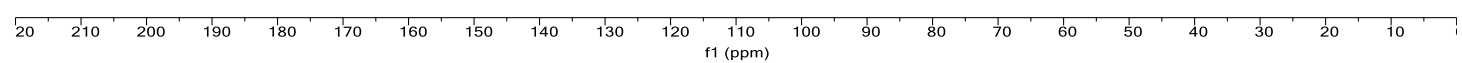


${ }^{19} \mathrm{~F} \mathrm{NMR}\left(376 \mathrm{MHz}, \mathrm{CDCl}_{3}\right)$

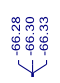<smiles>O=C(CCC(CCC(F)(F)F)c1ccccc1)c1ccc(-c2ccccc2)cc1</smiles>

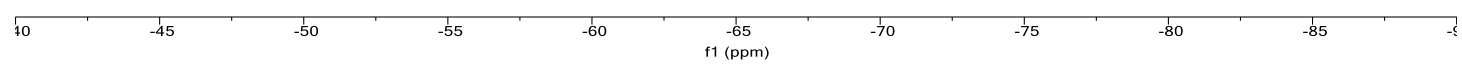

major product:

${ }^{1} \mathrm{H} \mathrm{NMR}\left(600 \mathrm{MHz}, \mathrm{CDCl}_{3}\right)$

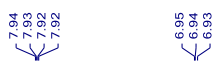

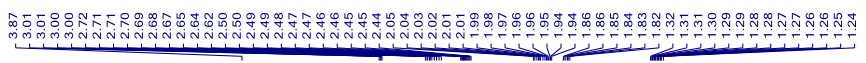

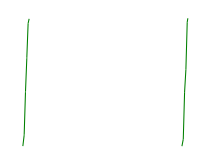<smiles>COc1ccc(C(=O)CC2CCC(C(F)(F)F)C2)cc1</smiles>

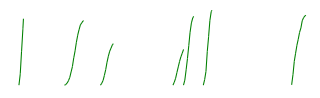

$32-1$

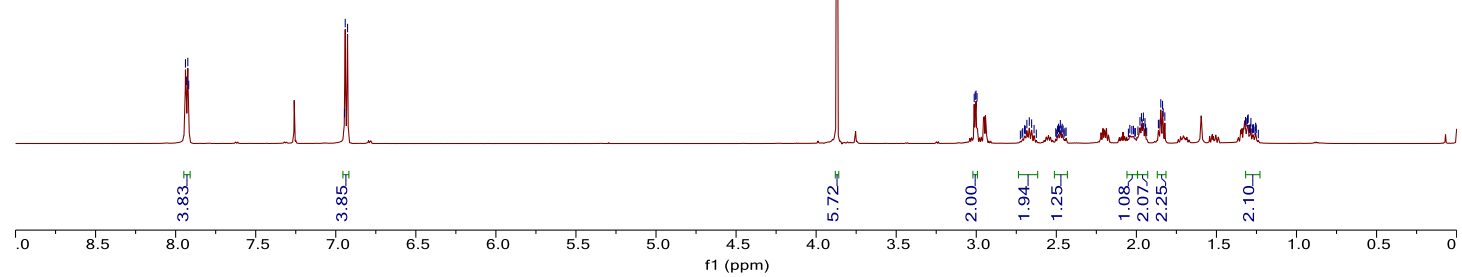


${ }^{13} \mathrm{C} \mathrm{NMR}\left(151 \mathrm{MHz}, \mathrm{CDCl}_{3}\right)$
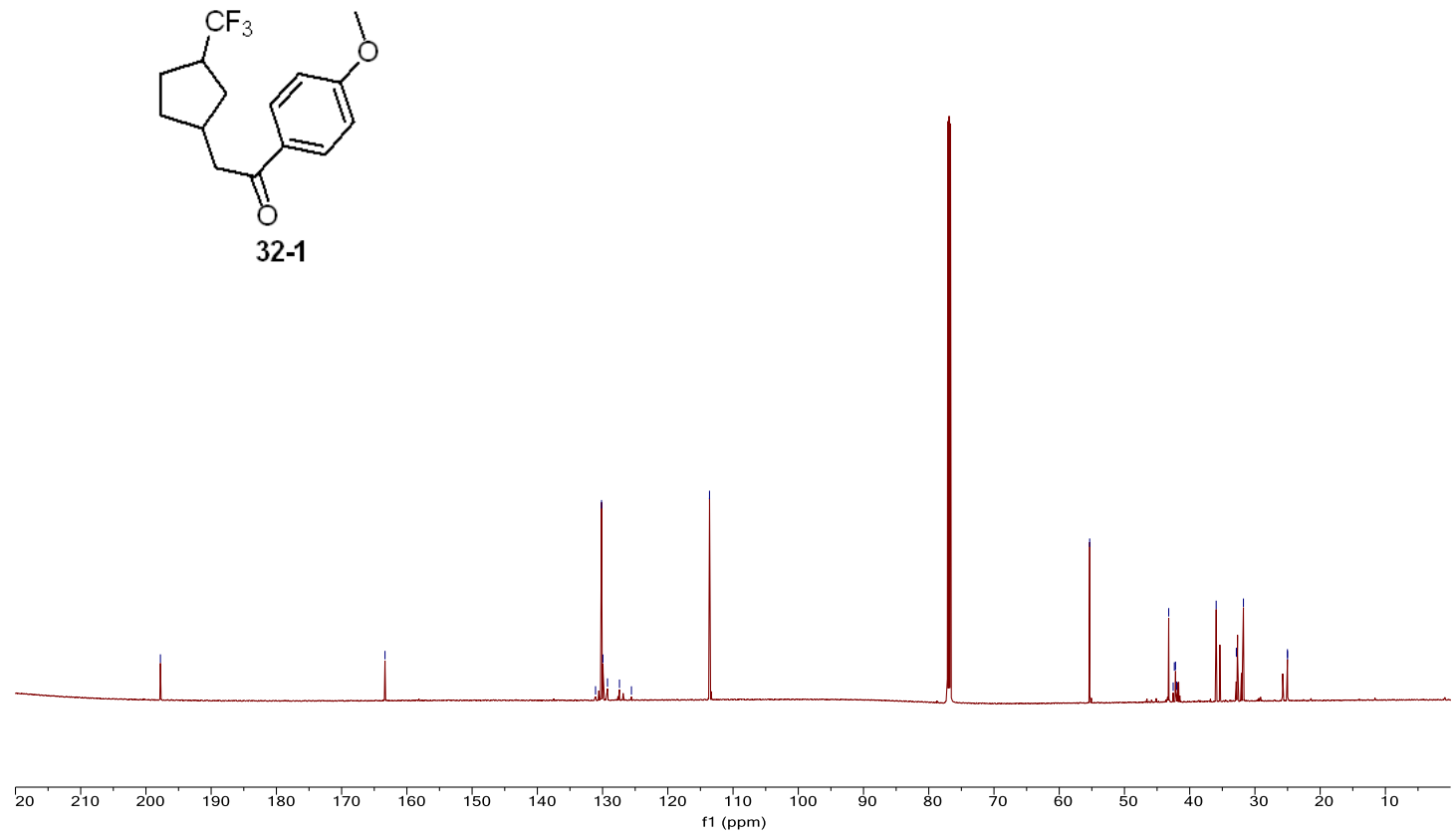

${ }^{19} \mathrm{~F}$ NMR (376 MHz, $\mathrm{CDCl}_{3}$ )

丩<smiles>COc1ccc(C(=O)CC2CCC(C(F)(F)F)C2)cc1</smiles>

10 $-50$ -65
f1 $(\mathrm{ppm})$ 


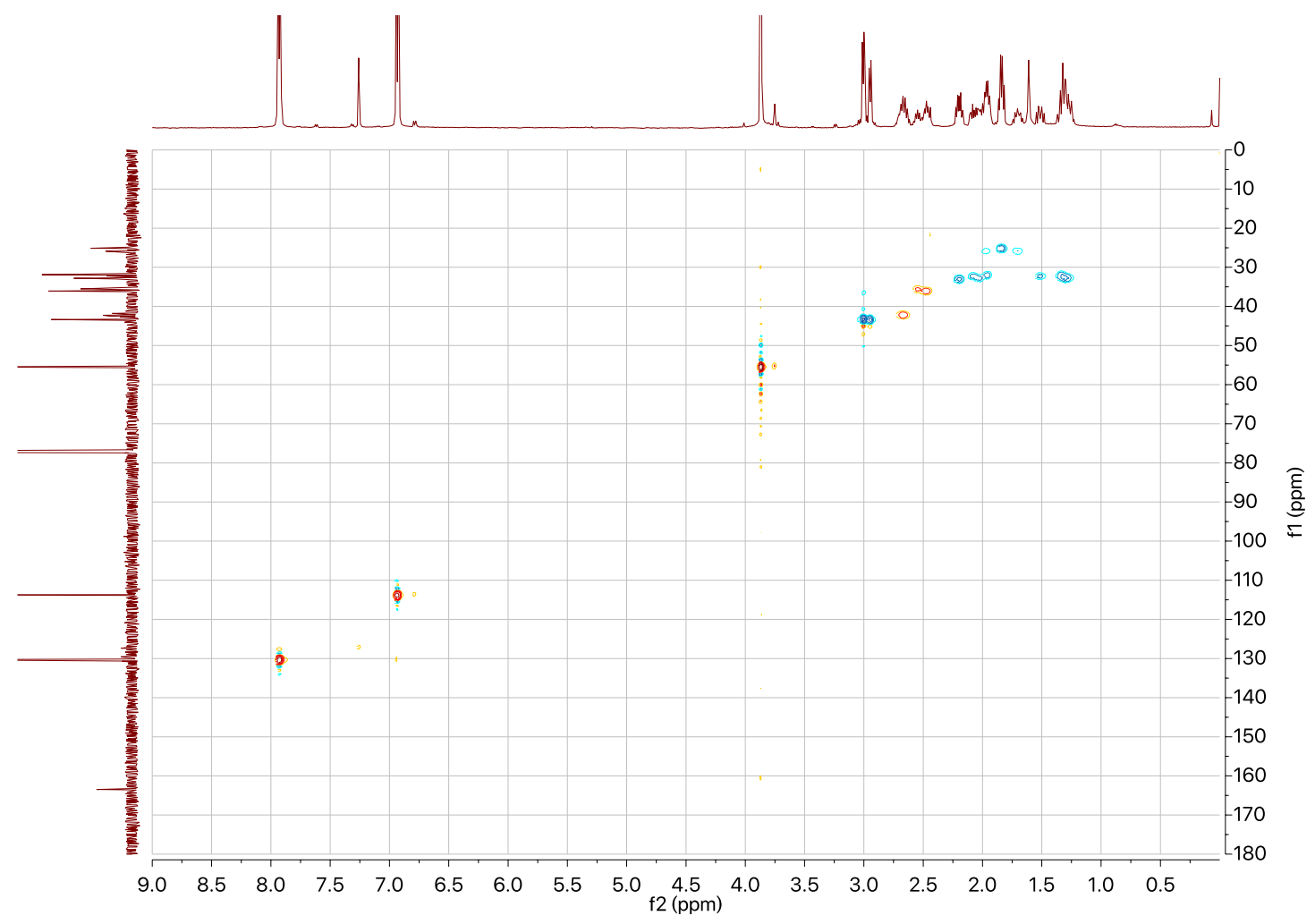

minor product:

${ }^{1} \mathrm{H} \mathrm{NMR}\left(600 \mathrm{MHz}, \mathrm{CDCl}_{3}\right)$

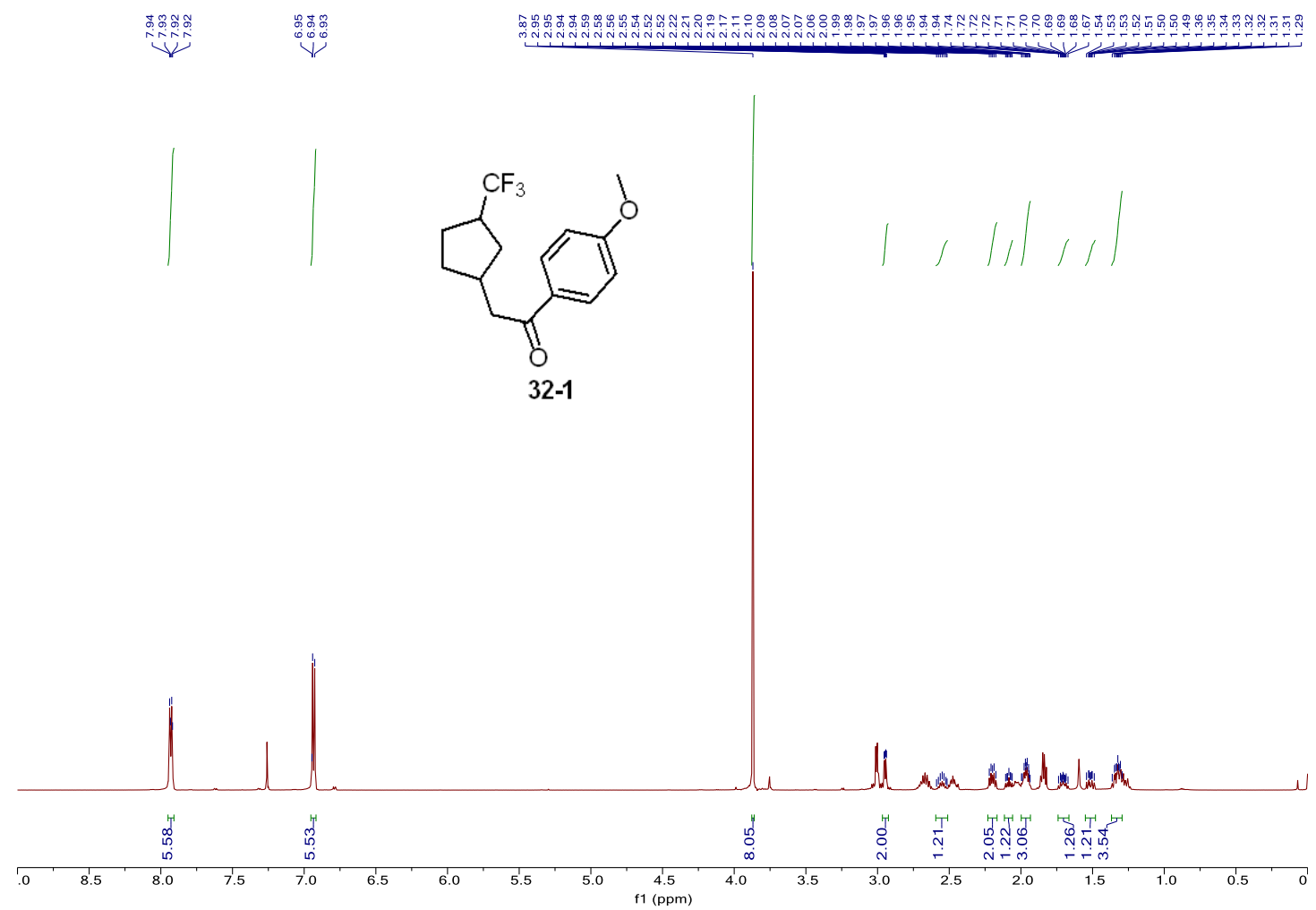


${ }^{13} \mathrm{C} \mathrm{NMR}\left(151 \mathrm{MHz}, \mathrm{CDCl}_{3}\right)$

$$
\text { 产 }
$$
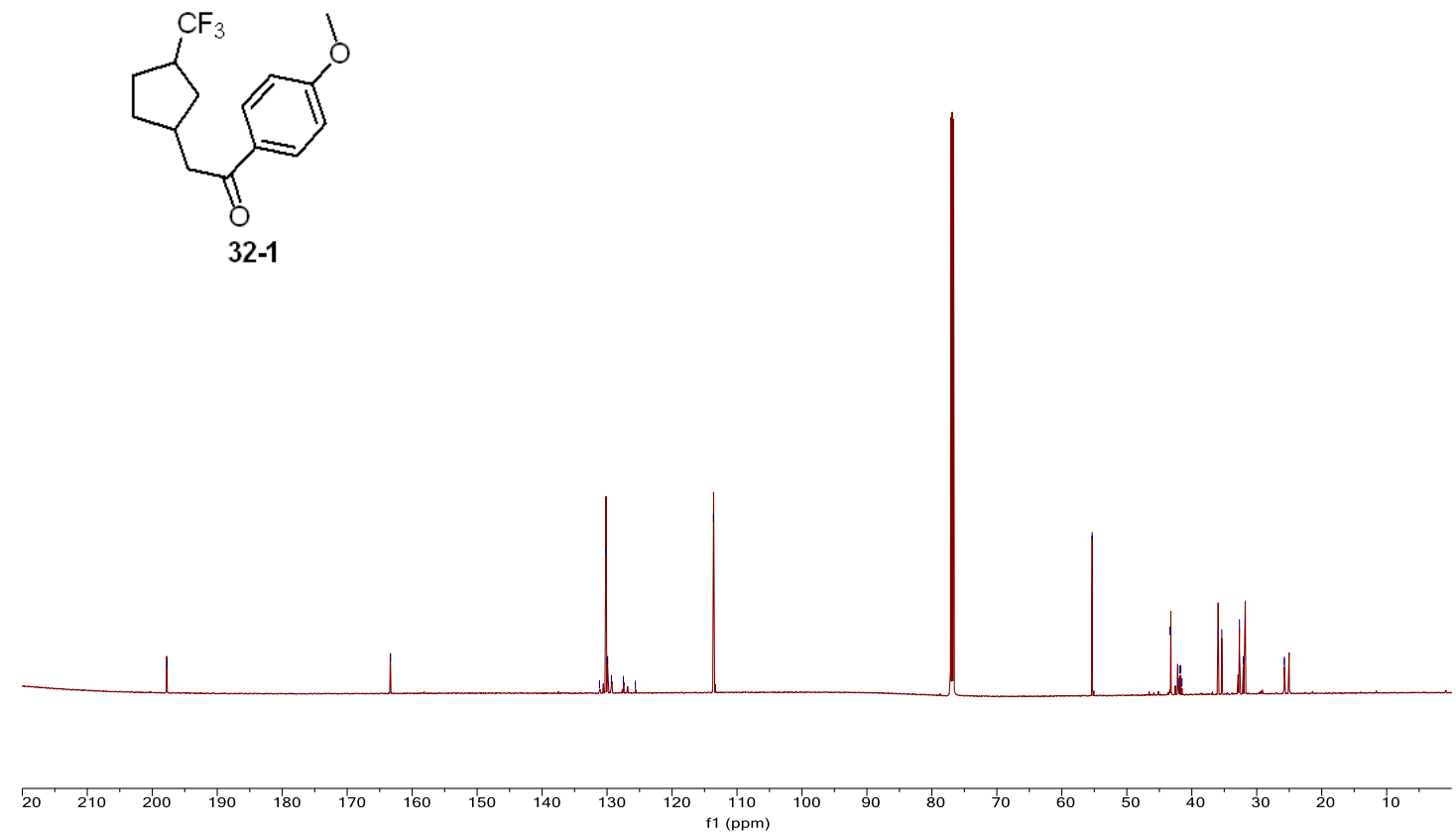

${ }^{19} \mathrm{~F}$ NMR (376 MHz, $\mathrm{CDCl}_{3}$ )
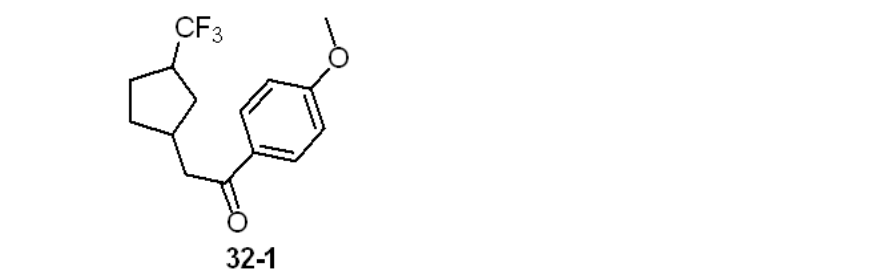

40

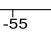

-70
$\mathrm{f} 1(\mathrm{ppm})$ 
${ }^{1} \mathrm{H}$ NMR $\left(600 \mathrm{MHz}, \mathrm{CDCl}_{3}\right)$

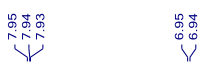

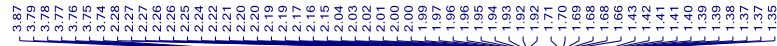
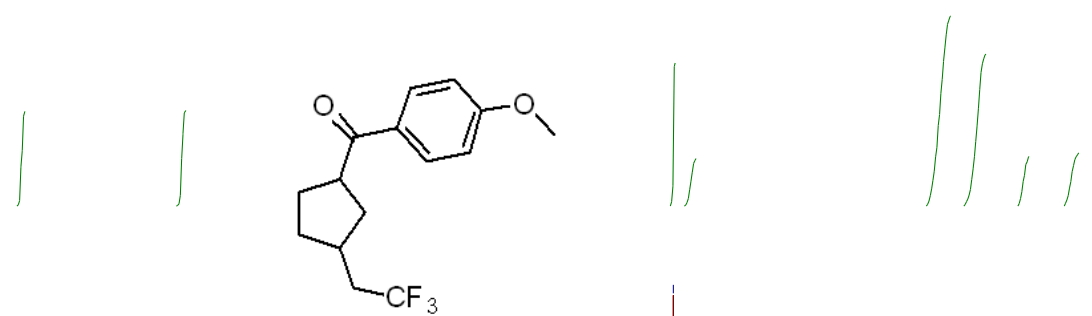

$32-2$
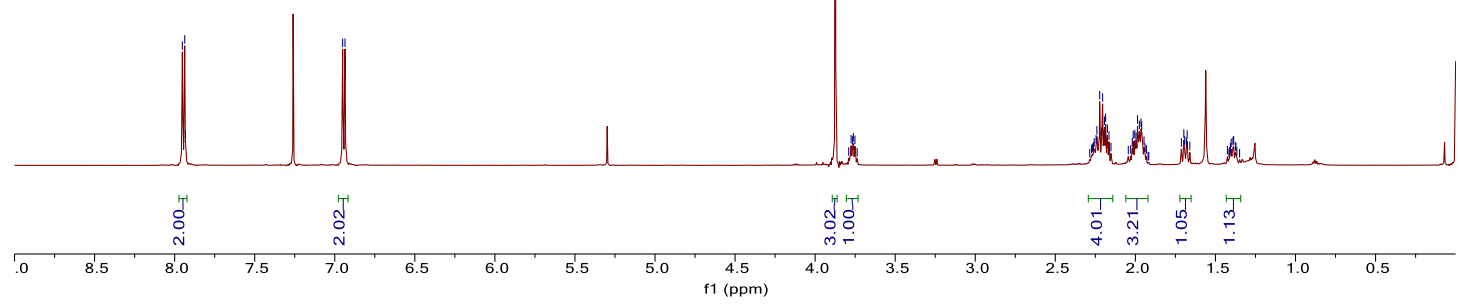

${ }^{13} \mathrm{C}$ NMR (151 MHz, $\mathrm{CDCl}_{3}$ )

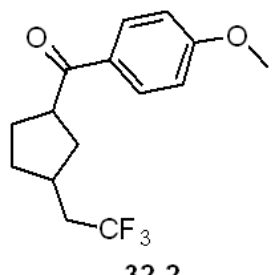

$32-2$

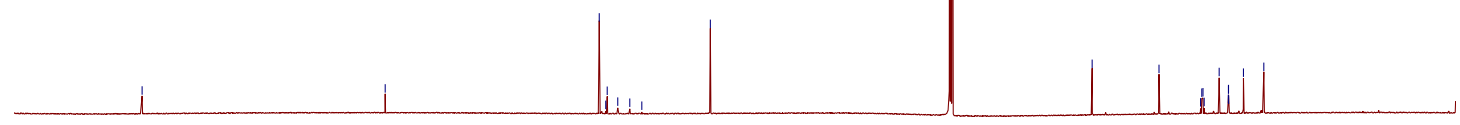

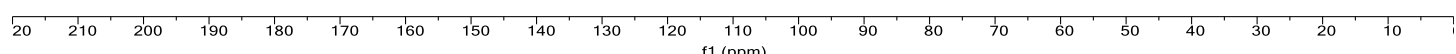


${ }^{19} \mathrm{~F}$ NMR $\left(376 \mathrm{MHz}, \mathrm{CDCl}_{3}\right)$

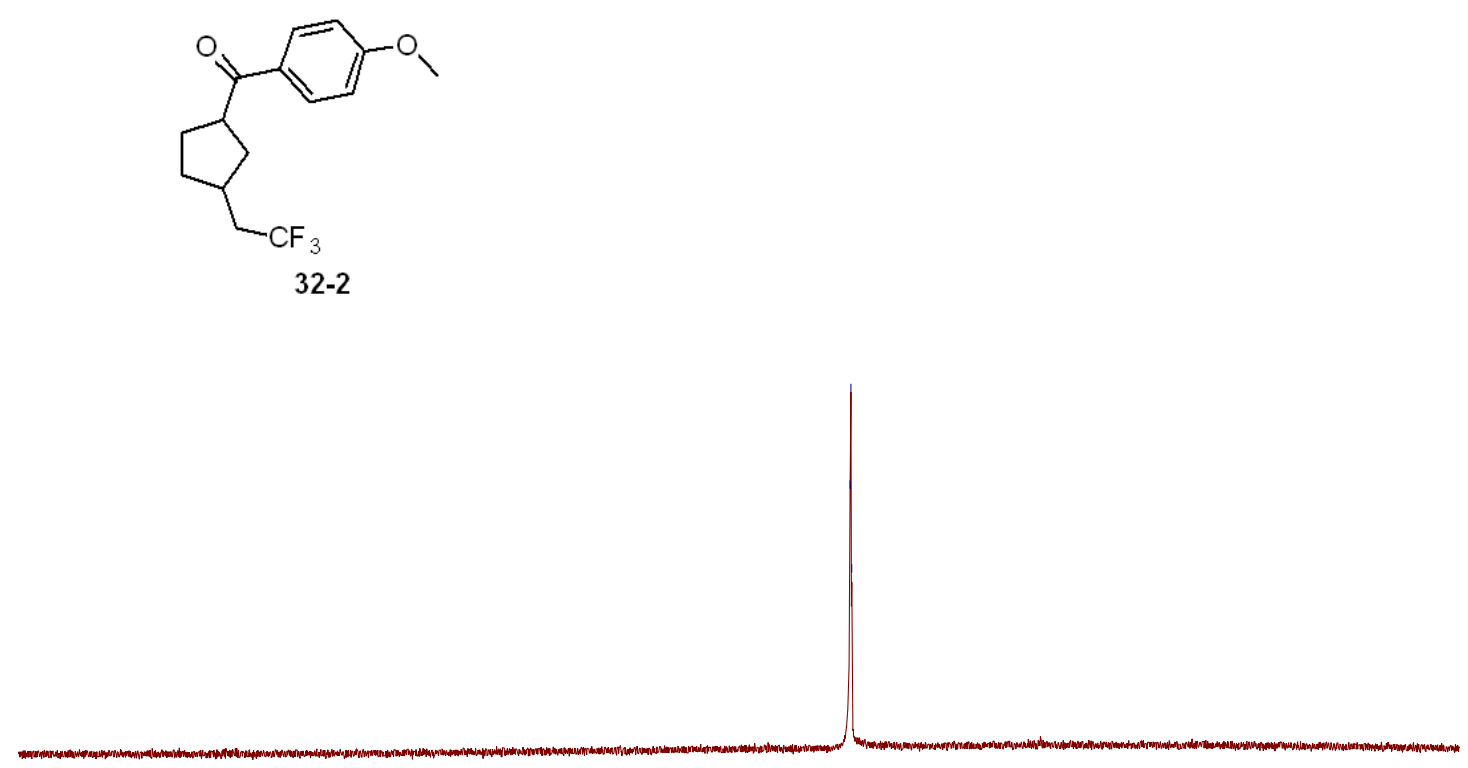

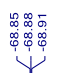

-45
$10 \quad+15$

$-50$

$-55$

$-60$

-65
$\mathrm{f} 1(\mathrm{ppm})$

$-70$

$-80$

$-85$

${ }^{1} \mathrm{H}$ NMR (600 MHz, $\left.\mathrm{CDCl}_{3}\right)$

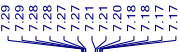

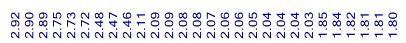

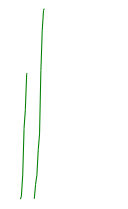<smiles>O=C(CCCC(F)(F)F)CCc1ccccc1</smiles>

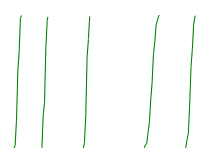

33

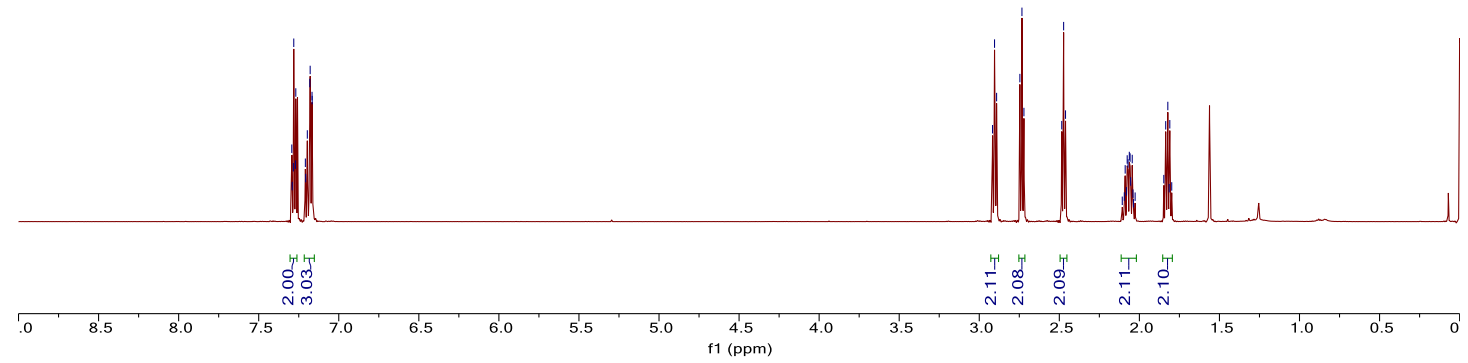


${ }^{13} \mathrm{C} \mathrm{NMR}\left(151 \mathrm{MHz}, \mathrm{CDCl}_{3}\right)$

萬<smiles>O=C(CCCC(F)(F)F)CCc1ccccc1</smiles>

33
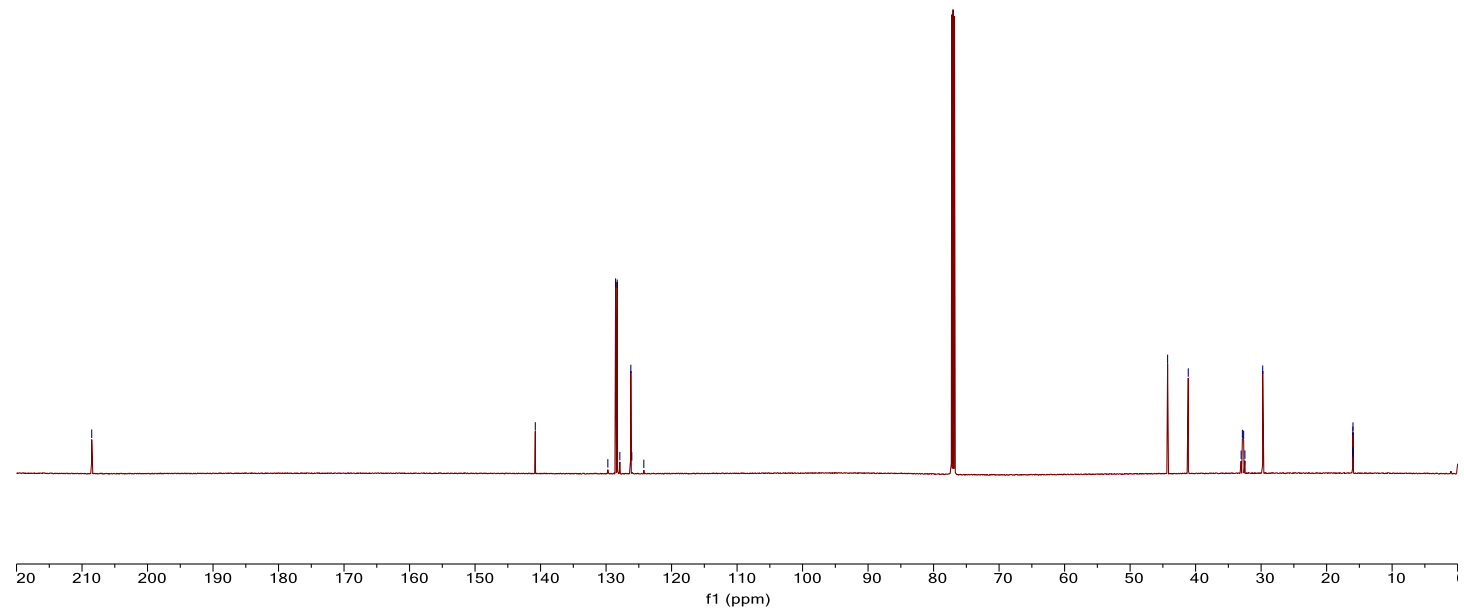

${ }^{19} \mathrm{~F}$ NMR $\left(376 \mathrm{MHz}, \mathrm{CDCl}_{3}\right)$

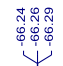<smiles>O=C(CCCC(F)(F)F)Cc1ccccc1</smiles>

33

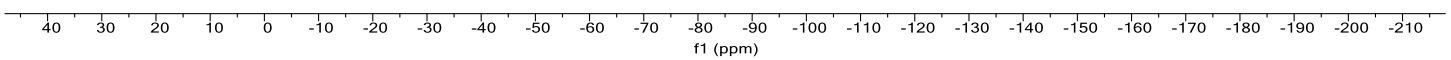


${ }^{1} \mathrm{H}$ NMR (600 MHz, $\left.\mathrm{CDCl}_{3}\right)$

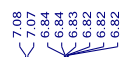

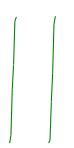

P

34

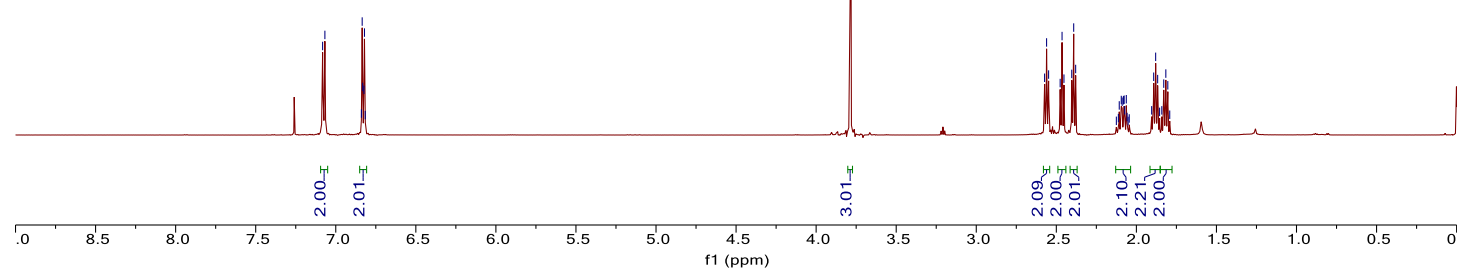

${ }^{13} \mathrm{C}$ NMR $\left(151 \mathrm{MHz}, \mathrm{CDCl}_{3}\right)$

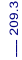<smiles>COc1ccc(CCCC(=O)CCCC(F)(F)F)cc1</smiles>

34

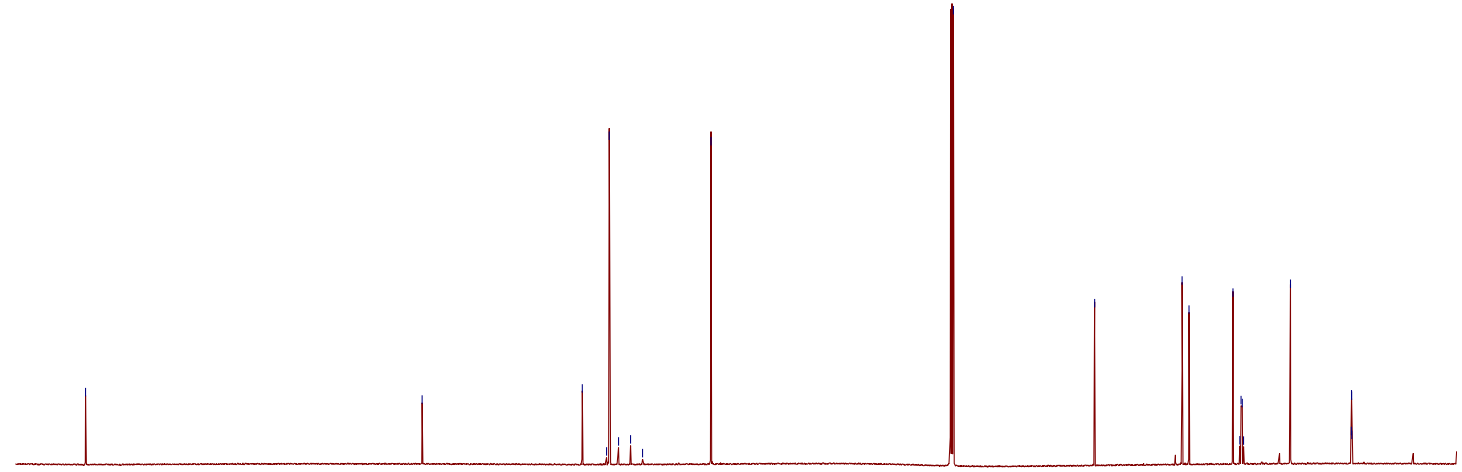

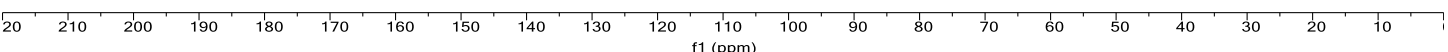


${ }^{19} \mathrm{~F} \mathrm{NMR}\left(376 \mathrm{MHz}, \mathrm{CDCl}_{3}\right)$<smiles>COc1ccc(CCCC(=O)CCCC(F)(F)F)cc1</smiles>

34

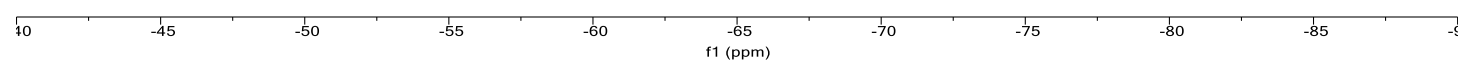

${ }^{1} \mathrm{H}$ NMR $\left(600 \mathrm{MHz}, \mathrm{CDCl}_{3}\right)$

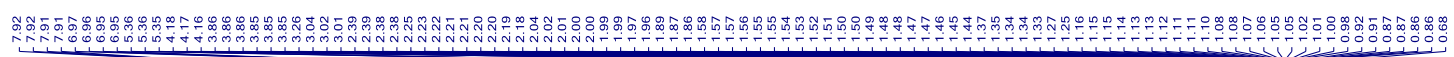

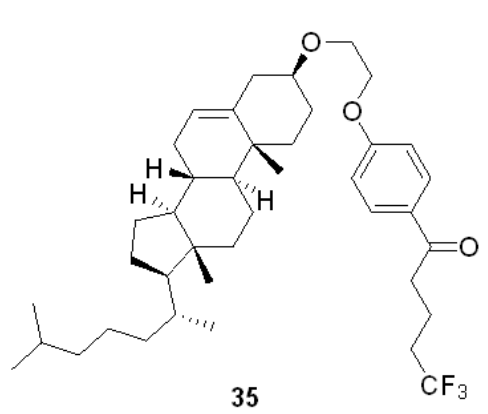

35
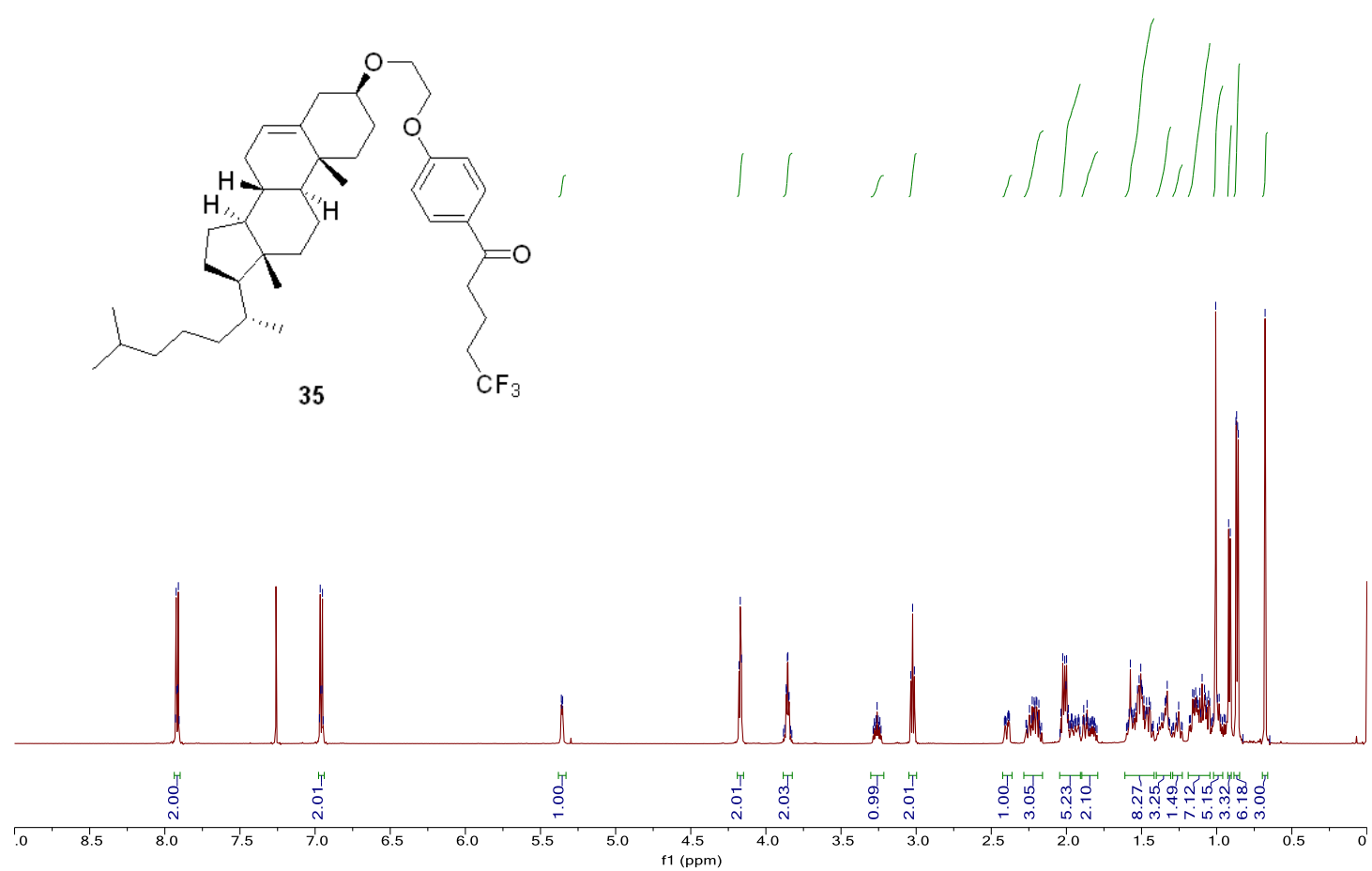
${ }^{13} \mathrm{C} \mathrm{NMR}\left(151 \mathrm{MHz}, \mathrm{CDCl}_{3}\right)$
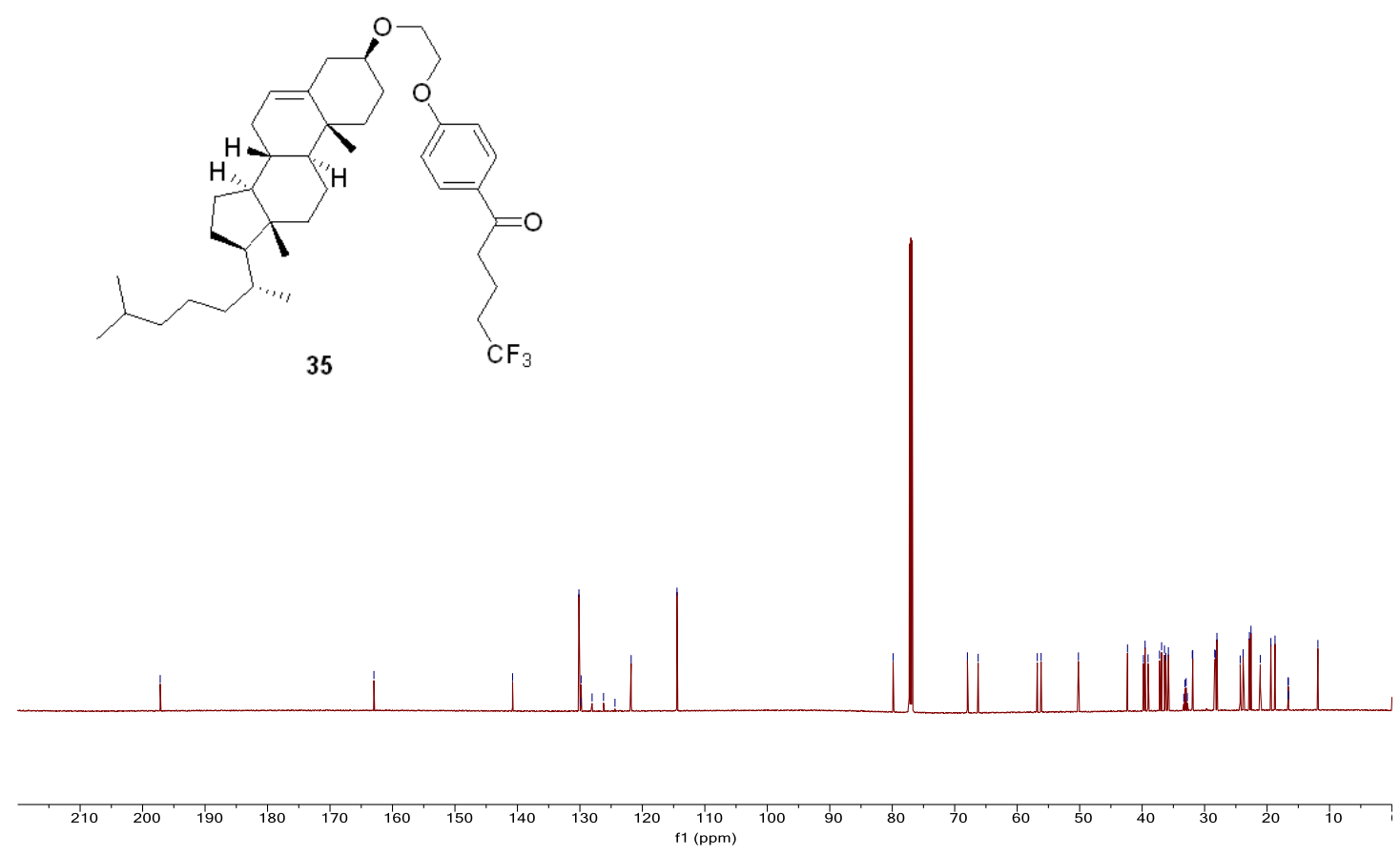

${ }^{19} \mathrm{~F}$ NMR (376 MHz, $\mathrm{CDCl}_{3}$ )

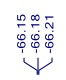

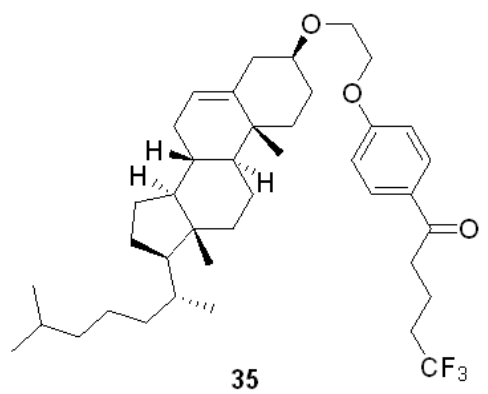

10

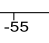

$-60$ -65
$\mathrm{f} 1(\mathrm{ppm})$ $-85$ 
${ }^{1} \mathrm{H}$ NMR (600 MHz, $\left.\mathrm{CDCl}_{3}\right)$

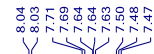

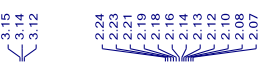

\|\| $\mid$<smiles>O=C(CCCC(F)(F)F)c1ccc(-c2ccccc2)cc1</smiles>

|

36

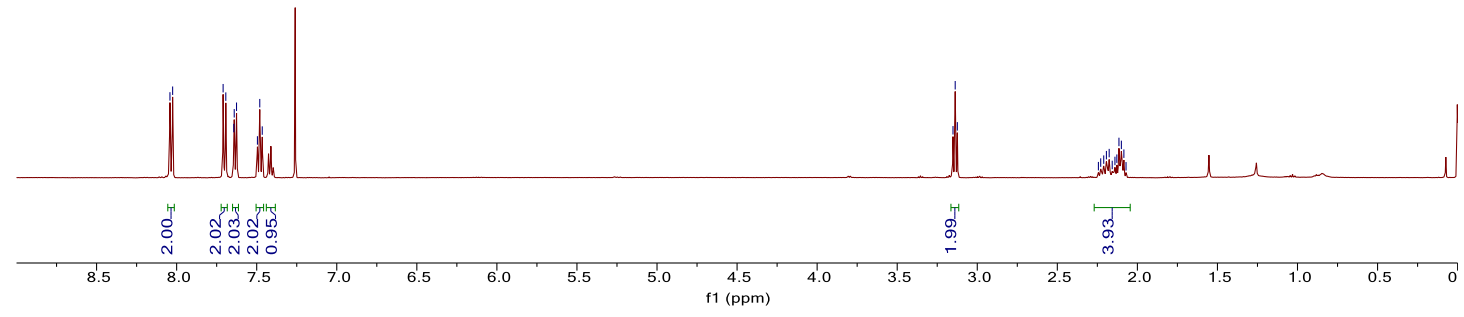

${ }^{13} \mathrm{C} \mathrm{NMR}\left(151 \mathrm{MHz}, \mathrm{CDCl}_{3}\right)$

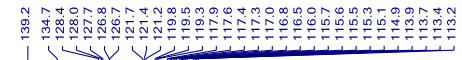
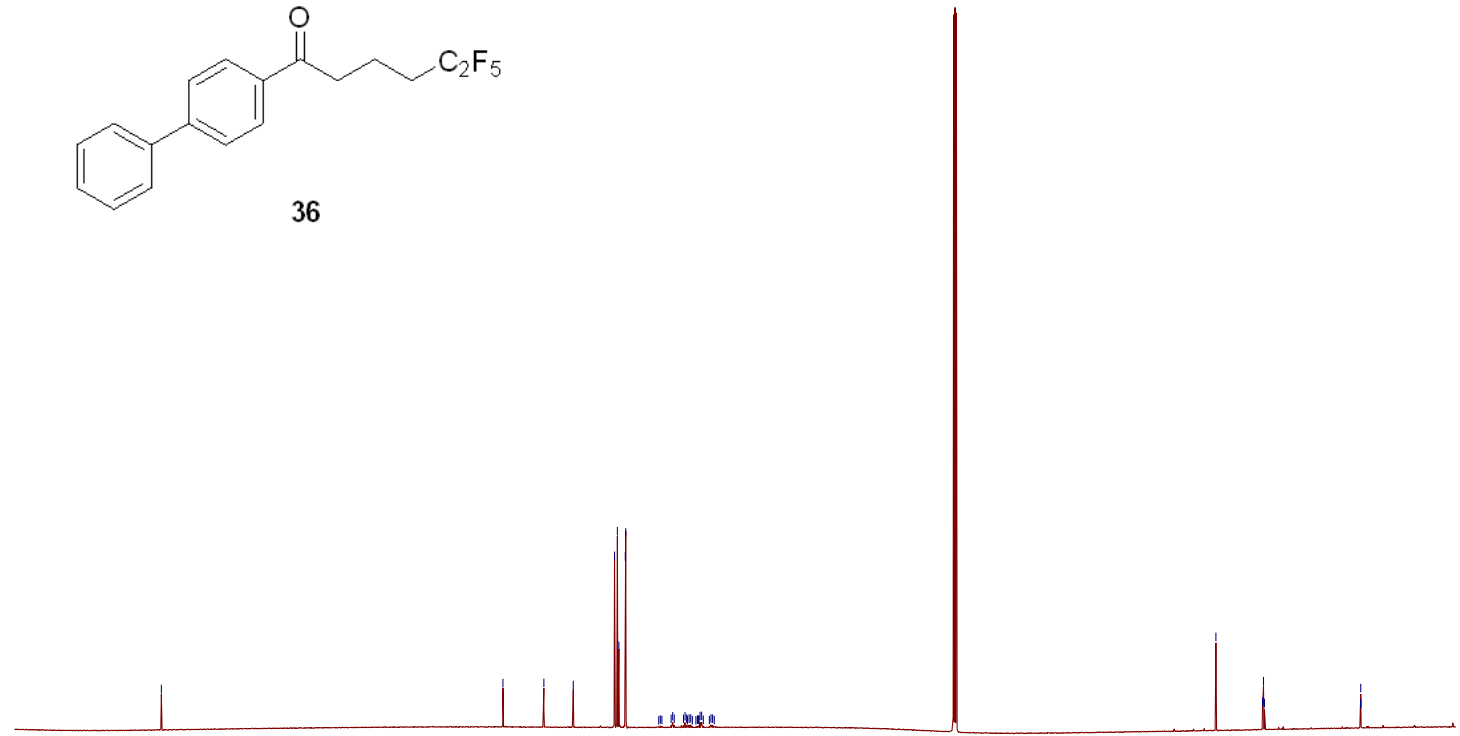

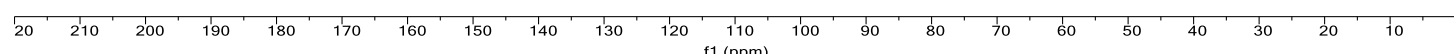


${ }^{19} \mathrm{~F} \mathrm{NMR} \mathrm{(376} \mathrm{MHz,} \mathrm{CDCl}_{3}$ )

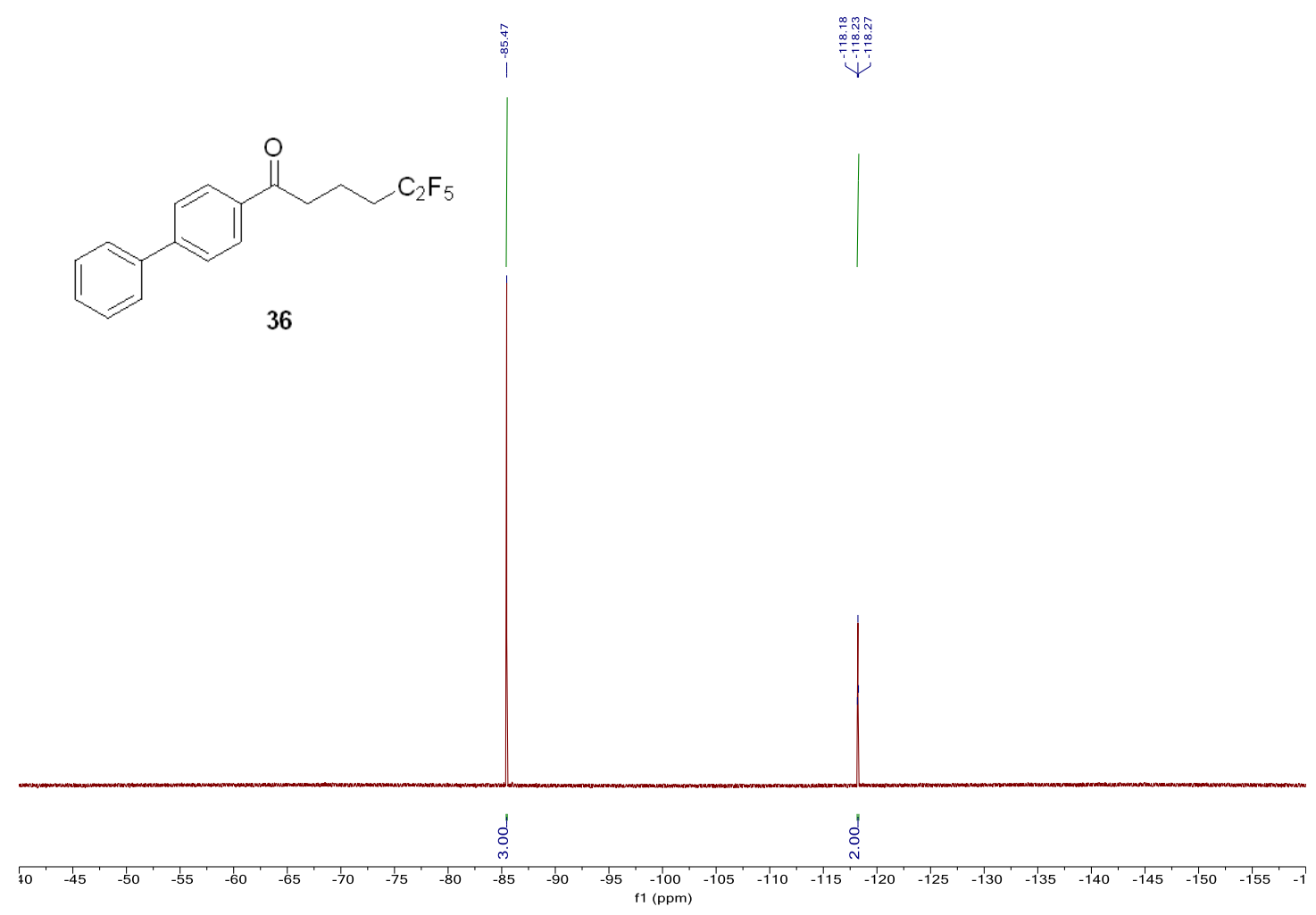

${ }^{1} \mathrm{H}$ NMR (500 MHz, $\left.\mathrm{CDCl}_{3}\right)$

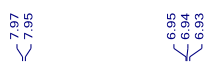

管

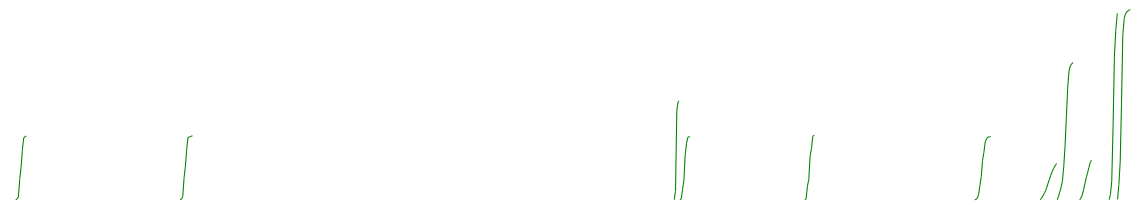<smiles>COc1ccc(C(=O)CCCON2C(C)(C)CCCC2(C)C)cc1</smiles>

37

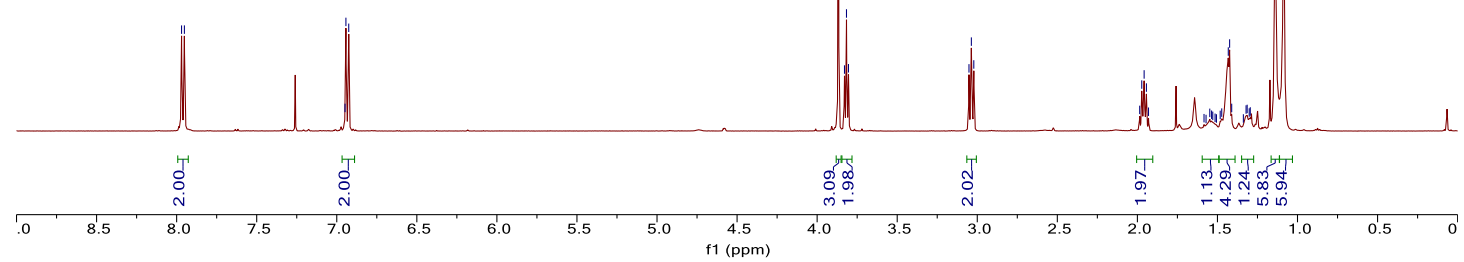


${ }^{13} \mathrm{C} \mathrm{NMR}\left(126 \mathrm{MHz}, \mathrm{CDCl}_{3}\right)$

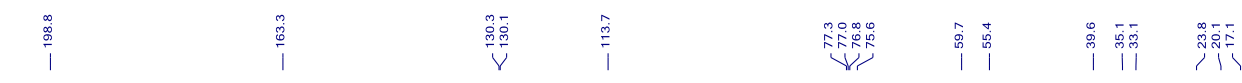<smiles>COc1ccc(C(=O)CCCON2C(C)(C)CCCC2(C)C)cc1</smiles>

37

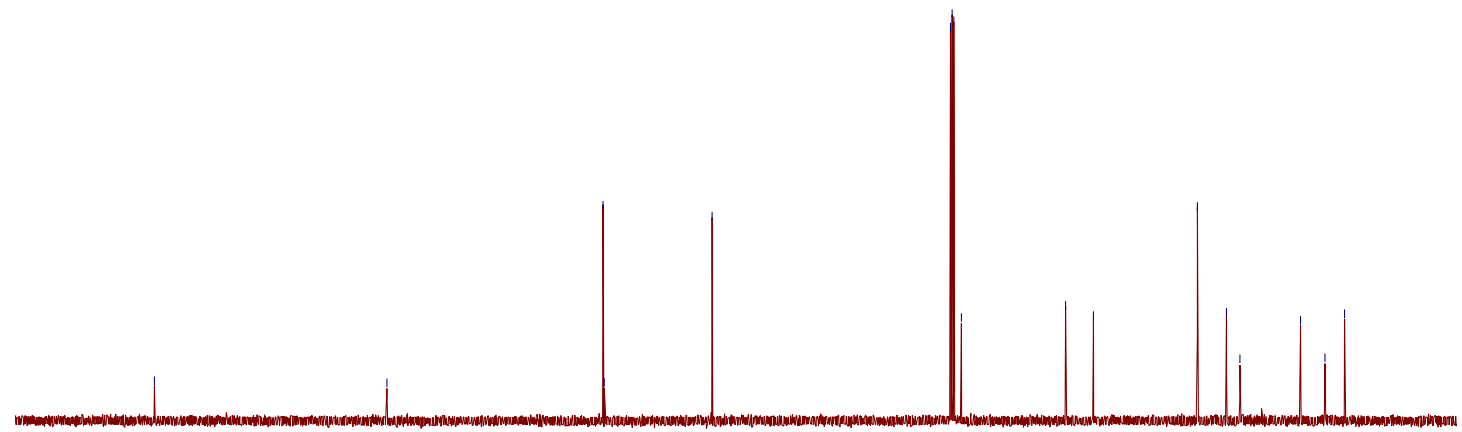

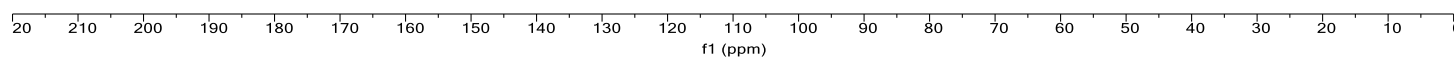

${ }^{1} \mathrm{H}$ NMR (600 MHz, $\left.\mathrm{CDCl}_{3}\right)$

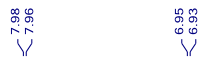

离

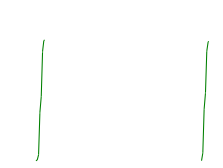<smiles>COc1ccc(C(=O)CCCBr)cc1</smiles>

39

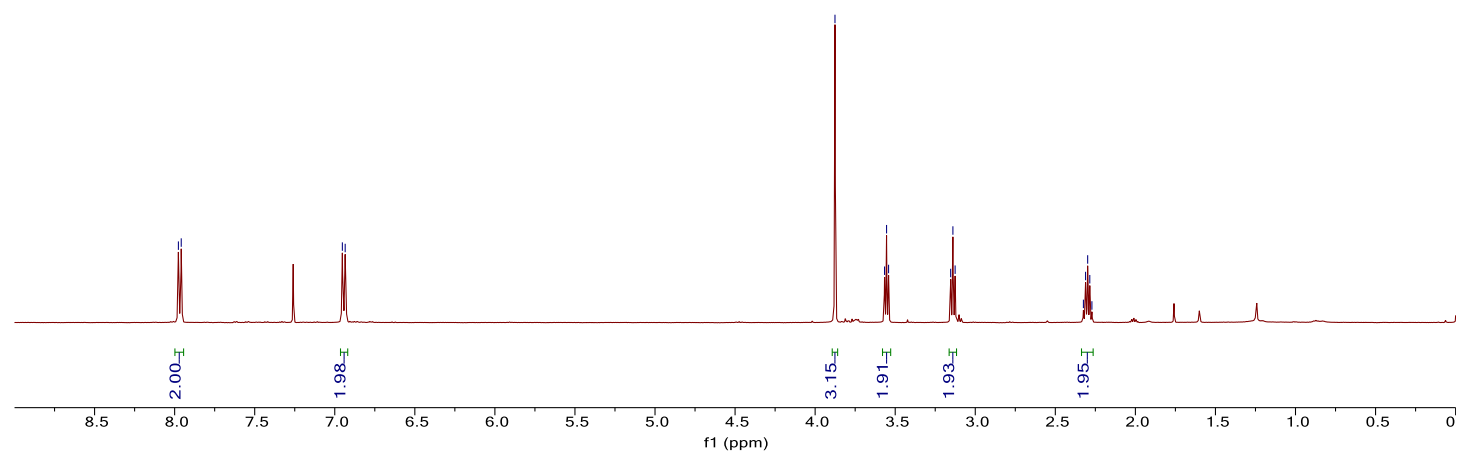


${ }^{13} \mathrm{C} \mathrm{NMR}\left(151 \mathrm{MHz}, \mathrm{CDCl}_{3}\right)$<smiles>COc1ccc(C(=O)CCCBr)cc1</smiles>

39

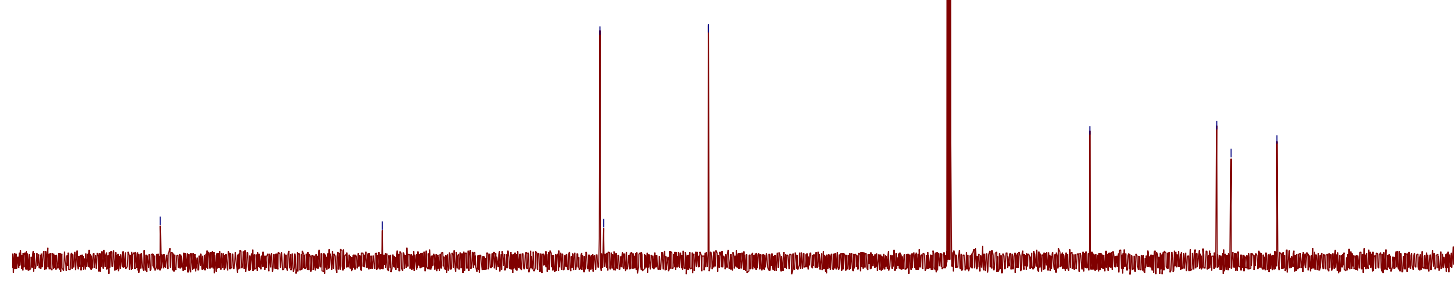

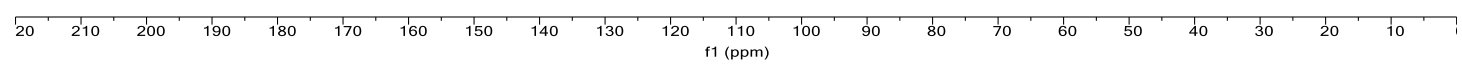

${ }^{1} \mathrm{H}$ NMR (600 MHz, $\left.\mathrm{CDCl}_{3}\right)$

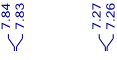

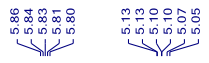

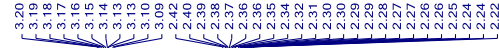
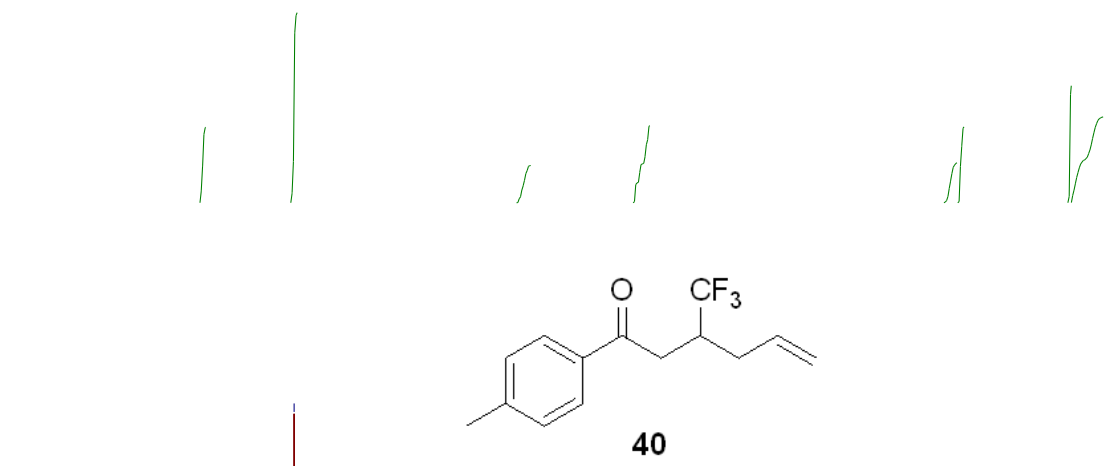

40

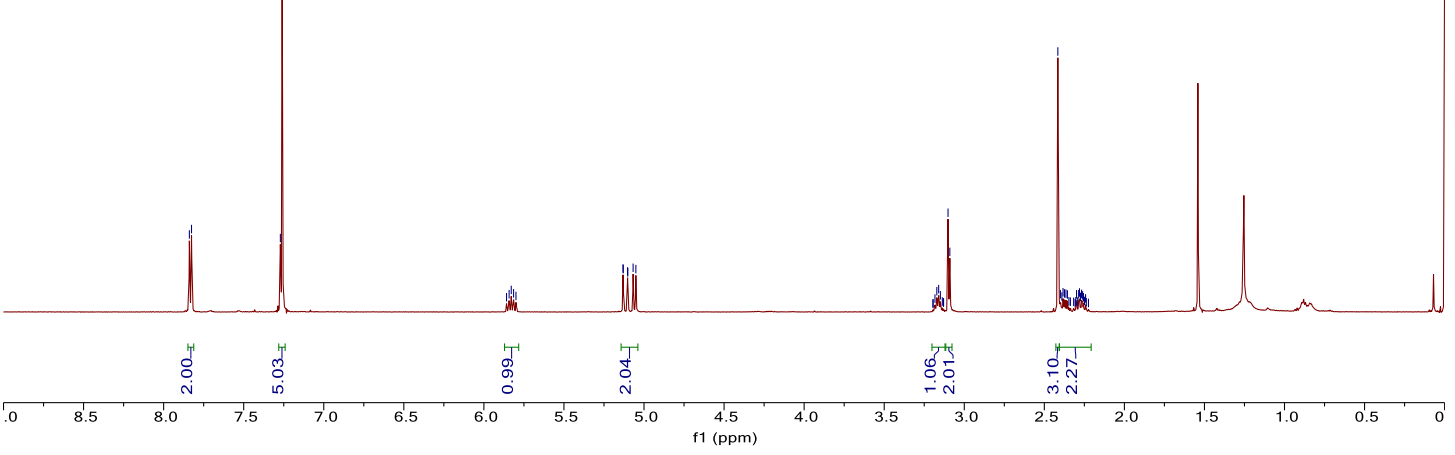


${ }^{13} \mathrm{C} \mathrm{NMR}\left(151 \mathrm{MHz}, \mathrm{CDCl}_{3}\right)$

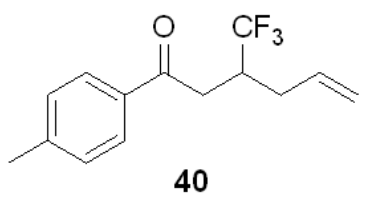

40
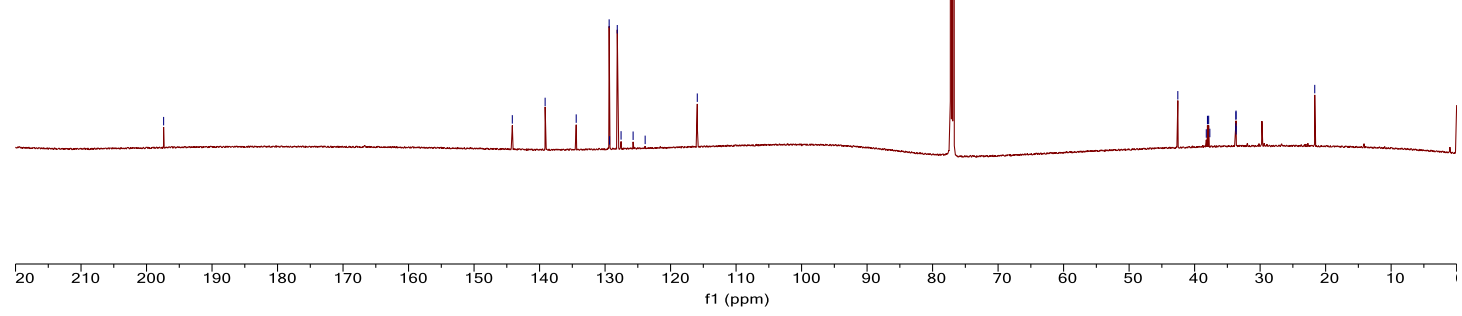

${ }^{19} \mathrm{~F}$ NMR (376 MHz, $\mathrm{CDCl}_{3}$ )
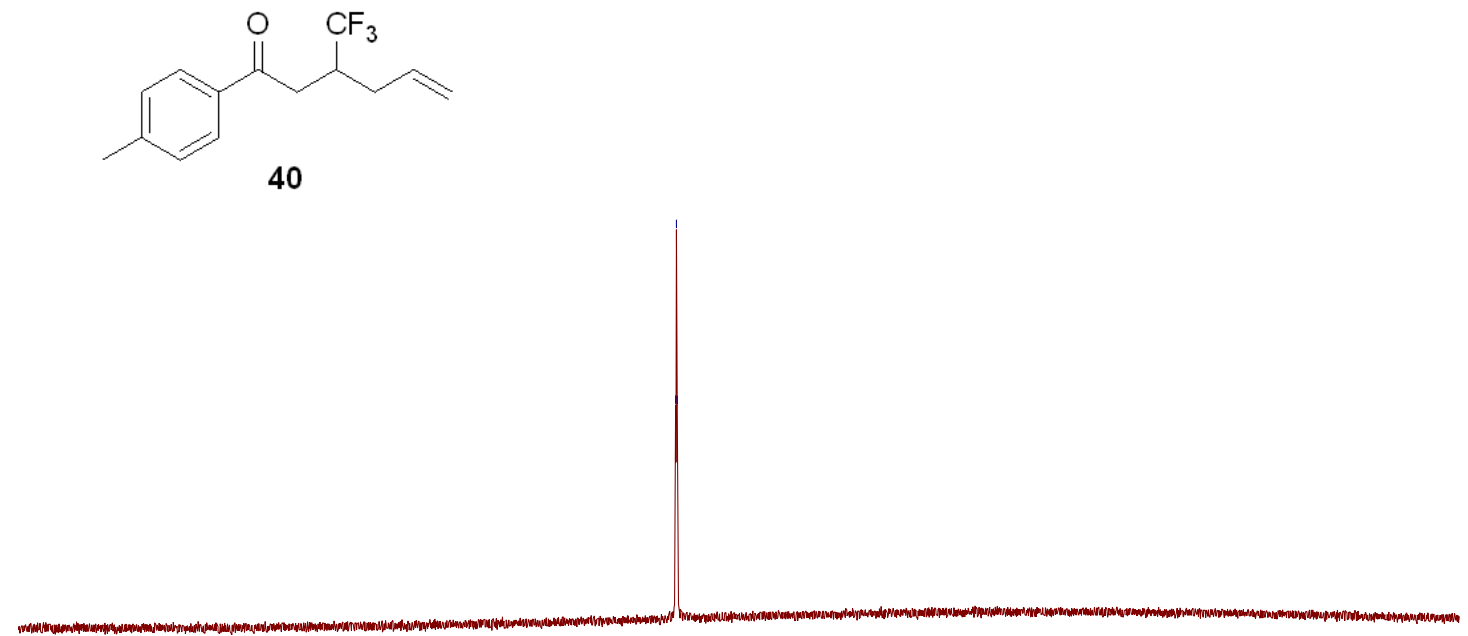

10 $-50$ -65
$\mathrm{f} 1(\mathrm{ppm})$ 
${ }^{1} \mathrm{H} \mathrm{NMR}\left(600 \mathrm{MHz}, \mathrm{CDCl}_{3}\right)$

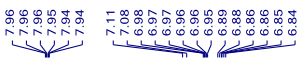

番
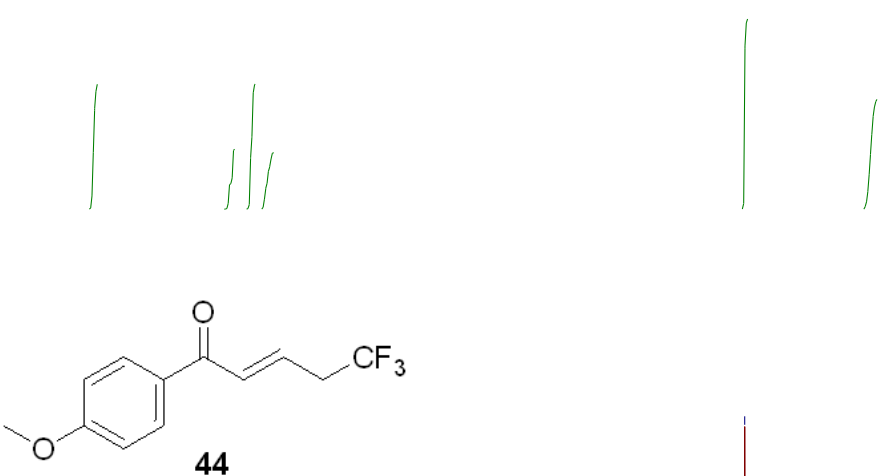

44

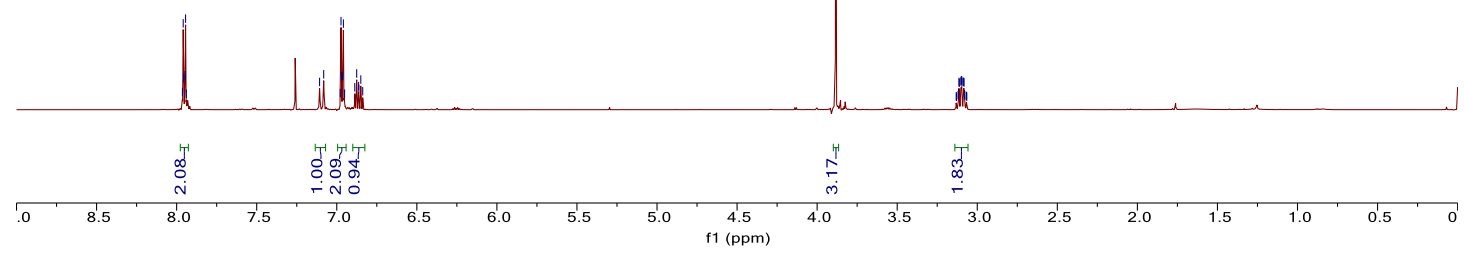

${ }^{13} \mathrm{C} \mathrm{NMR}\left(151 \mathrm{MHz}, \mathrm{CDCl}_{3}\right)$

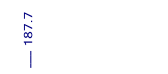

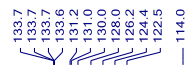

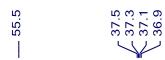

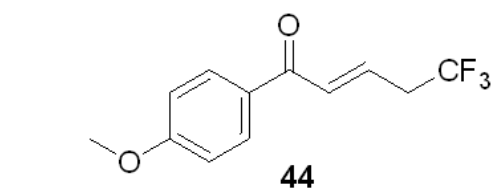

44

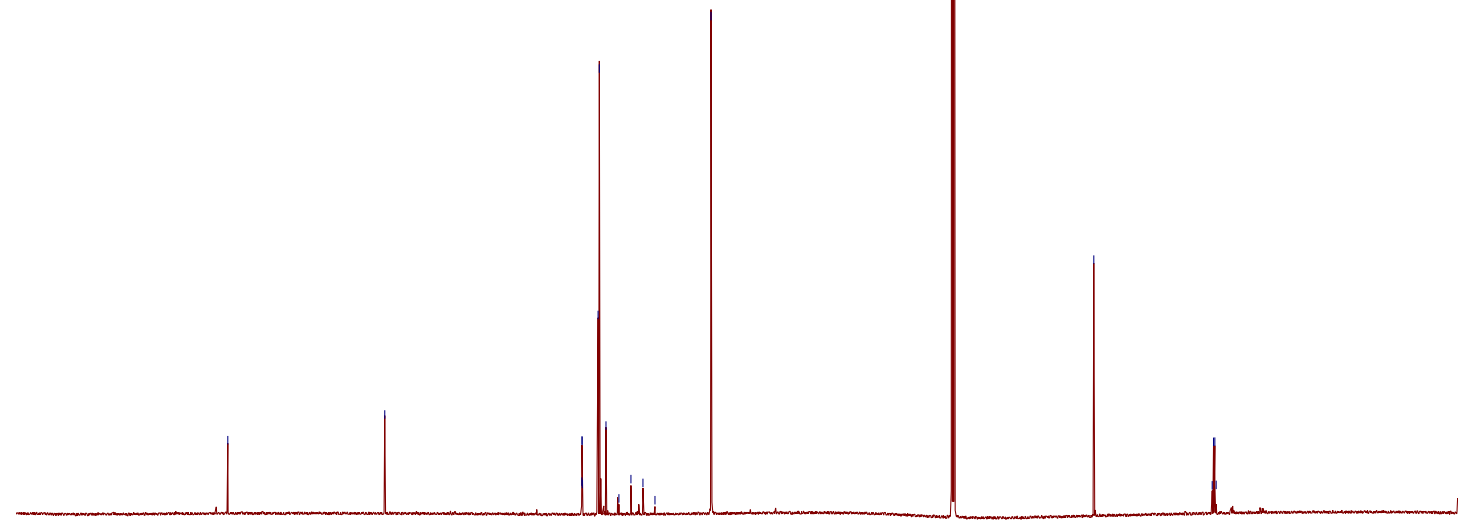

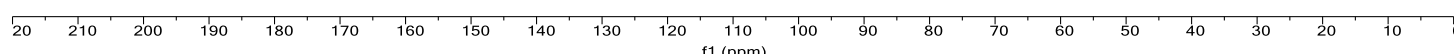


${ }^{19} \mathrm{~F} \mathrm{NMR} \mathrm{(376} \mathrm{MHz,} \mathrm{CDCl}_{3}$ )

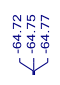<smiles>COc1ccc(C(=O)/C=C/CC(F)(F)F)cc1</smiles>

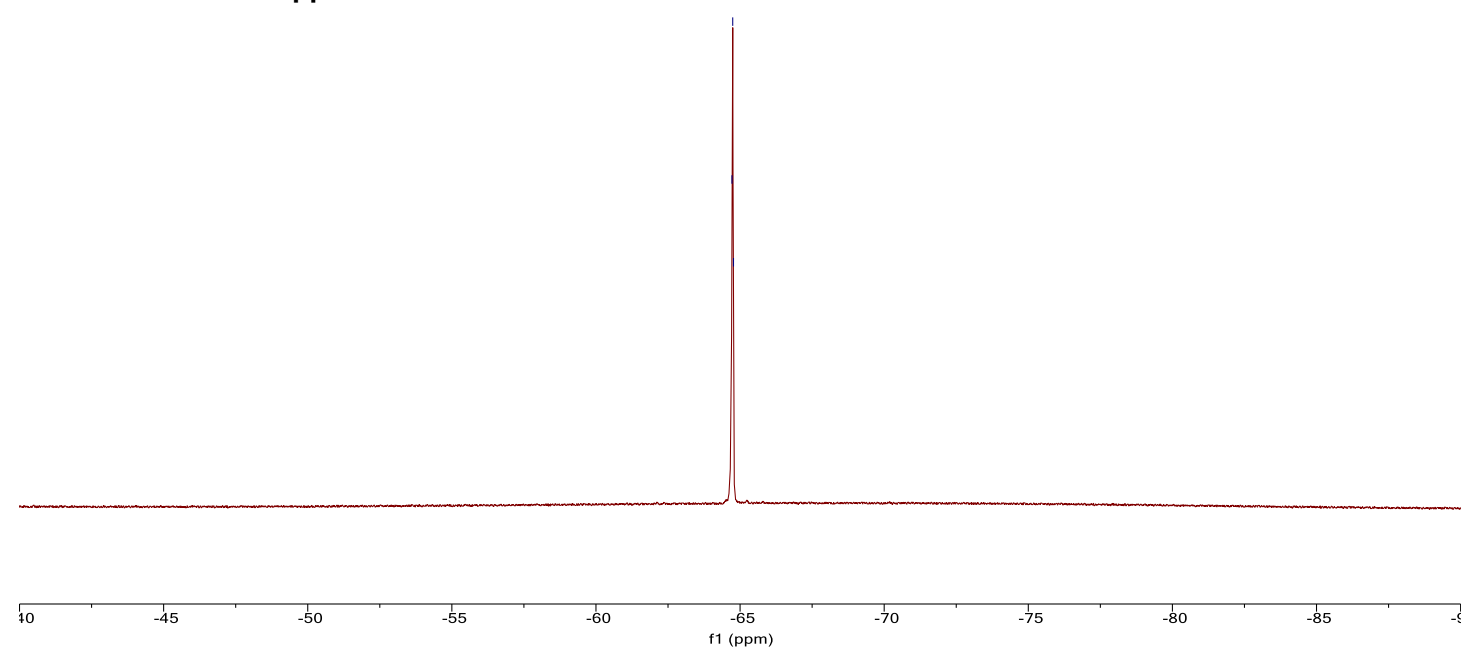

${ }^{1} \mathrm{H}$ NMR (600 MHz, $\left.\mathrm{CDCl}_{3}\right)$

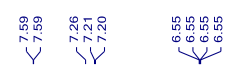

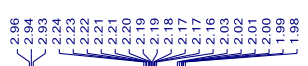

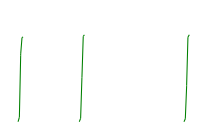<smiles>O=C(CCCC(F)(F)F)c1ccco1</smiles>

45

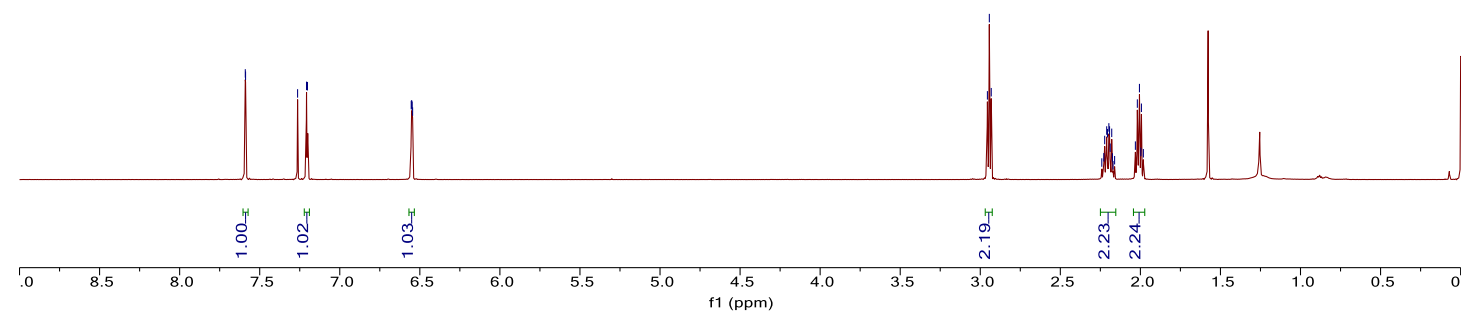


${ }^{13} \mathrm{C} \mathrm{NMR}\left(151 \mathrm{MHz}, \mathrm{CDCl}_{3}\right)$

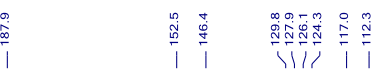

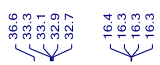<smiles>O=C(CCCC(F)(F)F)c1ccco1</smiles>

45

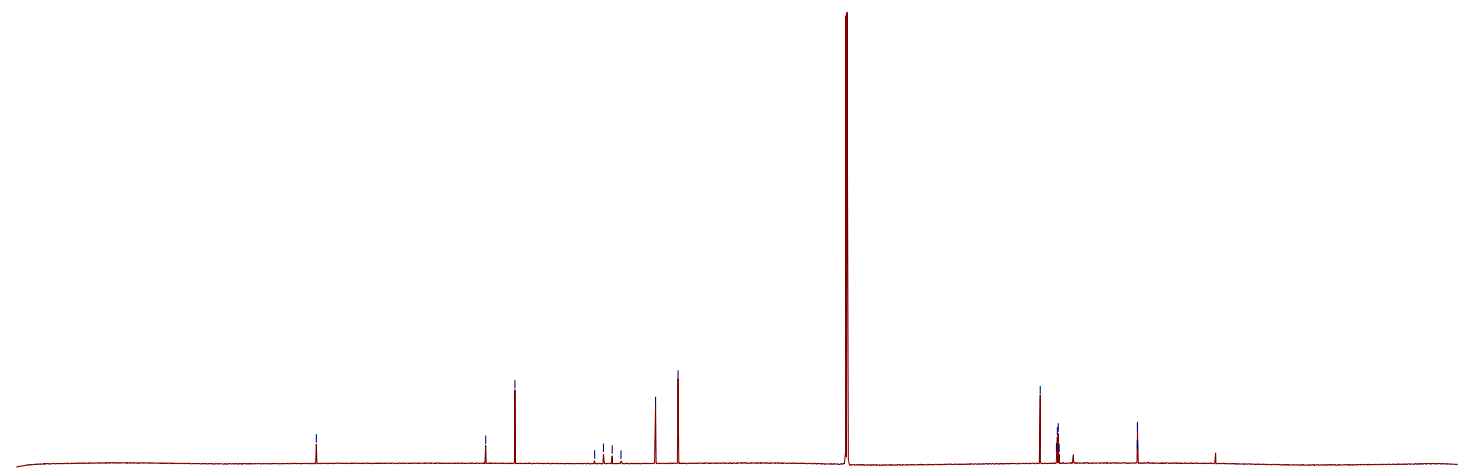

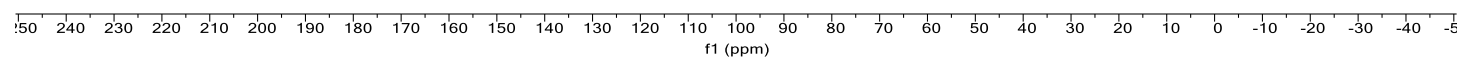

${ }^{19} \mathrm{~F}$ NMR $\left(376 \mathrm{MHz}, \mathrm{CDCl}_{3}\right)$

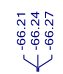<smiles>C[As](C)(F)(F)CCCC(=O)c1ccco1</smiles>

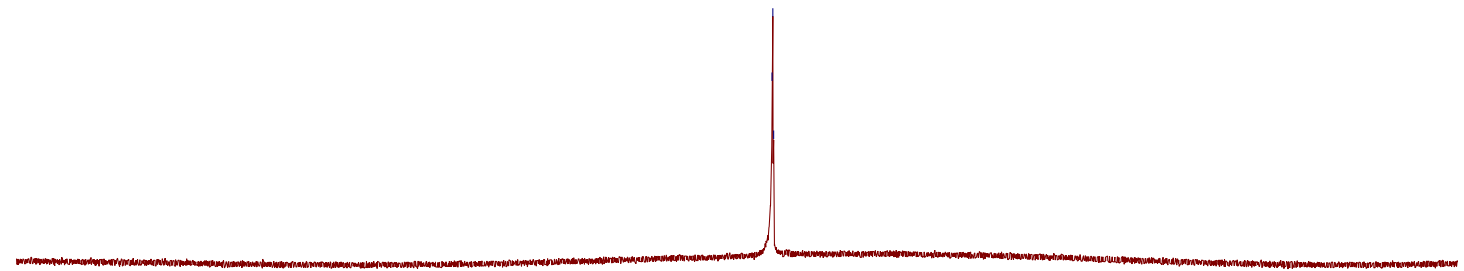




\section{Reference}

1. Wang, X.; Ye, Y.; Zhang, S.; Feng, J.; Xu, Y.; Zhang, Y.; Wang, J., CopperCatalyzed $\mathrm{C}\left(\mathrm{sp}^{3}\right)-\mathrm{C}\left(\mathrm{sp}^{3}\right)$ Bond Formation Using a Hypervalent Iodine Reagent: An Efficient Allylic Trifluoromethylation. J. Am. Chem. Soc. 2011, 133, 16410-16413.

2. Wang, D.; Mao, J.; Zhu, C., Visible light-promoted ring-opening functionalization of unstrained cycloalkanols via inert C-C bond scission. Chem. Sci., 2018, 9, 58055809.

3. Choi, G. J.; Zhu, Q.; Miller, D. C.; Gu, C. J.; Knowles, R. R., Catalytic alkylation of remote C-H bonds enabled by proton-coupled electron transfer. Nature 2016, 539, 268-271.

4. Ward, W. M.; Farnum, B. H.; Siegler, M.; Meyer, G. J., Chloride Ion-Pairing with Ru(II) Polypyridyl Compounds in Dichloromethane. J. Phys. Chem. A 2013, 117, 88838894.

5. Yayla, H. G.; Wang, H.; Tarantino, K. T.; Orbe, H. S.; Knowles, R. R., Catalytic Ring-Opening of Cyclic Alcohols Enabled by PCET Activation of Strong O-H Bonds. J. Am. Chem. Soc. 2016, 138, 10794-10797.

6. Nebra, N.; Grushin, V. V., Distinct Mechanism of Oxidative Trifluoromethylation with a Well-Defined Cu(II) Fluoride Promoter: Hidden Catalysis. J. Am. Chem. Soc. 2014, 136, 16998-17001.

7. Romine, A. M.; Nebra, N.; Konovalov, A. I.; Martin, E.; Benet, J.; Grushin, V. V., Easy Access to the Copper(III) Anion $\left[\mathrm{Cu}\left(\mathrm{CF}^{3}\right)_{4}\right]^{-}$. Angew. Chem., Int. Ed. 2015, 54, 2745-2749.

8. Wang, J.; Huang, B.; Shi, C.; Yang, C.; Xia, W., Visible-Light-Mediated RingOpening Strategy for the Regiospecific Allylation/Formylation of Cycloalkanols. $J$. Org. Chem. 2018, 83, 9696-9706.

9. Creary, X.; Hinckley, J.; Kraft, C.; Genereux, M., Photochemical Behavior of Cyclopropyl-Substituted Benzophenones and Valerophenones. J. Org. Chem. 2011, 76, 2062-2071.

10. Ren, R.; Zhao, H.; Huan, L.; Zhu, C., Manganese-Catalyzed Oxidative Azidation of Cyclobutanols: Regiospecific Synthesis of Alkyl Azides by C-C Bond Cleavage. Angew. Chem., Int. Ed. 2015, 54, 12692-12696. 
11. Jia, K.; Zhang, F.; Huang, H.; Chen, Y., Visible-Light-Induced Alkoxyl Radical Generation Enables Selective $\mathrm{C}\left(\mathrm{sp}^{3}\right)-\mathrm{C}\left(\mathrm{sp}^{3}\right)$ Bond Cleavage and Functionalizations. $J$. Am. Chem. Soc. 2016, 138, 1514-1517.

12. Natho, P.; Rouse, A. B.; Greenfield, J. L.; Allen, L. A. T.; White, A. J. P.; Yang, Z.; Parsons, P. J., Regioselective synthesis of 1- and 4-tetralones from heteroaryl-3cyclobutanols. Tetrahedron 2020, 76, 131636.

13. Fang, J.; Li, L.; Yang, C.; Chen, J.; Deng, G. J.; Gong, H., Tandem Oxidative RingOpening/Cyclization Reaction in Seconds in Open Atmosphere for the Synthesis of 1Tetralones in Water-Acetonitrile. Org. Lett. 2018, 20, 7308-7311.

14. Donald G. Farnum; Robert E. Botto; William T. Chambers; Lam, B., Structural Changes in 2-Arylnorbornyl and Other Aryl Carbocations with Increasing Electron Demand. ${ }^{13}$ C Nuclear Magnetic Resonance as a Probe. J. Am. Chem. Soc. 1978, 100, 3847-3855.

15. Song, L.; Zhu, L.; Zhang, Z.; Ye, J. H.; Yan, S. S.; Han, J. L.; Yin, Z. B.; Lan, Y.; $\mathrm{Yu}$, D. G., Catalytic Lactonization of Unactivated Aryl C-H Bonds with $\mathrm{CO}_{2}$ : Experimental and Computational Investigation. Org. Lett. 2018, 20, 3776-3779.

16. Huang, L.; Ji, T.; Rueping, M., Remote Nickel-Catalyzed Cross-Coupling Arylation via Proton-Coupled Electron Transfer-Enabled C-C Bond Cleavage. J. Am. Chem. Soc. 2020, 142, 3532-3539.

17. Canonne, P.; Bernatchez, M., A Highly Stereoselective Synthesis of Trans-1,2Disubstituted Cycloalkanols. J. Org. Chem. 1987, 52, 4025-4031.

18. Croft, R. A.; Dubois, M. A. J.; Boddy, A. J.; Denis, C.; Lazaridou, A.; VoisinChiret, A. S.; Bureau, R.; Choi, C.; Mousseau, J. J.; Bull, J. A., Catalytic Friedel-Crafts Reactions on Saturated Heterocycles and Small Rings for $\mathrm{sp}^{3}-\mathrm{sp}^{2}$ Coupling of Medicinally Relevant Fragments. Eur. J. Org. Chem. 2019, 5385-5395.

19. Huang, H. M.; Bellotti, P.; Pfluger, P. M.; Schwarz, J. L.; Heidrich, B.; Glorius, F., Three-Component, Interrupted Radical Heck/Allylic Substitution Cascade Involving Unactivated Alkyl Bromides. J. Am. Chem. Soc. 2020, 142, 10173-10183.

20. Muller, S.; Afraz, M. C.; de Gelder, R.; Ariaans, G. J. A.; Kaptein, B.; Broxterman, Q. B.; Bruggink, A., Design and evaluation of inclusion resolutions, based on readily available host compounds. Eur. J. Org. Chem. 2005, 1082-1096. 
21. Zheng, Y.; Liu, X.; Samoshina, N. M.; Samoshin, V. V.; Franz, A. H.; Guo, X., trans-2-Aminocyclohexanol-based amphiphiles as highly efficient helper lipids for gene delivery by lipoplexes. Biochim. Biophys. Acta 2015, 1848, 3113-3125. 